Graduate Theses, Dissertations, and Problem Reports

2020

Time-Dependent Reliability Framework for Durability Design of FRP Composites

Rajneesh Kumar Bharil

Follow this and additional works at: https://researchrepository.wvu.edu/etd

Part of the Polymer Science Commons, Probability Commons, Risk Analysis Commons, Structural Engineering Commons, and the Structural Materials Commons 


\title{
Time-Dependent Reliability Framework for Durability Design of FRP Composites
}

\author{
Rajneesh K. Bharil \\ Dissertation Submitted to The \\ College of Engineering and Mineral Resources at \\ West Virginia University in \\ Partial fulfillment of the requirements \\ For the degree of
}

\section{Doctor of Philosophy \\ In}

Civil and Environmental Engineering

\author{
Hota GangaRao, Ph.D., Chair \\ Nithi Sivaneri, Ph.D. \\ P. V. Vijay, Ph.D. \\ Ruifeng Liang, Ph.D. \\ Dimitra Pyrialakou, Ph.D.
}

\section{Department of Civil and Environmental Engineering}

\author{
Morgantown, West Virginia
}

May 2020

Keywords: Fiber-Reinforced Polymer, FRP Composites, Design Guidelines, TimeDependent Reliability, Framework, Durability, Aging, Creep, Sustained Stress, Environmental Effects, Accelerated Testing, Knock-Down Factors, Strength-Reduction Factors, Factors of Safety, Life Prediction

\section{Copyright 2020 Rajneesh K. Bharil}




\title{
ABSTRACT \\ Time-Dependent Reliability Framework for Durability Design of FRP Composites
}

\author{
Rajneesh K. Bharil, PE(CA,WA,OR,AK,IA) $\mathrm{SE}^{(\mathrm{CA}, \mathrm{WA})}, \mathrm{PMP}^{(\mathrm{PMI})}$
}

The life-cycle performance, durability, and aging characteristics of Fiber Reinforced Polymer (FRP or Structural Composites) have been of keen interest to the engineers engaged in the FRP design, construction, and manufacturing. Unlike conventional construction materials such as steel and concrete, the design guidelines to account for the aging of FRP are somewhat scattered or not available in an approved or consistent format. Loss of strength over time or aging of any structural material should be of concern to engineers as the in-service lifespan of many engineering structures is expected to exceed 100 years. Use of durability strength-reduction factors or factors of safety (aka knock-down factors) is a common way to account for the anticipated in-situ site conditions during the FRP design phase; however, the considerations for FRP service life is often ignored or smeared into knock-down or safety factors. The individual or combined effect of these factors can be arbitrary and can lead to the system's premature failure (or overdesigns), rendering FRP commercial application unreliable (or cost-prohibitive). Reliability or risk-based approach to the development of strength reduction factors has been successfully applied in modern Load, and Resistance Factor Design codes (e.g., highway bridge design specifications), and an original design framework (i.e., a set of ideas, tools or techniques that forms the basis for filling in the final details) incorporating the time-dependent behavior of FRP composites (e.g., decrease of mechanical strengths with an increase of variability with aging) is proposed.

The research presented herein utilizes available natural and accelerated aged test databases to develop a relationship between the probability of failures (using reliability index and confidence intervals to measure reliability) and the desired service life of FRP members. The proposed framework illustrates how to use time-dependent reliability techniques to account for environmental and physical effects. For environmental effects, developing a direct relationship of reliability index with time-dependent durability works better, and for physical effects, indirect inclusion of probability in projecting the time (or cycles) to failure is more effective. The techniques presented in this research, along with three real-life design examples and a case study 
(i.e., the basis of design), can be readily used by design professionals to ascertain an adequate life cycle performance of FRP while maintaining a consistent component or system-level reliability. The intent is to allow others to refine this knowledge bank and to further the professional FRP design practice in a consistent, rational manner leading to the adaptation of formal codes and specifications. Although the presented data and associated findings primarily refer to pultruded glass fiber reinforced polymers (GFRP) in Vinylester resin, the presented framework can be easily extended to other structural composites.

The report entails thorough documentation of published analytical and experimental formulations for various modes of FRP failures due to the typical aging process (e.g., moisture, temperature, alkalinity, and sustained loading, and a combination thereof) along with an associated sampling of durability strength reduction factors. Critical reviews of deterministic and stochastic methods are conducted, and gaps in the current approach to determining durability factors for FRP systems have been identified. A Basis of Design (BOD) for vinylester/polyester-based GFRP in a submerged marine condition using an accelerated test database with illustrative design examples has also been included for a better understanding of the proposed time-dependent reliabilitydurability concept.

Understanding how an FRP system's reliability changes over its life-span, designers will be able to confidentially choose the most suitable durability strength reduction factors, or factors of safety, that will meet their design's target service life-span without exceeding strength or service limit states. Since absolute safety is not possible, all FRP members must be designed for a specific acceptable risk of failure. The research illustrates a unique set of techniques for determining FRP composites' durability strength reduction factors, or threshold design values, by integrating durability characteristics developed in the laboratory tests with desired service lives and commonly acceptable risks of failure. Due to the limited availability of complete durability datasets, vast applications, varieties of FRP composites, and the enormity of calibration efforts required, this research proposes additional work to determine the final durability recommendations for the general use of FRP composites. However, this unique research forms a rational tool for designing specific FRP composites that are consistent with other modern design codes, takes into account their target service lives (e.g., 10, 50, 100 years), and bridges the gap between traditional deterministic FRP design methods and state of the art risked-based design philosophies. 


\section{ACKNOWLEDGMENTS}

The test data used in this report came from many previous research projects and various FRP manufacturers. This work would not have been possible without the prior work of those dedicated researchers whose work proceeded mine. This dissertation is dedicated to those who came before and allowed me to build upon their successes and failures.

I would like to foremost thank Dr. Hota GangaRao for providing me with the opportunity to conduct this intriguing research and for his overall guidance on FRP composites throughout my doctoral program. My gratitude also goes to many faculty members and colleagues especially: Dr. Dimitra Pyrialakou for guidance on the econometric analysis, Dr. Ruifeng Liang for constant encouragement and assistance, along with Mr. Praveen Majugappi and Ms. Maria Lorenzo, both WVU graduate students at that time for their help in sorting through the vast collection of prior WVU research on the aging of FRP. The prior contribution of many WVU graduate students and researchers in the fields of FRP composites and structural reliability has made this work possible, for which the author is grateful.

Special thanks go to my father-in-law, Dr. Srinivasa Iyer, professor emeritus of the Civil Engineering Department at South Dakota School of Mines and Technology, who always encouraged me throughout this doctoral program and was the first to introduce me to the field of structural composites early on my professional engineering career. I am also thankful for the support received from and hardship endured by my family, especially my wife, Kumari, during the pursuit of my doctorate.

Finally, I acknowledge the continued research funding from the US Army Corps of Engineers and financial support received from the Department of Civil and Environmental Engineer at West Virginia University. 


\section{TABLE OF CONTENTS}

\section{ABSTRACT}

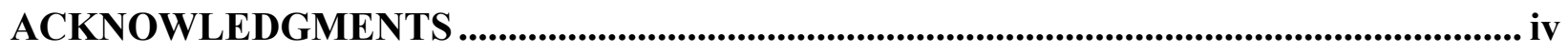

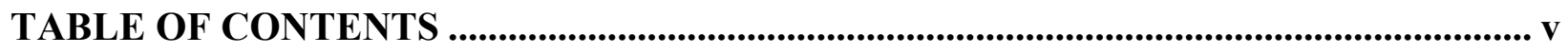

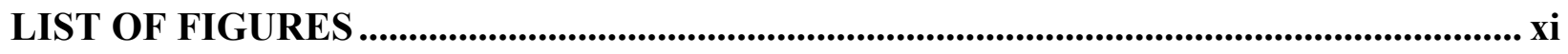

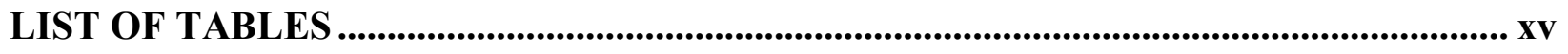

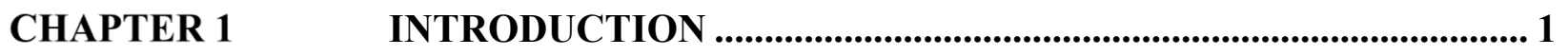

1.1 Background and Need Identification.................................................... 1

1.2 Research Objective and Scope ............................................................... 3

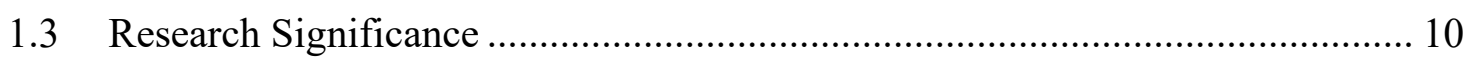

1.4 Limitation of Scope ............................................................................. 12

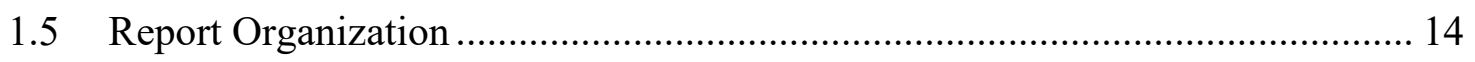

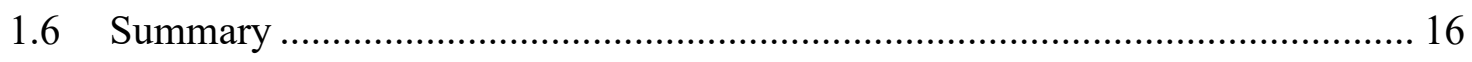

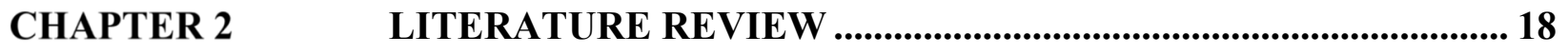

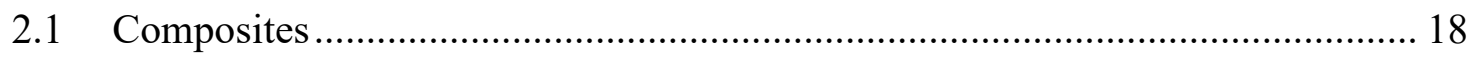

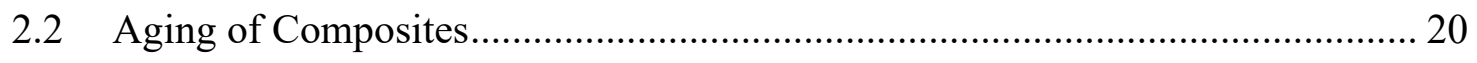

2.2.1 Time-dependent Effects on Durability Characteristics ............................. 22

2.2.2 Effect of Acidity and Alkalinity ........................................................ 28

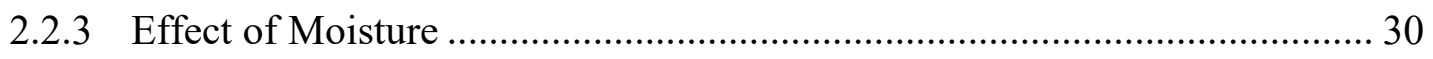

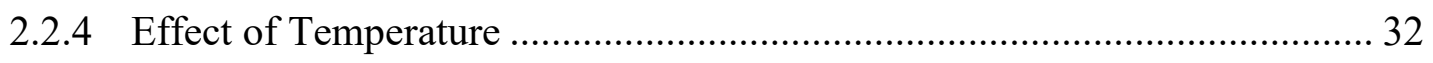




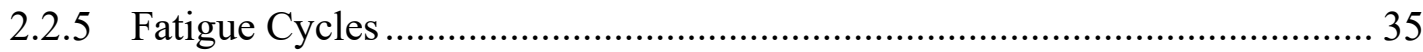

2.2.6 Sustained Stress or Creep .......................................................................... 36

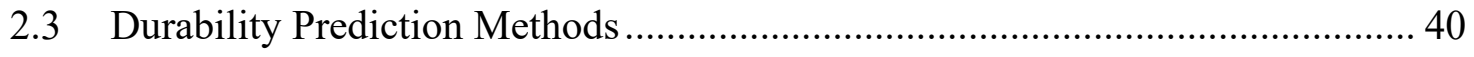

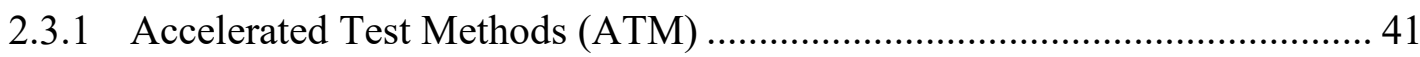

2.3.2 Natural Aging Methods ............................................................................. 41

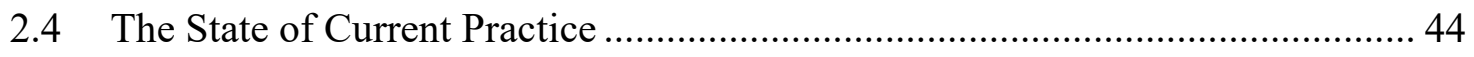

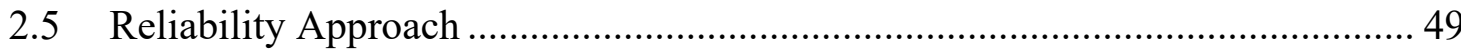

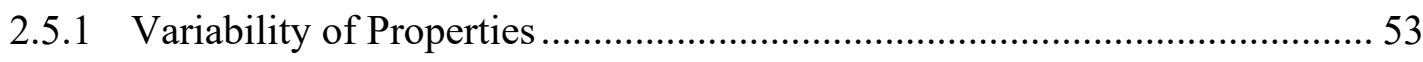

2.5.2 Interpreting Nominal Values or Characteristic Strengths ……………......... 55

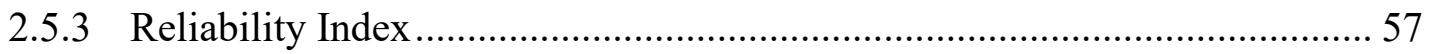

2.5.4 Design Guideline Development ............................................................... 58

2.6 Reliability Approach to Durability Predictions.................................................. 60

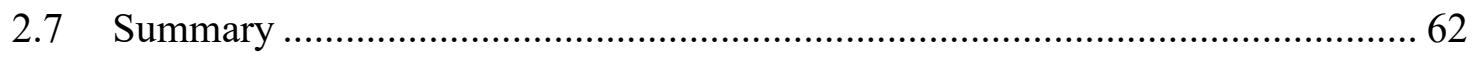

CHAPTER 3 TIME-DEPENDENT RELIABILITY FRAMEWORK FOR

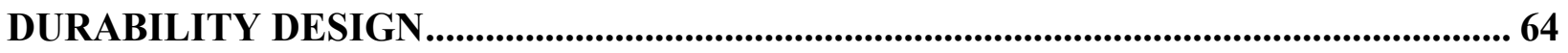

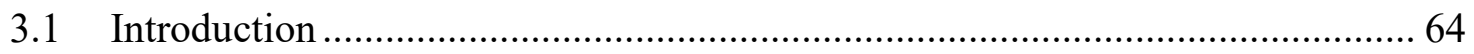

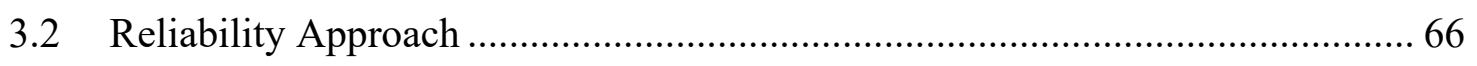

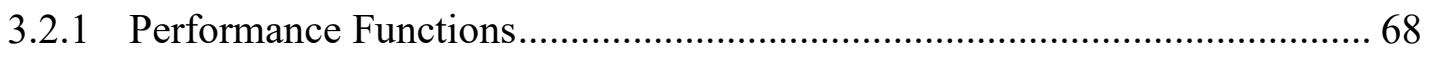

3.2.2 Strength Reduction Factors ..................................................................... 73

3.3 Reliability-Based Durability Framework …………......................................... 79 


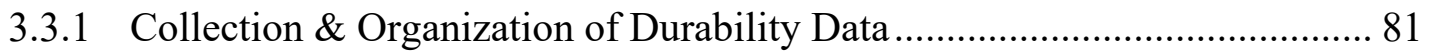

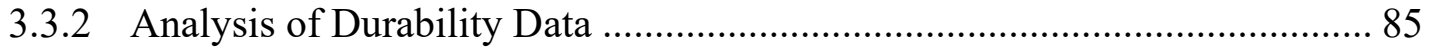

3.3.3 Development of Statistical Parameters .................................................. 91

3.3.4 Selection of Design Examples........................................................... 91

3.3.5 Development of Performance Functions............................................... 92

3.3.6 Selection of Target Reliability and Service Life ..................................... 92

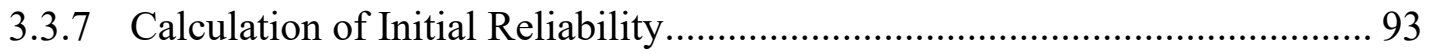

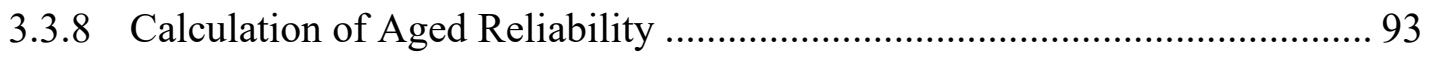

3.3.9 Calculation of Reliability-Based Durability Reduction Factors .................. 94

3.3.10 Calibration of Durability Reduction Factors ........................................... 94

3.3.11 Development of Recommendations or Basis of Design (BOD) ................ 95

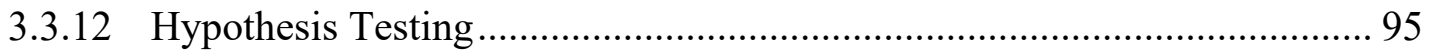

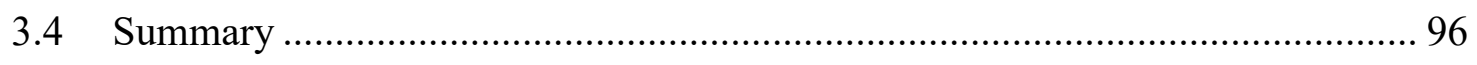

CHAPTER 4 ACCOUNTING FOR ENVIRONMENTAL EFFECTS................. 98

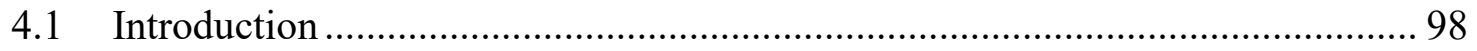

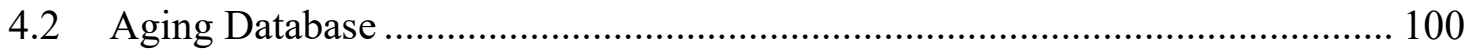

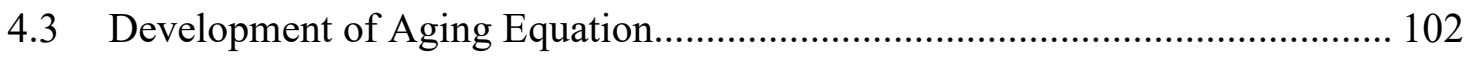

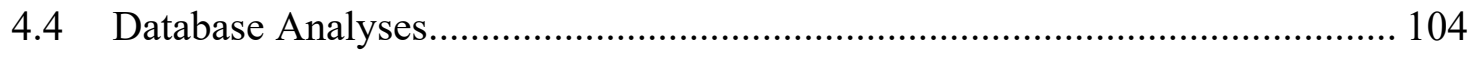

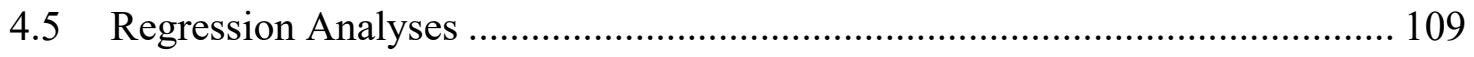

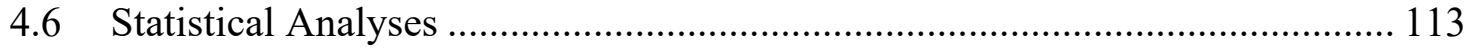




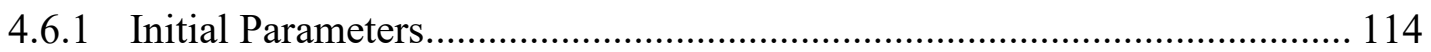

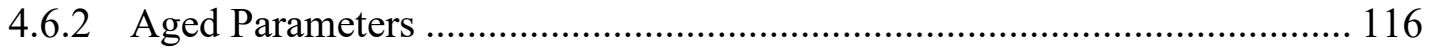

4.7 Time-dependent Reliability Approach ........................................................... 120

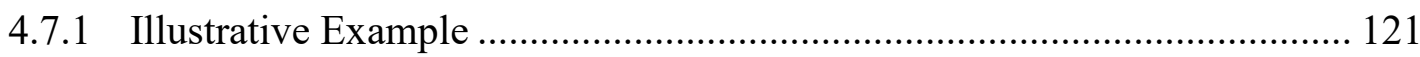

4.7.2 Selection of Target Reliability Index and Service Life............................... 123

4.7.3 Degradation of Reliability Index over Time .............................................. 124

4.7.4 Degradation of Factor of Safety (FOS) over Time ………........................ 126

4.7.5 Relationship of Reliability with Various Factors......................................... 127

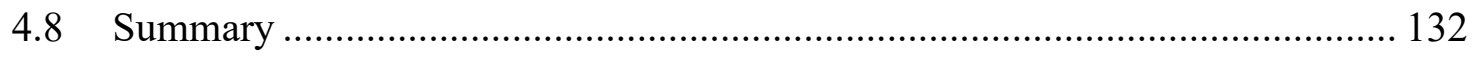

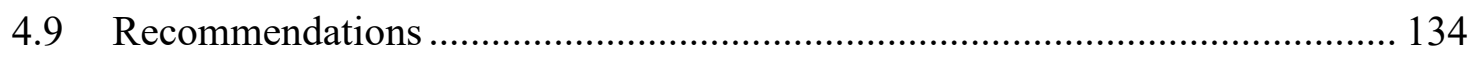

CHAPTER 5 ACCOUNTING FOR PHYSICAL EFFECTS ................................. 135

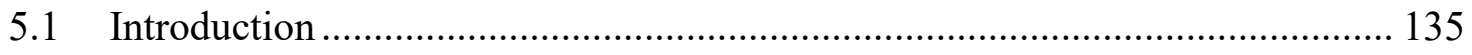

5.2 Importance of Limiting Sustained Stress Ratios ............................................. 138

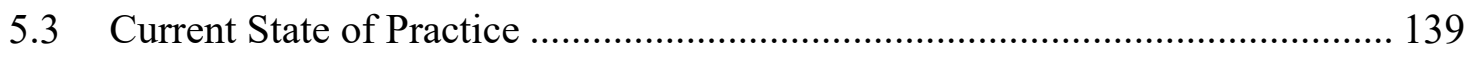

5.4 Development of Time-dependent Model for Sustained Stress........................... 142

5.4.1 Analytical Formulation of Creep............................................................ 142

5.4.2 Application of Findley's Power Law ....................................................... 145

5.4.3 Creep or Sustained Stress Database ......................................................... 150

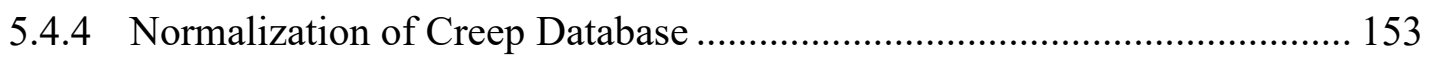

5.4.5 Development of Basic Creep Strain Coefficients ........................................ 159 
5.4.6 Estimation of Lower Bound Age 169

5.4.7 Determination of Rupture Strain 171

5.4.8 Accounting for Creep Deformation. 174

5.4.9 Correction for Nominal Strength 179

5.5 Applicability to Other Physical Effects:. 181

5.6 Summary 182

5.7 Recommendations 183

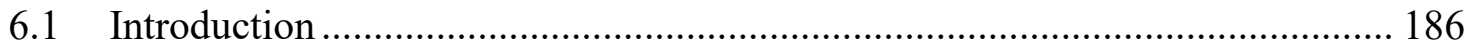

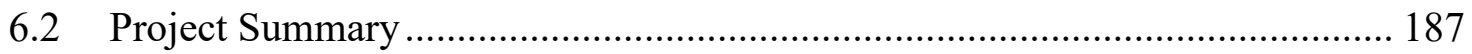

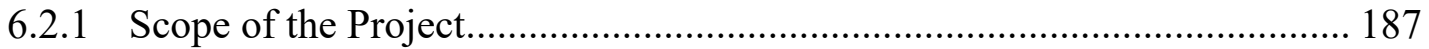

6.2.2 Aging Methods Used......................................................................... 189

6.2.3 Testing of Mechanical Properties.......................................................... 192

6.2.4 Moisture (Mass) Uptake Measurements ............................................... 195

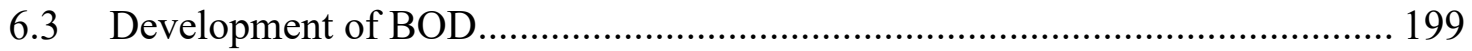

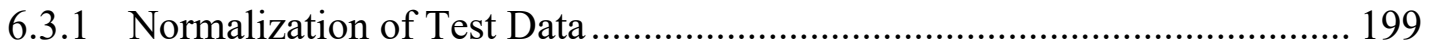

6.3.2 Regression for Degradation Rates................................................... 201

6.3.3 Calculation of Time Shift Factors (TSF)........................................... 203

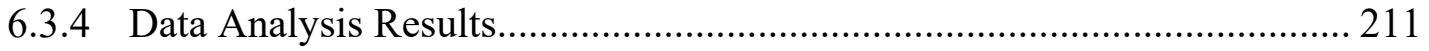

6.3.5 Development of Basis of Design (BOD) .............................................. 220 
6.4 Summary

6.5 Recommendations

235

CHAPTER 7

SUMMARY AND CONCLUSION 236

CHAPTER 8

RECOMMENDATIONS

243

REFERENCES. 246

APPENDICES 254

APPENDIX-A DESIGN EXAMPLES 258

A.1 Example 1 - Outdoor Vinylester GFRP Beam 258

A.2 Example 2 - Design of a Subsea Polyester GFRP Tension Bracing 267

A.3 Example 3 - Design of a Subsea Vinylester GFRP Beam 275

APPENDIX-B

FRP DURABILITY DATABASE. 283

APPENDIX-C

SUBSEA FRP DATABASE 299

APPENDIX-D

SUSTAINED STRESS DATABASE 319

APPENDIX-E

DURABILITY TEST DATABASE

331 


\section{LIST OF FIGURES}

Figure 1.1: Flowchart of Time-dependent Reliability-Durability Research .............................. 7

Figure 2.1: Typical "Bathtub” Curve of Mechanical Systems .............................................. 21

Figure 2.2: Durability Effects Involved in Natural Weathering of FRP Structures................... 23

Figure 2.3: FRP Nominal and Characteristics Values per ASTM D30 …............................... 54

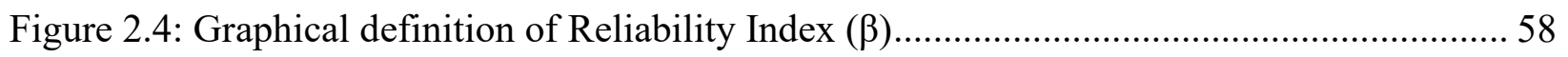

Figure 3.1: Illustration of Proposed "Reliability-Based Service Life" Curve ............................ 66

Figure 3.2: Probability of Failure with Load and Resistance Functions.................................. 70

Figure 3.3: Definition of Reliability Index with Performance Function ................................ 71

Figure 3.4: Flowchart of Reliability-Based Durability Framework........................................ 80

Figure 3.5: Ave. Strength Retention (SR) from Accelerated Testing Database ........................ 84

Figure 3.6: Fast Converging Steps used for Data Analysis ............................................... 88

Figure 4.1: Application of Environmental Effects on Outdoor Structures .............................. 98

Figure 4.2: Predicted Normalized Deterioration Curves for Various SRs.............................. 112

Figure 4.3: Predicted Normalized Increase in COV for Various SRs .................................. 113

Figure 4.4: Typical Degradation of PDF with Aging of FRP ........................................... 117

Figure 4.5: Typical Degradation of SR Mean Value and COV with Age .............................. 119

Figure 4.6: Schematic Loading Diagram of Example FRP Beam...................................... 121

Figure 4.7: The Probability of Failure and Reliability Index........................................... 123

Figure 4.8: Degradation of RelibilityIndex $(\beta)$ over Service Life ....................................... 126

Figure 4.9: Deterioration of Factor of Safety (FOS) over Service Life................................. 127

Figure 4.10: Variation of Reliability Index with Age for various Phi Factors ........................ 128

Figure 4.11: Variation of Reliability Indices with Phi Factors for Various Ages ................... 129 
Figure 4.12: Variation of Reliability Indices with Ages for various FOS 130

Figure 4.13: Variation of Reliability Indices with FOS for various Ages 131

Figure 4.14: Variation of Phi Factors with Ages for Various Target Reliability Indices 132

Figure 5.1: Physical Effects on Outdoor FRP Structures 138

Figure 5.2: Typical Time-Dependent Behavior of Strain due to Creep. 144

Figure 5.3: Comparison of Observed Test values Vs. Findley's Prediction Model ..... 150

Figure 5.4: Modelling of Experimental Data using Findley's Law..... 159

Figure 5.5: Logarithmic Transformation of Findley's Law..... 160

Figure 5.6: Total Sustained Strain Ratio (wrt $\varepsilon_{0}$ ) leading to Creep Rupture 165

Figure 5.7: Determination of Service Life for Various Initial Strain Ratios 168

Figure 5.8: Computation of Service life for an FRP Sample 170

Figure 5.9: Explanation of the Probability of Exceedance 171

Figure 5.10: Lower Bound Prediction of Service Life at Various Rupture Strengths..... 173

Figure 5.11: Average \% Strain Increase at Various Service Lives (Years). 176

Figure 6.1: Aged FRP Beams (Left); Wellhead Protection System (Right) 187

Figure 6.2: Beam Sample Extraction Locations and Orientation 194

Figure 6.3: Aging Environments and Degradation Coefficients for Subsea Project. 205

Figure 6.4: Histogram and P-P plot of Distribution of SR Residuals. 215

Figure 6.5: Vinylester Tensile Transverse SR data in Laboratory 219

Figure 6.6: Projected Vinylester Tensile Transverse SR data in Seawater. 220

Figure 6.7: Reliability Index and Strength Reduction Factors (Examples 2 and 3) 232

Figure 6.8: Reliability Index and Factor of Safety (Examples 2 and 3) 233

Figure 7.1: Concept of Time-Dependent Reliability \& Durability..... 236 
Figure B.1: Tested ATM Tensile Strengths of Vinylester GFRP …………………………....... 285

Figure B.2: Projected Normalized Tensile Strengths of Vinylester GFRP ………………........ 286

Figure B.3: Tested ATM Flexural Strengths of Vinylester GFRP ………………………….... 287

Figure B.4: Projected Normalized Flexural Strengths of Vinylester GFRP ............................... 288

Figure B.5: Tested ATM Shear Strengths of Vinylester GFRP............................................... 289

Figure B.6: Projected Normalized Shear Strengths of Vinylester GFRP ................................... 290

Figure B.7: Tested ATM Tensile Strengths of Polyester GFRP................................................ 291

Figure B.8: Projected Normalized Tensile Strengths of Polyester GFRP ................................. 292

Figure B.9: Tested ATM Flexural Strengths of Polyester GFRP .............................................. 293

Figure B.10: Projected Normalized Flexural Strengths of Polyester GFRP ............................... 294

Figure B.11: Tested ATM Shear Strengths of Polyester GFRP ……....................................... 295

Figure B.12: Projected Normalized Shear Strengths of Polyester GFRP .................................. 296

Figure B.13: Tested ATM Tensile Strengths of Epoxy GFRP …………………....................... 297

Figure B.14: Projected Normalized Tensile Strengths of Epoxy GFRP ...................................... 298

Figure C.1: Tested Tensile-Transverse Strengths of Vinylester GFRP ....................................... 301

Figure C.2: Projected Tensile-Transverse Strengths of Vinylester GFRP ................................ 302

Figure C.3: Tested Tensile-Longitudinal Strengths of Vinylester GFRP .................................... 303

Figure C.4: Projected Tensile-Longitudinal Strengths of Vinylester GFRP ................................ 304

Figure C.5: Projected Flexural-Transverse Strengths of Vinylester GFRP ................................ 305

Figure C.6: Projected Flexural-Longitudinal Strengths of Vinylester GFRP .............................. 306

Figure C.7: Projected Shear-Transverse Strengths of Vinylester GFRP ..................................... 307

Figure C.8: Projected Shear-Longitudinal Strengths of Vinylester GFRP ................................ 308

Figure C.9: Projected Tensile Modulus-Transverse Strengths of Vinylester GFRP .................. 309 
Figure C.10: Projected Tensile Modulus-Longitudinal Strengths of Vinylester GFRP

Figure C.11: Projected Tensile-Transverse Strengths of Polyester GFRP ................................ 311

Figure C.12: Projected Tensile-Longitudinal Strengths of Polyester GFRP .............................. 312

Figure C.13: Projected Flexural-Transverse Strengths of Polyester GFRP................................ 313

Figure C.14: Projected Flexural-Longitudinal Strengths of Polyester GFRP.............................. 314

Figure C.15: Projected Shear-Transverse Strengths of Polyester GFRP ................................... 315

Figure C.16: Projected Shear-Longitudinal Strengths of Polyester GFRP ……………............. 316

Figure C.17: Projected Tensile Modulus-Transverse Strengths of Polyester GFRP .................. 317

Figure C.18: Projected Tensile Modulus-Longitudinal Strengths of Polyester GFRP ............... 318 


\section{LIST OF TABLES}

Table 2.1: Natural Outdoor Aging Comparisons (Keller et al., 2007, 2016) ........................... 42

Table 2.2: Review of Current Practice of Strength Reduction Factors.................................... 45

Table 2.3: Comparison of the Ranges for Strength Reduction Factors ................................... 48

Table 3.2- Variables and Sources of FRP ATM Database for This Study .............................. 83

Table 4.1- Basic Aging Database Groups......................................................................... 101

Table 4.2- pH Group Combinations.......................................................................... 101

Table 4.3- Temperature Group Combinations ............................................................ 102

Table 4.5- Vinylester GFRP Group Degradation Coefficients in Lab (alab).......................... 106

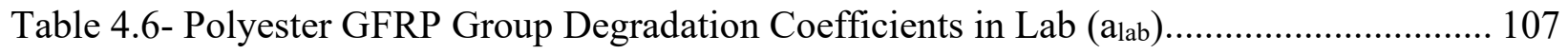

Table 4.7- Epoxy GFRP Group Degradation Coefficients in Lab $\left(\mathrm{a}_{\text {lab }}\right)$................................ 108

Table 4.8- Final Regression Summary of Normalized FRP Database (aref) .......................... 111

Table 4.9: Compilation of FRP Statistical Parameters for Reliability Analysis...................... 115

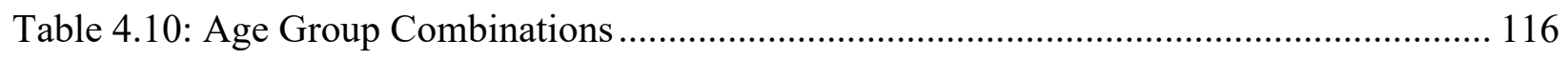

Table 5.1- Current Practices Regarding Sustained Stress Limitations .................................. 140

Table 5.2: Summary of Current Practices Regarding Sustained Stresses............................. 141

Table 5.3: Summary of GFRP Samples used in Creep Study ........................................ 151

Table 5.4: Modified Creep Database of GFRP Samples ................................................. 158

Table 5.5: Creep Rupture Test Results of GFRP Rebar Samples........................................ 164

Table 5.6: Maximum Allowable Sustained Stress Ratio ................................................... 184

Table 5.7: Approximate Creep Deformation Reduction Factor $(\gamma E)$ for Elastic Modulus........ 185

Table 6.1: Strength Retention due to Seawater Saturation and Aging ................................. 198

Table 6.2: Time Shift Factors used in This Study ......................................................... 207 
Table 6.3: Summary of Comparison of Distribution Type 214

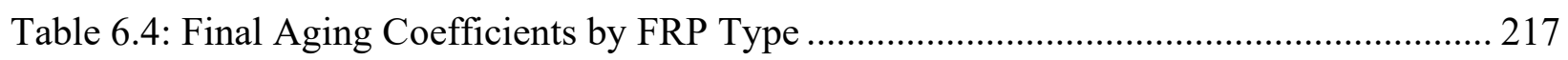

Table 6.5: Projected Strength Retentions (SR) by Age .......................................................... 218

Table 6.6: Average Tested GFRP Material Properties ……………………………………........ 223

Table 6.7: Nominal or Minimum Guaranteed GFRP Material Properties................................... 225

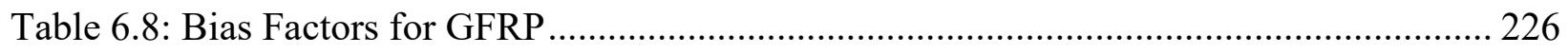

Table 6.9: Coefficient of Variations (COV) for Virgin and Aged Materials.............................. 227

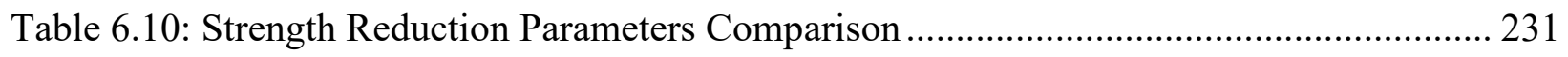

Table B.1: Sample of FRP Database created for this study ………......................................... 284

Table C.1: Sample of Original Dataset Provided by the FRP System Supplier .......................... 300

Table D.1: Listing of Test Database Used in Sustained Stress Study ..................................... 320

Table E.1: Listing of Test Database Used in Durability Study ............................................... 332 


\section{CHAPTER 1 INTRODUCTION}

\subsection{BACKGROUND AND NEED IDENTIFICATION}

The health of US infrastructure has been steadily declining for the past several decades. According to the American Society of Civil Engineers ("ASCE's Infrastructure Report Card," 2017), current infrastructure redevelopment needs to maintain a safe level of operations is estimated to be over 4 trillion dollars. The role of FRP composites (also known as FiberReinforced Polymer, FRP or Structural Composites) will continue to increase in rebuilding and rehabilitating US infrastructure due to their inherent advantages of high strength to weight ratio, high corrosion resistance, and adaptability to a specific site/use conditions as compared to other conventional construction materials.

Composites have already made significant headway as commodity materials into our society in the form of high-end cars, rocket fuselages, storage tanks, airplane bodies, and buried pipes, to name a few. They will continue to provide a viable solution to infrastructure applications and improvements for many years to come. These applications include replacement and rehabilitation of aging pipelines, dams, bridges, and buildings as well as extend into the manufacturing and operations of more efficient mechanical systems such as airplanes, spacecraft, automobiles, mass transits, and wind turbine blades. In many applications, every pound of weight saved and unit strength increased with enhanced durability, translates into significant dollar savings (e.g., increased payload, life-cycle cost, or fuel-efficiency). The fact remains that a future without the use of composite applications or composites in combination with conventional materials is almost unimaginable in every aspect of our daily lives. That is particularly true in light of rapidly aging US infrastructure due to insufficient funding and lack of suitable alternatives. 
Despite recent advances in the composite industry, practicing engineers can be apprehensive about the use of FRP primarily due to its lack of "acceptable" design, construction, and maintenance standards or specifications. The lack of such specifications, together with an inadequate understanding of complex long-term behavior of FRP under various environmental and loading conditions, often results in the higher initial cost or unacceptable life-cycle performance of FRP. The gaps in adequately quantifying the aging characteristics of FRP often result in minimally substantiated durability reduction factors and therefore uncertain outcomes. Unfortunately, such long-term performance uncertainty is more glaring despite FRP's material advantages over other construction materials. FRP's inherent advantages over commodity materials such as steel, aluminum, concrete, and wood, seem to diminish when the ambiguity of its design guidelines takes center stage.

One way to overcome uncertainties is to use a probabilistic or risk-based approach. What if an FRP member can be designed to last its specified service life-span with substantiated confidence (or a known risk) that the member's reliability (or its probability of failure) will continue to meet (or surpass)) the commonly accepted design, durability, and cost standards of other conventional construction materials throughout its service life? Do we have the tools to economically design an FRP member to perform adequately over a certain life-span, say 10 or 100-years? These answers lie in building a rational framework to allow the development of riskbased durability design factors to ascertain an accepted FRP system performance through its entire service life. Such a time-dependent reliability-based framework can render FRP designs that are highly efficient and consistently reliable. Thus, this research attempts to overcome the long-term performance uncertainties and presents FRP as a competitive and reliable construction material. 
This report focuses on providing experimental and analytical tools for building a risk-based framework for assessing the life-cycle performance of FRP Composites. The methodology presented in this report uses 1) accepted deterministic models to instill "certainty" in the design formulations, 2) reliability based probabilistic methods to address inherent system "risk" from the natural variability of loads and strengths, and 3) actual material aging parameters derived from the scores of durability studies and tests conducted (or in progress) to model the "time-dependent" effects. The primary focus is to reduce "uncertainty" in the design process by rationally quantifying the reliability-durability relationship for typical FRP applications encompassing the entire service life of the member. The method proposed herein is termed a "risk-based" framework or "reliability approach," which uses test derived "time-dependent" durability characteristics.

\subsection{RESEARCH OBJECTIVE AND SCOPE}

The primary objective of this research is:

To develop a rational framework of designing FRP composites for lifecycle performance using risk-based methodologies and time-dependent FRP durability characteristics for civil infrastructure applications.

A risk-based approach allows for the inclusion of inherent uncertainties of external loads, internal strengths, and aging characteristics in design with an intent to limit the probability of system failure to a level commonly accepted in the industry for similar applications. 
This research also forms an integral part of the National Science Foundation's IndustryUniversity Cooperative Research Centers (NSF-IUCRC) Program at West Virginia University Constructed Facility Center (CFC).

This research addresses the underlying issue of unrealistically high (or low) knock-down factors (i.e., strength reduction factors in load and resistance factor design or factors of safety in working stress design methods) that are often used by the designers to account for uncertainties of FRP durability/aging. The use of such factors, without the inclusion of risk, is perhaps one of the primary reasons preventing FRPs from becoming economical and reliable materials of choice among design, manufacturing, and construction sectors. This research is based on proven principles of reliability/probabilistic analyses, risk-based decision making, and failure acceptance levels using the existing test data and published durability/reliability reports from FRP manufacturers, WVU, and other research institutions around the world.

The durability of FRP composites can be described in terms of gradual losses of chemical, physical, and mechanical properties over time. The continuous degradation of FRP properties over time without a threshold value or endurance limit has been well documented by many researchers such as GangaRao (2007, 2011, 2017), Manalo (2020), Vijay (2000), Karbhari (2003), and Barker (2019). The aging process involves many external and internal factors such as moisture, $\mathrm{pH}$ levels, temperature variations, sustained loading (or static fatigue), cyclic loadings (i.e., harmonic vibrations, dynamic fatigue or common fatigue), ultraviolet (UV) exposures, FRP architecture (i.e., fiber orientation, fiber volume fraction, manufacturing process, etc.), internal defects (e.g., inadequate curing, improper bonding, resin-rich pockets, voids, etc.) and combinations of above factors such as thermo-mechanical stresses. The scope of this research is limited to FRP durability issues related to common civil engineering applications such as environmental effects from the 
outdoor temperatures and moisture, and typical physical effects such as constant static loading. Specific FRP architectures, fatigue loading, UV radiations, and harsh chemical environments are discussed briefly but not analyzed; although, the framework developed in this report can be extended to those conditions.

A case study illustrating the development of a Basis of Design (BOD) for a commercial Vinylester GFRP application in seawater with design examples (using an actual accelerated test data from a two-year sub-sea research project, Chapter 6) are provided to show the concept of time-dependent reliability and durability clearly. Normalized accelerated test data, natural aging data (when available) along with manufacturing data are used to develop aging performance (e.g. decay/deterioration) curves and to estimate statistical variations needed for probabilistic analysis. As a part of current research, test databases from about 20 separate studies spanning over the last two decades were collected and analyzed (Chapter 4).

Figure 1.1 illustrates the scope of work completed and the associated twelve-steps program used in this research. The research is conducted in the following phases: 1) conduct a comprehensive literature search and critical reviews of the previous work performed in the field of durability and reliability; 2) identify and collect relevant test databases from WVU, manufacturers, and other institutions worldwide; 3) normalize and analyze the collected raw test data using confidence intervals and stress-superimposition principle (i.e., Arrhenius principle) as applicable; 4) formulate reliability-based durability framework using the concept of timedependent reliability (see Chapter 3 for details); 5) based on step 4, develop a risk-based framework to account for "environmental" effects (e.g., outdoor applications); 6) Based on step 4, develop a risk-based framework to account for "physical" effects (e.g., sustained loads); 7) develop preliminary recommendations; 8) compare with industry standards and practices; 9) test and 
illustrate the proposed reliability-based approach for an actual 2-year accelerated test database of a GFRP product designed for submerged seawater applications; 10) test and demonstrate the proposed reliability-based approach with design examples and engineering calculations; 11) finalize conclusion and revisit prior recommendations (from step no. 7) and evaluate how stated recommendation holds for adoption in design codes or guidelines, and finally, 12) Closeout reliability-durability research by publishing research findings (i.e., this dissertation). 


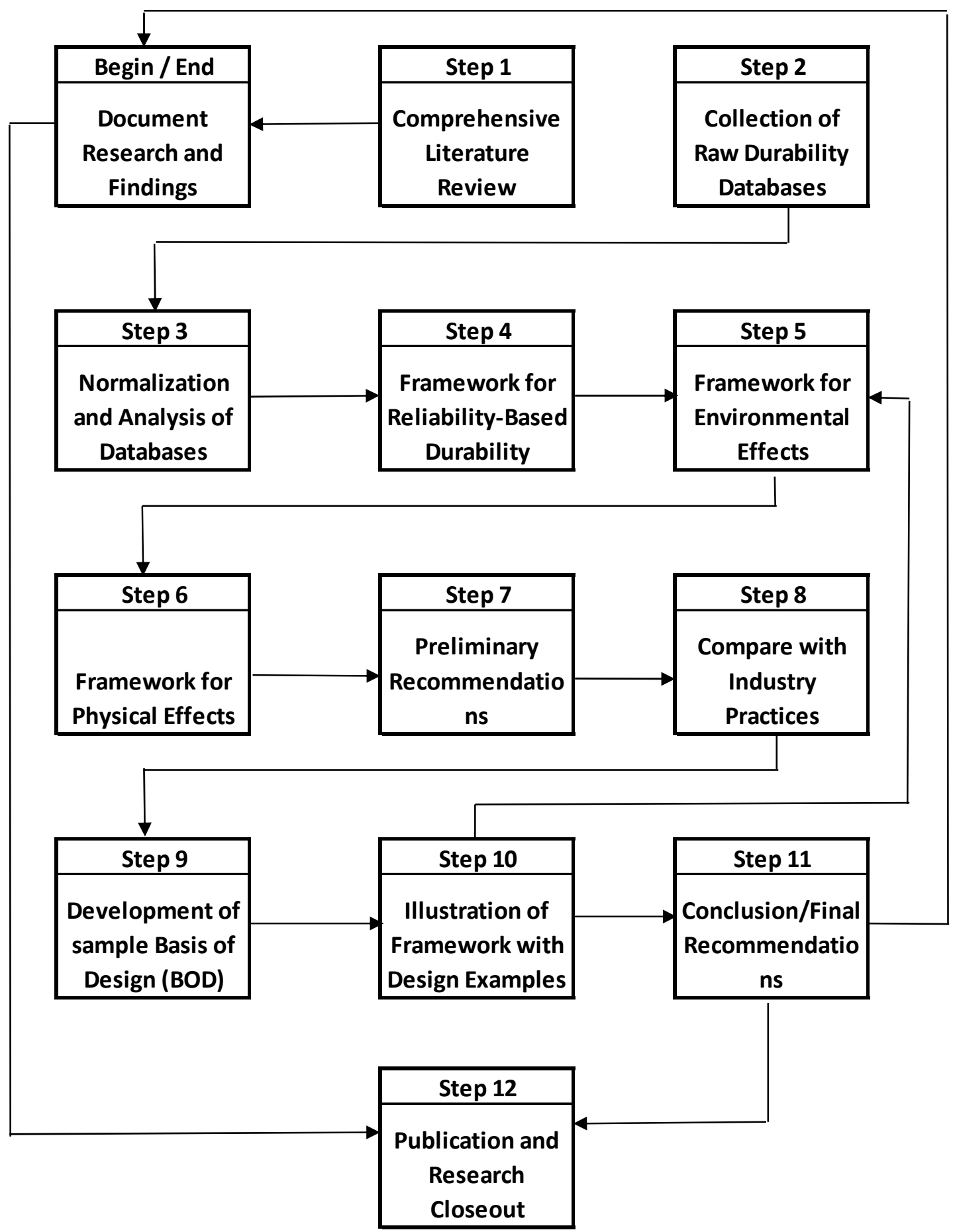

Figure 1.1: Flowchart of Time-dependent Reliability-Durability Research

This research faced many challenges, and some of them include: i) the collection, reduction, and normalization of a vast FRP test database (accelerated and natural) from different 
sources for deterministic and stochastic analyses, ii) the selection of appropriate performance functions, design examples, and deterioration models to capture various failure modes for calibrating test data and identifying the missing data, iii) computations of reliability indices (or probability of failures) for multiple modes of failures and virgin/aging parameters that can represent typical FRP applications; iv) Calibration of FRP's strength reduction factors necessary for formal design guidelines with the limited number of observations and scope of the data, and v) the lack of available examples or frameworks for developing rational durability strength reduction factors suitable for adaption in the US and international design codes.

This research overcame the above challenges by:

1. Carefully reviewing and scrutinizing all external data. For example, test data that was not complete or lacking essential information such as original strength was rejected. Rearranging all data obtained in a format that is more suited for extensive database analyses to evaluate the relevance to the project objectives and eliminate possible errors of re-coding. A wide variety of statistical functions and Add-ons (e.g., SPC for Excel) were employed to check the data validity and compute statistical parameters to catch errors and outliers. It must be stated that FRP database analyses were not performed to derive a set of regression equations for general use but to show how such analyses can be used in the development of a reliability-based durability framework. There is still much more work that can be done to refine such FRP databases.

2. Limiting the selection of performance functions (i.e., defining failure modes) to only a few failure modes in flexure and tension of Vinylester GFRP (for which adequate test data was available) by using specific design examples. The calibration of strength reduction factors for various FRP composites for general applications was considered out of scope because it will 
require similar computations for hundreds of design examples, more durability data, and use of refined and time-consuming simulation methods. By focusing on how to perform timedependent analyses with illustrated design examples opens the door to processing of a more extensive set of design examples, failure modes, deterioration models, and distribution functions for a wider variety of FRP composites than the current scope can reasonably allow.

3. Utilizing simple forms of design examples and reliability index formulations (first-order methods) to develop and test the framework. Fortunately, the results of the data analysis used in this research indicated that most probability density functions were following "normal" or "lognormal" distribution patterns, which allowed for the use of more straightforward framework reliability index calculation methods.

4. Focusing on building a framework that would open doors to more detailed refinements and future calibration of FRP durability strength reduction factors for industry-wide applications after the author realized the enormous data requirements in order to calibrate for the durability strength reduction factors of FRP composites. Calibration is considered out of scope.

5. Adding recommendations at chapter and report levels to provide a clear understanding of the framework presented. The final recommendations for code adoption are the ultimate goal of such research projects; however, it is still a vast area and must be deferred to the industry to follow the proposed framework and move towards developing a reliability-based design code for FRP. 


\subsection{RESEARCH SIGNIFICANCE}

The proposed research approach is unique and original due to the following reasons:

1. Introduction of "time-dependent" relationship between reliability and durability: This research questions the traditionally assumed linear relationship between reliability and durability and illustrates how traditional methods can fail to provide an acceptable level of structural safety as FRP members age. By introducing the concept of time dependency, one can safely design an FRP member to perform adequately till the end of a target service life (e.g., 25-year or 100year) - all within an acceptable risk (e.g., probability of failure). This "first published research" not only combines reliability and durability for FRP composites in the civil engineering/structural design field but also introduces the critical concepts of time-dependent functions for both. One of the unique findings is the discovery of an increase in the variability $(\mathrm{COV})$ of mechanical properties with aging. This type of time-dependency - although easily proven by deductive reasoning - often results in a non-linear relationship of the reliability with aging and challenges the traditional use of mean value (of aged mechanical properties) in age predictions. This unique relationship has not been explored in prior age prediction methodologies of civil infrastructure materials.

2. Establishment of a new "risk-based" framework: The framework proposed in this research is the first of its kind and can help researchers to rethink how they approach and test the durability of FRP's structural components and systems. This research allows the development of timedependent relationships between structural safety (i.e., probability of failure) and the age of FRP composites. Such a risk-based framework can allow FRP designers to choose appropriate strength reduction factors or factors of safety (or other design threshold values such as percentage of sustained stress or number of fatigue cycles) based on actual durability test data 
that account for deterioration and uncertainties of material strength/stiffness properties. For example, the research illustrates how a strength reduction factor of 0.3 for vinylester-based GFRP flexural members can be computed using accelerated and naturally aged testing data to provide 100 years of service life. Such results, although preliminary at this time, are close to what the FRP industry is experiencing (Barker, 2019, Manalo et al., 2020).

3. A "catalyst" for future code developments \& age-related research: The framework can spur additional research and similar data analyses on fatigue cycles, partial or full submergence in warm marine/freshwaters, chemicals, freeze and thaw cycles, extreme heat, UV radiations, and more. For example, the limits on the number of fatigue cycles for various target service lives can be derived like the method proposed for limiting sustained stress ratios using normalized fatigue test data. For example, using a sustained stress test database, this research illustrated how various initial sustained stress limits on vinylester-based GFRP rebars could be justified to design for $25,50,75$, or 100 years of service lives. The limits computed using the proposed framework match very well with the previously established limits and provided a consistent prediction of FRP service lives, which is often missing in the current practice. A similar approach to estimating fatigue life is also possible.

4. "Customization" of FRP design using BOD: One of the early steps towards the adoption of practical FRP design guidelines is the development of a Basis of Design (BOD, Chapter 6) that can close the gap between an accelerated testing program and its immediate practical applications. This work takes the raw accelerated test data, which was developed for a particular application of FRP (i.e., immersion into cold seawater at certain depth) and shows how the proposed time-dependent reliability framework can be used to migrate many research findings into immediate practical design use. The BOD case study offers a unique professional 
engineering practice perspective about how FRP members can be custom-designed for a particular application to last a certain life-span in a specific environment, both economically and reliably.

5. "Integration" of academic research into the professional practice of FRP: This research provides several illustrative design examples (Appendix A) to expand the understanding of time-dependent reliability further and how specific strength reduction factors for use in LRFD (load and resistance factor design) or factor of safety for use in WSD (working/allowable stress design) can be derived to provide a consistent but acceptable probability of failure. The framework helps in taking the guesswork out of durability testing, combines the reliability approach with derived/known durability characteristics, proposes a time-dependent reliabilitydurability design framework that can take into account changes in FRP's performance with age/service life, and bridges the gap between academic research and professional engineering practice using several illustrative examples and a BOD case study.

\subsection{LIMITATION OF SCOPE}

The primary intent of this report is to provide a reliability-based framework that can be applied to many facets of FRP applications and eventually allow the development of risk-based design guidelines or codes. The research findings, as presented in this report, are derived from a limited set of FRP test data, and results can vary (or refined) if additional test data is introduced. Proposed as a framework, it cannot possibly cover all possible FRP applications, or its design recommendations are considered as the final. 
The work conducted in this research is limited to:

1. Common structural engineering applications of pultruded "thermoset" composites comprising of continuous glass fibers and vinylester matrix with a fiber volume fraction of above $50 \%$ are included. Such vinylester-based GFRP sections are common for composite materials in civil engineering, and often there is enough durability testing data available. Polyester and epoxybased GFRP were also evaluated but only on a limited case by case basis. In all cases, naturally-aged test data are scarce for all FRP/resin types. More data (e.g., accelerated testing, naturally aged, manufacturer's quality testing, etc.) will undoubtedly help to refine further and finalize the new and aged durability characteristics presented in this research. Applicability to different fibers (e.g., Carbon and Aramid), thermoplastic resins, and other resin/fiber combination is possible, should more relevant test data become available; however, it is beyond the current scope.

2. Although the terms "aging" and "durability" are used interchangeably, there are minor differences (depending on the industry). The report focuses on the "durability" characteristics of FRP, which are common in most structural engineering applications (e.g., outdoor/indoor temperature ranges, alkalinity, moisture uptakes, and sustained loadings). FRP applications in extreme environments such as fire, very hot or extremely cold temperatures, highly corrosive environments, very high pressures, etc. are outside the scope of this report.

3. Composite applications where FRP is used in conjunctions with other materials (such as FRP reinforcing bars in concrete members, FRP wraps, or plates bonded to concrete, steel or timber members, FRP shells filled with concrete, etc.) or FRP produced with a very high level of QA/QC (e.g., in aerospace or automobile industries) are not considered in this report. The report focuses on standalone pultruded FRP members produced for civil engineering 
applications only. However, the proposed methodology may be extended to hybrid materials and other sophisticated FRP applications as more data and technology become available.

4. Although the age predictions, with the help of accelerated testing methods and the proposed framework, can be extended well beyond commonly used design spectrums, the applicability of this method outside the service life of 100 years is not yet verifiable. There is not enough calibration data available about the FRP mechanical properties or the applied external loads to support such predictions.

5. Due to the complexities involving anisotropic behavior of FRP composite and system-level reliability computations, the application of the proposed Framework is currently limited to the long-term prediction at "component" or "coupon" levels of "in-service" civil engineering structures only. System-wide predictions are highly complex, requiring the modeling of interactions among various components, connections, and different materials, and therefore, are considered outside of the scope.

\subsection{REPORT ORGANIZATION}

This report is organized as:

- Chapter 1 (this Chapter) provides the necessary background, needs identification, objectives, and scope, work plan, the significance of this work, and establishes the limitation for the scope of this research.

- Chapter 2 provides a necessary literature review of the topics related to this research. It discusses the aging of composites, durability characteristics, time-dependent effects, environmental effects, physical effects, natural weathering, standard durability prediction methods, and the state of current practice. Effects of acidity, alkalinity, moisture, temperature, 
fatigue, and sustained stress are also discussed. The reliability section includes a discussion on the variability of material properties, reliability index, development methods for design guideline and resistance factors, and reliability approach to durability predictions.

- Chapter 3 establishes the framework for the time-dependent reliability-based durability. Steps involve data collection, data analysis, selection of design examples, performance function, target reliability index and service life, calculations of as designed and aged reliability, formulation of reliability-durability relationship, development of reduction factors, and development of recommendations.

- Chapter 4 illustrates how to account for "environmental" effects using an extensive, combined accelerated test database. Using the Arrhenius principle, the accelerated test data is projected into the timeline of naturally aged data (thus matching the outdoor aging) or a reference environment (e.g., submergence in room temperature tap water) of various GFRP and associated reliability indices over time are formulated. An illustrative design example (Example 1 in Appendix A) is used to show all steps involved to develop reliability-based strength reduction factors as well as factors of safety.

- Chapter 5 illustrates how to account for "physical" effects using a sustained stress database. It discusses the importance of limiting sustained stresses, current practice, and development of time-dependent creep equation using Findley's power law. It proposes a rational method using confidence intervals to limit sustained stresses, serviceability checks for creep deformation, corrections for nominal values, and compares finding with industry practices.

- Chapter 6 is a case study illustrating the development of a basis of design (BOD) for real-life vinylester and polyester-based GFRP members in a submerged deep seawater environment for 25-years of desired service life. This chapter walks through various steps from analysis of a 
comprehensive 2-year test database to the final determination of strength reduction factors for a particular FRP use.

- Chapter 7 includes a summary of findings from all previous chapters

- Chapter 8 contains a summary of recommendations from all previous chapters

- Appendix A includes three design examples that illustrate the development of a timedependent reliability approach for the calculations of durability strength reduction factors for an outdoor beam, a submerged tension bracing, and a flexural beam. These examples are often referred to in previous chapters (Chapters 4, 5, and 6)

- Appendix B contains additional tables and graphs from collected and analyzed FRP databases (Chapters 3 and 4) that are not included in the main chapters.

- Appendix C contains data tables and graphs from the Case study used in the development of the Basis of Design (BOD) for Vinylester and Polyester GFRP's subsea applications (Chapter 6)

- Appendix D contains additional information about the database used in the sustained stress study (Chapter 5).

\subsection{SUMMARY}

Specific findings of this chapter can be summarized as follows:

1. The risk-based reliability approach, as proposed in this research, addresses shortcomings of current deterministic design practices, as well as gaps in the knowledge-base and data-base issues of FRP behavior with time/age and environmental exposures.

2. This research is significant due to i) introduction of time-dependent relationship between reliability and durability behaviors of FRP with age, ii) establishment of a risk-based 
framework to tie structural design with a certain probability of failure, durability characteristics, and target service life, iii) customization possibility for a specific FRP field application illustrated using a BOD case study, and iv) bringing professional engineering practice perspectives into the academia.

3. The research focuses on developing a rational framework for FRP composites that can allow the development of risk-based durability design factors to assure an acceptable level of performance throughout the target service life while providing material efficiencies and consistent reliability.

4. The study overcame many challenges ranging from combing diverse durability databases to defining performance functions. The limitation of the scope (e.g., available databases for certain FRP types, simple beam examples, normalization of data, removal of outliers, etc.) helped in reaching the desired outcome.

5. This research limits the work to pultruded sections of continuous glass fiber reinforced polymers (GFRP) in the thermoset matrix with a focus on Vinylester resin and fiber volume fraction above $50 \%$. The findings are presented for the durability/reliability of components or coupons of "in-service" civil engineering structures up to 100-years of service life. Load variations are considered in the analysis but are not the focus of this study. In addition, the findings are based on the limited FRP databases analyzed in this study. 


\section{CHAPTER 2 LITERATURE REVIEW}

\subsection{COMPOSITES}

Composite is a generic name for fabricated structural materials that contain two or more distinct components, all of which retain their original (at the time of manufacturing) properties in the final product. In the context of fiber-reinforced polymer (FRP) composites, they are made from engineered layers of glass, or carbon, or aramid fibers (or fabric) embedded in a polymer matrix to function as a structural member.

Composites are used in various industries because of:

1. High strength/stiffness to low weight ratio - many times superior when compared to many other structural materials including steel

2. Design flexibility - a wide variety of fibers can be added and oriented to meet almost any specific design requirement. Choices offered by FRP make it a perfect designers' material.

3. Fabrication flexibility - can be formed into virtually any shape, offers part consolidation by using moldings to replace assemblies, can be finished to almost any long-lasting appearance, and uses low tooling cost as compared to metals.

4. Dimensional stability - yield point to the breaking point

5. High dielectric strength - excellent electrical insulating properties

6. Corrosion resistance - proper resin systems with additives can provide long-term resistance to degradation from UV radiation, acids, alkalinity, to fire

The disadvantages of FRP include relatively complex design parameters (due to anisotropic behavior and lack of understanding about durability/aging), the variability of properties (due to 
many proprietary products and varying levels of QA/QC enforcement), limited recycling options (from a sustainability point of view), and potential for high cost with low reliability (often a result of the uncertainties involving its long-term performance and minimally substantiated design standards). Many benefits are often overlooked due to durability and reliability issues encompassing FRP's service life, and overcoming these obstacles is the primary motive for this research.

All composites go through a continuous change in their material properties from the time they are manufactured to the end of their useful service lives. After manufacturing, material strengths continue to increase for a few months to years (therefore, a 60 to 90 days waiting period is recommended to promote consistency in the reporting of virgin mechanical properties). In time, a general stabilization of mechanical properties occurs, and then a slow loss of material properties begins, which can last for a vast majority of years in service. Near the end of FRP composite useful life, a substantial and/or rapid loss of material strength may occur that often results in a failure by exceeding its serviceability or strength limit states. Durability studies look at how changes in FRP characteristic properties occur (e.g., reduction of FRP strengths/stiffnesses when subjected to various environments and loadings) while the reliability approach looks at how the distribution of FRP resistance (e.g., the variability of strength/stiffness from the mean value) and design loads affects the structural safety (e.g., probability of failure). A time-dependent approach evaluates the relationship between durability and reliability as FRP ages (e.g., over the service life).

The following is a brief overview of the published literature related to the durability and reliability aspects of FRP composites. 


\subsection{AGING OF Composites}

All construction materials age and lose their strength over time, and composites are no exception. The best way to explain such aging is by a "bathtub" performance curve (Figure 2.1), which shows varying failure rates of a system that uses three types of Weibull distribution (Weibull, 1939) to model its time-dependent relationship. The first (left) curve relates to a failure rate that decreases over time, the second (middle) line is when the failure rate is constant, and for the third curve (right), failure rate increases with time. The first curve relates to the maturity process (e.g., curing), the second one relates to the failure of matured systems due to inherent variations in material properties (manufacturing tolerances) and external factors (oversize loads), and the third curve relates to the natural aging process where material properties deteriorate over time. The final curve (top) is a composite curve that we actually observe over the systems' entire life-cycle. In this study, we will ignore the maturity curve, assuming that FRP manufacturing, quality control, and installation in service are appropriately done. The second curve considers the variability in loads, inherent material properties, and the environment. It also forms the "initial" basis of design, assuming a consistent pattern of member resistance and external loadings. The second curve has been the basis of modern codes, which implies a uniform failure rate or probability of failure. The third curve of increasing failure rate is probably the most important for the long-term study of FRP behavior since it accounts for "aging" or changes in material mechanical properties with time. The third curve-based responses are not well understood for materials under varying exposures, and often not included in the design on a scientific basis. The research objective is to closely match the fourth curve labeled "observed behavior" using the available known or derived data. Figure 2.1 illustrates a hypothetical "bath-tub" performance curve (probability distribution curve), which is created using various combinations of Weibull 
distribution parameters (e.g., scale and shape parameters). For "in-service" civil engineering structures (the primary focus of this research) only the middle portion of "observed behavior" applies. The early "wear-in" and later "wear-out" portions apply primarily to mechanical systems with moving parts. This concept is borrowed from the mechanical/industrial engineering field to develop a basic understanding of FRP behavior undergoing "aging" when subjected to various environmental and loading conditions.

If the probability distribution function (PDF) of all relevant mechanical properties follows Weibull distribution, the assumption of a time-dependent failure rate pattern will be logical. However, unless there can be enough data collected to correlate theoretical Weibull distribution through laboratory test results to (actual) natural aging, the research in this area will always be a work-in-progress.

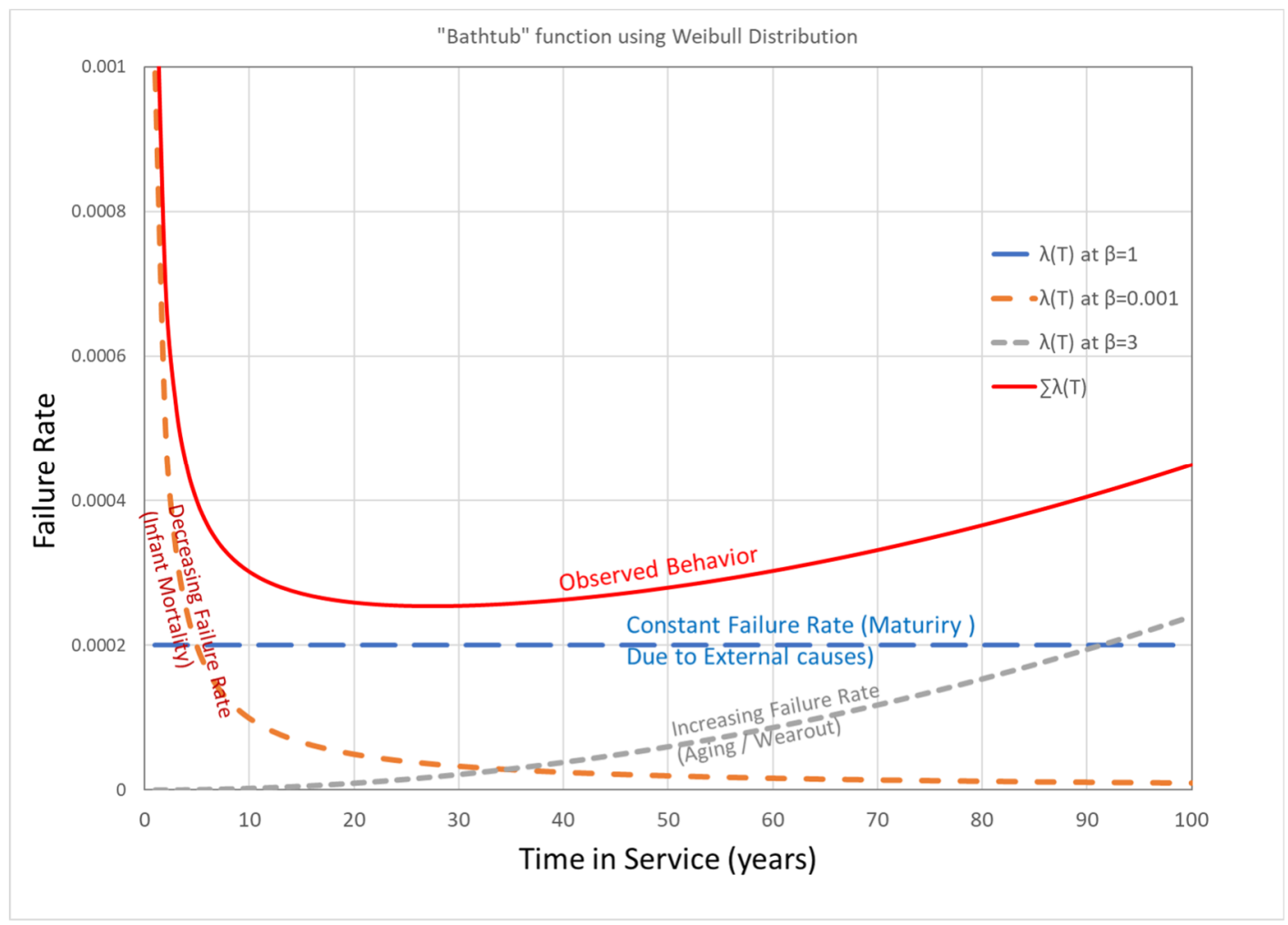

Figure 2.1: Typical "Bathtub" Curve of Mechanical Systems 
In the field of composites, words "durability" and "aging" are often used interchangeably; however, there is a small distinction. The durability of a material is defined as "its ability to resist cracking, oxidation, chemical degradation, delamination, wear, or the effects of foreign object damage for a specified period under specified environmental conditions" (Dutta, 2001). The aging (or ageing) is a more generic term that often refers to a time-dependent degradation of material properties (physical aging), usually under a service environment, that can shorten a component's useful service life. Even under the ambient conditions, although limited (maximum 20\% of original manufactured FRP properties), there will be some aging. Two are closely related, but the former is a natural material's characteristics, and the latter is a common way to evaluate the lifespan a component made using that material under different environmental conditions. For example, how quickly an FRP reinforcing bar "ages" depends on the material's "durability" characteristics. In simple terms, throughout this report, we will be focusing on the "durability" characteristics of FRP composites as it applies to the "aging" predictions of FRP components.

\subsubsection{Time-dependent Effects on Durability Characteristics}

All environmental and physical effects (see Fig. 2.2 for definition) impact the durability of FRP over time, so it is essential to consider the service life of the member when accounting for these effects. For example, it will be uncomical to design a member with a 10-year life using durability factors for 100-year of service life or unsafe if durability factor that was developed for 25 years life is used to design for a 75-year life. This report focuses on designing FRP for the desired service life subject to various external effects.

According to ISIS Canada Educational Module 8 (Bisby \& Eng, 2006), the external effects that impact the durability of FRP over time can be classified into two broad categories Environmental Effects and Physical Effects. This research focuses on the mechanical properties 
of FRP that are commonly needed for structural design, such as tensile strength, compressive strength, flexural strength, shear strength, and young's modulus. Durability considerations for other properties (e.g., abrasion resistance, hardness, damping, etc.) are also important; however, lack of adequate data and difficulties in defining suitable strength or service limit states (failure criterion) restrict proper reliability analyses. The application of the reliability approach is different for each effect; therefore, these effects must be studied separately. Figure 2.2 (inspired by ISIS Canada module 8 and Bisby, 2006) shows such effects on an outdoor bridge FRP beam.

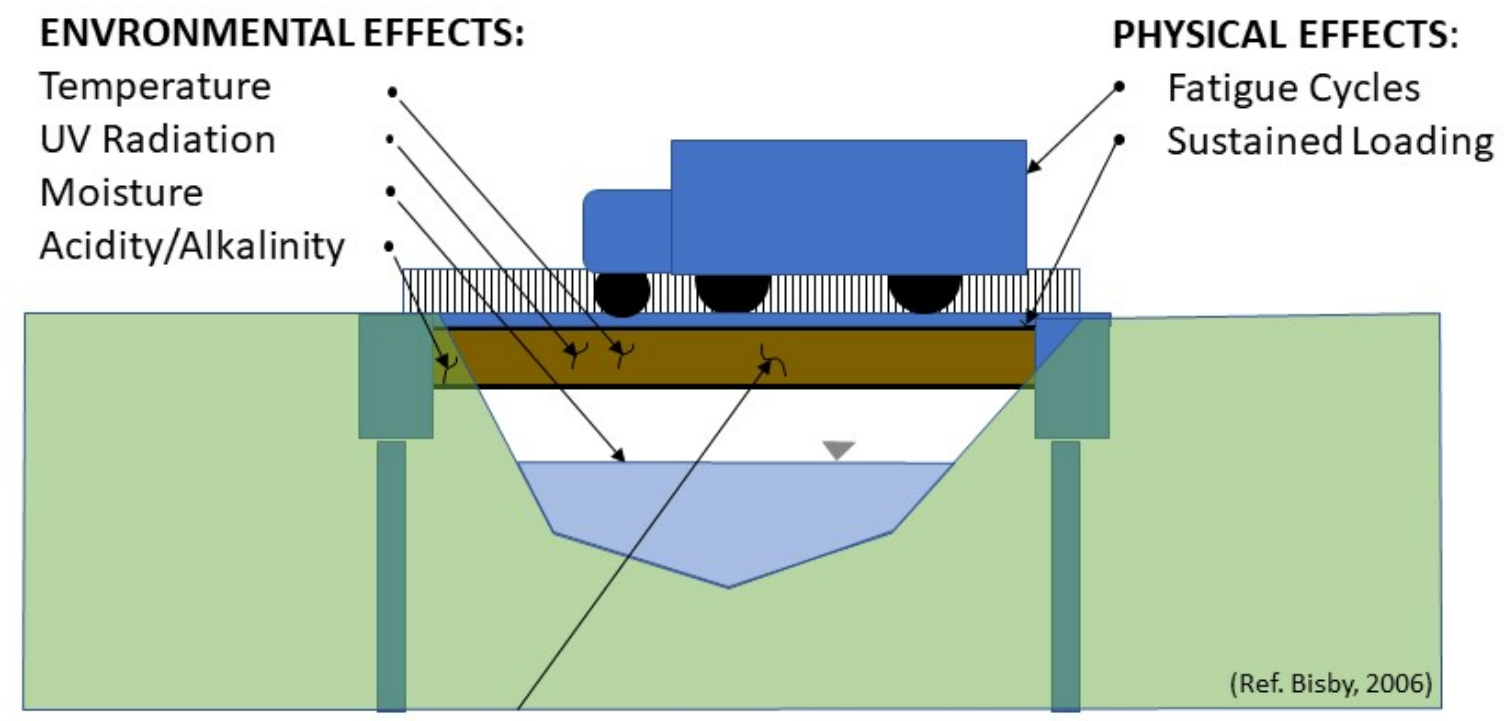

AFFECTED DURABILITY CHARACTERSTICS:

Tensile Strength, Compressive Strength, Flexural Strength, Shear Strength, Modulus

Figure 2.2: Durability Effects Involved in Natural Weathering of FRP Structures

Many researchers (Wang et al., 2016 \& 2015; Karbhari et al., 2003; Micelli \& Nanni, 2004) have identified several factors affecting the durability of FRP, such as moisture, $\mathrm{pH}$, temperature, sustained stress, UV radiation, and combinations of these factors. Exposure to these factors degrades FRP and lowers FRP's strength/stiffness (along with other mechanical properties) over time. Submergence in moisture and $\mathrm{pH}$ variations weakens FRP composite strengths more than its 
stiffness. Alkaline conditioning has more detrimental impacts than the acidic environment. The tensile strength decreases significantly once the temperature exceeds the glass transition temperature. Sustained loading tends to accelerate the degradation of mechanical properties of FRP, and UV radiation degrades the matrix-dominated transverse properties more than fiber dominated longitudinal properties. A combination of the above effects with time on FRP, over a long-term basis, is not well documented.

These primary effects are further described in the following sections.

\subsubsection{Environmental Effects}

Environmental effects include the following common effects derived from the environment in which an FRP member will remain in service for most of its useful life.

Moisture - from atmospheric air, sprays, or full or partial submergence. Moisture can be further subclassified as acidic, alkaline, salty/marine, or neutral (i.e., fresh bodies of water, rain exposures, or pipes carrying potable water).

Acidity/Alkalinity - is defined by $\mathrm{pH}$ value and often represents the submerged environment that a specimen is placed in for testing or service. The environment can be "wet" as described in an acidic chemical storage tank, or it can be "dry" as in FRP buried in dirt or contact with alkaline concrete. $\mathrm{pH}$ solutions are often used in a laboratory for accelerated testing along with temperature effects.

Temperature - it can be defined as freezing, cold, room temperature, hot, very hot, or even fire. Freeze and Thaw cycles are also included, but they act more like physical effects and treated separately as such.

Ultra-violet radiation - It is the natural sunlight outdoor or artificially created light with UV effect. 


\subsubsection{Physical Effects}

The name implies that it is created outside the environment and often introduced due to particular loading applications. Since it is always associated with applied loads, it is called load effects. However, in order to avoid confusion with a similar LRFD term (load effect Q, Section 2.5), we will refer to it as "Physical Effects" in this report. It is different from "physical aging," which is often the end result of the environment and time on FRP composites. Main categories are:

Sustained Load - mostly dead loads that are an essential part of civil engineering structures. It must be limited to improve serviceability and reduce the premature risk of failure. The "time to failure" under various initial sustained stress ratios is modeled as its effects.

Fatigue Cycles - it has specialized applications where load intensity varies in cyclic patterns from low to high. The "number of cycles" to failure under various fatigue stress range is modeled as its effects.

\subsubsection{Impact of Effects on Durability Characteristics}

It is helpful to clarify which environment or physical effects impact which FRP properties so that we can formulate a rational method to quantify them. Some properties depend on the internal architecture of the FRP component and its manufacturing process, so it is essential to make that distinction when applicable. Certain properties are dominated by the resin (e.g., interlaminar shear and strengths in the primarily transverse direction), and others are by the fiber (e.g., tensile and flexure and strength in the primarily longitudinal direction). To simplify, the critical mechanical properties of FRP that are affected by time-dependent effects are:

- Tensile strength - dominated by fibers, typically obtained from axial tensile tests such as ASTM D638 
- Compressive strength - dominated by resins, usually derived from combined loading compression (CLC) tests such as ASTM D6441

- Shear strength - also referred to as interlaminar shear strength and is dominated by the properties of matrix/resins, typically obtained from short beam test results such as ASTM D2344

- Flexural strength - dominated by fibers, usually derived from three-point beam test results such as ASTM D790

- Modulus of elasticity - dominated by fibers, traditionally derived from $(\sigma-\varepsilon)$ plots of the beam during its tensile or compressive strength tests. The effect is minor, but it must be accounted for in the serviceability checks (e.g., deflections)

Also, many other FRP characteristics are impacted by time-dependent effects, but they are generally not strength related or are dependent on additives or additional treatment. Some of them are color, bond strength, hardness, skid resistance, fire resistance, chemical resistance, etc. These properties are not studied in this research.

\subsubsection{Natural Weathering}

One of the objectives of this report is to predict the behavior of FRP under natural weathering processes that are found in common civil engineering applications. The change of mechanical properties of FRP with water absorption, solar rays, and cycles of low/high humidity can be observed using accelerated aging tests (Silva, Sena da Fonseca, \& Biscaia, 2014). One of the most critical types of natural weathering conditions that composites encounter during their service life is physical (e.g., stress cycles) and thermal (e.g., freeze-thaw) fatigue wherein fluid sorption-desorption has been found to influence durability response in the most severe manner. Fatigue damage in FRP composites is progressive and cumulative at a micro-level. 
Silva also concluded that UV tests in particular pose problems for correlating to actual operating conditions. It is possible to use Arrhenius equations to extend the results of accelerated aging (Silva et al., 2014). The results showed that i) Young's modulus suffers only minor changes; ii) moisture exposure by immersion in water causes only slight degradation; iii) thermal cycles of small amplitude, around room temperature, increased the ultimate strain; iv) salt fog spray cycles were most severe; v) selected dry-wet cycles caused higher degradation than the thermal cycles; and vi) cycles of UV radiation and flooding led to continuous decay of tensile strength.

The degradation of FRP composite properties under a wide variety of environments and service life (i.e., durability or aging) has been studied by many researchers (Dittenber \& Hota, 2010; Karbhari et al., 2003; Wang et al., 2016) in the past. Numerous past and current projects by GangaRao and his associates (Kajorncheappunngam et al., 2002; Vijay, 1999; Wang et al., 2016) have provided appropriate data on the durability performance of GFRP. Their experimental data, particularly the accelerated test data for the combined effect of moisture, $\mathrm{pH}$, and temperature variations (common conditions in outdoor FRP application), can be used to develop durability models and in-service life predictions.

Numerous environmental factors can adversely impact the original mechanical properties, and serviceability characteristics of FRP components and their impacts can vary significantly depending on the composition of the FRP. This research intends to investigate the effects of natural outdoor environments along with common load conditions that most common FRP components are likely to experience over their service life in a typical civil engineering application (e.g., highway bridges, buildings, industrial plants, dams, railroad ties, pipelines, etc.). Such applications may include outdoor exposure, permanent contacts with concrete or ground, sustained loading due to dead loads, and fatigue cycles from moving live loads. A typical FRP composition 
can be E-glass fibers in vinylester, polyester, or epoxy matrix. Some of the prevailing environmental exposures can be moisture, saltwater, temperature, ultraviolet radiation, and acidity/alkalinity. A combination of the above environments and loading conditions does affect the thermo-mechanical properties of composites, especially for 50 to 100 years of service life. The following is a further discussion of specific effects.

\subsubsection{Effect of Acidity and Alkalinity}

The impact of the $\mathrm{pH}$ value of the environment is prominent for FRP subjected to harsh environments such as FRP rebars embedded in concrete (which is highly alkaline). Varying pH is often used with varying temperatures in accelerated testing. However, for the natural weathering process, besides the use of FRP wraps or rebars with concrete or particular locations in alkaline mines (e.g., limestone), alkaline environments are not common. Acidic rain and acidic soils exist in some parts of the US, but the impact of acids on durability is minor. Marine water, which is slightly alkaline ( $\mathrm{pH}$ around 8.2), has its adverse effects when combined with temperature and is often studied separately. Accelerated testing is a big part of the extrapolating natural behavior of FRP; therefore, it is beneficial to utilize $\mathrm{pH}$ along with temperatures.

Saadatmanesh et al. (Saadatmanesh \& Tavakkolizadeh, 2010) evaluated the effect of the environment on a composite varies depending on FRP composition, especially the resin or sizing type. FRP coupons were immersed in different solutions to simulate typical harsh environments encountered in the field. CFRP and GFRP coupons were compared, and in general, CFRP indicated much better tensile strength durability for harsh environments. Similarly, a study conducted at WVU by Cho and Mounts (Cho \& Mounts, 2007) tested GFRP samples under sustained tensile loads and alkaline environment for six months and concluded that polymer matrix plays an essential role in property retention by protecting fibers from a harsh environment. 
Pre-existing flaws in FRP can also change the durability of FRP under harsh environments (Buck, Lischer, \& Nemat-Nasser, 2001). The curing temperature while manufacturing FRP composites can also alter durability. FRP cured at high temperatures $\left(200^{\circ} \mathrm{Celsius}\right.$ and above $)$ have more durability (e.g., tighter crosslinks leading to degradation of the matrix/chain scission, higher moisture absorption, and brittle fracture) related issues (Han \& Drzal, 2003). Such items add to the variability of FRP's mechanical properties in the context of this research.

Marru et al. (Marru et al., 2014) applied standard accelerated age test data to non-isotropic epoxy GFRP pipes to estimate lifespan, showing that the pipe degradation mechanism follows Arrhenius superimposition principle. Zhou et al. (Zhou, Chen, \& Chen, 2011) compared their test data on composites to steel and GFRP rebars for embedment into concrete under highly acidic conditions (e.g., acid rain environment). They used the Arrhenius principle to predict the durability of GFRP bars as compared to steel. Zhou et al. observed that FRP reinforcing bars have excellent durability characteristics showing $84 \%$ retention of strength after 34 years (projected), which seems to be a good match with a similar field investigation findings of Canada marine structures (Mulfi et al., 2007). A recent study of GFRP rebars (Manalo et al., 2020) conducted in an accelerated environment simulating concrete encasements concluded that the GFRP bars in actual concrete structures would retain up to $54 \%, 68 \%$, and $68 \%$ of their interlaminar shear strength (ILSS) after 100 years of service at an annual average temperature of $30^{\circ} \mathrm{C}$ when exposed to an alkaline environment, tap water, or saline solution, respectively. Similar results were also obtained in this research study (Chapter 4).

The effect of the aging environment on the degradation of GFRP was studied extensively by Kajorncheappunngam (Kajorncheappunngam \& Gupta, 1999; Kajorncheappunngam, Gupta, \& Gangarao, 2002). The strength of the composite Epoxy GFRP was reduced by more than $70 \%$ 
by the acid at room temperature and by the alkali at the elevated temperature. The worst cases of damage involved an attack on the glass fibers in acid at $60^{\circ} \mathrm{C}$ compared to room temperature. It concluded that reinforcing glass fibers have to be protected from attack by liquid media to improve the durability of composites. The report also questioned if accelerated testing at elevated temperatures is always appropriate.

\subsubsection{Effect of Moisture}

Typically, moisture in laboratory testing means total immersion, although partial submergence or sprays (e.g., piles or sea wall), atmospheric rain (e.g., bridges or rail ties), and high humidity conditions are also possible. Other variations are distilled/tap and marine/salt waters. Since saltwater is slightly alkaline, it has more detrimental effects than freshwater. Since immersion of samples for $\mathrm{pH}$ and temperature variations is necessary for accelerated testing, moisture uptake (by weight change and impacts on mechanical properties) is also often studied with temperature and $\mathrm{pH}$ in accelerated testing.

The durability of GFRP composites in both the saltwater and tap water immersions was investigated under different percentages of sustained loads of GFRP's ultimate tensile capacity by Wang et al. (Wang et al., 2015). The study revealed that the low-sustained load level (less than $20 \%$ ultimate strength) did not influence the trend of GFRP mass change curves, while higher load levels could lead to higher moisture uptake. All the test specimens showed a steep initial increase in the tensile strength and elastic modulus with immersion time, followed by a steady decrease. The synergistic effects of water immersion, temperature, and bending strain on the sorption behavior and tensile response of a unidirectional pultruded E-glass/Vinylester composite were investigated over two years by a study (Helbling \& Karbhari, 2008) which concluded that the tensile strength over an extended time, particularly under sustained high bending strain (35\% to 
$45 \%$ of ultimate tensile strain at failure under ambient conditions), decreases substantially and indicates an early and significant occurrence of irreversible damage.

Bian et al. (2012) observed that GFRP with higher fiber volume fraction absorbs more water and degrades more in tensile, flexural, and ILSS (interlaminar shear) strengths due to the presence of higher percentage of micropores inside the fiber bundles (likely from inadequate wetout). Similar conclusions were also reached by Karbhari et al. while conducting a test of high volume fraction carbon and glass composites (Karbhari \& Xian, 2008). The moisture uptake and degradation effect can be attributed to high levels of fiber loading with local fiber-to-fiber contact (i.e., inadequate protection and bond between fibers). Zafar et al. (Zafar, Bertocco, SchjødtThomsen, \& Rauhe, 2012) tested CFRP samples in seawater and demineralized water to study the long term effects of moisture and concluded that seawater immersion has a more detrimental effect (up to $35 \%$ reduction of ultimate tensile strength).

Kumosa et al. (Kumosa, Benedikt, Armentrout, \& Kumosa, 2004) studied the effect of moisture absorption in unidirectional GFRP samples in resin systems and concluded that Vinylester based materials had the most favorable moisture diffusion properties. The modified polyester-based composites exhibited the worst moisture absorption performance. The epoxybased materials had absorption; however, these materials did not reach equilibrium and kept slowly taking on more moisture in a non-Fickian manner. This conclusion is also supported by the subsea case study, as described in Chapter 6.

A study by Mourad et al. (Mourad, Abdel-Magid, El-Maaddawy, \& Grami, 2010) included glass/epoxy and glass/polyurethane composites were immersed in warm seawater $\left(23^{\circ} \mathrm{C}\right.$ and $\left.65^{\circ} \mathrm{C}\right)$ from the Arabian Gulf for up to 1 year. The effect of seawater differed with matrix materials and conditioning environments. In the glass/epoxy composite, the mode of failure changed from brittle 
matrix and ductile fiber to ductile matrix and brittle fiber, and in the glass/polyurethane composite the mode of failure was similar at room temperature, but at high temperature, it changed to brittle fiber primarily due to matrix becoming brittle with time and heat.

In general, in an accelerated testing environment of a laboratory, it is often difficult to separate the effects of immersion from the effects of temperature and $\mathrm{pH}$. Usually, the initial steep drop in strengths can be attributed to water (mass) uptake, which may take months or even years under a natural environment when compared to accelerated testing. In order to make accelerated tests more meaningful, the focus of strength measurements should be on the mechanical properties after the effect of immersion (saturation of moisture uptake) has subsidized

\subsubsection{Effect of Temperature}

The effect of temperature in natural weathering is significant. Depending on the location of FRP member and exposure, the local climate can change from freezing to extremely hot, and it may go through many freeze-thaw cycles, and even be exposed to fire. Temperatures along with moisture (tap water and saltwater immersion) and acid/alkaline additives are often used in many accelerated tests making up an essential part of databases used in aging studies.

Sauder et al. (Sauder, Lamon, \& Pailler, 2004) studied the effect of very high temperature (up to $2400^{\circ} \mathrm{C}$ ) on Carbon FRP and found that depending on the temperature, resin type, and fiber/resin composition, 1) temperature increase causes Young's modulus decrease and a tensile strength increase to a maximum value and dropping at a temperature above $1600^{\circ} \mathrm{C} ; 2$ ) a substantially linear elastic behavior from room temperature to intermediate temperatures up to $1400-1800^{\circ} \mathrm{C}$, then a nonlinear delayed elastic response at higher temperatures, and ultimately an inelastic response with permanent deformations at very high temperatures; and finally 3) elastic/inelastic transition stresses and temperature depend on fiber structure/texture. Adequate fire 
endurance of Glass FRP strengthens concrete beams that can be obtained by applying special insulations to get a fire rating of 4 hours or more (Williams et al., 2008). At very high temperatures, nearing about the glass-transition temperatures of the polymer matrix, the mechanical properties, especially the stiffness and the strength of the composites, decrease considerably. In general, the detrimental effect of high temperature on various FRP composites has been evaluated and well documented by many researchers (Cao, Wu, \& Wang, 2009; Hawileh, Abu-Obeidah, Abdalla, \& Al-Tamimi, 2015). For this research, the effect of temperature on mechanical properties is limited to a temperature well below the FRP glass transition temperature (Tg). However, before starting any accelerated testing program, it is crucial to determine the glass transition temperature of the FRP specimen so that Arrhenius stress superimposition principles will apply.

Aging tests conducted on epoxy-based GFRP by Eldridge et al. (Eldridge \& Fam, 2014) evaluated the effects of exposure to three different environments, namely $23^{\circ}, 40^{\circ}$, and $55^{\circ} \mathrm{C}$ water with $3 \%$ salt concentration, for up to 300 days. The samples exhibited 86,72 , and $61 \%$ ultimate tensile strength retentions, respectively. No reductions occurred to Young's moduli.

In general, there is plenty of literature about the effects of temperature on FRP properties, and they all point to a common theme: higher the temperature (closer to the glass transition temperature, Tg), lower will be the FRP strength. Cold temperatures do not seem to affect the FRP properties adversely and are not of significance in civil/structural applications. (See Chapter 6 for the aging of GFRP in a cold marine environment with hydrostatic pressure). However, alternating cold-warm temperatures can initiate freeze-thaw cycles, which can have a detrimental effect on FRP durability. (see Section 2.2.4.1 for further discussion). 


\subsubsection{Effect of Freeze-Thaw Cycles}

The effect of freeze-thaw (FT) cycles on FRP properties is considered detrimental in general. Shi et al. (2014) concluded that FT cycles reduce tensile properties of common CFRP and GFRP sheets, and the degradation increases with sustained loading. The damage of the fibermatrix interface is caused by thermal incompatibility and subsequent degradation of the constituent materials. Wu et al. (2006) studied FRP bridge decks extensively and found the FT effects to be not significant but also indicated more work with more cycles, and a more extensive range of FT temperatures might be warranted. After using accelerated testing methods and additional FT cycles, Wu \& Yan $(2013,2011)$ concluded that for a high number of FT cycles, the damage to the FRP deck surface could be significant mainly when composites are pre-loaded (e.g., sustained dead load or post-tensioning). They concluded that freeze-thaw increases the interlaminar shear stress between the FRP core and the skin leading to deck delamination and, thus, a shorter service life in the range of 20-30 years unless measures are taken to limit moisture ingress. In a critical review of durability and prediction models of FRP under various environmental conditions, Wang et. al (2016) discussed that freeze-thaw cycles initiates as microcracks are formed in resins during cold temperatures which in turn allow further absorption of surface water at higher temperatures. The cyclic expansion/contraction of this trapped water inside the FRP matrix feeds the FT cycles that ultimately result in plasticization, hydrolysis, and debonding. Temperature strongly affects the moisture absorption rate of FRP composites, and hygrothermal effects can decrease Tg of FRP due to its plasticization effects, so FT when combined with UV and extreme temperature swings can form a degradation spiral. Wang et al. (2016) hypothesized that limited FT cycles, as observed in many FRP bridge decks, has insignificant effects on tensile and flexural properties of FRP 
composites, and for outdoor conditions, FT effects can be best studied with UV radiation. However, additional long-term performance data is needed to validate the FT durability models.

\subsubsection{Fatigue Cycles}

Fatigue life prediction in composites is particularly complex due to the heterogeneity and anisotropy of the material. Fiber type, matrix type, reinforcement structure, stacking sequence, manufacturing quality, loading conditions, environmental conditions, boundary conditions, and long-term behavior can significantly affect fatigue behavior (Dittenber \& GangaRao, 2013). Dittenber's modifications have been suggested in "pre-standard" for load and resistance factor design (LRFD) standard for pultruded FRP structures to provide the strain energy-based fatigue life determination techniques. The following Dittenber-GangaRao fatigue equation (Equation 2.1) found to yield reasonably consistent results for most common GFRP fatigue life predictions for hollow core, pultruded GFRP sections, subjected to three-point bending:

$$
\Delta S=\left(\frac{\left(\sigma^{\mathrm{ult}}\right)^{2} \times \mathrm{IxL}}{\left(48 c^{2} \times \mathrm{E} \times \mathrm{axN}\right)}\right)^{\left(\frac{1}{\mathrm{~m}}\right)} \quad \ldots \mathrm{Eq}(2.1)
$$

Where: $\Delta S=$ Stress range; $\sigma^{\text {ult }}=$ Stress at Rupture; $I=$ Moment of Inertia of tie (expressed in terms of GFRP properties); L = Span length used in MOR calculations (typically from a 3-point modulus of rupture test or computed theoretically); $\mathrm{c}=$ Depth of neutral axis; $\mathrm{E}=$ Modulus of Elasticity of GFRP; $a=13$ (or 1463 for SI units) computed experimental value for GFRP box sections; $\mathrm{m}=10$, computed experimental value for GFRP (also designated as $\bar{m}$ ); $\mathrm{N}=$ Total number of fatigue cycles to failure.

Fatigue loading cycles are physical effects and are best modeled by strain energy methods ( similar to sustained loading). Unlike steel, there is no minimum S-N threshold for FRP. With each fatigue cycle, no matter how small, its impact on FRP strength can be cumulative. 


\subsubsection{Sustained Stress or Creep}

One of the early published investigations on the time-dependent creep of FRP was published by Holmes and Rahman (1980), who tested GFRP box sections for approximately 20 months and discovered that the midspan deflection (loaded at $1 / 3$ of ultimate value) had become roughly twice its elastic value. Mosallam and Bank (Bank \& Mosallan,1991; Mosallam \& Bank, 1991) investigated the viscoelastic behavior of pultruded vinylester GFRP wide-flange sections. After 10,000 hours of loading, the girder mid-span deflection had increased by approximately $22 \%$. The power-law model utilized in the study predicted a decrease of $35 \%$ in the elastic flexural modulus over ten years. Daniali reported on the short and long-term creep response behavior of vinylester and polyester GFRP Tee beams (Daniali, 1991). At the elevated temperatures, Polyester GFRP suffered drastic reductions in strength, shorter creep life, and more considerable creep deflections when compared with vinylester GFRP. McClure and Mohammadi (1995) investigated the compression creep behavior of pultruded FRP angle sections. The authors found little difference between the experimental and the predicted results for both the full-size angle and coupon experiments. A paper by Scott and Zureick (1998) presented the results of an experimental investigation about the long-term behavior (up to 10,000 hours) of pultruded FRP wide-flange beams subjected to longitudinal compressive loading. The strain measurements over time were recorded and compared to a power-law formulation, with good agreement.

A recent discussion about FRP's creep under sustained loading can be found in the Ph.D. dissertation by Youssef at the University of Sherbrooke (Youssef, 2010). Creep is defined as the increase in strain with time at a constant stress level. In polymers, creep occurs due to a combination of elastic deformation (from initial loading) and viscous flow, commonly known as viscoelastic deformation. Due to viscoelasticity, FRP's are more susceptible to creep than are the 
conventional materials of construction such as steel or concrete. Although FRP architecture (i.e., fiber volume fraction, the direction of fibers, etc.) has some influence, polymer matrix remains the primary cause of creep of FRP composites. Creep due to glass fibers is insignificant. Deformation of molecules with molecular segments changing conformations (sliding past one another) cause creep in polymeric materials. Large deformations also cause chain ruptures that are predominant in thermosets since chains are cross-linked into a network. As a result, mechanical properties tend to irreversibly change as a function of time for which load is applied. Temperature, moisture, and other environmental factors also alter the creep behavior due to their effect on molecular activity in polymers. High loading levels damage the fiber-matrix interfacial regime, leading to a slip between the matrix and fiber. Fiber ruptures cause high fiber stresses in the surrounding intact fiber leading to elongation and hence increases the rate of creep near the end of its life.

There have been various models developed to study creep in polymers, the models used in this study were developed by Miyano and Sa (Miyano, Nakada, \& Cai, 2008; Nakada \& Miyano, 2015; Sá et al., 2011). Increasing initial strain with harsh environments was used to determine the creep rupture properties. Findley's model was used to assess the change of modulus with time. A technical paper by GangaRao and Liang (2010) with Batra (2009) proposed a deterministic model based on strain energy to predict the creep life of GFRP samples subjected to tension and bending. They attributed an increase in creep strain to the interconnection of micro-voids in a composite and then matrix softening with time if a composite is in the presence of moisture or other solutions. A similar phenomenon has been noted when a specimen is exposed to temperatures higher than room temperatures. As the percent of initial sustained strain increase in relation to GFRP composite's static strain to failure, sustained strain to static failure strain ratio has been found to be increasing with time in a power-law form; thus, resulting in an accurate methodology of creep 
rupture time prediction for a given sustained strain. They concluded that the internal strain energy of the material is expended under the externally induced work due to the damage accumulated in the material through matrix cracking or de-bonding of sizing material of glass fibers from the vinylester matrix. As the damage gets accumulated, the flexural strain energy expended by GFRP samples is found to be a function of 1) Induced sustained strain level as a percent of static failure strain, 2) Time to failure under varying sustained strain levels, and 3) Mechanical properties of the GFRP composite material. The creep related mechanical properties were found to depend primarily on the constituents such as fiber and matrix, percent of voids, cure percent, and others.

Studies have indicated that if sustained stress is less than $60 \%$ of the average ultimate tensile strength $\left(f_{u, \text { ave }}\right)$, creep rupture is less likely to occur immediately. However, in practice, much lower numbers are used. For example, MIL-17 Composite Materials Handbook suggests limiting sustained stress to $35 \%$ or $40 \%$, with $30 \%$ being a commonly used number for glass FRP. Greenwood (2002) deduced through series of creep-rupture testing that the creep stress limit is 45 $\% \mathrm{f}_{\mathrm{u} \text {,ave }}$ for 50 -year structure survivability. The latter claim is supported by the findings of Youssef et al. (2010). No sign of creep rupture at $45 \% \mathrm{f}_{\mathrm{u}, \mathrm{ave}}$ was found and confirmed by microstructural analysis. Rupture susceptibility is evident at about $60 \% \mathrm{f}_{\mathrm{u}, \text { ave }}$ (tensile). However, flexural creep tests conducted at WVU-CFC (Batra, 2009) indicate rupture susceptibility at 50\%. See Chapter 5 for additional details about Youssef's sustained stress experiments, which are used to develop the sustained stress effect model of this research.

Like fatigue failure response of GFRP composites, creep response also has three stages of progression to failure (Batra, 2009). Stage I is identified by strain energy expended near instantaneously when the load is statically applied to reach a sustained value. Stage II signifies the propagation of creep deflection increase or energy consumption with time, primarily by matrix 
yielding or cracking, leading to interlaminar crack propagation and delamination across the GFRP composite member thickness. Stage II is of primary significance for creep life prediction. Stage III (aka tertiary creep) is identified with a rapid increase in expended energy. Stage III has been characterized as a very high-energy expenditure of a composite specimen per unit time, which is about 20 times higher (or even more in some cases) when compared with Stage II (see Section 5.4.1 and Figure 5.1 for further explanation about modeling for creep effects). Batra's study found that Findley's Power Law model works well for the viscoelastic behavior of FRPs under constant static stress.

Creep Behavior of polymer matrix or composite under uniaxial stress is represented by Creep Compliance $\mathrm{D}(\mathrm{t})$ (also defined as Creep Coefficient or Creep Rate) defined as:

Creep compliance $=\mathrm{D}(\mathrm{t})=\frac{\varepsilon(t)}{\sigma}$

Where $\sigma$ is the constant (sustained) stress applied and $\varepsilon(t)$ is the strain as a function of time. Generally, creep compliance increases with an increase in stress level, temperature, and time. It is also a function of the fiber orientation angle.

Findley's Power-law model was initially developed by Findley in 1944 (Findley, 1944, and Findley, 1987) to study the viscoelastic properties of polymers, since then this model has been usefully used to model creep properties of polymer composites. Findley's power-law models the creep strain $\varepsilon(t)$ using a form defined as:

$$
\varepsilon_{t}=\varepsilon_{0}+m\left(t / t_{0}\right)^{n} \text { or } \varepsilon_{t}=\varepsilon_{0}+m t^{n} \quad \ldots(E q .2 .2) \text {, when } t_{0}=1
$$

Where, $\mathrm{t}$ is the time, $\varepsilon_{\mathrm{t}}$ is the total strain at given time $\mathrm{t}, \varepsilon_{0}$ is a stress-dependent initial elastic strain, and " $\mathrm{m}$ " and " $\mathrm{n}$ " are the creep parameters which are determined experimentally. Typically, $t_{0}$ is the scaling factor and is taken as unity. The development of Findley's power function for reliability-durability modeling of creep effect is shown in detail in Chapter 5 . 
Compression creep of pultruded FRP composites made with Vinylester resin and unidirectional E-glass roving was also investigated using Findley power law by Scott and Zureick, which observed that at a stress level of $60 \%$ material begins to show nonlinear behavior (Scott \& Zureick, 1998). In addition to the Findley power-law model, Boltzmann-Volterra superposition principle and Schapery nonlinear viscoelastic theory have also been used to describe the viscoelastic behavior of polymers and polymer composites.

\subsection{DURABILITy PREDiction MEthods}

Prediction of FRP durability under common civil engineering applications has been at the forefront of numerous studies. Some of the early discussions about FRP durability predictions in the civil engineering field were conducted by Chin et al. (1997). The lack of understanding of longterm durability performance has been identified as a significant technical barrier towards high volume use infrastructure systems (Karbhari et al., 2003; Micelli \& Nanni, 2004). For relatively newer construction materials such as FRP, it may take decades of careful monitoring to evaluate and understand its performance under the natural environments, so it becomes critical to formulating accelerated means (e.g., ATM) to assess and predict their long-term durability.

Numerous past and current projects at WVU-CFC (Kajorncheappunngam et al., 1999; Vijay et al., 1999; Wang, GangaRao, Liang, \& Liu, 2016) have provided a vast amount of test data on the durability performance of FRP and have kept the durability debate at the center stage.

The conventional methods used to predict the durability of FRP under various environments are described in the following section. 


\subsubsection{Accelerated Test Methods (ATM)}

Due to time constraints, most new materials are tested in a lab using accelerated testing methods (ATM) to predict their long-term behaviors. The process used to determine various strength reduction factors in many LRFD design codes is typically based on conducting strength tests and recording the statistical variations of material properties of a vast number of structural components over a long period, often lasting the life span. For relatively newer materials such as FRP, strength test data over an extended period is not generally available or is cost-prohibitive to obtain. However, it is possible to simulate the lifetime aging of a component in laboratories, within a short amount of time, using accelerated aging tests. These tests have been proven to provide reasonably accurate estimates and can also be used to simulate the life span of FRP. Despite a wide-spread use of ATMs, the reproducibility of many accelerated test results has been a severe problem, mainly when tests are conducted at distant sites under varied laboratory conditions for different periods and particular purposes.

The use of ATM is well documented for FRP composites. There are several good books and numerous publications providing information about the use of accelerated testing methods, the Arrhenius principle, and time-super imposition methods. A paper published by the National Research Council (National Research Council, 1996) provides a good source of information about accelerating aging of materials and structures. A textbook by Hota et al. (2006) and paper by Vijay et al. (Vijay, 1999) also provides a step by step discussion on the aging of composite materials.

\subsubsection{Natural Aging Methods}

FRP has been used in the civil engineering field for well over two decades; however, there are only a few actual case studies available to illustrate the effect of aging on the built structures. One such study was undertaken by Keller et al. $(2007,2016)$ on two occasions that included a 
GFRP bridge and a GFRP building using the same materials under two different environments in about 15-17 years after their installations (Table 2.1). Field tensile strength data is consistent with the results of ATM tests conducted at WVU-CFC. The study concluded that there is not much change in the structural stiffness, and most of the material strength reduction can be attributed to freeze-thaw and UV irradiation effect. The report concluded that the structures still do meet all safety requirements and will continue to do so for an extended period. With the understanding that the prevalent conservative FRP design methodology uses generous safety factors and unusually low design reduction factors, this finding should not be a surprise. The study, however, questioned the correlation of many accelerated tests that often conducted at specimen level to FRP natural performance at full scale and under a natural outdoor environment.

Table 2.1: Natural Outdoor Aging Comparisons (Keller et al., 2007, 2016)

\begin{tabular}{|c|c|c|c|c|c|}
\hline \multicolumn{6}{|c|}{ Overall System Stiffness Degradation } \\
\hline Test Year & Age & $\begin{array}{c}\text { Bolted Span } \\
\text { Deflection (\%) }\end{array}$ & $\begin{array}{l}\text { Bonded Span } \\
\text { Deflection (\%) }\end{array}$ & \multicolumn{2}{|c|}{ Material Type } \\
\hline 1997 & 0 & $100 \%$ & $100 \%$ & \multirow{3}{*}{\multicolumn{2}{|c|}{$\begin{array}{c}\text { E-Glass, Pultruded, } \\
\text { Isophthalic Polyester, } \\
\text { FVF }=50 \%\end{array}$}} \\
\hline 2005 & 8 & $97 \%$ & $86 \%$ & & \\
\hline 2014 & 17 & $97 \%$ & $86 \%$ & & \\
\hline \multicolumn{6}{|c|}{ Material Strength Degradation } \\
\hline & \multicolumn{3}{|c|}{ Axial Young's Modulus } & \multicolumn{2}{|c|}{ Axial Tensile Strength } \\
\hline Test Year & Age & Ex (Flange) & $E x($ Web) & Fu (Flange) & $F u(W e b)$ \\
\hline 1997 & 0 & $100 \%$ & $100 \%$ & $100 \%$ & $100 \%$ \\
\hline 2005 & 8 & $103 \%$ & $100 \%$ & $82 \%$ & $87 \%$ \\
\hline 2014 & 17 & $98 \%$ & $95 \%$ & $68 \%$ & $83 \%$ \\
\hline
\end{tabular}

Another on-going long-term natural weathering study is at WVU Constructed Facility Center (Dittenber, GangaRao \& Liang, 2014), where naturally aged FRP samples (manufactured between 1991 and 2006) were tested to document their aging behavior against their original values after 10+ years. This work was further advanced by Barker (Barker, 2019) by conducting additional testing of the same FRP materials. The study indicates that for many FRP samples, 
particularly the older specimens, and material properties improved with aging, which can be potentially attributed to inadequate initial curing. Out of all properties evaluated, short beam shear strength appears to be the most sensitive to age-induced reduction since it correlates highly to known FRP matrix's degradation over time. The average flexural modulus and strength reduction were about $10 \%$ over a broad spectrum, but it improves to about $5 \%$ for FRP samples manufactured in the last decades (indicating a general improvement in the FRP manufacturing process including the quality of resins, curing methods, etc.).

Wu and Yan (Wu \& Yan, 2011, 2013) correlated laboratory tests of an FRP bridge deck with naturally aged field measurements for four years and predicted deck's short-term performance concerning ply failure, shear failure, local buckling failure, and excessive deflections. Various failure modes for long-term performance were also predicted mostly stemming from interlaminar shear degradation, and the study indicated limitations due to lack of sufficient lab and long-term field data. They correlated laboratory time scale with an in-service time scale to predict the changes in stiffness and deflection of a monitored FRP bridge deck over 4 years.

Barker (Barker, 2019) at WVU-CFC evaluated about 450 samples of 1/8" and 1/4" FRP (E-Glass Fibers in vinylester Resin, Fiber Volume Fraction of 0.34 by weight) for strength retention under accelerated testing ranging in duration from 150 days to 540 days, at temperatures of $-23,22$, and 71 degree Celsius, in environments of acidic (HCL solution in tap water at $\mathrm{pH}=3$ ), neutral (Tap water), and alkaline $(\mathrm{NaOH}$ solution in tap water at $\mathrm{pH}=13)$. Accelerated tests were calibrated using natural data from 11 to 18 -year old naturally aged samples (exposed to indoor, partially outdoor, and fully exposed to outdoor environment). The samples were primarily tested for interlaminar shear strength (ILSS) in fiber dominated longitudinal and matrix dominant transverse direction. The degradation rate was found to be much slower for thicker specimens 
than that of the thinner sample. As in many other studies, higher temperatures and higher $\mathrm{pH}$ are more detrimental to FRP than the room or lower temperatures and neutral or acidic environments. Over 11 to 26 years of WVU test data indicates that FRP retains strengths well under a neutral environment (i.e., exposed to indoor air, room temperature, and neutral $\mathrm{pH}$ ).

\subsection{The State of CURRENT PRACTICE}

Recommendations for strength reduction factors for various types of FRP composites under a wide variety of environmental conditions are loosely available. Some are derived based on a consensus of practicing professionals, and some came from specific work performed by individual researchers for particular applications.

Since there is on-going work on (long-term) durability of FRP composites being performed by researchers across the globe, Karbhari (V. M. Karbhari \& Lee, 2011) suggested creating a database of durability data or an integrated knowledge system to serve as a central repository for designers and users of the FRP. A summary of various reduction factors for multiple fibers and resins used worldwide was compiled Myers (Myers \& Viswanath, 2006), indicating the spread of the environmental reduction factors (Table 2.1). Specific loading conditions (e.g., bending, tension, shear, etc.) and the operating environment influence the selection. The reduction factors for FRP (Fiber Reinforced Polymers) proposed by various guidelines from countries including the USA, Japan, Canada, Great Britain, Norway, and Europe were assembled to form a database of current environmental reduction factors to review their appropriateness. These FRP materials included carbon, aramid, glass, and hybrid FRP's. The reduction factors obtained from the results

of the recent laboratory studies are compared with those provided by various codes on FRP around the world (Table 2.2). 
Various laboratory studies have shown that there is strength degradation in all FRP materials used in conjunction with concrete systems when exposed to different aggressive environments. The use of strength reduction factors is a common practice to account for a wide range of environmental and loading conditions, but it can also be misleading if not used in a correct context. It should be noted that this research focuses on the Pultruded FRP section only and does not include the application of FRP bars or bonded layers/sheets with concrete.

The following Tables (2.2,2,3, and 2.4) provide a comprehensive summary of commonly sed strength reduction factors found in published FRP literature across the globe. The state of current practice for sustained stress limits can be found in Chapter 5 .

Table 2.2: Review of Current Practice of Strength Reduction Factors

\begin{tabular}{|c|c|c|c|c|}
\hline FRP Use / Environmental Conditions & $\begin{array}{l}\text { Fiber } \\
\text { Type }\end{array}$ & Matrix Type & Limitations & Source \\
\hline \multicolumn{4}{|c|}{ Externally Bonded Systems (used in the strengthening of Concrete) } & \multirow{20}{*}{$\begin{array}{l}\mathrm{ACl}-440.2 \mathrm{R}-8 \\
(2008)\end{array}$} \\
\hline \multicolumn{4}{|c|}{ Interior Exposure } & \\
\hline & Carbon & & $C_{E}=0.95$ & \\
\hline & Glass & & $C_{E}=0.75$ & \\
\hline & Aramid & & $C_{E}=0.85$ & \\
\hline \multicolumn{4}{|c|}{ Exterior Exposure (Normal Outdoor such as bridges, open parking garages) } & \\
\hline & Carbon & & $C_{E}=0.85$ & \\
\hline & Glass & & $C_{E}=0.65$ & \\
\hline & Aramid & & $C_{E}=0.75$ & \\
\hline \multicolumn{4}{|c|}{ Aggressive Exposure (e.g., Chemical plants, wastewater treatment plants) } & \\
\hline & Carbon & & $C_{E}=0.85$ & \\
\hline & Glass & & $C_{E}=0.50$ & \\
\hline & Aramid & & $C_{E}=0.70$ & \\
\hline \multicolumn{4}{|l|}{ Sustained stress / cyclic loading } & \\
\hline & Carbon & & $f_{s}<0.55 f_{f u}$ & \\
\hline & Glass & & $f_{s}<0.20 f_{f u}$ & \\
\hline & Aramid & & $\mathrm{f}_{\mathrm{s}}<0.30 \mathrm{f}_{\mathrm{fu}}$ & \\
\hline \multicolumn{4}{|l|}{ External Shear Reinforcement } & \\
\hline Completely wrapped schemes & & & $\Psi_{\mathrm{f}}=0.95$ & \\
\hline $\begin{array}{l}\text { Three or two (opposite) sided wrapped } \\
\text { schemes }\end{array}$ & & & $\Psi_{f}=0.85$ & \\
\hline \multicolumn{4}{|c|}{ Internally Bonded Systems (used in original concrete construction) } & \multirow{3}{*}{$\begin{array}{l}\mathrm{ACl} 440.1 \mathrm{R}-17 \\
\text { (DRAFT) }\end{array}$} \\
\hline \multirow{2}{*}{$\begin{array}{l}\text { Concrete not exposed to earth or } \\
\text { weather }\end{array}$} & Carbon & & $C_{E}=1.0$ & \\
\hline & Glass & & $C_{E}=0.8$ & \\
\hline
\end{tabular}




\begin{tabular}{|c|c|c|c|c|}
\hline & Aramid & & $C_{E}=0.9$ & \\
\hline \multirow[t]{3}{*}{ Concrete exposed to earth and weather } & Carbon & & $C_{E}=0.9$ & \\
\hline & Glass & & $C_{E}=0.7$ & \\
\hline & Aramid & & $C_{E}=0.8$ & \\
\hline \multicolumn{4}{|l|}{ Creep Rupture stress limits } & \\
\hline & Carbon & & $f_{f, s}<0.55 f_{f u}$ & \\
\hline & Glass & & $f_{f, s}<0.20 f_{f u}$ & \\
\hline & Aramid & & $f_{f, s}<0.30 f_{f u}$ & \\
\hline \multicolumn{4}{|c|}{ Safety Factors (for Allowable Stress Design of Pultruded FRP Members) } & \multirow{7}{*}{$\begin{array}{l}\text { FRP Manufacture's } \\
\text { recommendations } \\
\text { (Bedford } \\
\text { Reinforced } \\
\text { Plastics, Creative } \\
\text { Pultrusions, } \\
\text { Strongwell, } \\
\text { Fiberline, etc.) }\end{array}$} \\
\hline Pultruded Beams - Flexural Strength & & & $\Omega \geq 2.5-3.0$ & \\
\hline Pultruded Beams -Shear Strength & & & $\Omega \geq 2.5-3.0$ & \\
\hline Pultruded Beams - Buckling Strength & & & $\Omega \geq 2.5$ & \\
\hline $\begin{array}{l}\text { Pultruded Columns - Compressive } \\
\text { Strength }\end{array}$ & & & $\Omega \geq 3.0$ & \\
\hline $\begin{array}{l}\text { Axial Tension Members - Tension } \\
\text { Strength }\end{array}$ & & & $\Omega \geq 2.0$ & \\
\hline \multirow[t]{2}{*}{$\begin{array}{l}\text { Joints and Connections - Bearing } \\
\text { Strength }\end{array}$} & & & $\Omega \geq 4.0$ & \\
\hline & & & & \multirow[t]{2}{*}{ General Practice } \\
\hline $\begin{array}{l}\text { Long-term use and harsh environment } \\
\text { (Knock-down factor) - Cumulative limit }\end{array}$ & & & $\Omega \geq 5.0$ & \\
\hline \multicolumn{4}{|c|}{ Resistance Factors for LRFD of Pultruded Members } & \multirow{22}{*}{$\begin{array}{l}\text { ACMA, 2010, Pre- } \\
\text { Standard for LRFD } \\
\text { of Pultruded FRP } \\
\text { Structures }\end{array}$} \\
\hline \multicolumn{4}{|c|}{\begin{tabular}{|l|l|} 
Flexural and Shear Members: & \\
\end{tabular}} & \\
\hline Rupture strength & & & $\phi=0.65$ & \\
\hline Local buckling strength & & & $\phi=0.65$ & \\
\hline Global buckling strength & & & $\phi=0.65$ & \\
\hline Local crippling & & & $\phi=0.65$ & \\
\hline Torsional strength & & & $\phi=0.65$ & \\
\hline \multicolumn{4}{|l|}{ Axial Compression Members: } & \\
\hline Flexural buckling strength & & & $\phi=0.65$ & \\
\hline Local buckling strength & & & $\phi=0.65$ & \\
\hline \multicolumn{4}{|l|}{ Axial Tension Members: } & \\
\hline Tension rupture strength & & & $\phi=0.65$ & \\
\hline \multicolumn{4}{|l|}{ Adjustment for Moisture: } & \\
\hline Strength & & Vinylester & $C_{M}=0.85$ & \\
\hline Strength & & Polyester & $C_{M}=0.80$ & \\
\hline Elastic Modulus & & Vinylester & $C_{M}=0.95$ & \\
\hline Elastic Modulus & & Polyester & $C_{M}=0.90$ & \\
\hline \multicolumn{4}{|l|}{$\begin{array}{l}\text { Adjustment for Temperature: } \\
\left(90^{\circ} \mathrm{F}<T<140^{\circ} \mathrm{F}\right)\end{array}$} & \\
\hline Strength & & Vinylester & $C_{\mathrm{T}}=1.7-0.008 \mathrm{~T}$ & \\
\hline Strength & & Polyester & $C_{T}=1.9-0.010 T$ & \\
\hline Elastic Modulus & & Vinylester & $C_{T}=1.5-0.006 \mathrm{~T}$ & \\
\hline Elastic Modulus & & Polyester & $C_{T}=1.7-0.008 T$ & \\
\hline
\end{tabular}




\begin{tabular}{|c|c|c|}
\hline Strength & $C_{L S}=1.2$ & \\
\hline Stiffness & $\mathrm{C}_{\mathrm{CA}}=1.2$ & \\
\hline \multicolumn{2}{|c|}{ Load Factors for LRFD of Pultruded Members } & \\
\hline 1.4D (permanent load) & $\lambda=0.4$ & \\
\hline $\begin{array}{l}1.2 \mathrm{D}+1.6 \mathrm{~L}+0.5(\mathrm{Lr} \text { or } \mathrm{S} \text { or } \mathrm{R}) \\
\text { when } \mathrm{L} \text { is from occupancy }\end{array}$ & $\lambda=0.8$ & \\
\hline $\begin{array}{l}1.2 \mathrm{D}+1.6 \mathrm{~L}+0.5(\mathrm{Lr} \text { or } \mathrm{S} \text { or } \mathrm{R}) \\
\text { when } \mathrm{L} \text { is from storage }\end{array}$ & $\lambda=0.6$ & \\
\hline $\begin{array}{l}1.2 \mathrm{D}+1.6 \mathrm{~L}+0.5(\mathrm{Lr} \text { or } \mathrm{S} \text { or } \mathrm{R} \\
\text { when } \mathrm{L} \text { is from impact }\end{array}$ & $\lambda=1.0$ & \\
\hline $1.2 \mathrm{D}+1.6(\mathrm{Lr}$ or $\mathrm{S}$ or $\mathrm{R})+(0.5 \mathrm{~L}$ or $0.5 \mathrm{~W})$ & $\lambda=0.75$ & \\
\hline $1.2 \mathrm{D}+1.0 \mathrm{~W}+0.5 \mathrm{~L}+0.5(\mathrm{Lr}$ or $\mathrm{S}$ or $\mathrm{R})$ & $\lambda=1.0$ & \\
\hline $1.2 \mathrm{D}+1.0 \mathrm{E}+0.5 \mathrm{~L}+0.2 \mathrm{~S}$ & $\lambda=1.0$ & \\
\hline $0.9 \mathrm{D}+1.0 \mathrm{~W}$ & $\lambda=1.0$ & \\
\hline $0.9 \mathrm{D}+1.0 \mathrm{E}$ & $\lambda=1.0$ & \\
\hline Fa (Flood loads) & $\lambda=0.75$ & \\
\hline Atmospheric ice loads & $\lambda=0.75$ & \\
\hline
\end{tabular}

\section{LEGEND:}

$C_{E}=$ Environmental reduction factor

$\mathrm{fs}=$ Service stress

$\mathrm{f}_{\mathrm{fu}}=$ Ultimate stress strength

$f_{f, s}=$ Stress level induced in FRP by sustained loads

$\Psi_{\mathrm{f}}=$ Additional reduction factor

$\mathrm{SF}=$ Safety Factor

$\Omega=$ Knock-down Factor (to account for environmental impact or aging of FRP)

$\phi=$ Resistance factor used to modify member strength in LRFD

$\lambda=$ Time effect factor used to modify load effect in LRFD

$\mathrm{C}_{\mathrm{M}}=$ Moisture condition factor

$C_{T}=$ Temperature factor

$\mathrm{C}_{\mathrm{LS}}=$ Load sharing factor

$\mathrm{C}_{\mathrm{CA}}=$ composite action factor

$\mathrm{D}=$ Dead Load

$\mathrm{L}=$ Live Load

$L_{R}=$ Live load on roof caused during ordinary maintenance

W $=$ Wind Load

$\mathrm{S}=$ Snow Load

$\mathrm{E}=$ Earthquake Load

$\mathrm{R}=$ Rain Load 
Table 2.3: Comparison of the Ranges for Strength Reduction Factors

\begin{tabular}{|l|l|l|l|l|}
\hline Criteria & $\begin{array}{l}\text { Type of Fibers } \\
\text { Used }\end{array}$ & $\begin{array}{l}\text { Highest Value } \\
\text { Used }\end{array}$ & $\begin{array}{l}\text { Median Value } \\
\text { Used }\end{array}$ & $\begin{array}{l}\text { Lowest Value } \\
\text { Used }\end{array}$ \\
\hline \multirow{2}{*}{$\begin{array}{l}\text { Reduction for Environmental } \\
\text { Degradation }\end{array}$} & CFRP & 1.00 & 0.88 & 0.60 \\
\cline { 2 - 5 } & GFRP & 0.80 & 0.70 & 0.14 \\
\cline { 2 - 5 } & AFRP & 0.90 & 0.85 & 0.31 \\
\hline \multirow{4}{*}{ Reduction for Sustained Stress } & GFRP & 1.00 & 0.95 & 0.90 \\
\cline { 2 - 5 } & GFRP & 1.00 & 0.90 & 0.80 \\
\cline { 2 - 5 } & AFRP & 1.00 & 0.85 & 0.70 \\
\hline \multirow{2}{*}{$\begin{array}{l}\text { Total Strength Reduction for } \\
\text { Sustained Stress }\end{array}$} & GFRP & 1.00 & 0.86 & 0.60 \\
\cline { 2 - 5 } & GFRP & 0.80 & 0.55 & 0.30 \\
\cline { 2 - 5 } & AFRP & 0.90 & 0.74 & 0.42 \\
\hline Creep Rupture Limits & GFRP & 0.55 & 0.55 & 0.55 \\
\cline { 2 - 5 } & GFRP & 0.22 & 0.22 & 0.22 \\
\cline { 2 - 5 } & AFRP & 0.30 & 0.30 & 0.30 \\
\hline \multirow{4}{*}{ Stress Limits for Permanent Load } & GFRP & 0.85 & 0.76 & 0.44 \\
\cline { 2 - 5 } & GFRP & 0.75 & 0.70 & 0.14 \\
\cline { 2 - 5 } & AFRP & 0.85 & 0.70 & 0.16 \\
\hline
\end{tabular}

Note: The above values are in use for various criteria. Recreated from a table compiled initially by Myers (Myers $\&$ Viswanath, 2006)

Table 2.4: Comparison of Code and Experimental Values of Reduction Factors

\begin{tabular}{|c|c|c|c|c|c|c|c|}
\hline \multirow[t]{2}{*}{ Criteria } & \multirow{2}{*}{$\begin{array}{l}\text { Type of } \\
\text { Fibers } \\
\text { Used }\end{array}$} & \multicolumn{2}{|c|}{ Highest Value Used } & \multicolumn{2}{|c|}{ Median Value Used } & \multicolumn{2}{|c|}{ Lowest Value Used } \\
\hline & & $\begin{array}{l}\text { Code } \\
\text { Specified }\end{array}$ & $\begin{array}{l}\text { Experimenta } \\
\text { lly } \\
\text { Determined }\end{array}$ & $\begin{array}{l}\text { Code } \\
\text { Specified }\end{array}$ & $\begin{array}{l}\text { Experimentall } \\
\text { y Determined }\end{array}$ & $\begin{array}{l}\text { Code } \\
\text { Specified }\end{array}$ & $\begin{array}{l}\text { Experime } \\
\text { ntally } \\
\text { Determin } \\
\text { ed }\end{array}$ \\
\hline \multirow{3}{*}{$\begin{array}{l}\text { Reduction for } \\
\text { Environmental } \\
\text { Degradation }\end{array}$} & CFRP & 1.00 & 1.00 & 0.88 & 0.90 & 0.60 & 0.67 \\
\hline & GFRP & 0.80 & 0.97 & 0.70 & 0.81 & 0.14 & 0.15 \\
\hline & AFRP & 0.90 & 0.98 & 0.85 & 0.69 & 0.31 & 0.20 \\
\hline
\end{tabular}

Note: The above values are in use for various criteria and compares Code Specified Environmental Reduction Factors with Experimentally Determined values. Values are recreated from a table compiled initially by Myers (Myers \& Viswanath, 2006). Similar results for GFRP composites are also found by Barker (2019), Manalo et al. (2020), and many other researchers. 


\subsection{RELIABILITY APPROACH}

The Classic definition of Reliability (McGraw-Hill Dictionary of Scientific and Technical Terms., 2003) is:

Reliability: the probability that a component part, equipment, or system will satisfactorily perform its intended function under given circumstances, such as environmental conditions, limitations as to operating time, and frequency and thoroughness of maintenance for a specified period of time.

In simple words, reliability can be measured as a (statistical) probability that an item or facility will perform its intended function for a specified period under well-defined (design) service conditions. In our case, the item or facility is a structural component, a structural member, or an entire structural system (note: members or systems are not included in this research). In studies related to "Structural Safety," reliability is measurable and quantifiable, and a structural component can be designed for target reliability (Schneider, 2006).

In general, mass-produced composites show more scatter in their material properties (along with many independent variables which are still evolving) than most commonly manufactured composite materials in construction (e.g., structural steel). Some composites can fail without much visual warning signs, or some can age in a traditional "wear-in" and "wear-out" curve (Figure 2.1). In order to account for these uncertainties, traditional design methodology uses a wide range of factors over the assumed fixed material properties (working stress design or deterministic approach), which may result in a significant reduction of composite strength (50\% or more). Often such a high safety margin generates a considerable increase of a structure's required dimensions 
(e.g., cross-section area) and strengths during the design phase. Such increases lead to the higher construction cost of structures made of composites than other traditional materials such as steel or concrete (Dehmous et al., 2008).

The concept of using a reliability approach to overcome similar difficulties in the design of conventional construction materials is not new. However, it is still relatively new for FRP composites due to the absence of performance data of composite components and systems over a longer service life (e.g., 100-year for many civil engineering structures). The need for a standard procedure to establish nominal strengths and stiffness for use in load and resistance factor design (LRFD) standards governing FRP composite design were identified in the early 1990s. Wetherhold and Ucci (1994) described that the reliability approach offers a useful tool for structural design, maintenance, risk management, and optimization of FRP composites. It treats the strengths and stresses as random variables, unlike the conventional working stress/deterministic design approach. This approach requires making three important choices of:

1. Statistical parameters (e.g., distribution type, mean, and standard deviation) which describe the various uncertainties involved at various periods during the service life of a composite structure, both in terms of loads and resistances.

2. Performance function (i.e., failure criterion and its relationship with time-dependent variables of loads and resistances) that mathematically defines the structural failure.

3. Failure probability measurement (e.g., Reliability Index or Reliability Coefficient) that serves as a quantitative measure of the structural reliability.

Note that step 3 uses statistical parameters (as defined in step 1) and performance function (as defined in step 2) to facilitate the probability of failure calculation. 
Wetherhold (1994) further described various methods of computing the structural reliability, which can be summarized as the following two general categories:

1. Simulation Methods: they give the most accurate results and can cover a wide variety of complex failure modes (when a direct solution to the calculations of failure probability is not possible or too cumbersome), provided an adequate number of simulations can be reasonably performed for various pre-defined failure criteria. Monte Carlo simulation is widely used in this category; however, other modified rapid converging simulation methods are also available.

2. Approximate Methods: they are also known as fast probability integration (FPI), which defines the failure threshold (limit surface) in a) a linear form using a first-order reliability moment (FORM), and b) in a curved form using second-order reliability moment (SORM) methods. First-order methods such as Linear Failure Plane, Hasofer-Lind, and Rackwitz-Fiessler methods are typically considered adequate for the most common applications in the civil engineering field. (Nowak \& Collins, 2013, Wetherhold, 1994)

Before formulating a probabilistic solution for FRP composites' structural components, understanding of a deterministic solution to the failure mechanisms is necessary. In addition to the theoretical formulation of various failure mechanisms, Weibull's distribution function is used for characteristic mechanical property determination of composites, since most composites, despite their higher strengths, tend to fail in a brittle manner (Soares, 1997). Weibull's weakest link theory (Weibull, 1939) applies to unidirectional composites whose fibers contribute to the majority of its strength (except shear where the contribution of the matrix is also significant); however, it fails to account for the effects of load sharing among the fibers due to the presence of a ductile matrix and for composites whose fibers are oriented in various directions. It is difficult to apply Weibull's weakest link theory to the more common type of composite that uses multi-directional fabric and 
behaves close to the pseudo-isotropic manner (but can be susceptible to stress concentration issues when the fabric is woven or braided).

Two-parameter, rather than three-parameter, Weibull distribution is used in the determination of the "characteristic strength" of composites (Zureick, Bennett, \& Ellingwood, 2006), but normal, log-normal, and other types of more straightforward probability density functions cannot be rejected (in statistical terms) either. Nonetheless, modern composite materials which are made up of a large number of woven fibers in multiple orientations (i.e., engineered fabric), encased in different types of matrices, manufactured in a wide variety of techniques ranging from manual labor to automated pultruding machines, and whose properties, dimensions, curing, aging, and initial defects can be best described by a probabilistic formulation (i.e., inadequate deterministic data due to many variables and processes). According to Central Limit Theorem, when a large, diverse independent population is present, as is the case with several FRP databases used in this research, the distribution tends to fit a normal or near-normal distribution. The above discussions are vital as we explore various reliability techniques in the context of FRP durability.

There is limited literature addressing the reliability aspects of FRP composites designs. Several papers by Karbhari et al. ( 2005, 2006, 2008, 2009, and 2011) provide good discussions about the variability of FRP properties but do not provide adequate insight into the reliability design development for FRP. More work in this field has been recommended by Karbhari et. al.

The use of a reliability approach has been successfully adopted in design codes for conventional materials such as steel and concrete for over two decades; however, as we know, that is not common with composite materials. Unlike age-old common construction materials such as steel, concrete, and timber, the FRP fabrication process employs many unique ingredients and 
methods. The current process is susceptible to inconsistent and varied manufacturing and curing processes. Even with strict quality control/quality assurance processes in place, FRP can have higher variability in the material properties of the finished products than what can be expected from conventional materials. This variability, in turn, renders composite materials as an appropriate choice to apply/implement a reliability approach in the development of design codes/guidelines. Current composite design practices borrow the formulations used for homogeneous isotropic materials (e.g., steel), which may lead to unusually conservative designs with widespread factors of safety for material strengths and individual components. The reliability approach, as proposed in this research, narrows this wide range of factors of safety by using the concept of reliability index (often described as the Greek letter " $\beta$ "). Furthermore, this research defines the reliability index as a time-dependent variable " $\beta \mathrm{t}$ " as compared to a fixed parameter " $\beta$ " used in the current LRFD codes for concrete and steel. This approach results in two distinct values of reliability indices, one at the design phase and another at the end of the target service life (Section 2.6).

\subsubsection{Variability of Properties}

Strength measurements on fiber-reinforced-polymer (FRP) composites taken over a long period exhibit large scatter mainly as a consequence of the complexity and interaction of failure modes, inherent inhomogeneity, anisotropy and manufacturing induced defects in either unidirectional or multidirectional laminated configurations (Philippidis \& Lekou, 1998). Therefore, a deterministic approach in design for the strength of FRP composite structures, which uses as characteristic values for strength (in various material symmetry directions), use of mean regressed values derived from accelerated or natural test results (without refinements) may lead to incorrect predictions concerning failure onset (Figure 2.3). Furthermore, such a methodology is 
not conclusive concerning the reliability level of the structural component under consideration due to the inherent variability of both the material properties and the load effects.

It is also essential to recognize that at present, it is very likely there is not enough FRP composite data available to fully establish a reliability approach that can lead to LRFD style deterministic design codes. However, based on limited data together with many quality improvements (e.g., resin quality, manufacturing process, additives) taking place rapidly, we can identify critical parameters affecting the FRP reliability and durability, make a reasonable assumption, and integrate deterministic methods with a probabilistic approach (Section 3.3).

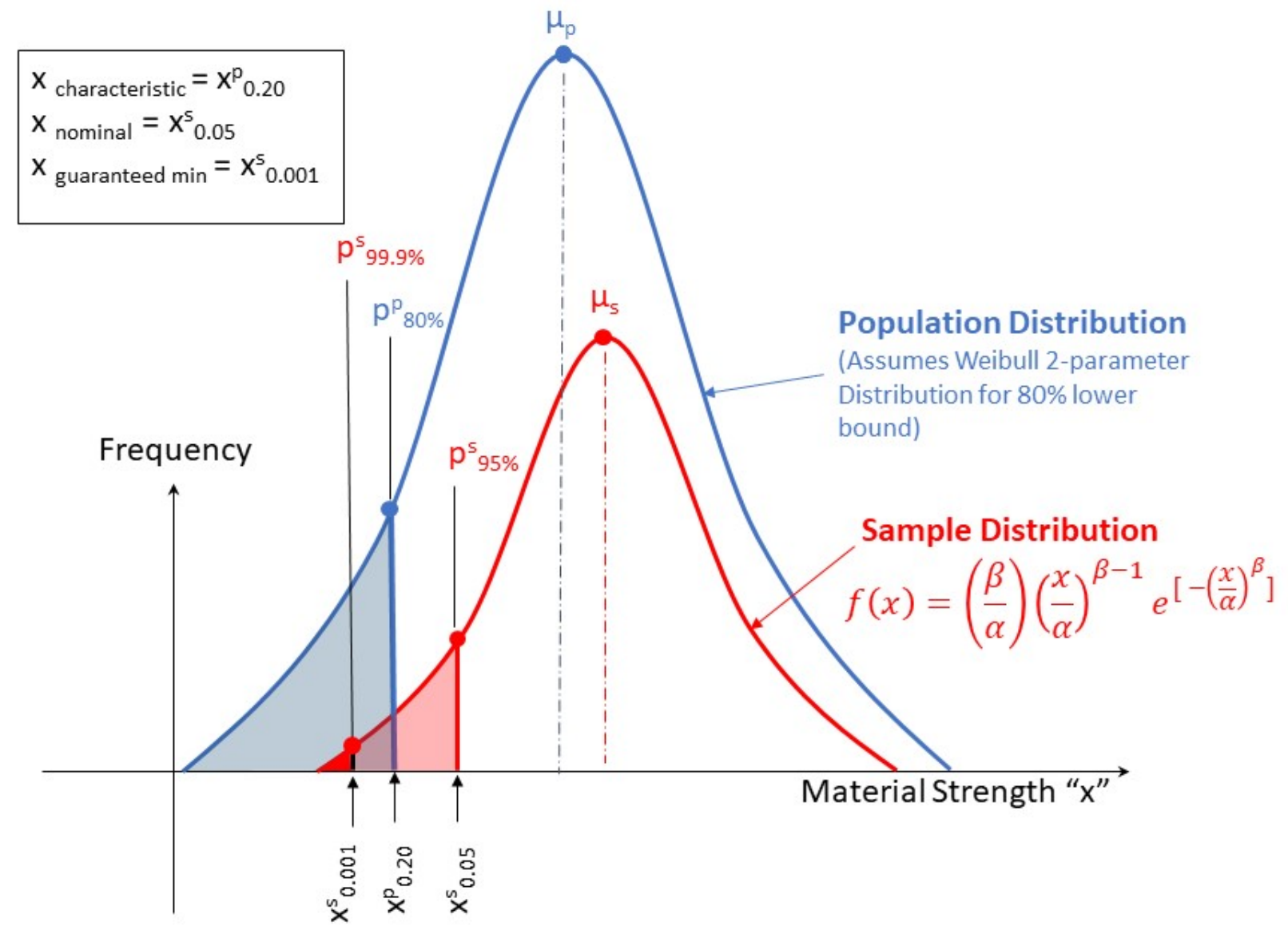

Figure 2.3: FRP Nominal and Characteristics Values per ASTM D30 


\subsubsection{Interpreting Nominal Values or Characteristic Strengths}

For deterministic design as described in many design codes, the material's nominal or characteristics strength (or resistance) is represented by a lower 5-percentile of tests while load effect is represented by upper 95-percentile to maintain an adequate factor of safety under normal circumstances. For example, ASTM Committee D30 on Composite Material ("Standard Test Method for Determining Tensile Properties of Fiber Reinforced Polymer Matrix Composites Used for Strengthening of Civil Structures, "2017) describes the characteristic value of FRP composites as a statistically-based material property representing the $80 \%$ lower confidence bound interval (or $20 \%$ significance level) on the $5^{\text {th }}$-percentile value of a specified population (n) and Coefficient of Variations (COV). The minimum sample size (n) generally stated as 10 , but 50 or more is preferred. As compared to sample distribution, we do not have enough FRP data for determining FRP population distribution, so several reasonable assumptions are made. The ASTM process is straight forward (for specifying and verifying the designated FRP properties, sample tests are typically performed by the manufacturers or builders) and requires the use of experimentally derived two-parameter Weibull distribution to compensate for the number of samples and lack of reliable population data. A nominal value is computed at $5 \%$ percentile using the maximum likelihood estimate (MLE) of shape and scale parameters. Then COV is calculated using Gamma function, and the characteristic value is obtained by further reducing the nominal value by a data confidence factor (selected from an $80 \%$ confidence table) to account for the uncertainties associated with the number of samples and COV obtained. Zureick et al. (2006) further described how various statistical analysis parameters could influence the FRP average value based on at least ten tests conducted by the manufacturer and why Weibull distribution was chosen even though a 
normal distribution also would have worked. This method is also prescribed in ASTM D7290-05 (2011) to provide a consistent way to quantify the highly varied material properties of FRP. In practice, there are never enough test results available, the three-sigma rule (sample mean - 3 x standard deviation or $\mu-3 \sigma$ ), which mostly applies to normal distributions and which discards all upper bound $99.9 \%$ values, is often used. Simple math will show that a $5 \%$ percentile of lower $20 \%$ is $1 \%$, which approximately translates to a nominal z-value (number of standard deviations from mean value) located at $2.33 \sigma$ (or $99.0 \%$ confidence interval) if a normal distribution is assumed. Therefore, the use of $3 \sigma$ for a "guaranteed minimum' will be conservative (i.e., $99.9 \%$ vs. $99.0 \%$ confidence interval) and can also serve as a "characteristics" value used in the reliabilitybased design. Besides, Weibull distribution is likely to have a shorter and heavier lower tail, which means that the lower $20 \%$ percentile of Weibull distribution will have more samples included when compared to a normal distribution. The use of three-sigma also helped conservatively define a "guaranteed minimum value" and often used instead of "nominal" strength in design. Atadero and Karbhari (2009) provide further discussion about $\mathrm{z}$-values and percentiles used in various codes around the world.

For reliability analysis presented in this report, no distinction is made between the characteristics value and nominal values, and they are often used interchangeably. The mean ultimate strength test value which is usually required for reliability analysis is "conservatively" interpreted by applying a bias factor to the nominal (or characteristic) value computed by threesigma method (assuming a normal distribution), five percentiles of lower $80 \%$ confidence interval (assuming Weibull distribution per ASTM method), or by using actual lab test data for mean ultimate strengths. In their work leading to the adoption of ASTM guidelines, Zureick (2006) mentioned that although Weibull's two-parameter distribution is recommended for the 
determination of characteristic or nominal strength from a large data set, it may not be the best fit and normal and lognormal distributions cannot be ruled out based on statistical probabilities.

\subsubsection{Reliability Index}

The design of a composite structural component to achieve a specified performance matrix can be achieved by using a target Reliability Index. Throft-Christensen $(2012,1982)$ and Nowak and Collins (2013) provide the concept of structural safety, structural reliability, and reliability index in great detail. The reliability index is defined as the minimal distance from a normalized origin to a specified limit state (failure criteria) in normalized space. Nowak (1995) and the follow-up NCHRP report discuss calibrating load and resistance factors (Nowak, 1999) and provide insights into how current load and resistance factor specifications for highway bridges were developed and calibrated using reliability index. Atadero and Karbhari (2007) also discussed the resistance factor calibration process and associated reliability indices for externally-bonded CFRP composite applications on existing reinforced concrete bridges. In the last two decades, the concept of the Reliability Index in the structural engineering field has been well documented and widely understood due to the adoption of LRFD codes for conventional construction materials. Additional information about various simple reliability techniques, load and resistance distributions, limit state functions, and associated probabilistic parameters can be found from Ghasami (2015).

The reliability index is defined as the inverse of the coefficient of variation of the Cumulative Distribution Function (CDF) of the limit state function. The graphical definition of the reliability index is the "shortest" distance from the origin in the reduced variables space state to the limit state function (see Figure 2.4, adopted from Nowak \& Collins, 2013). For instance, if $R$ 
represents the reduced variables of resistance, $Q$ indicates the reduced variables of load and the limit state function $\mathrm{g}(\mathrm{R}, \mathrm{Q})$ is defined as:

$$
g=R-Q \text { for } \mathrm{g}(\mathrm{R}, \mathrm{Q}) \geq 0 \text { or } \mathrm{g}=\mathrm{R} / \mathrm{Q} \text { for } \mathrm{g}(\mathrm{R}, \mathrm{Q}) \geq 1
$$

In Figure 2.4, $\mu_{\mathrm{R}}$ and $\mu_{\mathrm{Q}}$ are mean values, and $\sigma_{\mathrm{R}}$ and $\sigma_{\mathrm{Q}}$ of standard deviations of Resistance R and Load Q. The performance function and reliability index are further explained in Chapter 4.

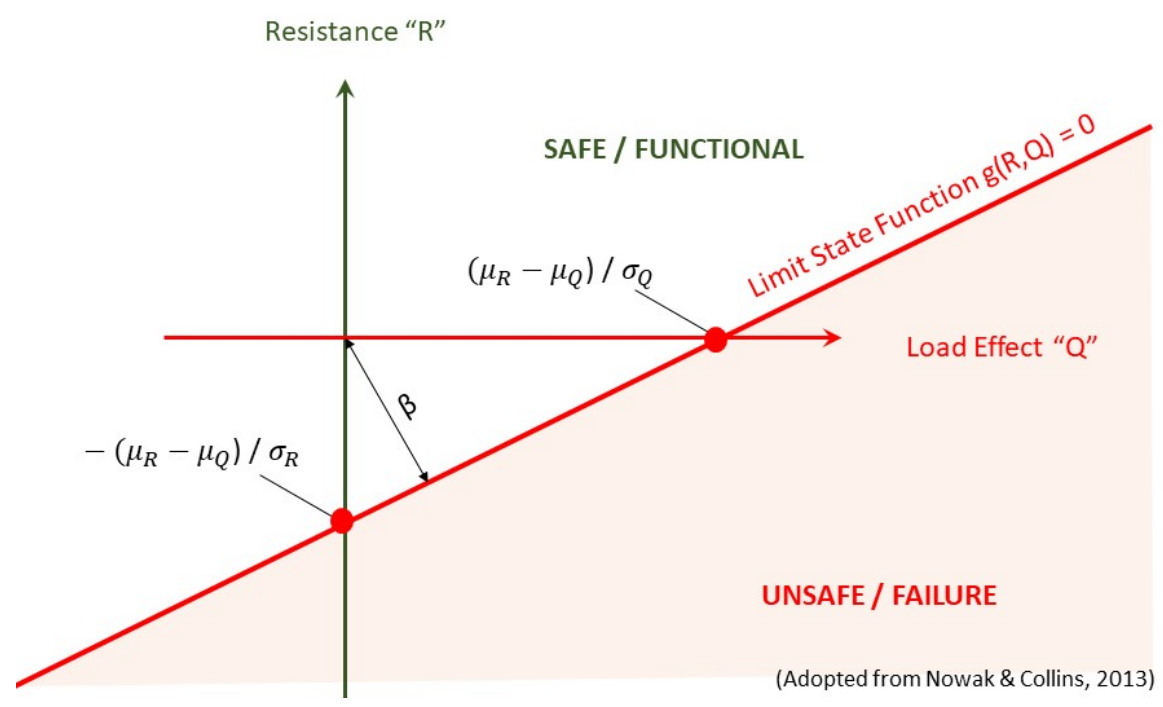

Figure 2.4: Graphical definition of Reliability Index ( $\beta$ )

\subsubsection{Design Guideline Development}

The load and resistance factors for current AASHTO LFRD bridge design specifications (2014) were calibrated by Nowak (1995) using the following primary steps: 1) Selection of representative bridges; 2) Establishment of a statistical database for load and resistance parameters; 3) Development of load and resistance models; 4) Development of reliability analysis procedures; 5) Selection of target reliability indices; 6) Calculation of load and resistance factors. The development of LRFD bridge specifications and associated calibration were massive undertakings that lasted almost two decades. A similar but more limited process can be applied to 
the development of the reliability-based framework for FRP design. Chapter 4 provides more details about this topic.

\subsubsection{Monte Carlo Simulations}

An alternate method to compute the probability of failure of a complex system is the Monte Carlo Simulation method. The maximum call number $(\mathrm{MCN})$ to limit state functions correlates to the cost/effort of any analysis. The MCN for Monte Carlo Simulations depends on the acceptable probability of failure. For example, if a failure probability value of $1 / 100$ to $1 / 1000$ is not acceptable, the MCN may exceed over millions while MCN for other simplified methods such as FORM or SORM does not depend on the target probability of failure but the number of random variables and the shape of limit state function. (Boyer et al., 1997). Despite the high cost of analysis, the Monte Carlo method (along with its rapid convergence versions) is still considered to be the most accurate and often used as a benchmark for critical risk-based design and development of modern LRFD design codes. The use of Monte Carlo simulations or similar techniques should be expected during the calibration of LRFD codes for FRP in the future.

\subsubsection{Model Specifications}

The proposed model specification for FRP composite materials for highway bridge applications (Bank et al., 2003) provides some insight into how specifications for FRP materials would look like and/or how they are developed. It used a combination of current specification sources of that era such as ASTM (American Society for Testing of Materials, 2000), AASHTO (American Association of State Highway and Transportation Officials, 2001), ICBO (International Conference of Building Officials, 1997), and MIL-HDBK (US Department of Defense Military Handbook, 1997) to address the issue of developing specifications for relatively new materials for 
use in public facilities. The goal was to provide uniform testing procedures and acceptance criteria for FRP materials for a minimum of 75-year service life in a structure. An important distinction was made between a coupon-level testing (e.g., a thin laminate) and element-level testing (e.g., a full-beam section) since the results from a coupon extracted from a component may not reflect the actual behavior of the structure in the same way as a full section. This kind of distinction is uniquely right for FRP due to its non-homogenous nature and complex interaction between the sub-parts (e.g., the architecture of a beam web is likely to be different from its flange). However, it should be duly noted that the data for system-level testing is hard to come by due to high cost, and therefore, coupon level data will have to be extrapolated until this gap is bridged in the future, concerning performance evaluation of in-service structures. The specification also describes the use of Arrhenius plots to project the results of accelerated test data (e.g., material property retention vs. time in the harsh environment) to compute design strength reduction values for FRP life-cycle in its design environment. The ultimate goal of a new design guideline should be to reach such a milestone.

\subsection{RELIABILITy APPRoACH to DURABILITy PREDICTIONS}

There is minimal literature available about the application of reliability to predict the durability or aging of FRP systems. Although plenty of separate publications on durability and reliability are available, the need for a systematic effort to develop reliability-based durability factors is unmet. Karbhari et al. (2006) provided a mechanism for enabling a reliability-based qualification of new FRP systems based on comparative requirements for the factor of safety. Several publications by Nowak et al. (1999, 2013) illustrated how reliability-based design guidelines and design codes can be developed for conventional construction materials (such as 
concrete and steel) and can apply to FRP. The use of the Markov chain and Weibull-based approach that use conditional probabilities between subsequent aging states to model deterioration function of various structural elements has also been successfully applied in modern bridge asset management systems (Agrawal et al., 2010). However, the lack of accurate sample survival data (e.g., a consistent number of samples at various discreet decay stages) is a crucial deterrent in the application of the Markov chain or Weibull survival function for a relatively new population of structures (as is the case with FRP).

The reliability approach helps in refining FRP strength reduction factors (phi values or “ф”), and durability study (using natural and accelerated aging test data with Arrhenius plots) helps in determining knock-down factors for intended lifespan and environment. For most permanent civil engineering structures, a design service life of 50 to 100 years can be safely assumed (with routine maintenance and repairs). The primary separation between civil engineering applications and other mechanical engineering disciplines is the continuous exposure of FRP to the outdoor environment over longer service life (e.g., 25-years for bridge deck and 100-years for bridge beam) when compared to temporary buildings (e.g., 10-years for a storage shed), automobiles (e.g., 5years for car bumpers), or machine parts (e.g., 5 to 15-years of replacement cycle). Besides, there is little expectation that routine maintenance/repair of FRP structures, once placed in service, is possible or will be performed.

In essence, available literature on the combined effects of reliability and durability on civil engineering structure is sparse, and therefore, relevant information from different disciplines (e.g., bath-tub curve functions from mechanical and industrial engineering) has been borrowed to complete the literature review on this topic. See Chapters 3 and 4 for a detailed explanation of the methods used in this report. 


\subsection{SUMMARY}

Specific findings of this chapter can be summarized as follows:

1. Composites offer many inherent advantages as a designer's choice material due to their high specific strength, high specific stiffness, design flexibility, dielectric, and corrosion resistance. The lack of reliable and durable performance guidelines often overshadows these advantages.

2. There is plenty of literature about the durability/aging of FRP and the reliability of structures (individually), minimal literature about the reliability of FRP, but almost none about the reliability-based approach to the durability of FRP. The durability test results, although plenty, often lack the necessary information needed for the time-dependent reliability analysis. However, the most existing durability data can be reanalyzed to provide the time-dependent and probabilistic parameters required for further analysis.

3. A "bathtub" performance curve can explain some of FRP life-cycle performance by changing the probability of failure starting from a maturity phase, aging phase, to wear-out phase.

4. Most external time-dependent effects on FRP durability characteristics can be modeled deterministically using the environment and physical effects. Although many properties are affected, the only mechanical properties studied in this research are tensile strength, compressive strength, flexural strength, shear strength, and elastic modulus. Reliability analysis for other properties (e.g., abrasion resistance, toughness, hardness, damping, etc.) is not performed due to a lack of adequate data and difficulties in clearly defining limit states (failure criteria).

5. Natural aging of FRP happens in the outdoor environment, and there are limited data and literature available about natural weathering. Under natural weathering, mechanical properties 
are affected by acidity, alkalinity, moisture, temperature, UV radiations, freeze-thaw cycles, fatigue cycles, and sustained stress.

6. Accelerate testing method is one of the most common durability prediction methods, which is often supplemented by the natural aging method for calibration.

7. The current practice to account for durability strength reduction factors can be confusing due to lack of uniform standards (e.g., Tables 2.2 and 2.3) and unsupported due to its failure to address structural safety (e.g., how safe is safe enough?).

8. The reliability approach offers a rational means to develop durability reduction factors. Simulation methods and approximate methods are often used to compute reliability.

9. The reliability approach uses an acceptable probability of failure, and the reliability index is used to measure the reliability of structures. The reliability approach can be modified (as shown in Chapter 3) to address the time-dependent behavior of FRP durability so that a minimum level of safety can be maintained as FRP ages. 


\section{CHAPTER 3 TIME-DEPENDENT RELIABILITY FRAMEWORK FOR DURABILITY DESIGN}

\subsection{INTRODUCTION}

For every structural design, there is always a chance that the structure, despite meeting all current code requirements, can still fail due to many unforeseen events unaccounted for in the design. This probability of failure is called "acceptable risk," which can be computed using probabilistic analysis. These failures can be attributed to the many inherent variations of design parameters that are taken as a deterministic value in the prevalent practice. For example, 1) the material strength as specified may not have reached a target value or the applied load was much higher than the design value; 2 ) the material dimension may not be as designed, or material flaws were introduced in the manufacturing process resulting in lower strength values, 3) simplified analysis method used could not have predicted secondary interactions among stresses from various load combinations, or 4) the resistance of the member deteriorated faster than anticipated due to some environmental conditions or human errors. Also, many current LRFD codes do not directly account for the time-dependent degradation of material properties and often assume that the routine maintenance and repairs can keep the material strength/stiffness par with the design values.

Theoretically speaking, with adequate data, most changes in material properties (e.g., section loss due to corrosion in steel, creep of concrete, loss of mechanical strength in FRP, etc.) with time can be estimated and accounted for in the design, directly (e.g., strength reduction factors) or indirectly (e.g., serviceability checks). For example, the failure mode - degradation of material strengths due to aging (or often referenced to as durability characteristics of FRP) - can be estimated using accelerated aging tests and from observations of natural weathering/aging of 
materials (Chapters 4 and 6), and a suitable strength/stiffness reduction factor (deterministic approach) can be formulated to design FRP composites. However, aging effects are often cumulative (e.g., damage accumulation due to fatigue) in FRP composites and follow a stochastic pattern to reach a state of failure with time. A reliability approach to durability estimation can provide a rational tool for designing FRP composites to last their intended service life. The reliability approach does use current deterministic methods as a start, and the end result is often a validated deterministic design method. For many well-founded deterministic methods, the change may not be noticeable.

In general, uncertainties due to inherent variabilities of member's resistance and load effects can be accounted for, and the risks associated with resulting failures can be addressed and mitigated using a reliability approach concerning the aging of FRP composites. The focus of this research is on how to take into account the inherent variations of FRP materials in rationally predicting strength degradation with time so that the risk of failure remains acceptable throughout the intended service lifespan of an FRP system. We know that absolute reliability (structural safety) is not possible (Nowak \& Collins, 2013) due to inherent variabilities of member's resistance and load (both are random variable even though design codes treat them as deterministic), so we must design structure with a certain probability of failure (or reliability, through $\beta$ factor). For this study, Figure 3.1 (based on the "bath-tub" curve, Figure 2.1) illustrates the increasing probability of failure (or decreasing reliability) as a member age and a computed $\beta$ $\mathrm{t}$ (Reliability vs. Age) design fit that can limit the risk of failure throughout its service life. It mainly shows that the initial reliability must be higher than the desired end of service life reliability, and the question is by how much? The time-dependent reliability framework - as explained in the 
following sections - can take the guesswork of such critical design decisions (e.g., varying strength reduction factors for a target service life of 5, 50, or 100 years).

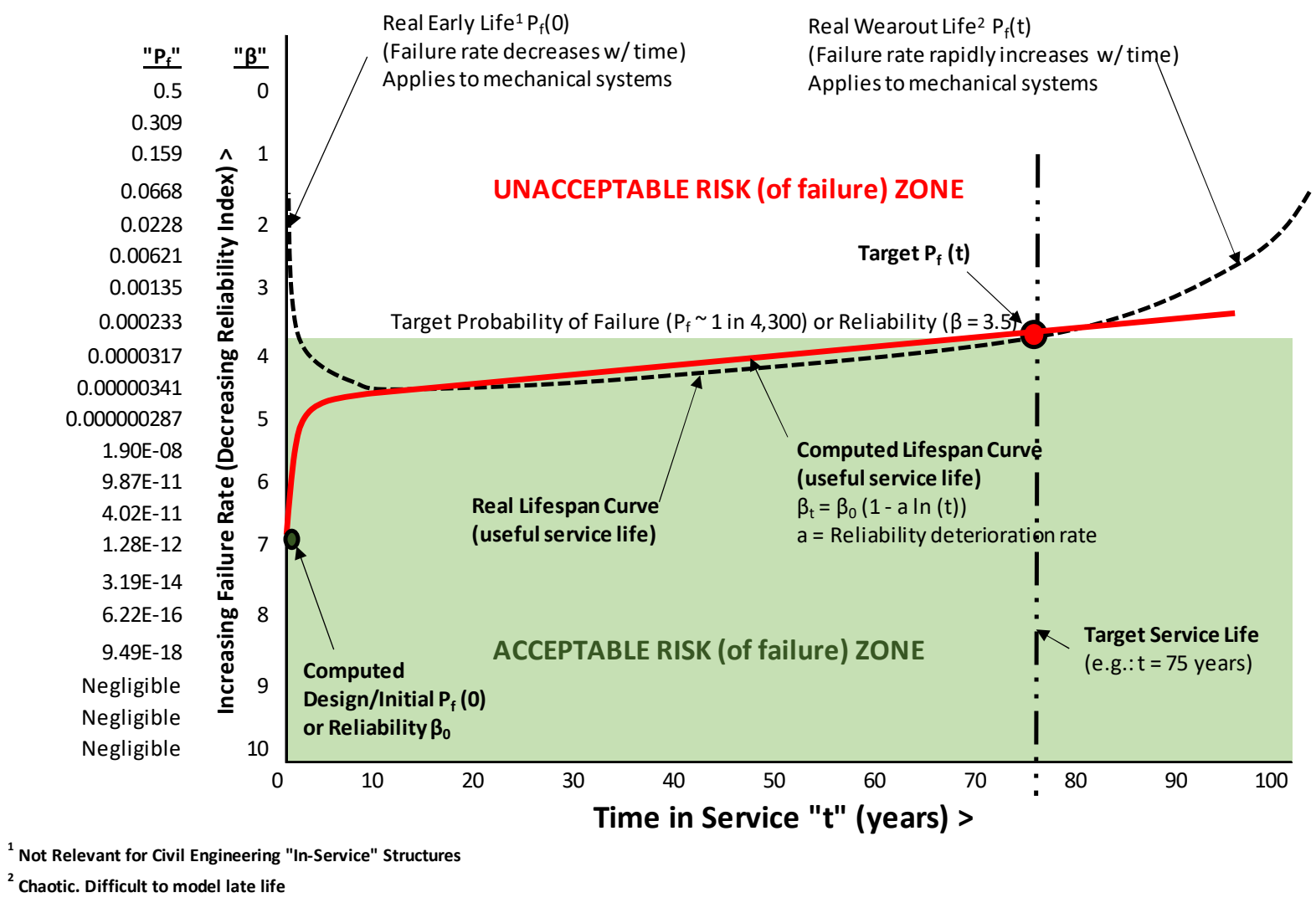

Figure 3.1: Illustration of Proposed "Reliability-Based Service Life" Curve

\subsection{RELIABILITY APPROACH}

Consider the reliability-based service life behavior of an FRP member (Figure 3.1), which is designed per today's practices of using a combination of strength reduction factors for stresses, usages, and environments of the structure. The solid curve represents the computed reliability-age relationship, while the dotted curve is the observed real-life behavior.

The initial probability of failure (due to inadequate curing, construction stability, improper construction techniques, under-prediction of loads, etc.) is high at the beginning, and so is its likelihood of failure. However, as the member is placed in service and begins to serve its function, 
the probability of failure stabilizes under-designed service conditions (due to gain in strengths from the post-curing process, corrective measures used to allow proper member functioning, etc.). This very short-term behavior is shown as "early life" and the first slope (shown as a steep dotted line with "real early life" label) of the service life of the member in Figure 3.1. This erratic behavior of FRP members at the very beginning of life is difficult to model and is not the focus of this research. However, after a member has been in service for some time (e.g., a few days to months), its degradation starts due to physical and chemical aging, and the member begins to lose its resistance slowly over its useful service life. This portion of curved is flagged as a "real lifespan curve" (shown as a solid line over member's target lifespan). It is of interest to our reliabilitydurability study and can be closely computed/modeled using a derived reliability-durability relationship. The rate of degradation accelerates near the end of its life (due to wear and tear, lack of maintenance, accumulated combined effects of environmental and physical factors, etc.), and its probability of failure again increases (often in a chaotic pattern) with time. This period is shown as a "wear-out" life, which is also difficult to model. The entire lifespan behavior is hence shaped like a "bath-tub" section (Figure 3.1, in comparison with Figure 2.1). Use of bathtub shape hazard function or failure rate (Weibull, 1939) to model the reliability of machinery is well-established in the mechanical/industrial engineering field; however, the above is an approximate adaptation of bathtub curve (shown in solid line and labeled "computed curve," Figure 3.1) that can be used to model the life-span of civil engineering FRP in-service structures.

Suppose that a designer decides the useful service life the FRP member will be 75-year of, and then the primary objective will be to use adequate aging reduction factors (or factor of safety) to provide an acceptable risk of failure until the member has served its design life (e.g., using a $\beta$ $\mathrm{t}$ relationship, $\beta$ design is 5.0 at $\mathrm{t}=0$ to ensure $\beta \min$ of 3.5 at age 75 ). It becomes evident that 
without a rational framework, one can easily over-design or grossly under-design a member without a manageable or consistent risk of failure. We will assume that there is no gross negligence on the designer's part or lack of understanding of structural behavior (e.g., aeroelastic failure of the original Tacoma Narrows Suspension bridge in 1940). This research proposes a rational framework, using a reliability-based approach, that can be used to optimally design an FRP member for specific applications (i.e., environments and loads), for the desired service life (i.e., $10,25,50$, to 100-years), and accounting for member's durability (i.e., time-dependent strength/stiffness degradation properties) and criticality (e.g., importance, ductility, redundancy, or tolerance for risk), all within a realm of acceptable, manageable risk of failure.

\subsubsection{Performance Functions}

First, it will be prudent to assume that the load factors (multipliers for design loads to account for various load combinations) for standard structural loads (often designated as "Q") are well established in the existing building or bridge codes (IBC, AASHTO, ACI, and AISC). Therefore, our focus will be on developing factors that are very specific to the design lifespans of FRP members under specified environmental or physical effects. Second, we will call these factors as "Durability Factors," and they apply to the "resistance" side (member capacity, often designated as " $R$ ") of the limit state function (failure criterion, usually designated as "g"). The primary intent is to design using a load value $(\gamma \mathrm{Q})$, which typically higher than the mean load value $(\mathrm{Q})$, and a resistance value $(\phi R)$, which is lower than the mean resistance value (R) to obtain the desired performance of the member. Figure 3.2 also illustrates the relationship between $\mathrm{Q}$ and R, which also forms the basis of most modern reliability-based LRFD (Load and Resistance Factor Design) codes. 
From Equation 2.2, we set the performance function "g" to define unacceptable performance as follows:

$$
\mathrm{g}=\mathrm{R}-\mathrm{Q}>0 \text { or } \mathrm{g}=\mathrm{R} / \mathrm{Q}>1.0
$$

Where " $\mathrm{g}$ " is the limit state function, $\mathrm{R}$ is resistance (i.e., load-carrying capacity), and Q is load effect. The probability of failure is defined as:

$$
\mathrm{P}_{\mathrm{F}}=\operatorname{Prob}(\mathrm{g}<0) \text { or Prob }(\mathrm{g}<1.0)
$$

Reliability index $\beta$ can be measured as an inverse standard normal function of $\mathrm{P}_{\mathrm{F}}$ :

$$
\beta=-\Phi^{-1}\left(P_{F}\right)
$$

The above concept is graphically illustrated in Figures 3.2 and 3.3 (adapted from Nowak \& Collins, 2013). Characteristic values of load $\left(Q_{\text {char }}\right)$ and associated load factors $\left(\gamma_{Q}\right)$ are well published in design codes (IBC, AASHTO, ACI, and AISC). Characteristic values of resistance ( $\mathrm{R}_{\text {char }}$ ) are provided by the material suppliers based on ASTM or similar standards. However, the information about Durability Strength Reduction factors or simply Resistance Factors $\left(\varphi_{R}\right)$ for FRP are often minimally substantiated or non-existent. It is critical to understand the difference between the mean average value and characteristic (or nominal and minimum guaranteed) values for performing any reliability analysis.

Figure 3.2 also shows the difference between LRFD and ASD/WSD philosophies. In LRFD, both the characteristic values of Resistance (R) and Load (Q) are modified by using strength reduction factor (phi or $\phi$ ) and load factor (gamma or $\gamma$ ) until they can satisfy the equation 3.1. In ASD/WSD, the ratio of characteristic values of Resistance and Load is expressed as the Factor of Safety (FOS). The proposed framework can be used to refine both $\phi$ and FOS. Load factor $(\gamma)$ can also be calculated (or refined), but for most common civil engineering structures and loading conditions, it is directly taken from the design codes. 


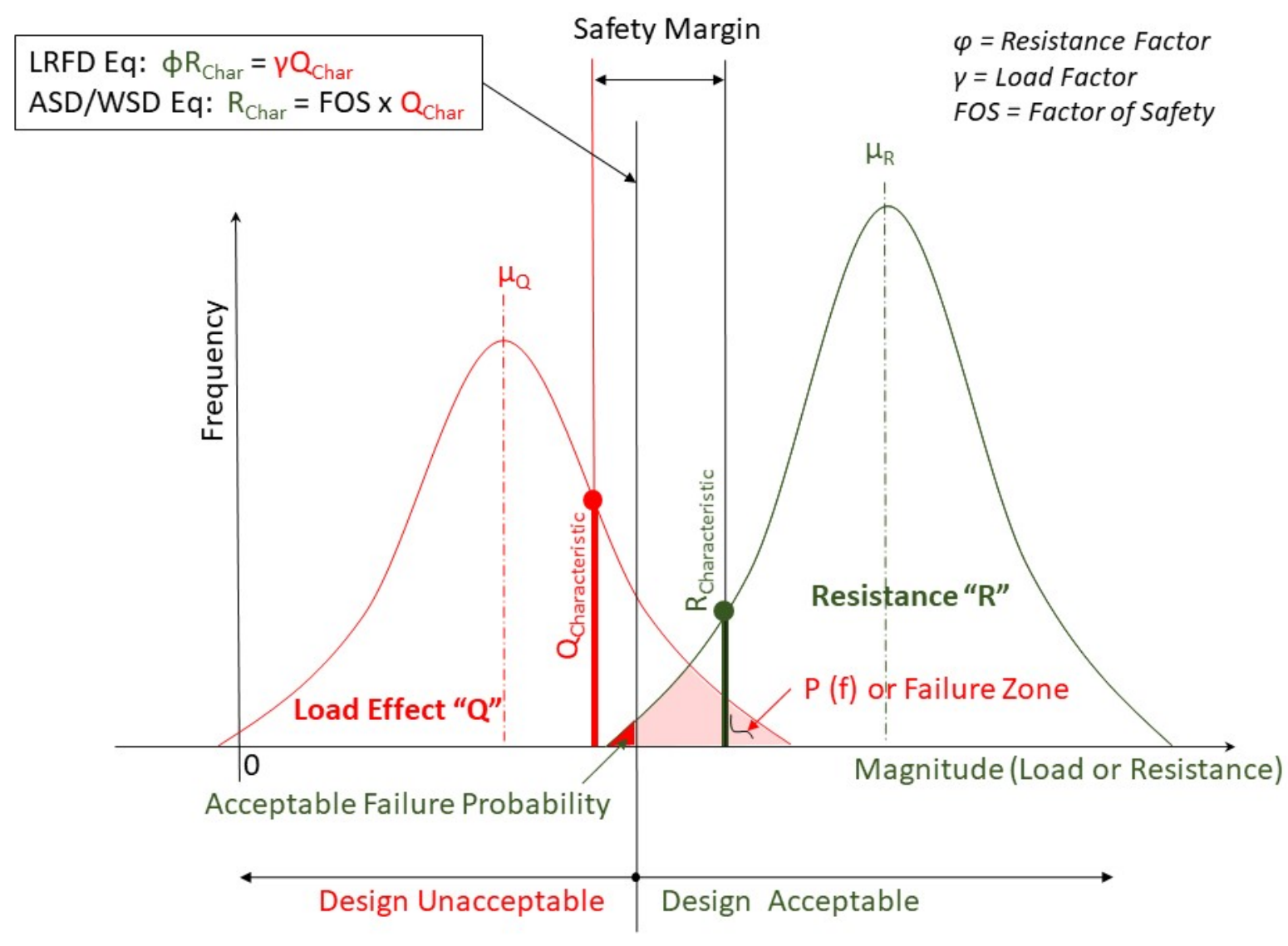

Figure 3.2: Probability of Failure with Load and Resistance Functions

The relationship between the $P_{F}$ and $\beta$ in terms of performance function $g(R, Q)$ is illustrated in Figure 3.3. The cross-over area of PDF of Q and Q represent the failure zone where the probability of failure $\left(\mathrm{p}_{\mathrm{f}}\right)$ falls below a defined acceptable level, characteristic values of Q and $\mathrm{R}$ are defined in Section 2.5.2, and the value separating the acceptable and unacceptable designs (per Code) is defined as "LRFD Design" line. In later chapters (Figures 4.5 and 7.1), we will evaluate how the resistance " $R$ " distribution parameters change with time and, in turn, impacts the probability of failure. 


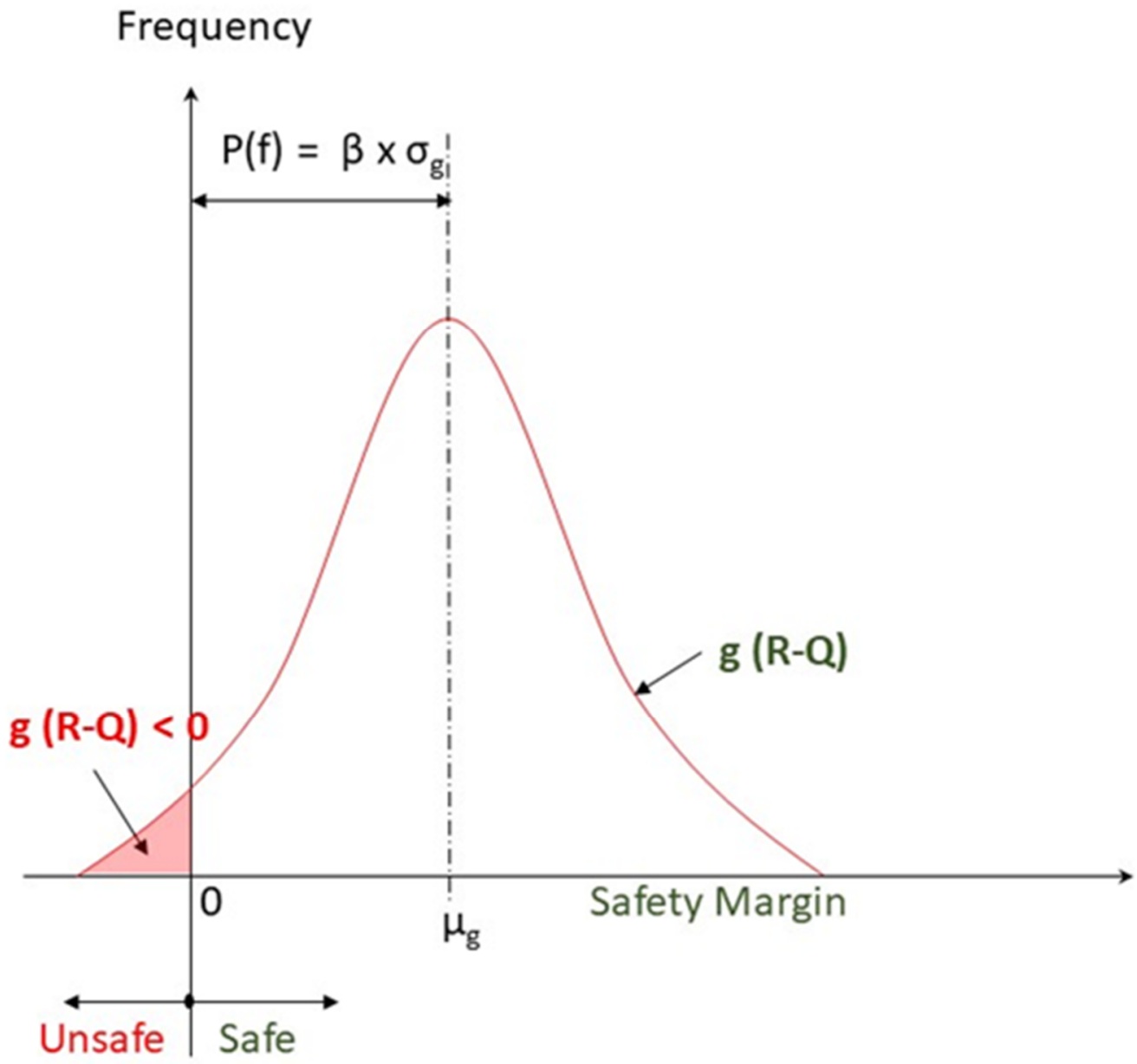

Figure 3.3: Definition of Reliability Index with Performance Function

If a normal distribution is assumed for $\mathrm{g}(\mathrm{R}, \mathrm{Q})$ function, the relationship between the $\mathrm{P}_{\mathrm{F}}$ and $\beta$ can be computed, as shown in Table 3.1. The probability of failure, as shown, can be easily computed from any normal distribution table or an Excel function (e.g., $\mathrm{P}_{\mathrm{f}}=$ NORM.S.DIST(- $\beta$, TRUE).) This table (3.1) reveals that any $\beta$ value below three or above five may be unreasonable from the design and cost viewpoints; however, the irony is that many FRP structures (new as well as old), if computed today, may have $\beta$ values outside this range for their target service lives. A 
practical value of target $\beta$ for various FRP applications is best determined during the calibration of LRFD codes/design guidelines.

Table 3.1- Probability of Failures Associated with Various Reliability Indices

\begin{tabular}{|c|c|c|}
\hline Reliability Index ( $\beta$ ) & Assessment & Probability of Failure $\left(P_{f}\right)$ \\
\hline 0 & Unacceptable & $0.5(1$ in 2$)$ \\
\hline 1 & \multirow{2}{*}{ Too Low } & $0.159(1$ in 6$)$ \\
\hline 1.5 & & 0.0668 (1 in 15) \\
\hline 2 & \multirow{2}{*}{ Low } & 0.0228 (1 in 44) \\
\hline 2.5 & & 0.00621 (1 in 161) \\
\hline 3 & \multirow{3}{*}{ Acceptable } & 0.00135 (1 in 741) \\
\hline 3.5 (common target value) & & 0.000233 (1 in 4,292) \\
\hline 4 & & 0.0000317 (1 in 31,546) \\
\hline 4.5 & \multirow{2}{*}{ High } & $0.0000034(1$ in 294,118$)$ \\
\hline 5 & & 0.000000287 (1 in 3.5 Million) \\
\hline 5.5 & \multirow{2}{*}{ Too high } & 0.0000000190 (1 in 53 Million) \\
\hline 6 & & 0.000000000987 (1 in 1 Billon) \\
\hline
\end{tabular}

Based on a comprehensive survey of the existing bridges during the development of Ontario and US codes (in the mid-' 80 s to late-90's), a value of $\beta$ between 3 and 4 was considered reasonable (Nowak, 1999). A design value of less than three can be regarded as too risky, and more than four may be too expensive for most permanent civil engineering applications. Based on previous work on the development of several LRFD codes, a target value of $\beta$ as 3.5 (relating to a probability of failure of approximately 1 in 4000 to 5000) is proposed for the development of the reliability-based framework. Atadero and Karbhari $(2008,2009)$ also discussed various target values of $\beta(2.5,3.0$, and 3.5) during their calibration of resistance factors for externally-bonded FRP composites on several existing reinforced concrete Tee-beam bridges. They provided design scenarios for the use of various values of $\beta$ based on the beam's existing condition (e.g., steel 
corrosion). However, it is not clear if the same discussion would apply to the design of new pultruded FRP members.

In theory, a higher or lower value of $\beta$ can be used for FRP design that will account for its aging uncertainties but the questions about what values should be used for different service lives, and which properties will take a bigger hit, where is the precedence, etc. may be difficult to answer. A rational method intends to promote a uniform and consistent probability of failures for all limit states (e.g., the failure probability of a beam in shear and bending failure should be similar) but allow enough flexibility to take into account other influencing parameters such as members' importance, modes of failure (brittle or ductile), or redundancies. Nevertheless, the target $\beta$ value of 3.5 can be further examined, should more evidence of the satisfactory long-term performance of FRP structures is found with a $\beta$ value of more (or less) than 3.5. In general, the use of a correct reliability index will result in the optimal cost of the structures over its life-cycle (initial plus upkeep), while a higher index will result in too much initial cost, and a lower index will cause the too high cost of maintenance and failure consequences.

\subsubsection{Strength Reduction Factors}

The process used to determine a strength reduction factor Phi " $\phi$ "in modern design codes is based on reliability-based probabilistic methods with a primary objective of providing a consistent level of "probability of failure, $\mathrm{P}_{\mathrm{f}}$ "" Original codes used deterministic methods such as factors of safety (FOS) resulting in allowable/working stress design (ASD/WSD) methods, which often resulted in inconsistent probabilities of failures (at individual material or component levels).

In essence, the structural reliability method attempts to select a strength reduction factor " $\phi$ " for the design strength parameter " $R$ " that will provide an acceptable and uniform probability 
of failure when subjected to the corresponding design load effect "Q" (Figure 3.2). The design requirement will be shown in the following form:

$$
\phi \mathrm{R} \geq \gamma \mathrm{Q} \text { or } \phi \mathrm{R}-\gamma \mathrm{Q} \geq 0 \quad \ldots \mathrm{Eq}(3.4)
$$

As mentioned before, the load effect $Q$ and its modification factor Gamma " $\gamma$ " are well established (per regulatory load and resistance factor design or LRFD codes); hence, the focus herein is on developing the appropriate $\phi$ factors for structural components involving relatively new FRP materials. The above values of $\mathrm{R}$ and $\mathrm{Q}$ are the characteristic values (often referred to as Nominal or guaranteed minimum values in this research) as found in design codes or material specifications. Equation 3.4 is also graphically represented as an optimal LRFD design situation where " $\phi \mathrm{R}-\gamma \mathrm{Q}=0$ " in Figure 3.2.

As mentioned in Section 3.2.1, during the calibration of replacing ASD/WSD methods with LRFD methods, most design codes in North America have adopted an acceptable probability of failure defined by a target reliability index $\beta$ value as 3.5 or higher. This index is a "notional" probability of failure, theoretically defined as the second moment of structural safety, the "shortest" distance from the origin of reduced variables space to the limit state function $g$ line (Figure 2.4), and approximately equates to a probability of failure of 1 in 4,300. The reason target $\beta$ is called notional is that since there is not enough evidence to verify how this theoretical probability of 1 in 4,300 applies to real-life situations except there is ample evidence that most conventional in-service structures with $\beta$ value of 3.5 or above have performed well to date. FRP being relatively a new material for construction, it will be preferable to err on the safe side in estimating the target $\beta$. For target $\beta$ to be above 3.5, further evidence is needed showing that structures designed using $\beta=3.5$ are not performing satisfactorily, thus warranting the use of higher $\beta$, or many well-performing structures already have $\beta$ of well above 3.5 . Keep in mind that 
our focus is to find the lowest acceptable value $\beta$ in all modes of failure at the end of a member's service life.

The method to determine $\phi$ can be summarized in the following steps.

\subsubsection{Development of Resistance $R$}

First, various parameters for computing the "R" value should be developed. As mentioned before, ASTM D7290-06 defines a method for selecting both the nominal and design values (referred to as characteristic value in ASTM) based on laboratory tests. In the absence of test data used to compute the $80 \%$ lower confidence limit on the $5^{\text {th }}$-percentile value, bias factors are often used to obtain the mean values that are commonly used in reliability analysis. As a minimum, an accurate estimation of mean value, bias factor, and standard deviations (or the coefficient of variations) are required for conducting reliability analysis. In the absence of information about the sample's probability distribution function (PDF) of Resistance R, a normal distribution can be assumed, at least in the preliminary analysis, for setting a reliability-based framework.

\subsubsection{Development of Strength Resistance Factor $\phi$}

The following process has been adopted from Nowak \& Collins (2013) and has been modified for FRP composites:

1. Select a target reliability index $\beta$. Typically, a value between 3 and 4 is selected, and as a minimum, a value of 3.5 or above is recommended.

2. Develop probability density functions (PDF) of material properties used to compute "R" using laboratory tests or manufacturer-supplied data. In lieu of testing and developing PDF for each material property (e.g., strengths, stiffness, length, thickness, depths, etc. contributing member resistance R), use published nominal/characteristic values. Use bias factors (=mean 
value/nominal value, generally in the range of 0.9 to 1.5 , if too high then the design value selected is too conservative) to obtain mean values and coefficient of variations (=standard deviation/mean value, generally in the range of $5 \%$ to $15 \%$ ) to get standard deviations based on data presented in this and other studies.

3. Select several common design situations (varying span lengths, loadings, member sizes, etc.) where the newly derived $\phi$ will be used. For example, if $\phi$ is to be developed for a simply supported wide flange or box Vinylester GFRP beam, select various spans 'L" and a range of unit weights "w" loading. Certain parameters will be deterministic (e.g., fixed span length L, beam section, etc.) and others will have probability distribution functions (e.g., variable ultimate flexural and shear strengths $f_{u}$, dead and live load $w$, etc.). Special design checks, stiffness limits, and corresponding limit states such as web crippling or compression flange buckling may govern the final design. If such conditions are applicable, they need to be addressed separately. (Outside the scope of this research).

4. Write the reduced limit state design equations (expand $\mathrm{R}$ and $\mathrm{Q}$ terms) as defined below in terms of variables $\mathrm{w}$ and $\mathrm{f}_{\mathrm{u}}$ to include all modes of failure or limit states. Define the performance function:

$$
\mathrm{G}\left(\mathrm{w}, \mathrm{f}_{\mathrm{u}}\right)=\phi \mathrm{R}-\gamma \mathrm{Q}=\phi \times\left(\mathrm{BF}_{\mathrm{R}} \times \mathrm{R}_{\text {char }}\right)-\gamma \times\left(\mathrm{BF}_{\mathrm{Q}} \times \mathrm{Q}_{\text {char }}\right)
$$

5. For selected examples, re-write the reduced limit state equation in terms of variables $a_{1}$ and $a_{2}$. The coefficient $a_{1}$ will be calibrated later as $\phi$, and $a_{2}$ will remain fixed (typically taken as a multiple of " $\gamma \times$ BF" where $\gamma$ is load factor shown in design codes for $\mathrm{Q}_{\text {char }}$ :

$$
\mathrm{G}\left(\mathrm{w}, \mathrm{f}_{\mathrm{u}}\right)=\mathrm{a}_{1} . \mathrm{f}_{\mathrm{u}}-\mathrm{a}_{2} \cdot \mathrm{w} \quad \ldots E q(3.6)
$$

6. Select a trial value of $\phi$. A good place to start is 1.0 to see what the absolute minimum value of $\beta$ can be. 
7. Using mean value and standard deviations of various parameters, compute $\beta$, and compare it to its target value of 3.5. For selected example ( $\sigma$ is the standard deviation, and $\mu$ is the mean value):

$$
\beta=\left(a_{1} \mu_{f u}-a_{2} \mu_{w}\right) / \sqrt{\left(a_{1} \sigma_{f u}{ }^{2}-a_{2} \sigma_{w}^{2}\right)} \quad \ldots E q(3.7)
$$

Where $\mu_{w}$ and $\mu_{f u}$ are the mean value of the load, and ultimate strength, $\sigma_{f u}$ and $\sigma_{w}$ are the standard deviation of ultimate strength and loads, respectively.

8. Adjust $\phi$ factor $\left(\mathrm{a}_{1}\right)$ accordingly and repeat steps 6 and 7 until the target $\beta$ value is achieved

9. Select another common design problem (e.g., different span and loading) and repeat above steps 3 to 8 to arrive at various $\phi$ values for various (example) designs. Select a conservative $\phi$ value yielding $\beta$ of 3.5 or more for all common examples.

10. Test the selected value for extreme and more sophisticated design cases to find thresholds (or limit) of the chosen $\phi$ where it may yield unacceptably higher or lower $\beta$ values than its target value. It may be worth developing separate $\phi$ for those special situations to provide more design flexibility.

\subsubsection{Calibration of Strength Reduction Factor $\phi$}

Use benchmark simulations techniques such as Monte Carlo Method (MCM) to build confidence in the use of new $\phi$ for more complex real-life design examples. Use appropriate PDFs, COVs, and mean values on both resistance and load sides (Ghasemi, 2015). Typically, the number and complexity of design examples used in the calibration phase will be much larger than a few simple examples used at the framework level. An industry-wide or institution level of efforts should be expected in the final calibration phase.

In simple terms, the calibration process can be defined as follows: 
1. Test the selected $\phi$ value on several standard new designs and reverse engineer (i.e., design) several existing structural systems to obtain the prevalent and built-in probability of failures (or reliability index $\beta$ ). Compare the built-in $\beta$ to the target reliability index to see how the change will impact the existing design practice. Often the existing $\beta$ will be more than 3.5 (i.e., overdesign) or less (i.e., under-designed) but will undoubtedly vary significantly for various limit state strength checks within a system (i.e., a common situation of the inconsistent probability of failures or non-uniform factors of safety in many old designs).

2. Redesign several existing structural components using the newly derived $\phi$ and using the current practice. Compare the two designs. If necessary, based on professional engineering judgment and prior knowledge of similar members' prior performance, tweak $\phi$ values to ensure the member will not be grossly "under-designed" (or over-designed) due to some unknown effect (that has not been yet well understood or evaluated). New $\phi$ factors, if they are yielding substantially lighter designs (as compared to traditional designs), should be carefully re-evaluated to see if there are some known serviceability/strength shortcomings that this new $\phi$ is going to mitigate.

A similar derivation about the factor of safety (FOS), as used in ASD/WSD methods, can also be presented that could allow us to obtain an acceptable level of safety. See chapters 4 and 6 for examples of FOS derivations using the proposed Framework.

It must be noted that due to the sheer amount of effort involved, and time/budget constraints, the calibration of strength/stiffness reduction factors is not performed by the author. It should be the task of the FRP industry to follow the framework established in this report and take upon the challenge of building an LRFD knowledge bank of civil infrastructure applications made of FRP composites. 


\subsection{RELIABILITY-BASED DURABILITY FRAMEWORK}

The reliability approach develops a set of rational design guidelines that can ensure satisfactory long-term performance of FRP composites. The reliability approach uses probabilitybased analysis to take into account the inherent variations of the component's resistance and external environment to limit the risk of failure. It often refines the existing deterministic methods to do the same. The use of a reliability approach to developing/refining design guidelines may be a relatively new concept in FRP but has been successfully applied to the development of new LRFD codes for bridges and buildings in the US.

As stated before, the primary objective of this research has been to establish a framework to allow the development of rationally derived strength reduction factors $(\Phi)$ to account for FRP aging from external physical and environmental effects that can be used in the design of FRP composites for civil engineering applications. A reliability-based approach is used in the development of these strength reduction factors to limit the probability of failure of FRP composites to an acceptable risk level. These factors are termed as "durability factors" to focus on aging aspects of FRP and are deemed "rational" for providing consistency and reproducibility for most practical applications. The research is focused on developing a basic framework of methodologies that can be practically applied and easily reproduced to account for various shortterm and long-term behavior of FRP composites from the time of placement to the end of useful life.

Various steps taken to develop the proposed reliability-based Durability Framework are described in the following sections. An associated step by step flowchart of the framework building process is shown in Figure 3.4. For additional explanation, see the article number corresponding to each box number in the following pages. 


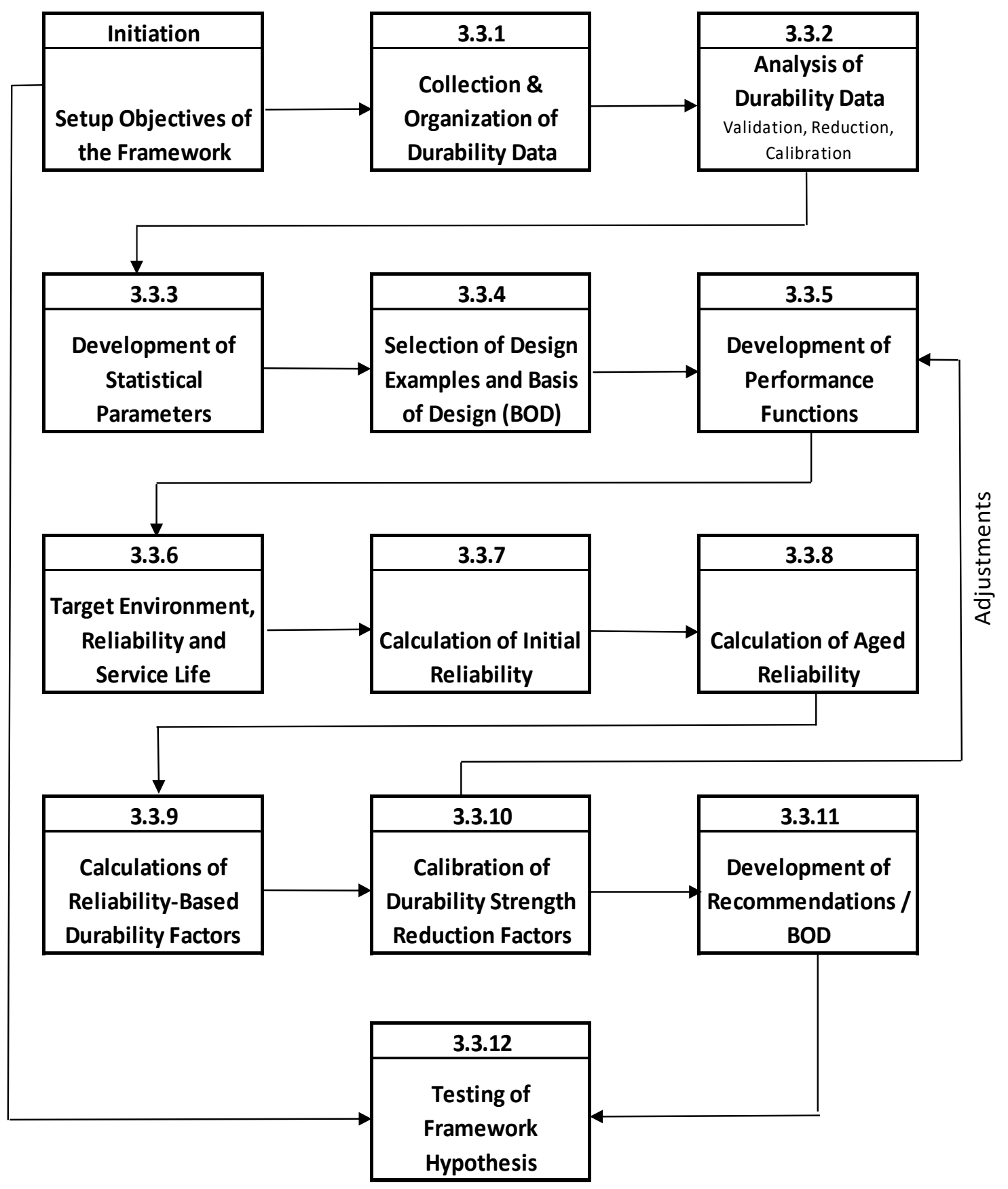

Figure 3.4: Flowchart of Reliability-Based Durability Framework 


\subsubsection{Collection \& Organization of Durability Data}

One of the most challenging and time-consuming tasks was to collect and organize the accelerated aging and natural aging test on a wide variety of FRP materials from WVU and other institutions. A reasonable amount of necessary aging data, mostly GFRP materials in vinylester and polyester resins, was collected before the start of this work thanks to the Master's thesis of several WVU graduate students (Barker, 2019; Lorenzo, 2018; Dittenber, 2012, and others).

It must be stressed that there is still much more durability data available that was not added in this evaluation, and more is becoming available every day. Further refinement of FRP deterioration rates will only further the primary objectives of this research. Expanding the durability data, hopefully in a more organized manner, will be a key take away from this research. The framework, as proposed, can be used by other researchers who are interested in developing specific FRP applications. For example, carbon fiber-reinforced polymer in epoxy resin for high fatigue outdoor applications can be tested in the laboratory and precise recommendations using reliability-based durability (or often referred to as Basis of Design or BOD) for certain life-span (say ten years), can be developed following the case study and examples provided in this research.

After data collection, the first step of organizing data was to normalize all virgin and aged material strengths as a percentage of the virgin strength (e.g., ultimate tensile, compressive, flexural, or shear strengths) in a "dry room temperature" environment after correcting for any short curing time. The next step was to organize the normalized data into a generic format to allow the use of statistical and regression software. A summary of FRP accelerated testing data used in this report is shown in Table 3.2 and Figure 3.5. Linear regression lines in Figure 3.5 encompassing all samples and shows only a general trend of various strengths in the different accelerated aging environments for sample ages ranging from 1 month to 18 months. Figure 3.5 shows the raw ATM 
data and is included for informational purposes only. Specific details and additional graphs can be found in Appendix B. After Arrhenius analysis of ATM data was performed for the referenced environment, the naturally aged data from WVU studies was used (Barker, 2019; Lorenzo, 2018; Dittenber, 2012) to project ATM data for the outdoor environment. A full listing of the final durability database (with data source) used in this study can be found in Appendix E. 


\section{Table 3.2- Variables and Sources of FRP ATM Database for This Study}

\begin{tabular}{|c|c|c|c|c|c|c|c|c|c|c|c|c|c|c|c|c|c|c|c|c|c|c|}
\hline \multicolumn{23}{|c|}{ Description of FRP Durability Database and Variables Collected for This Study } \\
\hline 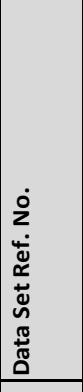 & 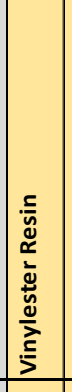 & 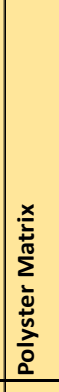 & 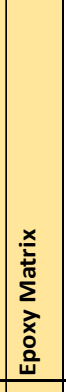 & 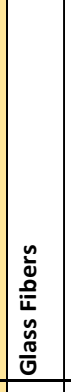 & 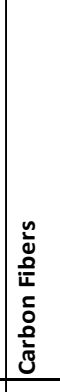 & 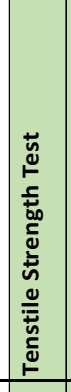 & 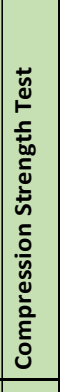 & 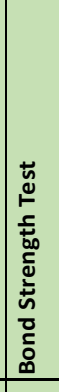 & 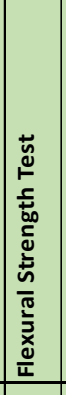 & 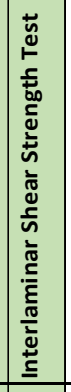 & 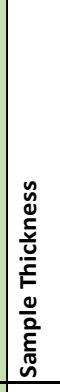 & 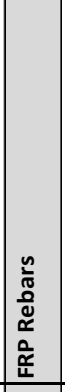 & 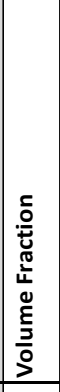 & 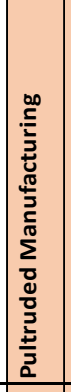 & 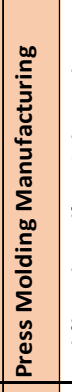 & 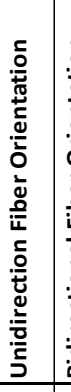 & 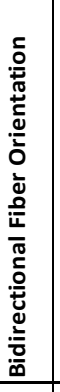 & 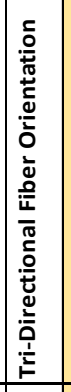 & 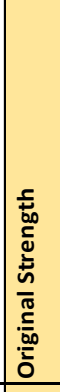 & 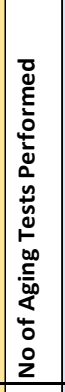 & 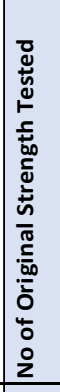 & \\
\hline$\sum_{\underline{\underline{x}}}^{\bar{x}}$ & $\stackrel{\mathbb{M}}{\underline{W}}$ & 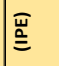 & $\stackrel{x}{\underline{\underline{x}}}$ & $\underline{\underline{\sigma}}$ & $\stackrel{\overline{\tilde{x}}}{\underline{y}}$ & $\underline{\underline{\underline{n}}}$ & $\underline{\underline{\underline{y}}}$ & 氫 & $\underline{\underline{\underline{n}}}$ & $\stackrel{\overline{\tilde{n}}}{\underline{\underline{n}}}$ & $\widehat{\hat{E}}$ & $\overline{\overline{\underline{a}}}$ & $\stackrel{\bar{M}}{2}$ & $\vec{a}$ & 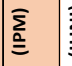 & $\underline{\underline{\underline{z}}}$ & $\overline{\overline{\underline{\omega}}}$ & $\overline{\overline{\underline{\underline{E}}}}$ & $\overline{\underline{s}}$ & & & List of Databases Compiled by Authors \\
\hline 1 & 71 & & & 71 & & 32 & 23 & 16 & & & & 71 & & \multicolumn{5}{|c|}{ Missing data } & & 20 & 4 & Vijay, P. V. (1999), Aging and Design of Concrete Members reinforced with GFRP bars, \\
\hline 2 & 16 & & & 16 & & & & 16 & & & & 16 & & 16 & & & & 16 & & 24 & 3 & Wang J. et al.,(2015), Durability and prediction models of fiber-reinforced polymer \\
\hline 3 & & & 48 & 48 & & 48 & & & & & & & & & 48 & \multicolumn{3}{|c|}{ Missing data } & & 36 & 2 & Kajorncheappunngam, S. (1999), The effects of environmental Aging on the Durability \\
\hline 4 & & & 30 & 30 & & & & & 30 & & & & \multicolumn{6}{|c|}{ Missing data } & & 6 & 1 & Marru, P. et al (2014), Lifetime Estimation of Glass Reinforced Epoxy Pipes in Acidic \\
\hline 5 & & & 48 & & & 48 & & & & & \multicolumn{8}{|c|}{ Missing data } & & 12 & 6 & Shi, J. et al (2011), Durability of wet lay-up FRP composites and their epoxy resins in \\
\hline 6 & 27 & & & & 27 & 9 & 18 & & & & & 27 & \multicolumn{6}{|c|}{ Missing data } & & 7 & 2 & Rivera, J. \& Karbhari, V.M. (2002), Cold-temperature and simultaneous aqueous \\
\hline 7 & 28 & 28 & & 56 & & 28 & & & 28 & & & & \multicolumn{7}{|c|}{ Missing data } & 8 & 0 & Sonawala, S.P., Spontak, R.J. (2017); Degradation kinetics of glass-reinforced \\
\hline 8 & 60 & 60 & & 120 & & & & & & 120 & & & & 120 & & 120 & & & & 24 & 0 & Chin, J.W. et al (2001), Elevated Temperature Aging of Glass Fiber Reinforced Vinyl \\
\hline 9 & 15 & & & 15 & & 15 & & & & & & 15 & \multicolumn{6}{|c|}{ Missing data } & & 3 & 1 & Chen Yi et al. (2006), Durability Prediction for GFRP Reinforcing Bars Using Short-Term \\
\hline 10 & 28 & & & 28 & & 28 & & & & & & 28 & \multicolumn{6}{|c|}{ Missing data } & & 4 & 1 & Won J.P. et al. (2007) The effect of exposure to alkaline solution and water on the \\
\hline 11 & 89 & & & 89 & & 89 & & & & & & 89 & \multicolumn{6}{|c|}{ Missing data } & & 10 & 2 & Kim H.Y. (2007), Short-term durability test for GFRP rods under various environmental \\
\hline 12 & 16 & & & 16 & & & & & 16 & & & 16 & \multicolumn{6}{|c|}{ Missing data } & & 14 & 1 & Robert M. (2010), Temperature as an Accelerating Factor for Long-Term DURABILITY \\
\hline 13 & 112 & 128 & & 240 & & 76 & & & 80 & 80 & & & & 80 & & \multicolumn{3}{|c|}{ Missing data } & & 42 & 6 & Cabral-Fonseca S. (2012), Artificial Accelerated Ageing of GFRP Pultruded Profiles \\
\hline 14 & & 40 & & 40 & & 20 & & & & 20 & & & & 20 & & 20 & & & & 8 & 2 & Grammatikos S.A. (2016), On the response to hygrothermal aging of pultruded FRPs \\
\hline 15 & 4 & & & 4 & & & & & 4 & & & 4 & \multicolumn{6}{|c|}{ Missing data } & & 1 & 1 & Sen R.(2002), Durability of E-Glass/Vinylester Reinforcement in Alkaline Solution, WVU \\
\hline 16 & 70 & & & 70 & & & & & & 70 & & & & issing da & & 70 & & & & 10 & 2 & Karbhari V.M., (2004); E-Glass/Vinylester Composites in Aqueous Environments: \\
\hline 17 & 24 & & & 24 & & 24 & & & & & & & \multicolumn{7}{|c|}{ Missing data } & 6 & 0 & Dejke V.; Durability and Service Life prediction of GFRP for Concrete reinforcement, \\
\hline 18 & 18 & & & 18 & & & & & 9 & 9 & & & & & 18 & & 18 & & & 6 & 2 & Hammami A. (2004); Durability and Environmental Degradation of Glass-Vinylester \\
\hline Total $=$ & $=578$ & 256 & 126 & 885 & 27 & 417 & 41 & 32 & 167 & 299 & & 266 & & 236 & 66 & 210 & 18 & 16 & & 241 & 36 & Approximately Total 960 Observations and 197 Aging Tests \\
\hline
\end{tabular}




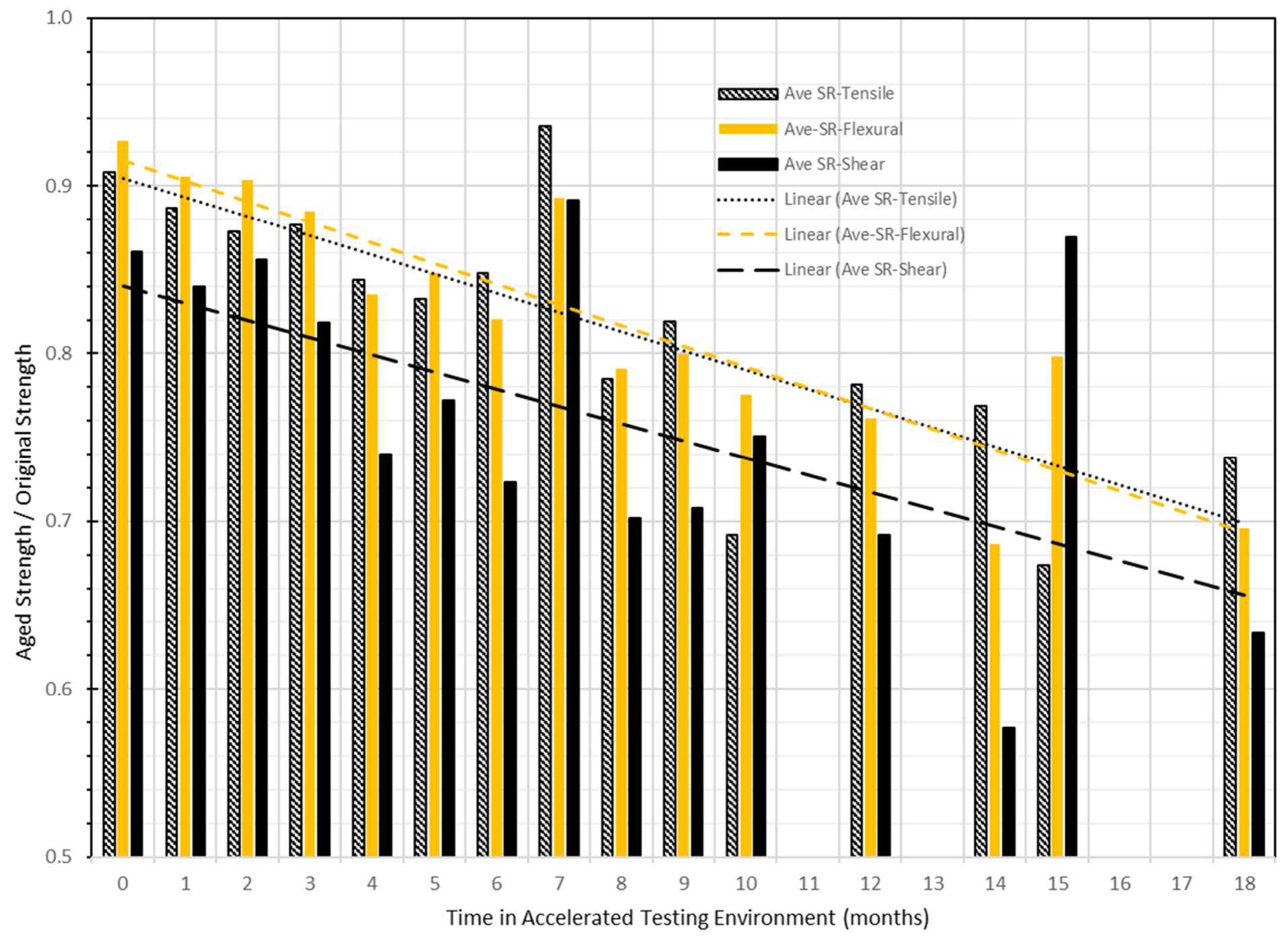

Figure 3.5: Ave. Strength Retention (SR) from Accelerated Testing Database 


\subsubsection{Analysis of Durability Data}

This portion of the work consisted of three main subtasks - data validation, reduction, and calibration. The process involved is described in the following sections.

\subsubsection{Data Validation}

Before any data analysis can be performed, data validation was carried out to remove incomplete, incorrect, and missing entries. The modifications were done carefully, avoiding the skewing of the results and included the following:

- A group of entries with an insufficient number of samples for Arrhenius analysis were removed. The data for carbon fibers and epoxy resins were minimal for accelerated test data and were not analyzed at the end.

- Test entries with missing information, such as the original or virgin strengths or type of resins or fibers, were not analyzed. In general, it was difficult to obtain additional information from researchers once their papers/reports have been published.

- Strength test entries, which were increasing after the previous readings, were removed under the assumption that some samples were still in a very prolonged curing process. Newer FRP samples seem to be fully cured, perhaps due to improved QA/QC at the manufacturing facilities, or they just have been stocked up too long in a virgin state before testing. Other readings of the same test sample set, which were sequentially decreasing in the accelerated environment, were kept after the entire test reading set was re-normalized with respect to the highest readings. For example, if the higher Strength Retention occurs in the third month 
(instead of day 1), it was assigned $100 \%$ SR with re-set of time $\mathrm{t}=0$, and subsequent SR readings and time were adjusted accordingly.

- The data analysis was used to fit various regression curves for a set of similar sets of data (e.g., tensile strength retention data of Vinylester GFRP at multiple ages). The regression curve was fitted using Excel's solver function using the method of least squares. Linear regression line $(y=m x+b$, where $x$ is the natural $\log$ of time and $y$, is SR), the $y$-intercept " $b$ " was forced at $100 \%$ to correspond with the normalized $100 \%$ dry strength of the virgin sample. The regression fit error was found to be minimal, but the regression equation provided a more realistic SR projection equation with time.

- Outliers were identified during regression by plotting and evaluating $95 \%$ confidence limits. The influence of outliers on the regression line was removed by nulling their least square sum. It is worth mentioning that the use of professional data analysis software can be of great help to simplify this tedious task, and the author wishes that more could have been done for this research database.

- During regression, there were large scatters and clusters of normalized data. If a reasonable justification was not found in test data reports, no action was taken. Manipulation of data was not done to allow the reproducibility of regression results presented in this report. Graphs of Arrhenius extrapolation and regression analyses performed for the FRP database can be found in Appendix B as Figures B.2 to B.12.

\subsubsection{Data Reduction}

The reduction of accelerated test data, which was normalized using the Arrhenius principle, was performed for various groups based on FRP systems (e.g., GRFP in Vinylester) and experimented with strength values (e.g., Flexural strength). The underlying concept of ATM is 
straightforward. It assumes that if we know the time (in lab or nature), it takes for a material to reach a specific strength reduction under a reference environment (e.g., $70^{\circ} \mathrm{F}$ outdoor air or immersion in $5^{\circ} \mathrm{C}$ seawater), we can prorate the lab time that it took for a similar material to reach the same strength reduction under much harsher environment condition of ATM (e.g., $120^{\circ} \mathrm{F}$ in lab or immersion in $60^{\circ} \mathrm{C}$ seawater). ATM allows the extension of test time obtained in the lab under a controlled harsh condition to a desired reference environment timeline using Time Shift Factor (TSF). In general, ATM shifts the lab timeline only (not the strengths), and many techniques can be used to compute TSF to a referenced environment. An accelerated testing and associated data reduction process seldom goes as planned; however, based on the experience of performing data reduction on a wide variety of databases spread over decades of research, an ideal ATM process can be briefly summarized as follows.

1. Prepare specimens for accelerated aging environments such as high temperature and alkaline solutions. Typically, selected environments should be much severe than the referenced (or natural) aging environment that a component will experience in its life span. Include at least one natural (or reference) environment for the baseline determination and final calibration of reduced accelerated test data. All virgin or new samples should be "cured" fully to avoid skewing of test data when aging. Due to a vast number of tests and preparation time involved, having a very detailed and staggered aging/testing schedule and test data management system is helpful to use time and labor involved efficiently. Best to plan in-depth first, then execute.

2. Conduct testing of specimens for various strength parameters before starting the test (virgin samples in a "dry" laboratory environment) and at preselected time intervals (aged samples in wet/saturated harsh environment). Since strength tests are destructive, a sufficient number of samples must be used in the planning. The test time intervals can range from months to years, 
say starting from $t_{0}$ to $t_{1000}$ (days). Perform prior DMA tests to ensure the glass transition temperatures will not be exceeded during aging tests. A good practice will be to keep the highest aging temperature at $20^{\circ} \mathrm{F}-30^{\circ} \mathrm{F}$ below the glass transition temperature. If time permits, conduct moisture uptake tests separately to tests for "saturated" strengths before placing samples in an accelerate aging environment (which is also a water-based solution).
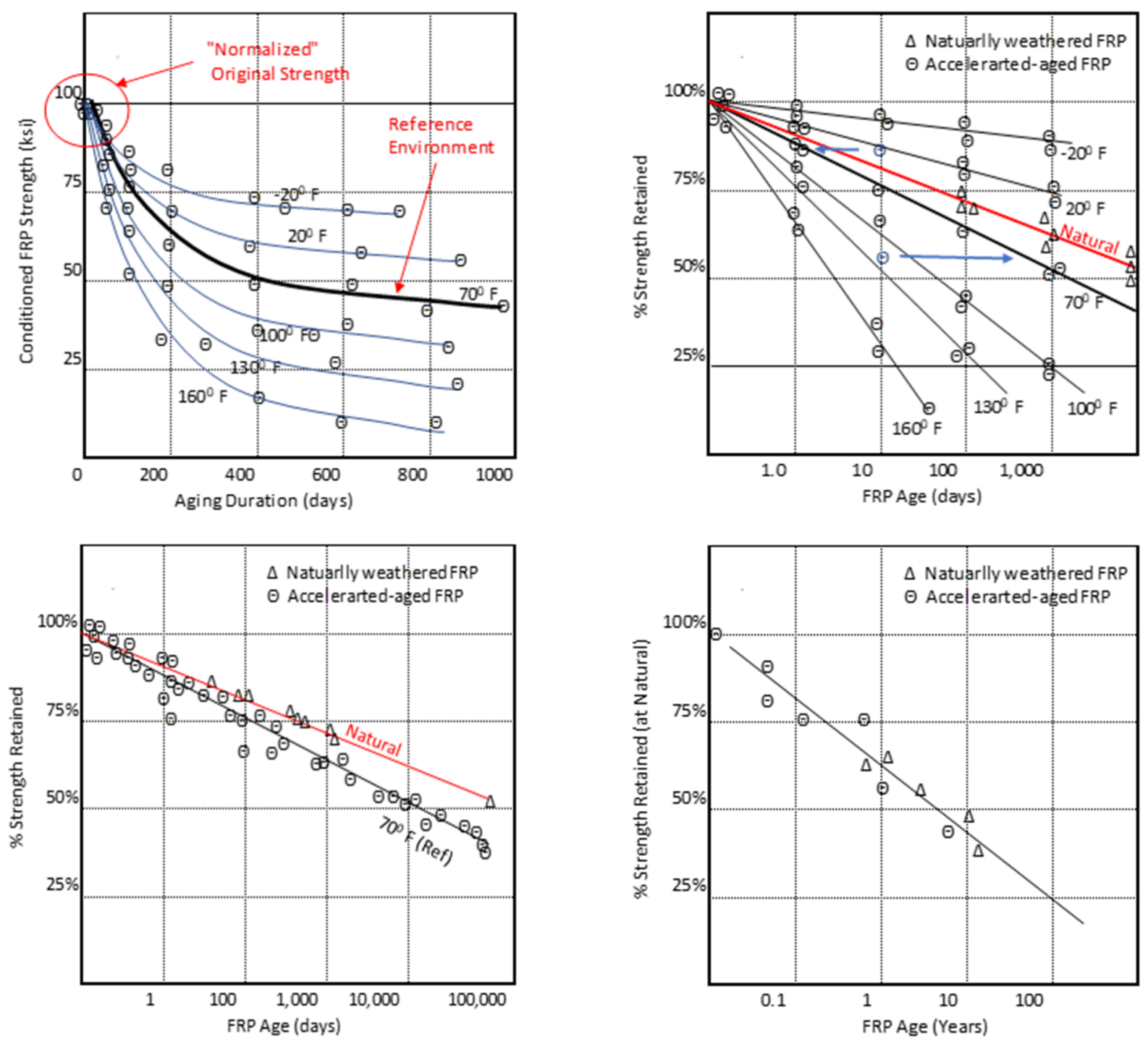

Figure 3.6: Fast Converging Steps used for Data Analysis 
3. For each environment tested, plot series of curves for strength loss (i.e., normalized percentage loss from $\mathrm{t}=0$ or conditioned strengths) for aged samples against the aging period (i.e., number of days, weeks, or months when the specimen was tested). See Figure 3.6 for illustrations. Plots are likely to be non-linear (use power or logarithmic functions for linear transformation), and the reduction in strength should taper off as the aging time increases. Using regression analysis, find the best-fit equations for each set of test readings (readings at $0 \mathrm{~d}, 1 \mathrm{~d}, 5 \mathrm{~d}, 30 \mathrm{~d}$, $6 \mathrm{~m}, 12 \mathrm{~m}, 2 \mathrm{y}$, etc.). Identify outliers using maximum normed residual (MNR) or many other statistical methods available. Even a simple visual comparison such as 2 or 3 standard deviations away from the mean regressed value will be helpful. After a set of tests (e.g., longitudinal tensile strength tests for Vinylester GFRP specimen in $80^{\circ} \mathrm{C}$ tap water after nine months aging period) are complete, find a best-fit equation (typically logarithmic) for that specific group using regression to identify outliers and missing data. Best to normalize the data with respect to dry virgin strength at time $t=0$ to render ATM data useful to others and for use in further reliability analysis. Expect steep drops in early strength test results as saturated strength due to moisture uptakes can significantly lower than the dry strength in the first few days or even hours.

4. Re-plot the same data for an Arrhenius-type relationship (e.g.: $A=A_{0} e^{(-\Delta E / R T)}$ to obtain linear plots. For each strength loss $(75 \%, 50 \%, 25 \%$, etc. $)$, the straight-line plot will be for the logarithm of time to reach a particular strength (or percentage reduction) against the inverse of temperature (in ${ }^{0}$ Kelvin or $\mathrm{T}=273^{0} \mathrm{~F}+\mathrm{t}_{\text {) }}$ ). The use of Arrhenius temperature-stress superimposition principle requires an understanding of activation energy $(\Delta \mathrm{E} / \mathrm{RT})$ and limitations which have been covered well in many published literature and reports(GangaRao et al., 2007; Lorenzo, 2018; Wang et al., 2015) and is not explained in this report. Other 
parameters, such as alkalinity/acidity (e.g., $\mathrm{pH}$ value) or a combination with two or more parameters, can also be used in combination with temperature. It helps to transform all test data under varying temperatures to a single reference temperature (typically room temperature) data and all $\mathrm{pH}$ tests to a single reference $\mathrm{pH}$ (typically tap water) data, and then transform reference $\mathrm{pH}$ data to the reference temperature data to obtain a single line of transformed data from many varied environments. In the Author's opinion, many subtasks listed in step 4 are not necessary, and transformation can be done directly based on the time required to reach various strengths under varied environments. Direct transformation helps to reduce unnecessary manipulation of test data and hampers the reproducibility of results.

5. Normalize accelerated test plots into a single straight-line plot by using logarithm or other time functions (for a given strength loss) for a selected temperature or another reference environmental parameter. For example, a reference parameter (e.g., $\left.70^{\circ} \mathrm{F}\right)$ can be chosen to represent the actual condition that an FRP component will be subjected to during its natural aging. If all regression equations (developed for varied environments) use the "dry" virgin sample strengths as the starting point (i.e., regression line y-intercept is at $100 \%$ ), it is easy to transform time by just using the line slope (i.e., degradation coefficient, "a") between the tested environments. Often multiple-step transformations are needed for a combination of $\mathrm{pH}$ and temperatures.

6. Once all strengths are normalized to a single referenced environment on a single timeline in the lab, the next step of calibration using Time Shift Function (TSF) can take place to transfer all strength retention results into a real-life reference timeline. See Chapter 4 for additional discussion about TSF. 


\subsubsection{Data Calibration}

Normalized data, as obtained from the reduction of accelerated testing data, must be calibrated with natural aging (when available) or with the final desired reference environment. The purpose is to transform the lab test data to actual site data so that strength retention predictions over the projected service life can be made. After such life prediction models were developed, the normalized data, along with other available data, was used to extract statistical data at various age intervals. This process can take a significant amount of effort to verify, check, and normalize data from diverse sources and varied periods. In this study, the analytical formulation of FRP deterioration curves based on logarithmic decay have been developed, and a detailed process is shown in Chapter 4 and Appendix B (Figures B.1 to B.12).

\subsubsection{Development of Statistical Parameters}

Since the mechanical properties of FRP members inherently vary from their time in placement in service to retirement from service, this step is vital for any reliability-based analysis. The method used in this report is described in detail in Chapter 4. All necessary descriptive statistics of the normalized calibrated data. (e.g., means, standard deviations, normality, and density distribution parameters) at initial and various aging intervals were obtained concerning the regressed mean degradation function. The intent is to get a material property variation (around the regressed time-dependent mean value) throughout FRP service life, not just at the beginning of its service life. Additional details can be found in Section 3.2.

\subsubsection{Selection of Design Examples}

A few simple design examples (flexural beams, tension bracings) were selected to allow the computation of reliability indices for the framework. The examples were chosen based on final 
normalized data available, the ability to form appropriate limit states equations for reliability analyses, and their potential use in civil engineering construction. The results of these examples are used in Chapter 4 for additional discussions. A sample Basis of Design (BOF) for a particular FRP application (Chapter 6) is also developed to illustrate how to bring such research findings to the professional practice of civil engineering. It must be noted that a wide variety of examples and many real-world examples must be tested to calibrate the code-adoptable design recommendations.

\subsubsection{Development of Performance Functions}

For the selected examples, performance equations were developed to determine its limit states for various failure modes (e.g., ultimate stress, deflections, etc.). A more detailed explanation can be found in Sections 3.2.

\subsubsection{Selection of Target Reliability and Service Life}

A target reliability index (an acceptable probability of failure) to ensure that the system will perform its function as designed must be selected upfront. The factor of safety is often used in the mechanical engineering field, and its non-linear relationship with the probability of failure is further studied in Chapter 4. The desired useful service life of the FRP member under consideration is also selected at this time. The desired service life for most sizeable civil engineering structures such as bridges and buildings should be 100 years. However, many design codes now require a minimum of 75 years of service life, while old codes required only a 50-year service life. Minor buildings (e.g., storage facilities) and special-purpose structures (e.g., offshore oil platforms) are often designed for 10 to 25 years of service. The smaller service life of many components in the mechanical engineering field seldom exceeds 30 years. This report includes 
service life comparisons for $10,25,50,75$, and 100 years to facilitate such selection. A discussion about the range of reliability indices and the service life is also provided in section 3.2

\subsubsection{Calculation of Initial Reliability}

Using the performance function and design variability of member and loadings shown in Section 3.2, initial or virgin reliability indices are computed. This value will relate to a member just placed in service $(\mathrm{t}=0)$, say within a reasonable time since manufacturing. This process has been well understood as LFRD codes in Canada/US were developed in the '80s and '90s.

The statistical variations of load effects Q will be based on available published data used in the development of other codes and assumed to not change over time. On the contrary, the statistical variation of member resistance $\mathrm{R}$ will be based on FRP data collected in previous steps and will vary depending on how long the member has been in service.

\subsubsection{Calculation of Aged Reliability}

Using a similar process as in the previous step, compute reliability at various commonly used service life intervals of $10,25,50,75$, and 100 years. Since most lab testing periods do not extend beyond 1-2 years and available natural aging data does not extend beyond 30 years, the normalized calibrated data from accelerated tests must be used for reliability-durability predictions. As more natural aging and accelerated test data become available, FRP resistance factors can be re-calibrated for longer known life spans. In this report, computed deterioration rates from normalized accelerated test data are used and supplemented with natural aging data. Use of Markov Chain and/or Weibull Survival functions were considered but discarded since adequate data for aged probabilities (e.g., FRP population survival statistics for intervals of 10, 25, 50,75 , and 100-year) is not yet available. 


\subsubsection{Calculation of Reliability-Based Durability Reduction Factors}

The reliability-durability curve was plotted for different life-spans and other parameters using reliability indices obtained at various aging intervals, and an equation to predict reliability at a given time interval was developed. Given the number of approximations and variability of both materials and loads, a simple design equation (such as linear or logarithmic curve) was preferred.

Overall, the process is simple to explain in a design example. The original durability factors used in the sample design (Example 1, Appendix A) were adjusted until its computed reliability indices are at or above its target values at the end of its design service life. Figures 3.1 and 4.8 reveal that the design reliability indices (at the time of the initial placement of members) must be higher than the in-service reliability indices near the end of a member's service life. However, in this research, we are seeking an answer to the question, "by how much?" The design intent should be to not over-design (or under-design) yet maintain a minimum acceptable probability of failure throughout a member's service life (e.g., a member designed for 50 years should still meet its target reliability indices till the end of its designed service life). The durability factors for various life spans are obtained by comparing its performance functions (e.g., reliability indices) at the initial and final stages.

\subsubsection{Calibration of Durability Reduction Factors}

This step involves computing durability factors for additional real-life examples (e.g., members designed using current codes and practices) and comparing them to the computed aged durability factors using the values obtained from this or similar durability research. This step often provides a good indication of where the gaps are in the current state of practice. 
This portion of work requires a vast amount of combined effort by industry professionals, researchers, and academia. This task must be taken under a separate large-scale endeavor and is considered outside of the scope of this report.

\subsubsection{Development of Recommendations or Basis of Design (BOD)}

This report makes conservative recommendations for new durability strength reduction factors along with appropriate life prediction models based on several databases analyzed (Chapter 4). A sample Basis of Design (BOD) case study is included to illustrate the process described in the Framework (Chapter 6). Even though more research is needed for calibration of reliabilitybased durability factors, these recommendations can help design an FRP member efficiently to serve its entire design service life within an acceptable risk of failure.

\subsubsection{Hypothesis Testing}

Typically, a null hypothesis is the best way to evaluate if the proposed hypothesis should be "kept" or "rejected" based on a pre-established measurement tool. The current hypothesis claims that "Reliability-based durability framework provides more consistent risk (measured as reliability indices at various service life intervals) for new FRP structures as compared to the existing traditional methods." In order to test the hypothesis, the next logical step will be to employ the framework in several long-term projects from start to finish. For example, a wide range and variety of FRP members can be designed using the framework recommendations, manufactured, tested in the laboratory under a controlled environment, and then their performance compared with actual members placed in service under similar loads and environments. It will not be a hypothesis testing from a statistical analysis sense, but an evaluation will indicate if the framework can stay 
as is, needs further modification, or be discarded entirely. This task is considered outside the scope of this research due to the extensive amount of time and effort involved.

\subsection{SUMMARY}

Specific findings of this chapter can be summarized as follows:

1. The use of ATM data and naturally aged data is critical in developing a reliability-based durability framework. The ATM process can be modified slightly to provide results needed for the reliability analysis.

2. The use of professional data analysis software is recommended to make the ATM and reliability data processing less tedious and more accurate. Most programs or Add-ons deal better with missing, erroneous, or outlier observations, regression analysis, distribution types, significance, confidence intervals, statistical parameters, and other many key parameters.

3. The use of a minimally corroborated deterministic approach may result in an overly expensive structure or grossly under-designed system, both are undesirable (assuming the test data is sparse for different material and environmental parameters). A reliability approach to durability estimation provides a systematic and rational tool for designing FRP composites to last their intended service life.

4. The reliability approach to FRP materials and members takes into account its inherent variability of properties, time-dependent degradation of FRP, brittle nature of failure, and acceptable risk or probability of failure to arrive at a rational design for the intended service life.

5. The formulation of correct performance function(s) based on the limit states (failure modes) is essential to compute the reliability index, which includes determination/selection probability 
distribution function. For a large sample, the normal distribution provides the simplest way to take reliability methods into account.

6. A well-accepted measure of the probability of failure can be approximated by the reliability index of 3.5, roughly translating into a probability of failure of 1 in 4,300. The development of the strength reduction factor is based on a pre-selected target reliability index.

7. There are many steps in the calculations of reliability index, strength reduction factor, timedependent durability, and service life predictions, and all are further illustrated via a case study (Chapter 6) and design examples (Chapter 4 and Appendix A). The calibration of strength reduction factors is the last step, and due to the level of efforts involved, it should be undertaken at an institution or industry level.

8. The entire process is explained with a flowchart with the use of an actual FRP database collected, normalized, and analyzed as a part of this research. The work is further carried into Chapters 4, and 6 illustrate how to account for environmental effects using the reliability-based durability framework proposed in this chapter. 


\section{CHAPTER 4 ACCOUNTING FOR ENVIRONMENTAL}

\section{EFFECTS}

\subsection{INTRODUCTION}

As mentioned previously in Section 2 that the durability of FRP is adversely affected by various environmental effects. This chapter discusses how a reliability-based framework can be used in the design of composite systems - accounting for multiple environmental effects from outdoor use - covering their entire service lives (Figure 4.1).

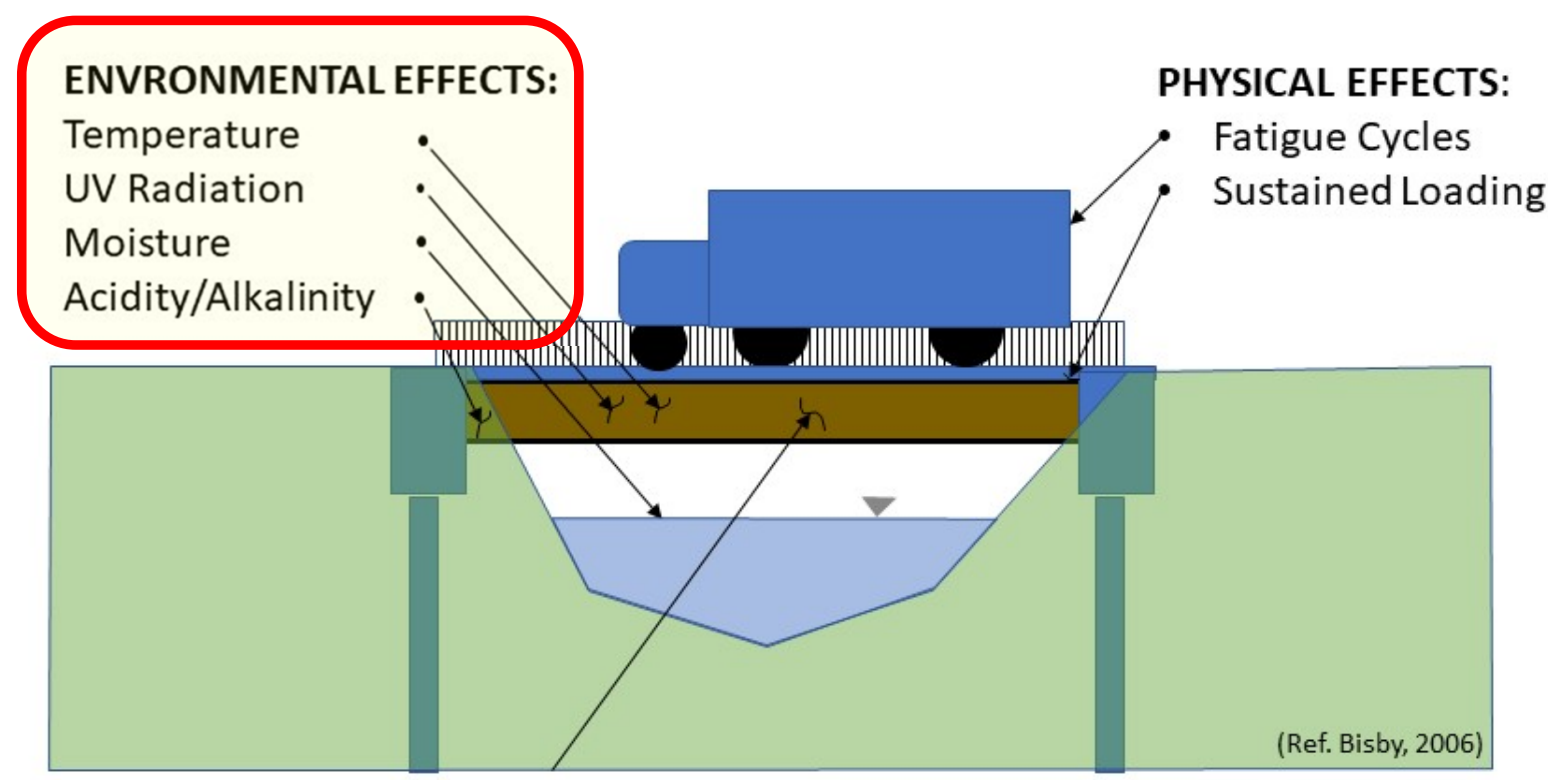

AFFECTED DURABILITY CHARACTERSTICS:

Tensile / Compressive / Flexural / Shear Strengths and Moduli

Figure 4.1: Application of Environmental Effects on Outdoor Structures

The database includes accelerated and natural tests performed by various researchers worldwide. Accelerated tests included a variety of temperatures, moistures, and $\mathrm{pH}$ variations, and natural aging included outdoor exposures. Some of the original test data were collected, 
organized, and presented by several graduate WVU students. Lorenzo (2018) compiled the original WVU-CFC database (consisting of accelerated testing and naturally aged data) from many researchers (Sonawala \& Spontak, 2017; Grammatikos, 2016; Wang et al., 2015; Marru et al., 2014; Shi et al., 2014; Cabral-Fonseca, 2012; Chin et al., 2010; Robert, 2010; Won et al., 2007; Kim, 2007; Chen et al., 2006; Hammami, 2004; Karbhari, 2004; Rivera \& Karbhari, 2002; Sen, 2002; Dejke, 2001; Vijay, 1999; and Kajorncheappunngam, 1999). Lorenzo's database was later reorganized, refined, and updated with additional naturally aged data (Barker, 2019 and Keller et al., 2016, 2007) and refined by the author, and has been used in this study. Barker (2019) performed additional tests on naturally aged and ATM samples for interlaminar shear; however, a direct comparison or correlation with previous natural aging data proved to be very difficult. Accelerated test databases from several other research institutions were also collected and analyzed. However, due to lack of proper natural (or reference) environments and insufficient data (for the formulation of a meaningful Arrhenius result), many of the accelerated test data could not be normalized or further analyzed for this report presentation. Another set of sizeable original natural and accelerate test data was provided by an FRP manufacturer whose name is not disclosed due to a Non-Disclosure Agreement or NDA. These data have been analyzed and used in a case study showing the development process for a basis of design (BOD) in Chapter 6. This chapter and Chapter 6 illustrate how to account for environmental effects in actual professional engineering practice.

The aging database used in this chapter was re-organized and analyzed using simplified linear regression methods (some of the ordinary least squares or OLS) to ensure reproducibility in the future work by other analysts. The primary object was to develop a formulation for various aging regression equations for mechanical strengths (e.g., tensile, compression, flexural, and shear 
strengths) of FRP samples in vinylester, polyester, and epoxy resins. Statistical variations of tested mechanical properties were also developed for use in the reliability analysis. Initially, aging regression equations for all four mechanical strengths are developed; but it is likely that in the future, this effort may be limited to interlaminar shear strength (ILSS) as the only controlling limit state because of its high sensitivity response to environmental exposure, and also ILSS forms the weak link in terms of force/strength transfer. However, proper correlations of various mechanical properties with ILSS strength/aging behavior are yet to be determined, and the reliability requires the entire distribution (not just the weak links), and therefore, for the time being, aging test data for all key mechanical properties are still needed.

\subsection{AGING DATABASE}

Since databases from various researchers contained a wide variety of combinations of fibers, resins, and environments, it was found necessary to organize the data into several common broader accelerated aging test groups to help the processing of extensive data, which is significant

for this study. These broad categories serve the purpose of building the proposed framework; however, specific subcategories can also be made if adequate data (number of valid test readings) is available. All original groups (as shown in Tables 4.1, 4.2, and 4.3) were organized initially by Lorenzo (2018) and are further refined by the author for this study. Chapter 6 describes a case study for the field application of a GFRP system, which is designed for a particular environment.

For normalization purposes, the primary aging database was divided into various $\mathrm{pH}$ and temperature groups, fibers, and resins, as summarized in Table 4.1. It should be noted that in the context of this research, the placement of test data into specific $\mathrm{pH}$ and Temperature groups (as shown in Tables 4.2 and 4.3) is not necessary, and perhaps, a direct Arrhenius conversion to the 
reference environment (using actual test environment $\mathrm{pH}$ and Temperature values) would have been more accurate and convenient.

Table 4.1- Basic Aging Database Groups

\begin{tabular}{llll} 
Fibers (FB): & Glass (GL) & Carbon $^{1}(\mathrm{CR})$ & \\
\hline Resin or Matrix (MX): & Vinylester (VE) & Polyester (PE) & Epoxy (EN)
\end{tabular}

\begin{tabular}{|c|c|c|c|}
\hline pH (P) Groups: & $\mathrm{P} 0, \mathrm{P} 1, \mathrm{P} 2, \mathrm{P} 3, \mathrm{P} 4$, and $\mathrm{P} 5$ & & \\
\hline Temperature (T) Groups: & $\mathrm{T} 0, \mathrm{~T} 1, \mathrm{~T} 2, \mathrm{~T} 3, \mathrm{~T} 4$, and $\mathrm{Tn}$ & & \\
\hline Strengths (SR) Tests: & $\begin{array}{l}\text { Compression } \\
\text { (CS) }\end{array}$ & Flexural (FS) & $\begin{array}{l}\text { Interlaminar Shear } \\
\text { (ILSS) }\end{array}$ \\
\hline
\end{tabular}

Note:

${ }^{1}$ Not enough data about Carbon fibers aging was collected to allow normalization/projections

$\mathrm{pH}(\mathrm{P})$ groups are described in Table 4.2. It should be noted that FRP immersion in the tap or distilled water (group P3) was often found to be the only "reference" environment for accelerated testing methods for specific properties and FRP types.

Table 4.2- pH Group Combinations

\begin{tabular}{|l|l|l|l|}
\hline Group Name & pH Range & Representative Value & Description \\
\hline Pn & 7 & 7 & Natural ${ }^{1}$ (Indoor/Outdoor in Air/Dirt) \\
\hline P1 & 0 to 4 & 2 & High Acidity (in Solution) \\
\hline P2 & 4 to 6 & 5 & Low Acidity (in Solution) $^{2}$ \\
\hline P3 & 6 to 8 & 7 & Neutral $^{2}$ (in Distilled/Tap Water) \\
\hline P4 & 8 to 10 & 9 & Low Alkaline $^{3}$ (in Solution) \\
\hline P5 & 10 to 13 & 13 & High Alkaline $^{4}$ (in Solution) \\
\hline
\end{tabular}

Notes:

${ }^{1}$ Natural environment is considered indoor/outdoor air with atmospheric moisture and dirt with freeze-thaw

${ }^{2}$ Neutral condition is considered "saturated" properties and P3 is used as the reference in lieu of natural data

${ }^{3}$ Low or slightly alkaline condition is typically found in marine water

${ }^{4} \mathrm{High}$ alkaline condition is generally found in concrete 
Temperature (T) groups are described in Table 4.3.

Table 4.3- Temperature Group Combinations

\begin{tabular}{|l|l|l|l|}
\hline $\begin{array}{l}\text { Group } \\
\text { Name }\end{array}$ & Temperature Range & $\begin{array}{l}\text { Representative } \\
\text { Value }\end{array}$ & Description \\
\hline Tn & $-5^{\circ}$ to $30^{\circ} \mathrm{C}\left(23^{\circ} \mathrm{F}\right.$ to $\left.85^{\circ} \mathrm{F}\right)$ & $15^{\circ} \mathrm{C}\left(60^{\circ} \mathrm{F}\right)$ & $\begin{array}{l}\text { Natural }{ }^{1} \text { (Indoor/Outdoor in Air/Dirt) } \\
\text { (Morgantown, West Virginia) }\end{array}$ \\
\hline $\mathrm{T} 1$ & $-10^{\circ}$ to $14^{\circ} \mathrm{C}\left(14^{\circ}\right.$ to $\left.57^{\circ} \mathrm{F}\right)$ & $5^{\circ} \mathrm{C}$ & Cold \\
\hline T2 & $15^{\circ}$ to $40^{\circ} \mathrm{C}\left(58^{\circ}\right.$ to $\left.104^{\circ} \mathrm{F}\right)$ & $25^{\circ} \mathrm{C}$ & Neutral ${ }^{2}$ (Indoor/Room Temperature) \\
\hline T3 & $41^{\circ}$ to $60^{\circ} \mathrm{C}\left(105^{\circ}\right.$ to $\left.140^{\circ} \mathrm{F}\right)$ & $45^{\circ} \mathrm{C}$ & Hot \\
\hline T4 & above $60^{\circ} \mathrm{C}\left(140^{\circ} \mathrm{F}\right)$ & $60^{\circ} \mathrm{C}$ & Very Hot \\
\hline T5 & $-10^{\circ}$ to $40^{\circ} \mathrm{C}\left(14^{\circ}\right.$ to $\left.104^{\circ} \mathrm{F}\right)$ & N/A & Freeze-Thaw cycles (in the lab) $)^{3}$ \\
\hline
\end{tabular}

Notes:

${ }^{1}$ Natural environment is the referenced outdoor environment outside of WVU CFC lab, Morgantown, WV

${ }^{2}$ Neutral environment is considered indoor/room temperature in the WVU CFC lab (with ample outdoor ventilation). $\mathrm{T} 2$ can also be used as the reference environment in lieu of natural data.

${ }^{3}$ Available freeze-thaw cycle data was not adequate for regression analysis

Description of the additional datasets studied for sustained stress and submerged seawater environment are included in Chapters 5 and 6, respectively.

\subsection{DEVELOPMENT OF AGING EQUATION}

FRP deteriorates over time in laboratory testing and also under natural environment (e.g., fluctuating temperatures, atmospheric moisture, water, UV, etc.), and loss of strength slows over time, as evident from many research findings. Long-term natural aging tests show that even under ambient conditions, up to $20 \%$ maximum loss of some mechanical properties can be expected. Logarithmic degradation equations (ln or natural log of exponent "e") chosen since it: a) provides the best fit for modeling strength retention curves for aged FRP due to environmental effects over a very long period ( 1 day to $100+$ years), b) matches well with exponential-based probability functions used in reliability analysis, c) allows single parameter linear transformation to a line equation format $(y=a x+b)$, and $d)$ has been extensively used in modeling Arrhenius relationship 
in many previous studies (Manalo et al., 2020). The aging strength retention (SR) equation selected for data reduction uses the following normalized format:

$\%$ Strength Retained SR $=100 \%$ - a $\mathrm{x} \ln$ (age in years "t" x 365+1) or

$\mathrm{SR}=1-\mathrm{a} \ln (365 \mathrm{t}+1)$ or it can be re-written as

$$
f_{S R}=1-a \ln (t)
$$

Where $f_{S R}$ is tested Strength or material property retained after $t$ days, and $a$ is the logarithmic degradation (slope) coefficient (also referred to as environmental degradation coefficient) At day one ( $\mathrm{t}=1$ day), the equation becomes:

$$
f_{S R}=f_{\operatorname{Virgin}(t=1)}
$$

Equation 4.1 can be rearranged to compute projected age t (in days) using a calibrated degradation coefficient " $a$ " for the referenced environment.

$$
t=e^{\frac{\left(1-f_{S R}\right)}{a}}
$$

Where: SR is percentage strength retained at the age of " $\mathrm{t}$ " year. "ln" is the natural log, and therefore the value of $t$ is assumed to be a minimum of 1 day (instead of 0 days). FRP Strength Retention (SR) coefficient or degradation coefficient "a" is derived using regression of accelerated and natural test data. All aged strengths are normalized with respect to the "dry" virgin material strength (typically ultimate mean strength) to allow consistency over various test periods and reproducibility of ATM results (e.g., all reported aging test results are normalized with respect to a single original test value). The default value provided by many regression solvers or trend lines is not used for the same reason.

The time shift factor (TSF) is used to transform the lab timeline to a reference environment timeline and can be computed, as shown below.

$$
T S F=\frac{t_{r e f}}{t_{l a b}} \quad \ldots E q(4.3)
$$


Assume that a degradation coefficient " $\mathrm{a}_{\mathrm{a} a \mathrm{~b}}$ " has been regressed for a tested lab environment $\mathrm{e}_{\text {lab. }}$. Now, we want to compute TSF that can be applied to the lab environment $\mathrm{e}_{\text {lab }}$ timeline to transform it into the timeline of a reference environment $\mathrm{e}_{\text {ref. }}$ It is assumed that the calculated degradation coefficient for the reference environment is $\mathrm{a}_{\text {ref. }}$ From Equation 4.3 substitutions of $t_{\text {ref }}$ and $t_{\text {lab }}$, we get:

$$
T S F=e^{\left(1-f_{S R}\right)\left(\frac{1}{a_{r e f}}-\frac{1}{a_{l a b}}\right)} \quad \ldots E q(4.4)
$$

$\mathrm{f}_{\mathrm{SR}}$ is the corresponding strength retention in the environment $\mathrm{e}_{\mathrm{lab}}$ timeline in lab $\mathrm{t}_{\text {lab }}$ which needs to be transformed to the timeline of the reference environment $\mathrm{e}_{\mathrm{ref}}$ by using the following TSF equation:

$$
t_{r e f}=t_{l a b} \times T S F \quad \ldots E q(4.4)
$$

After TSF transformation, $f_{S R}$ remains the same, but its timeline is now $t_{\text {ref }}$ (instead of $t_{\text {lab }}$ ) in the reference environment $e_{\text {ref. }}$ It is worth noting that once degradation coefficients (e.g., $a_{\text {ref, }}$ and $\mathrm{a}_{\mathrm{lab}}$ ) have been regressed for each environment, the TSF transformation is only one step process for each tested SR without going through other intermediate steps such as determination of activation energy or transforming temperatures to Kelvins or any other minor manipulations.

\subsection{DATABASE ANALYSES}

Tables 4.5, 4.6, and 4.7 list the regressed degradation coefficient " $\mathrm{a}_{\mathrm{lab}}$ " for accelerated and naturally aged environment tests for various combinations. Please note that due to the lack of adequate data, the regression coefficients for certain combinations could not be computed. There was no natural aging data available for Polyester GFRP and Epoxy GFRP for further calibration, 
so their reference environment remained as "fully immersed and saturated GFRP in a room temperature tap water." See Appendix A, Figures B.2 to B.6 for regression curves developed for Vinylester GFRP, Figures B7 to N.12 for Polyester GFRP, and B.13 and B.14 for Epoxy GFRP.

It must be noted that more data is still needed to compensate for insufficient, missing, highly skewed, or erroneous data. Further refinements of the ATM regressed degradation coefficients "a" (Tables 4.5, 4.6, and 4.7) are warranted to gain more confidence in the widespread use of these coefficients. Readers are cautioned that the computation of these degradation coefficients was performed only to illustrate the functioning of the proposed time-dependent reliability framework for FRP durability design, and better results can be obtained when tests are designed for a particular FRP application in a specific environment (instead of generic groups used in this Chapter). 
Table 4.5 shows the regressed coefficient " $\mathrm{a}_{\mathrm{lab}}$ " for individual ATM group combinations for "vinylester based GFRP" samples under ATM studies (max 18 months duration). For example, the tensile strength degradation coefficient is $6.8 \%$ for all vinylester GFRP samples in an environment that includes mildly alkaline $\mathrm{pH}$ group $\mathrm{P} 4$ with very hot Temperature Group T4.

Table 4.5- Vinylester GFRP Group Degradation Coefficients in Lab (a $\left.a_{\text {lab }}\right)$

\begin{tabular}{|c|c|c|c|c|c|c|c|c|}
\hline \multirow[b]{2}{*}{$\begin{array}{l}\text { Glass Fiber } \\
\text { (GL) Matrix }\end{array}$} & \multirow{2}{*}{\begin{tabular}{|l} 
Strength \\
Retention (SR) \\
Tests
\end{tabular}} & \multirow[b]{2}{*}{$\begin{array}{c}\text { Temperature } \\
\text { Groups }\end{array}$} & \multicolumn{6}{|c|}{ pH Groups } \\
\hline & & & $\begin{array}{c}\text { P1 } \\
\text { (Highly Acidic) }\end{array}$ & $\begin{array}{c}\text { P2 } \\
\text { (Mildly Acidic) }\end{array}$ & $\begin{array}{c}\text { P3 } \\
\text { (Neutral / } \\
\text { Tap Water) } \\
\end{array}$ & $\begin{array}{c}\text { P4 } \\
\text { (Mildy } \\
\text { Alkaline) }\end{array}$ & $\begin{array}{c}\text { P5 } \\
\text { (Highly } \\
\text { Alkaline) } \\
\end{array}$ & $\begin{array}{c}\text { Pn } \\
\text { ( Natural / } \\
\text { Outdoor) } \\
\end{array}$ \\
\hline \multirow{20}{*}{$\begin{array}{l}\text { Vinylester } \\
\text { (VE) }\end{array}$} & \multirow[t]{5}{*}{ Tensile (TS) } & T1 (Cold) & & & $0.3 \%$ & & & \\
\hline & & T2 (Room Temp) & & $2.1 \%$ & $2.4 \%$ & $3.2 \%$ & $3.8 \%$ & \\
\hline & & T3 (Hot) & & $2.6 \%$ & $3.4 \%$ & & $12.8 \%$ & \\
\hline & & T4 (Very Hot) & & $5.2 \%$ & $6.7 \%$ & $6.8 \%$ & $12.8 \%$ & \\
\hline & & Tn (Outdoor) & & & & & & $2.2 \%$ \\
\hline & \multirow[t]{5}{*}{ Compressive (CS) } & $\mathrm{T} 1$ (Cold) ${ }^{3}$ & & & & & & \\
\hline & & T2 (Room Temp) & & $5.5 \%$ & & & $4.8 \%$ & \\
\hline & & T3 (Hot) & & & & & & \\
\hline & & \begin{tabular}{|l} 
T4 (Very Hot) \\
\end{tabular} & & & & & & \\
\hline & & $\operatorname{Tn}$ (Outdoor) ${ }^{3}$ & & & & & & \\
\hline & \multirow[t]{5}{*}{ Flexural (FS) } & $\mathrm{T} 1$ (Cold) ${ }^{3}$ & & & & & & \\
\hline & & T2 (Room Temp) & $4.5 \%$ & $3.7 \%$ & $2.6 \%$ & & $6.2 \%$ & \\
\hline & & T3 (Hot) & & & $3.8 \%$ & & & \\
\hline & & T4 (Very Hot) & & & $4.2 \%$ & & & \\
\hline & & Tn (Outdoor) & & & & & & $3.0 \%$ \\
\hline & \multirow{5}{*}{$\begin{array}{l}\text { Interlaminar } \\
\text { Shear (ILSS) }\end{array}$} & T1 (Cold) & & & $1.8 \%$ & & & \\
\hline & & T2 (Room Temp) & $1.8 \%$ & $1.7 \%$ & $2.5 \%$ & & $10.3 \%$ & \\
\hline & & T3 (Hot) & & $3.1 \%$ & $4.2 \%$ & & $12.8 \%$ & \\
\hline & & T4 (Very Hot) & & & $6.9 \%$ & & $14.8 \%$ & \\
\hline & & In (Outdoor) & & & & & & $3.6 \%$ \\
\hline $\begin{array}{l}\text { Notes: } \\
\text { 1. Degrada } \\
\text { 2. Degrada } \\
\text { 3. Very lin } \\
\text { 4. No natu } \\
\text { 5. Data fou }\end{array}$ & $\begin{array}{l}\text { ation Equation: \% Str } \\
\text { ation Coefficient "a" } \\
\text { ited data available } \\
\text { ral data is available } \\
\text { und to be erroneous }\end{array}$ & $\begin{array}{l}\text { rength Retained (SR } \\
\text { is computed based } \\
\text { for regression } \\
\text { for calibration. Roo } \\
\text { for regression anal }\end{array}$ & $\begin{array}{l}=100 \%-a * \text { In } \\
\text { on th eregressi } \\
\text { ysis temperature }\end{array}$ & $\begin{array}{l}\text { (age in years*36 } \\
\text { ion of the norma } \\
\text { tap water is use }\end{array}$ & $\begin{array}{l}\text { 5+1) } \\
\text { ized test data } \\
\text { d as Reference }\end{array}$ & nvironment & & \\
\hline
\end{tabular}


Table 4.7 shows the regressed coefficient " $\mathrm{a}_{\mathrm{lab}}$ " for individual ATM group combinations for "epoxy-based GFRP" samples under ATM studies for up to 18 months of test durations. For example, the tensile strength degradation coefficient is $6.3 \%$ for all epoxy GFRP samples in an environment that includes mildly acidic $\mathrm{pH}$ group $\mathrm{P} 2$ with very hot Temperature Group T4. In general, ATM data used in this study for epoxy based GFRP was very limiting, and there was no external naturally aged data available.

Table 4.7- Epoxy GFRP Group Degradation Coefficients in Lab (alab)

\begin{tabular}{|c|c|c|c|c|c|c|c|c|}
\hline \multirow[b]{2}{*}{$\begin{array}{l}\text { Glass Fiber } \\
\text { (GL) Matrix }\end{array}$} & \multirow{2}{*}{\begin{tabular}{|l} 
Strength \\
Retention (SR) \\
Tests
\end{tabular}} & \multirow[b]{2}{*}{$\begin{array}{l}\text { Temperature } \\
\text { Groups }\end{array}$} & \multicolumn{6}{|c|}{ pH Groups } \\
\hline & & & $\begin{array}{c}\text { P1 } \\
\text { (Highly Acidic) }\end{array}$ & $\begin{array}{c}\text { P2 } \\
\text { (Mildly Acidic) }\end{array}$ & $\begin{array}{c}\text { P3 } \\
\text { (Neutral / } \\
\text { Tap Water) } \\
\end{array}$ & $\begin{array}{c}\text { P4 } \\
\text { (Mildy } \\
\text { Alkaline) }\end{array}$ & $\begin{array}{c}\text { P5 } \\
\text { (Highly } \\
\text { Alkaline) }\end{array}$ & $\begin{array}{c}\text { Pn } \\
\text { ( Natural / } \\
\text { Outdoor) }\end{array}$ \\
\hline \multirow{20}{*}{$\begin{array}{l}\text { Epoxy } \\
\text { (EN) }\end{array}$} & \multirow[t]{5}{*}{ Tensile (TS) } & $\mathrm{T} 1$ (Cold) $^{3}$ & & & & & & \\
\hline & & T2 (Room Temp) & & $8.0 \%$ & $4.5 \%$ & $5.4 \%$ & $5.3 \%$ & \\
\hline & & T3 (Hot) & & $5.9 \%$ & $3.9 \%$ & $11.5 \%$ & $7.3 \%$ & \\
\hline & & T4 (Very Hot) & & $6.3 \%$ & & & $3.0 \%$ & \\
\hline & & \begin{tabular}{|l|}
$\operatorname{Tn}$ (Outdoor) \\
\end{tabular} & & & & & & $4.5 \%$ \\
\hline & \multirow[t]{5}{*}{ Compressive (CS) } & $\mathrm{T} 1$ (Cold) ${ }^{3}$ & & & & & & \\
\hline & & T2 (Room Temp) ${ }^{3}$ & & & & & & \\
\hline & & T3 (Hot) ${ }^{3}$ & & & & & & \\
\hline & & T4 (Very Hot) ${ }^{3}$ & & & & & & \\
\hline & & $\operatorname{Tn}$ (Outdoor) $^{3}$ & & & & & & \\
\hline & \multirow[t]{5}{*}{ Flexural (FS) } & \begin{tabular}{|l} 
T1 (Cold) \\
${ }^{3}$
\end{tabular} & & & & & & \\
\hline & & T2 (Room Temp) & & $3.8 \%$ & & & $1.6 \%$ & \\
\hline & & T3 (Hot) & & $4.8 \%$ & & & $2.1 \%$ & \\
\hline & & \begin{tabular}{|l} 
T4 (Very Hot) $)^{5}$ \\
\end{tabular} & & $6.3 \%$ & & & $3.0 \%$ & \\
\hline & & $\operatorname{Tn}$ (Outdoor) $^{4}$ & & & & & & $3.8 \%$ \\
\hline & \multirow{5}{*}{\begin{tabular}{|l} 
Interlaminar \\
Shear (ILSS)
\end{tabular}} & $\mathrm{T} 1$ (Cold) ${ }^{3}$ & & & & & & \\
\hline & & T2 (Room Temp) ${ }^{3}$ & & & & & & \\
\hline & & T3 (Hot) ${ }^{3}$ & & & & & & \\
\hline & & T4 (Very Hot) ${ }^{3}$ & & & & & & \\
\hline & & $\operatorname{Tn}$ (Outdoor) $^{3}$ & & & & & & \\
\hline \multicolumn{9}{|c|}{ Notes: } \\
\hline \multicolumn{9}{|c|}{ 1. Degradation Equation: $\%$ Strength Retained $(S R)=100 \%-a * \ln ($ age in years $* 365+1$ ) } \\
\hline \multicolumn{9}{|c|}{ 2. Degradation Coefficient "a" is computed based on th eregression of the normalized test data } \\
\hline \multicolumn{9}{|c|}{ 3. Very limited data available for regression } \\
\hline \multirow{2}{*}{\multicolumn{9}{|c|}{ 4. No natural data is available for calibration. Room temperature tap water is used as Reference Environment }} \\
\hline & & for regression anal & & & & & & \\
\hline
\end{tabular}




\subsection{REGRESSION ANALYSES}

After the aging equations (i.e., lab regressed degradation coefficients as shown in Tables 4.5, 4.6, and 4.7) were established for each ATM environment group, the FRP data was normalized using Arrhenius stress superimposition principle. The normalization process included transforming each ATM environment group timeline into the referenced environment group timeline. The referenced environment group in this study was $\mathrm{P} 3$ tap water with a neutral $\mathrm{pH}$ of 7 and T2 representing indoor room temperature. The referenced group timeline was later calibrated with a natural weathering aging group $\left(\mathrm{P}_{\mathrm{n}}\right.$ and $\left.\mathrm{T}_{\mathrm{n}}\right)$. If natural weathering (typically outdoor) data was not available, the normalization process assumed a neutral $\mathrm{pH}$ at room temperature conditions (i.e., samples submerged in room temperature tap water or P3/T2 group).

Using the logarithmic deterioration equations as described in the previous section, the regressed values of decay coefficient "a" (i.e., strength retention deterioration coefficient) are listed in Table 4.8. Also, by grouping the aged strength from age 1 to 100 years, an increase in the Coefficient of Variation (COV) was estimated and is shown in Table 4.8. This increase in COV applies over the initial or virgin COV since al virgin strengths are normalized at $100 \%$ for time zero (Figure 4.3).

During the final analyses of the FRP database, it became clear that there is more room for improvements in the regression of degradation coefficients for several FRP types and material properties. It is noted that a substantial amount of test data from additional ATM and natural aging studies will undoubtedly help in offsetting the influence of a few skewed databases and will result in more representative degradation coefficients.

Table 4.8 is the normalized summary of all ATM and Natural data analyses conducted in this study. It shows the normalized coefficient for strength retention ("a") for computing the mean 
strength retention of various FRP samples at multiple ages. It uses equation $4.1\left(f_{S R}=1-\right.$ $a \ln (t))$ to compute retained strengths. It also shows the COV coefficient ("b") for computing $\mathrm{COV}_{\mathrm{t}}$ (Coefficient of Variation) of various FRP samples at various ages using equation $\left(\operatorname{COV}_{\mathrm{t}}=\right.$ $\left.\mathrm{COV}_{0}+\mathrm{b} \ln (t)\right)$, where $\mathrm{COV}_{0}$ is the "coefficient of variation" of the dry virgin samples. The aged $\mathrm{COV}_{\mathrm{t}}$ estimate is very approximate and may be further refined with additional ATM or natural data. All coefficient values listed in this table are also used in design examples (Appendix A). In addition to computing regression coefficients and projecting timelines, the time-dependent reliability analysis also requires an estimation of i) aged material property COV and ii) aged material property distribution type - both can be obtained from ATM data analysis. We are assuming that virgin material $\mathrm{COV}$ and distribution type is available by lab testing (or from the FRP manufacturer).

As mentioned before, there is room for the refinement of these coefficients/constants presented in Table 4.8, even though some of the \% strengths retained (SRs) shown in Table 4.8 match with other published studies. For example, the interlaminar shear strength of vinylester based glass fiber FRP (GL-VE-ILSS) in outdoor conditions is shown as $68 \%$ at 100 years, which compares well to an estimated $68 \%$ retained ILSS of GFRP rebars exposed to tap or saline water (Manalo et al., 2020). More ATM/naturally aged data are added, more representative these aging and COV coefficients will be of real FRP population in various environments. It should be noted that the primary purpose of this chapter is to show how to generate necessary parameters from ATM/naturally aged data for further reliability analysis. 
Table 4.8- Final Regression Summary of Normalized FRP Database ( $\left.a_{r e f}\right)$

\begin{tabular}{|c|c|c|c|c|c|c|c|c|}
\hline Designation & $\begin{array}{l}\text { Fiber } \\
\text { Type }\end{array}$ & $\begin{array}{l}\text { Resin } \\
\text { Type }\end{array}$ & $\begin{array}{c}\text { Strength } \\
\text { Type }\end{array}$ & Age $(y r s)^{1}$ & $\begin{array}{l}\text { Strength } \\
\text { Retension } \\
\text { Degradtion } \\
\text { Coefficient }{ }^{2} \text { "a" }\end{array}$ & $\begin{array}{c}\text { Estimated SR } \%^{3} \\
\text { (Outdoor }^{4} \\
\text { Environment) }\end{array}$ & $\begin{array}{l}\text { Coeffient of } \\
\text { Variation } \\
\text { (COV) } \\
\text { Constant }{ }^{5} \text { "b" }\end{array}$ & $\begin{array}{l}\text { Estimated } \\
\Delta \text { COV (over } \\
\text { Virgin COV) }\end{array}$ \\
\hline \multirow{7}{*}{ GL-VE-TS } & \multirow[t]{7}{*}{ Glass } & \multirow[t]{7}{*}{ Vinylester } & \multirow[t]{7}{*}{ Tensile } & \begin{tabular}{l|}
0 \\
\end{tabular} & \multirow{7}{*}{$2.2 \%$} & $100 \%$ & \multirow{7}{*}{$0.6 \%$} & $0 \%$ \\
\hline & & & & 1 & & $87 \%$ & & $3 \%$ \\
\hline & & & & 10 & & $82 \%$ & & $5 \%$ \\
\hline & & & & 25 & & $80 \%$ & & $5 \%$ \\
\hline & & & & 50 & & $78 \%$ & & $5 \%$ \\
\hline & & & & 75 & & $77 \%$ & & $6 \%$ \\
\hline & & & & 100 & & $77 \%$ & & $6 \%$ \\
\hline GL-VE-CS & Glass & Vinylester & \multicolumn{2}{|l|}{ Compressive } & \multicolumn{2}{|l|}{ Insufficient data } & \multicolumn{2}{|l|}{ Insufficient data } \\
\hline \multirow{7}{*}{ GL-VE-FS } & \multirow{7}{*}{ Glass } & \multirow{7}{*}{ Vinylester } & \multirow[t]{7}{*}{ Flexural } & 0 & \multirow{7}{*}{$3.0 \%$} & $100 \%$ & & $0 \%$ \\
\hline & & & & 1 & & $82 \%$ & & $8 \%$ \\
\hline & & & & 10 & & $75 \%$ & & $12 \%$ \\
\hline & & & & 10 & & $75 \%$ & $1.4 \%$ & $12 \%$ \\
\hline & & & & 10 & & $75 \%$ & & $12 \%$ \\
\hline & & & & 10 & & $75 \%$ & & $12 \%$ \\
\hline & & & & 100 & & $68 \%$ & & $15 \%$ \\
\hline GL-VE-ILSS & Glass & Vinylester & Interlaminar & 0 & & $100 \%$ & & $0 \%$ \\
\hline GL-VE-ILSS & alass & viпyеs tet & Shear & 1 & & $79 \%$ & & $5 \%$ \\
\hline & & & & 10 & & $70 \%$ & & $7 \%$ \\
\hline & & & & 25 & $3.6 \%$ & $67 \%$ & $0.9 \%$ & $8 \%$ \\
\hline & & & & 50 & & $64 \%$ & & $9 \%$ \\
\hline & & & & 75 & & $63 \%$ & & $9 \%$ \\
\hline & & & & 100 & & $62 \%$ & & $10 \%$ \\
\hline GL-PE-TS & Glass & Polyester & Tensile & 0 & & $100 \%$ & & $0 \%$ \\
\hline GL-PE-IS & viass & rolyester & 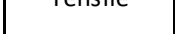 & 1 & & $90 \%$ & & $1 \%$ \\
\hline & & & & 10 & & $86 \%$ & & $1 \%$ \\
\hline & & & & 25 & $1.7 \%$ & $85 \%$ & $0.1 \%$ & $1 \%$ \\
\hline & & & & 50 & & $84 \%$ & & $1 \%$ \\
\hline & & & & 75 & & $83 \%$ & & $1 \%$ \\
\hline & & & & 100 & & $82 \%$ & & $1 \%$ \\
\hline GL-PE-CS & Glass & Polyester & Compressive & & Insufficient data & & Insufficient data & \\
\hline GL-PE-FS & Glass & Polyester & Flexural & 0 & & $100 \%$ & & $0 \%$ \\
\hline GL-PE-rS & Gidss & polyestel & riexulat & 1 & & $96 \%$ & & $4 \%$ \\
\hline & & & & 10 & & $94 \%$ & & $6 \%$ \\
\hline & & & & 25 & $0.7 \%$ & $94 \%$ & $0.8 \%$ & $7 \%$ \\
\hline & & & & 50 & & $93 \%$ & & $7 \%$ \\
\hline & & & & 75 & & $93 \%$ & & $8 \%$ \\
\hline & & & & 100 & & $93 \%$ & & $8 \%$ \\
\hline GL-PE-ILSS & Glass & Polyester & Interlaminar & 0 & & $100 \%$ & & $0 \%$ \\
\hline ULET-1LOS & & & Shear & 1 & & $82 \%$ & & $2 \%$ \\
\hline & & & & 10 & & $75 \%$ & & $3 \%$ \\
\hline & & & & 25 & $3.0 \%$ & $73 \%$ & $0.3 \%$ & $3 \%$ \\
\hline & & & & 50 & & $70 \%$ & & $3 \%$ \\
\hline & & & & 75 & & $69 \%$ & & $3 \%$ \\
\hline & & & & 100 & & $68 \%$ & & $4 \%$ \\
\hline GL-EN-TS & Glass & Epoxy & Tensile & 0 & & $100 \%$ & & $0 \%$ \\
\hline GL-EIN-IS & Gidss & Lpuxy & terisite & 1 & & $74 \%$ & & $5 \%$ \\
\hline & & & & 10 & & $63 \%$ & & $7 \%$ \\
\hline & & & & 25 & $4.5 \%$ & $59 \%$ & $0.8 \%$ & $7 \%$ \\
\hline & & & & 50 & & $56 \%$ & & $8 \%$ \\
\hline & & & & 75 & & $54 \%$ & & $8 \%$ \\
\hline & & & & 100 & & $53 \%$ & & $8 \%$ \\
\hline GL-EN-CS & Glass & Epoxy & Compressive & & Insufficient data & & Insufficient data & \\
\hline GI_FN_ES & Glass & Epoxy & Flexural & 0 & & $100 \%$ & & $0 \%$ \\
\hline GL-EIN-FS & & & & 1 & & $78 \%$ & & $4 \%$ \\
\hline & & & & 10 & & $69 \%$ & & $6 \%$ \\
\hline & & & & 25 & $3.8 \%$ & $65 \%$ & $0.8 \%$ & $7 \%$ \\
\hline & & & & 50 & & $63 \%$ & & $7 \%$ \\
\hline & & & & 75 & & $61 \%$ & & $8 \%$ \\
\hline & & & & 100 & & $60 \%$ & & $8 \%$ \\
\hline GL-EN-CS & Glass & Epoxy & Interlaminar Sh & ear & Insufficient data & & Insufficient data & \\
\hline $\begin{array}{l}\text { Notes: } \\
\text { 1. Due to loga } \\
\text { 2. Degradtion } \\
\text { 3. Equation fo } \\
\text { 4. Due to lack } \\
\text { b. tquatıon to }\end{array}$ & $\begin{array}{l}\text { ithmic eq } \\
\text { coefficien } \\
\text { \% Streng } \\
\text { of natural } \\
\Delta C \cup V \text { at }\end{array}$ & $\begin{array}{l}\text { tion, year "0" } \\
\text { a" is derived } \\
\text { Retained (at } \\
\text { ta, GL-PE-TS, } \\
\text { e "t" = b x In }\end{array}$ & $\begin{array}{l}\text { ' is entered as "1) } \\
\text { from Arrhenius } \\
\text { Outdoor Environ } \\
\text { FS, ILSS, and GL-E } \\
\text { ("t" age in years' }\end{array}$ & $\begin{array}{l}365 " \\
\text { orinciples and } \\
\text { ment) at age "t } \\
\text { N-FS were cali } \\
\text { " } 365 \text { days + 1); }\end{array}$ & $\begin{array}{l}\text { calibration with natur } \\
\text { 't" = } 100 \% \text { - a } \ln \text { ("t" a } \\
\text { ibrated for immersion } \\
\text { Aged CUV = Vırgın C }\end{array}$ & $\begin{array}{l}\text { aging data } \\
\text { ge in years* } 365 \text { days }+1) \\
\text { in tap water at room ter } \\
V+\Delta C U V\end{array}$ & mperature & \\
\hline
\end{tabular}


Once all accelerated test data have been normalized (with respect to a reference environment - outdoor exposure in this case), as shown in Table 4.8, aging curves for material strengths/stiffness and COV changes for various FRP type can be predicted (or plotted). Figure 4.2 graphically illustrates the decrease in strength retentions from $100 \%$ at time zero (day 1), and Figure 4.3 shows the increase in the coefficient of variation (COV) of aged FRP in addition to its initial COV. It indicates that there an increase in COV (an indicator of dispersion of strength values) as FRP ages as compared to COV of the virgin samples. In general, as FRP ages, about $10 \%$ maximum increase of aged COV over the original/virgin COV can be expected. A realistic estimation of aged COV along with aged mean strength is critical in reliability analyses. Additional discussion about COV increase with FRP age can be found in Section 4.6.2.

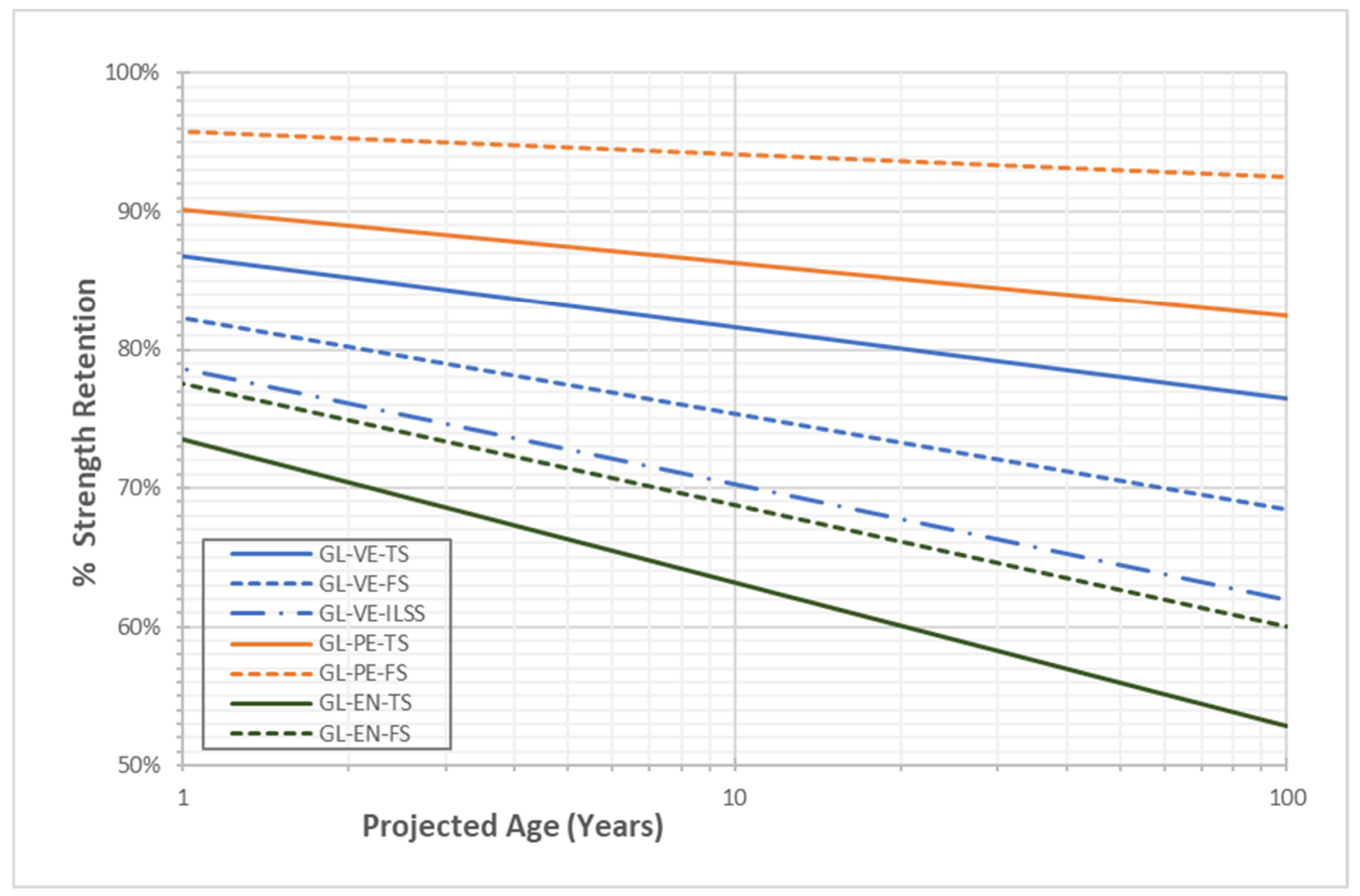

Figure 4.2: Predicted Normalized Deterioration Curves for Various SRs 


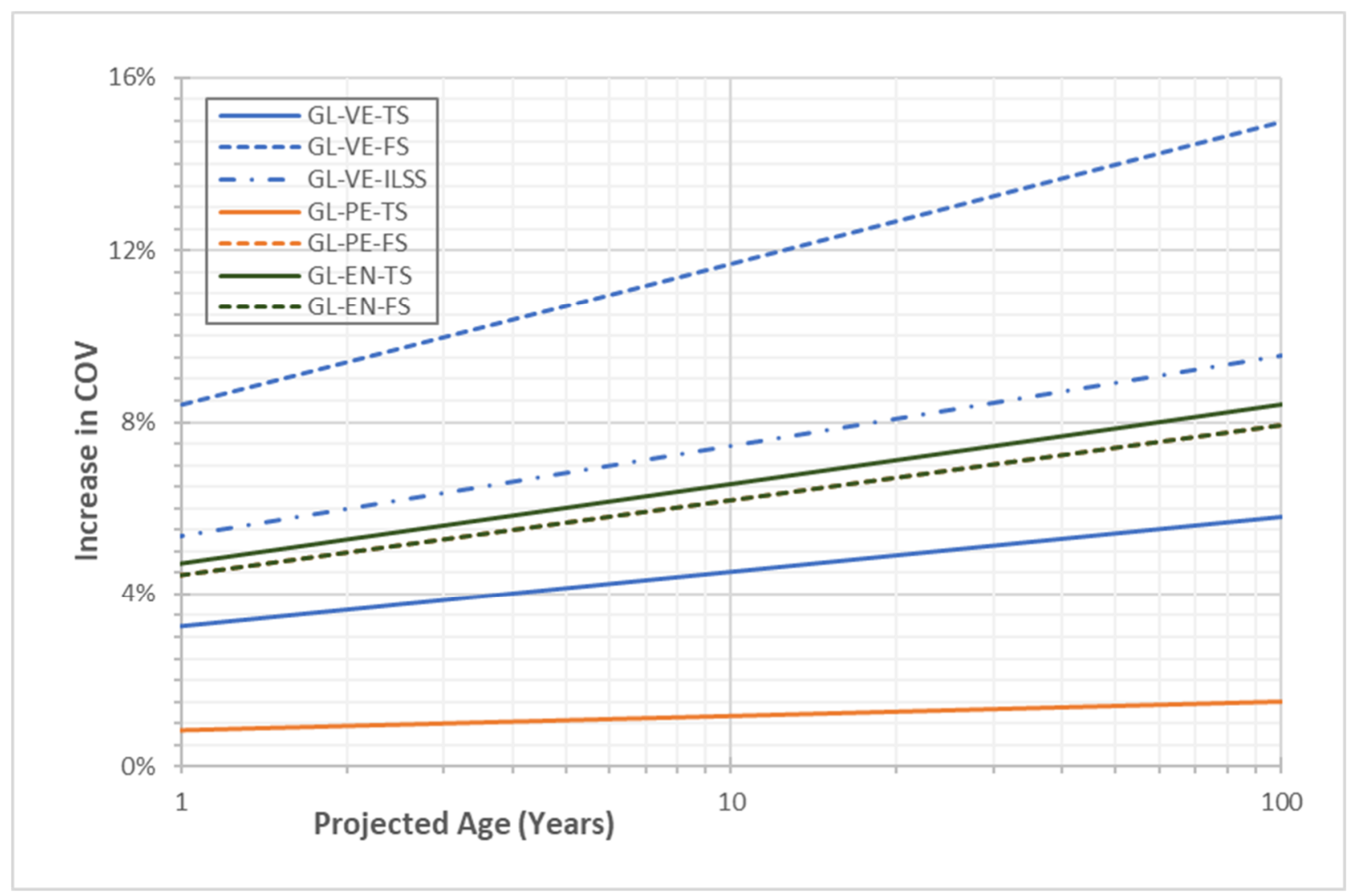

Figure 4.3: Predicted Normalized Increase in COV for Various SRs

\subsection{STATISTICAL ANAL YSES}

After database values were regressed and normalized with respect to a referenced environment (outdoor exposure), a simple statistical analysis was performed to obtain the distribution type, mean, and standard deviation (coefficient of variation is used as "normalized" standard deviation) for two timelines: Initial and Aged. Initial parameters are based on the original dry virgin material, and aged parameters are derived from normalized ATM/naturally aged data. The aged parameters should be computed in the range of estimated life (e.g., 1 to 25, 50, or 100 years) from the normalized data on an actual timeline; however, if enough aged data is not available, they can be roughly obtained for the entire group of aged samples. The primary intent is to account for increased FRP property variability with age. For this study, the FRP samples 
were grouped into the age groups between 1 to 100 years. There were not enough samples available to make smaller age groups for COV and distribution analysis. This COV estimate can be improved with additional data and similar examinations.

\subsubsection{Initial Parameters}

Initial design parameters are published or tested materials mechanical properties such as mean strength or stiffness and are obtained directly from FRP manufacturers, published literature (minimal information may be available due to proprietary nature of FRP), or independently tested in laboratories. They are often referred to as "virgin" or "initial" material properties. A typical statistical data collected from various manufacturers and reports summarized in Table 4.9. If we take into account the inherent variability of FRP and many approximations made in the reliability analyses, additional precision in calculating $\mathrm{COV}$ and $\mathrm{BF}$ is not required, nor will it change the outcome of the reliability analysis. For obtaining better representative values of COV and BF of a wide variety of FRP composites, published test databases for a large number (minimum 30 for each property) of FRP samples will be needed. 
Table 4.9: Compilation of FRP Statistical Parameters for Reliability Analysis

Materials: $\quad$ Glass Fibers in Vinylester (VE) \& Polyester (PE) Polymers

Manufacturers: Various

Source:

Bedford Plastics, University of Mississippi, West Virginia University

Date of Testing: $\quad$ 2007, 2011, 2016

\begin{tabular}{|c|c|c|c|c|c|}
\hline Material Type & Material Property & $\begin{array}{l}\text { Sample } \\
\text { Orientation }\end{array}$ & $\begin{array}{l}\text { Virgin } \\
\text { Coeff. of } \\
\text { Variation } \\
\text { (COV) }\end{array}$ & $\begin{array}{c}\text { Aged } \\
\text { COV } \\
\text { (Average) }\end{array}$ & $\begin{array}{l}\text { Bias Factor } \\
\text { (Virgin) }\end{array}$ \\
\hline \multirow{3}{*}{ Vinylester GFRP } & \multirow{3}{*}{$\begin{array}{l}\text { Ult. Flexural Strength } \\
\text { (FS) }\end{array}$} & Transverse & $8 \%$ & - & 1.3 \\
\hline & & \begin{tabular}{|l|} 
Longitudinal \\
\end{tabular} & $5 \%$ & - & 1.2 \\
\hline & & Average & $7 \%$ & - & 1.3 \\
\hline \multirow{3}{*}{ Vinylester GFRP } & \multirow{3}{*}{$\begin{array}{l}\text { Ult. Shear Strength } \\
\text { (ILSS) }\end{array}$} & Transverse & - & - & - \\
\hline & & Longitudinal & $3 \%$ & - & 1.1 \\
\hline & & Average & $4 \%$ & $8 \%$ & 1.1 \\
\hline \multirow{3}{*}{ Vinylester GFRP } & \multirow{3}{*}{$\begin{array}{l}\text { Ult. Tensile Strength } \\
\text { (TS) }\end{array}$} & Transverse & $7 \%$ & - & 1.3 \\
\hline & & Longitudinal & $5 \%$ & - & 1.2 \\
\hline & & Average & $6 \%$ & - & 1.2 \\
\hline \multirow{3}{*}{ Vinylester GFRP } & \multirow{3}{*}{$\begin{array}{l}\text { Elastic Modulus- } \\
\text { Tensile (MT) }\end{array}$} & Transverse & $16 \%$ & - & 2.1 \\
\hline & & Longitudinal & $8 \%$ & - & 1.4 \\
\hline & & Average & $12 \%$ & - & 1.7 \\
\hline \multirow{3}{*}{ Vinylester GFRP } & \multirow{3}{*}{$\begin{array}{l}\text { Elastic Modulus- } \\
\text { Flexural (MF) }\end{array}$} & Transverse & $4 \%$ & - & 1.1 \\
\hline & & Longitudinal & $5 \%$ & - & 1.2 \\
\hline & & Average & $4 \%$ & - & 1.2 \\
\hline \multirow{3}{*}{ Vinylester GFRP } & \multirow{3}{*}{$\begin{array}{l}\text { Ult. Compressive } \\
\text { Strength (CS) }\end{array}$} & Transverse & - & - & - \\
\hline & & Longitudinal & - & - & - \\
\hline & & Average & $8 \%$ & $10 \%$ & 1.3 \\
\hline \multirow{3}{*}{ Vinylester GFRP } & \multirow{3}{*}{$\begin{array}{l}\text { Elastic Modulus- } \\
\text { Compressive (MC) }\end{array}$} & Transverse & - & - & - \\
\hline & & Longitudinal & - & - & - \\
\hline & & Average & $12 \%$ & $13 \%$ & 1.5 \\
\hline \multirow{3}{*}{ Polyester GFRP } & \multirow{3}{*}{$\begin{array}{l}\text { Ult. Compressive } \\
\text { Strength (CS) }\end{array}$} & Transverse & - & - & - \\
\hline & & Longitudinal & - & - & - \\
\hline & & Average & $9 \%$ & $10 \%$ & 1.4 \\
\hline \multirow{3}{*}{ Polyester GFRP } & \multirow{3}{*}{$\begin{array}{l}\text { Elastic Modulus- } \\
\text { Compressive (MC) }\end{array}$} & Transverse & - & - & - \\
\hline & & Longitudinal & - & - & - \\
\hline & & Average & $15 \%$ & $23 \%$ & 1.8 \\
\hline
\end{tabular}

Notes:

1. Above values are compiled from various sources and not all values are available

2. When the one or more orienations are misisng, the average values are computed from a data source which did not provide sample orientations

3. Bias Factors $=$ Mean Values $/$ (Mean Value $-3 \times$ Std Deviation) 


\subsubsection{Aged Parameters}

Aged parameters are difficult to obtain since there is not much test data available beyond the initial/virgin values. Tables 4.8 and 4.9 show that, in general, standard deviations increases for aged materials due to different rate of deterioration in controlled (ATM lab) or uncontrolled (Nature/Outdoor) environments. The regression analysis can also provide data about the mean value for aged samples as well (an approximate estimation).

Once normalized deterioration equations are established, the data was analyzed to determine the statistical mean of Strength Retention and associated Standard Deviations for various age groups. Based on the common design lifespan of FRP elements, the age groups (more appropriately called Design Spectrum) can be made, as shown in Table 4.10. In this study, aged statistical parameters are derived for 10 to100 years range (by grouping aged samples by projected age). Computed aged statistical parameters will improve with more samples.

Table 4.10: Age Group Combinations

\begin{tabular}{|l|l|l|}
\hline Age Group & Design Domain & Common Applications \\
\hline 0 year & New & $\begin{array}{l}\text { Quality Control and Quality Assurance at the FRP } \\
\text { manufacturing facility and by FRP suppliers }\end{array}$ \\
\hline 0 to 1 year & Just placed in service & $\begin{array}{l}\text { In-Situ tested strength after installation, monitoring, and } \\
\text { testing }\end{array}$ \\
\hline 1 to 10 years & Short service life & $\begin{array}{l}\text { Temporary facilities: non-critical structures, storage units, } \\
\text { etc. }\end{array}$ \\
\hline 10 to 100 years & Normal service life & $\begin{array}{l}\text { Common permanent facilities: bridges, buildings, industrial, } \\
\text { pipelines, etc. The most common life spans are 10, 25, 50, } \\
75, \text { and } 100 \text { years. }\end{array}$ \\
\hline $100+$ years & Extended service life & $\begin{array}{l}\text { Critical permanent facilities: unusual structures, critical } \\
\text { facilities }\end{array}$ \\
\hline
\end{tabular}

Sample probability density functions (PDF) for the retained tensile strength of Glass Fibers in Vinylester Matrix (VE-GL-TS) at various ages are shown in Figure 4.4. Figure 4.4 shows how the PDF peak (mean value) downgrades and dispersion (standard deviation/COV) increases as the 
material ages. The curves have been derived from this study (form ATM/naturally aged data) and are included for illustration purposes only. Again, the quality of and the number of data points are critical in plotting these curves, and not all aged material property distribution curves will be the same. For a large number of aged data points, as FRP ages, the probability distribution of retained strength/stiffness tends to match "normal" (or "lognormal" for younger ages) distributions (shown as a 100-year solid line on Figure 4.4), even though at the time "zero," all material properties are normalized to $100 \%$ of virgin strength. This tendency further confirms that the seemingly random aged material properties can be modeled using appropriate probabilistic distribution functions, and reliability analysis can be used to durability design of FRP composites.

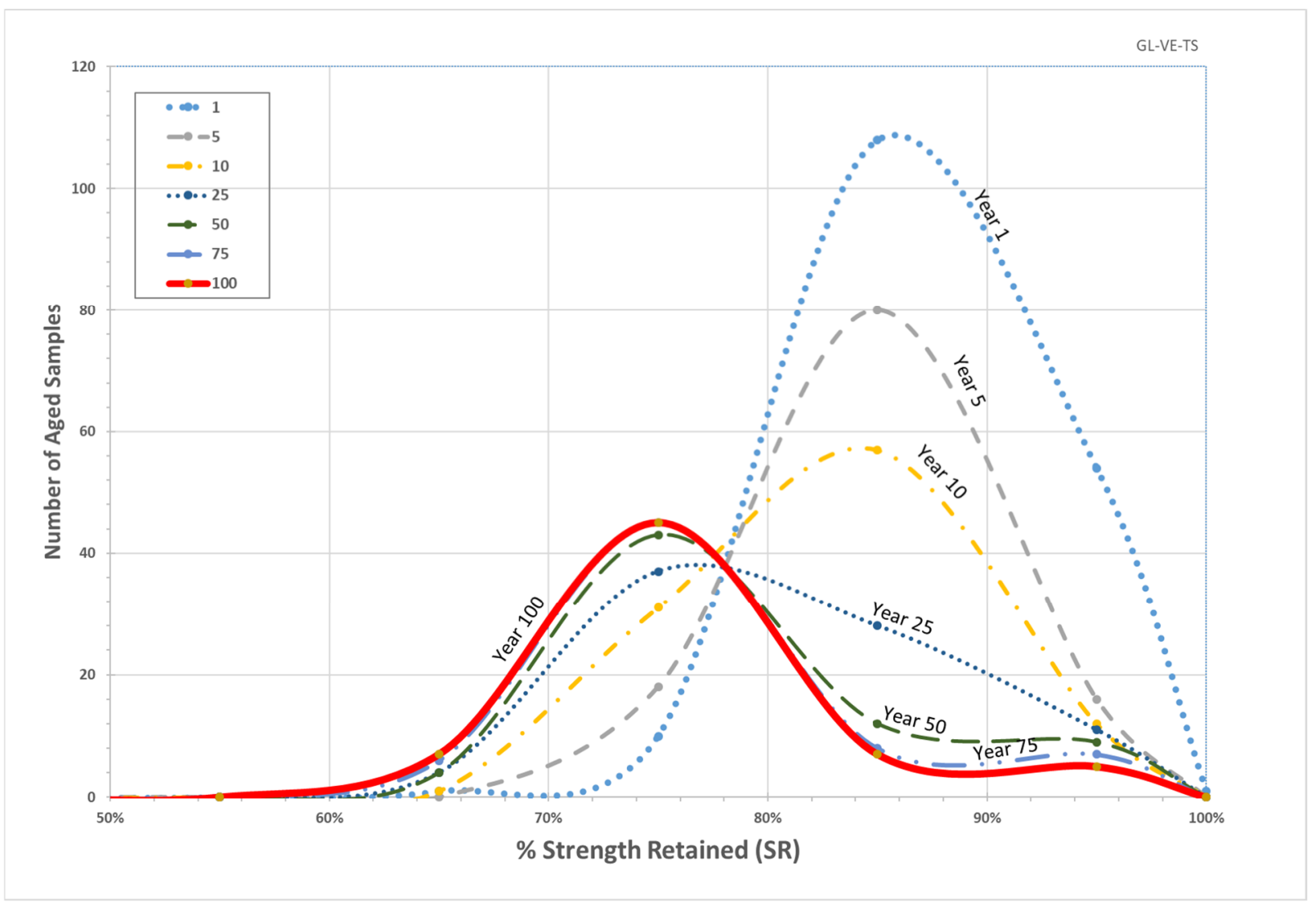

Figure 4.4: Typical Degradation of PDF with Aging of FRP 
Sample plots of aged mean retained tensile strength along with its aged COV of Glass Fibers in the polyester matrix (PE-GL-TS) for various ages are shown in Figure 4.5 (Additional strength retention curves for other FRP and properties can be found in Appendix B as Figures B.2 to B.12). The upper fitted line shows how mean tensile strength decreases from about $85 \%$ (at about $10-15$ years) to $75 \%$ in $50-100$ years from normalized strength of $100 \%$ at installation. The lower curve shows about $6 \%$ increase in aged standard deviation (or COV) at 100 years. It should be noted since all data were normalized with respect to $100 \%$ of mean strength, all standard deviations are shown as \% value of mean (i.e., $\mathrm{COV}$ and standard deviation are mostly the same and interchangeably used throughout this report). This increase in COV is also evident by comparing the COV of virgin samples with aged samples for data used in this study. The readers are cautioned that if the number of samples tested is too small, incorrect comparison of aged COV vs. virgin COV can be found (e.g., Table 6.9, where the virgin COV was based on only four samples).

The COV increase can be explained by using the following analogy. All samples are normalized to $100 \%$ of its mean ultimate strength at the start of ATM test (means COV is zero); however, each sample is slightly different from the others (e.g., fabric orientation, sample extraction method, manufacturing defects, resin impregnation, local ATM environment exposures, etc.), it will degrade at a similar, but slightly different/unique degradation rate and a scatter of aged properties will be found at the end of every ATM test. However, in an entirely deterministic world, the degradation rates of every sample in the ATM studies will be the same, and at the end of the ATM test (or test of naturally aged samples), there will be no data scatter. We know that is not the case in real life, so some increase in COV with age should always be expected. This logic applies 
to the aging of all construction materials, not just FRP, and forms the basis of this time-dependent reliability-durability research.

In this case, if COV of the original sample was $9 \%$, a $6 \%$ increase in value will render aged $\mathrm{COV}$ at $15 \%$ (i.e., $=9 \%$ original $+6 \%$ change) Although, an equation for aged $\mathrm{COV}$ can be developed (provided there are sufficient age data samples), it may be simpler to assign a life-cycle percentage change (assume 30\% increase over 100-year for this example). In this case, aged COV at 100 -year will be $=9 \%$ virgin $\operatorname{COV} \times 1.3=12 \%$, which can be pro-rated over its service life. Reliability calculations can be sensitive to COV, so care should be taken to assign a reasonable value based on data analysis and historical data.

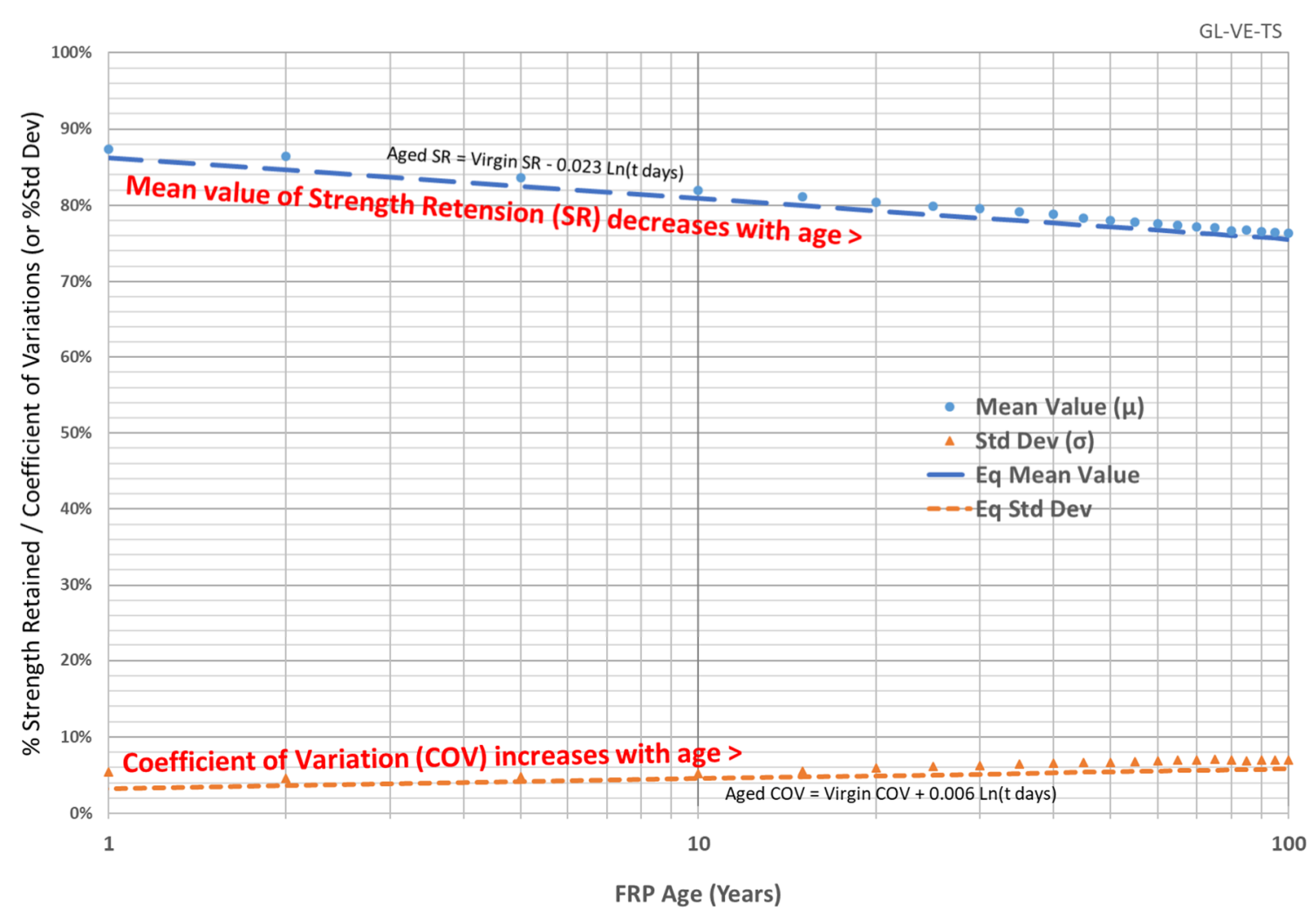

Figure 4.5: Typical Degradation of SR Mean Value and COV with Age 
Figure 4.5 shows the projected aged COV and projected aged SR in a graphical form for the tensile strength of vinylester-based GFRP samples. The top curve shows a \% gradual drop in strength retention, and the bottom curve shows the \% rise of COV with age based on regression analysis performed for this group (GL-VE-TS). Table 4.9 shows coefficients that can be used to approximately estimate mean strengths and associated statistical parameters for time-dependent reliability-durability analysis. The time-dependent effect of COV on the probability of failure can be graphically seen in Figure 7.1 .

\subsection{TIME-DEPENDENT RELIABILITY APPROACH}

This section summarizes the final step of time-dependent reliability analysis to show how the overall process to determine the strength reduction factor (or factor of safety) can work. The method employs durability deterioration data from the previous sections.

A hypothetical FRP beam is used as an illustrative example through the narrative part of this Chapter to promote a better understanding of the concept of time-dependent reliability. Design calculations are shown in Appendix A as Example No. 1. The step by step process shows how the reliability of a beam degrades over time and to safeguard the beam against potential failure (during its aging) how an adequate strength reduction factor (or factor of safety) can be incorporated into the initial design calculations. Initial values of ultimate mean strength and standard deviation are assigned to the new FRP beam based on statistical data, as shown in previous sections. The aged parameters are calculated based on various aging equations derived in previous sections. In the end, the reliability-based durability process consistently yields a minimum level of reliability (or probability of failure) throughout its service life. 
The non-linear relationship of reliability index, strength reduction factor, and factor of safety confirms some of the gaps (e.g., the variation of COV with time - see Section 6.4.2 for discussion) in the current FRP design practice. The objective of the proposed framework is to show the overall process of determining suitable strength reduction factors while accounting for FRP aging through its service life.

\subsubsection{Illustrative Example}

The primary objective of using examples is to illustrate the concept of time-dependent reliability and its potential uses in developing a consistent and rational methodology to design FRP members. Thus, FRP members can perform adequately not only at the beginning of the members' service lives but also at the end of the members' service lives. The underlying hypothesis is that once a rational process and relationship patterns are developed for this hypothetical example, it is very likely that other examples will follow a similar pattern, and then a broader prediction of reliability-durability relationship can be developed (i.e., time-based reliability and property variations). Keep in mind is that this example is included only to show the primary concept of reliability-based durability design.

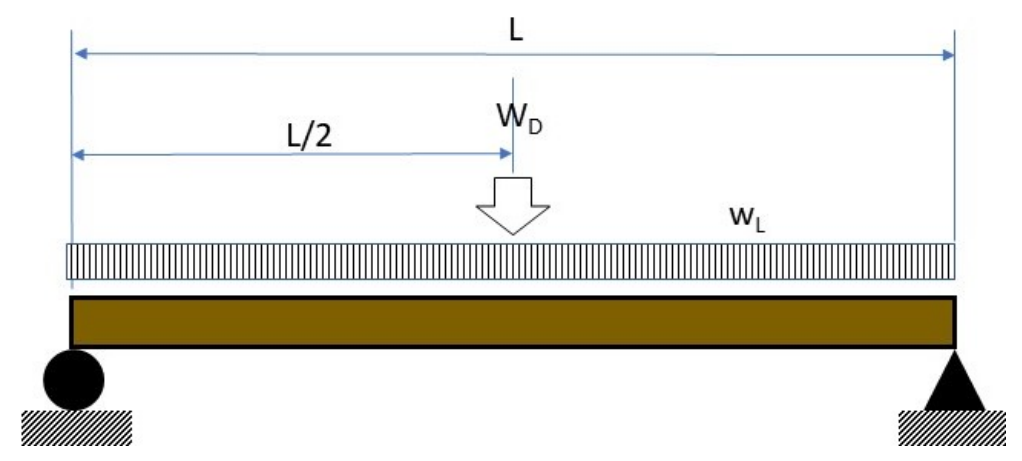

Figure 4.6: Schematic Loading Diagram of Example FRP Beam. 
Consider a simply supported vinylester-based GFRP beam, laterally-supported along its entire span length (L) of 18 feet, and loaded with uniformly distributed live load $\left(\mathrm{w}_{\mathrm{L}}\right)$ and a concentrated dead load $\left(\mathrm{W}_{\mathrm{D}}\right)$ at mid-span (Figure 4.6). This example format is taken from Nowak and Collins (2013). It uses a simple First Order Reliability Method (FORM) to compute the reliability of the beam. A complete design example deriving of the appropriate reliability-based strength reduction factor and factor of safety (FOS) is shown in Appendix A as Example No. 1. The example shows calculations of structural reliability index $(\beta)$ of the member at the time of that member's initial placement and the end of member's (useful) service life.

The serviceability checks for sustained loads and deflections are also performed (Example 1, Appendix A), but are not controlling. The beam is designed using an assumed factor of safety (working stress method) and strength reduction factor (load and resistance factor method), which was later calibrated to provide minimum reliability throughout its service span. To compare various scenarios equally, each design point was optimized (by varying the section modulus) to keep the capacity/demand ratio to be just at 1.0 so that beam design calculations will always be considered "acceptable" under the current design practice. 


\subsubsection{Selection of Target Reliability Index and Service Life}

A commonly acceptable range of Reliability Index $\beta$ and Probability of Failure $\mathrm{P}_{\mathrm{f}}$ for common engineering structures is shown in Figure 4.7.

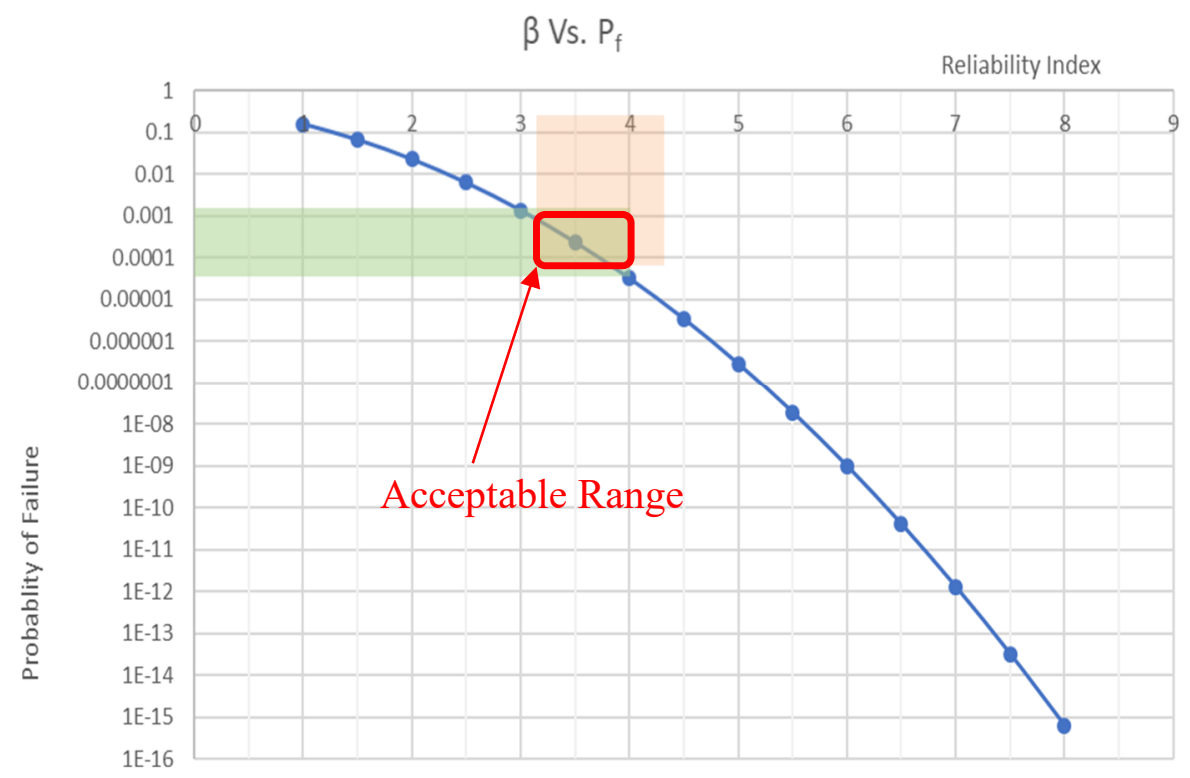

Figure 4.7: The Probability of Failure and Reliability Index

Based on a general understanding of LRFD codes, a target reliability index of 3.5 is selected. The service life of the beam is selected as 100-year even though the current design period today for many outdoor structures is 50 to 75 years (e.g., AASHTO LRFD specifications, 2017 , requires a minimum of 75 years of service life for permanent walls and bridges). A discussion about the selection of the target reliability index can be found in Section 2.5.3.

Refer to Example Number 1 in Appendix A (A.1) for the final design of this beam and the selection of a durability strength reduction factor. Once the basic design for the reliability-based durability process has been understood, a more in-depth analysis of the relationship of reliability index, the factor of safety, strength reduction factor, etc. using this example as the basis (shown in the following sections) can be presented. If a different example is selected, say a tension bracing 
or a compression-moment column, the following illustrations will be slightly different but is likely to show a similar pattern.

\subsubsection{Degradation of Reliability Index over Time}

For the beam example used in this report, the reliability index $(\beta)$ deteriorates from about 6.8 to 3.6 (Figure 4.8), and the factor of safety margin drops from 6 to 4 (Figure 4.9) from its time in placement at year 0 to 100 years of expected service life. A logarithmic degradation curve fits the best in this case. The loss of material strength is about $68 \%$ over a 100 -year, so a strength reduction factor of $30 \%$ is necessary to achieve adequate reliability (e.g., to provide an acceptable level of probability of failure till the end of its service life). See Example 1 calculation (Appendix A) for a check on i) increased live load deflection due to $5 \%$ loss of elastic modulus stiffness (against regulatory code requirements), and ii) initial creep strain due to sustained dead load (for computing built-in fabrication camber in the FRP beam). In this case, due to a very high depth/span ratio $(18$ feet $/ 24$ inch $=9)$ and low initial sustained dead load ratio (about 5\%), these severability checks are not critical but, in many cases, deflections and/or creep strains can drive the selection of final FRP beam size, strength, and/or stiffness. In Figure 4.8, it is worth noting that the deterioration rate for $\beta$ is steeper than that for material deterioration rate $(3.2 \%$ for $\beta$ Vs. $3 \%$ for $\mathrm{a}_{\mathrm{fs}}$ ). It merely means that the direct application of an estimated SR deterioration factor (say computed for projected 100-year life using mean values derived from Arrhenius regression equations) may not be adequate to maintain the desired reliability to the end of service life.

The reasons for the discrepancy between the mean value approach and the reliability approach are difficult to explain until more data is included, and a thorough calibration is performed. However, some possible explanations can be: a) the concept of LRFD is relatively new in the FRP industry and many reduction factors in use today were never calibrated using a 
reliability approach; b) the variability of FRP member aged strengths (over the initial placement values) is substantial but was not considered; c) although the age-related degradation of mean member's strength has been included in many reduction factors, the possible increase in the variability of strengths/stiffness with age and its effects have never been included in any previous work.

One way to overcome this drop in reliability with aging is to design FRP members with higher reliability at the time of placement, say $\beta=6.8$ instead of 3.6 (Figure 4.8). However, designing for target reliability is not straight forward and requires a good understanding of both reliability and durability fields. Figures 4.8 through 4.14 represent a single example (Appendix A), and figures (i.e., target $\beta, \phi$, FOS values) may look different for other combinations of loads, strengths, and geometrical configurations. In any case, we cannot realistically expect an FRP designer to know enough about the reliability methods (i.e., mean ultimate strength, initial or aged $\mathrm{COV}$, target reliability index, etc.) or durability predictions (i.e., accelerated test data, regression equations, Arrhenius stress superimposition principles, age projections, etc.) to make this critical choice. We can also safely assume that the designers will continue to prefer deterministic design methods. However, we can provide them with a set of fully calibrated and substantiated durability strength reduction factors (or factor of safety), say derived using the proposed time-dependent reliability-durability framework so that their designs can withstand the test of time. 


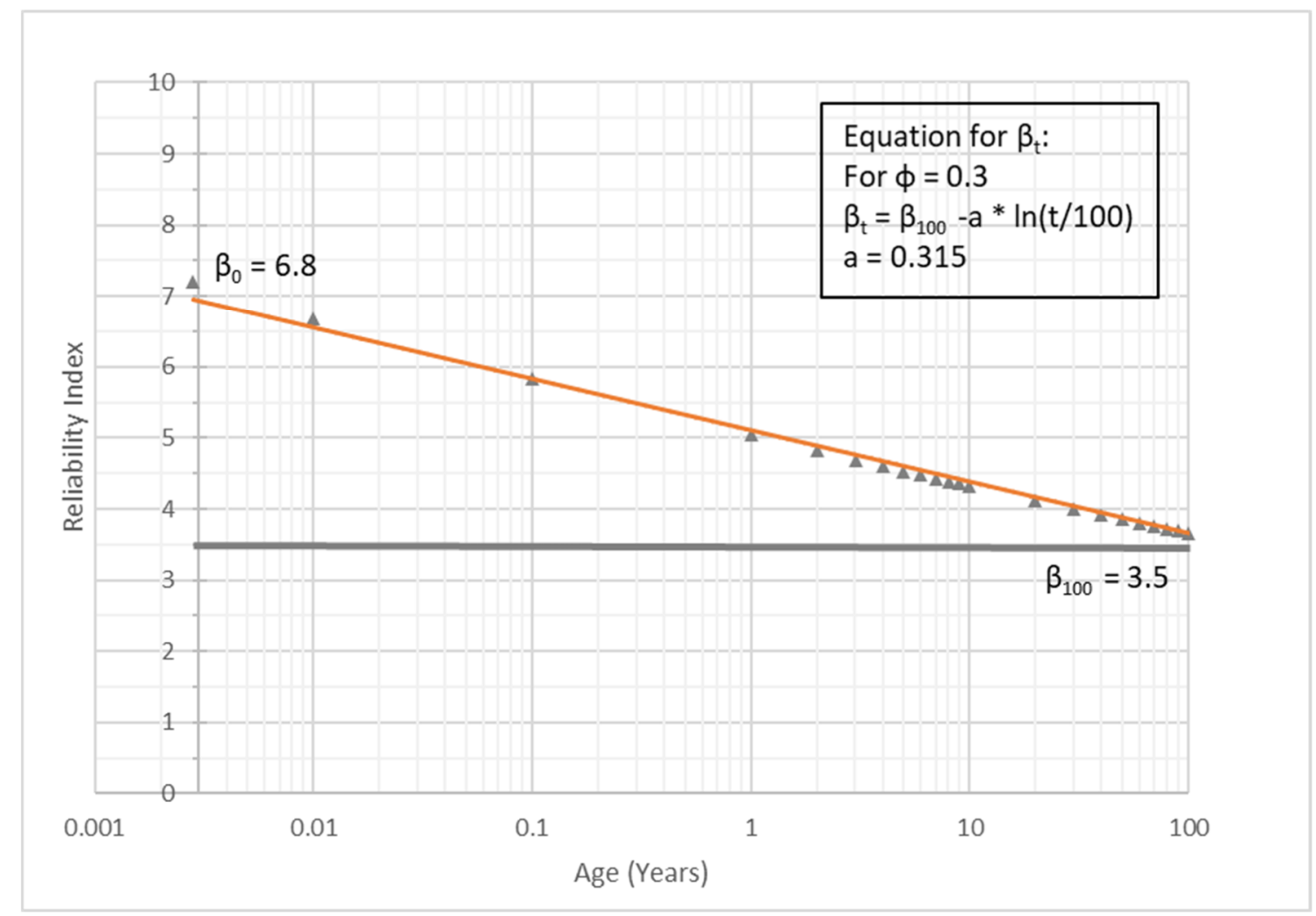

Figure 4.8: Degradation of RelibilityIndex (ß) over Service Life

\subsubsection{Degradation of Factor of Safety (FOS) over Time}

Similarly, the initial factor of safety deteriorates over time from 6.0 to 4.0 , as shown in the following Figure 4.9. Without the inclusion of the reliability index, it is difficult to ascertain how safe it is safe (e.g., is the aged FOS of 3.0 at 100 years adequate?) However, if the reliability is also included then a FOS of 4.0 will provide the reliability index of 3.5 (which is a generally accepted probability of failure for most civil engineering applications.) 


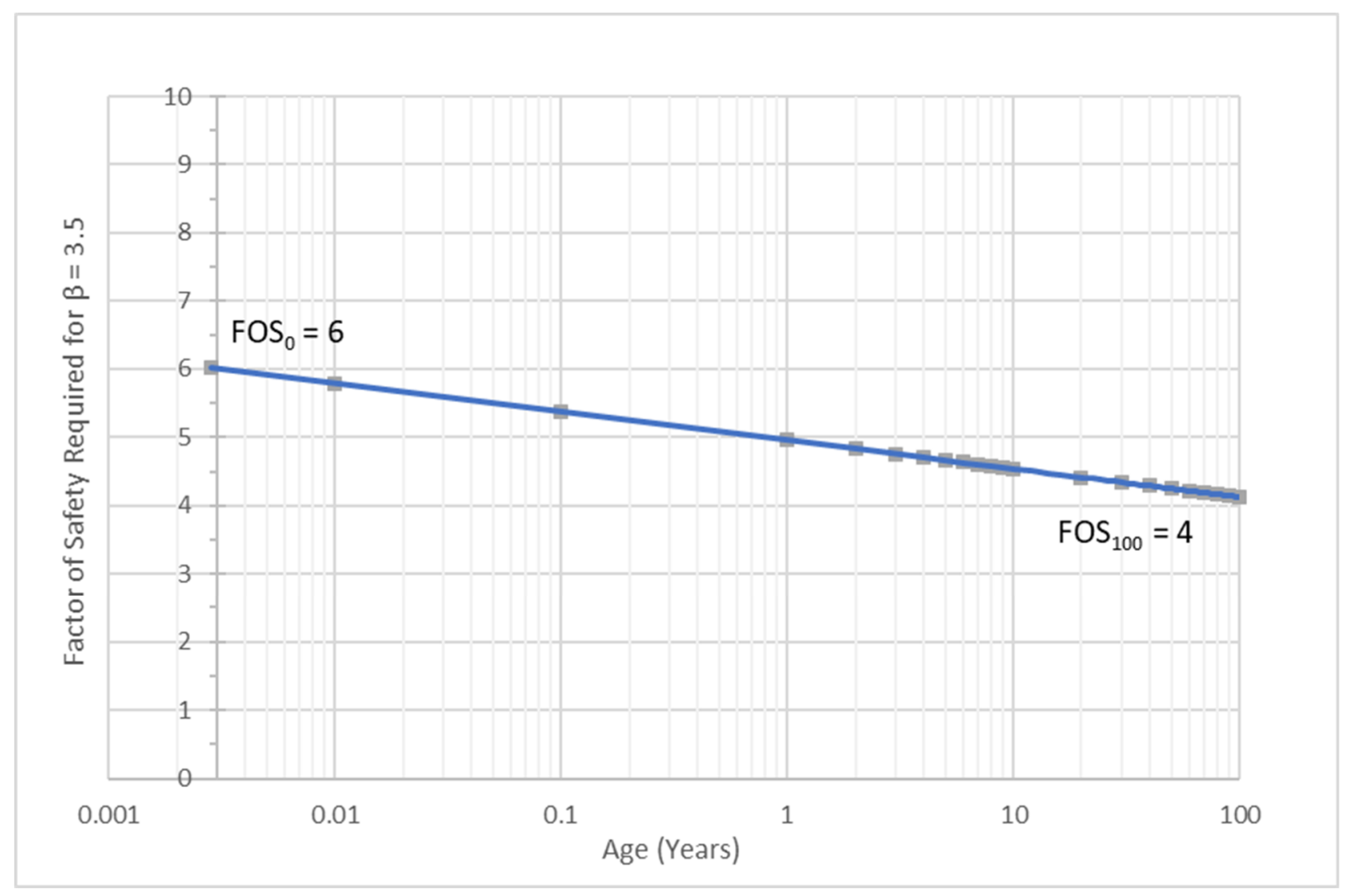

Figure 4.9: Deterioration of Factor of Safety (FOS) over Service Life

\subsubsection{Relationship of Reliability with Various Factors}

A brief parametric analysis was carried out to evaluate the relationship among reliability indices $(\beta)$, strength reduction factors $(\phi)$, factors of safety (FOS), and service lives (T). As a part of reduction factor calibration and basis of design (BOD) development, similar charts are generally developed and compared for varied ranges of design examples. Charts can be analyzed for various design parameters (e.g., span length, loads, etc.) to aid the designers without going through reliability calculations (only use $\phi$ and $\gamma$ ). These graphs can be used to set up a few basic equations (for the typical range of parameters) that can guarantee a minimum built-in structural safety. The following are illustrations of such relations developed explicitly for the illustrative example. 


\subsubsection{Reliability Index ( $\beta$ ) and Service Life (T) for various Strength Reduction Factors ( $\varphi$ )}

A set of $\varphi$ factors were used to compute section resistance that will provide capacity/demand ratio of 1.0 at the design phase $(t=0)$, along with computed reliability indices were calculated as shown in Figure 4.10. All graphs fit logarithmic deterioration pattern in general, and curves seem approximately parallel for various $\varphi$ values. This chart can help in selecting appropriate $\varphi$ value based on service life without doing the reliability calculations. For example, for a 100 -year life, $\varphi$ of 0.3 will be adequate, but for a 30 -year life, $\varphi$ of 0.4 may suffice. It may be worth noting that a $\varphi$ of 0.5 will only provide only five years of a safe life for this example. We are assuming that a designer will use the manufactured supplied virgin materials data, not the aged ATM data.

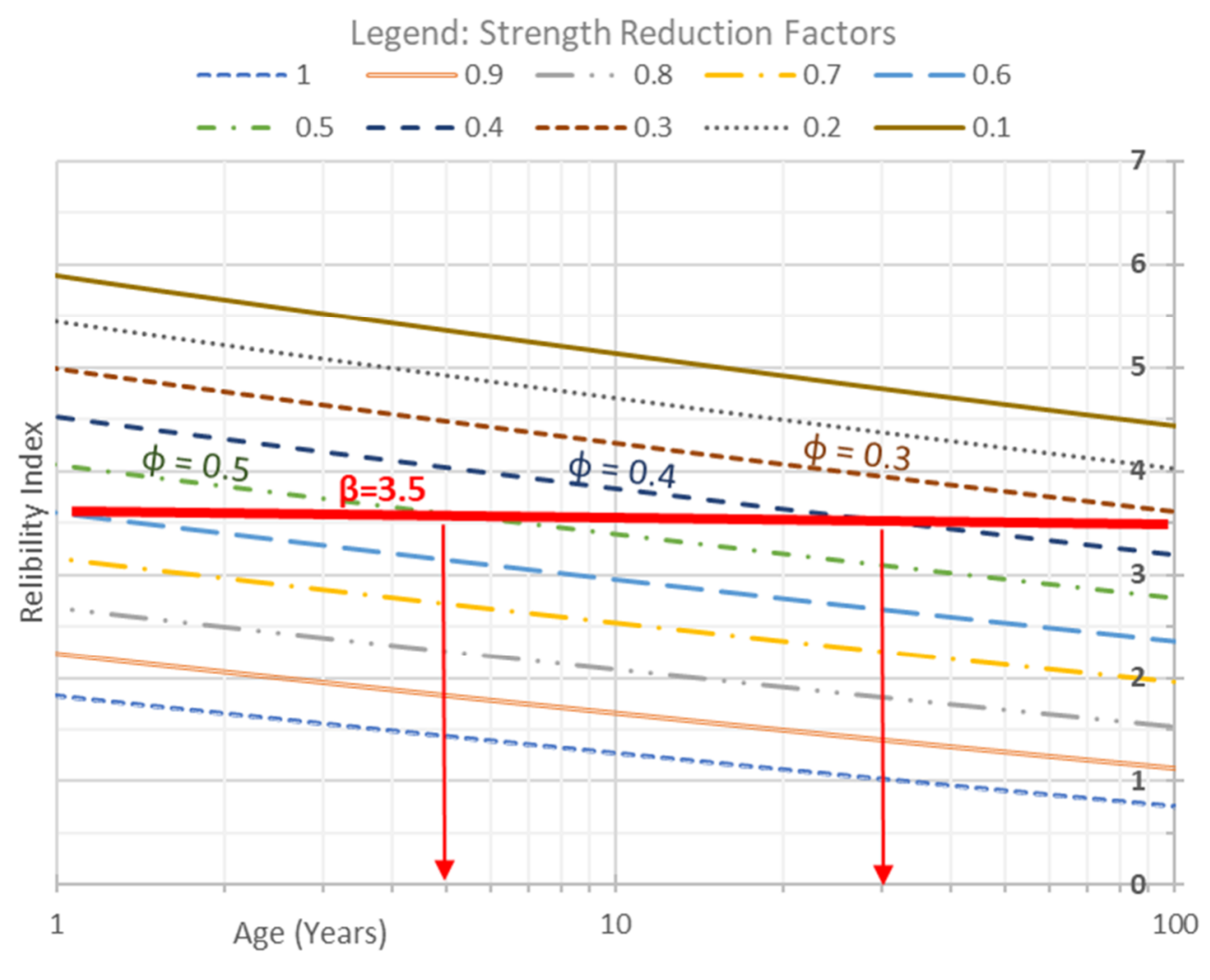

Figure 4.10: Variation of Reliability Index with Age for various Phi Factors 
4.7.5.2 Reliability Index ( $\beta)$ and Strength Reduction Factors $(\varphi)$ for various Service Lives (T)

Another way to look at the relationship is to develop curves for various life-spans and see what $\varphi$ values are needed to achieve the desired reliability index. Figure 4.11 shows such a relationship. For a 100-year life, one can pick a $\varphi$ value of 0.32 to get a target reliability index of 3.5 .

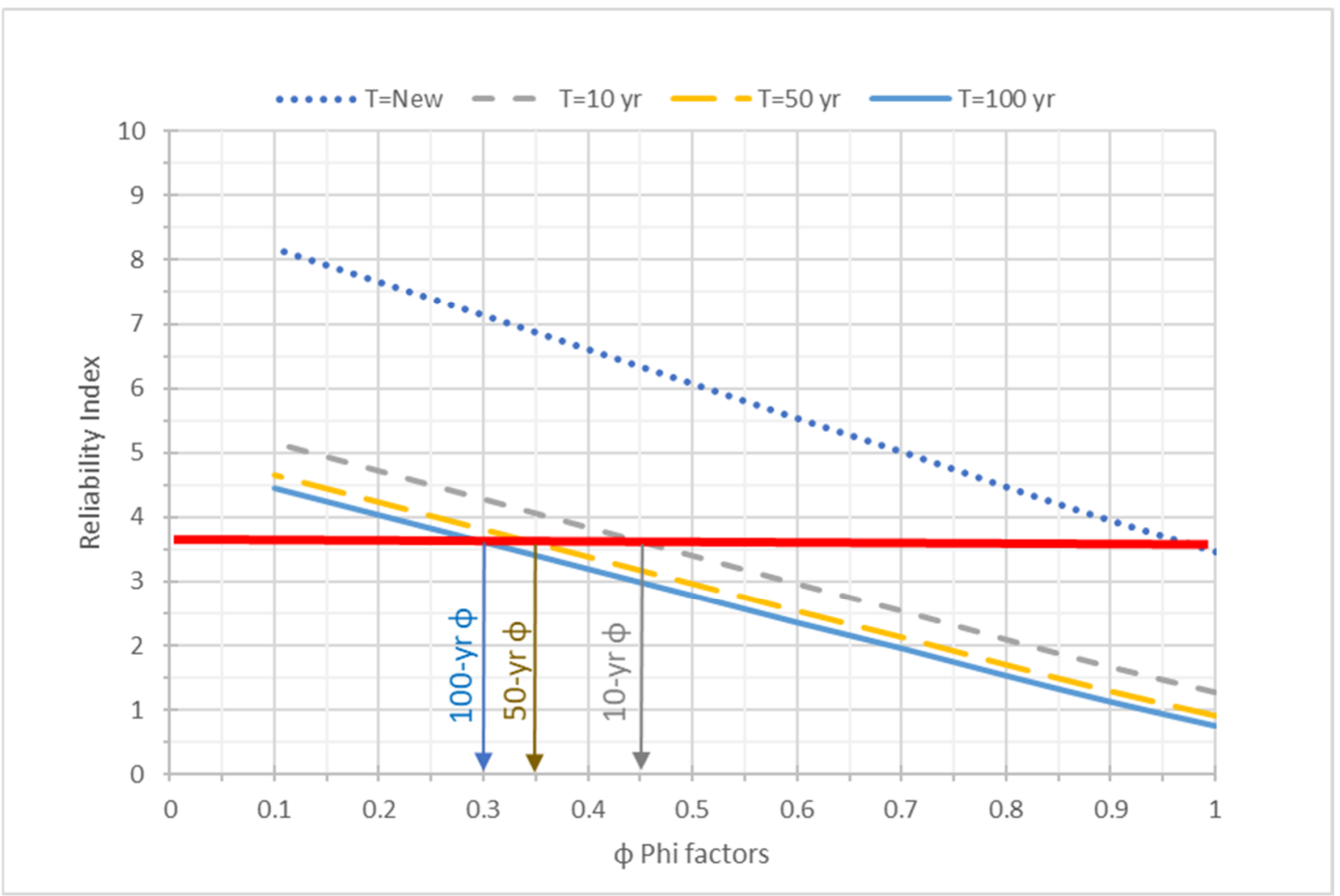

Figure 4.11: Variation of Reliability Indices with Phi Factors for Various Ages

\subsubsection{Reliability Index ( $\beta$ ) and Service Life (T) for various Factor of Safety (FOS)}

It is ubiquitous to use the factor of safety in many FOS applications, especially in the aerospace and mechanical engineering field. Reliability degradation curves for various factors of 
safety can be drawn and compared for safe and unsafe zones. Figure 4.12 will help in selecting an appropriate factor of safety for a specific service life. For example, for a 100-year life, FOS of 5 will suffice, but for a 10-year life, FOS of 3.0 may be adequate (Figure 4.12) for Example No. 1 (Appendix A). However, it is worth noting that very high FOS does not necessarily result in a proportional increase in reliability. In this case, FOS higher than 6, may not be justifiable and is not recommended. It should be noted that this evaluation is for bending strength only, and similar assessments about other properties (i.e., strengths and stiffnesses) and variations (loads and geometry) must be carried out before a general conclusion can be made.

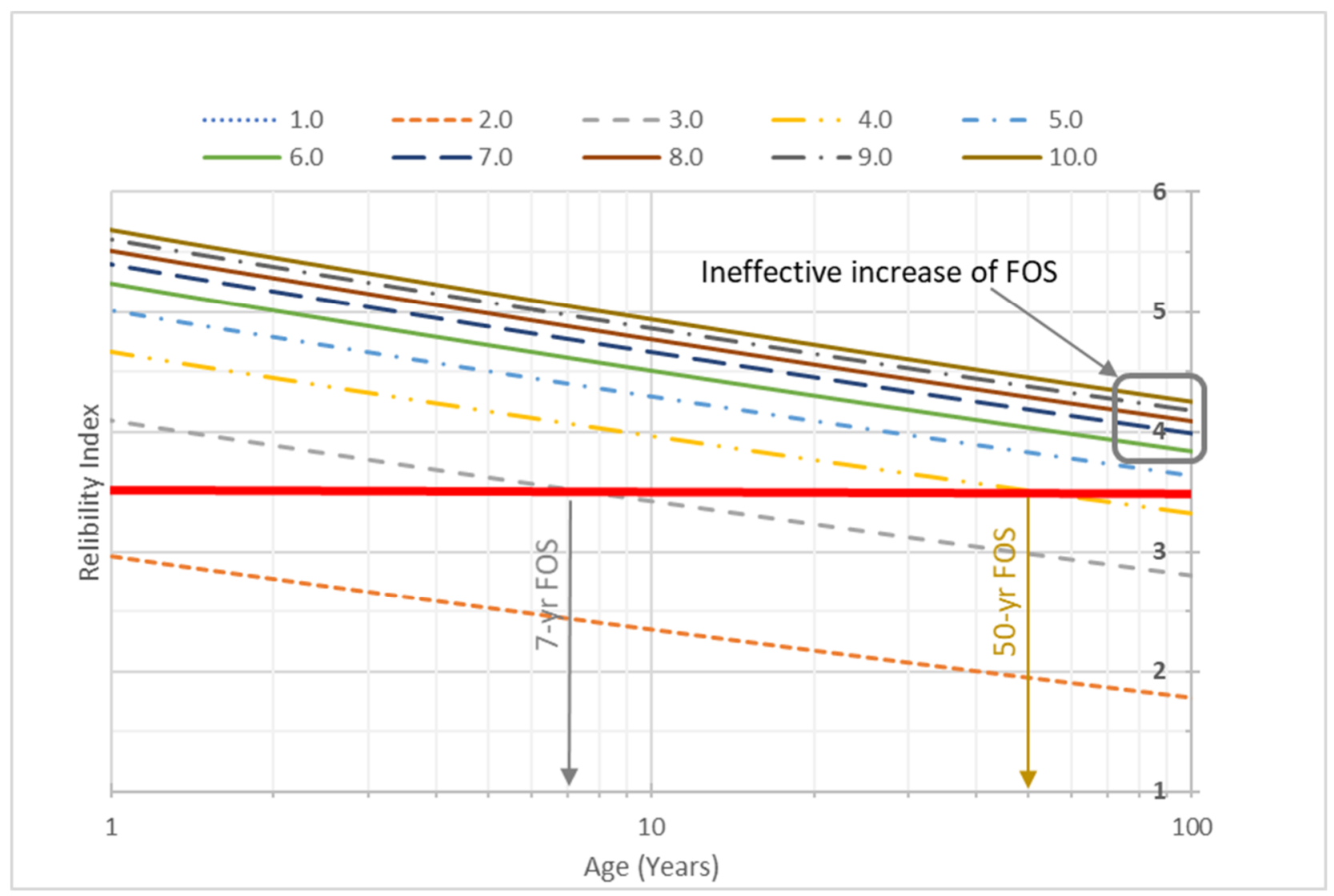

Figure 4.12: Variation of Reliability Indices with Ages for various FOS 


\subsubsection{Reliability Index ( $\beta$ ) and Factor of Safety (FOS) for various Service Life (T)}

Similarly, the factor of safety can be picked to obtain the desired degree of reliability, as shown in Figure 4.13 (a variation of Figure 4.12). The curve for FS is non-linear and does not fit any common trendline equations. For example, FOS of 3 can be picked for a 10-year service life that will provide a reliability index of 3.5 (calculated for Example No. 1, Appendix A).

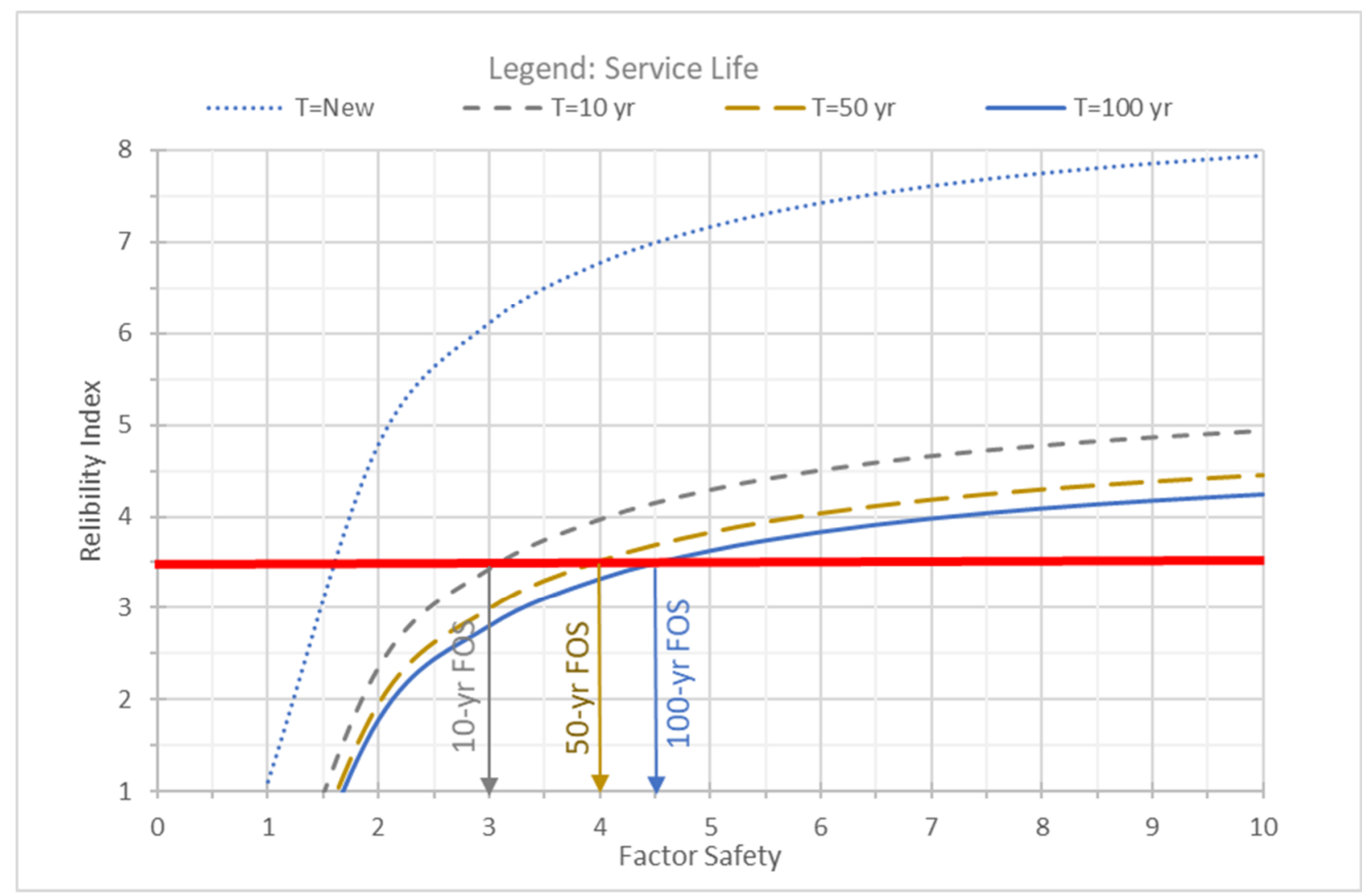

Figure 4.13: Variation of Reliability Indices with FOS for various Ages

\subsubsection{Strength Reduction Factors ( $\varphi$ ) and Service Life (T) for various Reliability Index ( $\beta$ )}

The following Figure 4.14 illustrates how $\varphi$ factor can be selected at the design phase that will provide the desired reliability index for different life spans. For example, a $\beta=3.0$ line shows what $\varphi$ factors need to be selected for various life span. For example, a $\varphi=0.58$ will provide a life of 10 years and maintain a $\beta$ value of 3.0. It should be noted that all the above design charts (Figures 
4.8 through 4.14) are developed only for Example No. 1 (Appendix A) and relate only to a specific FRP type, geometry, loading, and environmental condition.

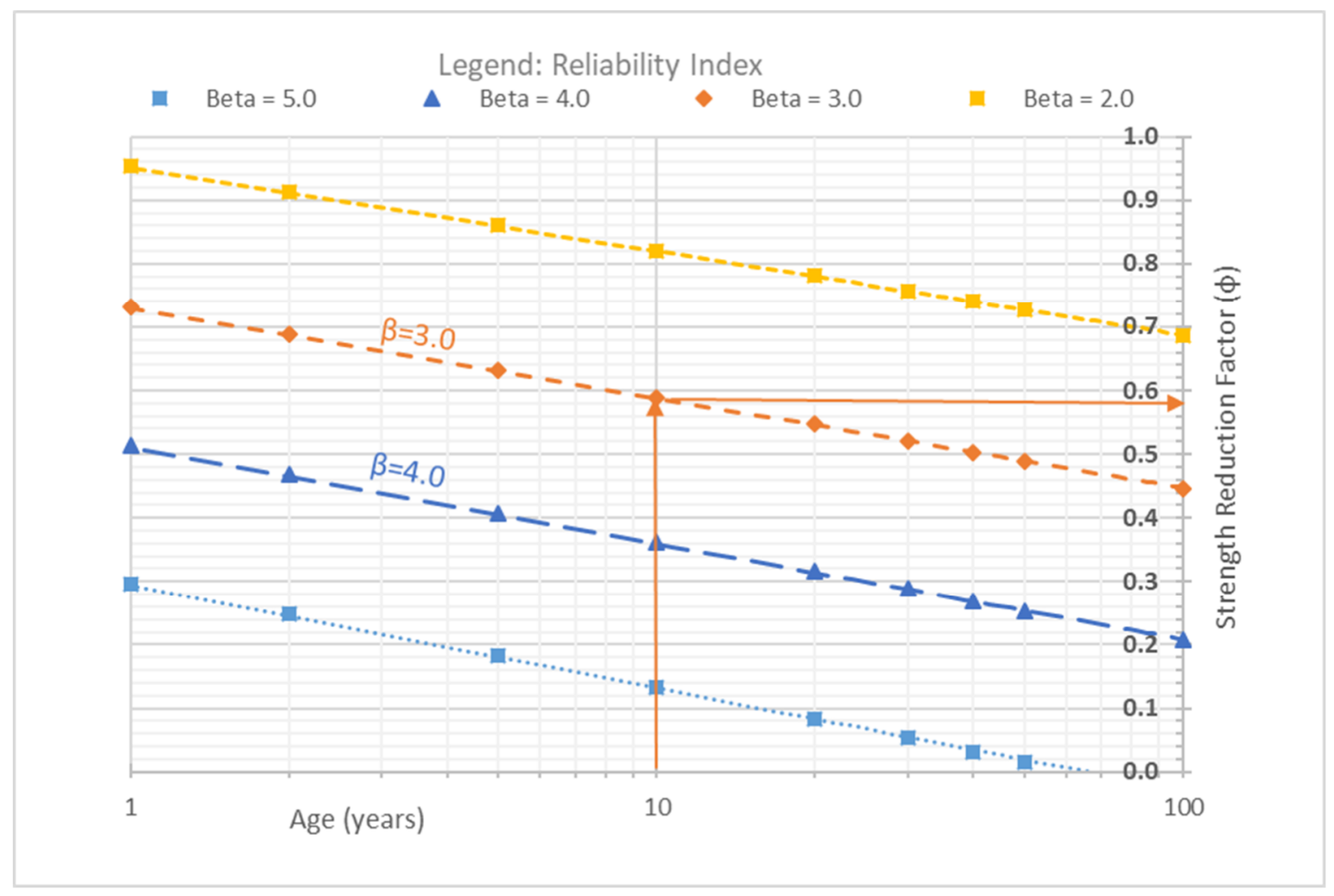

Figure 4.14: Variation of Phi Factors with Ages for Various Target Reliability Indices

\subsection{SUMMARY}

This chapter provides a workshop on the application of a reliability-based durability framework. Based on work performed in this chapter, an overview of findings are as follows:

1. There is a vast amount of published and unpublished aging data available on FRP that can be used in the development and the refinement of statistical data and aging equations presented in this chapter. The results presented in this report are based on the accelerated test method (ATM) to illustrate the use of a reliability-based durability framework only and not necessarily meant to compare or recommend final deterioration rates of various FRP systems or associated 
statistical parameters. In the author's opinion, the building of such a vast aging database for different FRP systems requires an industry-wide effort and is outside the scope of this research.

2. The selection of a proper reference environment in ATM allows the data to be readily available for real-life age prediction studies. Similarly, the reproducibility of ATM results is crucial in the development of the FRP aging database that can be used for the knowledge base necessary for the development of risk-based design guidelines. Simple modifications, as proposed in this chapter, can help in improving the effectiveness of ATM data for use in time-dependent reliability analyses.

3. There is undoubtedly a lack of enough available naturally aged FRP data (for various polymeric systems and fibers) to aid in the final calibration of ATM data to actual applications. The samples saturated in the room temperature tap water were used in the absence of naturally aged data. These results can be considered conservative for regular outdoor use in many parts of the US, but that may not be the case in the climates of high dessert temperatures or freezethaw cycles.

4. The inclusion of reliability in the durability studies helps answer the age-old question of how safe is safe? It also provides a rational means to use a strength reduction factor or factor of safety for the intended service life.

5. The non-linear relationship of the probability of failure with strength factors questions the validity of the current practice of using strength reduction factors as the mean strength retention value derived from Accelerated and Natural Tests. A reliability-based assessment may explain why some strength reduction factors used in current practice may not be adequate beyond 3040 years of service life. 
6. Based on the design examples illustrated in Appendix A, the proper choice of the relationship of reliability with service life and design factors is essential. The need for the development of a reliability-based durability framework should be deemed critical if a longer service life of FRP members (e.g., 100 years) is expected.

\subsection{RECOMMENDATIONS}

The following recommendations are made based on the findings presented in this Chapter:

1. The use of appropriate reference environments in an ATM study and normalization of ATM data will help create better data for reliability analyses. To keep ATM data reproducible, use more straightforward techniques, as explained in this chapter, avoid excessive manipulation of ATM data, and use proper regression techniques to weed out bad data.

2. Collection of statistical data about both virgin and aged FRP material properties (e.g., mean, nominal, and characteristic values, standard deviations, or the coefficient of variations, distribution type, etc.) will help advance the development of reliability-based durability strength reduction factor and factors of safety.

3. The calibration phase of the strength reduction factor is an essential final step in the development of reliability-based design guidelines or design codes for FRP and should be undertaken at an institution or industry level.

4. Development of the Basis of Design (BOD) or design examples after the conclusion of the ATM project should be encouraged to allow immediate practical use of the research. 


\section{CHAPTER 5 ACCOUNTING FOR PHYSICAL EFFECTS}

\subsection{INTRODUCTION}

This chapter summarizes the use of a time-dependent reliability-based method for accounting for the detrimental "physical" effects (Section 2.2.1.2) on FRP properties concerning FRP structural systems' service lives (e.g., time to failure). Physical effects are created outside the environment and often introduced due to particular loading applications such as sustained stress or stress cycles. It is different from "physical aging," which is usually the end result of both environmental and physical effects on FRP composites. In this chapter, a reliability-durability framework to account for sustained loading (or creep) in selecting appropriate initial stress/strain levels to reach different target service lives is illustrated. This method can be modified for other physical effects, such as determining the number of cyclic loadings (causing stress reversal or stress variations) for fatigue life estimation. The proposed method uses Findley's power law (or function) to model the time-dependent strain degradation behavior for estimating the creep strains to rupture and associated service life spans (time to failure) at select initial sustained stress levels and creep-rupture strain limits (also described as creep-rupture strengths).

Primary data used in the study was first developed at WVU during the 1996-98 period (Vijay, 1999). A more current and comprehensive creep database was developed at the University of Sherbrook (Youssef, 2010), which consisted of 10,000 hours of testing of 128 GFRP (in Vinylester matrix) reinforcing bars. The author acknowledges that although there are many sustained stress databases available, only the database from the University of Sherbrook (Youssef, 2010) is used in this study to illustrate the framework development process. 
Under this study, all original test data was modified by the normalization process and regrouping of test results based on initial sustained stress (or strain) levels (measured at the beginning of the test causing initial elastic deformation) and final strain levels (measured during creep tests at various time intervals). See Section 5.4.4 for a detailed discussion about the normalization process (including varied diameters and fiber volume fractions of rebars) used in this chapter. All strain values (at a given time "t" in hours) were normalized by dividing recorded strain $\left(\varepsilon_{\mathrm{t}}\right)$ by ultimate strain $\left(\varepsilon_{\mathrm{fu}}\right)$. Since "initial sustained stress ratio" and "initial sustained strain ratio" are in the elastic range (the maximum initial sustained strain tested was $80.90 \% \varepsilon_{\mathrm{fu}}$ when compared to $90-95 \% \varepsilon_{\mathrm{fu}}$, a threshold value for FRP elastic region) and virtually the same, these terms are often used interchangeably in this report. The term "initial" is not necessary to describe "sustained stress," but it is important to make this distinction for the "sustained strain" since the initial sustained strain will increase with time without any change to the sustained loading (causing an increase in sustained stress with time) due to the creep effect. From a designer's point of view, the calculation of mechanical stresses is direct and intuitive (since it is a function of the loading and easily calculated); therefore, the term "sustained stress ratio" (or "initial sustained stress ratio") is better suited to describe the creep-rupture limitations.

A relationship between strain degradation rates (creep coefficients) and initial sustained stress levels, was developed to illustrate the overall process of establishing the creep rupture phenomena as a function of FRP service life and initial sustained strain. Due to a large scatter of test data, a lower bound of "time to failure" (i.e., sample's service or creep life "tfailure") at various initial sustained stress ratios, was selected as the primary serviceability limit state (e.g., failure happens when $t_{\text {failure }}<t_{\text {service}}$ ). This method uses the mean value of degradation plus three and a half times the standard deviations $(\mu+3.5 \sigma)$ to capture the outliers and provide a confidence 
interval of minimum $99.977 \%$. The residuals (from the mean value) were tested for the best distribution fit, and the "normal" distribution seems to fit the best. Using Findley's power law, rupture times (i.e., time to failure) were computed for various initial sustained strain levels to reach a pre-selected creep rupture strain. In addition to Findley's power function, a single parameter logarithmic degradation relationship was also evaluated, which used a complex time step function to arrive at a similar conclusion. Findley's power law provided a direct solution of time to failure, and, therefore, is preferred in this report. Findley's power law also allows the formulation of an analytical solution based on strain energy principles and creep-rupture test data (GangaRao and Liang, 2010). The report compares the standard practices of limiting sustained stress levels with compute values and recommends sustained stress limits for 10,25, 50, 75, and 100-year design life-spans. It should be noted that the database used in this study primarily consisted of pultruded vinylester GFRP reinforcing bars subjected to a range of sustained axial tensile stress. Caution should be taken when applying the report findings to other FRP applications, such as a pultruded beam response under flexure.

This chapter illustrates a framework for taking design service life into account for selecting initial sustained strain (or sustained stress) limits based on a reliability-based time-dependent durability relationship. 


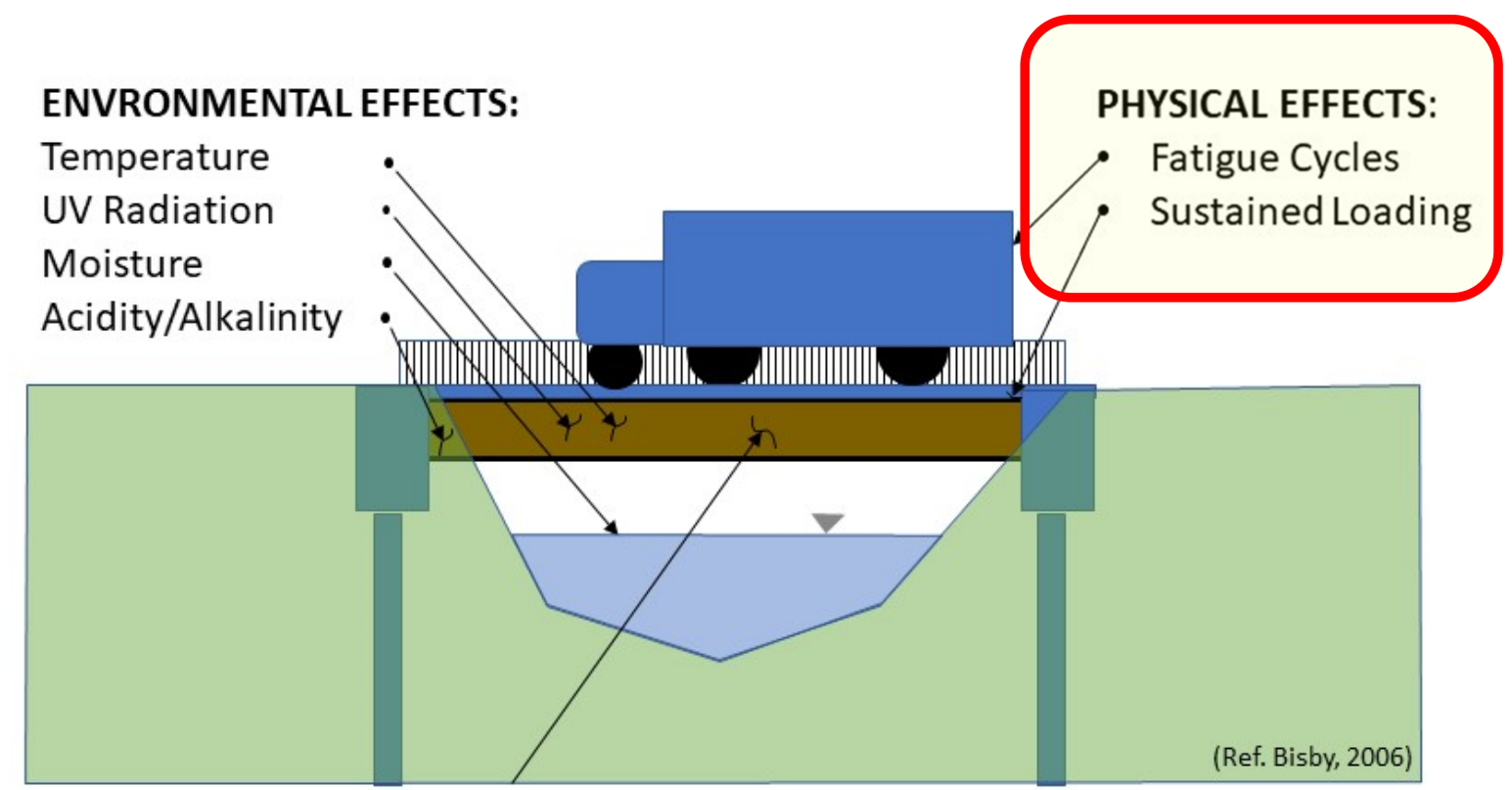

AFFECTED DURABILITY CHARACTERSTICS:

Tensile / Compressive / Flexural / Shear Strengths and Moduli

Figure 5.1: Physical Effects on Outdoor FRP Structures

\subsection{IMPORTANCE OF LIMITING SUSTAINED STRESS RATIOS}

Physical effects such as sustained load (or creep) and cyclic loading (or fatigue) have a detrimental impact on the durability of FRP (Figure 5.1). Almost all civil engineering structures carry some sustained stresses primarily due to dead loads (e.g., self-weight, flooring, wearing surface, railings, etc. on beams) or long-term live loads (e.g., winter snow on a forest service bridge or water stored inside tanks), and therefore, creep or sustained loading of FRP members is the primary focus of this report. For example, sustained loads can be almost $30 \%$ of the total service load on small-spans bridges and up to $80 \%$ on long-span bridges. Traditional codes often limit a design sustained stress ratio to $25 \%$ to $30 \%$ of ultimate design strength (for routine structures); however, they do not take into account: i) members' design life span (e.g., 10-year life vs. 100year life), ii) serviceability issues (e.g., deflection or camber) related to creep deformation of 
members, iii) the creep rupture limit/strength of the FRP material, or iv) substantiated design basis or justifications. If sustained stresses are not correctly accounted for in the design, FRP members can become prematurely non-serviceable due to large permanent deflections or fail spectacularly due to inelastic strain increase leading to creep-rupture failures.

A rational process to take in such common environmental conditions and material creep behavior can result in the reliable design of FRP structures. The purpose of this chapter is to provide a basis for a reliability-based durability framework that uses an actual large-scale creep test database to ensure safe but economical designs.

\subsection{Current State of Practice}

A vast majority of the current practices of limiting sustained load comes from concrete codes; however, other codes and industry practices also provide limited guidelines (e.g., MIL-17 Handbook) on pultruded FRP members. Table 5.1 is a summary of current design practice for strength reduction factors $(\phi)$ as adopted by various codes and reported by the University of Sherbrook Study (Youssef, 2010): 
Table 5.1- Current Practices Regarding Sustained Stress Limitations

\begin{tabular}{|c|c|c|c|c|c|}
\hline $\begin{array}{r}\text { M } \\
\text { (Referenc } \\
\end{array}$ & $\begin{array}{l}\text { um allowa } \\
\text { /CSA S806-( }\end{array}$ & $\begin{array}{l}\text { ensile stre } \\
\text { here } \mathrm{f}_{\mathrm{FRPu}}=\end{array}$ & $\begin{array}{l}\text { RP bars } \mathbf{} \\
\text { e failure str }\end{array}$ & $\begin{array}{l}\text { sustaine } \\
\text { FRP bar) }\end{array}$ & \\
\hline Glass FRP & & & & & \\
\hline $0.30 f_{F R P u}$ & & & & & \\
\hline $\begin{array}{r}\text { M } \\
\text { (Referenc } \\
\text { strength o }\end{array}$ & $\begin{array}{l}\text { um allowa } \\
\text { adian Highn } \\
\text { oar). Includ }\end{array}$ & $\begin{array}{l}\text { tress in FI } \\
\text { dge Design } \\
\text { nbined ser }\end{array}$ & $\begin{array}{l}\text { rs at servi } \\
\text { (CAN/CSA S } \\
\text { esses from }\end{array}$ & $\begin{array}{l}\text { ity limit } \\
\text { where } f_{F R I} \\
\text { ned loads }\end{array}$ & $\begin{array}{l}\text { cified tensile } \\
\text { loads }\end{array}$ \\
\hline Glass FRP & & Aramid FF & & Carbon F & \\
\hline $0.25 f_{\mathrm{FRPu}}$ & & $0.35 f_{F R P u}$ & & $0.65 f_{\mathrm{FRPu}}$ & \\
\hline $\begin{array}{l}\text { F } \\
\text { (Referenc } \\
\text { of the bar }\end{array}$ & $\begin{array}{l}\text { inforceme } \\
\text { 440.1R-06; } \\
\text { omputed as }\end{array}$ & $\begin{array}{l}\text { eep ruptu } \\
f_{f u}=\text { Enviro } \\
-3 \sigma)\end{array}$ & al reductior & C $C_{E} \times$ Gua & tensile stress \\
\hline Glass FRP & & Aramid FF & & Carbon $\mathrm{F}$ & \\
\hline $0.20 f_{f u}$ & & $0.30 f_{f u}$ & & $0.55 f_{f u}$ & \\
\hline $\begin{array}{r}\text { F } \\
\text { (Referenc } \\
\text { limit state } \\
\end{array}$ & inforceme & stained st & $\begin{array}{l}\text { mit (in Vir } \\
\text { bars CNR-D }\end{array}$ & $\begin{array}{l}\text { er or Ep } \\
\text { 2006; wh }\end{array}$ & $\begin{array}{l}\text { trix) } \\
\text { is serviceability }\end{array}$ \\
\hline Glass FRP & & Aramid FF & & Carbon $\mathrm{F}$ & \\
\hline $0.30 \mathrm{SLS}$ & & $0.50 \mathrm{SLS}$ & & $0.90 \mathrm{SLS}$ & \\
\hline $\begin{array}{r}\text { F } \\
\text { (Referenc } \\
\end{array}$ & $\begin{array}{l}\text { inforceme } \\
\text { ask group b } \\
\end{array}$ & $\begin{array}{l}\text { stained st } \\
40 ; \text { where }\end{array}$ & $\begin{array}{l}\text { mit } \\
\text { sign streng } \\
\end{array}$ & & \\
\hline Glass FRP & & Aramid FF & & Carbon $\mathrm{F}$ & \\
\hline 50 years & 100 years & 50 years & 100 years & 50 years & 100 years \\
\hline $0.30 f_{u}$ & $0.25 f_{u}$ & $0.45 f_{u}$ & $0.40 f_{u}$ & $0.80 f_{u}$ & $0.75 f_{u}$ \\
\hline
\end{tabular}

It is better to normalize Table 5.1 parameters to compare the above guidelines in terms of design service life. For comparison, we can assume that the guaranteed tensile design strength 
$\left(\mathrm{f}^{*} \mathrm{fu}\right)$ is always computed as a nominal strength $\left(\mathrm{f}_{\mathrm{fu}, \mathrm{ave}}-3 \sigma\right)$ where $\sigma$ is the standard deviation, and the design tensile strength $\left(\mathrm{f}_{\mathrm{fu}}\right)$ is calculated using an Environmental factor $\mathrm{C}_{\mathrm{E}}$ of $0.8 \mathrm{x} \mathrm{f}_{\mathrm{fu}}$ or 0.8 $x\left(f_{f u, a v e}-3 \sigma\right)$. (Also, see section 2.5.2 for discussion on characteristics strength.) For simplicity, we can assume that about $80 \%$ of total service stresses in FRP reinforcement in a concrete beam come from the sustained load. Aramid FRP has been removed from the following table since i) there is minimal creep data available, ii) it is generally more prone to creep effects (as compared to glass or carbon FRPs) and, iii) its use in the civil infrastructure field is limited due to its susceptibility to moisture. Various limits shown in Table 5.1 can be reorganized as Table 5.2 for a better comparison by using the above normalization process.

Table 5.2: Summary of Current Practices Regarding Sustained Stresses

\begin{tabular}{|c|c|c|c|c|}
\hline \multicolumn{5}{|c|}{ Maximum Allowable Sustained Stress Ratio ${ }^{3}$} \\
\hline Fiber Type & \multicolumn{2}{|c|}{ Glass FRP } & \multicolumn{2}{|c|}{ Carbon FRP } \\
\hline CAN/CSA S806-062 & \multicolumn{2}{|l|}{$30 \%$} & & \\
\hline CAN/CSA S6-06 note 1 & \multicolumn{2}{|l|}{$31 \%$} & \multicolumn{2}{|l|}{$81 \%$} \\
\hline $\mathrm{ACl} 440.1 \mathrm{R}-06^{\text {note }} 2$ & \multicolumn{2}{|l|}{$31 \%$} & \multicolumn{2}{|l|}{$86 \%$} \\
\hline CNR-DT 203/2006 & \multicolumn{2}{|l|}{$30 \%$} & \multicolumn{2}{|l|}{$90 \%$} \\
\hline \multirow{2}{*}{ fib task bulletin 40} & $50-y r$ & $100-y r$ & $50-y r$ & $100-y r$ \\
\hline & $30 \%$ & $25 \%$ & $80 \%$ & $75 \%$ \\
\hline
\end{tabular}

${ }^{1}$ Assumes $80 \%$ of total service stresses in FRP reinforcing bar come from initially sustained loads (common in large structures such as major bridges or parking structures, much lower for other structures such as concrete slabs). The values shown may or may not provide 50 or 100 years of service life (no information about service life is available.) However, these values can be calibrated to provide the desired service life based on the reliabilitybased framework presented in this chapter.

${ }^{2}$ Assumes $C_{E}$ (Environmental Reduction Factor) is 0.8 , and $80 \%$ of stresses in FRP reinforcing bar are from sustained loads.

${ }^{3}$ The maximum allowable sustained stress ratio is defined as $f_{s s} / f^{*} f_{f u}$, where $f_{s s}$ is sustained stresses (e.g., service stresses from dead loads), and $\mathrm{f}_{\mathrm{fu}}$ is "guaranteed tensile strength" or characteristic strength (computed as $0.8 \mathrm{x}$ ( $\left.f_{f u, a v e}-3 \sigma\right)$. It should be noted that the ultimate strain $\left(\varepsilon_{f u}\right.$, ave $)$ is typically obtained at $90 \%$ to $95 \%$ value of $f_{f u, a v e}$ during testing. 


\subsection{DEVELOPMENT OF TIME-DEPENDENT MODEL FOR SUSTAINED STRESS}

Since the creep behavior of FRP is time and material dependent, and non-linear (or perhaps somewhat inconsistent) particularly at the tertiary stages (Stage III, Figure 5.2), it is reasonable to conduct a time-dependent reliability analysis of sustained strain period (Stage II, Figure 5.2) to arrive at service life recommendations. The initial stage (Stage I) happens instantly upon loading, is linear, and can be computed based on conventional stress-strain relationship. To evaluate the time-dependent relationship between durability and reliability related to sustained stress (or creep strain) - the primary objective of this chapter - the extensive rebar test data from the University of Sherbrook was normalized (e.g., final strains were expressed as a ratio for initial strain levels, initial stresses were computed as a ratio of initial stress over ultimate stress, etc.) for comparisons and statistical analysis (also see Section 5.4.4 for the normalization process used in this chapter). The underlying hypothesis is that once a rational process and relationship patterns are developed for a group of GFRP reinforcing bars in vinylester matrix (or in epoxy or polyester), it is very likely that other samples will follow a similar pattern and then a more comprehensive prediction of reliability-durability relationship can be made.

\subsubsection{Analytical Formulation of Creep}

An increase in creep strain is attributed to the interconnection of micro-voids in a composite, followed by softening of resin with time if a composite is in the presence of moisture (or other solutions), or when exposed to temperatures higher than room temperatures (Batra, 2009; GangaRao \& Liang, 2010), or due to the failure of the local bond between fibers and resins. As the percent of sustained strain increases in relation to an FRP composite material's static strain to failure, sustained strain to static failure strain decays with time in a power-law form, which can provide a direct equation of creep rupture time prediction for a given sustained strain. 
Under sustained stress conditions such as axial force or bending, internal strain energy (stored energy) of the material is expended under the externally induced work (e.g., axial elongation or flexural/shear deflections) and due to the damage accumulated in the material thru matrix cracking or de-bonding of sizing material of glass fibers from the resin. As the damage gets accumulated, the total energy expended by FRP is found to be a function of i) induced initial sustained strain level as a percent of static failure strain (e.g., linear strain value typically corresponding to ultimate stress of 0.90 to $0.95 \mathrm{f}_{\mathrm{pu}}$ ), ii) time to failure under initial sustained strain level and iii) mechanical properties affected by intrinsic variations of the FRP composites (e.g., fiber and matrix ratio, resin impregnation, internal defects, void percent, cure percent, etc.) leading to an ultimate creep rupture strain value $\left(\varepsilon_{\mathrm{r}}\right)$.

The sustained strain-time response of FRP composites is similar to their fatigue phenomenon, except that the creep behavior studies focus on "time" to failure at given sustained stress while fatigue failure is measured as a function of the "number of cycles" to failure. Typically, creep effects under fatigue (due to mean fatigue stress) are neglected due to the short duration of time to fatigue failure in lab experiments. Similar to the fatigue failure response of FRP composites, creep response also has three distinct stages of progression to failure. As previously explained in Section 2.2.6, Stage I is identified by strain energy expended near instantaneously when the load is statically applied to reach a sustained value (elastic deformation). Stage II is marked by slow creep deformation due to initial sustained load, and Stage III, also known as "tertiary creep," is identified with a rapid increase in expended energy (and strain) within the short time duration to the final creep strain rupture (failure). The modeling of actual response in Stage III has little practical/design significance, instead of a strain value designated as "ultimate rupture strain," it is assumed to mark the start of stage III, leading to eventual catastrophic failure (Figure 
5.2). The tertiary strain (Stage III) is difficult to model in practice; however, the secondary inelastic strain increase (stage II) can be modeled using a logarithmic or Findley's power laws over the sample's life span to near failure. Since Findley's power law is simple in approach and has been widely used to predict nonlinear viscoelastic creep behavior of composites, Findley's power law is used in this research.

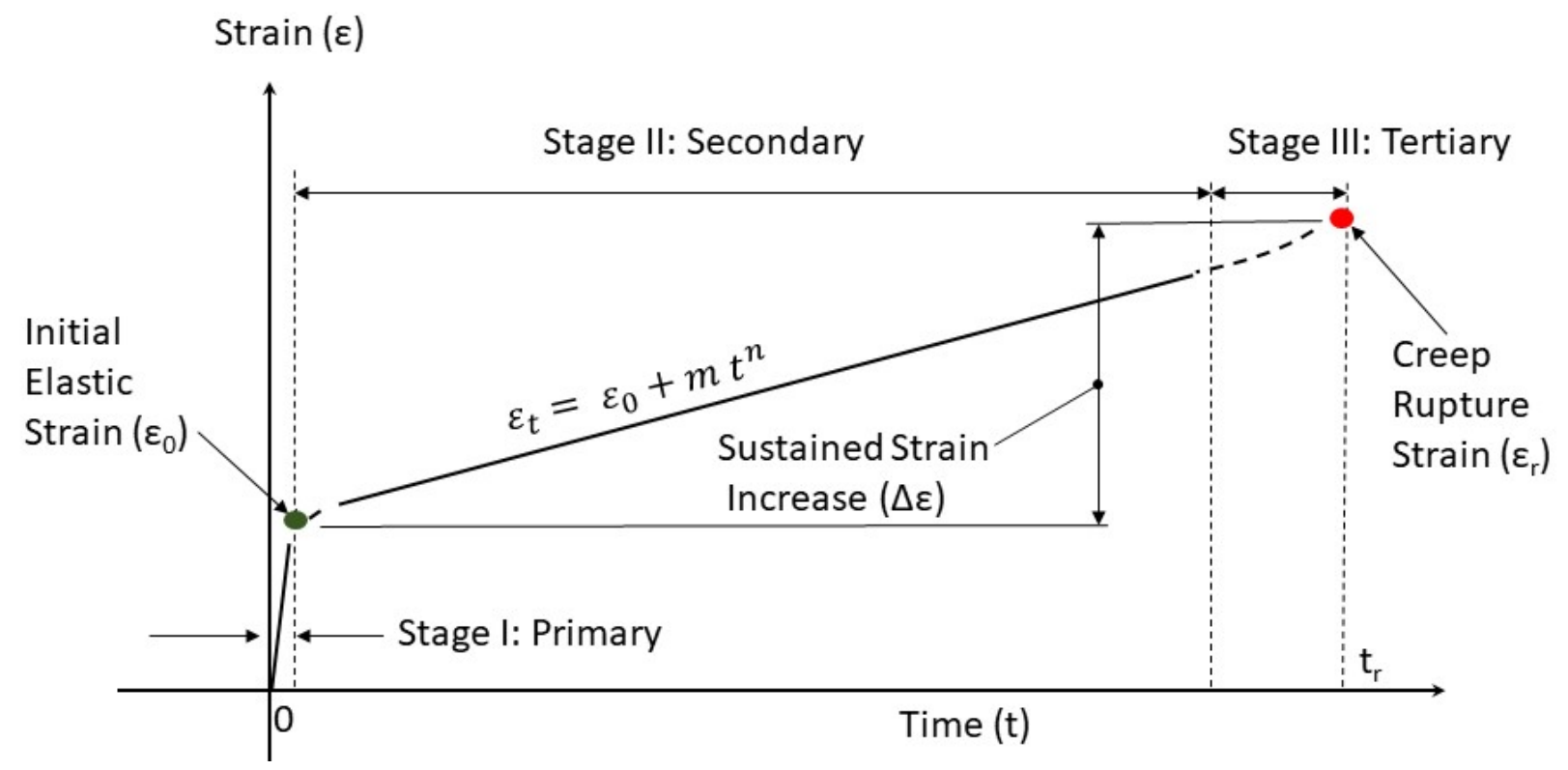

Figure 5.2: Typical Time-Dependent Behavior of Strain due to Creep

Proper modeling of Stage II creep is important as it signifies the propagation of creep deformation increase (or strain energy consumption with time), primarily by matrix yielding, cracking or local debonding of fibers from the matrix, leading to interlaminar crack propagation and delamination across the entire FRP composite member cross-section. The expended energy with time is almost linear until about $90-95 \%$ of the time to failure. This expended strain energy (induced by inelastic deformation) is a function of initial static strain, applied load (initial stress), and intrinsic material properties of GFRP, and it can be represented by the coefficients of Findley's 
power law (or other modified forms of Findley's power law). The studies conducted at WVU (including this study) have found that Findley's Power Law model works well for the viscoelastic behavior of FRPs under constant static stress/strain.

The energy release rate in Stage II (obtained experimentally) for a composite of certain constituents, is found to be constant and is a characteristic of the constitutive material under similar loading conditions (i.e., bending or compression or tension). The energy release rate increases with an increase in sustained load fraction of static failure load, but the total energy release to failure (over the induced static energy) remains constant for a given cross-section.

The variation in energy release rates $\left(\mathrm{dU}_{t} / \mathrm{dt}\right)$ can be plotted as a function of initial sustained strain $\left(\varepsilon_{0}\right)$, final strain $\left(\varepsilon_{t}\right)$, strain energy $\left(U_{t}\right)$ expended at time $t$, internal FRP parameter $C_{t}$ which is affected by time t, and creep coefficients " $a$ " and " $b$ " resembling Findley's power law as shown below:

$\frac{\mathrm{du}_{t}}{d t}=\mathrm{a} f\left(\varepsilon_{0}, \varepsilon_{\mathrm{t}}, U_{t}, C_{t}\right)^{b}$

Once a fundamental analytical relationship has been identified, the final form of Findley's equation and coefficients can be experimentally determined, as shown in the next section (GangaRao \& Liang, 2010).

\subsubsection{Application of Findley's Power Law}

Definitions:

$\varepsilon_{0}=$ Initial Sustained Strain (iSS) at $(t=0)$ for an applied sustained stress

$\varepsilon_{t}=$ Final Sustained Strain $(f S S)$ at time $(t)$

$\varepsilon_{f u}=$ Average,Ultimate Strin (coresponding to Ultimate Strength $f_{u, \text { ave }}$ )

(Often determined at $0.90-0.95$ of the ultimate stress) 


$$
\begin{aligned}
& \varepsilon_{r}=\text { Creep Rupture Strain (cRS) at time } t=t_{r} \\
& t=\text { Time from Initial Strain (hours }- \text { based on test data) } \\
& t_{r}=\text { Time to Creep Rupture Failure } \\
& \varepsilon_{0}^{\prime}=\text { Initial Sustained Strain Ratio with respect to Ultimate }(\text { iSSR })=\frac{\varepsilon_{0}}{\varepsilon_{f u}} \\
& \varepsilon_{t}^{\prime}=\text { Creep Sustained Strain Ratio w.r.t. Initial }(\text { CSSR })=\frac{\Delta \varepsilon_{t}}{\varepsilon_{0}}=\frac{\left(\varepsilon_{t}-\varepsilon_{0}\right)}{\varepsilon_{0}} \\
& \varepsilon_{t u}^{\prime}=\text { Final Sustained Strain Ratio w.r.t. Ultimate }(f S S R=i S S R+c S S R)=\frac{\varepsilon_{t}}{\varepsilon_{f u}} \\
& \varepsilon_{t 0}^{\prime}=\text { Total Sustained Strain Ratio w.r.t. Initial }(t S S R=f S S R / i S S R)=\frac{\varepsilon_{t}}{\varepsilon_{0}} \\
& \varepsilon_{r}^{\prime}=\text { Creep Rupture Strain w.r.t.Ultimate }=\frac{\varepsilon_{r}}{\varepsilon_{f u}} \\
& m^{\prime}=\text { Normalized Creep Intercept Coefficient }=\frac{m}{\varepsilon_{0}} \\
& \quad \text { (Often determined at } 0.90-0.95 \text { of the ultimate rupture value }) \\
& m=\text { Creep Intercept Coefficient }
\end{aligned}
$$

For experimental determination of sustained stress parameters, Findley power law (Eq. 5.1) can be written as

$$
\varepsilon_{t}=\varepsilon_{0}+m t^{n} \ldots E q(5.2)
$$

Where "m" and " $n$ " are creep coefficients which are a function of FRP material properties and sustained loading. In this study, creep coefficients " $m$ " and " $n$ " are two experimentally derived unique pairs of variables that are regressed for the best curve fitting of each set of experimental 
data (i.e., a sample strain reading at 1, 1000,3000, and 10,000 hours). No meaningful pattern for either " $m$ " or " $n$ " values was found in this test database, indicating that mean values of " $m$ " or " $n$ " cannot be used to make any reasonable prediction for "time to failure" due to creep. The previous flexure creep tests conducted at WVU-CFC (Batra, 2009, GangaRao \& Liang, 2010) on 1/4" and $1 / 2$ " Vinylester-based GFRP laminates have indicated the mean value of " $\mathrm{m}$ " to be constant for various sustained stress ratios $(35 \%, 50 \%$, and $65 \%)$ and the mean value of " $\mathrm{n}$ " to be proportional to the increase in the initial sustained strain ratio and depends on constituent material properties. Without additional supporting data, this disparity of results cannot be explained except for the fact that the Youssef (2010) data contained more "low initial sustained strain" test values (over 100 sets of observations), and only five creep rupture values. Nonetheless, Youssef data serves as a valuable resource for lower sustained stress values that are more common in practice.

The two-parameter Findley's power Equation 5.2 can be re-written as:

$$
\begin{gathered}
\varepsilon_{t}-\varepsilon_{0}=m t^{n} \text { or } \\
\frac{\varepsilon_{t}-\varepsilon_{0}}{\varepsilon_{0}}=\frac{m}{\varepsilon_{0}} t^{n} \text { or } \\
\frac{\Delta \varepsilon_{t}}{\varepsilon_{0}}=\left(\frac{m}{\varepsilon_{0}}\right) t^{n} \text { or } \\
\varepsilon_{\mathrm{t}}^{\prime}=m^{\prime} t^{n} \quad \ldots E q(5.3)
\end{gathered}
$$

Where $\varepsilon_{t}^{\prime}$ is normalized creep strain ratio $\left(\frac{\Delta \varepsilon_{t}}{\varepsilon_{0}}\right)$, also referred to as creep sustained strain ratio with respect to the initial sustained strain ratio (cSSR). The coefficient $m^{\prime}$ is a normalized creep coefficient $\left(\frac{m}{\varepsilon_{0}}\right)$. Coefficients $m^{\prime}$ and $\mathrm{n}$ can be obtained by the power-law regression method or as a straight line by taking the natural or conventional log of equation 5.3, as shown below.

$$
\ln \left(\varepsilon_{\mathrm{t}}^{\prime}\right)=\ln \left(m^{\prime}\right)+n \ln (t) \quad \ldots E q(5.4)
$$


Equation 5.4 is an equation of a straight line with $\ln \left(m^{\prime}\right)$ as the y-axis intercept and line slope "n." Coefficients " $\ln \left(m^{\prime}\right)$ " and "n" are computed using linear regression of test data comprised of normalized creep sustained strain ratio $\left(\varepsilon_{\mathrm{t}}^{\prime}=\left(\frac{\Delta \varepsilon_{t}}{\varepsilon_{0}}\right)\right)$ and associated time $(\mathrm{t})$.

Re-writing Findley equation (5.2) after dividing by $\varepsilon_{0}$ to obtain the normalized final strain $\varepsilon_{t 0}^{\prime}$ (also referred to as $\mathrm{tSSR}$, total sustained strain ratio divided by initial sustained strain ratio) gives:

$$
\begin{gathered}
\frac{\varepsilon_{t}}{\varepsilon_{0}}=1+\left(\frac{m}{\varepsilon_{0}}\right) t^{n} \text { or } \\
\varepsilon_{t 0}^{\prime}=1+m^{\prime} t^{n} \text { or simply, } \quad \varepsilon_{t 0}^{\prime}=1+\varepsilon_{\mathrm{t}}^{\prime}
\end{gathered}
$$

Similarly, coefficients $m^{\prime}$ and $n$ can be obtained using experimental test data. If the rupture strain ratio $\left(\varepsilon_{r}^{\prime}\right)$ is known or can be estimated, the time to reach that rupture strain can be derived directly by dividing Equation 5.2 with $\varepsilon_{f u}$ as shown below and substituting $\mathrm{m}\left(=m^{\prime} x \varepsilon_{0}\right)$ :

$$
\begin{gathered}
\frac{\varepsilon_{t}}{\varepsilon_{f u}}=\frac{\varepsilon_{0}}{\varepsilon_{f u}}+\frac{m}{\varepsilon_{f u}} t^{n} \text { or } \\
\varepsilon_{t u}^{\prime}=\varepsilon_{0}^{\prime}+m^{\prime} \frac{\varepsilon_{0}}{\varepsilon_{f u}} t^{n} \quad \text { or } \\
\varepsilon_{t u}^{\prime}=\varepsilon_{0}^{\prime}+m^{\prime} \varepsilon_{0}^{\prime} t^{n} \quad \ldots \text { Eq }
\end{gathered}
$$

Where $\varepsilon_{t u}^{\prime}$ is the final sustained strain ration (fSSR) and is obtained after dividing the final sustained strain by average ultimate strain.

We understand that the final rupture strain $\left(\varepsilon_{r}\right)$ can be reached only in Stage III, and Stage III cannot be correctly modeled using Fiddley's power law. Since there are not enough experimental data points available, we can also assume that Stage III begins at about 0.90 to $95 \varepsilon_{\mathrm{r}}$. Theoretically speaking, the value of strain $\varepsilon_{t}$ computed using Findley's power law is correct only up to 0.90 to $95 \varepsilon_{\mathrm{r}}$. However, the ultimate static strain $\left(\varepsilon_{f u}\right)$ is also taken as 0.90 to 0.95 of ultimate 
values, and due to the use of ratios in this chapter, the fraction (0.90 to 0.95$)$ cancels out. We are assuming that this fraction is similar for both the static ultimate and final rupture strains. The error introduced due to the above assumption can be considered within the context of the proposed reliability approach.

Equation 5.6, after substituting for time $\left(t=t_{r}\right)$ and strain $\left(\varepsilon^{\prime}{ }_{t u}=\varepsilon_{r}^{\prime}\right)$ at rupture failure, can be written to obtain $\mathrm{t}_{\mathrm{r}}$ (or time to rupture or time to failure).

$$
\begin{gathered}
\varepsilon_{r}^{\prime}=\varepsilon_{0}^{\prime}+m^{\prime} \varepsilon_{0}^{\prime}\left(t_{r}\right)^{n} \text { or } \\
\left(t_{r}\right)^{n}=\frac{\varepsilon_{r}^{\prime}-\varepsilon_{0}^{\prime}}{m^{\prime} \varepsilon_{0}^{\prime}} \text { or } \\
t_{r}=\left(\frac{\varepsilon_{r}^{\prime}-\varepsilon_{0}^{\prime}}{m^{\prime} \varepsilon_{0}^{\prime}}\right)^{\frac{1}{n}} \quad \ldots E q(5.7)
\end{gathered}
$$

The above equation uses only strain ratios and does not require knowledge of actual strain measurements. However, if actual strain measurements of initial and final sustained strains $\left(\varepsilon_{0}, \varepsilon_{r}\right)$ are known, then Equation 5.7 can be re-written to compute time to rupture failure $\left(t_{r}\right)$ :

$$
t_{r}=\left(\frac{\varepsilon_{r}^{\prime}-\varepsilon_{0}^{\prime}}{\mathrm{m}}\right)^{\frac{1}{n}}=\left(\frac{\varepsilon_{t}-\varepsilon_{0}}{\mathrm{~m}}\right)^{\frac{1}{n}} \quad \ldots E q(5.8)
$$

Once a relationship of time to rupture $t_{r}$ with rupture strain ratio $\varepsilon_{r}^{\prime}$ and initial strain ratio $\varepsilon_{0}^{\prime}$ has been experimentally established (Equations 5.6 and 5.8), a reliability-based time-dependent framework can be developed (as shown in the following sections - starting with 5.4.3 - for a sustained stress test database) for common commercially available FRP composites. The effectiveness of the Findley model can be seen from the comparison of test data observations used in this report versus estimated values (at $t=10,000$ hours), as shown in Figure 5.3. 


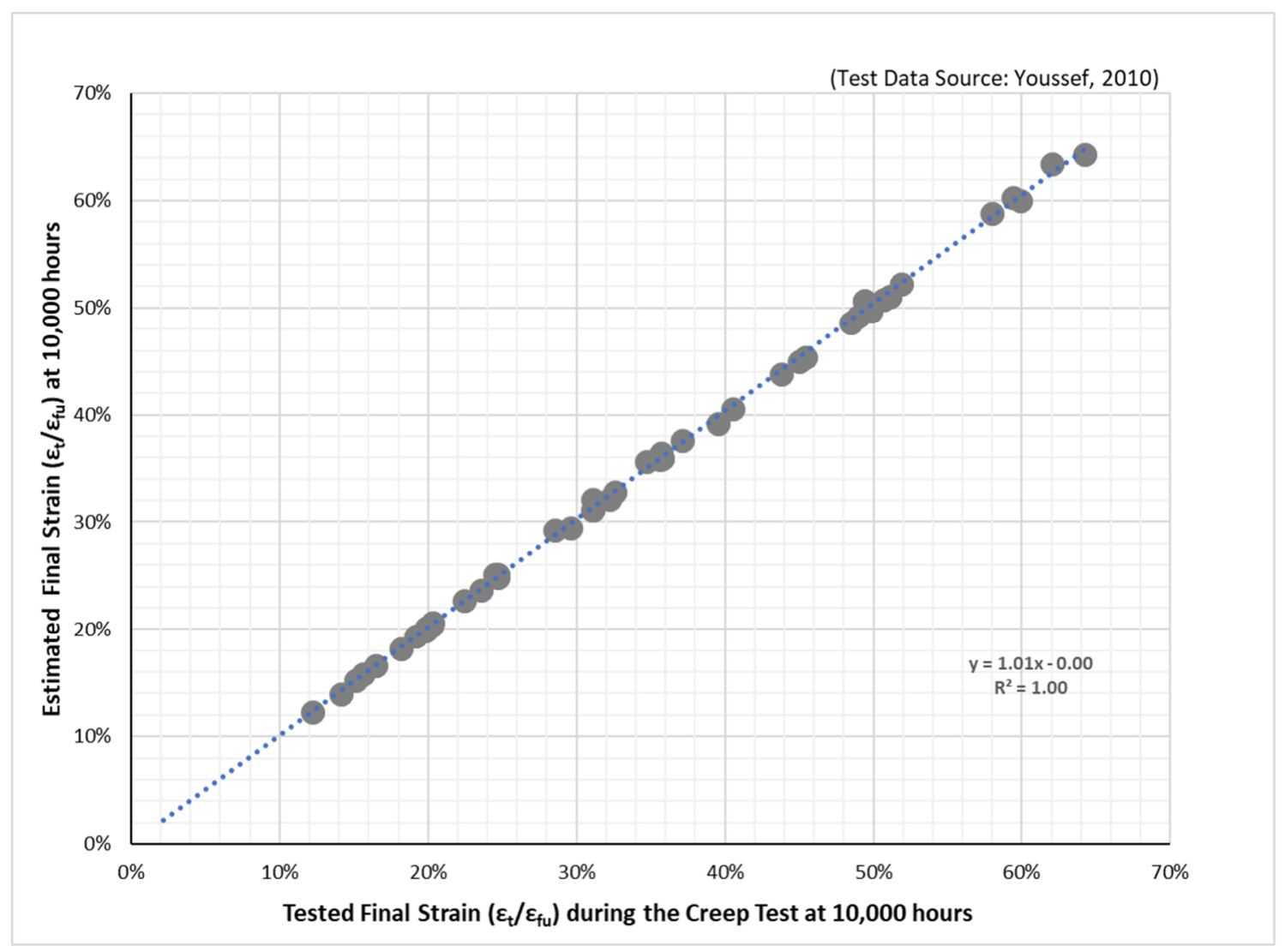

Figure 5.3: Comparison of Observed Test values Vs. Findley's Prediction Model

\subsubsection{Creep or Sustained Stress Database}

As mentioned before, one of the recent large-scale databases on the effects of sustained stress on FRP was published by the University of Sherbrook FRP Durability Facility (Youssef, 2010). In the study, creep behavior tests were conducted on fifty-two GFRP bar-samples, of six different commercial brands, over 10,000 hours (417 days). The glass/Vinylester GFRP bars (of diameters $9.5 \mathrm{~mm}, 12 \mathrm{~mm}, 12.7 \mathrm{~mm}$ and $15.9 \mathrm{~mm})$, were tested at room temperature $\left(23 \pm 3^{\circ} \mathrm{C}\right)$ and subjected to constant sustained load service levels, The E-glass/Vinylester GFRP bars, $9.5 \mathrm{~mm}$ in diameter, were tested in the air $\left(23 \pm 3^{\circ} \mathrm{C}\right)$ at different levels of sustained axial load, nominally $\left(15 \%, 30 \% 45 \%\right.$ and $60 \%$ of the average ultimate tensile strength $f_{u}$, ave). 
Table 5.3: Summary of GFRP Samples used in Creep Study

\begin{tabular}{|c|c|c|c|c|c|c|c|c|c|c|c|c|c|c|}
\hline Bar Designation: & \multicolumn{2}{|c|}{ GFRP-1 } & \multicolumn{2}{|c|}{ GFRP-2 } & \multicolumn{2}{|c|}{ GFRP-3 } & \multicolumn{2}{|c|}{ GFRP-4 } & \multicolumn{2}{|c|}{ GFRP-5 } & \multicolumn{2}{|c|}{ GFRP-6 } & \multicolumn{2}{|c|}{ RANGE / AVE } \\
\hline Bar Diameter (mm): & \multicolumn{2}{|c|}{9.5} & \multicolumn{2}{|c|}{9.5} & \multicolumn{2}{|c|}{12.7} & \multicolumn{2}{|c|}{12} & \multicolumn{2}{|c|}{15.9} & \multicolumn{2}{|c|}{15.9} & \multicolumn{2}{|c|}{$9.5-15.9$} \\
\hline Bar Cross-Sectional Area $\left(\mathrm{mm}^{2}\right)$ & \multicolumn{2}{|c|}{71} & \multicolumn{2}{|c|}{71} & \multicolumn{2}{|c|}{127} & \multicolumn{2}{|c|}{113} & \multicolumn{2}{|c|}{199} & \multicolumn{2}{|c|}{199} & \multicolumn{2}{|c|}{$71-199$} \\
\hline Bar Type (Sand-Coated, GL-VE): & \multicolumn{2}{|c|}{ Sand-Coated Only } & \multicolumn{2}{|c|}{$\begin{array}{l}\text { Helically } \\
\text { Wrapped }\end{array}$} & \multicolumn{2}{|c|}{ Sand-Coated Only } & \multicolumn{2}{|c|}{ Ribbed } & \multicolumn{2}{|c|}{ Sand-Coated Only } & \multicolumn{2}{|c|}{$\begin{array}{l}\text { Helically } \\
\text { Wrapped }\end{array}$} & & \\
\hline Fiber Fraction (By Volume): & \multicolumn{2}{|c|}{$56.8 \%$} & \multicolumn{2}{|c|}{$50.6 \%$} & \multicolumn{2}{|c|}{$64.5 \%$} & \multicolumn{2}{|c|}{$75.2 \%$} & \multicolumn{2}{|c|}{$66.6 \%$} & \multicolumn{2}{|c|}{$57.5 \%$} & \multicolumn{2}{|c|}{$51 \%-75 \%$} \\
\hline TESTED PROPERTIES & $\begin{array}{c}\text { As } \\
\text { Tested } \\
\end{array}$ & \begin{tabular}{|c} 
Normali \\
zed
\end{tabular} & $\begin{array}{c}\text { As } \\
\text { Tested } \\
\end{array}$ & \begin{tabular}{|c} 
Normali \\
zed
\end{tabular} & $\begin{array}{c}\text { As } \\
\text { Tested }\end{array}$ & \begin{tabular}{|c|}
$\begin{array}{c}\text { Normali } \\
\text { zed }\end{array}$ \\
\end{tabular} & $\begin{array}{c}\text { As } \\
\text { Tested } \\
\end{array}$ & \begin{tabular}{|c} 
Normali \\
zed
\end{tabular} & $\begin{array}{c}\text { As } \\
\text { Tested }\end{array}$ & \begin{tabular}{|c|}
$\begin{array}{c}\text { Normali } \\
\text { zed }\end{array}$ \\
\end{tabular} & $\begin{array}{c}\text { As } \\
\text { Tested }\end{array}$ & \begin{tabular}{|c|}
$\begin{array}{c}\text { Normali } \\
\text { zed }\end{array}$ \\
\end{tabular} & $\begin{array}{c}\text { As } \\
\text { Tested } \\
\end{array}$ & $\begin{array}{c}\text { Normali } \\
\text { zed }\end{array}$ \\
\hline Ultimate Tensile Strength $\mathrm{f}_{\mathrm{u}}(\mathrm{MPa})$ & 854 & $100 \%$ & 828 & $100 \%$ & 774 & $100 \%$ & 1410 & $100 \%$ & 748 & $100 \%$ & 783 & $100 \%$ & 900 & $100 \%$ \\
\hline Guaranteed Tensile Strength $f^{*}{ }_{u}=f_{u}-3 \sigma(M P a)$ & 752 & $88 \%$ & 714 & $86 \%$ & 660 & $85 \%$ & 1341 & $95 \%$ & 643 & $86 \%$ & 722 & $92 \%$ & 805 & $89 \%$ \\
\hline Std. Deviation $\sigma_{\mathrm{fu}}(\mathrm{MPa})=\left(\mathrm{f}_{\mathrm{u}}-\mathrm{f}^{*}{ }_{\mathrm{u}}\right) / 3$ & 34 & $4 \%$ & 38 & $5 \%$ & 38 & $5 \%$ & 23 & $2 \%$ & 35 & $5 \%$ & 20 & $3 \%$ & 31 & $4 \%$ \\
\hline Bias Factor $B F_{f u}=f_{u} / f^{*}{ }_{u}$ & 1.1 & 1.1 & 1.2 & 1.2 & 1.2 & 1.2 & 1.1 & 1.1 & 1.2 & 1.2 & 1.1 & 1.1 & 1.1 & 1.1 \\
\hline Modulus of Elasticity $E_{f}(G P a)$ & 46.9 & & 44.8 & & 49.9 & & 66.5 & & 42.5 & & 41 & & 48.6 & \\
\hline Ultimate Tensile Strain $\varepsilon_{\mathrm{fu}}(\mu \varepsilon)$ & 18232 & $100 \%$ & 18484 & $100 \%$ & 15620 & $100 \%$ & 21368 & $100 \%$ & 17712 & $100 \%$ & 19270 & $100 \%$ & 18448 & $100 \%$ \\
\hline Guaranteed Tensile Strain $\varepsilon_{f u}^{*}=\varepsilon_{f u}-3 \sigma(\mu \varepsilon)$ & 15931 & $87 \%$ & 15880 & $86 \%$ & 12752 & $82 \%$ & 16565 & $78 \%$ & 14467 & $82 \%$ & 17647 & $92 \%$ & 15540 & $84 \%$ \\
\hline Std. Deviation $\sigma_{\varepsilon f u}(\mu \varepsilon)=\left(\varepsilon_{\mathrm{fu}}-\varepsilon_{\mathrm{fu}}^{*}\right) / 3$ & 767 & $4 \%$ & 868 & $5 \%$ & 956 & $6 \%$ & 1601 & $7 \%$ & 1082 & $6 \%$ & 541 & $3 \%$ & 969 & $5 \%$ \\
\hline Bias Factor $B F_{\varepsilon f u}=\varepsilon_{f u} / \varepsilon_{f u}^{*}$ & 1.1 & 1.1 & 1.2 & 1.2 & 1.2 & 1.2 & 1.3 & 1.3 & 1.2 & 1.2 & 1.1 & 1.1 & 1.2 & 1.2 \\
\hline Number of Sustained Stress Observations & 87 & 63 & 52 & 44 & 43 & 31 & 36 & 24 & 32 & 32 & 32 & 32 & 282 & 226 \\
\hline
\end{tabular}

Data Source: University of Sherbrook (Youssef, 2010).

Note: Bias Factors are computed based on computed guaranteed tensile strain values and standard deviations, as shown. Ribbed bar type (GFRP-4) has the highest Bias Factor due to large standard deviations and should be further examined to see if the inclusion of this bar type is necessary for future research. The values presented in this Table are derived or directly taken from the published work of Youssef (2010), and any discrepancy, if present, was not investigated in this study. 
Table 5.3 summarizes all relevant creep tests conducted at the University of Sherbrook (Youssef, 2010), and the author acknowledges that some entries (e.g., rebar properties) as listed in the table may or may not be correct or relevant to the context of this study. For example, the rebar diameters and cross-sectional areas could have been modified to account for the presence of ribs and shear lags in different bar sizes. Also, the modulus of elasticity for some rebars with higher fiver volume fraction (fvf) is lower than bars with lower fvf (e.g., GFRP-3 vs. GFRP-4, etc.). However, detailed information about the samples is not available for further verification, and in the context of this chapter's objectives, such steps do not change the outcome of the analysis. The table is included here for the sake of reproducibility of results and to help the readers in understanding the scope of work involved in the original creep tests (Youssef, 2010). See the additional discussion about the normalization of the tabulated values in Section 5.4.4.

It should be noted that the theoretical validation of creep data using Findley's power law is limited to $90 \%$ to $95 \%$ of the ultimate values of stress or strains ( $f_{u}$ or $\varepsilon_{u}$.). However, due to limited availability of experimental test data for Stage III behavior and to maintain the reproducibility of such results, all ultimate values, as reported by Youssef (2010) in Table 5.3, are used in this chapter. See the additional discussion about this assumption in Section 5.4.2.

The University of Sherbrook researchers conducted microstructural analysis on tested commercial bars that were under $(25 \%, 30 \%$, and $45 \%) f_{u \text {, ave }}$ for 10,000 hours. They did not find any microcrack confirming no degradation in GFRP bars, meaning that $45 \% \mathrm{f}_{\mathrm{u} \text {, ave }}$ may be an acceptable upper creep rupture limit for the bars, in air, suitable for 50-years of service life (no evidence was provided how 50-year of service life was derived). The study, however, indicated that a logarithmic equation could approximate total strain $\varepsilon(\mathrm{t})$ at a time $(\mathrm{t})$ of the entire test duration as a function of the initial (elastic) strain and a creep rate parameter (or creep coefficient). The 
traditional method using Findley's power law was not used. The author's critical review of the University of Sherbrook report (Youssef, 2010) indicates that the irregular nature of data collected for rebar sustained stress study was found to be not suitable for deterministic analyses, and perhaps, therefore, no specific recommendations for the sustained stress degradation model could have been made.

The extensive test data collected on the long-term behavior of GFRP bars under sustained loads (particularly at the lower initial sustained strain of $15 \%$ to $30 \%$ ) provided a basis for this reliability-based study in this report for pultruded GFRP members in vinylester resins. It should be noted that about $23 \%$ of sustained load data from Youssef's study (2010) were rejected for use in this Report (Table 5.3). The primary reason for rejection was the Findley's equation for the time to failure/rupture (Equation 5.6) was found to be invalid due to negative or extremely flat slopes (i.e., unreasonable rate of increase in sustained strain with time). The weighted FVF (based on normalized sustained data used for the formulating results) is estimated to be $60 \%$, which encompasses an FVF range of $51 \%$ to $75 \%$.

\subsubsection{Normalization of Creep Database}

All data related to sustained load testing of FRP bars from the University of Sherbrook was first tabulated and then normalized. See Table 5.4 for a sample data page (Youssef, 2010) and Appendix D for the entire dataset. All measured strain values were re-tabulated as a percentage ratio of i) initial sustained strain/ultimate strain (iSSR $=\varepsilon_{0}^{\prime}=\frac{\varepsilon_{0}}{\varepsilon_{\mathrm{fu}}}$ ), ii) creep strain increase over initial sustained strain/initial sustained strain $\left(C S S R=\varepsilon_{\mathrm{t}}^{\prime}=\frac{\Delta \varepsilon_{\mathrm{t}}}{\varepsilon_{0}}=\frac{\left(\varepsilon_{\mathrm{t}}-\varepsilon_{0}\right)}{\varepsilon_{0}}\right)$, iii) final sustained strain/ultimate strain $\left(f S S R=\varepsilon_{\mathrm{to}}^{\prime}=\frac{\varepsilon_{\mathrm{t}}}{\varepsilon_{\mathrm{fu}}}\right)$, and iv) total sustained strain/initial sustained strain $\left(t S S R=\varepsilon_{\mathrm{tu}}^{\prime}=\frac{\varepsilon_{\mathrm{t}}}{\varepsilon_{0}}\right) . \quad$ Data related to the creep testing of concrete beams was not used. 
Normalization was necessary to conduct statistical analysis and to arrive at deterioration response for the general population of FRP rebar samples and to develop recommendations. Initial stress levels are expressed as a percentage of average ultimate stress, and the final strains are expressed as a percentage of initial strain levels. Several normalized parameters such as Coefficient of Variations (instead of standard deviations) and Bias Factors (instead of ultimate and nominal stresses or strains) are also developed and used from this database (see Table 5.3).

From a professional design practice point of view, the mean or average ultimate stress, $\mathrm{f}_{\mathrm{u}, \mathrm{ave}}$ or strain, $\varepsilon_{u, a v e}$ are not readily available to the designers; however, nominal or guaranteed ultimate stress, $f_{u}^{*}$ or strains $\varepsilon^{*}{ }_{u}$ are typically published (or provided) by the FRP manufacturers. Since most research is based on actual tested average ultimate stress or strains, appropriate Bias Factors should be multiplied with known/published nominal strengths to obtain the ultimate strength values and to apply the proposed research findings correctly. Again, in the absence of any other reliable information, the Bias Factor of 1.0 can be used conservatively.

When a product is placed in service (time $=0$ ), the initial sustained stress ratio and initial sustained strain ratio are the same and can be used interchangeably, provided the strains are within elastic ranges of the FRP member's response. It should be noted that the exact time when elastic strain stops and creep strain begins to accumulate (i.e., $t=0$ ) is difficult to identify in practice. The transition between the two stages (Stage I and Stage II) can be best estimated by projecting the Stage II curve backward to meet Stage I curve (or simply the $\mathrm{y}$-axis at $\mathrm{t}=0$, Figure 5.2). Nonetheless, this timeline error (a few minutes to an hour) due to this approximation is not of much practical value when projecting service life in years. Typically, a linear relationship between stress and strain exists up to $90 \%-95 \%$ of the test range for most FRPs. Plots and tables are developed for mean or average ultimate (stress or strain) strengths; however, a conversion from 
nominal, characteristic, or published strengths to mean ultimate strength is needed as also shown (using Bias Factors) in Section 5.4.9 of this report. In general, published strengths are much lower than the tested mean strengths; therefore, a conversion is needed to use the research data in professional practice.

Please note for creep test data (as presented in Table 5.3, source: Youssef, 2010) of various FRP rebar samples, a correction for fiber volume fraction (FVF), and thickness of samples (rebars diameter in this case) may also be required. Since we know that creep is higher in a resin-rich specimen (low FVF) as compared to a fiber-rich specimen (high FVF), the creep strain test data can be normalized with respect to FVF of a selected sample as a reference. The correction for thickness (or bar diameter) is not as straight forward due to shear lag (i.e., inversely parabolic of bar diameter) but can be applied after adjusting for the presence of ribs. For FVF correction, the initial sustained strain on a specimen will remain the same, but the subsequent increase in strain can be adjusted by the ratio of the specimen's FVF to the reference specimen's FVF. For example, if GFRP-2 rebar with the lowest FVF of $51 \%$ is chosen as the reference specimen, the measured creep strains (while keeping the elastic initial sustained strain as measured) in GFRP-4 rebar with the highest FVF of $75 \%$ can be adjusted by multiplying it by an FVF correction factor of 1.47 ( = $75 \% / 51 \%$ ). Such normalization will result in a creep database for an FVF of $51 \%$, which can be further calibrated for different FVFs. Another correction for rebar diameter and rebar surface types should also be applied to reflect typical rebar types in practice. For example, all tabulated values can be normalized to a typical $9.5 \mathrm{~mm}$ or $3 / 8$ " diameter sand-coated rebar with FVF of $60 \%$ as the primary reference.

Understanding that the purpose of this study is to illustrate the use of the time-dependent reliability-durability framework in predicting life-cycle performance for sustained stress in 
general, the above refinements are considered outside the scope of this study and normalization for FVFs or rebar diameters is not carried out. The results obtained under this study can be regarded as generic (covers a broad range of FVF from 51\% to 75\%, and rebar diameter from 9.5 $\mathrm{mm}$ to $15.9 \mathrm{~mm}$ ) and are used to illustrate the framework process only. The weighted FVF of data used in this study is $60 \%$, so the results presented here can be generally applied to FRP members with similar FVF. It must be noted that the author revisited the analysis of creep data by normalizing various FVFs to the lowest FVF of 51\% (GFRP-2 samples), but the final results did not change much. The scatter of creep coefficients $m$ and $n$ remained similar (since most of creep data is coming from GFRP samples $1 \& 2$, which has an average FVF of 55\%) and the change in the sustained strain limit recommendations were found to be insignificant. Although, adjustments for various rebar diameters or rebar surface types were not made, based on the results of FVF normalization, and it is reasonable to conclude that the results will not be much different. Due to the reasons explained, additional normalization of the Youssef (2010) database for FVF, rebar diameter, or rebar surfaces is not presented or used in this Chapter. However, the study keeps the options open for further refinements and customizations related to other FVFs, pultruded sections, thicknesses, surfaces, load types, and other applications in the future.

The following discussions are based on analyses of a vast creep test database developed at the University of Sherbrook (Youssef, 2010), and all figures and tables presented in this chapter are derived from that dataset. The author acknowledges that many more available creep test databases could have been used in this study to confirm/improve the results presented in this chapter. However, the purpose of this study is to illustrate the application of the proposed framework using an actual creep test database; the use of additional creep test data is considered outside the scope of current work. 
Table 5.4 shows about 8 test readings conducted at 0 hours (listed as 1 hour for ease in equation modeling options), then at 1000, 3000, and 10,000 hours. See Appendix D for the complete listing of the University of Sherbrook (Youssef, 2010) data points used in this study. Columns in gray color are listed for reference only and not used in this study. A typical bar designation such as GFRP1-30-2 will indicate that it is the second test of GFRP 1 bar with an approximate $30 \%$ of the initial sustained strain ratio. In this study, the actual initial sustained strain ratio (e.g., $31.10 \%$ ) computed with reference to the ultimate strain (listed in the original report) have been used. 
Table 5.4: Modified Creep Database of GFRP Samples

\begin{tabular}{|c|c|c|c|c|c|c|c|}
\hline \multicolumn{8}{|c|}{ Modified Data - Re-Tabulated \& Normalized Creep Test Data (Youssef, 2010) } \\
\hline & & & & $\varepsilon_{0}^{\prime}$ & $\varepsilon_{t}^{\prime}$ & $\varepsilon_{\mathrm{tu}}^{\prime}$ & $\varepsilon_{\mathrm{t} 0}^{\prime}$ \\
\hline & & $(\mathrm{t})$ & $\left(t^{\prime}\right)$ & $\left(\varepsilon_{0} / \varepsilon_{\mathrm{fu}}\right)$ & $\left(\Delta \varepsilon_{t} / \varepsilon_{0}\right)$ & $\left(\varepsilon_{\mathrm{t}} / \varepsilon_{\mathrm{fu}}\right)$ & $\left(\varepsilon_{\mathrm{t}} / \varepsilon_{0}\right)$ \\
\hline & Sample \# & thrs & t yrs & iSSR & CSSR & fSSR & $\mathrm{tSSR}$ \\
\hline $\begin{array}{c}\text { Test } \\
\text { Data } \\
\text { Set No. }\end{array}$ & $\begin{array}{l}\text { GFRP Rebar } \\
\text { Sample } \\
\text { Designation }\end{array}$ & \begin{tabular}{|l} 
Test Time \\
(hrs)
\end{tabular} & $\begin{array}{l}\text { Test Time } \\
\text { (Years) }\end{array}$ & $\begin{array}{c}\text { Initial } \\
\text { Sustained } \\
\text { Strain Ratio } \\
\text { (based on } \\
\text { Ultimate } \\
\text { Strain) }\end{array}$ & $\begin{array}{l}\% \text { Creep } \\
\text { Strain } \\
\text { Increase } \\
\text { Ratio }\end{array}$ & $\begin{array}{c}\text { Final } \\
\text { Sustained } \\
\text { Strain Ratio } \\
\text { (based on } \\
\text { Ultimate) }\end{array}$ & $\begin{array}{c}\text { Total } \\
\text { Sustained } \\
\text { Strain } \\
\text { Increase over } \\
100 \% \text { initial } \\
\text { (based on } \\
\text { Ultimate) }\end{array}$ \\
\hline 1 & GFRP1-15-1 & 1 & 0.0001 & $12.40 \%$ & $0.00 \%$ & $12.40 \%$ & $100.0 \%$ \\
\hline 1 & GFRP1-15-1 & 1000 & 0.1142 & $12.40 \%$ & $2.00 \%$ & $14.40 \%$ & $116.1 \%$ \\
\hline 1 & GFRP1-15-1 & 3000 & 0.3425 & $12.40 \%$ & $3.00 \%$ & $15.40 \%$ & $124.2 \%$ \\
\hline 1 & GFRP1-15-1 & 10000 & 1.1416 & $12.40 \%$ & $4.10 \%$ & $16.50 \%$ & $133.1 \%$ \\
\hline 2 & GFRP1-15-2 & 1 & 0.0001 & $12.40 \%$ & $0.00 \%$ & $12.40 \%$ & $100.0 \%$ \\
\hline 2 & GFRP1-15-2 & 1000 & 0.1142 & $12.40 \%$ & $1.20 \%$ & $13.60 \%$ & $109.7 \%$ \\
\hline 2 & GFRP1-15-2 & 3000 & 0.3425 & $12.40 \%$ & $4.50 \%$ & $16.90 \%$ & $136.3 \%$ \\
\hline 2 & GFRP1-15-2 & 10000 & 1.1416 & $12.40 \%$ & $7.80 \%$ & $20.20 \%$ & $162.9 \%$ \\
\hline 3 & GFRP1-15-3 & 1 & 0.0001 & $14.40 \%$ & $0.00 \%$ & $14.40 \%$ & $100.0 \%$ \\
\hline 3 & GFRP1-15-3 & 1000 & 0.1142 & $14.40 \%$ & $0.00 \%$ & $14.40 \%$ & $100.0 \%$ \\
\hline 3 & GFRP1-15-3 & 3000 & 0.3425 & $14.40 \%$ & $6.90 \%$ & $21.30 \%$ & $147.9 \%$ \\
\hline 3 & GFRP1-15-3 & 10000 & 1.1416 & $14.40 \%$ & $5.00 \%$ & $19.40 \%$ & $134.7 \%$ \\
\hline 4 & GFRP1-15-4 & 1 & 0.0001 & $12.60 \%$ & $0.00 \%$ & $12.60 \%$ & $100.0 \%$ \\
\hline 4 & GFRP1-15-4 & 1000 & 0.1142 & $12.60 \%$ & $0.30 \%$ & $12.90 \%$ & $102.4 \%$ \\
\hline 4 & GFRP1-15-4 & 3000 & 0.3425 & $12.60 \%$ & $3.30 \%$ & $15.90 \%$ & $126.2 \%$ \\
\hline 4 & GFRP1-15-4 & 10000 & 1.1416 & $12.60 \%$ & $7.20 \%$ & $19.80 \%$ & $157.1 \%$ \\
\hline 5 & GFRP1-15-5 & 1 & 0.0001 & $14.80 \%$ & $0.00 \%$ & $14.80 \%$ & $100.0 \%$ \\
\hline 5 & GFRP1-15-5 & 1000 & 0.1142 & $14.80 \%$ & $5.70 \%$ & $20.50 \%$ & $138.5 \%$ \\
\hline 5 & GFRP1-15-5 & 3000 & 0.3425 & $14.80 \%$ & $5.40 \%$ & $20.20 \%$ & $136.5 \%$ \\
\hline 5 & GFRP1-15-5 & 10000 & 1.1416 & $14.80 \%$ & $8.80 \%$ & $23.60 \%$ & $159.5 \%$ \\
\hline 6 & GFRP1-30-1 & 1 & 0.0001 & $20.90 \%$ & $0.00 \%$ & $20.90 \%$ & $100.0 \%$ \\
\hline 6 & GFRP1-30-1 & 1000 & 0.1142 & $20.90 \%$ & $-0.20 \%$ & $20.70 \%$ & $99.0 \%$ \\
\hline 6 & GFRP1-30-1 & 3000 & 0.3425 & $20.90 \%$ & $1.30 \%$ & $22.20 \%$ & $106.2 \%$ \\
\hline 6 & GFRP1-30-1 & 10000 & 1.1416 & $20.90 \%$ & $1.20 \%$ & $22.10 \%$ & $105.7 \%$ \\
\hline 7 & GFRP1-30-2 & 1 & 0.0001 & $31.10 \%$ & $0.00 \%$ & $31.10 \%$ & $100.0 \%$ \\
\hline 7 & GFRP1-30-2 & 1000 & 0.1142 & $31.10 \%$ & $-2.90 \%$ & $28.20 \%$ & $90.7 \%$ \\
\hline 7 & GFRP1-30-2 & 3000 & 0.3425 & $31.10 \%$ & $-2.30 \%$ & $28.80 \%$ & $92.6 \%$ \\
\hline 7 & GFRP1-30-2 & 10000 & 1.1416 & $31.10 \%$ & $-2.90 \%$ & $28.20 \%$ & $90.7 \%$ \\
\hline
\end{tabular}

Data Source: University of Sherbrook (Youssef, 2010).

Single sustained stress test data set, at $1,1000,3000$, and 10,000 hours 


\subsubsection{Development of Basic Creep Strain Coefficients}

Once the data (Youssef, 2010) was normalized, deterioration equation for creep strain value (i.e., an increase in strain over the initial strain) was developed using a Findley's power law, as shown in Section 5.4.1. Findley's equation uses two experimentally determined coefficients: “n” and "m." In this study, the coefficient " $m$ " was normalized by dividing " $m$ " by the initial strain ratio. The coefficients " $\mathrm{m}$ " and " $\mathrm{n}$ " are illustrated in Figures 5.4 and 5.5 for a single creep test (for specimen GFRP-1, a 9.5 mm diameter, sand-coated rebar with 57\% FVF).

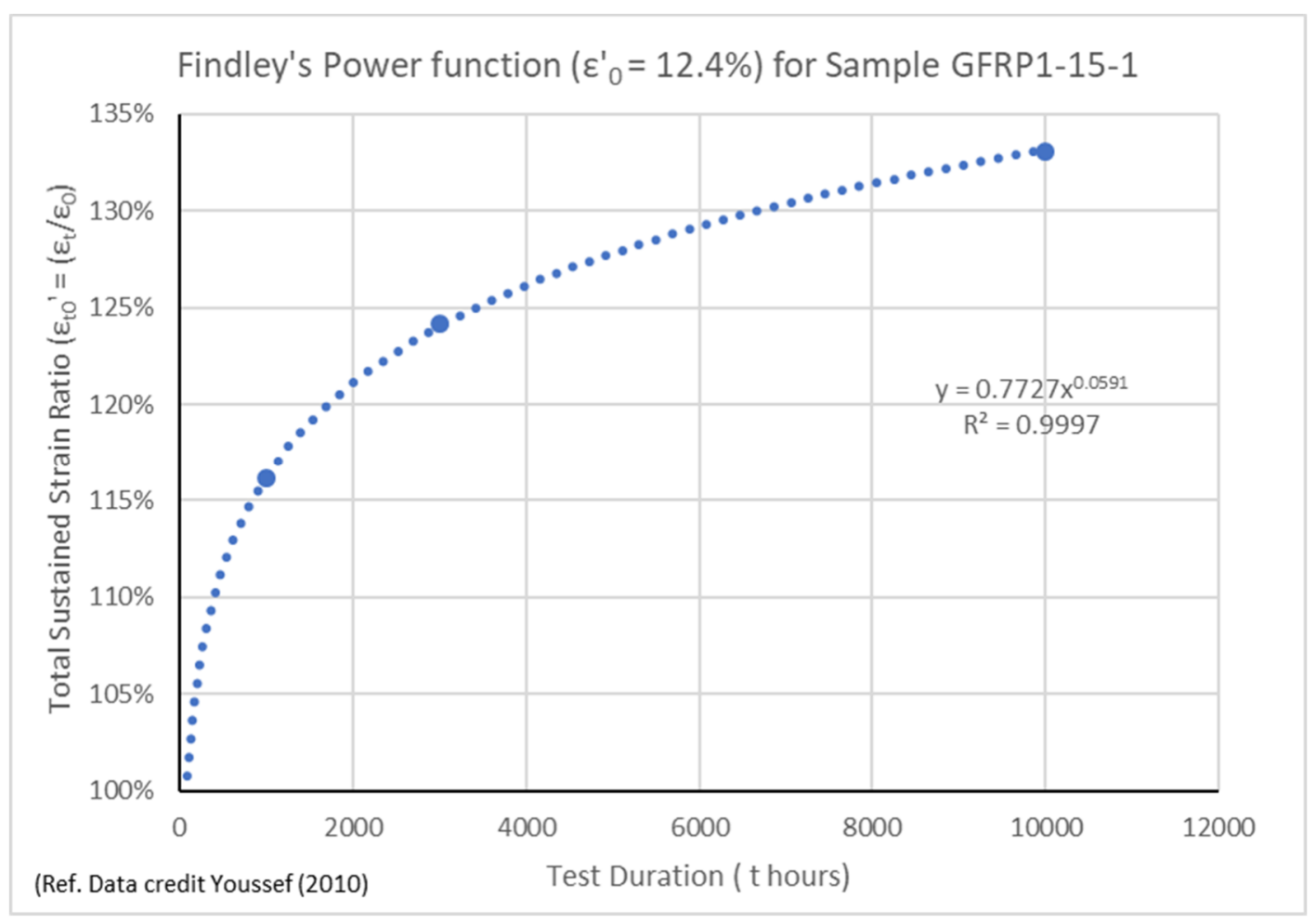

Figure 5.4: Modelling of Experimental Data using Findley's Law 
Findley's Power Function (Ln transformation) for GFRP1-15-1

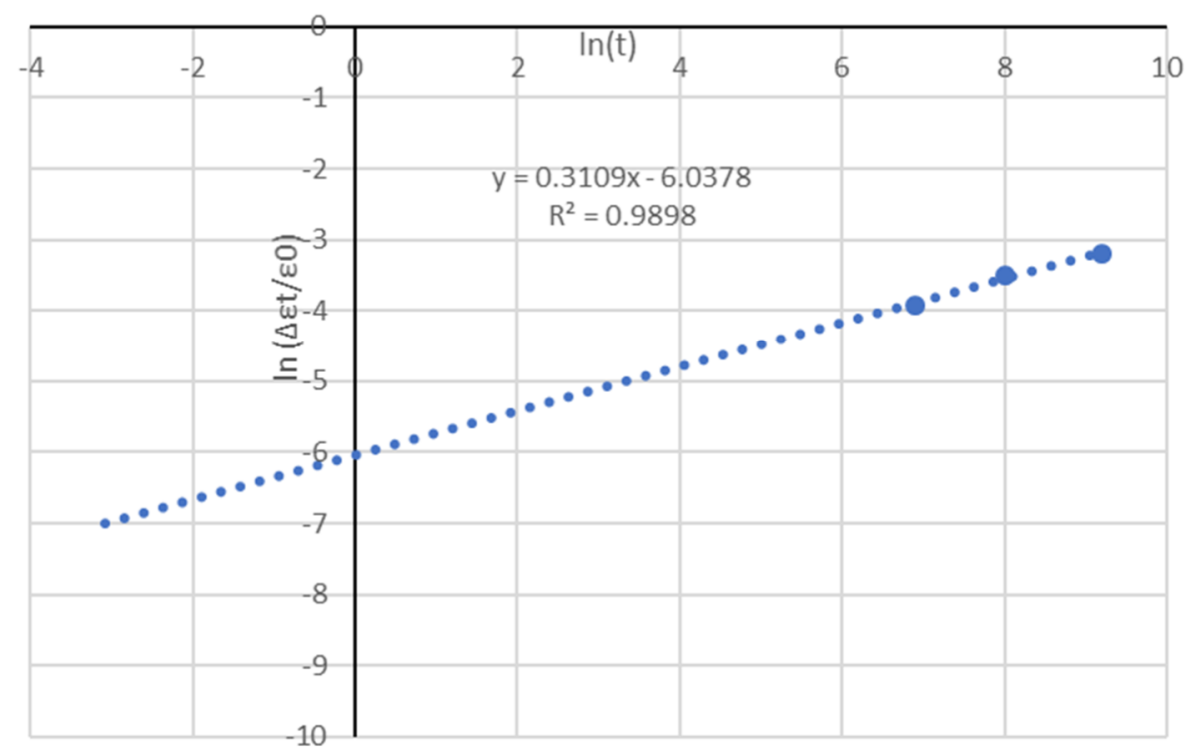

(Ref. Data credit Youssef (2010)

Figure 5.5: Logarithmic Transformation of Findley's Law

Typical data management software (including Excel) allows the regression for two or three-parameter equations (linear, power, logarithmic, polynomial, etc.), and care must be taken when the total number of datapoints is same or less than the number of parameters to be regressed. Figure 5.4 shows the basic power law for an initial sustained strain ratio of $12.4 \%$, which shows how the total sustained strain ratio (with respect to initial sustained strain ratio) increases with time $\mathrm{t}$ in the lab for a specimen (GFRP-1). 100\% strain is the initial strain at the start of the test. It should be noted that Figures 5.4 and 5.5 show only a set of creep test readings and are included only to illustrate Findley's power law application to creep test data. Creep constants "m" and " $n$ " as interpreted from these figures are specific to this particular test reading only.

Figure 5.5 helps in understanding the meaning of coefficients " $\mathrm{m}$ " as the transformed straight-line intercept and slope "n," as defined in Equation 5.4. In Figure 5.5, the x-axis is the 
natural $\log$ of $\mathrm{t}$ (test hour), and the $\mathrm{y}$-axis is the natural $\log$ of $\mathrm{cSSR}$ or creep sustained strain ratio defined with respect to the initial sustained strain ratio. Normalized strain ratios are defined either with respect to ultimate strain value or initial sustained strain.

The use of a transformed logarithmic straight-line equation is essential since it allows the use of linear regression techniques for estimating upper and lower bounds using frequently used confidence intervals or significant levels. The selection of confidence intervals is explained in section 5.4.6.

A direct comparison of regressed values of " $m$ " and " $n$ " from Youssef data (2010) with previous creep studies conducted at WVU-CFC (Batra, 2009; GangaRao \& Liang, 2010) was not possible due to a very wide scatter of values obtained at lower initial sustained strain ratios. The reasons for the wide scatter of computed/regressed coefficient values of Youssef data (2010) are not known but by inspection can be attributed to: i) coefficients are regressed for a wide variety of rebars consisting of varying FVFs, diameters, and coatings, ii) creep tests were conducted for different initial sustained strains, and most were not carried out to the final rupture, iii) there are only 5 creep rupture tests with missing intermediate readings to allow the regression of unique Findley's coefficients, and iv) possible fluctuation of temperatures or the environment in the lab (not recorded, unknown). The author attempted to normalize the test data to a single reference (i.e., 51\% FVF of GFRP-2 rebar, $9.5 \mathrm{~mm}$ diameter, and sand-coated surface), the scatter got slightly smaller, but no clear pattern emerged. The scatter (of "m," "n," or computed service life) is typically broader for lower initial sustained strain ratios and somewhat narrower for higher ratios. It can be interpreted that at lower sustained strains, the regression of creep coefficients can be very sensitive to the experimental values, which can result in higher uncertainties in the predictions of the final strains (concerning any projected time outside the test duration). 
For each complete sustained stress test with three or more data points, a power curve can be regressed to find degradation coefficients "m" and "n." Since each test is different, a broad set of " $m$ " and " $n$ " coefficients will be found. As mentioned before, unlike previous studies, Youssef's (2010) data yielded a very large scatter of " $m$ " and " $n$ " coefficients, which rendered the use of mean values to be of minimal benefits in computing service life estimates. Findley's dependence on these two "coupled" parameters creates some complexities in the direct application of these coefficients in a reliability-based approach, and therefore, a slightly modified method is proposed in this report.

Findley's power equation fits the time-dependent nature of this study and confirms the test results within reasonable accuracy. The degradation coefficients m' and $\mathrm{n}$ are computed to produce the best fit for each set of the reading (sample or spot values). Figures 5.6 and 5.7 are only provided to illustrate the basic concept of Findley's power law and to show how large the data scatter is. For reliability analyses, we will not be using the mean value of $m$ and $n$ coefficients individually (they are used in pair to produce time to failure using Equation 5.6), so there is no need to improve the goodness of fit $\left(\mathrm{R}^{2}\right)$ for " $\mathrm{m}$ " or " $\mathrm{n}$ " by eliminating the local outliers. The outliers are only removed when fitting the modified Fiddley's power law Equation 5.5 to the experimental data and then confidence intervals used for Equation 5.6. Grouping the specimen by initial strain ratios $(15 \%, 30 \%$, etc.) to arrive at mean coefficients did not yield good results, and the regression of mean values of all valid coefficients $m$ and $n$ (as shown in Figures 5.6 and 5.7) did not provide reasonable results. As also evident from Figures 5.6 and 5.7, a direct correlation between the regressed values of coefficients " $m$ " and " $n$ " and the initial sustained strain ratios - purely based on this creep test results - is difficult to find. This lack of correlation holds even for a single rebar type (GRRP-1 or GFRP-2) when no corrections are needed for FVF, rebar diameter, or surface 
type. In short, a deterministic solution for computing creep life using mean regressed values of " $m$ " and " $n$ " coefficients is not possible, at least from this database. In the end, the only viable option available found was to directly regress the "time to failure" to arrive at reliability-based recommendations.

The original database was reduced from its 286 strain observations to 226 observations by removing inconsistent data based on the following techniques:

- Individual sample sets with negative slopes (coefficient " $n$ ") and very flat slopes were considered anomalies and were removed. Such readings will yield infinite (or near-infinite) lives under sustained loads and will skew the results for time to failure.

- If any sequential strain readings $\left(\varepsilon^{\prime}\right.$ to $)$ of a creep data set is found to be lower than the previous readings (e.g., a set of readings of $100 \%, 98.6 \%, 99.7 \%$, and $103 \%$ for $0,1000,3000$, and 10,000 hours), those readings (e.g., $98.6 \%$ and $99.7 \%$ in the above set) were removed. However, if more than two readings out of 4 readings were removed (e.g., as will be the case with this data set), the entire data set was eliminated. The reasons are i) negative creep strain is not realistic, and ii) the regression of two coefficients of power-law requires at least three lab readings. However, this has an exception, as explained in the next bullet.

- When there were only two strain readings taken, as were the case with six sets of data leading to rupture, the value of " $m$ " was fixed, and only " $n$ " was regressed. The value of $m$ from a nearby data set of similar strain ratio was used in those cases. In any case, rupture data sets had minimal impacts on the proposed methodology except to help in deciding what creep rupture strain value to use in computing the time to failure. 
Table 5.5 shows the results of 5 rebar test samples that reached creep rupture. Due to limited test readings, it is difficult to predict the final shape of the creep-rupture curve or when the transition from Stage II or Stage III occurred. The final sustained strain ratio $\left(\varepsilon^{\prime}\right.$ tu), as shown in Table 5.5, is the rupture strain ratio $\left(\varepsilon_{\mathrm{r}}^{\prime}\right)$. Since both the ultimate strain $\left(\varepsilon_{\mathrm{fu}}\right)$ and rupture strain $\left(\varepsilon_{\mathrm{r}}\right)$ are typically estimated at $90 \%$ to $95 \%$ of ultimate static failure values, no correction to rupture strain ratio $\left(\varepsilon_{r}^{\prime}\right)$ is needed for all practical purposes. Figure 5.6 graphically depicts the strain vs. time curve where the y-axis starts at $100 \%$ strain, which is also the normalized initial strain ratio $\left(\varepsilon^{\prime} 0\right)$ for all samples. The tested ultimate strain $\left(\varepsilon_{f u}\right)$ for specimen GFRP1 was $18,232 \mu \varepsilon$, and specimen GFRP2 was 18,484 $\mu \varepsilon$ (as also shown in Table 5.3, source: Youssef, 2010).

Table 5.5: Creep Rupture Test Results of GFRP Rebar Samples

\begin{tabular}{|c|c|c|c|c|c|}
\hline \multicolumn{6}{|c|}{ Creep Rupture Test Data (Source: Youssef, 2010) } \\
\hline \multirow[b]{3}{*}{ Sample \# } & \multirow[b]{3}{*}{$(t)$} & $\varepsilon_{0}^{\prime}$ & $\varepsilon_{\mathrm{t}}^{\prime}$ & $\varepsilon^{\prime}{ }_{\text {tu }}$ & $\varepsilon_{\text {t0 }}^{\prime}$ \\
\hline & & $\left(\varepsilon_{0} / \varepsilon_{\mathrm{fu}}\right)$ & $\left(\Delta \varepsilon_{t} / \varepsilon_{0}\right)$ & $\left(\varepsilon_{\mathrm{t}} / \varepsilon_{\mathrm{fu}}\right)$ & $\left(\varepsilon_{\mathrm{t}} / \varepsilon_{0}\right)$ \\
\hline & & iSSR & cSSR & fSSR & tSSR \\
\hline $\begin{array}{l}\text { GFRP Rebar } \\
\text { Sample } \\
\text { Designation }\end{array}$ & $\begin{array}{l}\text { Test } \\
\text { Time } \\
\text { (hrs) }\end{array}$ & $\begin{array}{c}\text { Initial Sustained } \\
\text { Strain Ratio (based } \\
\text { on Ultimate Strain) }\end{array}$ & $\begin{array}{l}\text { \% Creep Strain } \\
\text { Increase Ratio }\end{array}$ & $\begin{array}{c}\text { Final Sustained } \\
\text { Strain Ratio } \\
\text { (based on } \\
\text { Ultimate) } \\
\end{array}$ & $\begin{array}{c}\text { Total Sustained } \\
\text { Strain Increase } \\
\text { over } 100 \% \\
\text { initial (based } \\
\text { on Ultimate) } \\
\end{array}$ \\
\hline GFRP2-60-4 & 1 & $58.60 \%$ & $0.00 \%$ & $58.60 \%$ & $100.0 \%$ \\
\hline GFRP2-60-4 & 54 & $58.60 \%$ & $6.40 \%$ & $65.00 \%$ & $110.9 \%$ \\
\hline GFRP2-60-2 & 1 & $62.30 \%$ & $0.00 \%$ & $62.30 \%$ & $100.0 \%$ \\
\hline GFRP2-60-2 & 56.8 & $62.30 \%$ & $1.30 \%$ & $63.60 \%$ & $102.1 \%$ \\
\hline GFRP1-60-3 & 1 & $63.40 \%$ & $0.00 \%$ & $63.40 \%$ & $100.0 \%$ \\
\hline GFRP1-60-3 & 1000 & $63.40 \%$ & $3.40 \%$ & $66.80 \%$ & $105.4 \%$ \\
\hline GFRP1-60-3 & 2964 & $63.40 \%$ & $4.30 \%$ & $67.70 \%$ & $106.8 \%$ \\
\hline GFRP2-60-3 & 1 & $75.70 \%$ & $0.00 \%$ & $75.70 \%$ & $100.0 \%$ \\
\hline GFRP2-60-3 & 231 & $75.70 \%$ & $10.60 \%$ & $86.30 \%$ & $114.0 \%$ \\
\hline GFRP2-60-1 & 1 & $80.90 \%$ & $0.00 \%$ & $80.90 \%$ & $100.0 \%$ \\
\hline GFRP2-60-1 & 13.8 & $80.90 \%$ & $2.20 \%$ & $83.10 \%$ & $102.7 \%$ \\
\hline
\end{tabular}




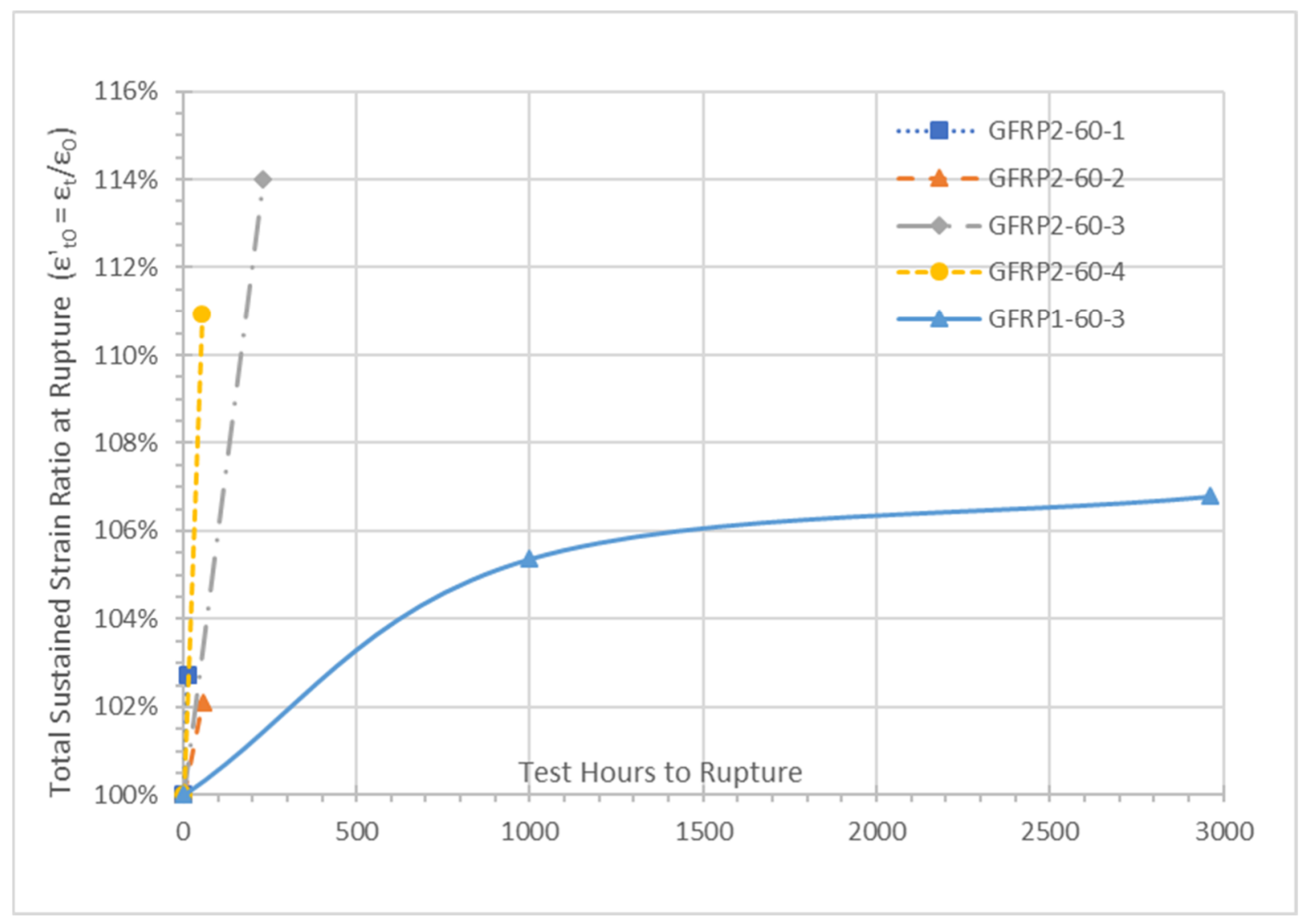

Figure 5.6: Total Sustained Strain Ratio (wrt $\left.\varepsilon_{0}\right)$ leading to Creep Rupture

In normalization process, initial sustained strain $\left(\varepsilon_{0}\right)$, final sustained strain $\left(\varepsilon_{\mathrm{tu}}\right)$, and rupture failure strain $\left(\varepsilon_{\mathrm{r}}\right)$ are expressed as a percentage of ultimate static strain $\varepsilon_{\mathrm{fu}}$ (e.g., initial sustained strain ratio $\varepsilon^{\prime}$, final sustained strain ratio $\left(\varepsilon_{\text {'tu }}^{\prime}\right)$, and rupture strain ratio $\left.\varepsilon_{r}^{\prime}\right)$. Creep strain ratio $\left(\varepsilon^{\prime} t\right)$, and total sustained strain ratio $\left(\varepsilon^{\prime}{ }^{\prime} 0\right)$ are expressed as a percentage of initial sustained strain $\left(\varepsilon_{0}\right)$. The time $\mathrm{t}$ for initial sustained strain $\left(\varepsilon_{0}\right)$ is assumed to be 0 hours, and the creep begins after time zero, i.e., soon after the static load begins to exert its influence on the fiber/resin architecture of the test specimen.

Once the entire sustained database was cleaned up of anomalies and outliers (as described in this section), sample coefficients $\mathrm{m}^{\prime}\left(=\mathrm{m} / \varepsilon_{0}\right)$ and n were regressed for each rebar testing, and specific rupture times $\left(t_{r}\right)$ for each sample was computed using Equation 5.6. A wide scatter in the 
computed rupture age for various strain ratios (Figure 5.7) was observed, and test data initially seems random. An analogy will be akin to regressing a compression strength test data of 1000 concrete cylinders at different curing times (e.g., one day, 14 days, 28 days, and three months) but samples were prepared at various concrete mix plants located in different states using their state standard specifications for highway bridge decks. It is possible to reduce the scatter of $\mathrm{m}$ and $\mathrm{n}$ coefficients should the data be normalized for FVF and/or bar diameter. However, a large spread (scatter) can still be found within a single bar creep test data (GFRP-1 or GFRP-2), indicating a need for a different approach that does not rely solely on the regression of creep coefficients.

A closer examination of FRP rebar data revealed that a mean value of $t_{f}$ (time to failure) can be regressed using test-derived Findley's power law coefficients ( $m$ and $n$ ) and results plotted on a logarithmic timescale. An appropriate age for which a vast majority of samples are not likely to fail can be selected for a given initial strain ratio and vice versa by using an appropriate confidence interval (or significance level). In this case, we want to focus on the lower bound estimation of the age (which is appropriate for practical ranges of sustained stress in the design practice) so that we can safely pick the maximum initial sustained stress that we want to limit our design value to obtain the desired service life.

Since we are introducing a reliability concept for creep life estimation, additional discussion is necessary. First, we are not doing a deterministic determination of sustained stress service life, creep coefficients, or validating a creep theory. Instead, we already know that the FRP sustained stress data is somewhat random, and it is difficult to make a generic model; therefore, we are taking a probabilistic (reliability) approach to find a reasonably safe estimate for the service life for a given initial stress. To explain the concept better, let us revisit the same concrete cylinder testing analogy, as was discussed in the previous paragraph. Assume that the Federal Highway 
Administration (FHWA) wants to know what should be a reasonable minimum bridge deck concrete strength at 14 days (to remove falsework) and 28 days (to open bridge deck the traffic) in mid-western states. FHWA asks State Departments of Transportation (DOTs) to send their actual concrete compression test results from bridge deck pour projects completed in the last year. The snapshot of the database received shows about $1000+$ results of concrete compression test value (f'c) taken at one day to 3 months range. There is no other information available. It will not be prudent to pick the minimum $\mathrm{f}_{\mathrm{c}}$ value at 14 and 28 days or take the average $\mathrm{f}_{\mathrm{c}}{ }_{\mathrm{c}}$ value. There are also a few cylinders with very low strengths, and there seems no good pattern to model strength increase from 14 days to 28 days. To solve this dilemma, FHWA regresses the mean and lower / upper bounds of $f_{c}$ and uses a standard $95 \%$ confidence interval. In the end, FHWA picks the lower bound value for 14 days and 28 days to make their final recommendations. FHWA assumed that even though there were a few strengths that were well below the recommended amount, the statistical probability of actually having those low value is so small that those values are almost insignificant. In our case for FRP rebars, picking the "time to failure" (derived based 282 test readings on actual creep test of 74 samples) is very similar to finding a reasonable minimum value for f'c based on the data.

In Figure 5.7, each data point represents a computed age (shown in years, along the y-axis) to reach a target creep rupture strain of $60 \%$ (with respect to ultimate strain) for a given initial sustained stress/strain ratio (shown as a percentage of ultimate strain, along the x-axis). Three solid lines are statistically computed upper, average, and lower confidence limits based on all data points computed for this particular sustained strain/creep database (Youssef, 2010) with various key parameters listed in Table 5.2. The data points (of creep-rupture tests) and lines below 1-year life are shown for completeness of the regressed data only and have no practical significance. 
Life Expectancy (years) under Sustained Load

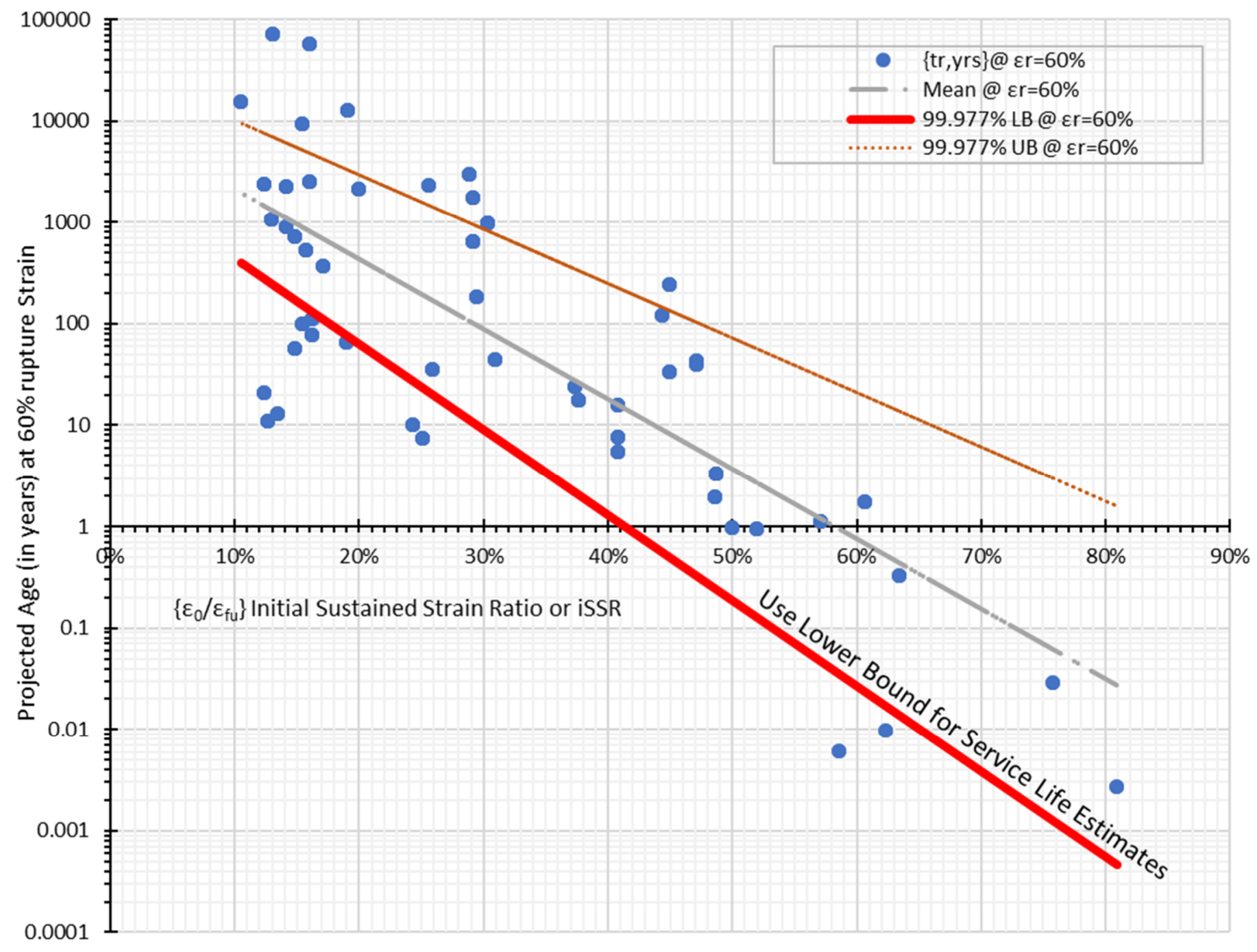

Oriiginal Data Source: Youssef (2010)

Figure 5.7: Determination of Service Life for Various Initial Strain Ratios 


\subsubsection{Estimation of Lower Bound Age}

The residual of age differential (i.e., the difference between each test-based projected age and the computed mean regressed age) calculated at $60 \%$ rupture strain was analyzed for probability distribution type. This distribution will indicate how the test-based age points vary from the mean value equation of all data points (a measure of central tendency). In general, the distribution was found to be skewed towards higher values (very high projected age based on all test results). A three-parameter Gamma or three-parameter Lognormal distribution was found to be a relatively reasonable fit. The solution for Weibull distribution did not converge, and normal distribution did not fit well either (due to the elimination of several data points yielding unrealistic estimates during the normalization process). Since the distribution is skewed towards the higher values, and we are interested in a lower bound value, the use of typical confidence interval provided adequate results, which could be considered to be on the conservative side.

Figure 5.8 (original data source: Youssef, 2010) shows an actual single specimen test data extrapolated using Findley's power law coefficients to estimate the service life for a given initial strain ratio. In this case, we are assuming that when the sample's final creep strain reaches a preselected rupture strain ( $60 \%$ of ultimate strain in this case), its service life ends (which will be 24 years for this sample). It should be noted that Figure 5.8 is just one datapoint computed for time to failure, and we would do the same for all samples using their unique regressed " $m$ " and " $n$ " coefficients. The 24-year is not the representative time to failure for an FRP rebar at $37.3 \%$ initial strain, but just an illustration. It also shows that the projected age beyond the lab test duration can be sensitive to regressed creep coefficients $m$ and $n$. Once all data points for time to failure have been computed, the next step is to find a reasonably safe estimate for time to failure for a given initial strain ratio and rupture strain. 


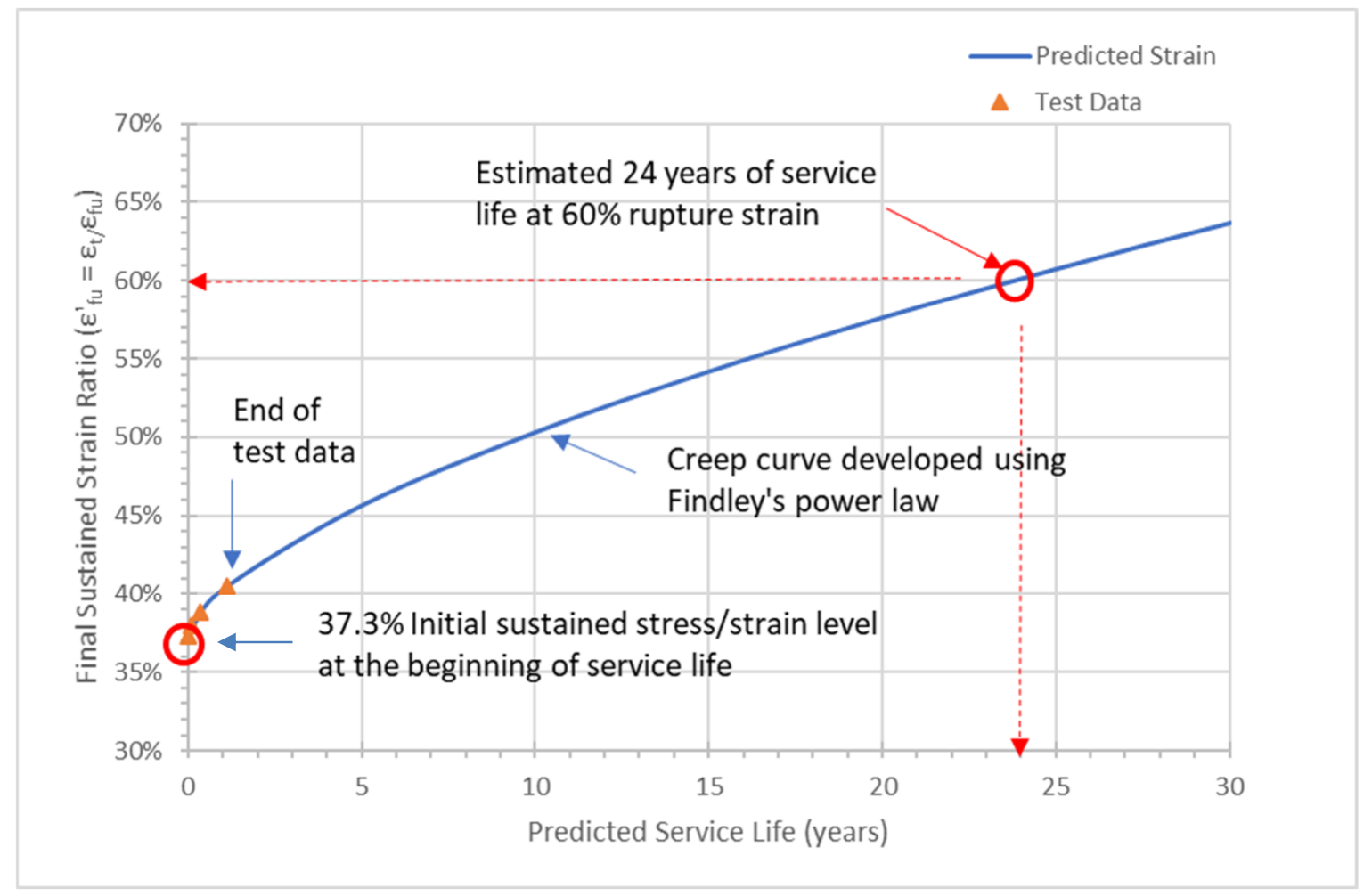

Figure 5.8: Computation of Service life for an FRP Sample

A confidence interval of $99.977 \%$ (or a significance level of $0.023 \%$ ) was selected to compute the lower bound threshold of the linear regression equation. This interval closely relates to the probability of exceedance (i.e., the chance that actual sustained life will be less than the computed lower bound age) to 1 in 4,300 which is very similar to the value provided by $\mathrm{z}$ value of 3.5 (i.e.: $\mu-3.5 \sigma$ ) and produced by the reliability index of 3.5 .

This lower bound value for time to failure can be explained as a predicted age value that will include $99.977 \%$ of all possible actual age values (for a given initial strain ratio) or only $0.023 \%$ age (time to failure) will be less than the predicted value. Figure 5.9 graphically explains the above parameters for a normal distribution. For a skewed distribution such as Gamma or Lognormal, the lower bound estimates will also be conservative. It should be noted that upper and lower bound linear regression parameters (i.e., intercepts and slope - both are obtained by 
transforming Findley's power law to a logarithmic straight-line function) for the confidence interval of $99.977 \%$ were computed by a commercially available regression (Excel's regressions add-on). For creep deformation (e.g., deflections or elongation) estimates, the average final strain is used, which is also calculated at a $50 \%$ confidence interval.

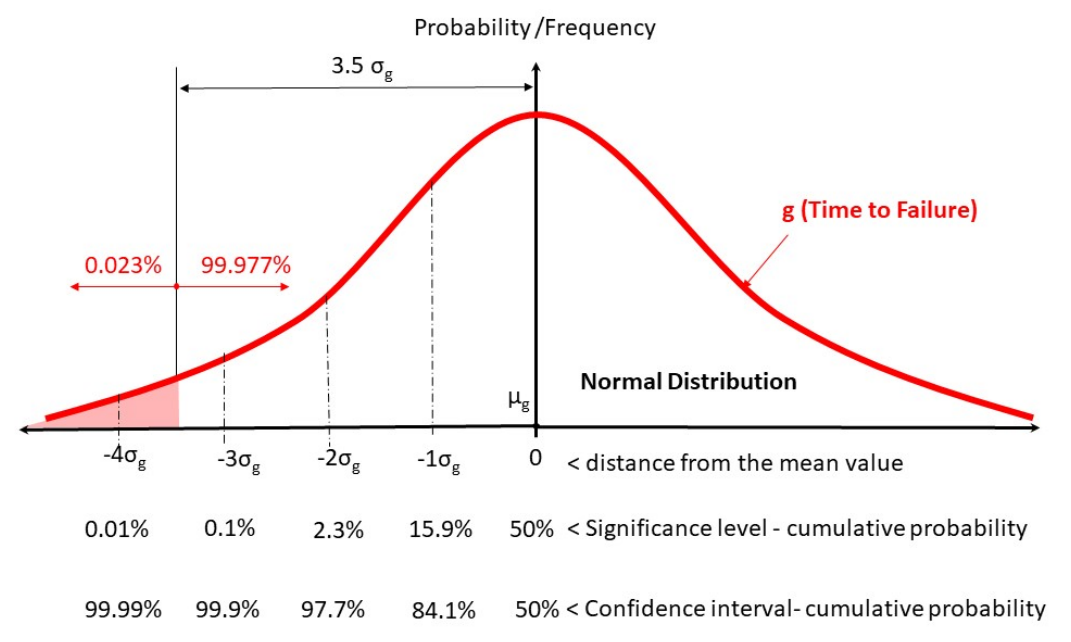

Figure 5.9: Explanation of the Probability of Exceedance

\subsubsection{Determination of Rupture Strain}

One of the primary challenges in long term projections is how to estimate the life-span of the samples when most tests conducted were limited to a maximum of 10,000 hours (417 days or little over a year). Physical effects do not adapt well to the Arrhenius principle or other similar superimposition techniques (as often used in accelerated testing to forecast future timeline), so the projection of time to failure from a set of initial strain, final strain, and lab test durations alone is not straightforward. The use of a single parameter logarithmic degradation equation becomes very complicated and requires a time-step function to model the changes in degradation rate as the sustained stress increases. Strain energy-based methods (Batra, 2009; GangaRao and Liang, 2010) 
work well but require meticulous scrutiny of the test data. Findley's power law method produces a direct estimation of predicted age, but it requires experimental determination for rupture failure strain with adjustments for materials aging under harsh environment and sets of reliable long-term creep test data.

The primary reasoning here is that there is no minimum threshold in FRP composites for sustained strain or fatigue cycles. The specimen will continue to degrade until failure, perhaps at a slower rate for lower sustained strains when compared with higher sustained strains when close to the failure (Stage III). Using Findley law, the final strain in a sample can be computed to a critical strain level (e.g., 30\% initial strain will reach a $70 \%$ final strain at 75 years) where creep rupture becomes a real possibility. The University of Sherbrook data has five tests that produce creep ruptures and provide a valuable resource to predict possible life-span thresholds (e.g., all samples at $60 \%, 70 \%$, and $80 \%$ initial sustained stress ratios failed). Other reports and tests show a wide range of creep rupture limits even in the same material, and therefore, creep rupture ratio is not a fixed number and is not typically found in published data from the FRP manufacturers (as is the case for ultimate stress, strain, and Young's moduli values). In lieu of testing for actual creep rupture limits in the laboratory, a proper estimate of creep rupture limit (also described as strength) is essential since it can significantly impact the estimation of service life, as also shown in Figure 5.10 (original data source: Youssef, 2010). For example, if the creep rupture strain $\left(\varepsilon_{\mathrm{r}}\right)$ ratio (with reference to ultimate strength) is assumed to be $80 \%$, the sustained stress ratio should be limited to $23 \%$ (with reference to ultimate strength) to reach a target 100-year life. Also, assuming a $60 \%$ rupture strain ratio, the sustained stress ratio should be limited to $18 \%$ to obtain the same target service life, and vice versa (Figure 5.10). 


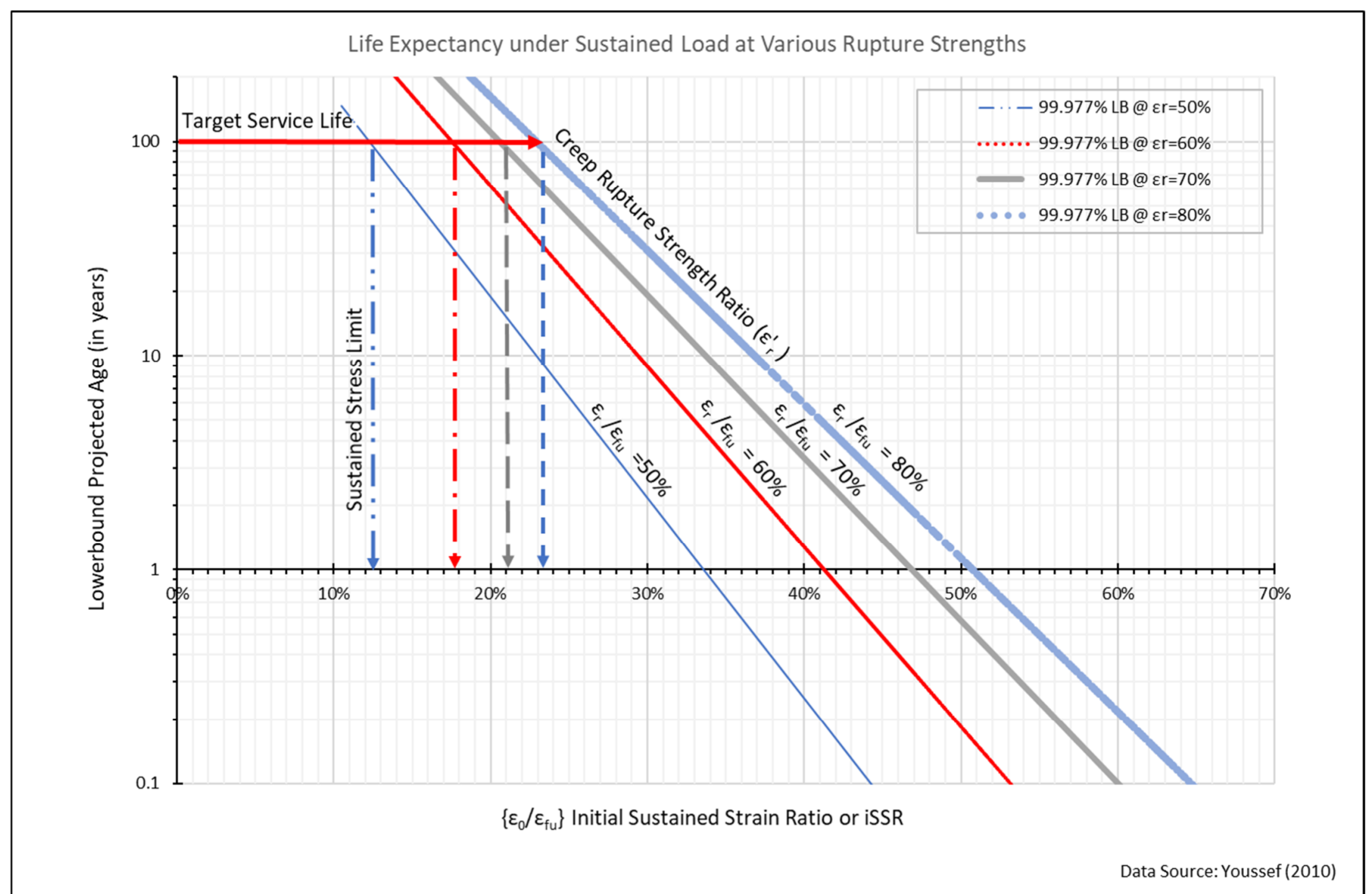

Note: Creep life is computed at a lower bound confidence limit of $99.977 \%$ (or at $3.5 \sigma$ from the mean) for various assumed creep rupture ( $\varepsilon_{r}$ ) ratios. The above figure is based on the reliability analysis of a long-term sustained stress experiment carried out by Youssef (2010) for vinylester-based GFRP rebars of fiber volume fractions ranging from $51 \%$ to $75 \%$ and rebar diameters from $9.5 \mathrm{~mm}$ to $15.9 \mathrm{~mm}$ with different surface treatments.

Figure 5.10: Lower Bound Prediction of Service Life at Various Rupture Strengths 
A deterministic estimation of creep rupture life is theoretically possible using a deterministic estimate of $\mathrm{m} \& \mathrm{n}$ coefficients or strain energy method. However, this study intends to estimate the creep rupture life with an acceptable probability of failure when an FRP is subjected to a certain amount of initial sustained strain. The proposed reliability-durability framework indicates that a safe estimation of design creep life is possible from Findley's creep coefficients (as $m$ and $n$-a pair of variables derived from creep test data) and final creep rupture strength/strain limit (as an estimation or derived from creep rupture tests).

In the University of Sherbrook's study, the lowest value of rupture strain (expressed as $\left.\varepsilon_{\mathrm{r}}\right) / \varepsilon_{\mathrm{u}}$ ) was $64 \%$, the highest value was $86 \%$, and the average value was $73 \%$. A range of life-spans can be computed using various thresholds, and a conservative value (say between 60 to $70 \%$ unless otherwise supported by more test data) can be chosen for life-span. The use of $70 \%$ seems to produce similar results as used in current practice and is recommended in lieu of a large-scale test study to determine a more reliable creep rupture value. Figure 5.10 can be considered to provide a conservative (i.e., lower) estimate of creep life when compared to current codes.

\subsubsection{Accounting for Creep Deformation}

Life-span prediction computed for an assumed (or tested) creep rupture strain must also be checked for serviceability so that a member can serve its intended function over its service life, such as limiting excess deflection. Many studies have indicated that modulus of elasticity does not deteriorate over time due to the environment or after the removal of a sustained load in the lab. However, in real life, the member will have to perform its design function, carrying some sustained loads until the member's retirement from the service or its premature failure. Based on experimental data, several studies have suggested a wide variety of methods to account for creep deformations in FRP reinforced concrete beams; however, more studies are needed to estimate the 
long-term deformation of pultruded FRP sections accurately. In lieu of detailed studies or sophisticated methods, a good starting point can be a simple method that accounts for the increase in sustained strain with time (service life) by adjusting the Modulus of Elasticity. The readers are cautioned that the simple method proposed in this section is based on the reliability analysis of a single FRP rebar database (Youssef, 2010) and does not replace more suitable deflection calculation methods should more relevant data become available.

Estimated deformation from sustained loads are often used for fabrication tolerances (e.g., built-in camber to obtain the vertical profile within a year of placement), serviceability (e.g., drainage of flat roof or bridges), the visual appearance of safe structure (e.g., the plumpness of walls), and many other non-critical functions. Unlike sustained stresses, the deformation estimation needs to be in an "average" domain. A very rough estimation of deformation near the end of life can be made from the average estimated final strains using the Findley's power-law relationship developed in this research. The author admits that there is a need for more research in this long-term deformation field for pultruded FRP sections. However, in lieu of deterministic and experimental data on the long-term deflection of standalone FRP sections (excluding reinforced concrete sections), the following sections present a probabilistic approach to computing the approximate final strains near the end of member's service life, which in turn can be used to calculate a preliminary estimate of creep induced inelastic deflections (based on the data analysis conducted in this chapter). No attempt is made here to provide broader recommendations about long-term deflection computation methods; however, the author strongly feels that the issue of long-term deflections of FRP members subjected to sustained loads must be addressed during its design phase. 
Figure 5.11 (original data source: Youssef, 2010) shows the estimated increase in final strain for the commonly designed initial sustained stress ratios shown for various values of design service lives. The probabilistic method used is described in the following paragraphs.

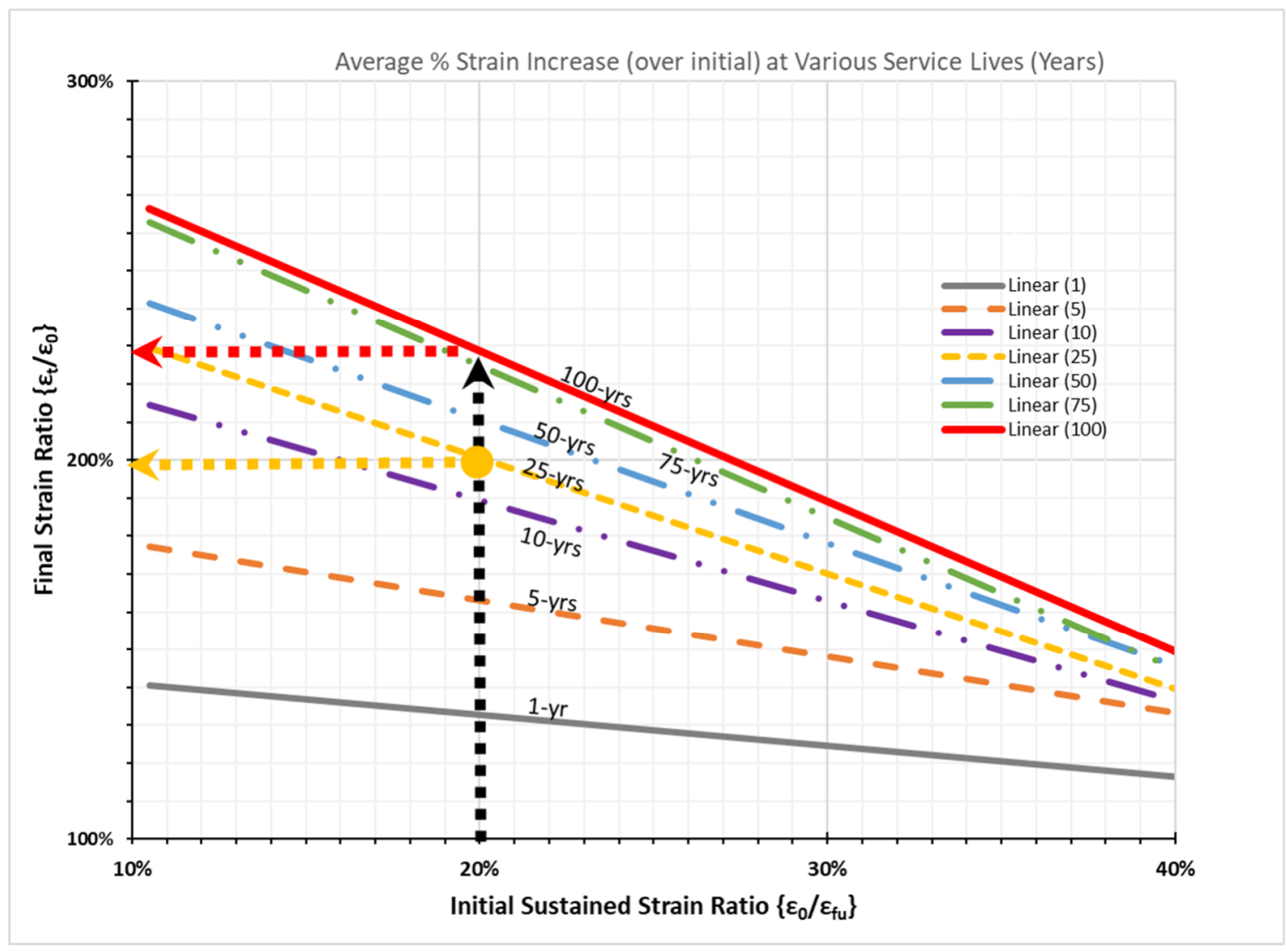

Figure 5.11: Average \% Strain Increase at Various Service Lives (Years).

Since we do not have a mean value of Findley's coefficients (m or $n$ ) to compute the final strain from initial strain, we will first calculate the final strains for each set of test data (based on regressed local coefficients) and plot them at various design timelines $(1,5,10,25,50,75$, and 100 years). Since there is a large scatter of projected final strain values, only a simple mean value line (linear trend lines) for each service life is shown in Figure 5.11. All final strain increases are computed with respect to the initial strain (taken as 100\%) and do not require the knowledge of creep rupture strain. For example, for a $20 \%$ initial sustained strain, on average, one can expect 
the final strains to be double the initial strain (i.e., $40 \%$ of ultimate strain from its original sustained strain of $20 \%$ ) at the end of 25 -year service life, and $230 \%$ at the end of 100 -years (a minimal increase). It is interesting to note that for lower initial strain ratios, the percentage early increase in the final strain (over initial strain) is much higher, but it diminishes as the member ages. This finding, though seems counterintuitive, is similar to Batra's (2009) and Zhai's (2018) findings. The author acknowledges that past studies do not have enough published data to make a conclusive finding in this regard (i.e., the rate of strain increase is inversely proportional to initial sustained strain ratio), and more research will be helpful. Batra (2009) indicated a similar trend in strain increase but an opposite (or non-conclusive) trend in the deflection measurements. We do not know the exact reasons for such pattern, but the overall trend is difficult to ignore due to the large size of the data particularly at lower strain levels as compared other test data (Batra, 2009 and Zhai, 2018) which focused on higher strain ratios and were carried out to creep rupture failures. Nonetheless, this trend indicates that even at very low strain ratios, it is crucial to account for the increased deformation to ensure FRP members will remain serviceable until the end of its service life. In the absence of more detailed studies, a very rough estimate of the final strain increase for FRP members (with a target service life of 5 to 100 years) over the initial sustained strain can be obtained from Figure 5.11.

As creep strain increases over the initial sustained strain, additional deformation over the initial elastic deformation will also increase and can result in the serviceability failures of FRP members. From a professional practice point of view, all creep strain-induced deformation must be accounted for in the design and often may be a controlling factor in the sizing of FRP members. For example, similar to steel or concrete members, FRP members can cambered (e.g., roof beams or slabs) correctly to account for short-term elastic deflections; however, the long-term sustained 
load deflections are not accounted for (by cambering geometric members' profile and starting with low sustained stresses), creep deformation over time may result in noticeable sags or cracks in secondary members. Another example can be a narrow FRP pedestrian through truss bridge, located in a remote park, can be subjected to a large amount of trapped snow/ice during winter months, and fail in serviceability limit state due to its excessive deflection, which was not taken into account during its design.

As mentioned before, there is not enough long-term experimental data to support a prediction of the long-term deflection of pultruded GFRP members; therefore, we will make a very rough attempt to estimate the final deflection using the prorated experimental data from this report. The computed final strain near the end of the desired service life (over the initially designed sustained strain level) can be used to modify the original elastic modulus approximately. The readers are cautioned that the approximate method, as presented herein, is derived from a creep testing database of vinylester-based GFRP rebars subjected to tensile sustained loads only. In general, pultruded GFRP shapes in flexure and subjected to other complex modes of failure will require additional computations and adjustments. The intent is to show the general application of the reliability framework for the durability design of FRP members and to bring the adverse effects of sustained stress in estimating long-term deflections to the center stage. In general, excessive deformation not necessarily means the fracture or failure (strength limit states), so it is not necessary to envelop a full range of possible creep deformations. For simplicity, we will use the mean value encompassing about $50 \%$ of the samples.

Once and the relationship between initial sustained ratio and final strain has been established, the effective elastic modulus can be modified by the ratio of the estimated final strain over the initial strain. 


$$
E_{\text {eff }}=E_{\text {elastic }} x \frac{\text { Initial Strain }}{\text { Final Strain }}=\gamma_{E} x E_{\text {elastic }} \ldots E q(5.9)
$$

Where creep deformation reduction factor $\left(\gamma_{E}\right)$ for Elastic Modulus is defined as:

$$
\gamma_{E}=\frac{\varepsilon_{0}}{\varepsilon_{t=\text { service life }}} \quad \ldots E q(5.10)
$$

Based on Figure 5.11, the average final strain ratio $\left(\frac{\varepsilon_{t}}{\varepsilon_{0}}\right)$ at the end of 25-year service life is estimated at $200 \%$ of an initial sustained strain ratio of $20 \%$ (and $230 \%$ for 100 -year service life). The reduction factor $\left(\gamma_{E}\right)$ for elastic modulus due to creep can be calculated as follows:

$$
\gamma_{E}(25 \mathrm{yr})=\frac{1}{\left(\frac{\varepsilon_{t}}{\varepsilon_{0}}\right)}=\frac{1}{2.0}=0.50
$$

For 100-year service life, this factor slightly decreases to:

$$
\gamma_{E}(100 \mathrm{yr})=\frac{1}{\left(\frac{\varepsilon_{t}}{\varepsilon_{0}}\right)}=\frac{1}{2.3}=0.43
$$

Cautionary Note: The above method for estimating creep deflections in stand-alone pultruded FRP members is preliminary and is included for information only. For complex FRP shapes subject to flexure, the shear may influence the deformation substantially, and modulus of elasticity for flanges and web can substantially differ, all of which further complicate the longterm estimation. However, the author strongly feels that a preliminary mean to compute long-term serviceability of FRP members (under sustained loads) during the design phase is critical, and in the absence of more refined methods or supporting experimental test data (for predicting longterm deflection), the above approximation can serve as a starting point.

\subsubsection{Correction for Nominal Strength}

As mentioned before that often, the nominal or guaranteed minimum strength (also referred to as Characteristic Strength) is provided by the FRP suppliers. Without additional laboratory 
testing, it is difficult to estimate average or mean ultimate strength (which is commonly referenced in most research findings). In lieu of test data, Bias Correction Factor $\left(\gamma_{\mathrm{BF}}\right)$ for converting between ultimate and nominal values can be defined as follows:

$$
\text { Bias Correction Factor }\left(\gamma_{\mathrm{BF}}\right)=\frac{\text { Average Ultimate Strength }\left(f_{\left.u, \text { ave or } \varepsilon_{u, \text { ave }}\right)}\right.}{\text { Nominal or Guranteed Min.Strength }\left(f_{u}^{*} \text { or } \varepsilon_{u}^{*}\right)} \ldots E q
$$

It is customary to express design stress or strain ratios as a function of ultimate strength which is defined as:

$$
\begin{aligned}
& \text { Ultimate Design Ratio }=\frac{\text { Sustained Stress or Strain }}{\text { Average Ultimate Strength }}=\frac{f_{0} \text { or } \varepsilon_{0}}{\gamma_{\mathrm{BF}} x f_{u}^{*} \text { or } \varepsilon_{u}^{*}} \ldots E q \\
& \text { Or } \\
& \text { Ultimate Design Ratio }=\frac{1}{\gamma_{\mathrm{BF}}} x \text { Nominal Design Ratio } \ldots E q(5.13) \\
& \text { Or }
\end{aligned}
$$

$$
\text { Nominal Design Ratio }=\gamma_{\mathrm{BF}} x \text { Ultimate Design Ratio } \ldots E q(5.14)
$$

Based on creep test data, we inferred that Bias Factor for the strain is 1.09 to 1.29 , therefore, by substituting and an average Bias Factor value 1.2 is used,

$$
\text { Nominal Design Ratio }=1.2 \times \text { Ultimate Design Ratio }
$$

For example, based on Figure 5.10, for a desired 100-year service life and assuming a rupture strain of $70 \%$, the $99.977 \%$ lower bound value of maximum sustained stress ratio is $20.5 \%$ (based on ultimate strength). To find the maximum allowable sustained stress ratio based on "nominal "strength, multiply the ultimate design ratio of $20.5 \%$ by the bias factor of 1.2 to obtain $24.5 \%$ as the nominal design ratio. It should be noted that creep rupture strain is a function of FRP material properties, including initial sustained strain, internal defects, environment, etc. 
It is an important distinction since the design sustained stress ratio will be about $20 \%$ more than the value computed using mean ultimate strength value. All graphs in this report are developed for various rupture strength (maximum rupture strain limit) and sustained stress ratios based on the "ultimate" strength, so a bias correction of 1.2 is necessary.

This study did evaluate both the use of nominal and ultimate values as the basis of the recommendations; however, the mean or average ultimate values are a more accurate representation of FRP mechanical properties and should be used to develop initial design recommendations. To take into account the nominal values, designers can further apply a general Bias correction factor of 1.2 to the sustained stress ratios values obtained from these charts. In the absence of more data, a value of 1.0 can be cooperatively used for Bias Factor. No Bias Factor correction is typically necessary for Elastic Modulus.

\subsection{APpliCability to Other Physical EFfects:}

The concept of the lower bound estimate to make a safe design value is not new. The lower bound estimate method works well when it is difficult to formulate a failure limit state accurately. For example, the failure state for sustained stress is when the member reaches the rupture strain (ultimate limit state), or its deformation reaches a pre-defined value (a serviceability limit state). Both limit states are difficult to formulate in terms of equations that include all variables involved. Although not investigated in this report, fatigue damage is cumulative over a member's life span and is difficult to formulate. An evaluation of fatigue life using the proposed framework, perhaps very much possible using a similar technique used for sustained stress; however, it is considered outside the scope of this research. 


\subsection{SUMMARY}

Based on the information presented in this report, the following can be inferred regarding glass fiber reinforced polymers (GFRP) in vinylester resin members subjected to sustained stresses:

1. The use of normalization of strain data was found to be very helpful in analyzing the complex strain database. Normalization of initial strain with respect to mean ultimate strain value (at $90 \%$ to $95 \%$ of static ultimate strength) and final strains in terms of initial strain makes the data easy to visualize and use in regression analysis for general purpose use.

2. Findley's law fits nicely for modeling sustained stress and allows a direct equation to compute the time to failure. Other regression equations such as logarithmic equations can also fit the test data, but a direct solution for time to failure can be cumbersome. The use of Findley's power law (or similarly modified forms) is recommended.

3. Since sustained stress limitation is not subject to any limit state function describing the failure mode, the use of lower bound values of "time to failure" is preferred for employing reliability principles to this physical effect. The statistical analysis of Findley's prediction model indicated the normal/lognormal distribution of residuals, which further justifies the use of lower bound values using a standard deviation of 3.5, which roughly equates to the target reliability index of 3.5. One of the definitions of the reliability index is the z-value measured from the mean value (typically " 0 ") of the residuals in normalized space.

4. Limitation of sustained stress, as computed for various service lives in this chapter, matches well with the existing recommendations, but provide a rational methodology for deriving those limitations from an experimental database. 
5. Creep can render an FRP structure dysfunctional if inelastic FRP deformations (due to sustained loads) are not accounted for in the original design. The framework suggested in this chapter provides the rationale for accounting for increased strains (based on a $50 \%$ confidence interval) to address this issue (for simple shapes with a uniform modulus of elasticity). Additional research is needed to address the long-term deflection of complex FRP shapes subjected to a wide variety of loading conditions.

\subsection{RECOMMENDATIONS}

Based on the conclusion reached in this chapter, the following are the recommendations specific to current practice:

1. Limit creep rupture ratio to $70 \%$ of the average ultimate strain to obtain a service life of 25 to 100 -years. If nominal values are used, limit rupture strain to $80 \%$ of nominal value. Based on the creep test results (of the database used in this chapter), a minimum value of creep rupture strain of $60 \%$ can be safely used.

2. Use $\gamma_{\mathrm{BF}}$ as the bias correction factor of 1.2 to compute maximum allowable sustained stress ratios $\left(\varepsilon_{0} / \varepsilon_{f u}\right)$ to be applied when using nominal values (or guaranteed minimum strength) instead of ultimate strength values. Where $\varepsilon_{0}$ is the initial sustained strain and $\varepsilon_{f u}$ is the ultimate static strain (corresponding to $\mathrm{f}_{\mathrm{u}, \mathrm{ave}}$ ).

3. Limit maximum allowable initial sustained strain ratio or sustained stress ratio $\left(\frac{\varepsilon_{0}}{\varepsilon_{f u}}\right.$ or $\left.\frac{\sigma_{0}}{\sigma_{f u}}\right)$ to a value defined in Table 5.6. Where:

$$
\text { maximum allowable }\left(\frac{\varepsilon_{0}}{\varepsilon_{f u}} \text { or } \frac{\sigma_{0}}{\sigma_{f u}}\right)=\gamma_{B F} x \frac{f_{s s}}{f_{u}^{*}}=\gamma_{B F} x \eta_{s s}^{*} \ldots E q
$$

$\gamma_{B F}=$ Bias correction factor, use 1.2 in lieu of test data 
$\eta_{s S}^{*}=$ sustained stress ration based on nominal strength as defined below

$f_{s s}=$ design sustained stresses (e.g., service stresses from dead loads) and

$f_{u}^{*}=$ "guaranteed tensile strength" typically provided by the FRP manufacturer

(also referred to as "nominal" strength of FRP member and can be computed as

$\left.f_{u}^{*}=f_{u, a v e}-3 \sigma\right)$

$f_{u, a v e}=$ Average or mean ultimate tensile strength

$\sigma=$ standard deviation obtained from testing of FRP samples for $f_{u, \text { ave }}$

Table 5.6: Maximum Allowable Sustained Stress Ratio

\begin{tabular}{|l|l|l|l|l|l|}
\hline \multirow{2}{*}{$\begin{array}{c}\left\{\boldsymbol{\varepsilon}_{r}\right\} \text { or Rupture } \\
\text { Strain }\end{array}$} & \multicolumn{5}{|c|}{$\boldsymbol{\eta}_{\boldsymbol{S S}}^{*}$} or Maximum Allowable Sustained Stress Ratio at Service Life at \\
\cline { 2 - 6 } & At 10-year & At 25-Year & At 50-Year & $\mathbf{7 5 - y e a r}$ & $\mathbf{1 0 0 - y e a r ~}$ \\
\hline $\mathbf{6 0 \%}$ & $35 \%$ & $30 \%$ & $25 \%$ & $23 \%$ & $21 \%$ \\
\hline $\mathbf{7 0 \%}$ & $40 \%$ & $34 \%$ & $29 \%$ & $27 \%$ & $25 \%$ \\
\hline $\mathbf{8 0 \%}$ & $44 \%$ & $38 \%$ & $33 \%$ & $30 \%$ & $28 \%$ \\
\hline
\end{tabular}

Note: Rupture strain limit $\left(\varepsilon_{r}\right)$ is the ratio of final rupture strain during sustained load tests over ultimate strain during material's stress-strain tests. Average rupture strain limit of $70 \%$ and Bias Correction Factor of 1.2 have been used to compute above maximum allowable sustained stress ratio based on nominal strength. The results are based on sustained strain tests conducted at the University of Sherbrook (Youssef, 2010) and are presented for pultruded vinylester-based GFRP sections of average FVF of 0.60 and an average thickness of $12 \mathrm{~mm}$.

Cautionary Note: Table 5.6 and 5.7 values are derived assuming a normal probability distribution (with a lognormal timeline component for regression) and assume that the number of data points (about 226) is adequate to support such assumption. More representative results can be obtained analyzing additional databases and using a specially fitted distribution type (such as Gamma, Lognormal, or Weibull) to compute lower confidence limits of "time to failure."

4. In the absence of experimental data or research on long-term creep deformation of standalone pultruded FRP members, the following approximate method (based on experimental data 
analyzed in this report) can be used as a "starting point" to estimate creep deformation at the end of member's service life. Roughly speaking, the creep deformation reduction factor $\left(\gamma_{E}\right)$ as defined below and further shown in Table 5.7, can be used for "simple shapes" with "uniform modulus of elasticity." Where

$$
E_{\text {eff }}=E_{\text {elastic }} \times \frac{\text { Initial Sustained Strain }}{\text { Final Strain }}=\gamma_{E} \times E_{\text {elastic }}
$$

$E_{\text {elastic }}=$ Nominal (or guaranteed minimum) Modulus of Elasticity as derived from stress \& strain tests

$E_{e f f}=$ Effective Modulus of Elasticity taking into account creep deformation due to sustained stress on FRP member over time

Table 5.7: Approximate Creep Deformation Reduction Factor $\left(\gamma_{E}\right)$ for Elastic Modulus

\begin{tabular}{|l|l|l|l|l|l|}
\hline \multirow{2}{*}{$\begin{array}{l}\left(\frac{\varepsilon_{0}}{\varepsilon_{f u}}\right) \text { or Initial Sustained } \\
\text { Stress Ratio }\end{array}$} & \multicolumn{4}{|l|}{$\gamma_{E}$} or Creep Deformation Reduction Factor for Service Life at \\
\cline { 2 - 6 } & At 10-year & At 25-Year & At 50-Year & 75-year & 100-year \\
\hline $\mathbf{1 0 \%}$ & 0.46 & 0.43 & 0.41 & 0.38 & 0.37 \\
\hline $\mathbf{2 0 \%}$ & 0.53 & 0.50 & 0.47 & 0.44 & 0.44 \\
\hline $\mathbf{3 0 \%}$ & 0.61 & 0.59 & 0.56 & 0.54 & 0.53 \\
\hline $\mathbf{4 0 \%}$ & 0.73 & 0.72 & 0.69 & 0.69 & 0.67 \\
\hline
\end{tabular}

Note: The above values were computed using the mean value ( $50 \%$ confidence interval) of estimated final strains based on the University of Sherbrook rebar creep test data (Youssef, 2010). For more conservative results, upper bound values (at higher confidence intervals) can be used. Also, the above values are provided only as a starting point for the approximate estimate of deflections of pultruded vinylester based GFRP standalone sections with a fiber volume fraction (FVF) of 0.60. Long-term deflection estimates computed using the above values should be supported by additional experimental or field test data or more refined methods, if available.

See cautionary note shown under Table 5.6.

Sample design calculations showing sustained stress checks are provided in Appendix A as Example 1. 


\section{CHAPTER 6 BASIS OF DESIGN (BOD) CASE STUDY}

\subsection{INTRODUCTION}

In order to illustrate a real-life application of the proposed framework, this chapter develops the basis of design (BOD) for the Glass FRP in a submerged marine environment. The in-service data from a two-year research project (2016-2018) that evaluated the effect of aging on the mechanical properties of the vinylester and polyester-based GFRP composite materials in the cold (about $5^{\circ} \mathrm{C}$ ) marine water immersion applications is used. Full set of test data, accelerated testing plan, details of samples tested, and research report (referred to as the "project report" in this chapter) were obtained for this purpose. This project has been referred to as the "subsea research project" or the "project" in this chapter due to its possible FRP applications in oil \& gas offshore exploration. All researchers who originally worked on the project have been referred to as "project researchers (or PR)" in this chapter. This BOD case study reanalyzes the raw test data from a subsea research project which is referred to as the "modified data," and this case study is referred to as the "study" or the "basis of design (BOD)" to illustrate the professional application of time-dependent reliability-based durability framework presented in the previous chapters (Chapter 3 and 4). The data reduction methods, conclusions, and recommendations are similar but slightly different from the details shown in the Project reports. This chapter presents the next logical step towards the development of professional design practice based on the proposed reliability-durability framework that uses actual research ATM data.

Due to a non-disclosure agreement (NDA) executed by WVU, the identities of the manufacturer, owners, researchers, and various commercial vendors associated with the project cannot be revealed in this report. Nevertheless, the author sincerely acknowledges the project 
researchers' contributions to FRP knowledge bank and willingness to share their test database for this BOD case study.

\subsection{PRoJeCt SUMmaRY}

A summary of the original subsea research project, along with specific modifications used for this study, is presented in this section to set up the context of this BOD case study.
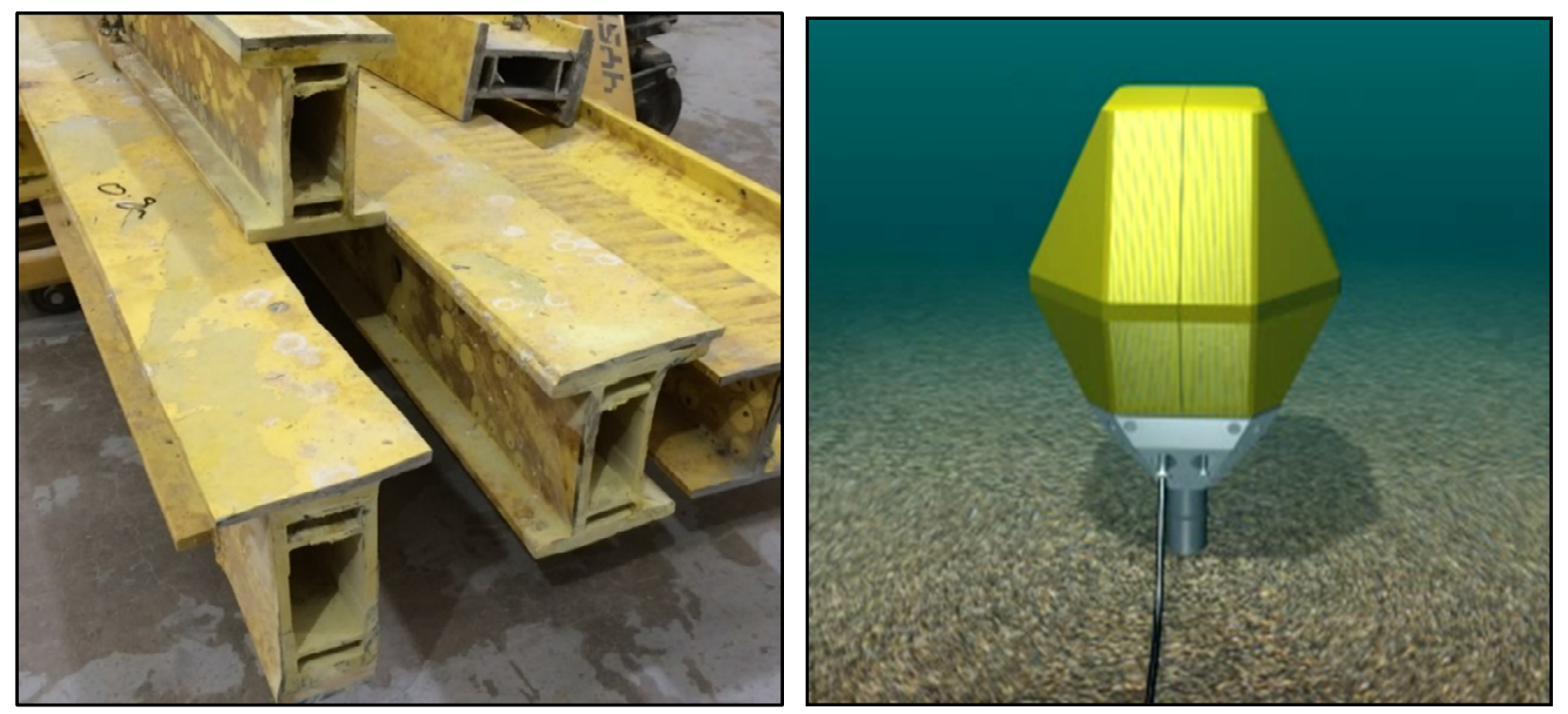

Figure 6.1: Aged FRP Beams (Left); Wellhead Protection System (Right)

\subsubsection{Scope of the Project}

In the project, two types of FRP samples were tested: i) virgin material, which was the composite panels as manufactured for subsea applications, and ii) aged material, which was retrieved from a fully immersed in a subsea application (e.g., Cocoon/shell for an oil wellhead) after seven years in service (see Figure 6.1). The natural environment for aged material was estimated to have an average temperature of 5 degrees Celsius and a seawater depth of approximately 300 feet (94 meters). The project researchers used accelerated testing method 
(ATM) to saturate and age both virgin and aged (Cocoon) vinylester (VE) materials (coupons extracted from FRP beam sections) in synthetic seawater at $55^{\circ} \mathrm{C}$. For polyester (PE) material, two different temperatures $\left(30^{\circ} \mathrm{C}\right.$ and $\left.40^{\circ} \mathrm{C}\right)$ were used. They evaluated the collected accelerated test data using the Arrhenius approach (see Section 6.2.2 for details). The project report did not mention the use of any hydrostatic head in lab testing or any relation to it. The report also did not mention saturation conditions (except for duration it took to reach mass uptake equilibriums). Extracted coupons (from both virgin and aged beams, Figure 6.2) were tested for tension, flexure, and short beam shear properties at predefined intervals to quantify possible changes in the material's performance as a result of the subsea aging process. No original test data (i.e., pre-age properties at the time of placement in service of the cocoon) about the aged materials were provided.

\subsubsection{Aging Process Used}

This particular FRP subsea component is designed to work in a subsea environment (e.g., submerged structures used by the oil and gas industry for offshore work) at $5^{\circ} \mathrm{C}$ temperature for at least 25 years at a depth of $300 \mathrm{ft}$. A simple Arrhenius approach was used by the project researchers (PR) to plan the experiment and simulate the anticipated life span of 25 years in laboratory conditions. In order to complete the aging exposure within a reasonable length of time, PR chose the aging temperature to be $55^{\circ} \mathrm{C}$, which approximately equated to an exposure period of about ten months in the lab. This exposure period for experimental planning purposes can be computed based on a simple rule-of-thumb Arrhenius approach, as proposed by PR and is further described in Section 6.2.2.1. For example, to simulated 25 years of aging in a natural subsea environment of $5^{\circ} \mathrm{C}$ with $55^{\circ} \mathrm{C}$ seawater in the lab, $\mathrm{n}=(55-5) / 10=5$ (Eq. 6.3), TSF $=2^{\mathrm{n}}=32$ (Eq. 6.2), which can provide the minimum required time in the lab as $=(25 \times 12) / 32=9.4$ months (from 
Eq. 6.1). It should be noted that the selected temperature of $55^{\circ} \mathrm{C}$ was well below the glass transition temperature of the dry vinylester material (i.e., $115^{\circ} \mathrm{C}$ as derived from DMA tests by PR) to limit any potential chemical aging mechanisms caused by the high temperature. However, due to the lower glass transition of wet (saturated) polyester material (i.e., $56^{\circ} \mathrm{C}$ ), the accelerated aging temperature was lowered to limit any potential chemical aging mechanisms. Hence the project researchers decided to age this material at two different temperatures of $30^{\circ} \mathrm{C}$ and $40^{\circ} \mathrm{C}$. It should be noted that the reported value of the glass transition temperature of $56^{\circ} \mathrm{C}$ for polyester is considered too low.

\subsubsection{Mechanical Properties Evaluated}

To evaluate the effect of marine water aging on the mechanical properties of the material, retrieved specimens at predefined intervals were tested to evaluate their tensile, flexure, short beam shear, impact, hardness and glass transition properties. The mechanical properties (i.e., tensile, flexure, and short beam shear) were performed in two principal directions (i.e., longitudinal and transverse to fiber direction) to provide a better understanding of the effects of the aging (Figure 6.2). Hardness measurements are not used in the proposed BOD. All test data, as provided by project researchers, has been used in the development of the BOD.

\subsubsection{Aging Methods Used}

\subsubsection{Vinylester GFRP}

To age vinylester-based FRP representing its 25-year design working life in a reasonable accelerated laboratory time, the project researchers immersed the specimens in synthetic seawater at $55^{\circ} \mathrm{C}$. To estimate the time for accelerated testing in the lab, the following approximate 
Arrhenius equation (or rule-of-thumb, see the following discussion in this section) was used by the project researchers.

$$
t_{\text {Age in Service }}=t_{\text {Age in Lab }} \times \text { TSF }
$$

Where:

$\mathrm{TSF}=$ Time Shift Factor (define by the project researchers) as:

$$
T S F=2^{n}
$$

$\mathrm{n}=\mathrm{TSF}$ coefficient (defined by the project researchers) as:

$$
n=\left(T_{\text {Temp in Lab }}-T_{\text {Temp in Service }}\right) / 10 \quad \ldots \text { Eq (6.3) }
$$

$t_{\text {Age in Service }}=$ Projected Age in the service environment (days)

$t_{\text {Age in Lab }}=$ Actual Age in the laboratory under Accelerated Aging environment (days)

$T_{\text {Temp in Lab }}=$ Temperature in the laboratory (Celsius)

$T_{\text {Temp in Service }}=$ Expected temperature in the service environment (Celsius) or the reference temperature

For 25 years in service at $5^{\circ} \mathrm{C}$ in seawater with a lab temperature of $55^{\circ} \mathrm{C}$, the coefficient " $\mathrm{n}$ " is computed to be 5 for Vinylester GFRP. In order to complete the aging exposure tests within a reasonable length of time, project researchers used a "rule of thumb" method for TSF calculations that implies that the rate of aging is doubled for every $10^{\circ} \mathrm{C}$ increase in temperature. This rule of thumb TSF justifies for the use of $n=10$ (i.e., for every $10^{\circ} \mathrm{C}$ increase) and base $=2$ (i.e., rate of aging doubles), as shown in Equations 6.2 and 6.3. However, the author feels that the TSF equation used by the project researchers is oversimplified concerning the final data analysis needs of a reliability framework; therefore, it is not used in this chapter. At the same time, the author acknowledges that the TSF calculations for this project are very complicated and almost 
impossible to accurately compute due to i) the lack of accounting for hydrostatic pressure (i.e., 300 feet depth) conditions in the saturation and accelerated testing condition of the lab, ii) missing original and aged mechanical properties of aged (cocoon) materials before starting saturation in the lab (see Section 6.3 .3 for TSF used by the author), and iii) unknown lab conditions such as excessive mass uptake of samples well beyond saturation phase into the aging process.

To represent the actual working environment of the material, the seawater environment was approximated by synthetic seawater using ASTM D1141-98 Section 6 (Synthetic Seawater prepared without heavy metals). The $\mathrm{pH}$ was adjusted to 8.2 using a $0.1 \mathrm{~N}$ Sodium Hydroxide solution.

Following the immersion of all composite specimens in appropriate containers, the volume was filled with synthetic seawater and the lids secured. The use of any hydrostatic head is not mentioned in the project report. The containers were then placed in an air circulating oven, which was set at a temperature of $55^{\circ} \mathrm{C}$.

\subsubsection{Polyester GFRP}

For the polyester (PE) material aging at $30^{\circ} \mathrm{C}$ and $40^{\circ} \mathrm{C}$, using PR methods (Eq. 6.1, 6.2, and 6.3), the calculated TSF will be 2.5 and 3.5, respectively. In order to normalize the PE data in this report to a stable $30^{\circ} \mathrm{C}$ environment, the time in the lab for $40^{\circ} \mathrm{C}$ was multiplied by a factor 2 based on TSF (rule of thumb which is the same as used for vinylester) used by the project researchers. It is the opinion of the author that TSF used by the project researchers appears to be oversimplified for polyester-based GFRP and more detailed calculations with the help of additional data (see discussion in the previous section 6.2.2.1) could have provided more realistic results. 


$$
\begin{aligned}
& n=\frac{40-30}{10}=1 \\
& T S F=2^{1}=2.0
\end{aligned}
$$

The aging environment is similar to that used for Vinylester, seawater environment based on ASTM D1141-98 Section 6 (i.e., synthetic seawater prepared without heavy metals).

\subsubsection{Testing of Mechanical Properties}

About 882 samples were extracted with about 314 samples came from a retrieved aged wellhead cocoon, and the remaining samples came from a similar virgin (dry) material. About 200 samples were used for Hardness, Impact, and Glass Transition Temperature tests (not reported or used in this report). The entire testing program lasted about two years, with about 10-12 months in the actual accelerated aging environment.

For flexure, the 3-point bend tests were performed following the principles of the ASTM D790 standard test method in both the longitudinal and transverse directions.

The tensile tests were performed in longitudinal and transverse directions following the guidance of the ASTM D638M and ASTM D3039. To conduct the short beam shear tests, ASTM D2344 was followed.

Also, tests for hardness (ASTM D2583), impact resistance (ASTM D7136), and glass transition temperature (using DMA 8000 under ASTM D7028) were conducted as listed in the following paragraphs. It should be noted that these properties are not used in formulating the basis of design (BOD) presented in this report.

No information about the fiber volume fraction (FVF), sample timelines (e.g., manufacturing, placement in service, extraction, storage, etc.), or the constituents/architecture of the virgin/aged sections was provided. In retrospect, the author feels that the prior knowledge of 
some of the missing details would have helped in developing a more relevant BOD along with a better explanation of test findings, reliability analysis results, and the final recommendations.

Figure 6.2 shows the location and sample extractions on virgin and aged sections, relevant section properties of vinylester and polyester sections, and explains the sample orientations for the testing of longitudinal and transverse properties. Arrows show the approximate directions of test loads (tensile and flexural tests). In the absence of the details regarding FRP architecture, beam geometry in the subsea wellhead, and final loading orientation, BOD assumes that longitudinal mechanical properties will form the member's primary resistance against the load effects. 
$\mathrm{P}_{\text {Transverse }}=$ Axial Tension Test Load for transverse sample

$P_{\text {Longitudinal }}=$ Axial Tension Test Load for longitudinal sample

$\mathrm{W}_{\text {Transverse }}=$ Flexural Test Load for transverse sample

$\mathrm{W}_{\text {Longitudinal }}=$ Flexural Test Load for longitudinal sample

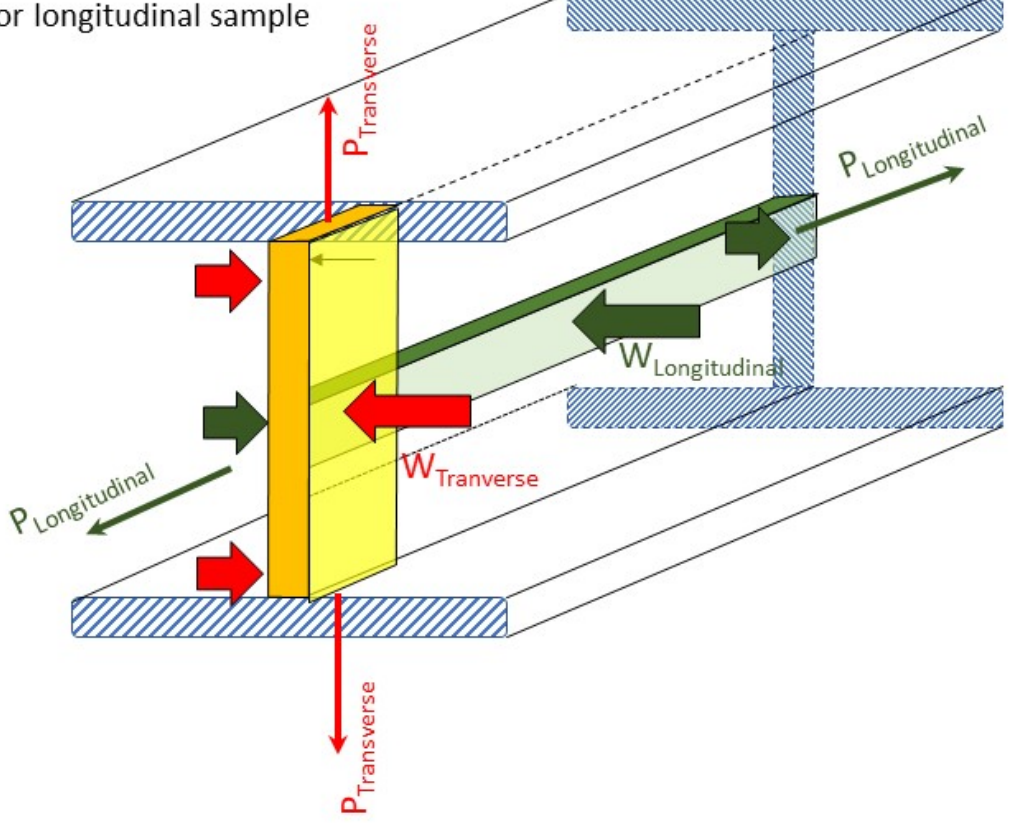

\section{Location of Transverse and Longitudinal Samples}

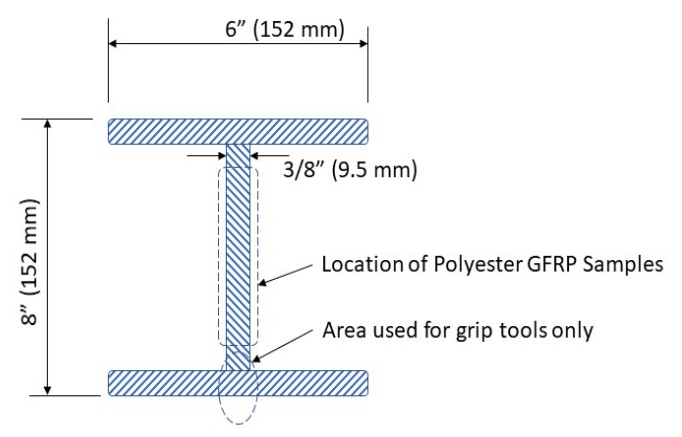

6" Wide Flange Beam Section

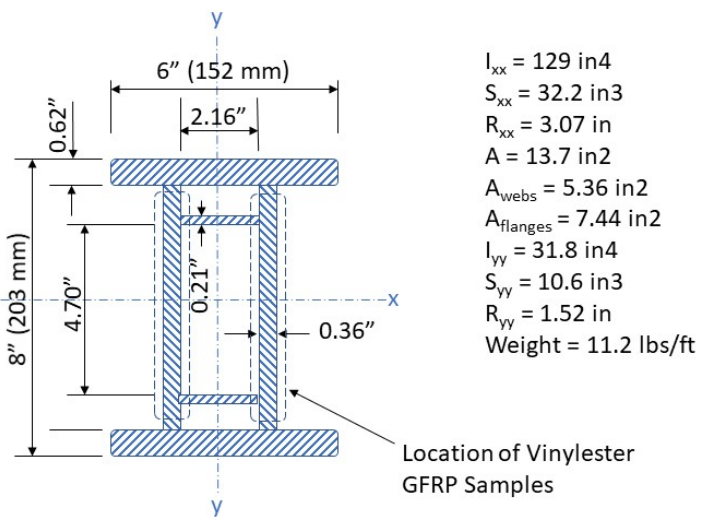

8" Double Web Box Beam Section

Figure 6.2: Beam Sample Extraction Locations and Orientation 


\subsubsection{Moisture (Mass) Uptake Measurements}

Due to the permanently submerged application of GFRP, the project researchers included a very comprehensive mass uptake testing program to prepare samples for accelerated testing. A summary of strength reductions is provided in Table 6.1 since moisture absorption forms an integral part of GFRP applications in a subsea environment.

The primary difference in this Chapter methodology is that the project researchers set the time for degradation to "zero" only after the saturation of samples (assume room temperature seawater without any hydrostatic pressure) has been reached, whereas, the modified data as presented in this report considers the time for the degradation to "zero" at the beginning of sample immersion into the synthetic seawater (i.e., the start of the saturation process in the lab). The primary reason for not using the same timeline is that the aging begins at the time when specimens are submerged in the accelerated environment solution (room temperature synthetic seawater as compared to colder seawater in this case), so saturation and aging have the same starting timeline and it is complicated to find an exact timeline separating these two. The project test results show that even after the theoretical period of saturation ended (when the equilibrium of mass change reached or when project researchers noted negligible weight gain), all samples continue to absorb water till the project completed in about two years (see the following paragraphs for more details). If the saturation process would have been carried out in the referenced natural environment $\left(5^{\circ} \mathrm{C}\right.$ seawater and 300 feet of hydrostatic pressure), it could have been much different. This distinction is necessary to formulate the aging timeline and aging equation for the proper establishment of a reliability-durability framework. Some of the advantages in using a single timeline are: i) easy to formulate a single-parameter degradation equation with respect to the time after the placement in service (i.e., starting time is zero), ii) use of the dry ultimate strength as the primary reference (at 
time zero), and iii) precision at the early/intermediate stages (saturation stops but the aging continues) of degradation is not required for the reliability analysis which focuses on the end of service life.

The project developed two separate reduction factors- one for moisture uptake with reference to dry virgin sample properties and other for degradation with reference to the saturated sample properties, while this research report provides just one degradation equation with reference to the dry virgin sample. In this case, the computed factors for immersion are included with the aging factors (Table 6.1).

The mass uptake of the vinylester (VE) material throughout aging in seawater is significant, especially under the hydrostatic pressure of a 300 feet column of seawater (due to nano-diffusion of seawater particles into GFRP). Both virgin and cocoon materials reached an effective mass equilibrium state (at $0.55 \%$ and $0.45 \%$ mass gains for virgin and cocoon samples respectively) after about 65 days in room temperature $\left(25^{\circ} \mathrm{C}\right)$ seawater. This point was then considered as the start of the aging process (i.e., Time Zero) in the project. However, during the aging process, the material continues to absorb seawater. At the end of the test periods (365 days), the virgin material had absorbed $10 \%$, and the cocoon material $25 \%$ more than the values recorded at the theoretical start (of the aging) assumed by the project researchers. This finding further confirms that a hard timeline separating the effects of the moisture and the accelerated environment is challenging to estimate in a laboratory setting.

The mass uptake of the polyester (PE) material throughout aging is much more significant than vinylester (VE). The cocoon material has reached an effective equilibrium state (at $1.05 \%+$ and $0.65 \%$ mass gains for virgin and cocoon, respectively) after 100 days at $30^{\circ} \mathrm{C}$ while the virgin material has reached an effective equilibrium state after 268 days. This point was then considered 
as the start of the aging process (i.e., Time Zero) in the project. By the end of the study (582 days), the virgin material has absorbed another $14 \%$ and the cocoon material another $10 \%$ seawater compared to the values recorded at the theoretical end of saturation (or the start of the theoretical aging). The author acknowledges the fact that the above values of mass uptake (in both virgin and aged samples, particularly for polyester specimens) are considered high and the reasons for which cannot be explained with information available.

There was a noticeable drop in the samples' strengths after the mass uptake process was concluded at 65 days for both VE cocoon and VE virgin materials, and 100 days for PE Cocoon and 268 days for PE Cocoon. The results of all saturation tests (as reported by the project researchers) with reference to dry virgin strengths are summarized in Table 6.1. It should be noted that some of the results may appear out of place, particularly for aged materials (e.g., transverse shear strength for aged materials is $105 \%$ of dry strength of virgin material) which can be contributed to possible differences in the original mechanical properties of virgin and aged materials and perhaps to an inadequate number of samples (only five tested per property). Similar inconsistencies can be seen for aged samples in Table 6.1. Besides, since the original test data for the aged material (at the time of placement in service) was not available, the project researchers assumed the same original dry strengths for both (aged and virgin) materials.

The author would like to emphasize that the intent of this chapter is to illustrate a case study of the BOD development process using the time-dependent reliability-based framework, as presented in previous chapters. The readers are cautioned that the test database, as provided by the project researchers, is used to illustrate the basic concept only. The validation of test data provided, interpretation of test results, or applicability of TSF equations used by the project researchers is outside the scope of this report. 
Table 6.1: Strength Retention due to Seawater Saturation and Aging

\begin{tabular}{|c|c|c|c|c|c|c|c|}
\hline \multirow[t]{2}{*}{ 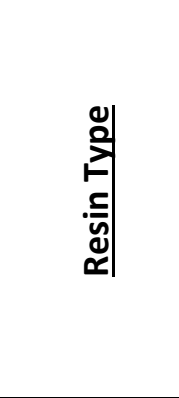 } & \multirow{2}{*}{ 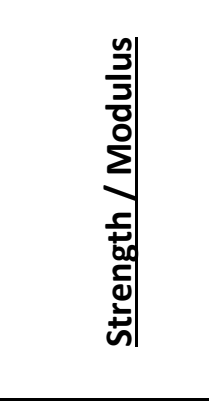 } & \multirow[t]{2}{*}{ 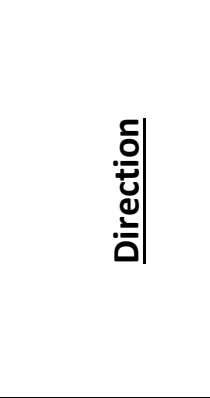 } & \multirow[t]{2}{*}{ 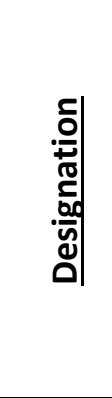 } & \multicolumn{2}{|c|}{$\begin{array}{c}\text { Note } 1 \\
\frac{\text { Strength Retention }}{\text { (SR) at the end of }} \\
\begin{array}{l}\text { Saturation } \\
\text { (with reference to } \\
\text { dry virgin strength) }\end{array}\end{array}$} & \multicolumn{2}{|c|}{$\begin{array}{c}\text { Note } 2 \\
\frac{\text { Strength Retention }}{\text { (SR) at the end of }} \\
\frac{\text { Aging Period }}{\text { (with reference to dry }} \\
\frac{\text { virgin strength) }}{}\end{array}$} \\
\hline & & & & $\begin{array}{l}\text { Virgin } \\
\text { Samples }\end{array}$ & $\begin{array}{l}\text { Aged } \\
\text { Samples }\end{array}$ & $\begin{array}{l}\text { Virgin } \\
\text { Samples }\end{array}$ & $\begin{array}{l}\text { Aged } \\
\text { Samples }\end{array}$ \\
\hline \multirow{8}{*}{ Vinylester } & Tensile SR & Transverse & VE-TT & $86.6 \%$ & $74.6 \%$ & $81.3 \%$ & $56.1 \%$ \\
\hline & Tensile SR & Longitudinal & VE-TL & $80.4 \%$ & $82.3 \%$ & $52.3 \%$ & $57.3 \%$ \\
\hline & Flexural SR & Transverse & VE-FT & $84.3 \%$ & $67.0 \%$ & $73.0 \%$ & $54.4 \%$ \\
\hline & Flexural SR & Longitudinal & VE-FL & $85.6 \%$ & $67.8 \%$ & $63.4 \%$ & $61.5 \%$ \\
\hline & Shear SR & Transverse & VE-ST & $90.6 \%$ & $105.0 \%^{1}$ & $76.3 \%$ & $96.4 \%$ \\
\hline & Shear SR & Longitudinal & VE-SL & $86.5 \%$ & $93.9 \%$ & $72.3 \%$ & $88.5 \%$ \\
\hline & Tensile Mod & Transverse & VE-ET & $97.5 \%$ & $91.1 \%$ & $85.4 \%$ & $89.9 \%$ \\
\hline & Tensile Mod & Longitudinal & VE-EL & $97.5 \%$ & $96.0 \%$ & $96.0 \%$ & $94.9 \%$ \\
\hline \multirow{8}{*}{ Polyester } & Tensile SR & Transverse & PE-TT & $94.1 \%$ & $84.3 \%$ & $78.1 \%$ & $70.6 \%$ \\
\hline & Tensile SR & Longitudinal & PE-TL & $93.4 \%$ & $81.0 \%$ & $98.2 \%$ & $69.9 \%$ \\
\hline & Flexural SR & Transverse & PE-FT & $90.9 \%$ & $89.2 \%$ & $85.0 \%$ & $76.4 \%$ \\
\hline & Flexural SR & Longitudinal & PE-FL & $86.4 \%$ & $68.3 \%$ & $81.2 \%$ & $63.3 \%$ \\
\hline & Shear SR & Transverse & PE-ST & $81.8 \%$ & $78.4 \%$ & $75.3 \%$ & $63.2 \%$ \\
\hline & Shear SR & Longitudinal & PE-SL & $87.7 \%$ & $80.1 \%$ & $78.2 \%$ & $63.8 \%$ \\
\hline & Tensile Mod & Transverse & PE-ET & $97.5 \%$ & $71.4 \%$ & $75.1 \%$ & $61.8 \%$ \\
\hline & Tensile Mod & Longitudinal & PE-EL & $97.5 \%$ & $93.3 \%$ & $102.9 \%$ & $95.2 \%$ \\
\hline
\end{tabular}

Note 1: The reported saturation period for virgin samples was 65 days for vinylester and 268 days for polyester. Similarly, the reported saturation period for aged samples was 65 days for vinylester and 100 days for polyester. It should be noted that aged (7 years of subsea aging as a wellhead cocoon) saturated samples showed much higher shear strength than dry virgin strength. This discrepancy could be due to diffusion of seawater nano-particles under hydrostatic pressure or simply due to dry cocoon material aged sample had a higher shears strength than the virgin sample provided. This value was adjusted in Arrhenius analysis, reflecting higher original shear strength of aged samples. The timeline of full saturation stages is assumed to be reached by the project researchers, as discussed in Section 6.2.4.

Note 2: Aged and virgin sample strengths are measured as a percentage of the dry virgin sample strengths at the end of the aging period. The reported total aging period (including saturation) for virgin samples was 368 days for vinylester and 568/571 days for polyester. Similarly, the total reported aging period for aged samples was 368 days for vinylester and 400 days for polyester. 


\subsection{DEVELOPMENT OF BOD}

In order to develop a generic basis of design (BOD) for subsea applications, the following steps were adopted under this study.

\subsubsection{Normalization of Test Data}

All original test data from Subsea Project were normalized with reference to the dry strength of virgin material. The mean ultimate strengths (tensile, flexural, and shear) and tensile elastic modulus of virgin materials in the transverse and longitudinal directions are considered at $100 \%$ value to provide a primary reference for developing BOD. Aged strengths at other test periods are computed as a percentage of the dry virgin strengths. In order to perform regression analysis, the following assumptions are made:

- The dry virgin material property represents the current state of the art GFRP with both the vinylester and polyester matrices, and all other design parameters are to be calculated based on this value. The rate of degradation computed for the virgin material also applies to the aged cocoon material as well. The "dry" mechanical property generally refers to average or mean ultimate strengths or stiffnesses, at the time of placement in service, based on quality assurance testing or manufactured supplied data.

- Aged cocoon material may or may not have the same dry mechanical properties as of the dry virgin materials. Adjustments to the saturated strength of cocoon materials were made to normalize the data with reference to dry virgin material. This adjustment did create a few additional steps in TSF calculations (see section 6.3.3) but helped normalize the entire dataset with reference to virgin dry strengths or stiffnesses. To arrive at the normalized values of saturated cocoon material properties in the aging process, it was assumed that its rate of loss of strength/stiffness from the dry state to the saturated state is the same as that of dry virgin 
material. Also, the timeline for cocoon material was adjusted to account for the natural aging of cocoon materials for 7 years in seawater prior to the aging test, so that all values are finally computed with reference to the dry virgin material. The effect of hydrostatic pressure, although important for both saturation and aging, was ignored due to a lack of data.

In this study, the following equation was used to adjust for the lab time of cocoon materials (so that cocoon timeline can be normalized with respect to the timeline of the virgin material before the start of the aging process) before starting regression analysis:

$$
t_{\text {Adjusted Lab }}=\frac{\left(t_{\text {Natural Env. }}+t_{\text {Actual Lab }}\right)}{T S F}
$$

(For example, $t_{\text {Natural Env. }}$ will be 7 years x 365 or 2,555 days, and $t_{\text {Actual Lab }}$ will be 65 days for vinylester cocoon material at the end of saturation. TSF is reiterated to obtain the final value. As an example, for vinylester GFRP tensile transverse strength (GL-VE-TT) calculations, TSF computed is 36 (see Table 6.2), and therefore, $t_{\text {Adjusted Lab }}$ will be 72.8 days instead of 65 days)

- The rate of degradation of cocoon material in its designed environment (5-degree seawater) was found to be slightly different (both higher and lower based on which property was being compared) than that of virgin materials. Since the primary assumption is that the degradation rate of the virgin material is the controlling reference, the timeline for cocoon material was adjusted to fit the degradation rate of the virgin material. Again, no correction for hydrostatic pressure was made.

- The start time for the aging of all samples began $(\mathrm{t}=0)$ when the samples were placed in a synthetic seawater saturation chamber and continued into an elevated temperature aging environment. Information about the saturation environment is not provided in the report, so it is assumed to be the same as the aging environment. If saturation is performed in room 
temperature seawater, there will be some error introduced. As mentioned before, the early or intermediate phase aging is not critical in the reliability analyses, so some loss of accuracy from this approximation will be acceptable.

- The author acknowledges that due to the reasons explained in Section 6.2.2.1 (i.e., not enough data for equations to solve the unknowns), it will be almost impossible to compute an accurate value of TSF in this case study. Attempts have been made to calculate a reasonable value of TSFs using the test data available (as also explained in sections 6.2.2.1, 6.3.1, and 6.3.3), but the author's confidence in computed TSF remains low. Again, the readers' attention is directed to the overall understanding of the framework process than the database itself.

\subsubsection{Regression for Degradation Rates}

A regression analysis was performed on dry and saturated test data to evaluate the type of curve that can fit not only the 10-12-month lab test data but also allow to forecast for 20-40 years in its natural/reference environment. First, a linear degradation relationship, as used in the Project, was investigated. Separate degradation rates for "dry to saturated" and then "saturated to aged" as used in the project were evaluated. The project's linear regression did not fit well in either case and particularly for forecasting and aging of ATM lab data. Also, a single equation for drysaturated-aged conditions as used in the project did not work for a linear relationship. Since the objective of this research is to develop guidelines for the newly manufactured material, which will be in a "dry" state (for fabrication, QA/QC testing, delivery, etc.) until placed in service, it makes sense to use the dry properties as the primary basis of a BOD. It is the author's understanding that the dry properties of the virgin samples were tested close to the time of samples placement in a saturation environment at the project laboratory. Tests confirm a steep initial drop in strength from 
dry to fully saturated conditions followed by a gradual (less steep) degradation after saturation (up to $19.6 \%$ drop for virgin samples and $33 \%$ for aged samples at the end of saturation period; and a total reduction strength reduction up to $47.7 \%$ for virgin samples and $45.6 \%$ for aged samples at the end of the aging period, see Table 6.1); therefore, a log curve was found to be the best fit. Power functions and polynomial curves were also evaluated but did not provide any better fit than a log curve. Due to the close relationship of the natural log curves with previously established durability work at WVU-CFC, a single degradation curve using the "natural log" equation (or reverse exponential relation) is proposed. Mathematically, log equations suit well to timedependent slowing rates of degradation over an extended range of time, say from a few days (6080 days) to $100+$ years.

Assuming all test data is normalized to $100 \%$ of dry strength (i.e., $\mathrm{SR}=100 \%$ at $\mathrm{t}=0$ ), the basic degradation equation, to compute Strength Retention (SR) at a given time $\mathrm{t}$ (or $S R_{t}$ ), or time $\mathrm{t}$ to reach a certain $S R_{t}$ at a degradation rate $C_{\text {Aging }}$ can be written in various forms as follows:

$$
\begin{array}{r}
S R_{t}=1-C_{\text {Aging }} x \operatorname{Ln}(t) \\
t=e^{\frac{(1-S R(t))}{\text { CAging }}} \\
C_{\text {Aging }}=\frac{\left(1-S R_{t}\right)}{\operatorname{Ln}(t)}
\end{array}
$$

The combined degradation coefficient $C_{\text {Aging }}$ includes the effects of service temperatures and seawater in the lab. There is no information available if any hydrostatic pressure was used in the lab environments, so it is prudent to assume that there was none. Since we will be normalizing all lab data to a subsea natural environment $\left(5^{\circ} \mathrm{C}\right.$ seawater at 300 feet depth) timeline, theoretically speaking, the effects of hydrostatic pressure should also be included. In general, hydrostatic pressures can accelerate FRP moisture absorption and subsequent degradation; however, at the 
same time, cooler temperatures tend to slow down the rate of degradation. The author acknowledges that errors can be introduced (due to reasons explained in sections 6.2.2.1, 6.2.4, 6.3.1, and 6.3.3) when combing the effect of three aging environments: temperature, $\mathrm{pH}$ (seawater), and hydrostatic pressure (missing in the project report) into a single coefficient $C_{\text {Aging }}$. However, in the context of this study, the primary objective is to show the BOD development process with an underlying assumption that any significant inaccuracies or inconstancies found in the test dataset, if significant, would have been mitigated by the project researchers.

\subsubsection{Calculation of Time Shift Factors (TSF)}

Once all the subsea research data has been normalized to the "dry" strength of the Virgin material in the lab, an appropriate Time Shift Factor (TSF) (or factors) must be computed. In simple terms, TSF is a multiplier for the accelerated time in the lab $t_{\text {lab env }}$ to obtain equivalent time $t_{\text {natural env }}$ in a natural (or reference) environment to achieve the same reduction in strength as it would be the situation under lab conditions.

$$
T S F=\frac{t_{\text {natural env }}}{t_{\text {lab env }}}
$$

The project researchers used a simplified approach to compute TSF as a binary power function of temperature difference in lab and service. However, for this research, a more comprehensive approach is used to arrive at a reasonable TSF for the modified data used in this report. The approach to computing TSF as illustrated in this section applies to this database only due to the following unique conditions: i) dry strength of cocoon material was not tested at the start of the aging process and its placement in service; therefore, it is unknown (possible explanation is that cocoon material was "partially" saturated from being in sea for seven years at 300 feet depth), ii) saturated strength/stiffness of cocoon material was tested only after 65 to 100 
days in a saturation environment (no data on strength/stiffness at the start of saturation), and iii) there is no indication if the project researchers took into account seven years of hydrostatic pressure in the lab environment (during saturation or aging processes). Assume no hydrostatic pressure was applied in lab testing.

TSF for this report is computed based on many assumptions made during the analysis of the test data for both virgin and cocoon materials. Since the cocoon material was already saturated/aged for seven years at $5^{\circ} \mathrm{C}$ seawater at 300 feet depth and was again saturated/aged for 65 to 100 days in the lab (without hydrostatic pressure after its extraction from sea bed), it can provide some basis for computing the TSF for virgin materials (as explained in the following paragraphs). The concept is that if both virgin and cocoon materials have the same initial dry strength, both would have the same SR at saturation, otherwise adjust cocoon's SR values under saturated and aged conditions up or down to be comparable to virgin material's dry SR. It should be noted that even after going through all the following steps, the computation of TSF in this situation is still going to be approximate, so extra precision (in Arrhenius analysis of test data or reliability analysis of projected data) will not improve the overall results and could compensate for the missing cocoon's dry virgin strength/stiffness (original and after extraction) and hydrostatic pressure.

Figure 6.3 shows the complexity of accurately computing various levels of TSFs required for this BOD case study. In order to compute TSF, an estimate of degradation rates in various environments is needed. With several key values unknown, it is almost impossible to compute accurate TSF. In addition, the only natural aging reference we have in the seven years in the subsea environment and with no knowledge of its dry strength at the time of installation and later before the start of saturation. Project researchers assumed that dry strengths of virgin and cocoon are the 
same; however, this assumption could not be verified in the subsequent test results. A graphical representation of strength retentions (SR) and timelines, as interpreted by the author, is shown in Figure 6.3 (no scale).

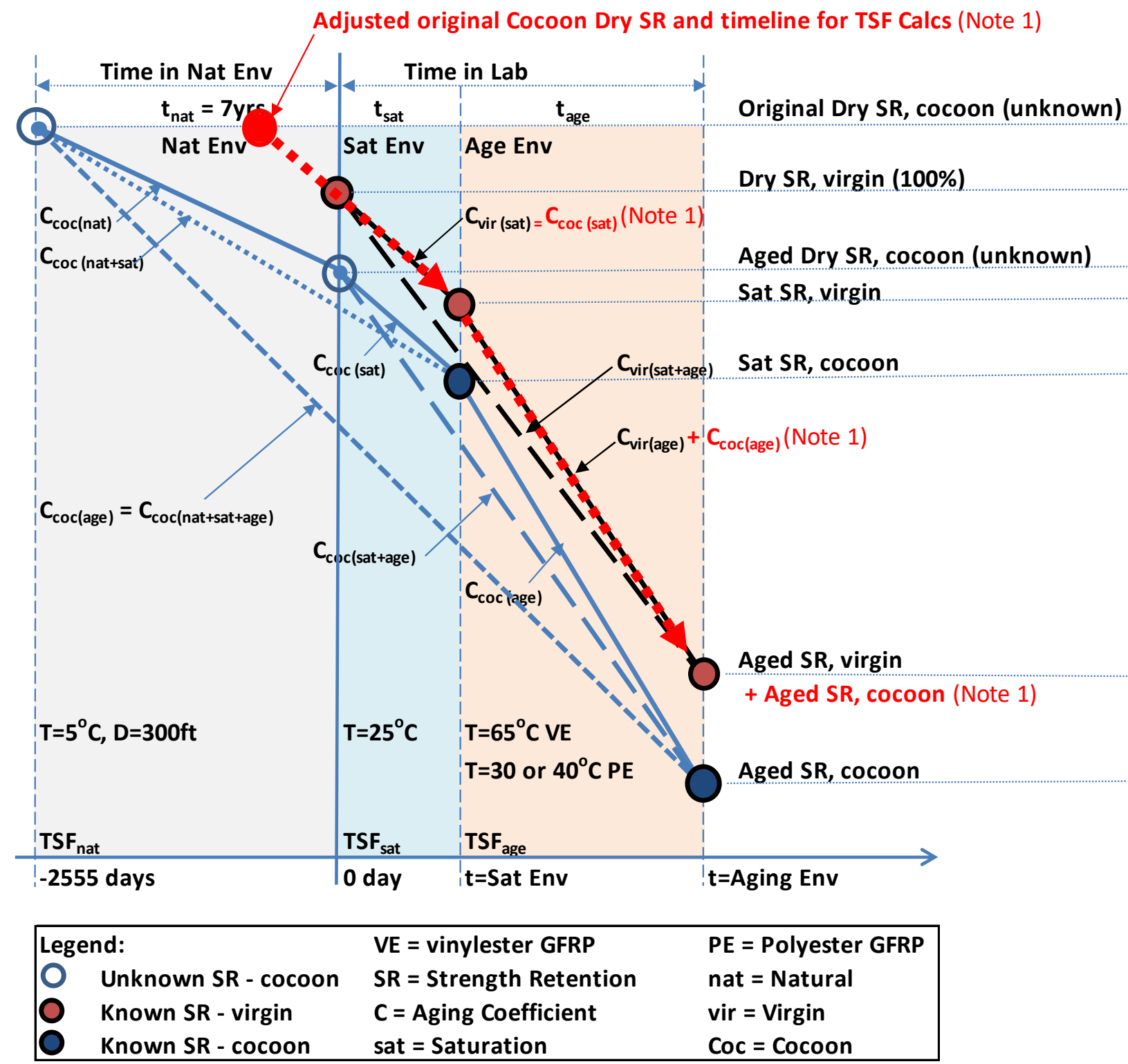

Note 1: Indicates the process used in this study (i.e., adjustment of cocoon's original dry SR and combining aging test results for both virgin and cocoon samples)

Figure 6.3: Aging Environments and Degradation Coefficients for Subsea Project 
Figure 6.3 shows the complexities involved in calculating correct TSFs for various properties in the subsea accelerated experiment. First, the cocoon material naturally ages (applicable aging coefficient $\mathrm{C}_{\mathrm{coc}(\mathrm{nat})}$ ) in a subsea environment at $5^{0} \mathrm{C}$ and 300 feet seawater depth for seven years until its extraction. Samples from the extracted aged cocoon are taken and are placed in another saturation seawater lab environment at $25^{\circ} \mathrm{C}$ but without the hydrostatic pressure

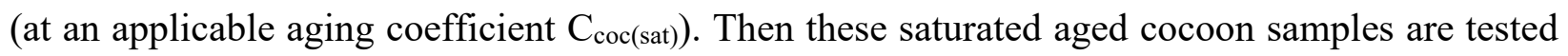
(to determine Saturated $\left.\mathrm{SR}_{\text {cocoon }}\right)$ and aged further in higher temperatures $\left(55^{\circ} \mathrm{C}\right.$ for vinylester and $30^{\circ} \mathrm{C} / 40^{\circ} \mathrm{C}$ for polyester) environments of the lab (applicable aging coefficient $\mathrm{C}_{\text {coc(age) }}$ ). After the completion of aging, these samples are tested to determine aged $\mathrm{SR}_{\text {cocoon. }}$ Without the knowledge

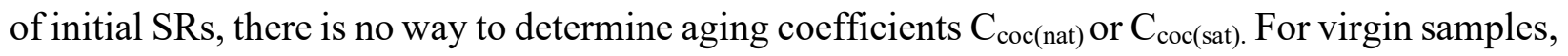
we have all information about the aging coefficients and various SRs, but we must make several assumptions to correlate virgin SR to cocoon SR to compute TSFs for use in this study. The dotted red line with Note 1 in Figure 6.3 graphically depicts the simplified process used in this study and is further explained in the following paragraphs.

Table 6.2 shows the TSFs used in this research. The TSF computation assumes that the degradation rates for cocoon samples are the same as virgin samples except for the dry strengths of cocoon may be different from the dry strength of virgin samples. Once all cocoon sample strengths have been normalized with reference to dry virgin sample strengths, we can compute TSF by comparing the time to reach a particular strength in the lab (for both virgin and cocoon) and natural (for cocoon only) environments. The TFS values computed seems to be high when compared to common GFRP accelerated testing experiments. However, realizing the limitations of the test data, we will proceed with these values. 
Table 6.2: Time Shift Factors used in This Study

\begin{tabular}{|c|c|c|c|c|c|c|}
\hline \multirow{2}{*}{ 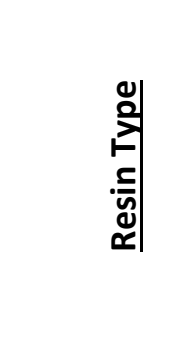 } & \multirow{2}{*}{ 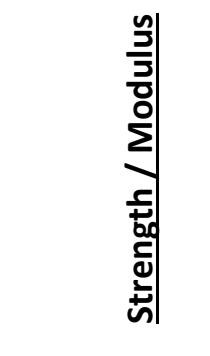 } & \multirow{2}{*}{ 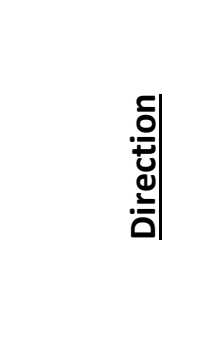 } & \multirow{2}{*}{ 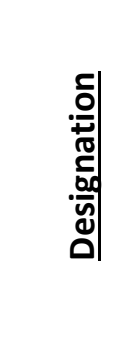 } & \multirow{2}{*}{ 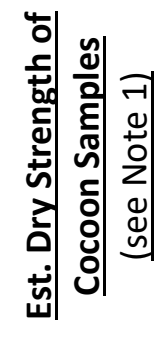 } & \multicolumn{2}{|c|}{$\begin{array}{c}\text { TSF } \\
\frac{\text { Time Shift Factors }}{\text { (see Note } 2 \text { ) }}\end{array}$} \\
\hline & & & & & Raw & Final \\
\hline \multirow{8}{*}{ Vinylester } & Tensile SR & Transverse & VE-TT & $112.0 \%$ & 40.3 & 36.0 \\
\hline & Tensile SR & Longitudinal & VE-TL & $98.2 \%$ & 40.3 & 41.1 \\
\hline & Flexural SR & Transverse & VE-FT & $117.3 \%$ & 40.3 & 34.4 \\
\hline & Flexural SR & Longitudinal & VE-FL & $117.8 \%$ & 40.3 & 34.2 \\
\hline & Shear SR & Transverse & VE-ST & $85.6 \%$ & 40.3 & 47.1 \\
\hline & Shear SR & Longitudinal & VE-SL & $92.6 \%$ & 40.3 & 43.5 \\
\hline & Tensile Mod & Transverse & VE-ET & $106.3 \%$ & 40.3 & 37.9 \\
\hline & Tensile Mod & Longitudinal & VE-EL & $101.5 \%$ & 40.3 & 39.7 \\
\hline \multirow{8}{*}{ Polyester } & Tensile SR & Transverse & PE-TT & $110.8 \%$ & 26.6 & 24.0 \\
\hline & Tensile SR & Longitudinal & PE-TL & $113.5 \%$ & 26.6 & 23.4 \\
\hline & Flexural SR & Transverse & PE-FT & $103.3 \%$ & 26.6 & 25.7 \\
\hline & Flexural SR & Longitudinal & PE-FL & $120.5 \%$ & 26.6 & 22.0 \\
\hline & Shear SR & Transverse & PE-ST & $106.5 \%$ & 26.6 & 24.9 \\
\hline & Shear SR & Longitudinal & PE-SL & $109.7 \%$ & 26.6 & 24.2 \\
\hline & Tensile Mod & Transverse & PE-ET & $126.5 \%$ & 26.6 & 21.0 \\
\hline & Tensile Mod & Longitudinal & PE-EL & $104.6 \%$ & 26.6 & 25.4 \\
\hline
\end{tabular}

Note 1: The dry strength of cocoon or aged samples is computed (with reference to dry virgin strength as $100 \%$ ), assuming that the rate of degradation of cocoon samples is the same as the rate of degradation of virgin samples.

Note 2: Due to missing test data of cocoon samples, several assumptions, as listed in section 6.3.3, have been made to arrive at TSF values for all samples. It should be noted that TSF computed are approximate and only shown for the illustration of the BOD development process based on the proposed reliability framework.

The following steps show the TSF computation process used in this study:

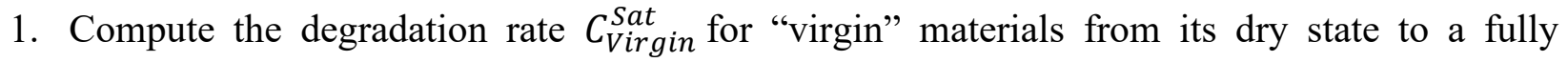
saturated state. Project researchers measured the time (t) required for this state transition as 65 days for virgin VE and 228 days for virgin PE. 


$$
C_{\text {Virgin }}^{\text {Sat }}=\frac{\left(1-S R_{\text {Virgin }}^{\text {Sat }}\right)}{\operatorname{Ln}(t)} \quad \ldots E q(6.9)
$$

Note: As an example, for vinylester GFRP tensile transverse strength, using $\mathrm{t}=65$ and $S R_{\text {Virgin }}^{\text {Sat }}=86.6 \%$, will provide $C_{\text {Virgin }}^{\text {Sat }}$ of $3.206 \%$.

2. Using the full saturation degradation rate computed in the previous step, calculate the SR for virgin material at the time when "cocoon" material reaches the saturated state. Project researchers measured this time (t) as 65 days for aged VE and 100 days for aged PE.

$$
S R_{\text {Virgin }}^{\text {Sat }}=1-C_{\text {Virgin }}^{\text {Sat }} x \operatorname{Ln}(t) \ldots E q(6.10)
$$

Note: As an example, for vinylester GFRP tensile transverse strength, using $\mathrm{t}=65$ and $C_{\text {Virgin }}^{\text {Sat }}$ of $3.206 \%$, the computed $S R_{\text {Virgin }}^{\text {Sat }}$ will be $86.6 \%$. In this case, results from Eq. 6.9 and Eq. 6.10 are the same since the time $(\mathrm{t})$ is 65 for both vinylester GFRP virgin and cocoon samples; however, that is not the case with polyester GFRP.

In the following steps, we will make the following three key assumptions: i) the rate of degradation for cocoon is the same from a dry state of the virgin to a saturated state of the virgin $\left(C_{\text {Virgin }}^{\text {Dry }}=C_{\text {Cocoon }}^{\text {Dry }}\right)$, ii) the influence of hydrostatic pressure is not significant in calculating the final saturated and aged degradation rates. However, very high hydrostatic pressure has been known to accelerate the degradation of certain properties, and the overall degradation rates can be adjusted to account for hydrostatic pressures (between natural aging and test environments) with additional test data and/or prior established relationships. However, the test data does not account for this condition, and minimal information about natural and lab hydrostatic environments is available (assume no hydrostatic pressure was used in lab testing). The author acknowledges that 
this assumption can introduce errors, and iii) this TSF calculation exercise intends to extract/project aged strength/stiffness data solely to illustrate the use of the proposed framework. Inaccuracies in computing TSF, although critical in professional practice, are considered acceptable within the context of this report. With the above assumptions, adjust (i.e., increase/decrease) the cocoon's material SR to be comparable to virgin materials.

3. Compute the difference between saturated SR virgin and SR cocoon from the test data.

$$
\Delta S R_{\text {Cocoon }}^{\text {Sat }}=S R_{\text {Virgin }}^{\text {Sat }}-S R_{\text {Cocoon }}^{\text {Sat }}
$$

Note: Assume that virgin and cocoon samples were subjected to saturation from all sides during natural and lab conditions. As an example, for vinylester GFRP tensile strength, for the tested values of $S R_{\text {Virgin }}^{\text {Sat }}=86.6 \%$ and $S R_{\text {Cocoon }}^{\text {Sat }}=74.6 \%, \Delta S R_{\text {Cocoon }}^{\text {Sat }}$ can be computed as $12 \%$.

4. Estimate original dry cocoon strength as compared to $100 \%$ of virgin dry strength

$$
\begin{aligned}
& S R_{\text {Cocoon }}^{\text {Dry }}=S R_{\text {Virgin }}^{\text {Dry }}+\Delta S R_{\text {Cocoon }}^{\text {Sat }} \text { or } \\
& S R_{\text {Cocoon }}^{\text {Dry }}=100 \%+\Delta S R_{\text {Cocoon }}^{\text {Sat }}
\end{aligned}
$$

Note: All SRs are normalized with reference to the dry virgin SR $\left(S R_{\text {Virgin }}^{\text {Dry }}\right)$ and expressed as percentages while dry virgin SR is $100 \%$. Based on the property under consideration, the estimated dry strength of cocoon can be higher or lower than the dry strengths of virgin material. For our example of vinylester GFRP tensile strength, the estimated (or adjusted) $S R_{\text {Cocoon }}^{\text {Dry }}$ will be $112 \%$ 
5. Adjust all tested saturated/aged SRs of cocoon samples $\left(S R_{\text {Cocoon }}^{\text {Test }}\right)$ at the various aging periods to be comparable with virgin material (since all values are normalized to $100 \%$ dry strengths, adjusted values can be found by direct multiplications):

$$
\text { Adjusted SR } R_{\text {Cocoon }}^{\text {Aged }}=S R_{\text {Cocoon }}^{\text {Dry }} \times S R_{\text {Cocoon }}^{\text {Test }}
$$

Note: Above is an approximation to compensate for the unknown dry strength of the cocoon at the beginning of the saturation/aging tests, as well as the time of its original placement in service, about seven years ago in a subsea environment. For our example of vinylester GFRP for tensile strength, all tabulated strengths of cocoon samples will be multiplied by $112 \%$ so that they can be regressed with virgin samples to obtain overall aging coefficients.

6. If dry strengths of virgin and cocoon were precisely the same, a raw TSF could be computed easily. For $t_{\text {natural env }}$ or the time in the natural environment (which is seven years) and $t_{l a b}$ is the duration of testing, compute raw TSF factor as:

$$
T S F_{\text {raw }}=\frac{\left(t_{\text {natural env }}+t_{\text {lab }}\right)}{t_{\text {lab }}}
$$

Note: This TSF primarily accounts for the aging timeline due to saturation since this is the only value for which both virgin (in the lab environment) and cocoon samples (in natural and lab environments) can be correlated. It is a very approximate estimate given the nature of many missing key parameters. For our example of vinylester GFRP for tensile strength, $t_{\text {natural env }}$ is $7 \times 365=2555$ days, number of saturation days in the lab $t_{l a b}$ is 65 , and $T S F_{\text {raw }}$ is 40.3 .

7. Since TSF is used with reference to the $100 \%$ of the dry strengths of the virgin materials, normalize TSF to account for the adjusted dry SR of cocoon material: 


$$
T S F=T S F_{\text {raw }}-S R_{\text {Cocoon }}^{\text {Dry }}
$$

Note: This TSF accounts for any adjustment needed if the computed dry strength of cocoon is lower or higher than $100 \%$ value of dry strength of virgin samples. In our example of vinylester GFRP tensile strength, the estimated $S R_{\text {Cocoon }}^{\text {Dry }}$ is $112 \%$, and adjusting it to the $100 \%$ of dry virgin SR will provide a final TSF value of 36 . The author acknowledges that TSFs computed in this study seem to be on the higher side, and it would have been beneficial to have more data available to validate the computed TSFs further.

8. Projected time in lab's accelerated testing environment for both virgin and cocoon materials properties (adjusted properties as discussed in previous steps) to the time in the referenced natural environment (i.e., $5^{\circ} \mathrm{C}$ in seawater at a depth of 300 feet):

$$
t_{\text {natural env }}=t_{\text {lab }} \times T S F
$$

Note: For many reasons cited in previous paragraphs, the computed final TSF will still be approximate. Without new information, it will be almost impossible to accurately compute TSF values for various properties tested under the project accelerated test environments. For our example of vinylester GFRP tensile strength, 65 days in the lab can be projected to $t_{\text {natural env }}=$ 2339 days for both virgin and cocoon samples. For polyester, $t_{\text {natural env }}$ will be different since $t_{l a b}$ is different for virgin and cocoon samples. Table 6.2 shows the final TSF used in this study.

\subsubsection{Data Analysis Results}

The following is a summary of data analysis and corresponding results for the original test data provided and as modified in this report. A limited comparison with Subsea Research Project 
is also made where appropriate. More details of regression analyses for various FRP type and material strengths can be found in Appendix C (Figures C.1 to C.18). The measured and projected coupon-based values of ultimate tensile, flexural, and shear strengths $\left(f_{u}\right.$, ave $)$ and average elastic modulus $\left(\mathrm{E}_{\mathrm{ave}}\right)$ of the specimens for both virgin and cocoon material at different aging intervals are presented in a graphical format for both transverse and longitudinal directions (Figure 6.5 and 6.6, and Appendix A). Since our objective is to develop tools (e.g., Framework) for conducting time-dependent reliability-based durability analysis, besides removing outliers and incomplete datasets, no attempt has been made to justify the reasons for the data scatter and the low number of data points.

It should be noted that the initial drop in the strength due to saturation with seawater is considered as a part of aging in seawater starting at time "zero" and is included in these graphs. All data is represented as a percentage of dry virgin sample strength at the beginning of the test. Due to the use of logarithmic equations to model degradation, the onset of aging time is presented as day 1 (or 0.003 year) instead of "zero." The graphs are self-explanatory and are presented to illustrate the process used to obtain projected average ultimate strengths for different service years, and associated standard deviations (often referred to as Coefficient of Variation or COV when normalized data is used) over the projected life span.

Most data fits within the mean \pm 3 standard deviations ( $99.9 \%$ confidence limits) range, and only a few outliers outside this range were found and ignored. It should be noted that the Project used mean \pm 2 standard deviations (64.9\% confidence limits) range to remove outliers. Simple regression using the sum of least squares was used to keep the benchmark strength at $100 \%$ at the beginning of the aging period. 
To provide a better understanding of the performance of the material over time and ease the comparison of data, all the experimental points (i.e., virgin and cocoon material) were regressed and plotted together. Then, the best fit (solid line) of the virgin material data (mean value) was produced, followed by two lines (dashed lines) representing a distance of $\pm 3 \times$ standard deviation from the mean.

\subsubsection{Verification of Distribution Type}

For reliability analysis, the type of distribution (e.g., normal, lognormal, or Weibull) can be significant. So far, we have assumed that the resulting distribution type is "normal." In this case, we are assuming that aged strength retention values (over the samples' service lives) deviate from the regressed mean values in the "normal" distribution pattern. In general, if there are enough data points (test readings), most distributions tend to follow a normal distribution. Weibull (two or three parameters) or lognormal distributions also have been used extensively for FRP properties (see discussion about Central Limit Theorem in Section 2.5). Use of "normal" density function generally simplifies the reliability analysis, and given many approximations already made in arriving at the projected life spans from accelerated testing, the use of more complex distribution types may not yield more representative results. In order to justify the use of the normal distribution, an Excel Add-On "SPC for Excel 6" was used to check the best distribution fit for the residuals. The program uses Maximum Likelihood Estimation (MLE) technique, AndersonDarling (AD) statistics with p-value, and Akaike Information Criterion (AIC). In this case, AIC was considered the primary measure of goodness of fit (GOF), and all other measurements also support a similar conclusion. About 650 residual strength retention values (difference from actual testing strength of all samples to the regressed mean value over samples life-span) were analyzed for the best distribution fit. The "normal" distribution was found to be the best overall fit, followed 
by three-parameter Lognormal and Weibull. Table 6.3 shows a summary of the comparison using the descriptive statistics of the residuals in a goodness of fit matrix.

Table 6.3: Summary of Comparison of Distribution Type

\begin{tabular}{|c|c|c|c|c|c|c|}
\hline \multirow[b]{2}{*}{ Distribution Type Tested } & \multirow[b]{2}{*}{$\begin{array}{c}\text { GOF } \\
\text { Ranki } \\
\text { ng } \\
\end{array}$} & \multicolumn{5}{|c|}{ The Goodness of Fit (GOF) Measures } \\
\hline & & $\begin{array}{l}\text { Log- } \\
\text { Likelihood }\end{array}$ & $\begin{array}{l}\text { Anderson- } \\
\text { Darling (AD) }\end{array}$ & p-Value & $\begin{array}{l}\text { Likeliho } \\
\text { od Ratio } \\
\text { Test } \\
\text { (LTR) }\end{array}$ & $\begin{array}{l}\text { Akaike } \\
\text { Informati } \\
\text { on } \\
\text { Criterion } \\
\text { (AIC) }\end{array}$ \\
\hline Normal & 1 & 769.1 & 0.348 & 0.477 & & -1534.3 \\
\hline LogNormal - 3 Parameter & 2 & 769.2 & 0.376 & 0.410 & 0.000 & -1532.4 \\
\hline Logistic & 3 & 767.5 & 0.520 & 0.147 & & -1531.0 \\
\hline Weibull - 3 Parameter & 4 & 763.9 & 0.916 & 0.021 & 0.000 & -1521.8 \\
\hline Largest Extreme Value & 5 & 713.5 & 9.390 & $<0.01$ & & -1423.0 \\
\hline Smallest Extreme Value & 6 & 702.8 & 9.792 & $<0.01$ & & -1401.6 \\
\hline Gamma - 3 Parameter & 7 & 692.9 & 8.213 & $<0.005$ & 0.000 & -1379.8 \\
\hline Exponential - 2 Parameter & No Fit & 283.6 & 143.0 & $<0.001$ & 0.000 & -563.1 \\
\hline
\end{tabular}

Note: All of the above values are dimensionless.

\begin{tabular}{clllllll}
\multicolumn{2}{l}{ Descriptive Statistics } \\
\hline Count & Mean & StDev & Median & Min & Max & Skew & Kurtosis \\
\hline 659 & -0.00143 & 0.0754 & -0.000586 & -0.241 & 0.252 & 0.0336 & 0.328 \\
\hline
\end{tabular}

A histogram with a normal PDF of residual SR with P-P (theoretical probability vs. experimental data probability) plot is also shown in Fig 6.4. The near straight line of the P-P plot represents a strong indication of normal distribution. 

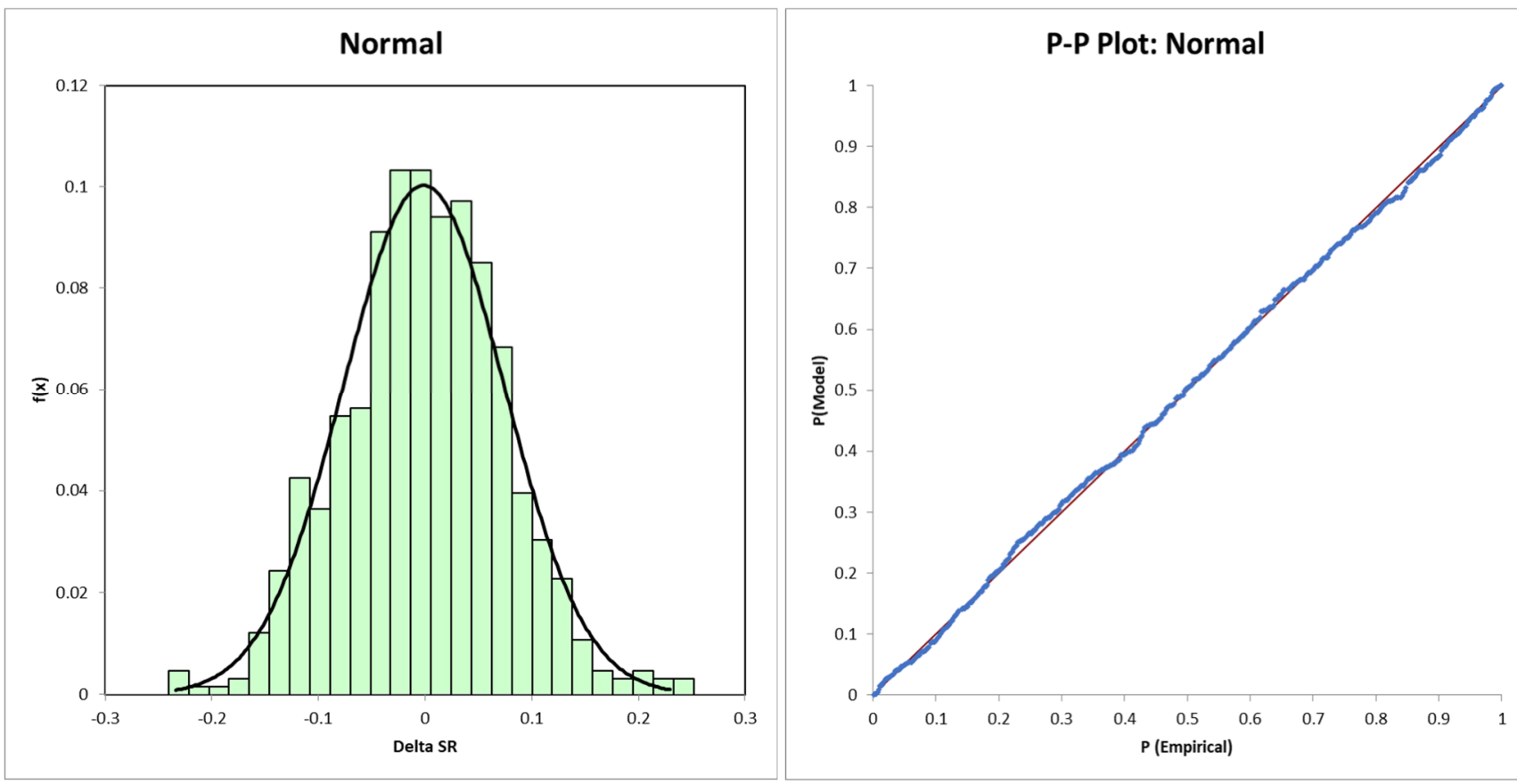

Figure 6.4: Histogram and P-P plot of Distribution of SR Residuals

The descriptive statistics (Table 6.3) are analyzed for the normality of the data (Figure 6.4). It was concluded for a comprehensive normalized test dataset, the difference between the actual and projected (mean) values can be represented using a "normal" distribution for reliability analysis. There is a large scatter of data, a few outliers, and often not enough data points for precise property plots; however, if all residual data are combined (i.e., number of observations increases above 100 and aging period is in order of 10 to 100 years), the distribution is mostly "normal" within the context of the data available for this study.

\subsubsection{Determination of Material Degradation Coefficients}

After distribution types were verified, the results of regression analysis were normalized using TSF on a single timeline (projected years) for immersion use in 5 degrees $\mathrm{C}$ seawater at 300 
feet depth. Various final results are shown in Tables 6.4 and 6.5. It should be noted that the data presented in these tables are based on project-specific ATM tests of 5 samples only and have not been verified for consistency with commonly known FRP behaviors and other published information. After the completion of the regression and timeline project, it is a good practice to review the details. For example, matrix dominated properties (shear) and cross-fiber orientations (typically in the transverse direction) generally have higher degradation coefficients than fiber dominated properties (tension or flexure) and along the primary fiber orientations (typically in the longitudinal direction). If fiber volume fraction (fvf) of a sample is highly skewed in favor of the longitudinal orientation of the sample, the difference between the transverse and longitudinal properties can be substantial. Similarly, for a member with a balanced fvf, the average transverse and longitudinal properties can be used. In the author's opinion, it is a safe practice to use the lower value unless the information about the member's FRP architecture with respect to the loading is available.

If the computed degradation coefficients (as shown in Tables 6.4 and 6.5) do not match these trends, they should be further reviewed for errors in data collecting, reporting, analyzing, regression, and Arrhenius projections. However, we do not have access to such information, and we will proceed with the assumptions that these values are acceptable within the context of this study and will use them to develop sample BOD details. 
Table 6.4: Final Aging Coefficients by FRP Type

\begin{tabular}{|c|c|c|c|c|c|}
\hline Resin & $\begin{array}{l}\text { Property } \\
\text { Description }\end{array}$ & Direction & Designation & $\begin{array}{l}\text { Projected } \\
\text { Years }\end{array}$ & $\begin{array}{l}\text { Degradation } \\
\text { Coefficients }\end{array}$ \\
\hline \multirow{8}{*}{ Vinylester } & \multirow{2}{*}{$\begin{array}{l}\text { Tensile Strength } \\
\text { Degradation Rate }\end{array}$} & Transverse & \multirow{2}{*}{$\mathrm{C}_{\mathrm{age} \text {, tensile SR }}$} & 38 & $2.70 \%$ \\
\hline & & Longitudinal & & 42 & $3.96 \%$ \\
\hline & \multirow{2}{*}{$\begin{array}{l}\text { Flexural Strength } \\
\text { Degradation Rate }\end{array}$} & Transverse & \multirow{2}{*}{$C_{\text {age, flexural SR }}$} & 37 & $2.59 \%$ \\
\hline & & Longitudinal & & 36 & $2.96 \%$ \\
\hline & \multirow{2}{*}{$\begin{array}{l}\text { Shear Strength } \\
\text { Degradation Rate }\end{array}$} & Transverse & \multirow{2}{*}{$\mathrm{C}_{\text {age, shear SR }}$} & 47 & $1.81 \%$ \\
\hline & & Longitudinal & & 44 & $2.37 \%$ \\
\hline & \multirow{2}{*}{$\begin{array}{l}\text { Elastic Modulus } \\
\text { Degradation Rate }\end{array}$} & Transverse & \multirow{2}{*}{$C_{\text {age, elastic mod }}$} & 39 & $0.72 \%$ \\
\hline & & Longitudinal & & 41 & $0.75 \%$ \\
\hline \multirow{8}{*}{ Polyester } & \multirow{2}{*}{$\begin{array}{l}\text { Tensile Strength } \\
\text { Degradation Rate }\end{array}$} & Transverse & \multirow{2}{*}{$\mathrm{C}_{\mathrm{age} \text {, tensile } \mathrm{SR}}$} & 64 & $2.39 \%$ \\
\hline & & Longitudinal & & 64 & $1.48 \%$ \\
\hline & \multirow{2}{*}{$\begin{array}{l}\text { Flexural Strength } \\
\text { Degradation Rate }\end{array}$} & Transverse & \multirow{2}{*}{$C_{\text {age, flexural } S R}$} & 68 & $2.01 \%$ \\
\hline & & Longitudinal & & 58 & $3.10 \%$ \\
\hline & \multirow{2}{*}{$\begin{array}{l}\text { Shear Strength } \\
\text { Degradation Rate }\end{array}$} & Transverse & \multirow{2}{*}{$\mathrm{C}_{\text {age, shear SR }}$} & 66 & $3.55 \%$ \\
\hline & & Longitudinal & & 64 & $3.03 \%$ \\
\hline & \multirow{2}{*}{$\begin{array}{l}\text { Elastic Modulus } \\
\text { Degradation Rate }\end{array}$} & Transverse & \multirow{2}{*}{$\mathrm{C}_{\text {age, elastic mod }}$} & 56 & $2.34 \%$ \\
\hline & & Longitudinal & & 67 & $0.40 \%$ \\
\hline
\end{tabular}

Notes: Degradation Equation: Strength Retained (tyrs) = Virgin Dry Strength $x$ (1 - Cage $x \ln ($ tyrs x 365) ) The readers are cautioned that the above values are developed for a project-specific use and have not been validated or verified with other published data. Verification of all tabulated values for use in design (or fabrication) is highly recommended. 
Table 6.5: Projected Strength Retentions (SR) by Age

\begin{tabular}{|c|c|c|c|c|c|c|c|c|c|}
\hline \multirow{2}{*}{$\begin{array}{l}\text { Resin } \\
\text { Type }\end{array}$} & \multirow{2}{*}{$\begin{array}{c}\text { Strength Retention (SR)/ } \\
\text { Elastic Modulus }\end{array}$} & \multirow{2}{*}{ Designation } & \multicolumn{7}{|c|}{$\begin{array}{c}\text { Estimated \% Ultimate Strength Retained } \\
\left\{f_{u, \text { ave }}\right\} \text { (or \%Elastic Modulus }\left\{E_{\text {ave }}\right\} \text { ) at } \\
\text { Service Age (Years) }\end{array}$} \\
\hline & & & 1 & 5 & 10 & 25 & 50 & 75 & 100 \\
\hline \multirow{8}{*}{ Vinylester } & Tensile SR-Transv. & VE-TT & 84 & 80 & 78 & 75 & 74 & 72 & 72 \\
\hline & Tensile SR-Longit. & VE-TL & 77 & 70 & 68 & 64 & 61 & 60 & 58 \\
\hline & Flexural SR-Transv. & VE-FT & 85 & 81 & 79 & 76 & 75 & 74 & 73 \\
\hline & Flexural SR-Longit. & VE-FL & 83 & 78 & 76 & 73 & 71 & 70 & 69 \\
\hline & Shear SR-Transv. & VE-ILSS-T & 89 & 86 & 85 & 83 & 82 & 82 & 81 \\
\hline & Shear SR-Longit. & VE-ILSS-L & 86 & 82 & 81 & 78 & 77 & 76 & 75 \\
\hline & Tensile Modulus -Transv. & VE-ET-T & 96 & 95 & 94 & 93 & 93 & 93 & 92 \\
\hline & Tensile Modulus-Longit. & VE-ET-L & 96 & 94 & 94 & 93 & 93 & 92 & 92 \\
\hline \multirow{8}{*}{ Polyester } & Tensile SR-Transv. & PE-TT & 86 & 82 & 80 & 78 & 77 & 76 & 75 \\
\hline & Tensile SR-Longit. & PE-TL & 91 & 89 & 88 & 86 & 85 & 85 & 84 \\
\hline & Flexural SR-Transv. & PE-FT & 88 & 85 & 84 & 82 & 80 & 79 & 79 \\
\hline & Flexural SR-Longit. & PE-FL & 82 & 77 & 75 & 72 & 70 & 68 & 67 \\
\hline & Shear SR-Transv. & PE-ILSS-T & 79 & 73 & 71 & 68 & 65 & 64 & 63 \\
\hline & Shear SR-Longit. & PE-ILSS-L & 82 & 77 & 75 & 72 & 70 & 69 & 68 \\
\hline & Tensile Modulus -Transv. & PE-ET-T & 86 & 82 & 81 & 79 & 77 & 76 & 75 \\
\hline & Tensile Modulus-Longit. & PE-ET-L & 98 & 97 & 97 & 96 & 96 & 96 & 96 \\
\hline
\end{tabular}

Note All aged material properties shown as a percentage of manufactured properties initially before placement in service in $5^{\circ} \mathrm{C}$ seawater up to 300 feet depth. In the author's opinion, the projected strength retentions values (except stiffness) computed for this table seems to be on a higher side than typically found in long-term natural and accelerated test data for vinylester and polyester-based GFRPs. The readers are cautioned that the above data is derived based on project-specific accelerated tests and should not be used for any other purposes without a thorough review and verifications.

The following are a few graphic representations (Figures 6.5 and 6.6) of the above data with both test data for virgin and cocoon samples. The mean value of SR (strength/stiffness retention) aging equation (for the lab as well as projected life-span) for the "dry" virgin material property is shown as a solid line, and upper bound and lower bound limits of SR equations are shown as dashed lines. Upper and lower bound lines were plotted by adding/subtracting three 
times the computed standard deviation (of the dataset population) to the mean values. The purpose of plotting the upper and lower bound lines was to identify any outlier and skewness of the data visually. By inspection, it appears that except for a few outliers, the lower and upper bound lines could have been narrowed to two times the standard deviation around the mean (i.e., $\mu \pm 2 \sigma$ ).

Limited datasets in a graphical format are included in the main report to avoid clutter. See Appendix $\mathrm{C}$ for tables and figures for all relevant ATM test results and projected Arrhenius curves (Table C.1 and Figures C.1 to C.18).

Figure 6.5: Vinylester Tensile Transverse SR data in Laboratory

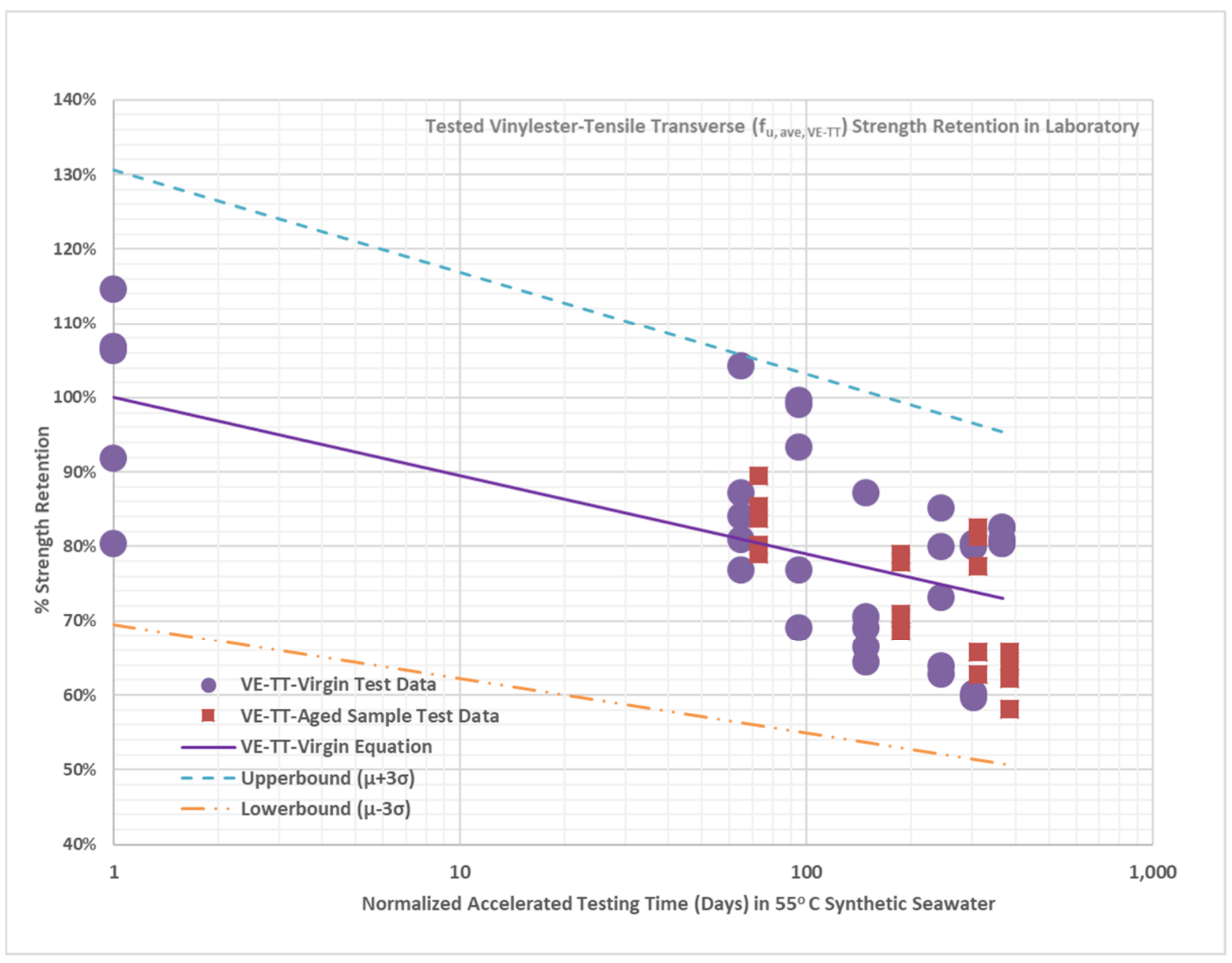




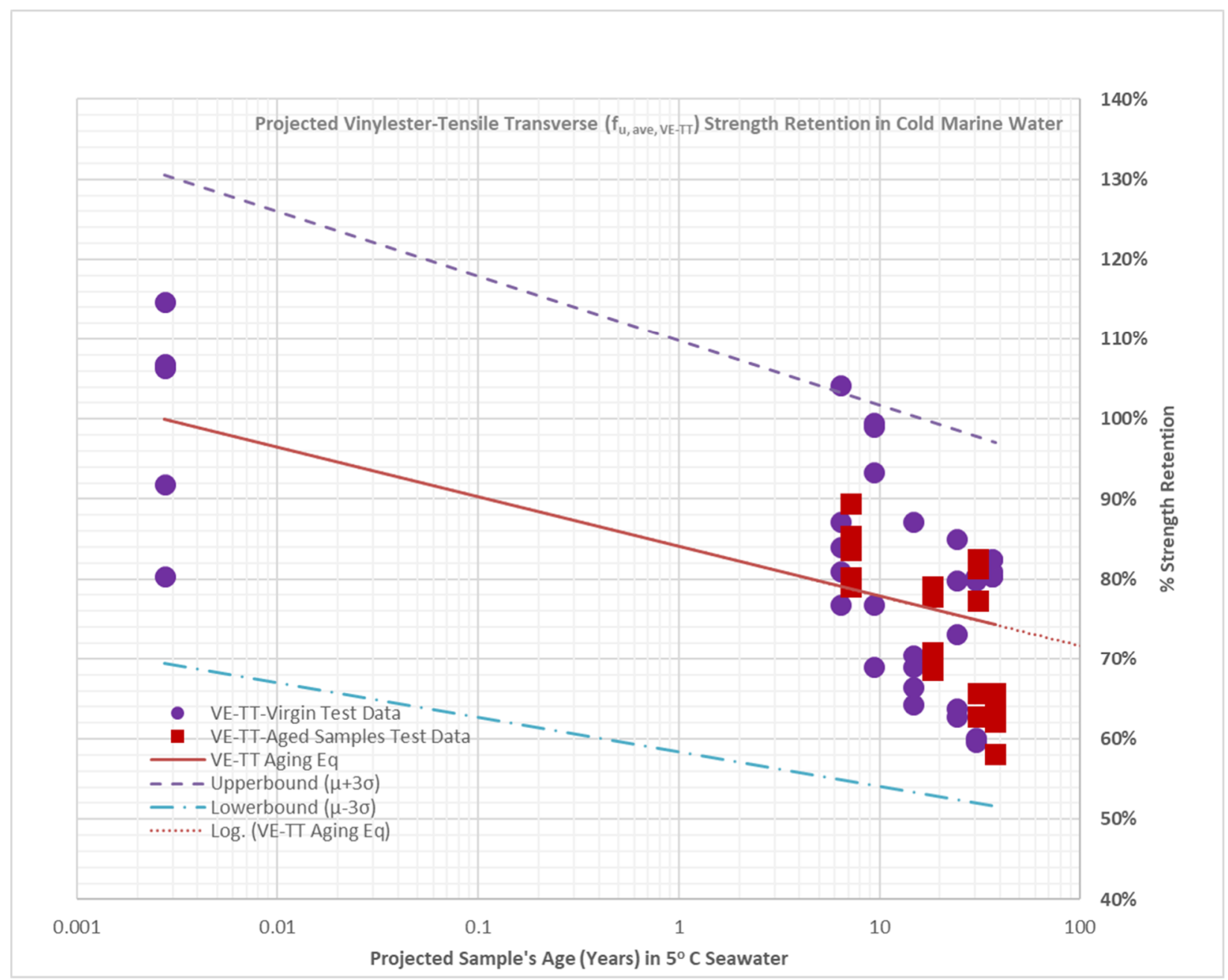

Figure 6.6: Projected Vinylester Tensile Transverse SR data in Seawater

\subsubsection{Development of Basis of Design (BOD)}

Basis of Design (BOD) is a professional tool that is used by design professionals based on the research findings. The BOD technical documentation identifies how the design provides the performance and operational requirements of a project. The BOD is developed by the architect/engineer (A/E) early in the project development stage, in technical terms, based on the owner's project requirements, technical approach planned, and the proposed design parameters. It 
is not a binding document such as (final specifications or contract agreement), but its primary purpose is to aid designers, often at the beginning stages of a project, to understand and document the technical parameters that will be used to develop the final design and seek acceptance with the project stakeholders (e.g., owners, end-users, manufacturers, fabricators, constructors, maintenance personnel, etc.).

The BOD provides designers with necessary technical information about (or a validated estimate of) the material properties, strength reduction factors, factors of safety, design methodology, etc. to meet the components' expected service life and functional requirements. Once the project sponsors have accepted the BOD, designers can proceed to design a structure/system with the confidence that their design will serve the intended narrowly focused purposes. Generally, the development of BODs can be considered to be the first logical step towards the development of industrywide practice guidelines or design codes.

The following illustrates the BOD process for GFRP with vinylester or polyester resin systems (located at seafloor) in a submerged environment of cold marine water.

\subsubsection{Average Mean Material Properties}

Most ASTM methods prefer a minimum of 30 samples for determining the average mean value. This information, although known to the manufacturer but is not easy to obtain by a designer. If this information is not available from a manufacturer or the project budget (or schedule) does not allow the laboratory testing, then pre-established Bias Factors (BF), as shown in Section 6.3.5.3 and Table 6.8, can be used.

In our case, only five tests of each virgin material property are available, which is a small number for more representative statistical results. If a member were isotropic (e.g., steel), it would have been acceptable to combine longitudinal and transverse property test results to obtain a more 
significant sample population. However, FRP is an anisotropic material with properties designed for a specific direction of loading, so that option typically not available (as is the case with study). It is important to keep in mind how the FRP member is loaded with respect to the transverse or longitudinal test properties (see Figure 6.2 for the location of test samples). Since FRP members have a predominant strength direction (e.g., longitudinal properties can be approximately 2-3 times greater than the transverse properties), coupon tests in the longitudinal direction provide reasonable indications regarding "fiber dominated" properties (e.g., tensile, flexure), and likewise, coupon tests in the transverse direction show similar trends for "matrix dominated" properties (e.g., shear).

In general, beams, columns, or bracings are placed to take advantage of their strong directional properties; therefore, the use of stronger "longitudinal" properties will be appropriate. In Example No. 2 (Appendix A), we can assume that both the web and flanges have similar properties and loaded along its length and in Example No. 3, the tension bracing is in tension along its length (pulling at ends), then the use of "longitudinal" properties will be appropriate for both. However, if we do not have information about the FRP member architecture or loading orientation with respect to the member, it may be conservative (perhaps, too conservative) to use the "lower" properties to allow any generic loading and component orientation. Averaging of properties (from multiple orientations) is not recommended for FRP composites.

Table 6.5 shows the average or mean value of various mechanical properties of vinylester and polyester-based dry virgin GFRP composites (6" and 8" beams, Figure 6.2) as tested by the project researchers. Based on the disparity of results between the transverse and longitudinal properties (Tables 6.6, 6.7, and 6.8), it can be concluded that fiber volume fraction is not balanced, particularly for polyester samples. Custom FRP sections are often designed to take advantage of 
their stronger section properties in primary loading directions, and such an imbalance in FVF is expected. The actual FVF details of both vinylester or polyester-based GFRP sections as used in the project testing are not available, and this BOD is explicitly developed for specific tested sections for specific subsea applications; therefore, no adjustment to the test results reported by the project researchers is made.

Table 6.6: Average Tested GFRP Material Properties

\begin{tabular}{|c|c|c|c|c|}
\hline Resin & Property Description & Direction & $\begin{array}{l}\text { Designation } \\
\text { (Units) }\end{array}$ & $\begin{array}{c}\text { Mean Value } \\
(\mu)\end{array}$ \\
\hline \multirow{8}{*}{ Vinylester } & \multirow{2}{*}{$\begin{array}{l}\text { Ave. Ultimate Tensile } \\
\text { Strength }\end{array}$} & Transverse & \multirow{2}{*}{$\begin{array}{c}\mathrm{f}_{\mathrm{u}, \text { ave, tensile }} \\
(\mathrm{MPa})\end{array}$} & 193 \\
\hline & & Longitudinal & & 302 \\
\hline & \multirow{2}{*}{$\begin{array}{l}\text { Ave. Ultimate } \\
\text { Flexural Strength }\end{array}$} & Transverse & \multirow{2}{*}{$\begin{array}{c}\mathrm{f}_{\mathrm{u}, \text { ave,flexural }} \\
(\mathrm{MPa})\end{array}$} & 372 \\
\hline & & Longitudinal & & 438 \\
\hline & \multirow{2}{*}{$\begin{array}{l}\text { Ave. Ultimate Shear } \\
\text { Strength }\end{array}$} & Transverse & \multirow{2}{*}{$\begin{array}{c}\mathrm{f}_{\mathrm{u}, \text { ave,shear }} \\
(\mathrm{MPa})\end{array}$} & 28 \\
\hline & & Longitudinal & & 30 \\
\hline & \multirow{2}{*}{$\begin{array}{l}\text { Ave. Tensile Elastic } \\
\text { Modulus }\end{array}$} & Transverse & \multirow{2}{*}{$\begin{array}{c}\text { Eave,tensile } \\
\text { (GPa) }\end{array}$} & 16 \\
\hline & & Longitudinal & & 20 \\
\hline \multirow{8}{*}{ Polyester } & \multirow{2}{*}{$\begin{array}{l}\text { Ave. Ultimate Tensile } \\
\text { Strength }\end{array}$} & Transverse & \multirow{2}{*}{$\begin{array}{c}\mathrm{f}_{\mathrm{u}, \text { ave, tensile }} \\
(\mathrm{MPa})\end{array}$} & 78 \\
\hline & & Longitudinal & & 294 \\
\hline & \multirow{2}{*}{$\begin{array}{l}\text { Ave. Ultimate } \\
\text { Flexural Strength }\end{array}$} & Transverse & \multirow{2}{*}{$\begin{array}{c}\mathrm{f}_{\mathrm{u}, \text { ave,flexural }} \\
(\mathrm{MPa})\end{array}$} & 118 \\
\hline & & Longitudinal & & 356 \\
\hline & \multirow{2}{*}{$\begin{array}{l}\text { Ave. Ultimate Shear } \\
\text { Strength }\end{array}$} & Transverse & \multirow{2}{*}{$\begin{array}{c}\mathrm{f}_{\mathrm{u}, \text { ave, shear }} \\
\text { (MPa) }\end{array}$} & 19 \\
\hline & & Longitudinal & & 27 \\
\hline & \multirow{2}{*}{$\begin{array}{l}\text { Ave. Tensile Elastic } \\
\text { Modulus }\end{array}$} & Transverse & \multirow{2}{*}{$\begin{array}{c}\text { Eave,tensile }_{\text {a }} \\
\text { (GPa) }\end{array}$} & 10 \\
\hline & & Longitudinal & & 21 \\
\hline
\end{tabular}

Note: The readers are cautioned that the above data is derived based on project-specific accelerated environment testing of about five samples for each listed property, as reported by the project researchers. The significant difference between longitudinal and transverse properties indicates the presence of an unbalanced fiber volume fraction (fvf). The above table values should not be used without a thorough review and verification of the data for the project-specific conditions. 


\subsubsection{Minimum Guaranteed or Nominal Material Properties}

It should be noted that the baseline references for any design are the material properties that are derived from ready-to-ship, dry, recently manufactured (within reasonable curing and wait period after manufacturing), unused (i.e., virgin), and approved under an acceptable manufacturer's Quality Control and Quality Assurance (QC/QA) program. Such nominal or characteristic material properties information is widely available and published by the FRP manufacturer or can be determined experimentally in a qualified testing laboratory. For special FRP applications such as subsea cocoon applications, it may be difficult to find published material data, and lab testing may be the only way to obtain these values. In this case, since we have the necessary material testing data, we will determine this value manually.

Since a set of tested material properties are available, a distribution curve for that property can be developed, and the nominal or characteristic properties can be determined using ASTM D7290-05 (2011) and also explained in Section 2.5.2. Often, there are not an adequate number of test results available to decide on the $80 \%$ lower confidence bound ( $20 \%$ significance) on the $5^{\text {th }}$ percentile value of a specified population using two-parameter Weibull distribution; instead, normal distribution function with 3 sigma (i.e., average mean value $-3 \times$ standard deviation) approach can be used to remove the $99.9 \%$ of upper-bound values for a minimum guaranteed strength (based on our test data). Additional discussion about the standard deviation multipliers that have been used to calculate characteristic values (for externally bonded FRP) is provided by Atadero and Karbhari (2006). Perhaps, if FRP member failure (for the property under discussion) does not lead to a catastrophic failure or the quality control in the manufacturing process is relatively consistent (e.g., resulting in very low and consistent COV), a lower multiplier may be used. In this case, due to the lack of an adequate number of tests or other relevant data, we will 
use the 3-sigma approach, as shown in Table 6.7 (values are based on virgin GFRP samples tested in this project).

Table 6.7: Nominal or Minimum Guaranteed GFRP Material Properties

\begin{tabular}{|c|c|c|c|c|c|c|}
\hline Resin & $\begin{array}{l}\text { Property } \\
\text { Description }\end{array}$ & Direction & $\begin{array}{l}\text { Designation } \\
\text { (Units) }\end{array}$ & $\begin{array}{c}\text { Mean } \\
\text { Value } \\
(\mu)\end{array}$ & $\begin{array}{c}\text { Std Dev } \\
(\sigma)\end{array}$ & $\begin{array}{c}\text { Nominal or } \\
\text { Min. } \\
\text { Guaranteed } \\
\text { Strength } \\
(\mu-3 \sigma) \\
\end{array}$ \\
\hline \multirow{8}{*}{ Vinylester } & \multirow{2}{*}{$\begin{array}{l}\text { Nominal Ultimate } \\
\text { Tensile Strength }\end{array}$} & Transverse & \multirow{2}{*}{$\begin{array}{c}\mathrm{f}^{*}{ }_{\mathrm{u} \text {,tensile }} \\
(\mathrm{MPa})\end{array}$} & 193 & 26 & 113 \\
\hline & & Longitudinal & & 302 & 17 & 251 \\
\hline & \multirow{2}{*}{$\begin{array}{l}\text { Nominal Ultimate } \\
\text { Flexural Strength }\end{array}$} & Transverse & \multirow{2}{*}{$\begin{array}{c}f^{*}{ }_{u, f l e x u r a l} \\
(\mathrm{MPa})\end{array}$} & 372 & 45 & 237 \\
\hline & & Longitudinal & & 438 & 39 & 322 \\
\hline & \multirow{2}{*}{$\begin{array}{l}\text { Nominal Ultimate } \\
\text { Shear Strength }\end{array}$} & Transverse & \multirow{2}{*}{$\begin{array}{l}f^{*}{ }_{u, \text { shear }} \\
(\mathrm{MPa})\end{array}$} & 28 & 1 & 25 \\
\hline & & Longitudinal & & 30 & 2 & 24 \\
\hline & \multirow{2}{*}{$\begin{array}{l}\text { Nominal Tensile } \\
\text { Elastic Modulus }\end{array}$} & Transverse & \multirow{2}{*}{$\begin{array}{l}E^{*} \text { tensile } \\
(\mathrm{GPa})\end{array}$} & 16 & 1 & 12 \\
\hline & & Longitudinal & & 20 & 1 & 17 \\
\hline \multirow{8}{*}{ Polyester } & \multirow{2}{*}{$\begin{array}{l}\text { Nominal Ultimate } \\
\text { Tensile Strength }\end{array}$} & Transverse & \multirow{2}{*}{$\begin{array}{l}\mathrm{f}^{*}{ }_{\mathrm{u} \text { tensile }} \\
\text { (MPa) }\end{array}$} & 78 & 4 & 65 \\
\hline & & Longitudinal & & 294 & 22 & 228 \\
\hline & \multirow{2}{*}{$\begin{array}{l}\text { Nominal Ultimate } \\
\text { Flexural Strength }\end{array}$} & Transverse & \multirow{2}{*}{$\begin{array}{c}f^{*}{ }_{u, f l e x u r a l} \\
\text { (MPa) }\end{array}$} & 118 & 6 & 101 \\
\hline & & Longitudinal & & 356 & 43 & 228 \\
\hline & \multirow{2}{*}{$\begin{array}{l}\text { Nominal Ultimate } \\
\text { Shear Strength }\end{array}$} & Transverse & \multirow{2}{*}{$\begin{array}{l}\mathrm{f}_{\mathrm{u}, \text { shear }} \\
(\mathrm{MPa})\end{array}$} & 19 & 1 & 17 \\
\hline & & Longitudinal & & 27 & 2 & 21 \\
\hline & \multirow{2}{*}{$\begin{array}{l}\text { Nominal Tensile } \\
\text { Elastic Modulus }\end{array}$} & Transverse & \multirow{2}{*}{$\begin{array}{l}E^{*} \text { tensile } \\
(\mathrm{GPa})\end{array}$} & 10 & 1 & 7 \\
\hline & & Longitudinal & & 21 & 4 & 9 \\
\hline
\end{tabular}

Note: The readers are cautioned that the above data is derived based on project-specific accelerated environment testing of about five samples for each listed property, as reported by the project researchers. The significant difference between longitudinal and transverse properties indicates the presence of an unbalanced fiber volume fraction (fvf). The above table values should not be used without a thorough review and verification of the above data for the project-specific conditions.

\subsubsection{Bias factors}

As explained in Section 2.5.2, establishing bias factors helps to back convert the nominal material property to the mean value or vice versa without a detailed material test program. We 
will calculate Bias Factors for each combined property as follows. Table 6.8 shows the bias factors computed for the virgin GFRP materials that can be used for this project.

Table 6.8: Bias Factors for GFRP

\begin{tabular}{|c|c|c|c|c|c|c|}
\hline Resin & $\begin{array}{l}\text { Property } \\
\text { Description }\end{array}$ & Direction & $\begin{array}{l}\text { Designation } \\
\text { (Units) }\end{array}$ & $\begin{array}{c}\text { Mean } \\
\text { Value } \\
(\mu)\end{array}$ & $\begin{array}{c}\text { Nominal } \\
(\mu-3 \sigma)\end{array}$ & $\begin{array}{c}\text { Bias } \\
\text { Factor } \\
(B F) \\
(\mu /(\mu-3 \sigma)\end{array}$ \\
\hline \multirow{8}{*}{ Vinylester } & \multirow{2}{*}{$\begin{array}{l}\text { Bias Factor for } \\
\text { Tensile Strength }\end{array}$} & Transverse & \multirow{2}{*}{$\begin{array}{l}\mathrm{BF}_{\text {tensile }} \\
(\mathrm{MPa})\end{array}$} & 193 & 113 & 1.7 \\
\hline & & Longitudinal & & 302 & 251 & 1.2 \\
\hline & \multirow{2}{*}{$\begin{array}{l}\text { Bias Factor for } \\
\text { Flexural Strength }\end{array}$} & Transverse & \multirow{2}{*}{$\begin{array}{l}\mathrm{BF}_{\text {flexural }} \\
(\mathrm{MPa})\end{array}$} & 372 & 237 & 1.6 \\
\hline & & Longitudinal & & 438 & 322 & 1.4 \\
\hline & \multirow{2}{*}{$\begin{array}{l}\text { Bias Factor for } \\
\text { Shear Strength }\end{array}$} & Transverse & \multirow{2}{*}{$\begin{array}{l}\mathrm{BF}_{\text {shear }} \\
(\mathrm{MPa})\end{array}$} & 28 & 25 & 1.1 \\
\hline & & Longitudinal & & 30 & 24 & 1.2 \\
\hline & \multirow{2}{*}{$\begin{array}{l}\text { Bias Factor for } \\
\text { Elastic Modulus }\end{array}$} & Transverse & \multirow{2}{*}{$\begin{array}{l}\text { BF }_{\text {Modulus }} \\
\text { (GPa) }\end{array}$} & 16 & 12 & 1.3 \\
\hline & & Longitudinal & & 20 & 17 & 1.1 \\
\hline \multirow{8}{*}{ Polyester } & \multirow{2}{*}{$\begin{array}{l}\text { Bias Factor for } \\
\text { Tensile Strength }\end{array}$} & Transverse & \multirow{2}{*}{$\begin{array}{l}\mathrm{BF}_{\text {tensile }} \\
(\mathrm{MPa})\end{array}$} & 78 & 65 & 1.2 \\
\hline & & Longitudinal & & 294 & 228 & 1.3 \\
\hline & \multirow{2}{*}{$\begin{array}{l}\text { Bias Factor for } \\
\text { Flexural Strength }\end{array}$} & Transverse & \multirow{2}{*}{$\begin{array}{l}\mathrm{BF}_{\text {flexural }} \\
(\mathrm{MPa})\end{array}$} & 118 & 101 & 1.2 \\
\hline & & Longitudinal & & 356 & 228 & 1.6 \\
\hline & \multirow{2}{*}{$\begin{array}{l}\text { Bias Factor for } \\
\text { Shear Strength }\end{array}$} & Transverse & \multirow{2}{*}{$\begin{array}{l}\mathrm{BF}_{\text {shear }} \\
(\mathrm{MPa})\end{array}$} & 19 & 17 & 1.1 \\
\hline & & Longitudinal & & 27 & 21 & 1.3 \\
\hline & \multirow{2}{*}{$\begin{array}{l}\text { Bias Factor for } \\
\text { Elastic Modulus }\end{array}$} & Transverse & \multirow{2}{*}{$\begin{array}{l}\text { BF Modulus } \\
\text { (GPa) }\end{array}$} & 10 & 7 & 1.3 \\
\hline & & Longitudinal & & 21 & 9 & 2.2 \\
\hline
\end{tabular}

Note: The readers are cautioned that the above data is derived based on project-specific accelerated environment testing of about five samples for each listed property, as reported by the project researchers. The significant difference between longitudinal and transverse properties indicates the presence of an unbalanced fiber volume fraction (fvf). The above table values should not be used without a thorough review and verification of the above data for the project-specific conditions.

\subsubsection{Coefficient of Variation (COV)}

There are two types of COV computed in this report: 1) COV of virgin material as calculated in previous sections and 2) COV of aged cocoon material over its life span. First, COV is easy to obtain from test results of the supplied virgin material, but the determination of aged $\mathrm{COV}$ is much more complicated. It requires a detailed analysis of test data, knowledge of the 
material tests performed, and Arrhenius projections from time-consuming accelerated testing programs. In the absence of detailed aged COV calculations, as a minimum, it should be assumed the same as virgin COV. However, in this case, since we have the data from aged samples, we will use the computed aged COV as also shown in Table 6.9 (for this project only).

Table 6.9: Coefficient of Variations (COV) for Virgin and Aged Materials

\begin{tabular}{|c|c|c|c|c|c|}
\hline Resin & Property Description & Direction & Designation & $\mathrm{COV}_{\text {virgin }}$ & $\operatorname{cov}_{\text {aged }}$ \\
\hline \multirow{8}{*}{ Vinylester } & \multirow{2}{*}{ COV for Tensile Strength } & Transverse & \multirow{2}{*}{$\mathrm{COV}_{\text {tensile }}$} & $14 \%$ & $10 \%$ \\
\hline & & Longitudinal & & $6 \%$ & $11 \%$ \\
\hline & \multirow{2}{*}{ COV for Flexural Strength } & Transverse & \multirow{2}{*}{$\mathrm{COV}_{\text {flexural }}$} & $12 \%$ & $8 \%$ \\
\hline & & Longitudinal & & $9 \%$ & $10 \%$ \\
\hline & \multirow{2}{*}{ COV for Shear Strength } & Transverse & \multirow{2}{*}{$\mathrm{COV}_{\text {shear }}$} & $3 \%$ & $5 \%$ \\
\hline & & Longitudinal & & $6 \%$ & $7 \%$ \\
\hline & \multirow{2}{*}{ COV for Tensile Elastic Modulus } & Transverse & \multirow{2}{*}{$\mathrm{COV}_{\text {modulus }}$} & $8 \%$ & $11 \%$ \\
\hline & & Longitudinal & & $4 \%$ & $14 \%$ \\
\hline \multirow{8}{*}{ Polyester } & \multirow{2}{*}{ COV for Tensile Strength } & Transverse & \multirow{2}{*}{$\mathrm{COV}_{\text {tensile }}$} & $5 \%$ & $7 \%$ \\
\hline & & Longitudinal & & $8 \%$ & $8 \%$ \\
\hline & \multirow{2}{*}{ COV for Flexural Strength } & Transverse & \multirow{2}{*}{$\mathrm{COV}_{\text {flexural }}$} & $5 \%$ & $6 \%$ \\
\hline & & Longitudinal & & $12 \%$ & $6 \%$ \\
\hline & \multirow{2}{*}{ COV for Shear Strength } & Transverse & \multirow{2}{*}{$\mathrm{COV}_{\text {shear }}$} & $3 \%$ & $6 \%$ \\
\hline & & Longitudinal & & $7 \%$ & $6 \%$ \\
\hline & \multirow{2}{*}{ COV for Tensile Elastic Modulus } & Transverse & \multirow{2}{*}{$\mathrm{COV}_{\text {modulus }}$} & $9 \%$ & $6 \%$ \\
\hline & & Longitudinal & & $18 \%$ & $10 \%$ \\
\hline
\end{tabular}

Note: The readers are cautioned that the above data is derived based on project-specific accelerated environment testing of about five samples for each listed property, as reported by the project researchers. The significant difference between longitudinal and transverse properties indicates the presence of an unbalanced fiber volume fraction (fvf). The above table values should not be used without a thorough review and verification of the above data for the project-specific conditions. In addition, the virgin COV is computed based on a very limited number of samples (total 4), and a large number of samples would have provided more representative values. 


\subsubsection{Expected Service Life}

The project researchers reported that a new subsea submerged cocoon is expected to last at least 20 years. For the study purposes, we will assume the expected design life for subsea immersion to be 25 years.

\subsubsection{Reliability-Based Design Method}

There are many ways to design this component using the conventional allowable/working stress design (ASD) method or a more current load and resistance factor (LRFD) design method. As also described in Chapter 4 and illustrated Examples (1-3 in Appendix A), ASD uses a factor of safety to reduce the ultimate mean strength to arrive at working strength, while LRFD uses various load factors to modify loads and strength reduction factors to reduce ultimate mean strength so that a probability of failure can be acceptable. In the previous chapters 3 and 4 , we have proven that without the application of the reliability-durability framework to an FRP design criteria development, we have no consistent way to determine if either the factor of safety (for use in WSD or working stress design) or the strength reduction factors (for use in LRFD or load and resistance factor design) will provide the minimum required structural safety.

In the absence of any new information, we will assume that a reliability index $(\beta)$ of 3.5 or more is acceptable, which roughly translates to a probability of failure of 1 in $4,300^{+}$. Modern codes have extensively used this value for the bridges, concrete, and steel in the last two decades, so it is a good start (see section 3.2.1. for additional discussion about the selection of an acceptable reliability index). Once an appropriate reliability index $(\beta)$ has been selected, a factor of safety or strength reduction factor can be computed to match the target probability of failure (Table 3.1). The reliability approach to BOD development refines the existing strength reduction factors (or factor of safety) to provide consistent reliability to all modes of failures (i.e., probability of failure 
will be consistent based on the modes of failures resulting from tension, compression, flexure, shear, etc.). Although FRP materials have lower shear strength (due to the faster aging of resin under varying environments) and exhibit highly anisotropic behavior (as compared to steel), the BOD process is the same as it is for tension or flexure.

Without the knowledge of detailed geometry or loading information on cocoon's structural framework (and sea undercurrent, fabrication/delivery requirements, installation conditions, etc.), it is difficult to determine controlling load effect and associated required GFRP section resistance. For simplicity of illustrating the reliability-durability design process, many assumptions about the load conditions and section property orientations are made. In practice, the designers will establish a BOD for particular requirements.

The illustrated examples (No. 2 and 3, Appendix A) show a step-by-step process to determine design parameters for the particular load cases shown for each member (i.e., tensile, flexural, shear, and elastic modulus). The example shows that the tension design of a bracing and flexural/shear design of a beam. With some additional details (e.g., loading combinations, frame geometry, connection details, etc. that the author does not have access to), design examples controlled by shear strength can be added to cover a more encompassing range of options. Keep in mind that a general guideline development for various specific subsea applications will require numerous iterations and many examples, and is considered out of scope for this study.

In a conventional professional practice (perhaps, sometime in the future for FRP materials), the designers are not needed to perform any testing or analysis to determine 1) the factor of safety or strength reduction factors, the average mean properties of virgin material, 2) accelerated testing and development of Arrhenius equations for strength reductions, or 3) statistical analysis to determine the mean, coefficients of variations for virgin or aged properties, and the bias factors. 
They are only expected to know the nominal virgin material properties and strength reduction factors (or allowable stresses with the built-in factor of safety for working stress design) associated with the required service life, under-designed environmental conditions, as stipulated for a structure by the owner.

\subsubsection{Recommended Design Parameters}

Refer to the illustrative examples no. 2 and 3 (Appendix A) for a step-by-step process to arrive at the final strength reduction factors for these specific examples for the subsea environment. It should be noted that if the loading case is different (span length, loads, section size, etc.), these values will also be different. Table 6.10 shows the final design parameters for different service lives, including 25 years.

Example no. 2 illustrates a design of tension bracing using 6" polyester GFRP I-beam as the tension bracing. Example 3 shows a design of a flexural beam using 8 " $x 8$ " vinylester double web GFRP beam as the beam subjected to uniform loads. In both instances, we first select the strength reduction factor $(\phi)$ based on known strength retention (SR) value from Table 6.4 for the service life of $t=25$ years (i.e., $\phi=S R$ is a good starting point), and check to make sure that this strength reduction factor $(\phi)$ provides adequate reliability index $(\beta)$ of 3.5 at the end of the life span, and if not, adjust $\phi$ till $\beta$ of at least 3.5 is obtained. Depending on a wide variety of factors such as loads, structural geometry, Bias factors, coefficient of variations, and material degradation rate, the relationship between the $\phi$ and $\beta$ may not be linear, and further adjustments to $\phi$ may be needed.

Refer to Table 6.10 for a brief comparison of strength reduction factors based on the mean value and reliability-based value for these examples. An ATM-based strength reduction factor is derived using the regressed mean value projected at a 25 -year timeline divided by the ultimate 
value (i.e., $\varphi$ is the same as SR). However, a reliability-based strength reduction factor approach will begin further refining the ATM-based strength reduction factor approach until a reliability index of 3.5 is obtained at the end of 25 -year service life (i.e., $\varphi$ may or may not be the same as SR). In this case, both factors are the same $(\varphi=$ SR) for the vinylester-based GFRP flexure beam (Example no. 3, Appendix A), but that is not the case for the polyester-based GFRP tension bracing (Example no. 2, Appendix A). The tension bracing designed using the deterministically derived strength reduction factor of 0.82 will not provide adequate structural safety at the end of its service life. However, one can argue that the 0.82 factor is very high, and only a maximum value of 0.60 (or even less) will be used, then the question is, "how safe is safe enough?"

Table 6.10: Strength Reduction Parameters Comparison

\begin{tabular}{|c|c|c|c|c|}
\hline $\begin{array}{c}\text { Design Example } \\
\text { No. } \\
\text { (Appendix A) }\end{array}$ & Material & Member Type & \multicolumn{2}{|c|}{ Strength Reduction Factor ( $\boldsymbol{\phi})$} \\
\cline { 4 - 5 } & & & Based on ATM & Based on Reliability \\
\hline $\mathbf{2}$ & Polyester GFRP & Tension Bracings & 0.82 & 0.70 \\
\hline $\mathbf{3}$ & Vinylester GFRP & Flexural Beam & 0.75 & 0.77 \\
\hline
\end{tabular}

Strength reduction factors in Table 6.10 are computed based on the optimal design of keeping capacity-demand ratio to 1.0 ; however, in practice, other parameters such as live loaddeformation, sustained stress, and erection considerations may govern the minimum size of a member under design considerations. The non-linear relationship between the reliability index and strength reduction factor for these two examples can be plotted, as shown in Figure 6.7. The reliability-based strength reduction factors for 25 -year of service life shown in Table 6.10 can be extracted from Figure 6.7. Based on the durability-reliability relationship developed in this chapter, 
the safer strength reduction factors will be based on reliability, which can be picked based on the desired reliability index (i.e., an acceptable probability of failure) and service life expectations.

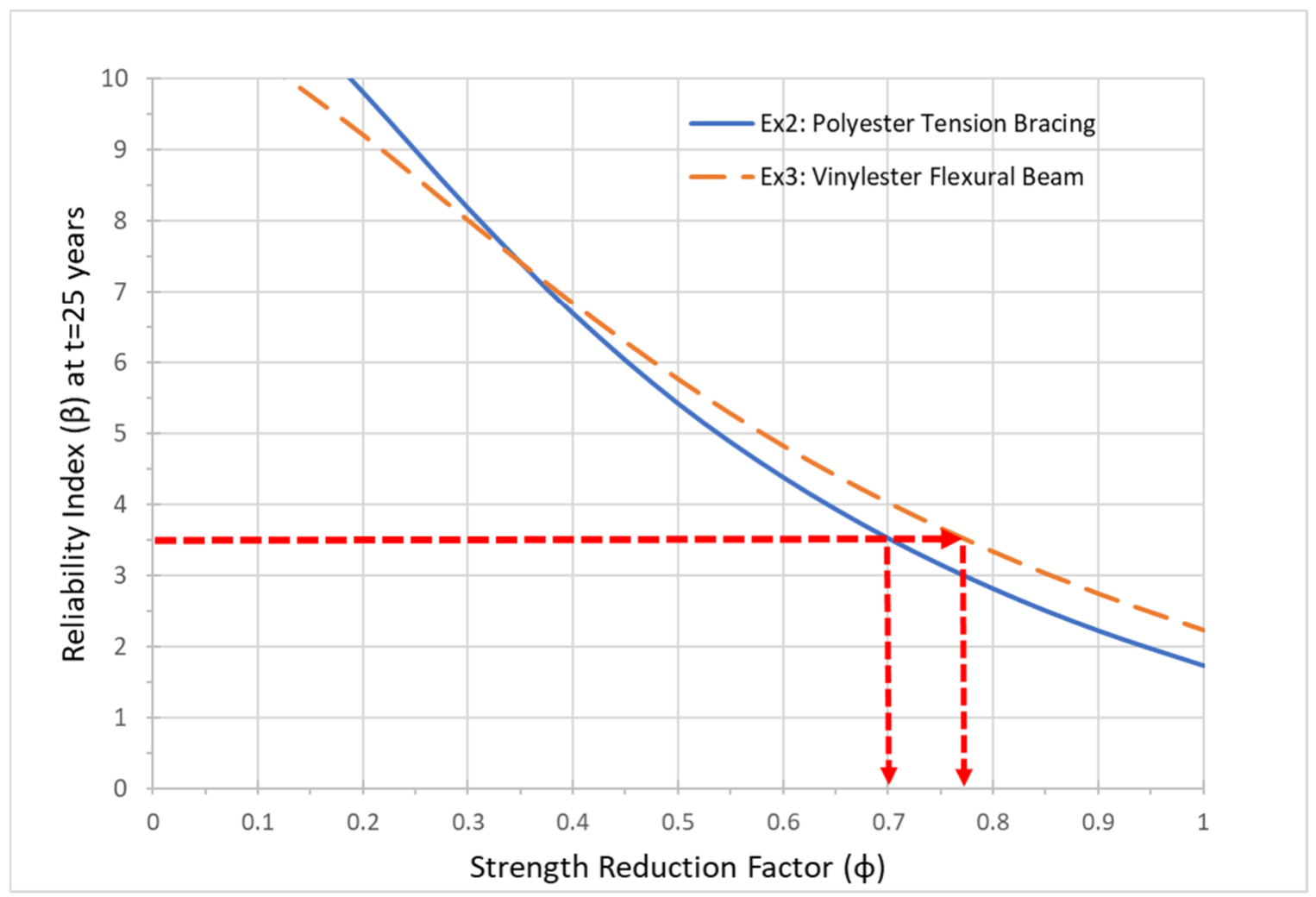

Figure 6.7: Reliability Index and Strength Reduction Factors (Examples 2 and 3)

Similarly, a non-linear relationship plot between the factor of safety and reliability index can also be plotted (Figure 6.8) to facilitate a suitable factor of safety (FOS) that will provide an appropriate structural safety over the product service life. For a reliability index of 3.5 , the estimated FOS for the vinylester flexural beam is 2.0 and 2.25 for polyester tension bracing. The graph also shows that the use of high FOS provides a diminishing return in terms of structural safety. 


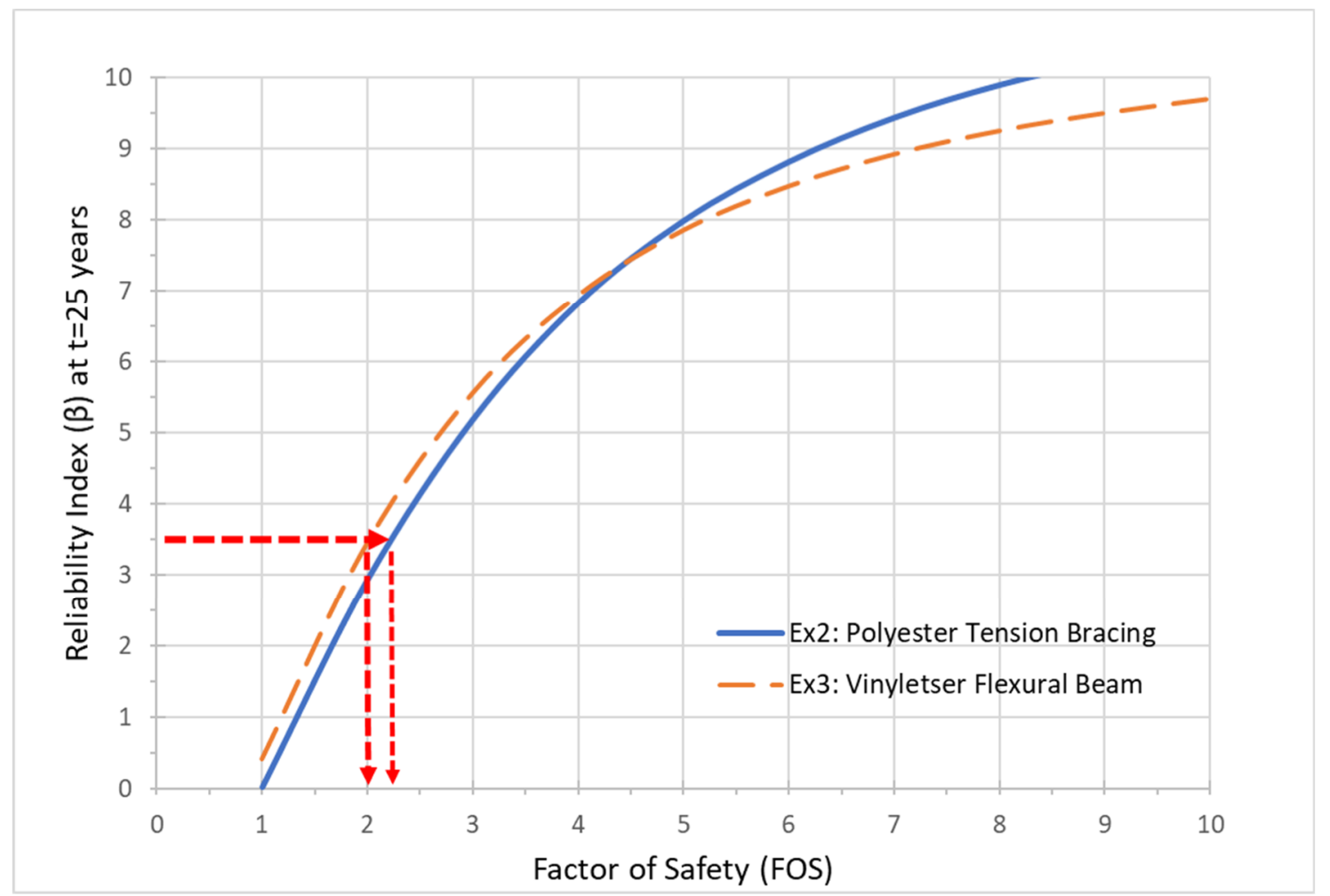

Figure 6.8: Reliability Index and Factor of Safety (Examples 2 and 3)

It should be noted that the applicability of a BOD can be greatly improved by adding more figures and design charts showing the relationships among service life, reliability index, strength reduction factors, and factor of safety (very similar to the Figures 4.10 to 4.14 used for design example no. 1 in Chapter 4). Providing more options for loading, geometry, and design parameters to FRP designers can result in significant cost savings without the loss of reliability. Additional figures are not repeated for the above design example no. 2 and 3 for the sake of brevity.

\subsection{SUMMARY}

The following broader conclusions can be drawn developing BOD for this application and from the data analysis of virgin and cocoon vinylester and polyester-based GFRP materials used in the Subsea Project Study: 
1. The Basis of Design for the subsea project, as presented in this chapter, can be derived from the project research documents and ATM data analysis.

2. BOD can also serve as systematic design-focus documentation of project research and can allow key research findings to immediate practical use.

3. Both VE and PE materials are generally degrading as a result of seawater saturation and environmental aging, all of which must be taken into account in the product design.

4. Additional reviews of the ATM data analysis results are necessary to ensure that BOD recommendations are in line with the known research findings for similar materials.

5. The BOD confirms that the use of mean values can result in inadequate structural safety as FRP member ages since the relationship between the reliability index/strength reduction factor/factor of safety and age of the member is not linear.

6. Based on project test data and assumed loading and geometry conditions of design examples no. 2 and 3 (Appendix A), the strength reduction factors, computed using the reliabilitydurability framework established in this report, will be 0.70 for tensile and 0.75 for flexural strengths. The author acknowledges that computed strength reduction factors seem to be reversed for tensile and flexural strengths. In general, one would expect to see a lower design factor for flexural strength than tensile strength. These factors are the computed theoretical values based on many assumptions stated in this chapter, and professional engineering judgment should be used when using these factors in the design of structures. 


\subsection{RECOMMENDATIONS}

The following recommendations are based on the conclusion of this chapter:

1. Development of the Basis of Design should follow the completion of an ATM project to allow immediate practical use of the research. BOD can i) promote confidence in the research efforts, ii) develop cost-effective, safe designs without the detailed knowledge of reliability or durability principles, iii) serve as a risk management tool for the owners, and iv) help improve the overall product development process.

2. Dry virgin strength measurements of all materials used in ATM is essential and should be performed early on to avoid making critical assumptions later in data analysis, particularly for calculating TSF. All aged properties should be referenced (normalized) with reference to the dry virgin properties,

3. A significant drop of strength in a saturated sample should be accounted for in the design of FRP in submerged conditions. Depending on the FRP type and test environment, it may take a substantial amount of time for full saturation to occur, so the use of a combined (i.e., saturation and aging) accelerated environment is recommended.

4. The development of BODs should be supported with design examples/charts/graphs to provide the necessary building blocks for the development of reliability-based codes and design guidelines for industrywide use, with time-dependency as a function of the proposed approach, herein. 


\section{CHAPTER 7 SUMMARY AND CONCLUSION}

The development of a time-dependent reliability framework for the durability design of FRP composites involves many facets of durability, FRP characteristics, data analyses, reliability theory, and a combination thereof. Due to the complexities of diverse subjects used in this report, individual summaries have been provided at the end of each chapter. An overall summary of this research project is as follows:

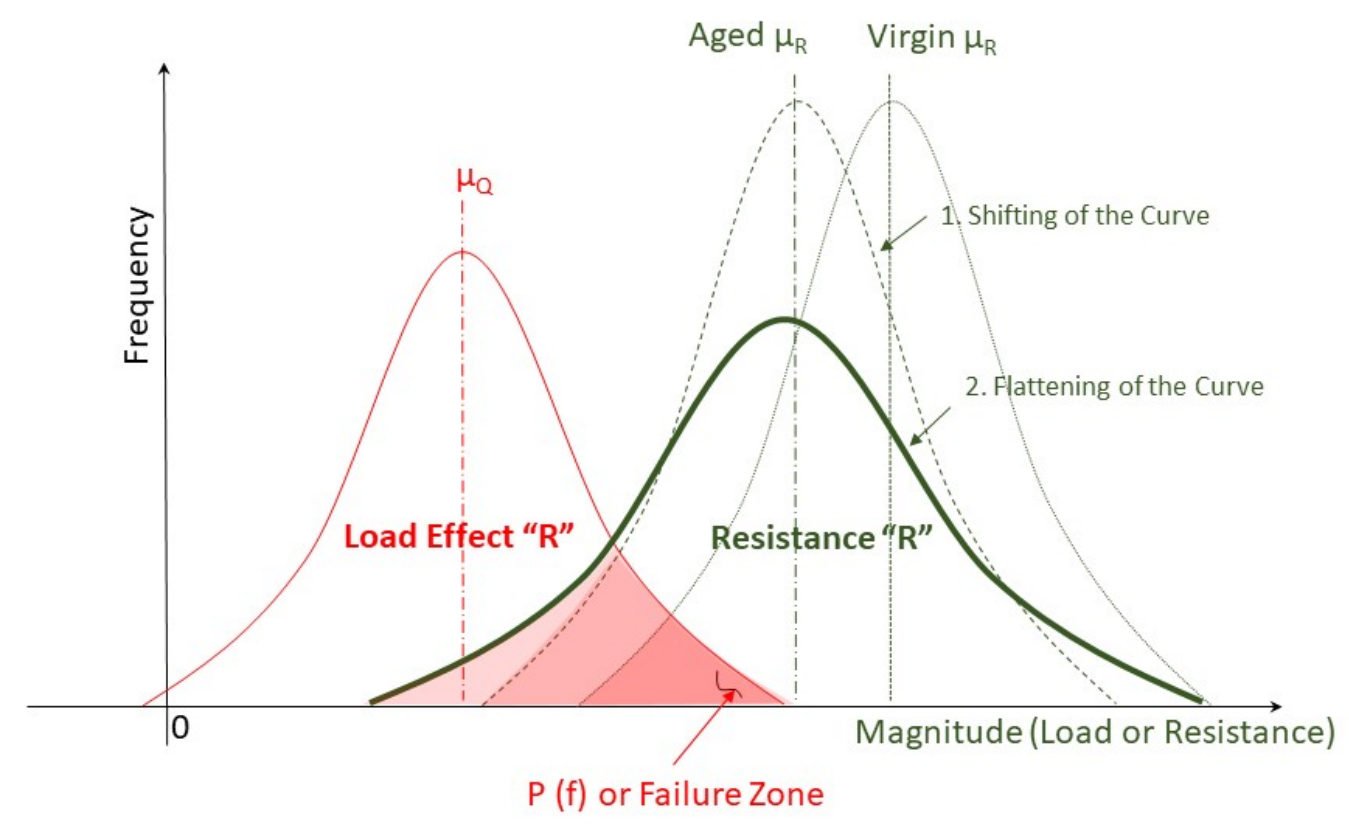

Figure 7.1: Concept of Time-Dependent Reliability \& Durability

The degradation of average strength and stiffness properties of FRP composites under outdoor environmental exposure with time (i.e., durability characteristics) using accelerated testing methods (ATM) and calibrating ATM results with naturally aged test data has been welldocumented by many researchers. However, the changes in the probability distribution functions (i.e., based on reliability principles) of the mechanical properties of FRP composites with time has not. Figure 7.1 shows that as FRP members age, the probability distribution function (PDF) of 
their mechanical properties changes, the mean strength decreases (i.e., shifting of PDF curve) and the coefficient of variation (COV) increases (i.e., flattening of PDF curve) due to inherent varying rates of degradations. This flattening of the curve leads to a further increase in the probability of failure (i.e., less reliability) with aging, which is best modeled by time-dependent reliability methods. By combing the two primary effects of aging, 1) shifting of the mean value curve, and 2) flattening of the PDF curve, the proposed Framework provides a rational means to predict the service life with confidence.

The fact is that the only scenario possible for COV to not increase with age is that all members must deteriorate at the same rate. However, the notion of a uniform rate of degradation for all samples is highly unlikely as we already know from naturally aged and accelerated test data. This time-dependent behavior of increasing COV when combined with decreasing average strength/stiffness of mechanical properties often leads to an increase of uncertainties (or unmitigated increase of risk) of structures in service, which has not been adequately addressed by conventional design practices. By accounting for the time-dependent behavior of both strength/stiffness reductions and statistical parameters changes in the FRP member design, an acceptable level of structural safety (i.e., a target probability of failure) can be achieved within known confidence levels (or mitigated risks) for the entire spectrum of the member's target service life (not just at the time of placement in service). This report formulates a reliability-durability based framework that couples the detrimental time-dependent behaviors (e.g., decrease of strength/stiffness due to environmental degradation and increase in sustained strain with time) into a design basis of FRP members. Using the proposed framework, FRP design can be customized for a specific environment or under specific sustained stress to perform adequately over a target service life (e.g., 10, 25, 50, 75, or 100-year). Although the focus of this report has been on 
pultruded vinylester and polyester-based GFRP members for civil infrastructure applications, the framework proposed herein can be used for developing broader FRP design guidelines and other specific FRP applications on a wide variety of service environments, loading conditions, and desired longevities.

This research is significant due to i) introduction of a new time-dependent relationship between the structural reliability (i.e., an increase in the probability of failure with the aging of FRP) and durability characteristics (i.e., a decrease of strengths/stiffness under service environments with time) of FRP composites, ii) establishment of a new risk-based framework to tie the structural design with the probability of failure, durability characteristics, and target service life, iii) unlimited customization possibilities of civil engineering structural designs through a specific FRP field application as illustrated using a BOD case study (Chapter 6), and iv) integration of professional engineering practice perspectives into academic research to further advance the knowledge bank and the design practice of FRP composites.

The key conclusions from this research project are as follows:

1. The lack of long-term performance guidelines on the durability and reliability of FRP members often overshadows the advantages of structural composites as a designer's choice material. The current practice does not adequately address the structural safety over the member's life cycle (e.g., how safe is safe enough when designing a member or how to rationally design a member to perform adequately till the end of its service life?)

2. The risk-based probabilistic approach, when combined with time-based durability characteristics of FRP composites, can be used to develop rational performance guidelines on FRP members. This approach has been termed as "Time-dependent Reliability Framework for 
Durability Design of FRP Composites," "Time-Dependent Reliability-Durability Framework," or "The Framework" in this report (Chapter 3).

3. The time-dependent reliability-durability framework can allow the development of appropriate durability design factors (and/or limitations) for FRP design to assure an acceptable level of performance throughout the target service life while providing material efficiencies and consistent reliabilities (Chapters 4, 5 and 6).

4. The durability test results (from accelerated testing methods which may or may not be calibrated with test results of naturally aged materials), although plenty, often lack the necessary information needed for conducting the time-dependent reliability analysis. However, the most existing durability data can be re-analyzed to extract the necessary time-dependent and probabilistic parameters (Chapters 3, 4, 5, and 6).

5. The framework offers a rational means to develop durability reduction factors under various environmental and loading conditions. The reliability approach to FRP materials and members takes into account its initial (at the time of placement in service) inherent variability of properties, time-dependent degradation of FRP properties (under certain environmental or loading conditions), time-dependent changes to its property characteristic distributions (coefficient of variations or distribution type), and acceptable design risk (or target probability of failure) to arrive at a set of rational design factors (e.g., strength reduction factors, factors of safety, or loading limitations) for the intended service life. Once the time-dependent lifecycle performance (in terms of structural safety) of FRP structures is understood, then a minimum level of safety can be maintained as FRP members age. A "bathtub" performance curve (Chapter 2) can explain such life-cycle performance as a member's reliability (structural safety, risk, or probability of failure) changes starting from its maturity phase (manufacturing, 
curing and construction), aging phase (in service with/without minor repairs/maintenance), to wear-out phase (out of service due to failures/poor performance). The intent is that once design factors have been substantiated and calibrated, no knowledge of reliability analysis or durability testing will be required by the FRP designers.

6. The use of material property test data obtained from manufacturers, laboratory testing (using accelerated test method), and natural aging is critical in developing a reliability-based durability framework. The ATM can be modified to provide aged durability characteristics needed for the reliability analysis. The use of professional data analysis software is necessary to make the ATM and reliability data processing less tedious and more accurate.

7. The formulation of correct performance functions (based on limit state functions of strengths, stiffness, and serviceability) along with the time-dependent statistical properties of FRP materials such as mean, standard deviations, distribution types is required to compute the reliability index " $\beta$ " at a given age. For large samples, and as the member's age, the variations of material's mechanical properties are more likely to follow a normal distribution.

8. In lieu of further research and availability of long-term in-service performance of FRP composites, a well-accepted measure of the probability of failure (in civil infrastructure applications) can be approximated by the reliability index of 3.5 , roughly translating into a probability of failure of 1 in 4,300. As the risk-based FRP design philosophy advances into the professional practice, this value should be further examined for adequacy. For example, a higher index above 3.5 may be considered given the brittle nature of FRP failures. In any case, the development of the risk-based durability design must be based on a pre-selected target reliability index to provide a consistent and quantitative measure of structural safety. 
9. The selection of a proper reference environment in ATM studies can allow the test data to be readily adjustable for real-life age predictions (e.g., outdoor conditions, alkaline environments, high temperatures, freeze and thaw cycles, etc.). The reproducibility of ATM results is crucial in the development of the FRP aging database that can be used for the knowledge base necessary for the development of risk-based design guidelines. Simple modifications (e.g., normalization of data, removal of outliers, measuring age-related standard deviations, etc.) can help in improving the effectiveness of ATM data for use in time-dependent reliability analyses.

10. The non-linear relationship between the probability of failure and strength reduction factors questions the validity of the current practice of using strength reduction factors as the "mean" strength retention value derived from Accelerated and Natural Tests. Alternatively, in simple words, increasing or decreasing strength reduction factors or factors of safety does not result in a proportional change in structural safety. A reliability-based assessment can explain why some strength reduction factors, as used in current design practice (without the inclusion of age and structural safety measures), may not be adequate beyond certain service periods. The need for the development of a reliability-based durability framework should be deemed critical, particularly if a longer service life of FRP members (e.g., 75-100 years) is expected.

11. Findley's law fits nicely for modeling physical effects such as sustained stress data and allows the direct computation of the time to failure (which can be later regressed for the desired confidence intervals). Other regression equations such as natural logarithmic equation fit well to model environmental effects data (which typically derived from accelerated or natural test methods) allow service life (and associated time-dependent statistical parameters) predictions using Arrhenius principles. 
12. Since physical effects do not easily allow a direct formation of a limit state function describing a particular failure mode, the use of lower bound values (e.g., time to failure " $t$ " for the given sustained stress) may be the preferred way to incorporate a reliability approach to account for the influence of physical effects on service life. The statistical analysis also indicates a normal/lognormal distribution of residuals, which further justifies the use of lower bound values at a standard deviation of -3.5 , which can be roughly equated to the target reliability index of 3.5.

13. Limitation of sustained stresses as derived for different service lives in this research not only matches well with the existing recommendations but also provides an association with service life. This proposed framework illustrates in detail how similar (target service life-based) recommendations can be developed for other sections, loading conditions, fiber volume fractions, and FRP types using other test databases.

14. Estimation of long-term creep deformation can be complicated, and the proposed framework provides a starting point in systematically addressing this adverse effect for various target service lives for a given sustained stress level.

15. Most critical information for building a Basis of Design (BOD) can be derived from the available research documents and ATM data analysis. A BOD developed using the proposed framework can serve as methodical design-focus documentation of research (or end-users' tool) and can render key research findings to immediate practical use. 


\section{CHAPTER 8 RECOMMENDATIONS}

This research project provides a framework for a reliability-based durability framework for FRP that can build the foundation for the development of risk-based guidelines and design codes for FRP structures. Due to the varied range of subject matters covered in this research, specific recommendations have been provided at the end of several chapters.

The following are the key recommendations of this research:

1. Industry-wide efforts are needed to develop a knowledge base of statistical parameters of new and aged FRP that can be used for reliability calculations. Such parameters should be normalized to provide: bias factors, coefficient of variations, material property distribution types, etc. Normalization can help in protecting proprietary information - a common practice in the FRP industry.

2. Additional efforts are needed to collect naturally aged test databases of a wide variety of FRP types so that the results from accelerate test methods (ATM) can be appropriately calibrated for time-dependent reliability analysis.

3. Accelerated Testing Methods can be modified to serve as an effective tool in the development of the reliability-based FRP durability database. The following are specific recommendations in that regards:

- Researchers must be mindful of using correct reference environments so that the harsh environment test results can be transformed into useful data for service life predictions without excessive manipulation of ATM data.

- A simple degradation equation (derived from the regression analysis that reduces the influence of outliers) to compute time shift factors directly (as illustrated in this report) is 
recommended to promote the reproducibility of ATM results across the various disciplines, FRP industry, and research institutions.

- A time-dependent degradation equation must be developed with reference to the dry virgin material properties (so that at age $=0$ or near 0 , the Strength Retained is $100 \%$ ). The use of program-generated trend line equations without proper statistical analysis will not allow for the correct reference to virgin material properties.

- Proper planning of ATM efforts is highly recommended to account for the laboratory time (from days to years) to reach a full saturation/aging state from the dry state.

4. Since the absolute structural safety is not possible, all structures must be designed for a particular tolerable risk of failures. In the absence of adequate data about the long-term performance of FRP members in service (i.e., due to the lack of data about very long-term performance data of in-service FRP members), it is recommended that a well-accepted measure of the acceptable probability of failure of civil engineering structures, a reliability index $(\beta)$ of 3.5 (which roughly translates to a probability of failure of 1 in 4,300) should be used. Without proper justification, the use of other $\beta$ values at this time will be considered arbitrary.

5. The use of several FRP databases, although cumbersome to manage and analyze, can be rewarding when proper normalization techniques are used to encompass a wide variety of FRP applications. The test findings presented in this report are based on a few selected databases, and one may reach different conclusions/trends if more data is added or entirely different databases are used. More data should be considered beneficial in off-setting the effects of skewed or poor-quality test data.

6. The calibration phase of the strength reduction factors is an essential final step in the development of reliability-based design guidelines or deign codes for FRP; however, it must 
be undertaken at an institution (e.g., National Institute of Standards and Technology or NIST) or industrywide level (e.g., American Composites Manufacturers Associations or ACMA) due to an enormous amount of effort involved.

7. The framework developed for physical effects such as creep life can substantiate design limitations imposed for various desired service life with the desired degree of confidence. Perhaps, other physical effects such as fatigue life (e.g., finding the number of cycles to failure with the corresponding modulus of rupture values) can also be evaluated. With additional data and efforts, the framework can be used to develop general or specific creep and fatigue design recommendations that include service life predictions.

8. Development of the Basis of Design after the conclusion of the ATM project should be encouraged to allow immediate practical use of the research. It promotes confidence in the research efforts and helps improve the product development processes addressing the parameters used in the BOD. Besides, the development of BODs can provide the necessary building blocks for the development of reliability-based codes and design guidelines for industry-wide use.

9. Understanding of the mean/average ultimate, nominal, characteristic, and the guaranteed minimum value is essential for bringing research findings into professional engineering practice. In the absence of adequate material property test data, the use of bias factors and coefficient of variations may be the quickest way to find the appropriate material properties when using a research database.

10. The proposed framework in this report should be considered as one of the early milestones towards the long journey of creating the risk-based design guidelines/codes for FRP composites, acknowledging that there is still much work to be done. 


\section{REFERENCES}

Agrawal, A., Kawaguchi, A., \& Chen, Z. (2010). Deterioration Rates of Typical Bridge Elements in New York. Journal of Bridge Engineering, 15(4), 419-429.

ASCE's Infrastructure Report Card. (2017). Retrieved May 13, 2019, from http://www.infrastructurereportcard.org/

Atadero, R. A., \& Karbhari, V. M. (2006). Development of load and resistance factor design for FRP strengthening of reinforced concrete bridges (No. FHWA/CA/ES-2006/11). California. Dept. of Transportation.

Atadero, R. A., \& Karbhari, V. M. (2008). Calibration of resistance factors for reliability based design of externally-bonded FRP composites. Composites Part B: Engrg, 39(4), 665-679.

Atadero, R. A., \& Karbhari, V. M. (2009). Sources of uncertainty and design values for field-manufactured FRP. Composite Structures, 89(1), 83-93.

Atadero, R., Lee, L., \& Karbhari, V. M. (2005). Consideration of material variability in reliability analysis of FRP strengthened bridge decks. Composite Structures, 70(4), 430-443.

Bank, L. C., Gentry, T. R., Thompson, B. P., \& Russell, J. S. (2003). A model specification for FRP composites for civil engineering structures. Construction and Building Materials, 17, 405-437.

Bank, L. C., \& Mosallam, A. S. (1992). Creep and failure of a full-size fiber-reinforced plastic pultruded frame. Composites Engineering, 2(3), 213-227.

Barker, W. T. (2019). Short Beam Shear Strength Evaluations of GFRP Composites: Correlations Through Accelerated and Natural Aging. (Master's Thesis). West Virginia University.

Batra, S. (2009). Creep Rupture and Life Prediction of Polymer Composites. (Master's Thesis). West Virginia University.

Bian, L., Xiao, J., Zeng, J., \& Xing, S. (2012). Effects of seawater immersion on water absorption and mechanical properties of GFRP composites. Journal of Composite Materials, 46(25), 3151-3162.

Biondini, F., \& Frangopol, D. M. (2016). Life-cycle performance of deteriorating structural systems under uncertainty. Journal of Structural Engineering, 142(9), F4016001.

Bisby, L. A., \& Eng, P. (2006). ISIS Educational Module 8: Durability of FRP Composites for Construction. ISIS Canada, A Canadian Network of Centres of Excellence on Intelligent Sensing for Innovative Structures. University of Winnipeg, Manitoba, 20p 
Boyer, C., B6akou, A., \& Lemaire, M. (1997). Design of a composite structure to achieve a specified reliability level. Reliability Engineering and System Safety, 56, 273-283.

Buck, S. E., Lischer, D. W., \& Nemat-Nasser, S. (2001). Mechanical and microstructural properties of notched E-glass/vinyl ester composite materials subjected to the environment and a sustained load. Materials Science and Engineering: A, 317(1-2), 128-134.

Cabral-Fonseca, S., Correia, J. R., Rodrigues, M. P., \& Branco, F. A. (2012). Artificial accelerated ageing of GFRP pultruded profiles made of polyester and vinylester resins: characterization of physical-chemical and mechanical damage. Strain, 48(2), 162-173.

Cao, S., Wu, Z., \& Wang, X. (2009). Tensile Properties of CFRP and Hybrid FRP Composites at Elevated Temperatures. Journal of Composite Materials, 43(04), 315-330.

Chen, Y., Davalos, J. F., \& Ray, I. (2006). Durability prediction for GFRP reinforcing bars using short-term data of accelerated aging tests. Journal of Composites for Construction, 10(4), 279-286.

Chin, J. W., Hughes, W. L., \& Signor, A. (2001). Elevated temperature aging of glass fiber reinforced Vinylester and Isophthalic polyester composites in water, saltwater, and concrete pore solution. American Society for Composites, 16th technical conference. Proceedings (pp. 1-12).

Chin, J. W., Nguyen, T., \& Aouadi, K. (1997). Effects of environmental exposure on fiberreinforced plastic (FRP) materials used in construction. Journal of Composites, Technology and Research, 19(4), 205-213.

Cho, E. H., \& Mounts, J. L. (2007). Durability of glass-fiber-reinforced polymer composites in an alkaline environment. Journal of Vinyl \& Additive Technology, 13(4), 221-228.

Dehmous, H., Ene Welemane, H., Karama, M., \& Tahar, K. A. (2008). Reliability approach for fibre-reinforced composites design. International Journal of Simulation and Multidisciplinary Optimization.

Dejke, V., \& Tepfers, R. (2001, July). Durability and service life prediction of GFRP for concrete reinforcement. Proceedings, 5th Int. Conf. on Fiber-Reinforced Plastics for Reinforced Concrete Structures (FRPRCS-5) (Vol. 1, pp. 505-516). London: Thomas Telford.

Daniali, S. (1991, February). Short-term and long-term behavior of two types of reinforced plastic beams. In 46th Annual Conference, Composites Institute.

Dittenber, David B, \& GangaRao, H. V. S. (2013). Fatigue equation considerations for the American Society of Civil Engineers pre-standard for load and resistance factor design of pultruded FRP structures. Journal of Composite Materials, 47(16), 1943-1949. 
Dittenber, David Brian, \& Hota, G. V. S. (2010). Evaluation of a Life Prediction Model and Environmental Effects of Fatigue for Glass Fiber Composite Materials. Structural Engineering International, 20(4), 379-384.

Eldridge, A., \& Fam, A. (2014). Environmental Aging Effect on Tensile Properties of GFRP Made of Furfuryl Alcohol Bioresin Compared to Epoxy. Journal of Composites for Construction, 18(5), 04014010.

Findley, W. N. (1987). 26-Year creep and recovery of poly (vinyl chloride) and polyethylene. Polymer Engineering \& Science, 27(8), 582-585.

GangaRao, G.V.S., Taly, N., \& Vijay, P.V. (2007). Reinforced concrete design with FRP composites. Boca Raton: CRC Press.

GangaRao, G.V.S., (2017), Design and Analysis of Fiber Reinforced Polymers. Unpublished Textbook\& Notes (CE 768). West Virginia University.

GangaRao, G.V.S. \& Liang, R. (2011). Creep Response Characterization and Life Prediction of GFRP Composites under Bending. US-NSF I/UCRC Center for Integration of Composites into Infrastructure (CICI).

Ghasemi, S. H. (2015). Target reliability analysis for structures (Doctoral dissertation). University of Auburn.

Grammatikos, S. A., Evernden, M., Mitchels, J., Zafari, B., Mottram, J. T., \& Papanicolaou, G. C. (2016). On the response to hygrothermal aging of pultruded FRPs used in the civil engineering sector. Materials \& Design, 96, 283-295.

Greenwood, M. (2002, May). Creep-rupture testing to predict long-term performance. In Proceedings of the 2nd International Conference on Durability of Fibre-Reinforced Polymer (FRP) Composites for Construction, Montreal, Quebec (pp. 29-31).

Hammami, A., \& Al-Ghuilani, N. (2004). Durability and environmental degradation of glass-Vinylester composites. Polymer composites, 25(6), 609-616.

Han, S. O., \& Drzal, L. T. (2003). Water absorption effects on hydrophilic polymer matrix of carboxyl functionalized resin and epoxy resin. European Polymer Journal, 39(9), 1791-1799.

Hawileh, R. A., Abu-Obeidah, A., Abdalla, J. A., \& Al-Tamimi, A. (2015). Temperature effect on the mechanical properties of carbon, glass and carbon-glass FRP laminates. Construction and Building Materials, 75, 342-348.

Helbling, C. S. S., \& Karbhari, V. M. M. (2008). Investigation of the Sorption and Tensile Response of Pultruded E-Glass/Vinylester Composites Subjected to Hygrothermal Exposure and Sustained Strain. Journal of Reinforced Plastics and Composites, 27(6), 613-638.

Holmes, M., \& Rahman, T. A. (1980). Creep behaviour of glass reinforced plastic box beams. Composites, 11(2), 79-85. 
Kajorncheappunngam, S. (1999). The Effects of Environmental Aging on the Durability of Glass/Epoxy Composites. (Doctoral dissertation) West Virginia University.

Kajorncheappunngam, S., Gupta, R. K., \& Gangarao, H. V. S. (2002). Effect of Aging Environment on Degradation of Glass-Reinforced Epoxy. ASCE Journal of Composites for Construction Vol. 6, Issue 1 (February 2002), 6(1 (February)), 61-69.

Karbhari, V. M. (2004). E-Glass/Vinylester composites in aqueous environments: effects on short-beam shear strength. Journal of Composites for Construction, 8(2), 148-156.

Karbhari, V. M., \& Abanilla, M. (2006). Design factors, reliability, and durability prediction of wet layup carbon/epoxy used in external strengthening. Composites Part B: Engineering, 10(23), 10-23.

Karbhari, V. M., Chin, J., Hunston, D., Benmokrane, B., Juska, T., Morgan, R., ... Reynaud, D. (2003). Durability Gap Analysis for Fiber-Reinforced Polymer Composites in Civil Infrastructure. Journal of Composites for Construction, 7(3)(August 2003), 238-247.

Karbhari, V. M., \& Lee, L. (2011). Service Life Estimation and Extension of Civil Engineering Structures. (V. Karbhari \& L. Lee, Eds.), Woodhead Publishing. Woodhead Publishing.

Karbhari, V. M., \& Xian, G. (2008). Hygrothermal effects on high V F pultruded unidirectional carbon/epoxy composites: Moisture uptake. Composites Part B, 40, 41-49.

Keller, T., Bai, Y., \& Vallée, T. (2007). Long-Term Performance of a Glass FiberReinforced Polymer Truss Bridge. Journal of Composites for Construction, 11(1), 99-108.

Keller, T., Theodorou, N. A., Vassilopoulos, A. P., \& de Castro, J. (2016). Effect of Natural Weathering on Durability of Pultruded Glass Fiber-Reinforced Bridge and Building Structures. Journal of Composites for Construction, 20(1), 04015025.

Kim, H. Y., Park, Y. H., You, Y. J., \& Moon, C. K. (2008). Short-term durability test for GFRP rods under various environmental conditions. Composite structures, 83(1), 37-47.

Kumosa, L., Benedikt, B., Armentrout, D., \& Kumosa, M. (2004). Moisture absorption properties of unidirectional glass/polymer composites used in composite (non-ceramic) insulators. Composites Part A: Applied Science and Manufacturing, 35(9), 1049-1063.

Lee, L. S., Atadero, B., Karbhari, V. M., \& Sikorsky, C. (2004). Remaining life of FRP rehabilitated bridge structures. In Composite Technologies for 2020 (pp. 1012-1017).

Batra, S. (2009). Creep Rupture and Life Prediction of Polymer Composites. (Master's Thesis). West Virgina University.

Lorenzo, M. (2018). Durability of GFRP composites under harsh environments: Effect of pH and Temperature. (Master's Thesis). West Virginia University. 
Manalo, A., Maranan, G., Benmokrane, B., Cousin, P., Alajarmeh, O., Ferdous, W., ... \& Gangarao, H. (2020). Comparative Durability of GFRP Composite Reinforcing Bars in Concrete and in Simulated Concrete Environments. Cement and Concrete Composites, 103564.

Marru, P., Latane, V., Puja, C., Vikas, K., Kumar, P., \& Neogi, S. (2014). Lifetime estimation of glass reinforced epoxy pipes in acidic and alkaline environment using accelerated test methodology. Fibers and Polymers, 15(9), 1935-1940.

McGraw-Hill Dictionary of Scientific and Technical Terms. (2003). McGraw-Hill.

McClure, G., \& Mohammadi, Y. (1995). Compression creep of pultruded E-glassreinforced-plastic angles. Journal of materials in civil engineering, 7(4), 269-276.

Micelli, F., \& Nanni, A. (2004). Durability of FRP rods for concrete structures. Construction and Building Materials.

Miyano, Y., Nakada, M., \& Cai, H. (2008). Formulation of Long-term Creep and Fatigue Strengths of Polymer Composites Based on Accelerated Testing Methodology. Journal of Composite Materials, 42(18), 1897-1919.

Mosallam, A. S., \& Bank, L. C. (1991). Creep and recovery of a pultruded FRP frame. In Advanced composites materials in civil engineering structures (pp. 24-35). ASCE.

Mourad, A.-H. I., Abdel-Magid, B. M., El-Maaddawy, T., \& Grami, M. E. (2010). Effect of Seawater and Warm Environment on Glass/Epoxy and Glass/Polyurethane Composites. Applied Composite Materials, 17(5), 557-573.

Mufti, A. A., Banthia, N., Benmokrane, B., Boulfiza, M., \& Newhook, J. P. (2007). Durability of GFRP composite rods. Concrete international, 29(2), 37-42.

Myers, J. J., \& Viswanath, T. (2006). A Worldwide Survey of Environmental Reduction Factors for Fiber Reinforced Polymers (FRP). Structures Congress 2006, 40889 (2006), 1-8.

Nakada, M., \& Miyano, Y. (2015). Advanced accelerated testing methodology for longterm life prediction of CFRP laminates. Journal of Composite Materials, 49(2), 163-175.

National Research Council. (1996). Accelerated Aging of Materials and Structures. Washington, D.C.: National Academies Press.

Nowak, A. S. (1995). Calibration of LRFD Bridge Code. Journal of Structural Engineering, 121(8), 1245-1251.

Nowak, A. S. (1999). Calibration of LRFD bridge design code. NCHRP Report. Transportation Research Board, National Research Council. Project C12-33 FY'88-'92.

Nowak, A. S., \& Collins, K. R. (2013). Reliability of structures (Second). CRC Press. 
Philippidis, T. P., \& Lekou, D. J. (1998). Probabilistic Failure Prediction for FRP Composites. Composites Science and Technology, 58(12), 1973-1982.

Rivera, J., \& Karbhari, V. M. (2002). Cold-temperature and simultaneous aqueous environment related degradation of carbon/Vinylester composites. Composites Part B: Engineering, 33(1), 17-24.

Robert, M., Wang, P., Cousin, P., \& Benmokrane, B. (2010). Temperature as an accelerating factor for long-term durability testing of FRPs: Should there be any limitations? Journal of Composites for Construction, 14(4), 361-367.

Sá, M. F., Gomes, A. M., Correia, J. R., \& Silvestre, N. (2011). Creep behavior of pultruded GFRP elements - Part 2: Analytical study. Composite Structures, 93(9), 2409-2418.

Saadatmanesh, H., \& Tavakkolizadeh, M. (2010). Environmental effects on mechanical properties of wet lay-up fiber-reinforced polymer. ACI Materials.

Sauder, C., Lamon, J., \& Pailler, R. (2004). The tensile behavior of carbon fibers at high temperatures up to $2400{ }^{\circ} \mathrm{C}$. Carbon, 42(4), 715-725.

Schneider, J. (2006). Introduction to safety and reliability of structures. Zürich: International Association for Bridge and Structural Engineering.

Sen, R., \& Mullins, G. (2003). Durability of E-Glass/Vinylester reinforcement in alkaline solution. ACI structural journal, (2), 265-266.

Shi, J.-W., Zhu, H., Wu, G., \& Wu, Z.-S. (2014). Tensile behavior of FRP and hybrid FRP sheets in freeze \& thaw cycling environments. Composites Part B: Engineering, 60, 239-247.

Shi, J., Zhu, H., \& Wu, Z. (2011, October). Durability of wet lay-up FRP composites and their epoxy resins in alkaline environment. 4th International symposium on innovation \& sustainability of structures in civil engineering (ISISS 2011) (pp. 1129-1135).

Silva, M., Sena da Fonseca, B., \& Biscaia, H. (2014). On estimates of durability of FRP based on accelerated tests. Composite Structures, 116(2014), 377-387.

Scott, D. W., \& Zureick, A. H. (1998). Compression creep of a pultruded E-glass/vinylester composite. Composites Science and Technology, 58(8), 1361-1369.

Soares, C. G. (1997). Reliability of components in composite materials. Reliability Engineering and System Safety, 55(17), 171-177.

Sonawala, S. P., \& Spontak, R. J. (1996). Degradation kinetics of glass-reinforced polyesters in chemical environments. Journal of materials science, 31(18), 4745-4756.

Standard Test Method for Determining Tensile Properties of Fiber Reinforced Polymer Matrix Composites Used for Strengthening of Civil Structures 1. (2017). In ASTM International D7565/D7565M-10 (Reapproved 2017). 
Thoft-Christensen, P., \& Baker, M. J. (2012) (1982). Structural Reliability Theory and its Applications. Springer Science \& Business Media.

Vijay, P. V., \& GangaRao, H. V. S. (1999). Accelerated and natural weathering of glass fiber reinforced plastic bars. Special Publication, ACI. 188, 605-614.

Vijay, P. (1999). Aging and Design of Concrete Members Reinforced with GFRP bars. (Doctoral Dissertation) West Virginia University.

Wang, J., GangaRao, H., Liang, R., Zhou, D., Liu, W., \& Fang, Y. (2015). Durability of glass fiber-reinforced polymer composites under the combined effects of moisture and sustained loads. Journal of Reinforced Plastics and Composites, 34(21), 1739-1754.

Wang, J., GangaRao, H., Liang, R., \& Liu, W. (2016). Durability and prediction models of fiber-reinforced polymer composites under various environmental conditions: A critical review. Journal of Reinforced Plastics and Composites, 35(3), 179-211.

Weibull, W. (1939). A statistical theory of the strength of materials. Stockholm: Generalstabens litografiska anstalts förlag.

Wetherhold, R. C., \& Ucci, A. M. (1994). Probability Techniques for Reliability Analysis of Composite Materials. Retrieved from

Williams, B., Kodur, V., Mark, G., \& Bisby, L. (2008). Fire Endurance of Fiber-Reinforced Polymer Strengthened Concrete T-Beams. ACI Structural Journal ACI Structural Journal MS No. $S, 105(1), 2006-2370$.

Won, J. P., Lee, S. J., Kim, Y. J., Jang, C. I., \& Lee, S. W. (2008). The effect of exposure to alkaline solution and water on the strength-porosity relationship of GFRP rebar. Composites Part B: Engineering, 39(5), 764-772.

Wu, H.-C., Fu, G., Gibson, R. F., Yan, A., Warnemuende, K., \& Anumandla, V. (2006). Durability of FRP composite bridge deck materials under freeze-thaw and low temperature conditions. Journal of Bridge Engineering, 11(4), 443-451.

Wu, H.-C., \& Yan, A. (2011). Time-dependent deterioration of FRP bridge deck under freeze/thaw conditions. Composites Part B: Engineering, 42(5), 1226-1232.

Wu, H.-C., \& Yan, A. (2013). Durability simulation of FRP bridge decks subject to weathering. Composites Part B: Engineering, 51, 162-168.

Youssef, T. A. (2010). Time-dependent behavior of fiber reinforced polymers (FRP) bars and FRP reinforced concrete beams under sustained load. (Ph.D. Dissertation). The University of Sherbrooke. 
Zafar, A., Bertocco, F., Schjødt-Thomsen, J., \& Rauhe, J. C. (2012). Investigation of the long term effects of moisture on carbon fibre and epoxy matrix composites. Composites Science and Technology, 72(6), 656-666.

Zhai, Z., Jiang, B., \& Drummer, D. (2018). Tensile Creep Behavior of QuasiUnidirectional E-Glass Fabric Reinforced Polypropylene Composite. Polymers, 10(6), 661.

Zureick, A.-H., Bennett, R., \& Ellingwood, B. (2006). Statistical Characterization of FiberReinforced Polymer Composite Material Properties for Structural Design. Journal of Structural Engineering, 132(8), 1320-1327. 


\section{APPENDICES}




\section{TABLE OF CONTENTS - APPENDICES}

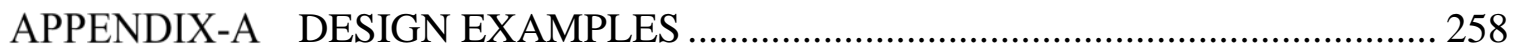

A.1 Example 1 - Outdoor Vinylester GFRP Beam ........................................ 258

A.2 Example 2 - Design of a Subsea Polyester GFRP Tension Bracing ................ 267

A.3 Example 3 - Design of a Subsea Vinylester GFRP Beam.............................. 275

APPENDIX-B FRP DURABILITY DATABASE …........................................... 283

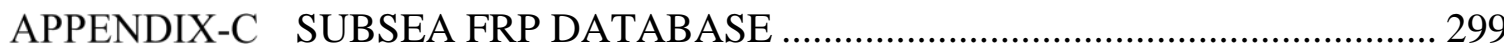

APPENDIX-D SUSTAINED STRESS DATABASE ........................................... 319

APPENDIX-E DURABILITY TEST DATABASE …....................................... 331

\section{List of Tables - Appendices}

Table B.1: Sample of FRP Database created for this study ........................................ 284

Table C.1: Sample of Original Dataset Provided by the FRP System Supplier ............. 300

Table D.1: Listing of Test Database Used in Sustained Stress Study ........................... 320

Table E.1: Listing of Test Database Used in Durability Study ................................... 332 


\section{List of Figures - Appendices}

Figure B.1: Tested ATM Tensile Strengths of Vinylester GFRP ................................ 285

Figure B.2: Projected Normalized Tensile Strengths of Vinylester GFRP .................. 286

Figure B.3: Tested ATM Flexural Strengths of Vinylester GFRP .............................. 287

Figure B.4: Projected Normalized Flexural Strengths of Vinylester GFRP .................. 288

Figure B.5: Tested ATM Shear Strengths of Vinylester GFRP................................. 289

Figure B.6: Projected Normalized Shear Strengths of Vinylester GFRP ..................... 290

Figure B.7: Tested ATM Tensile Strengths of Polyester GFRP ................................. 291

Figure B.8: Projected Normalized Tensile Strengths of Polyester GFRP .................... 292

Figure B.9: Tested ATM Flexural Strengths of Polyester GFRP ............................... 293

Figure B.10: Projected Normalized Flexural Strengths of Polyester GFRP................. 294

Figure B.11: Tested ATM Shear Strengths of Polyester GFRP ................................ 295

Figure B.12: Projected Normalized Shear Strengths of Polyester GFRP ...................... 296

Figure B.13: Tested ATM Tensile Strengths of Epoxy GFRP ................................... 297

Figure B.14: Projected Normalized Tensile Strengths of Epoxy GFRP....................... 298

Figure C.1: Tested Tensile-Transverse Strengths of Vinylester GFRP ........................ 301

Figure C.2: Projected Tensile-Transverse Strengths of Vinylester GFRP ................... 302

Figure C.3: Tested Tensile-Longitudinal Strengths of Vinylester GFRP ..................... 303

Figure C.4: Projected Tensile-Longitudinal Strengths of Vinylester GFRP ................. 304

Figure C.5: Projected Flexural-Transverse Strengths of Vinylester GFRP ................... 305 
Figure C.6: Projected Flexural-Longitudinal Strengths of Vinylester GFRP 306

Figure C.7: Projected Shear-Transverse Strengths of Vinylester GFRP. 307

Figure C.8: Projected Shear-Longitudinal Strengths of Vinylester GFRP 308

Figure C.9: Projected Tensile Modulus-Transverse Strengths of Vinylester GFRP ..... 309

Figure C.10: Projected Tensile Modulus-Longitudinal Strengths of Vinylester GFRP . 310

Figure C.11: Projected Tensile-Transverse Strengths of Polyester GFRP 311

Figure C.12: Projected Tensile-Longitudinal Strengths of Polyester GFRP 312

Figure C.13: Projected Flexural-Transverse Strengths of Polyester GFRP. 313

Figure C.14: Projected Flexural-Longitudinal Strengths of Polyester GFRP 314

Figure C.15: Projected Shear-Transverse Strengths of Polyester GFRP. 315

Figure C.16: Projected Shear-Longitudinal Strengths of Polyester GFRP 316

Figure C.17: Projected Tensile Modulus-Transverse Strengths of Polyester GFRP ...... 317

Figure C.18: Projected Tensile Modulus-Longitudinal Strengths of Polyester GFRP... 318 


\section{APPENDIX-A DESIGN EXAMPLES}

\section{A.1 EXAMPLE 1 - OUTDOOR VINYLESTER GFRP BEAM}

This example illustrates a general application of reliability-based design to provide an acceptable level of failure probabilities of a Vinylester GFRP beam in the natural outdoor environment. This example provides a basis for discussion in Chapter 4 . 


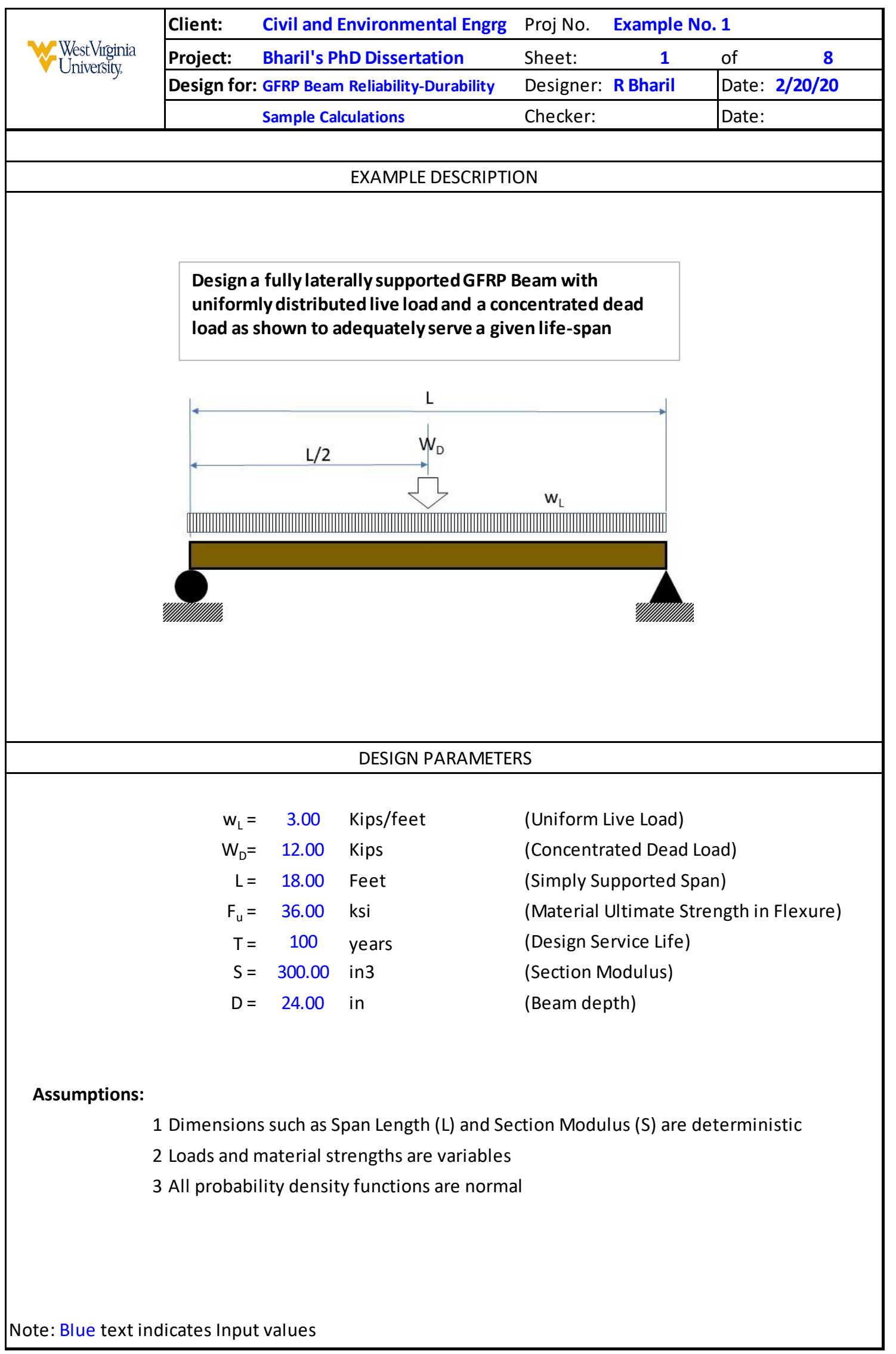




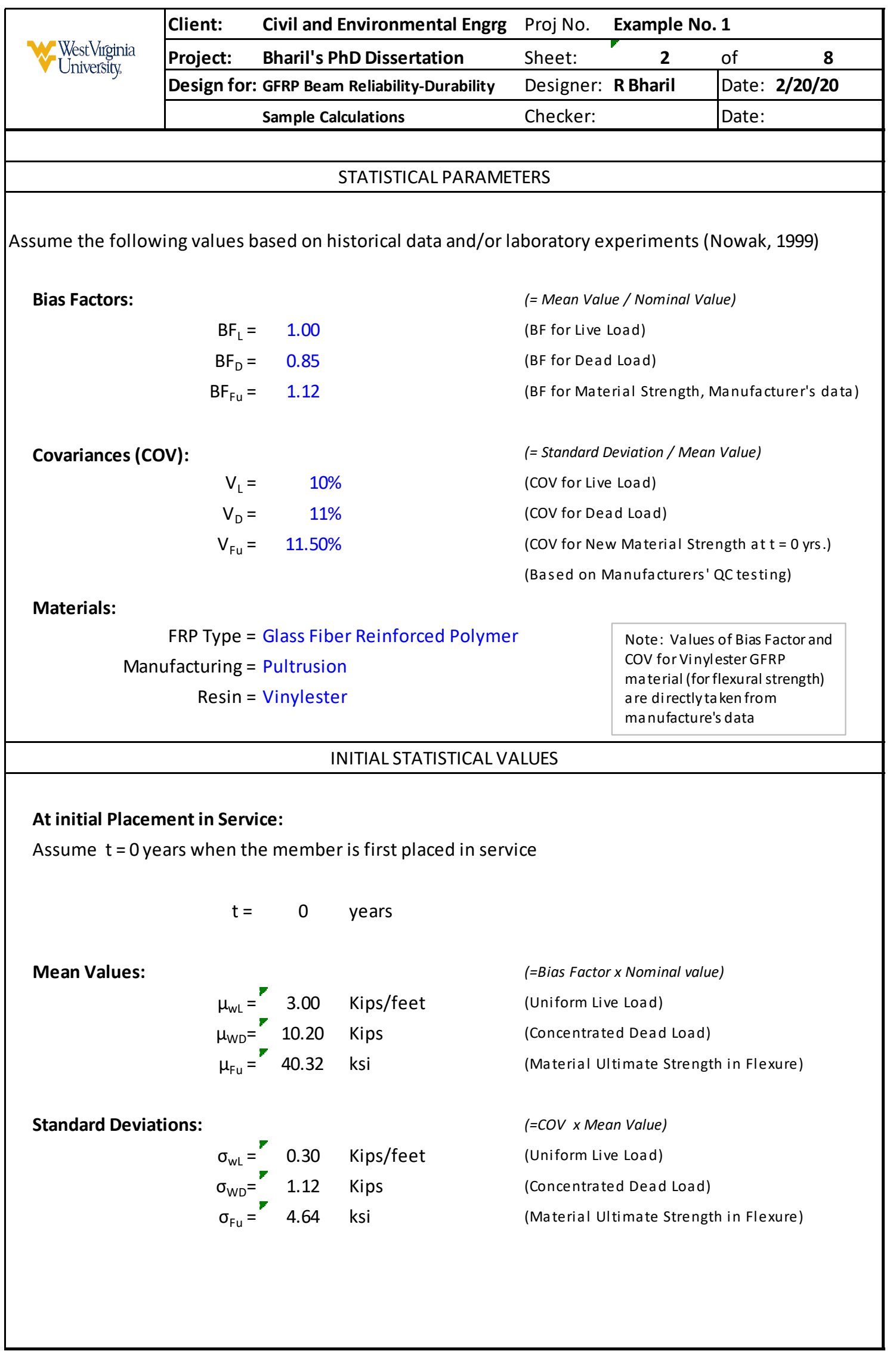




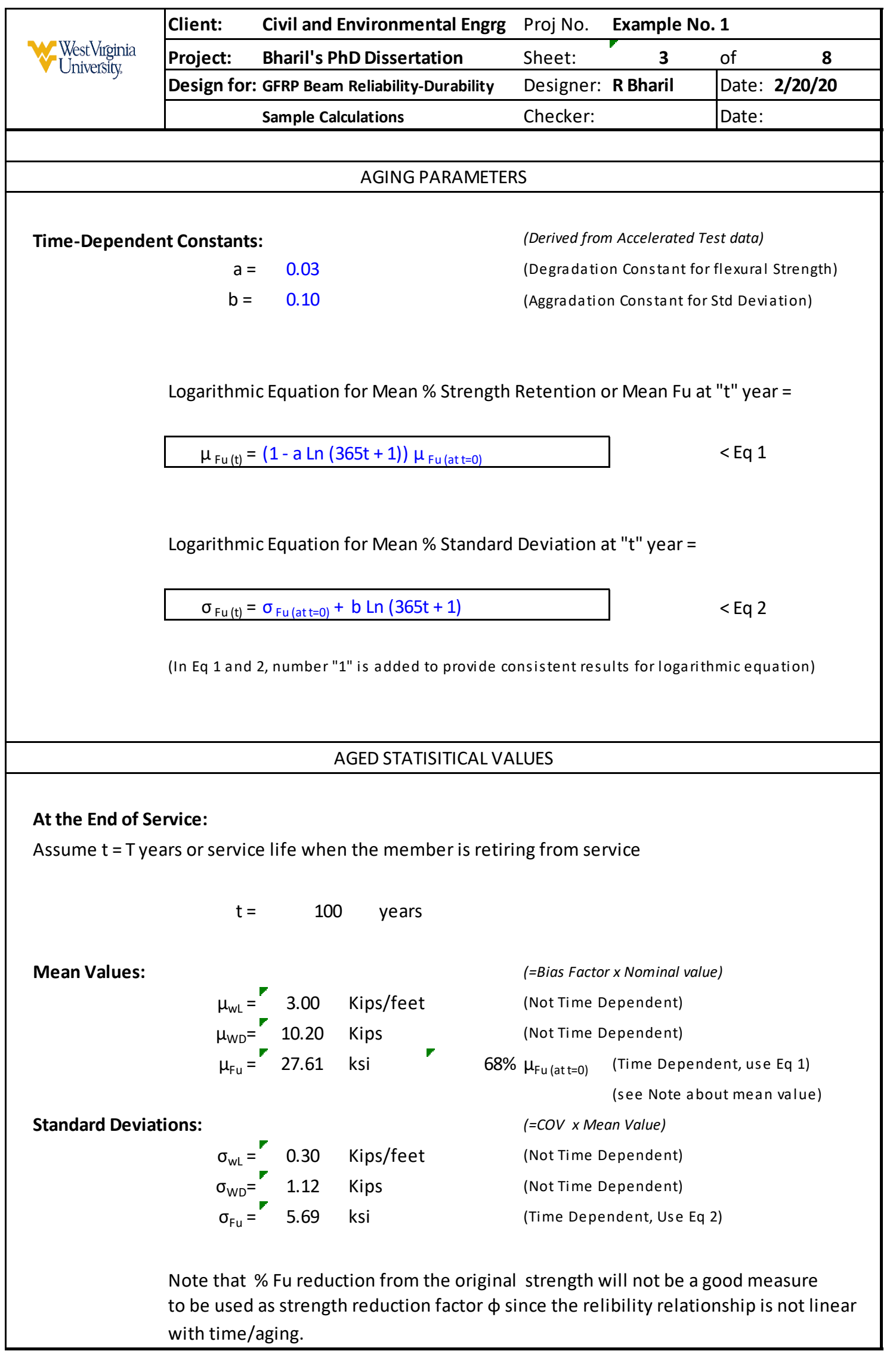




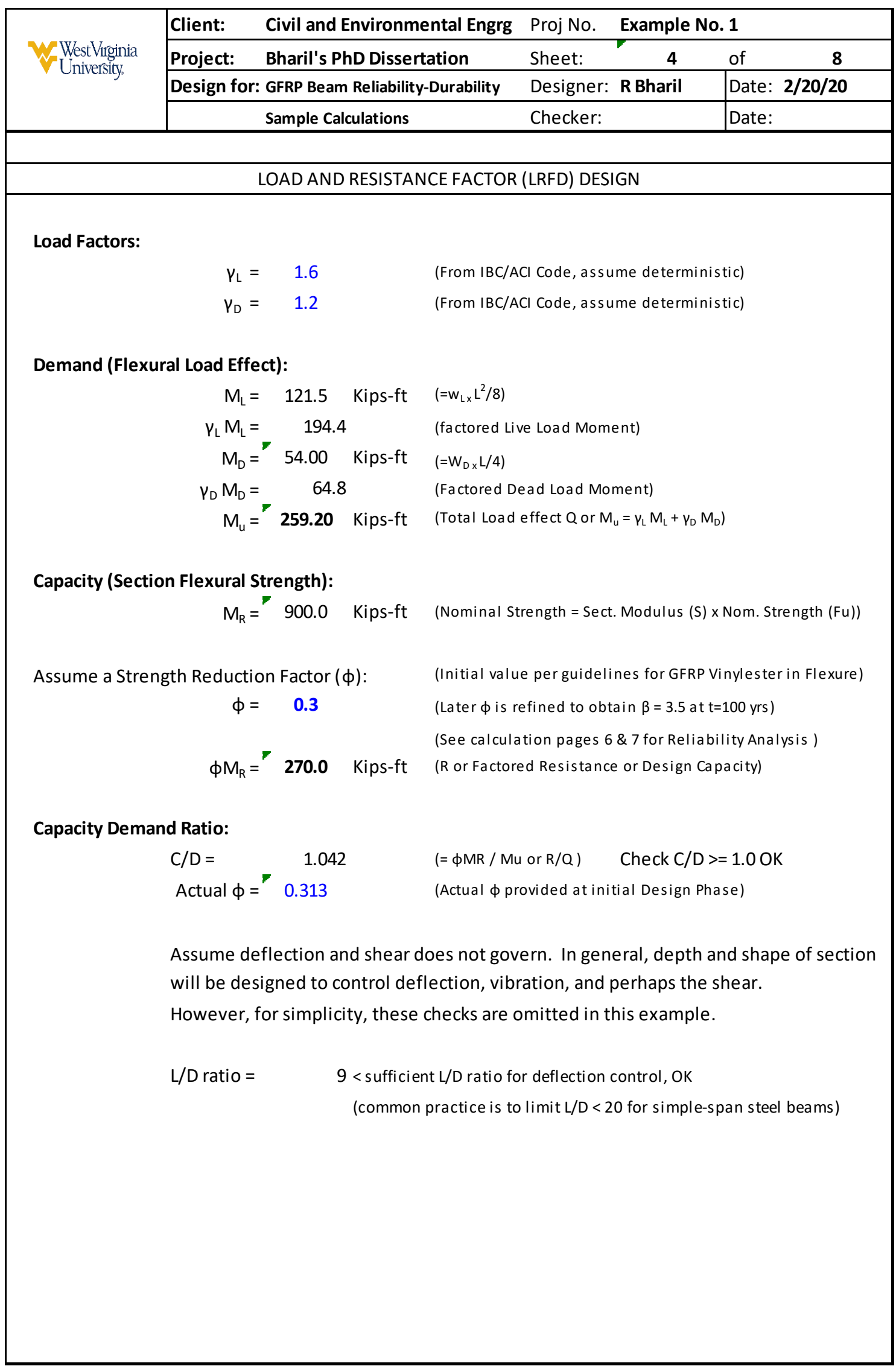




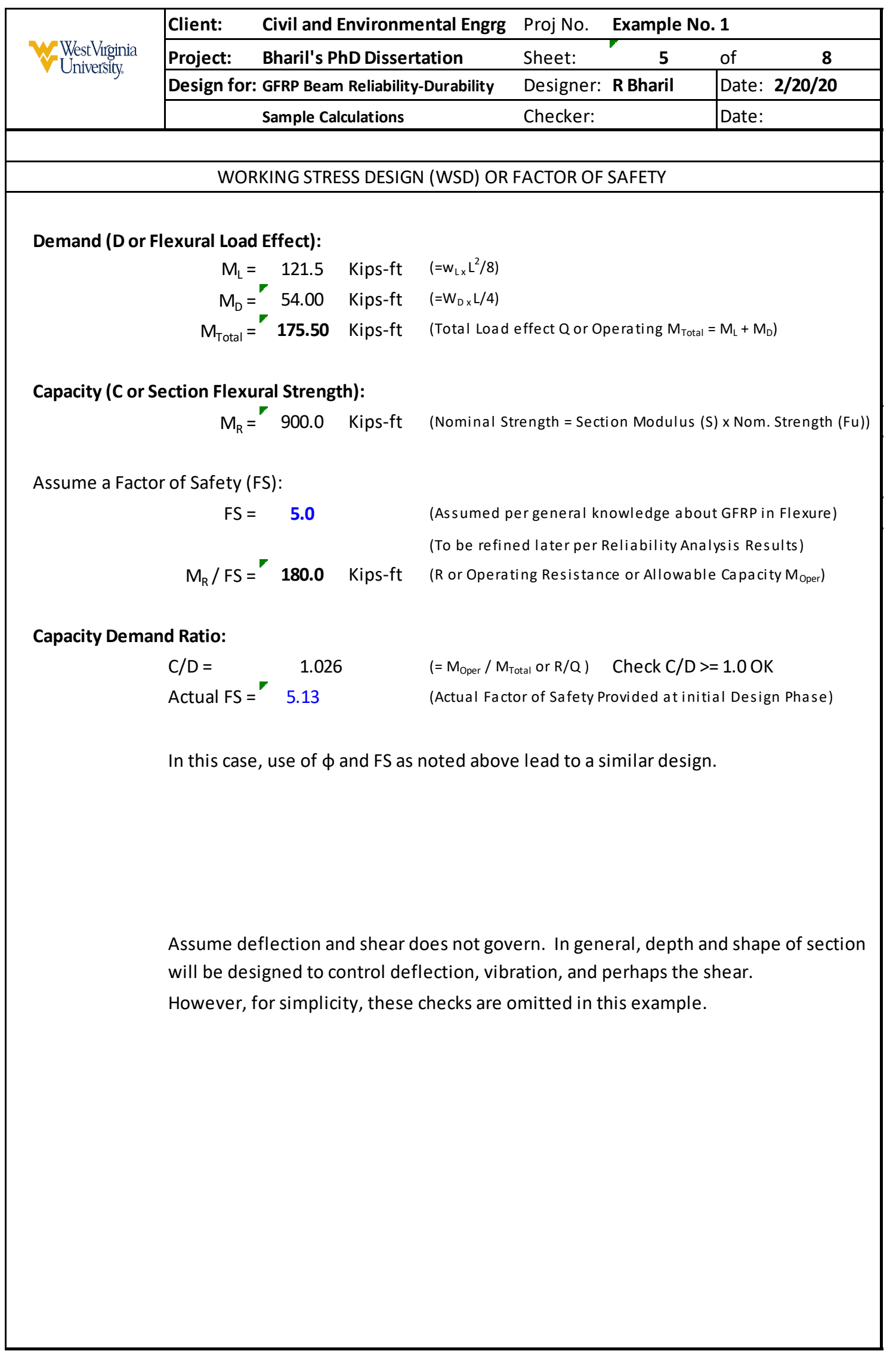




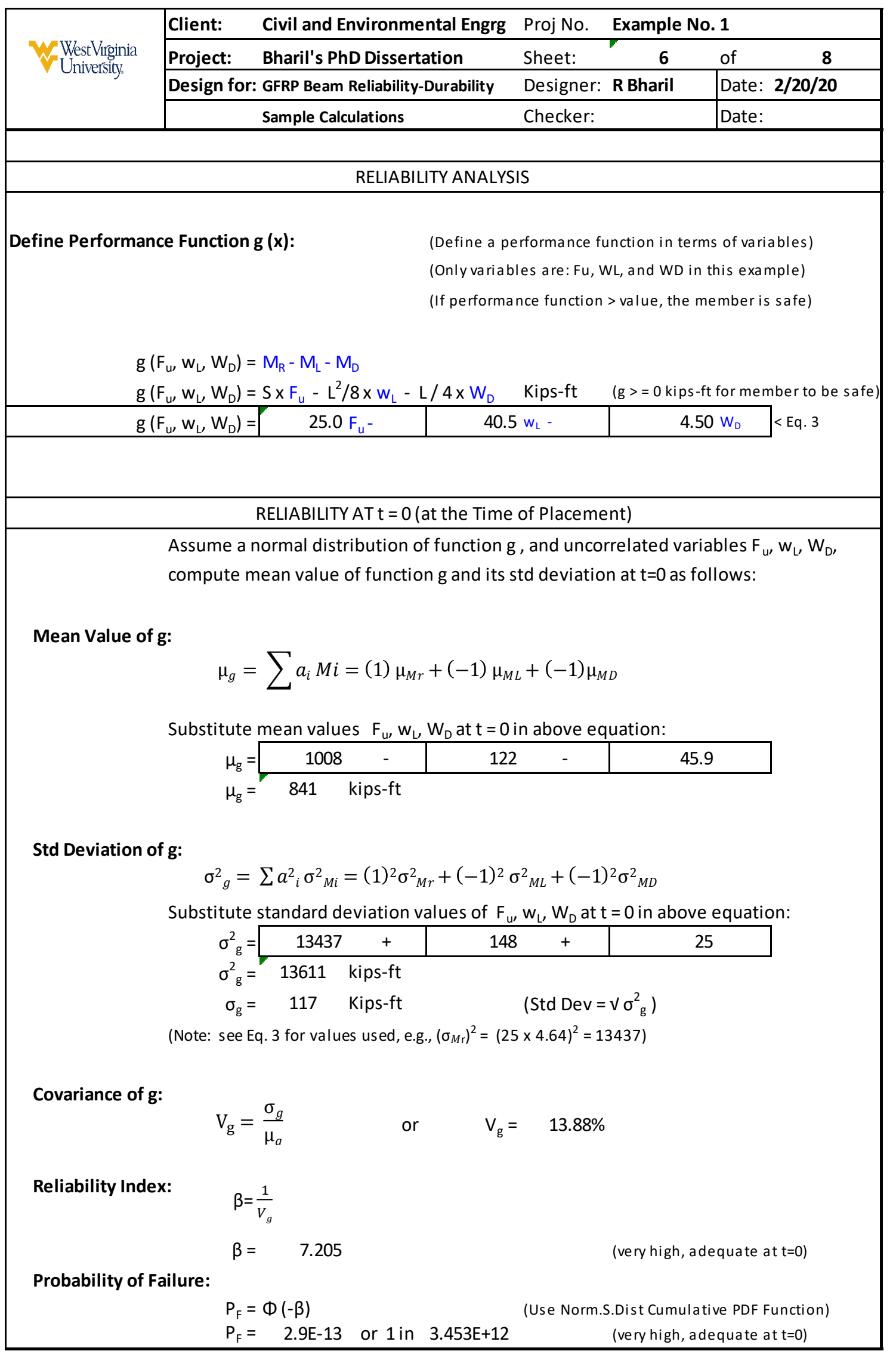




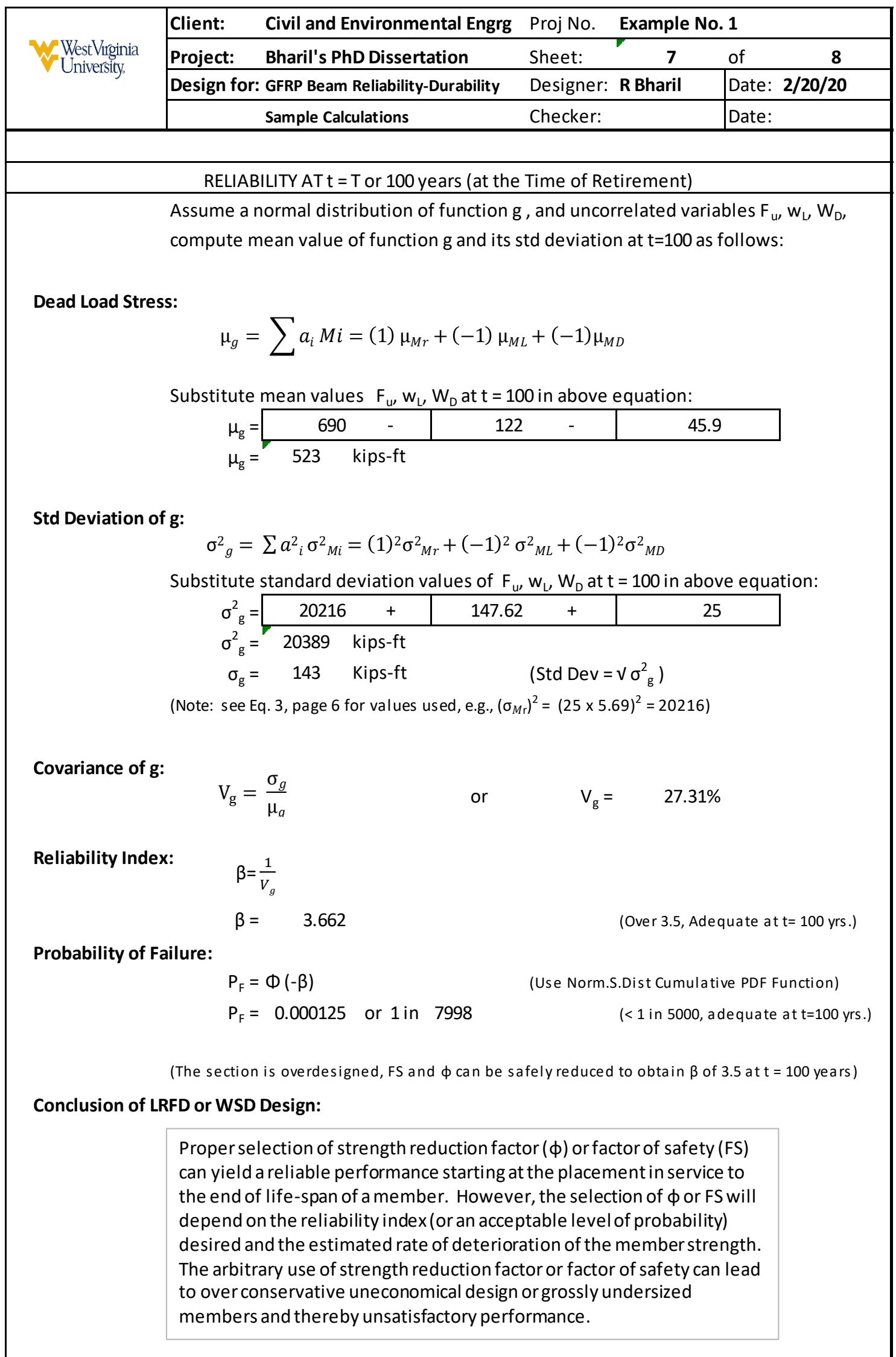




\begin{tabular}{|c|c|c|c|c|c|}
\hline \multirow{4}{*}{$\begin{array}{l}\text { WestVirginia } \\
\text { University. }\end{array}$} & \multirow{2}{*}{\begin{tabular}{|l|} 
Client: \\
Project: \\
\end{tabular}} & \multirow{2}{*}{\multicolumn{2}{|c|}{$\begin{array}{l}\text { Civil and Environmental Engrg } \\
\text { Bharil's PhD Dissertation }\end{array}$}} & \multirow{2}{*}{$\begin{array}{l}\text { Proj No. } \\
\text { Sheet: }\end{array}$} & Example No. 1 \\
\hline & & & & & of \\
\hline & \multicolumn{3}{|c|}{ Design for: GFRP Beam Reliability-Durability } & Designer: R Bharil & Date: $\mathbf{2 / 2 0 / 2 0}$ \\
\hline & \multicolumn{3}{|c|}{ Sample Calculations } & Checker: & Date: \\
\hline \multicolumn{6}{|c|}{ SUSTAINED STRESS / DEFLECTION CHECKS } \\
\hline \multicolumn{6}{|c|}{ 1) Perform a check of maximum sustained stress level (due to dead load) } \\
\hline \multicolumn{6}{|c|}{ Service Load Moments: } \\
\hline & $\mathrm{M}_{\mathrm{L}}=$ & \multicolumn{2}{|c|}{121.5 Kips-ft $\quad\left(=w_{L x} L^{2} / 8\right)$} & & \\
\hline & $M_{D}=$ & \multicolumn{2}{|c|}{54.00 Kips-ft $\quad\left(=W_{D x} L / 4\right)$} & & \\
\hline & $\mathrm{M}_{\text {Total }}=$ & 175.50 Kips-ft & \multicolumn{3}{|c|}{ (Total Load effect $Q$ or Operating $M_{\text {Total }}=M_{L}+M_{D}$ ) } \\
\hline \multicolumn{6}{|c|}{ Service Stresses: } \\
\hline & $f_{d}=$ & $2.2 \mathrm{ksi}$ & \multicolumn{3}{|c|}{ ( = Sustained Stress Moment (M) / Section Modulus (S)) } \\
\hline & $\mathrm{Fu}=$ & $36.00 \quad \mathrm{ksi}$ & \multicolumn{3}{|c|}{ (= Nominal Ultimate Flexural Strength) } \\
\hline & $\mathrm{BF}_{\mathrm{FU}}=$ & 1.12 & \multicolumn{3}{|c|}{ (=Bias Factor for Material Strength) } \\
\hline \multicolumn{2}{|c|}{ Sustained Stress Ratio $=$} & $5 \%$ & \multicolumn{3}{|c|}{$\left(=f_{d} / \mu_{\mathrm{Fu}}\right)$} \\
\hline \multicolumn{2}{|r|}{$\mathrm{T}=$} & 100 year & \multicolumn{3}{|c|}{ (=service life) } \\
\hline \multicolumn{2}{|c|}{ Max Allowable Ratio = } & $21 \%$ & (=from table & 5.6 in Chapter 5) & \\
\hline BF x Max Allow & able Ratio $=$ & $24 \%$ & $>$ Computed & Sustained Stress Ratic & \\
\hline 2) Check Dead & oad Deflectio & ons: & & & \\
\hline Elastic & Modulus $\mathrm{E}=$ & $3000 \mathrm{msi}$ & $(=$ value obt & ined form FRP manufa & \\
\hline Creep M & dification = & 0.37 & (=from Table & 5.7 , use $10 \%$ initial str & ratio) \\
\hline Effective $\mathrm{N}$ & odulus $\mathrm{E}_{\mathrm{eff}}=$ & $1110 \mathrm{msi}$ & ( $=$ Creep Moc & ification x Elastic Mod & \\
\hline & $\mathrm{I}=$ & 3600 in 4 & (=Moment o & Inertia of 24 " deep I-b & \\
\hline & $\Delta_{\mathrm{D}}=$ & 0.630486 in & $\left(=W_{D} L^{3} / 48 E_{e f}\right.$ & & \\
\hline & & $343 \mathrm{~L} / \Delta$ Ratio & (Allowance & or pre-camber) & \\
\hline 3) Check for Liv & Load Deflect & ction at the end of $L$ & ife: & & \\
\hline Aging Coeff $\mathrm{fc}$ & r Modulus = & $95 \%$ & (from natura & ( aging data) & \\
\hline Aged M & dulus $\mathrm{E}_{\mathrm{age}}=$ & $2850 \mathrm{msi}$ & & & \\
\hline & $\Delta_{\mathrm{L}}=$ & 0.7 in & $l=5 / 384 \times W_{L} L$ & $\left.4 / E_{\text {aged }} I\right)$ & \\
\hline & & $313 \mathrm{~L} / \Delta$ Ratio & $(<\mathrm{L} / 240 \mathrm{limi}$ & ts, Aged modulus used & \\
\hline Shear & Modulus G = & $0.5 \mathrm{msi}$ & $(=$ value obt & ined form FRP manufa & er or computed) \\
\hline Aged Shear & lodulus $\mathrm{G}_{\mathrm{A}}=$ & $0.475 \mathrm{msi}$ & (=use same & modification factor as & \\
\hline Area & f Web Aw = & 24 in2 & (=assume 2 & deep beam $\times 1$ " thick & \\
\hline Shear Defl from & Jead Load = & 0.06 in & $\left(=W_{D} L /\left(4 G_{A} A\right.\right.$ & w), from FRP design bo & rnotes, Ref. Dr. Hota) \\
\hline Shear Defl fror & Live Load = & 0.13 in & $\left(=\mathrm{w}_{\mathrm{L}} \mathrm{L}^{2} /\left(8 \mathrm{G}_{\mathrm{A}} \mathrm{A}\right.\right.$ & & \\
\hline Total Live Load & Deflection = & 0.8 in & & of L, Still OK & \\
\hline CONCLUSION: & Note: Check & ks for number of fat & igue cycles & and vibration (floor $\mathrm{c}$ & are not performed. \\
\hline & Beams passe & ses typical checks fo & r strength al & Id serviceability limi & ates for 100 -year life \\
\hline
\end{tabular}




\section{A.2 Example 2 - Design Of a SubSea Polyester GFRP Tension Bracing}

This example illustrates a specific reliability-based design to provide an acceptable level of failure probabilities for a Polyester GFRP tension bracing in a long-term fully immerged marine environment. This example provides a basis for discussion of a Case Study in Chapter 6. 


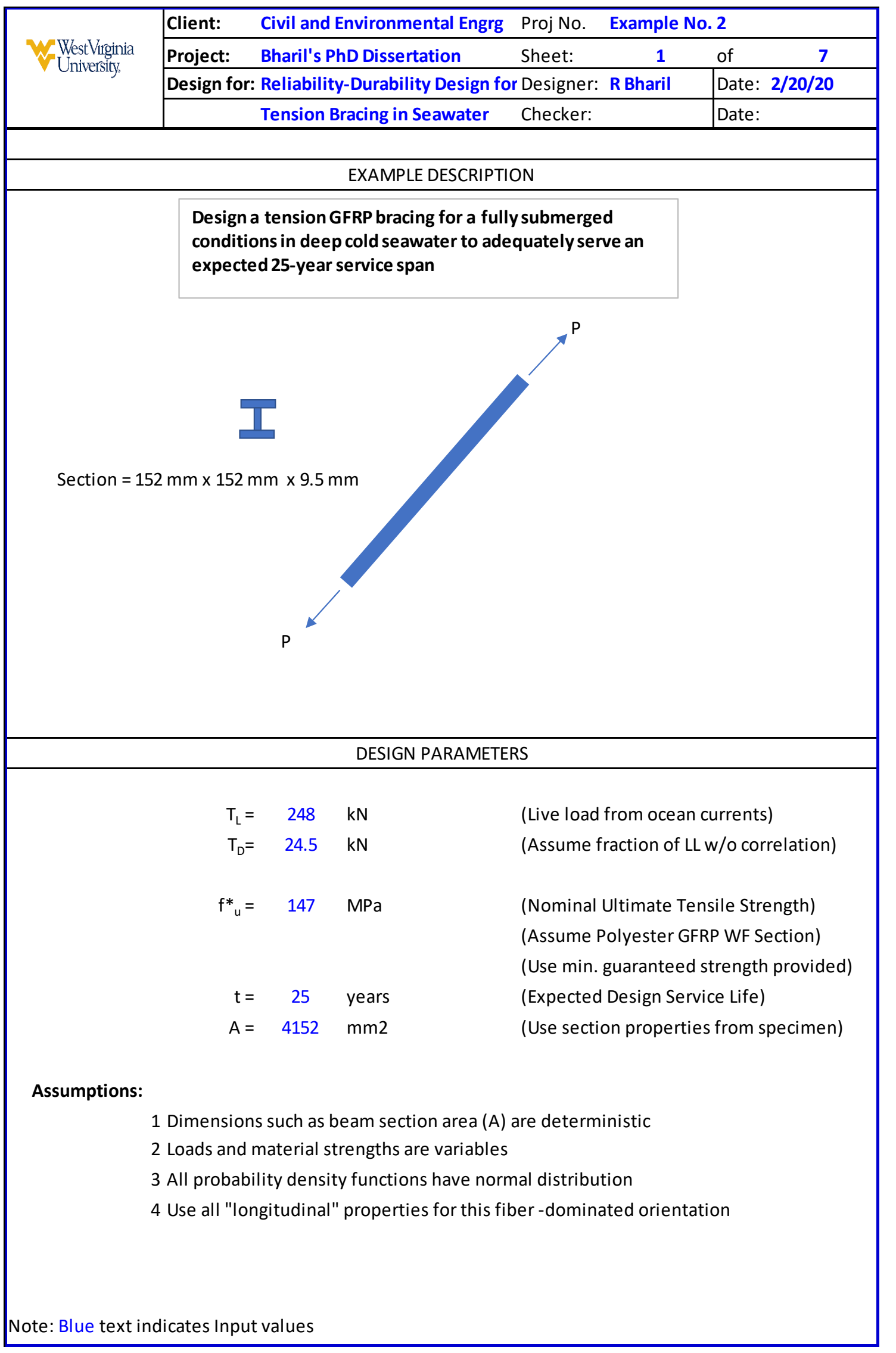




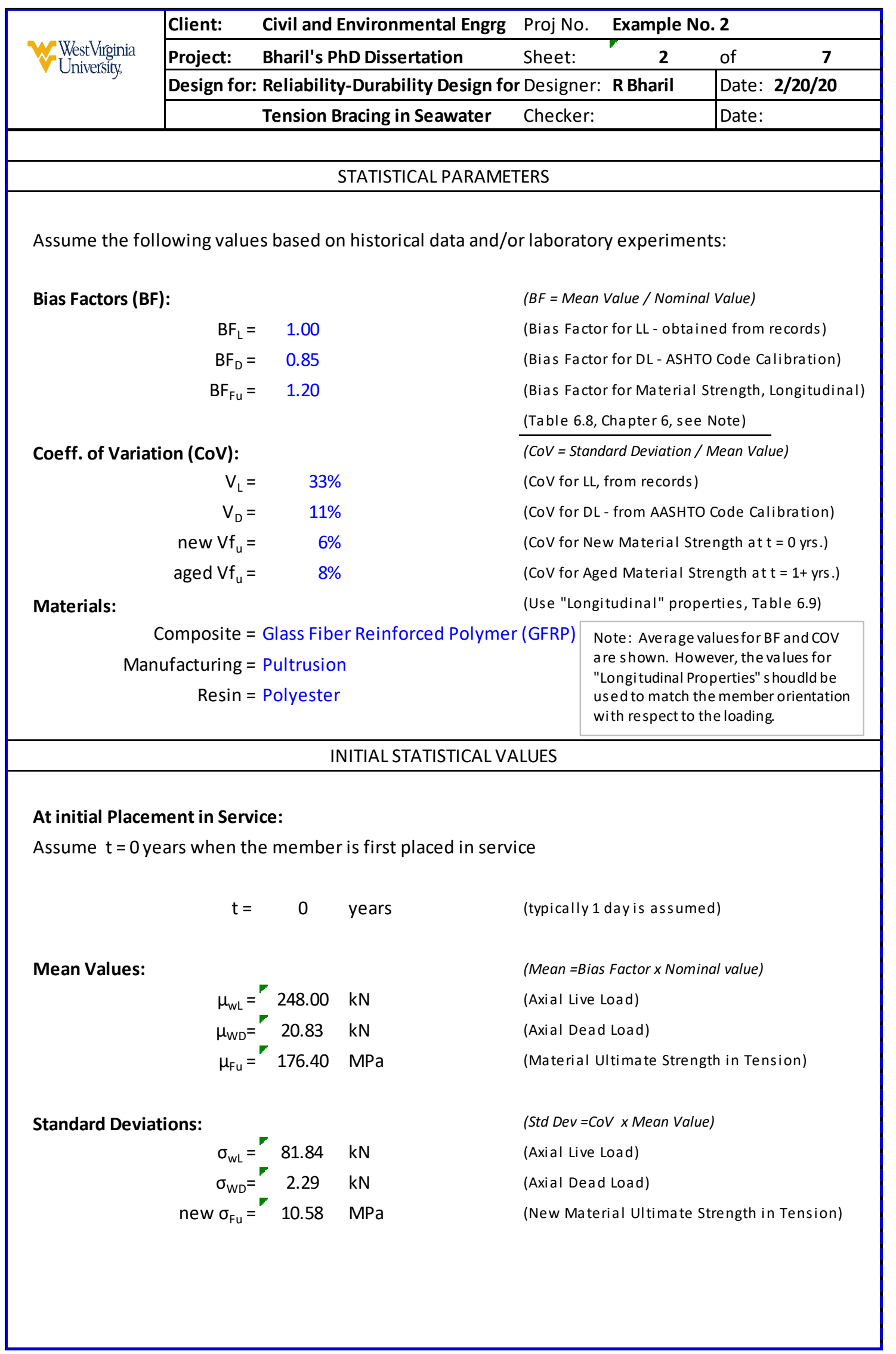




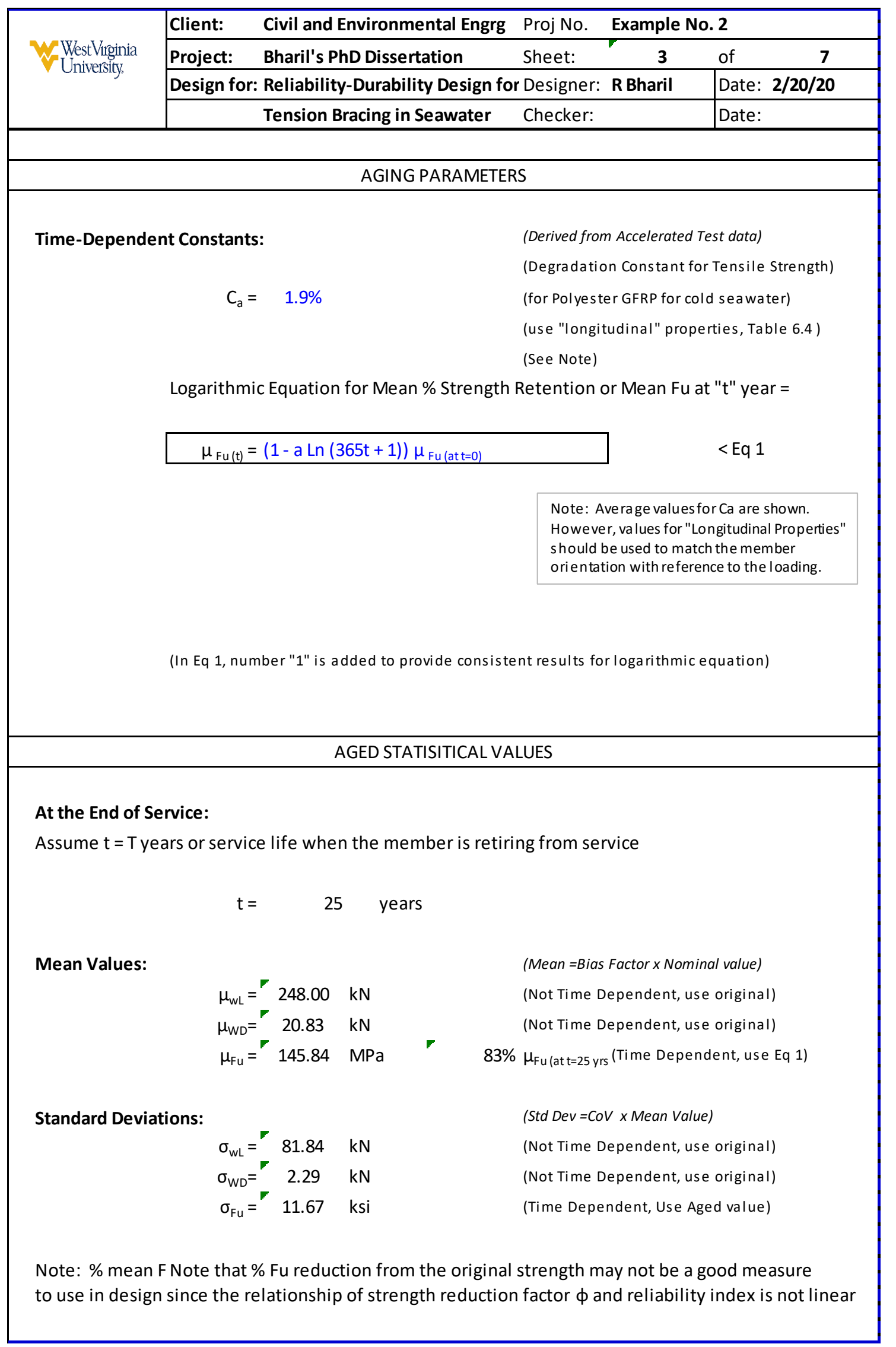




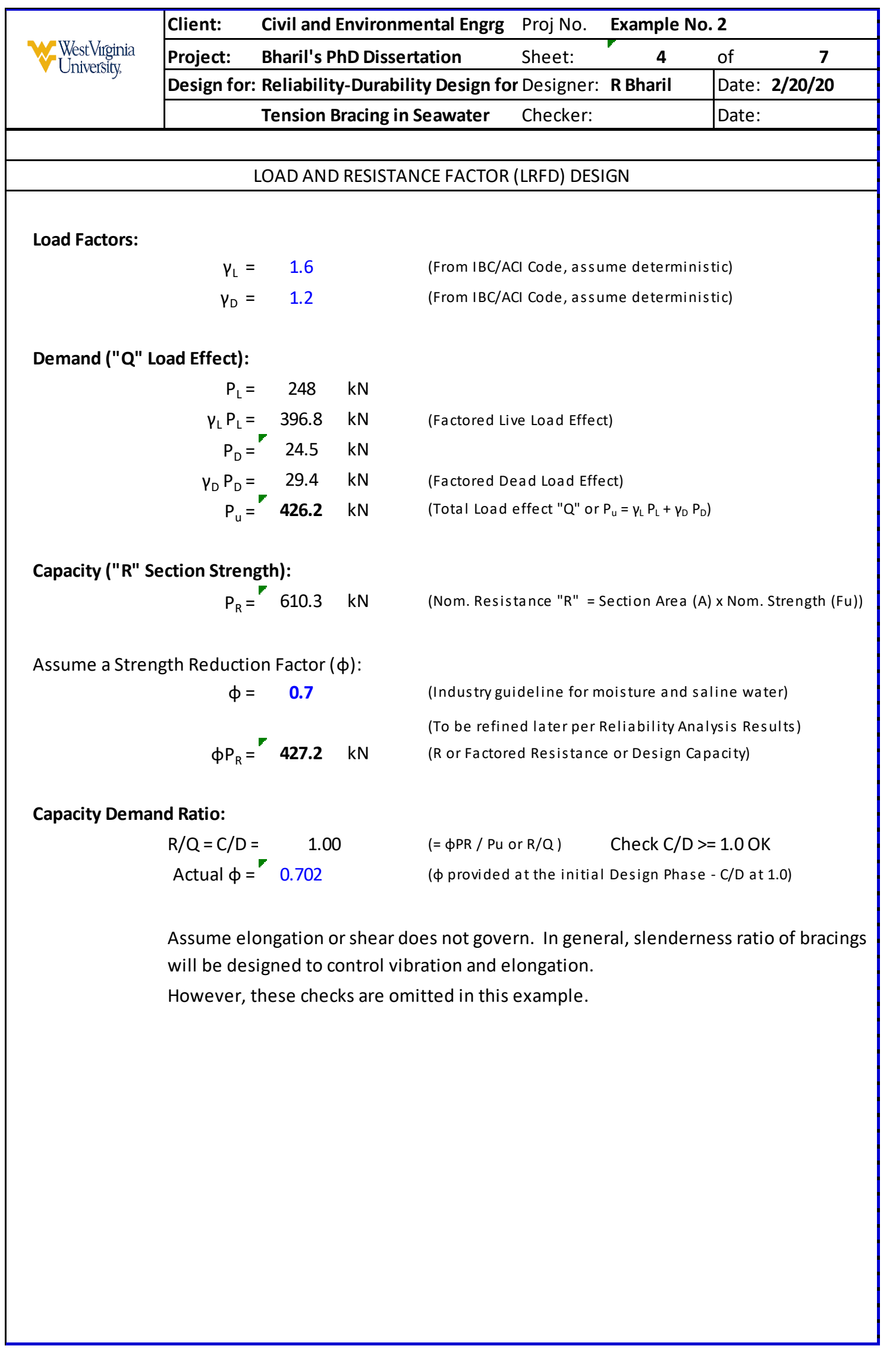




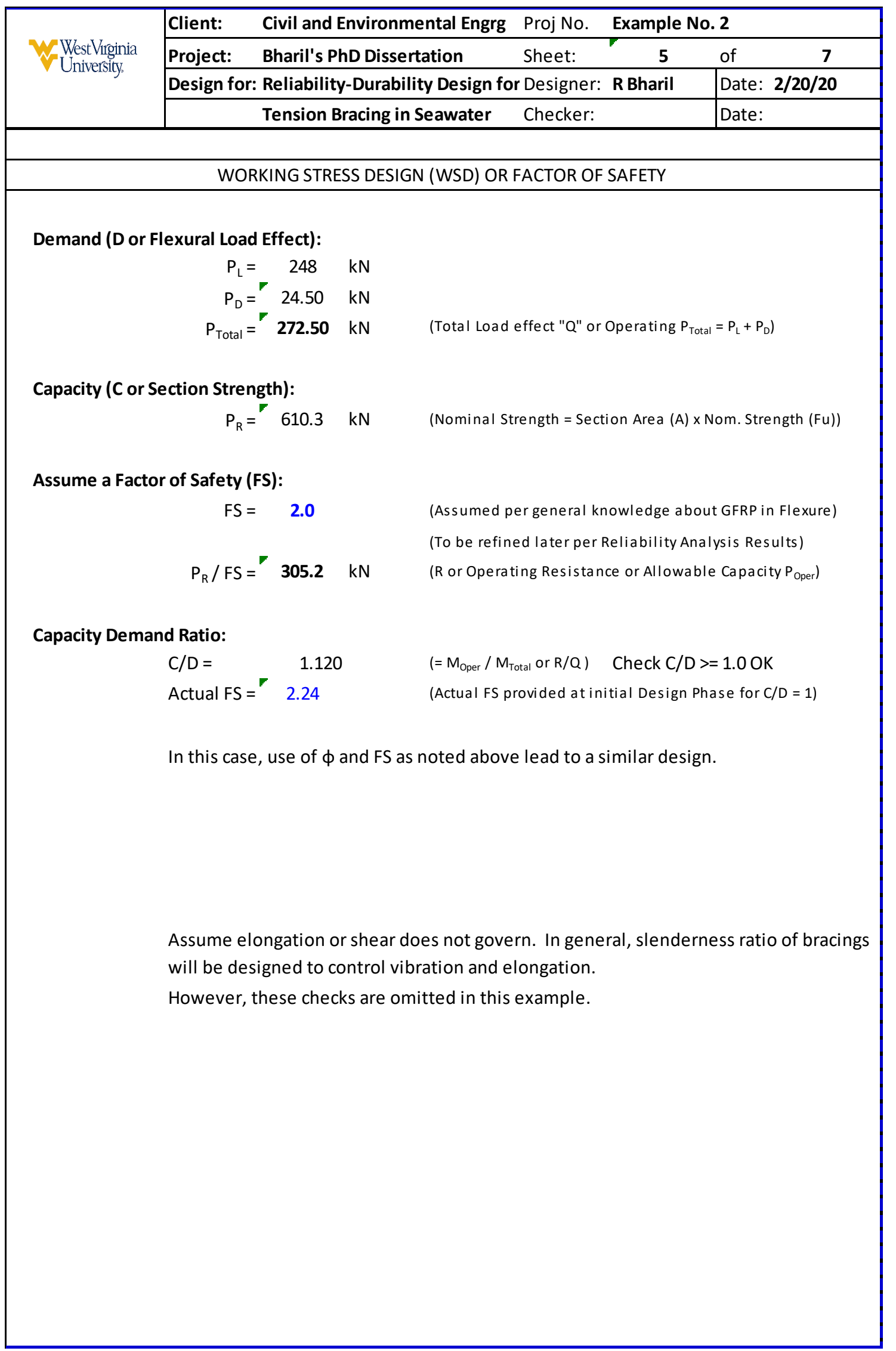




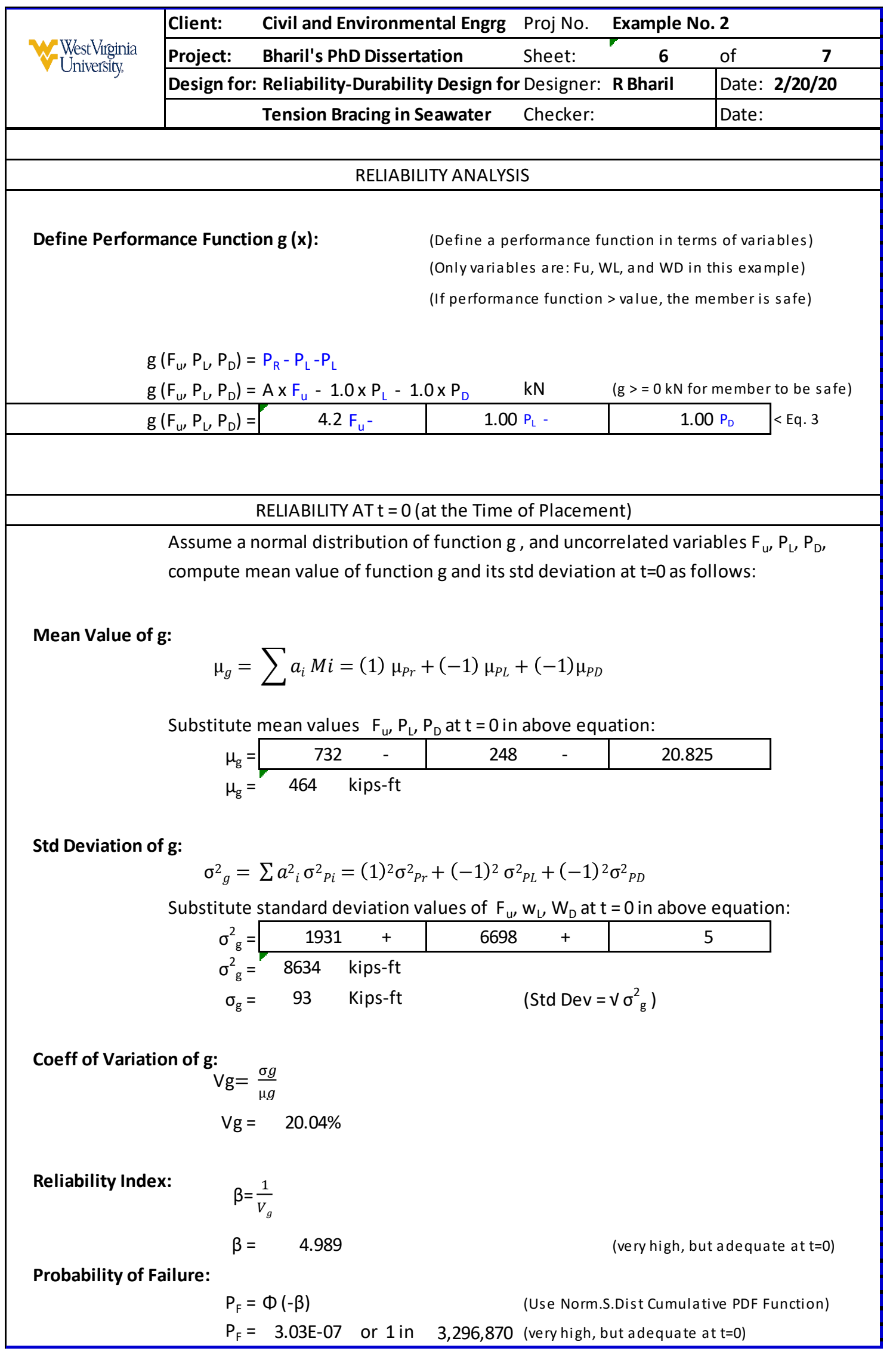




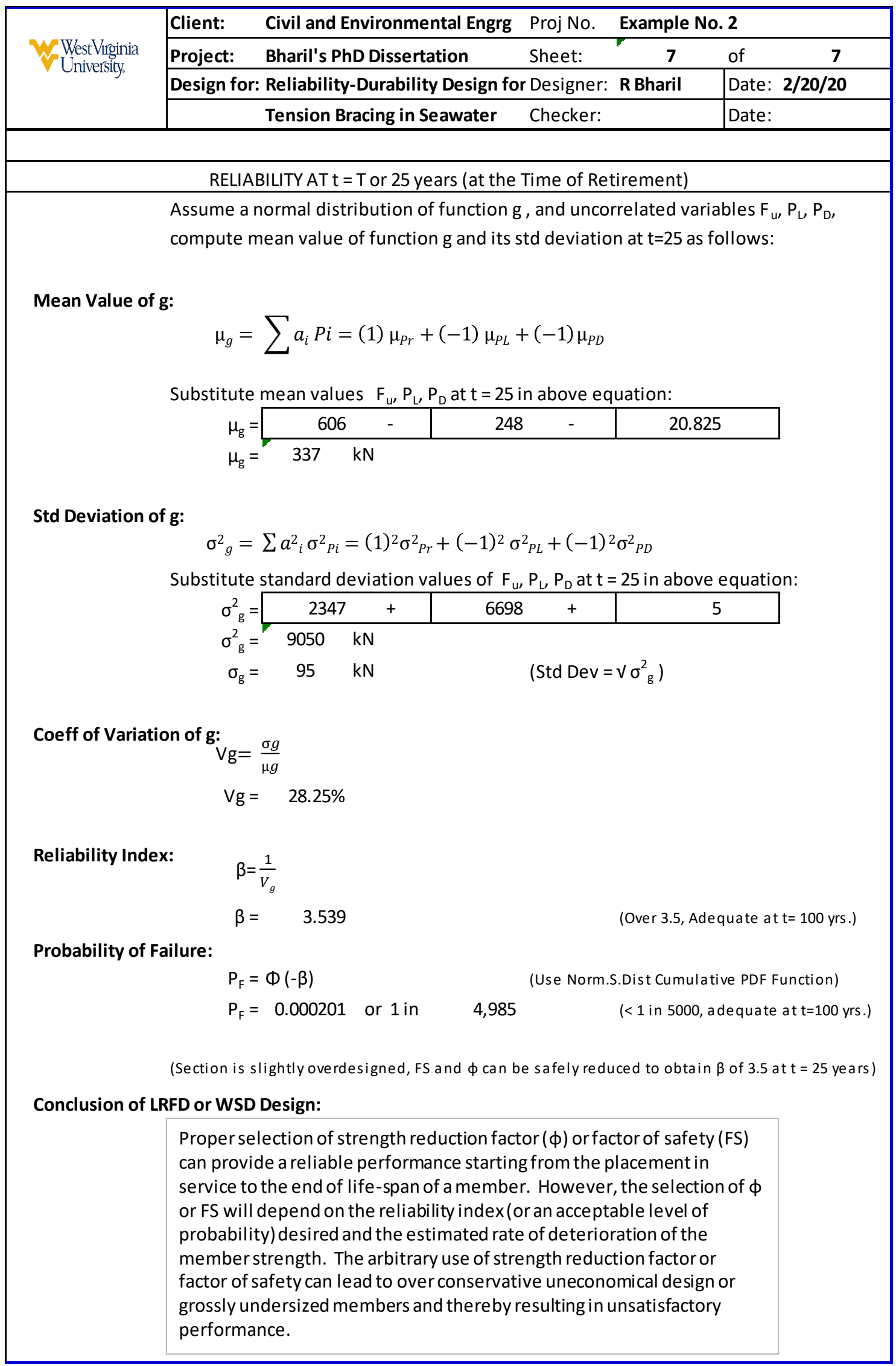




\section{A.3 EXAMPLE 3 - DESIGN OF A SUBSEA VINYLESTER GFRP BEAM}

The example illustrates a specific reliability-based design to provide an acceptable level of failure probabilities for a Vinylester GFRP beam in a long-term fully immerged marine environment. This example provides a basis for discussion of a Case Study in Chapter 6. 


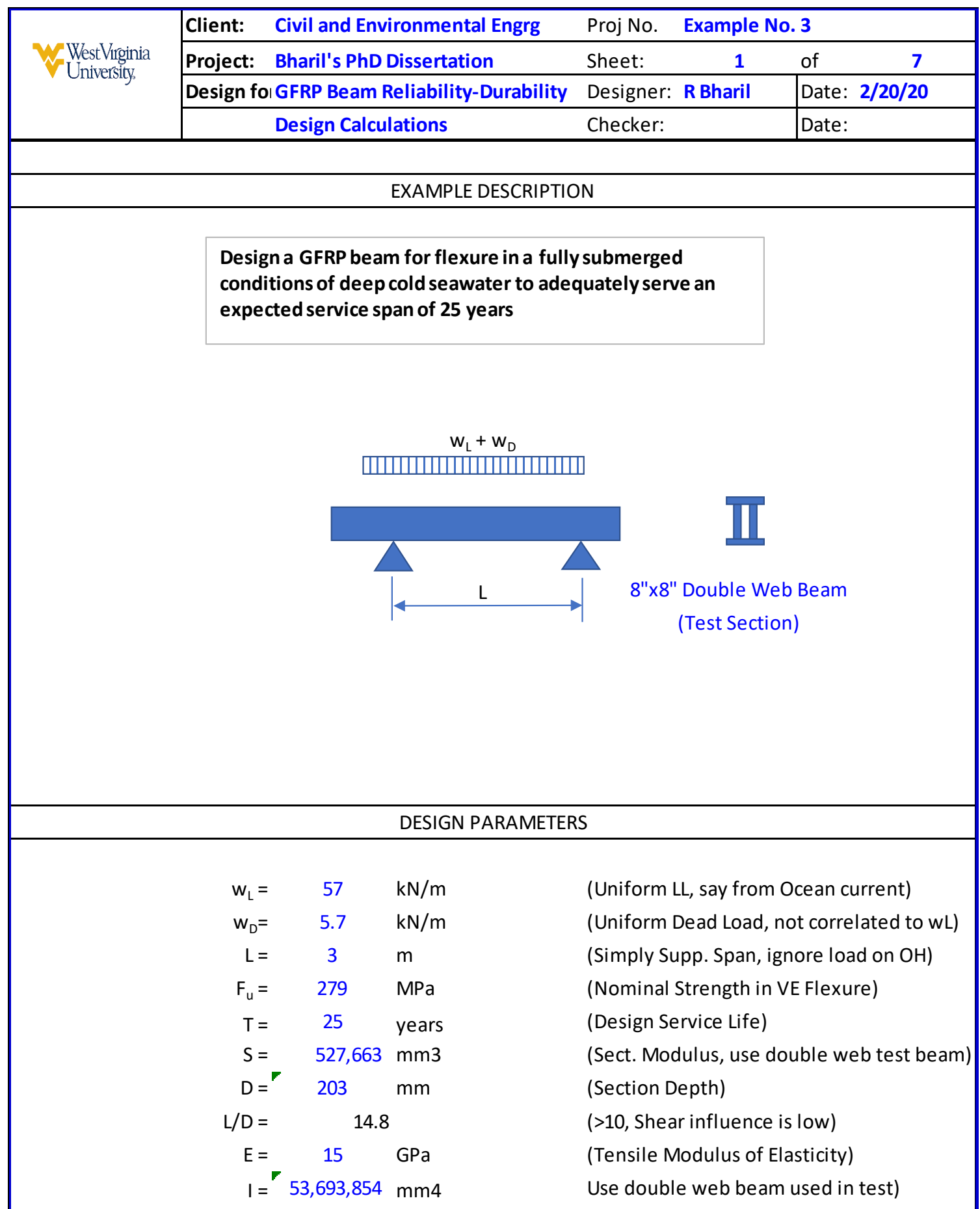

Assumptions:

1 Dimensions such as Span Length (L) and Section Modulus (S) are deterministic

2 Loads and material strengths are variables

3 All probability density functions are normal

4 Use "longitudinal" properties for bending, and "transverse" for shear

Note: Blue text indicates Input values 


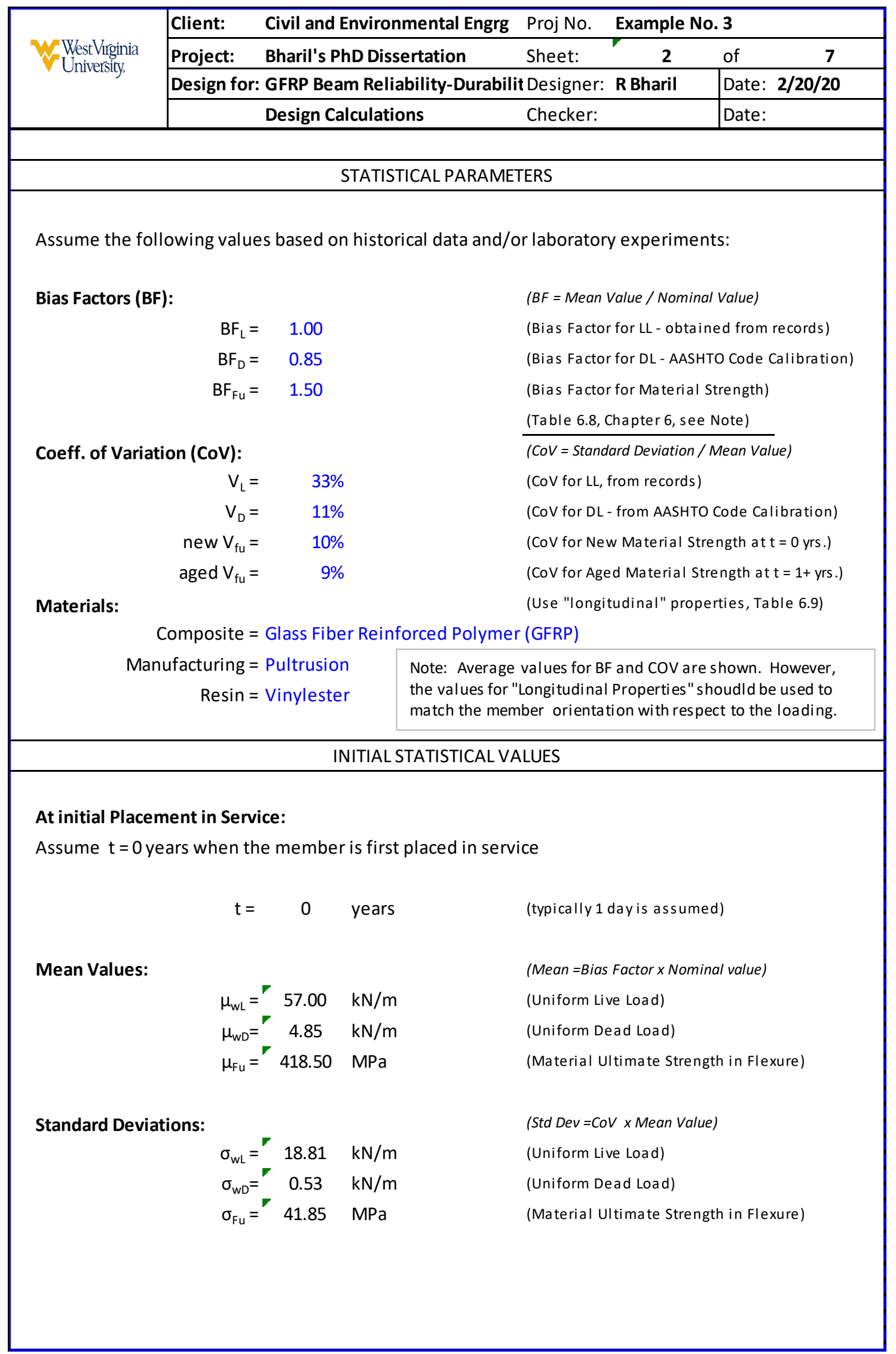




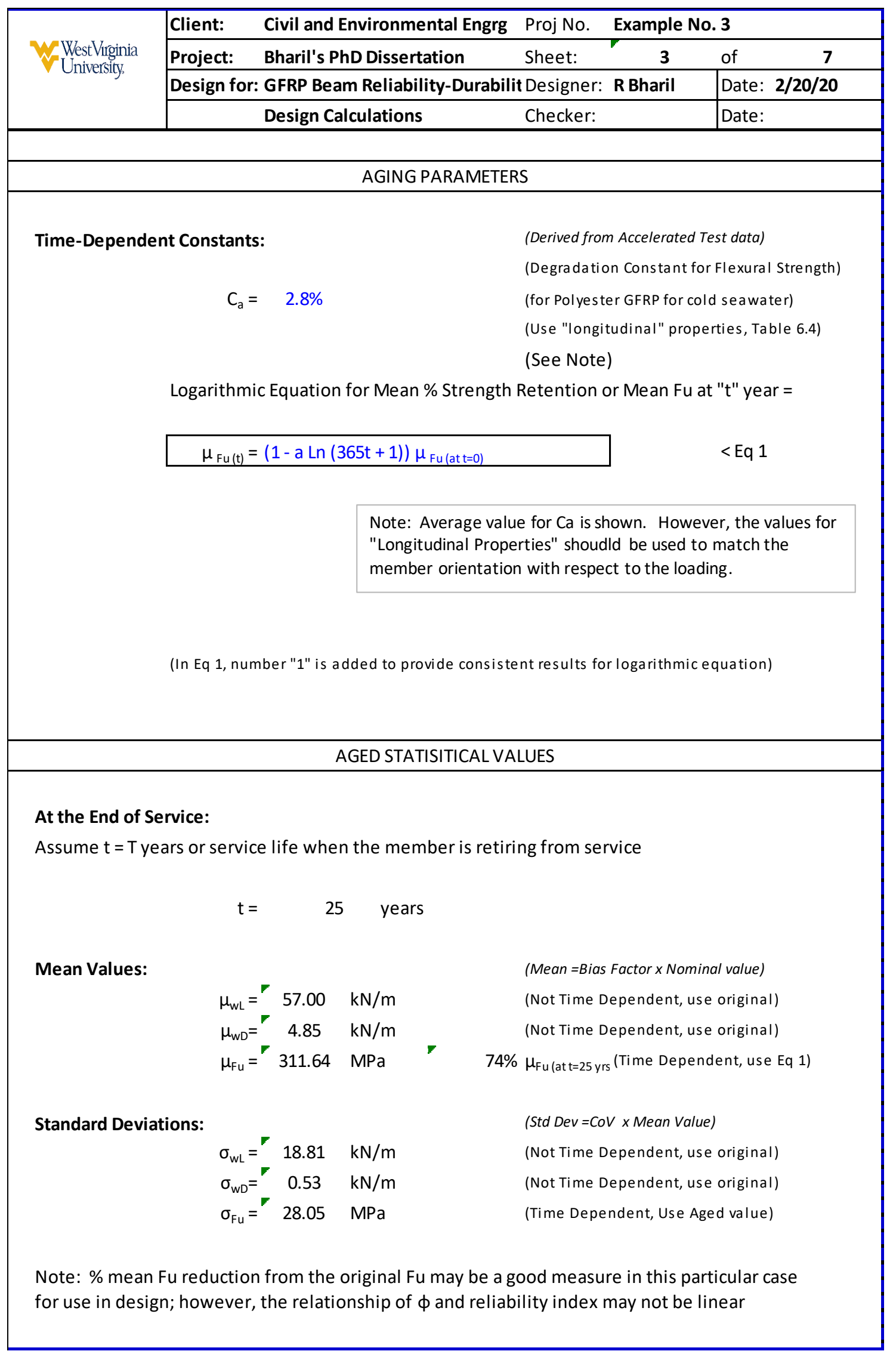




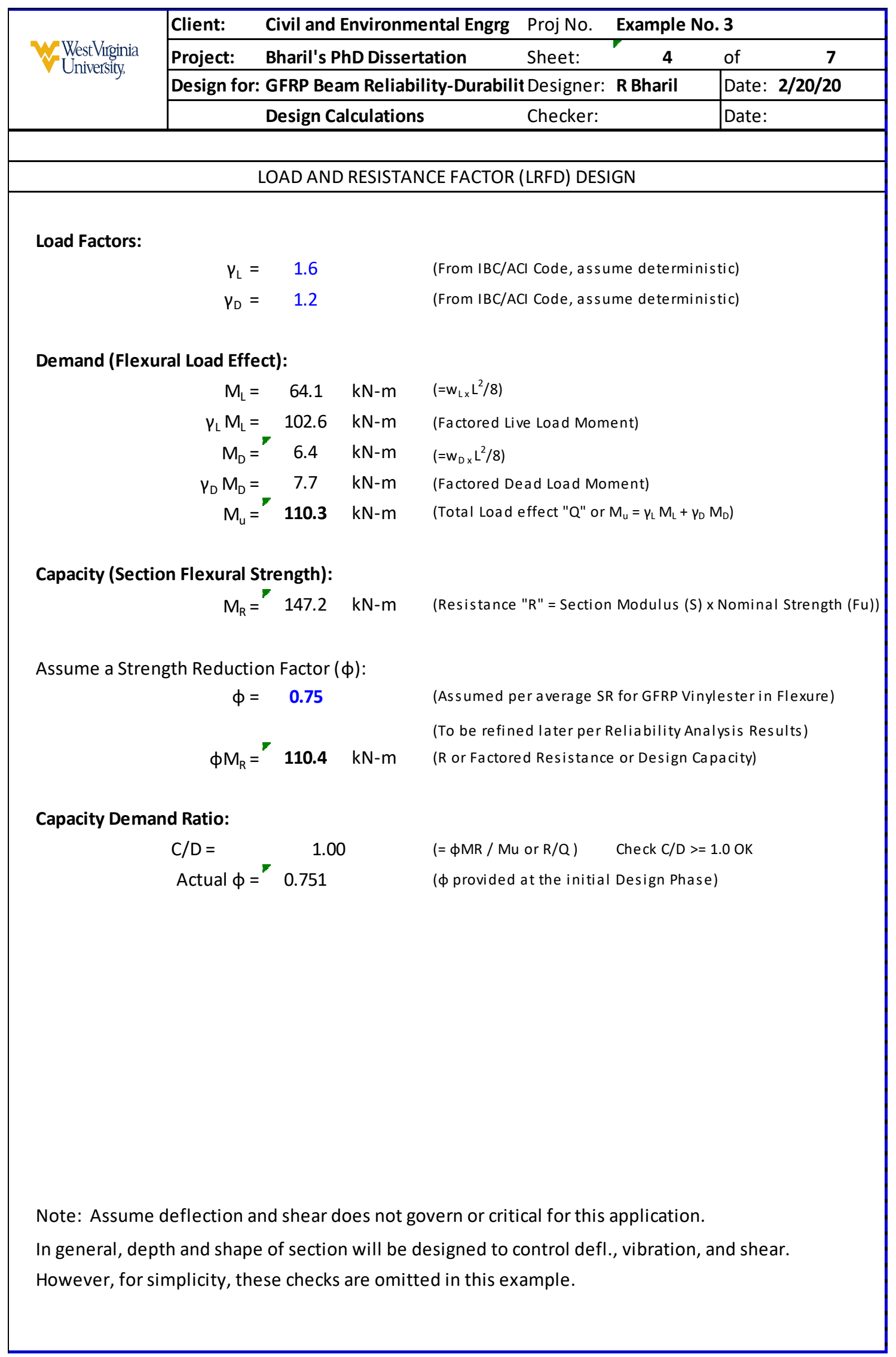




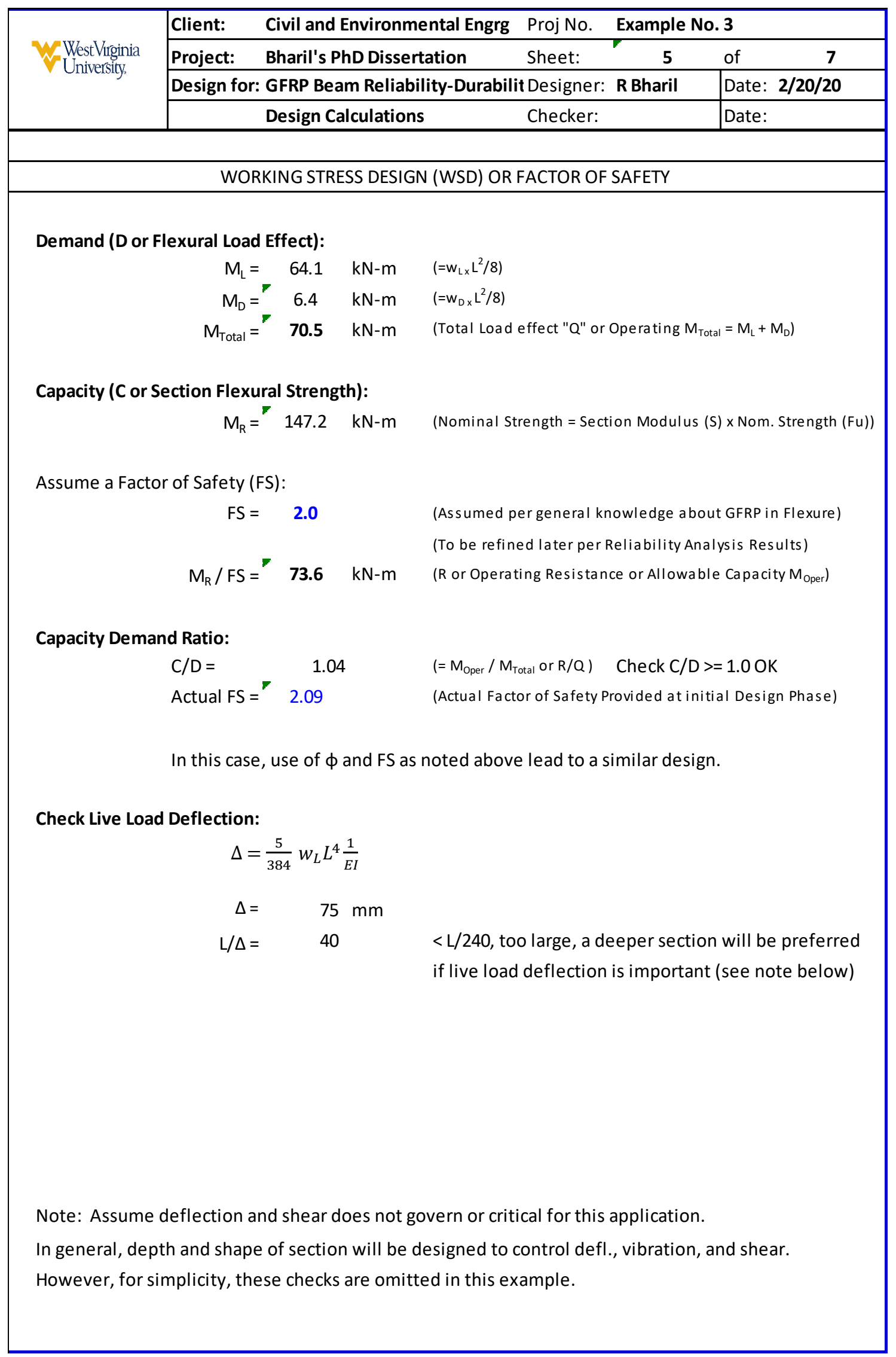




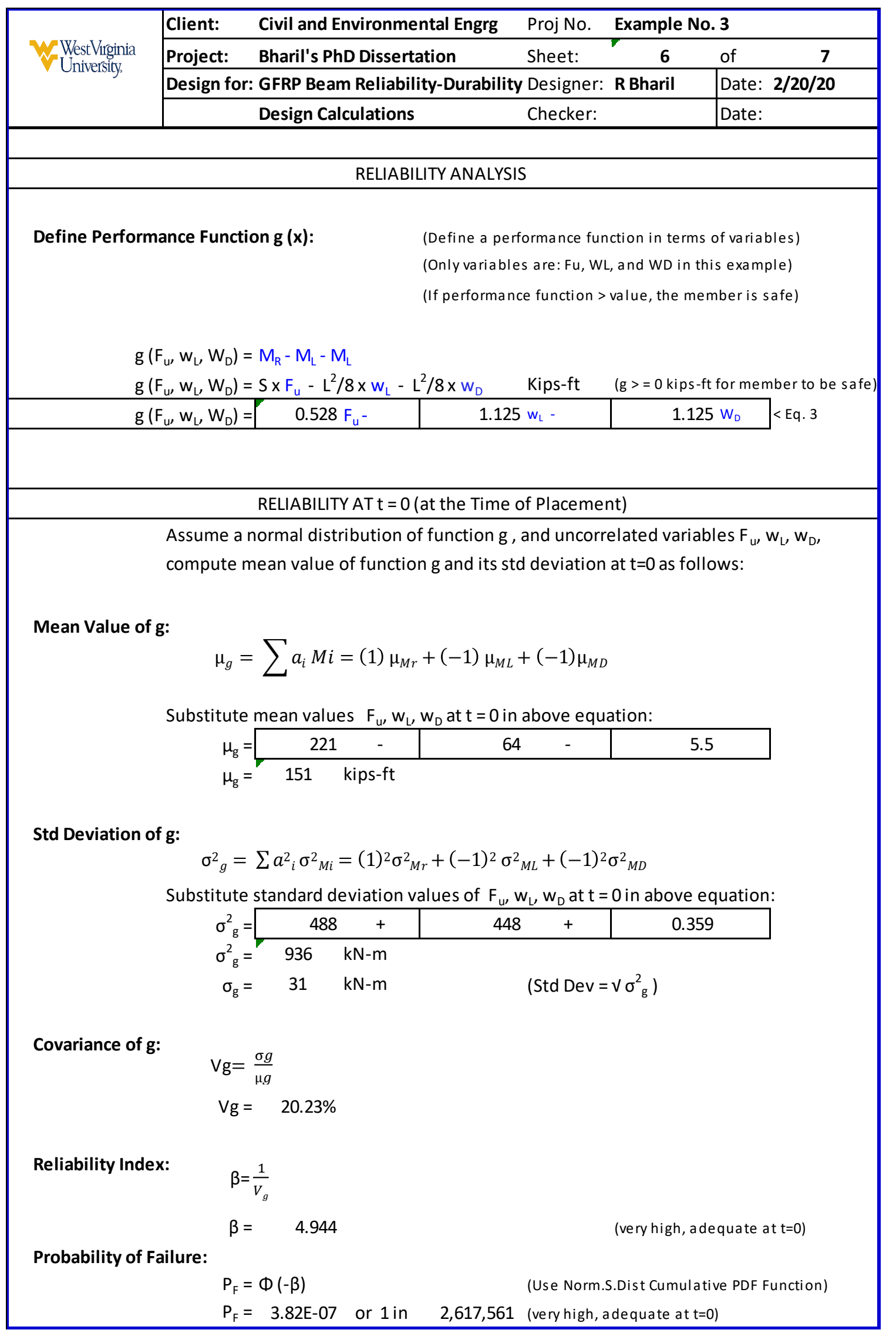




\begin{tabular}{|c|c|c|c|c|c|}
\hline \multirow{4}{*}{$\begin{array}{l}\text { WestVirginia } \\
\text { University, }\end{array}$} & \multirow{2}{*}{\begin{tabular}{|l|} 
Client: \\
Project:
\end{tabular}} & \multirow{2}{*}{$\begin{array}{l}\text { Civil and Environmental Engrg } \\
\text { Bharil's PhD Dissertation }\end{array}$} & \multirow{2}{*}{$\begin{array}{l}\text { Proj No. } \\
\text { Sheet: }\end{array}$} & \multicolumn{2}{|c|}{ Example No. 3} \\
\hline & & & & 7 & of \\
\hline & \multicolumn{4}{|c|}{ Design for: GFRP Beam Reliability-Durabilit Designer: R Bharil } & Date: $\mathbf{2 / 2 0 / 2 0}$ \\
\hline & & Design Calculations & Checker: & & Date: \\
\hline \multicolumn{6}{|c|}{ RELIABILITY AT $\mathrm{t}=\mathrm{T}$ or 25 years (at the Time of Retirement) } \\
\hline & \multicolumn{5}{|c|}{$\begin{array}{l}\text { Assume a normal distribution of function } g \text {, and uncorrelated variables } F_{u}, W_{L}, W_{D} \text {, } \\
\text { compute mean value of function } g \text { and its std deviation at } t=25 \text { as follows: }\end{array}$} \\
\hline
\end{tabular}

Mean Value of $g$ :

$$
\mu_{g}=\sum a_{i} M i=(1) \mu_{M r}+(-1) \mu_{M L}+(-1) \mu_{M D}
$$

Substitute mean values $F_{u}, W_{L}, w_{D}$ at $t=25$ in above equation:

\begin{tabular}{|c|c|c|c|c|c|}
\hline$\mu_{g}=$ & 164 & - & 64 & - & 5.45 \\
\hline
\end{tabular}

Std Deviation of $\mathbf{g}$ :

$$
\sigma_{g}^{2}=\sum a_{i}^{2} \sigma_{M i}^{2}=(1)^{2} \sigma_{M r}^{2}+(-1)^{2} \sigma^{2}{ }_{M L}+(-1)^{2} \sigma^{2}{ }_{M D}
$$

\begin{tabular}{|c|c|c|c|c|c|}
\hline$\sigma_{g}^{2}=$ & 219 & + & 447.80 & + & 0.36 \\
\hline$\sigma_{g}^{2}=$ & 667 & $\mathrm{kN}-\mathrm{m}$ & \multirow{2}{*}{\multicolumn{3}{|c|}{$\left(\right.$ Std Dev $\left.=\sqrt{ } \sigma_{g}^{2}\right)$}} \\
\hline$\sigma_{g}=$ & 26 & $\mathrm{kN}-\mathrm{m}$ & & & \\
\hline
\end{tabular}

Substitute standard deviation values of $F_{u}, w_{L}, w_{D}$ at $t=25$ in above equation:

Covariance of g:

$$
\begin{aligned}
& V g=\frac{\sigma g}{\mu g} \\
& V g=\quad 27.23 \%
\end{aligned}
$$

Reliability Index:

$$
\begin{aligned}
& \beta=\frac{1}{V_{g}} \\
& \beta=\quad 3.673
\end{aligned}
$$

(Over 3.5, Adequate at $\mathrm{t}=25 \mathrm{yrs}$.)

Probability of Failure:

$$
\begin{array}{ll}
P_{F}=\Phi(-\beta) & \text { (Use Norm.S.Dist Cumulative PDF Function) } \\
P_{F}=0.00012 \text { or } 1 \text { in } \quad 8,335 \quad(<1 \text { in } 5000 \text {, a dequate at } t=25 \text { yrs.) }
\end{array}
$$

(The section is slightly overdesigned, FS and $\phi$ can be adjusted to obtain $\beta$ of 3.5 at $t=25$ years)

\section{Conclusion of LRFD or WSD Design:}

Proper selection of strength reduction factor $(\phi)$ or factor of safety (FS) can provide a reliable performance starting from the placement in service to the end of life-span of a member. However, the selection of $\phi$ or FS will depend on the reliability index (or an acceptable level of probability) desired and the estimated rate of deterioration of the member strength. The arbitrary use of strength reduction factor or factor of safety can lead to over conservative uneconomical design or grossly undersized members and thereby resulting in unsatisfactory performance. 


\section{APPENDIX-B FRP DURABILITY DATABASE}

This Appendix supplement the information presented in Chapters 3 and 4.

The following pages contain a sample FRP database developed for this study, and various data reduction plots for the following FRP/Strength Retention combinations:

Glass Fiber in Vinylester Matrix:

VE-TS for Tensile Strength

VE-FS for Flexural Strength

VE-ILSS for Interlaminar Shear Strength

Glass Fiber in Polyester Matrix:

PE-TS for Tensile Strength

PE-FS for Flexural Strength

PE-ILSS for Interlaminar Shear Strength

Glass Fiber in Epoxy Matrix:

EN-TS for Tensile Strength

See Appendix E for a full listing of durability databases (with data source references) used in this study. 
Table B.1: Sample of FRP Database created for this study

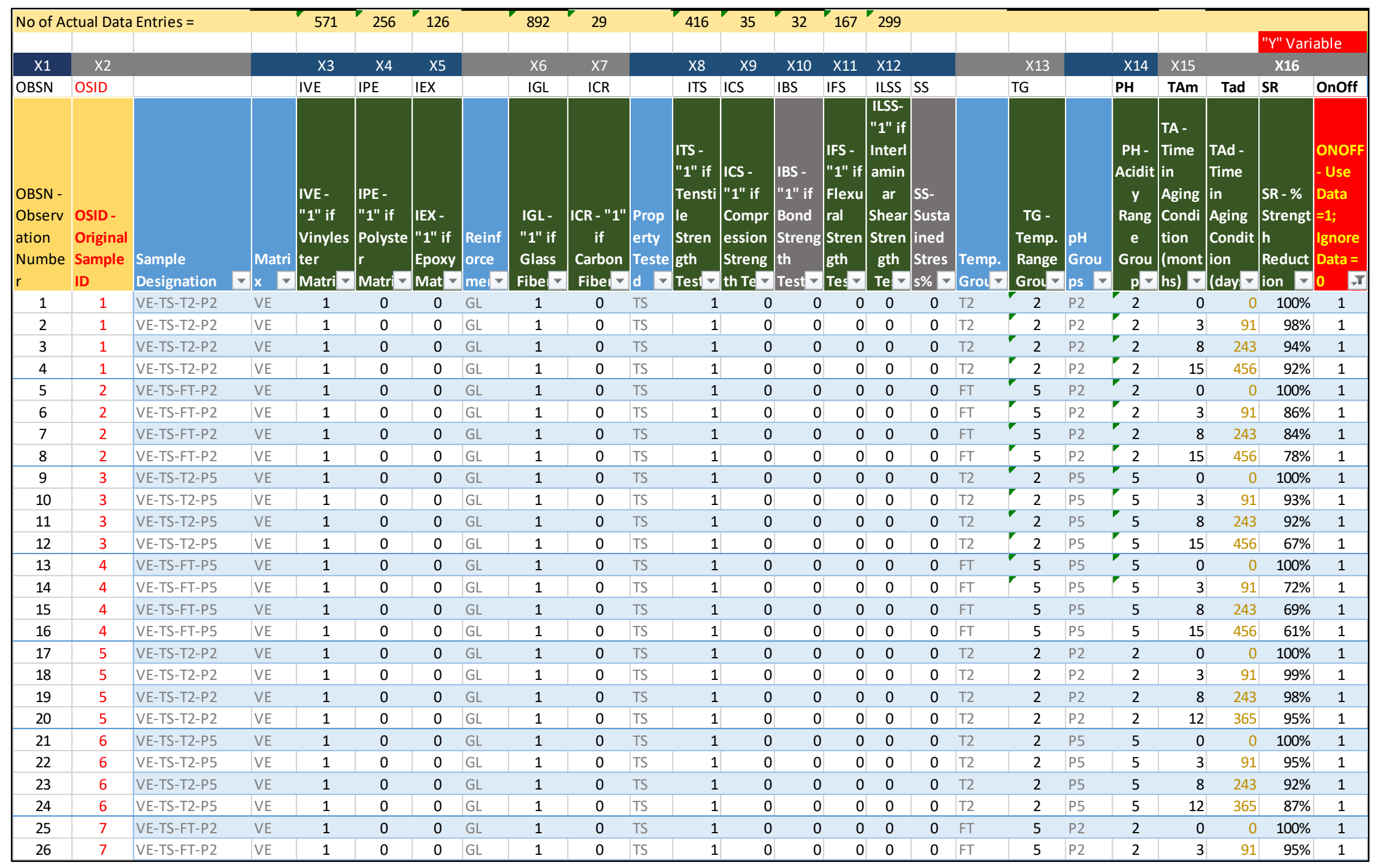

Note: See Appendix E for the full list with data source references. 
Figure B.1: Tested ATM Tensile Strengths of Vinylester GFRP

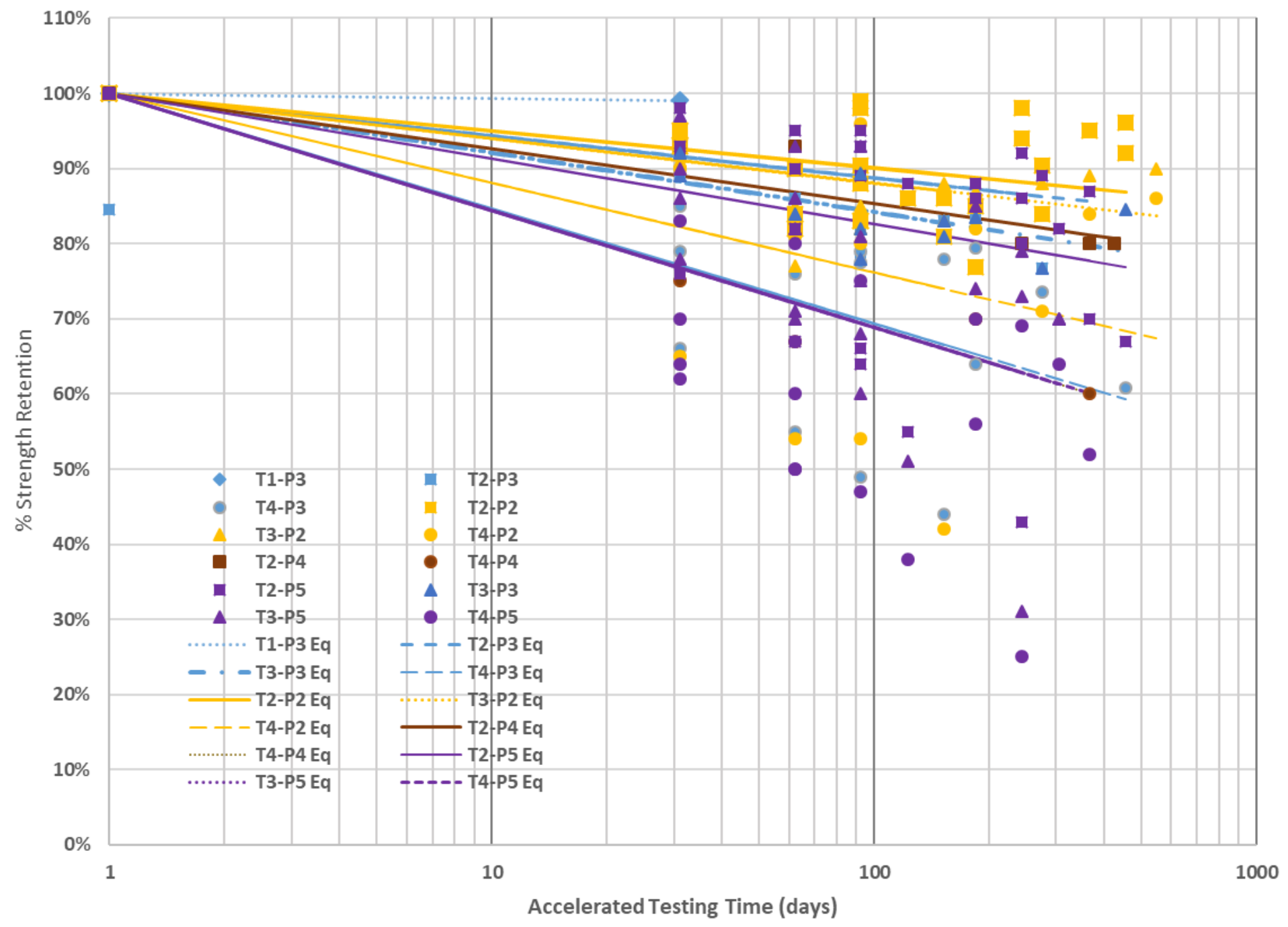


Figure B.2: Projected Normalized Tensile Strengths of Vinylester GFRP

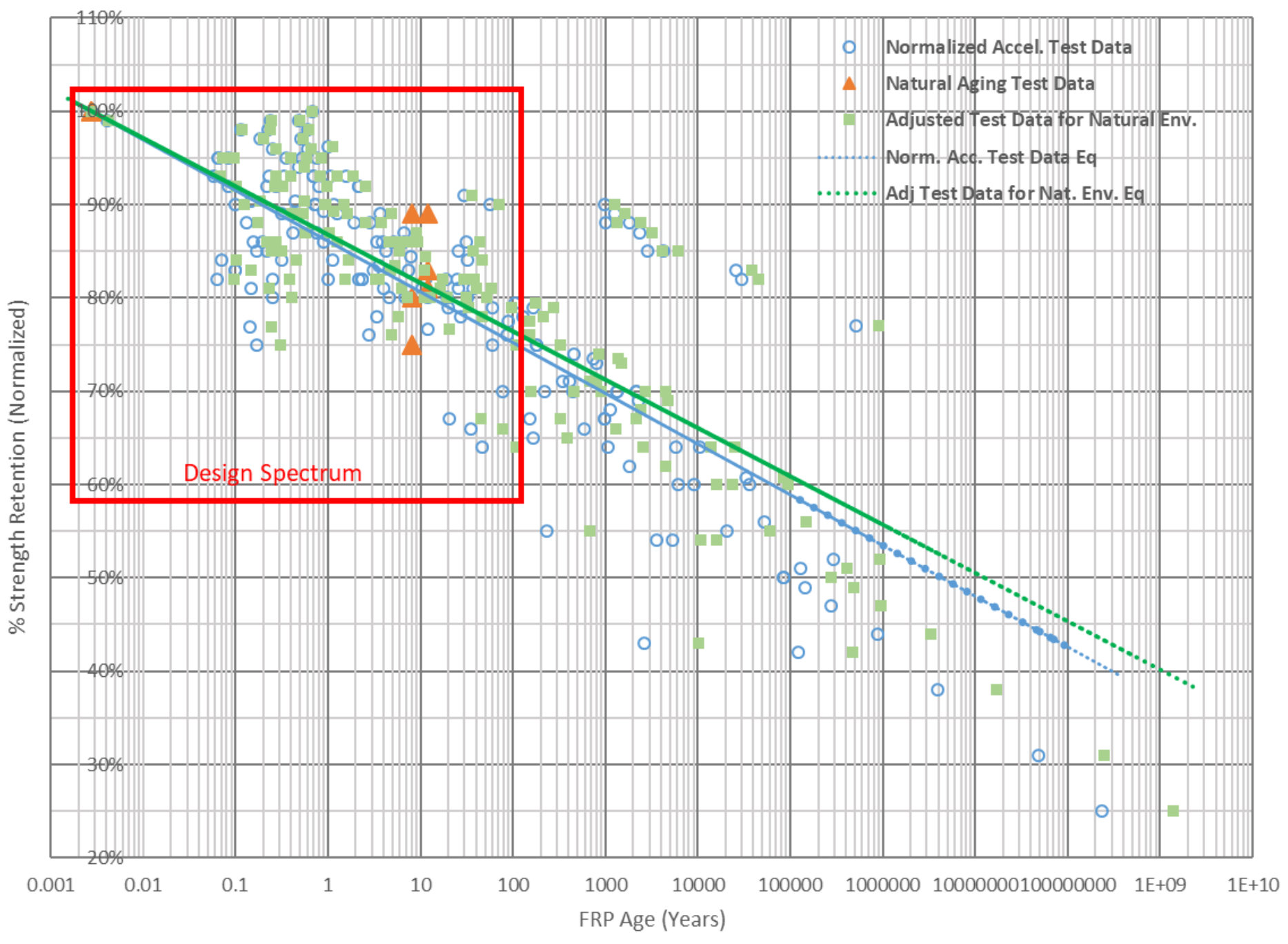

Note: Further refinement of the exiting data and/or additional data will benefit this projection. 
Figure B.3: Tested ATM Flexural Strengths of Vinylester GFRP

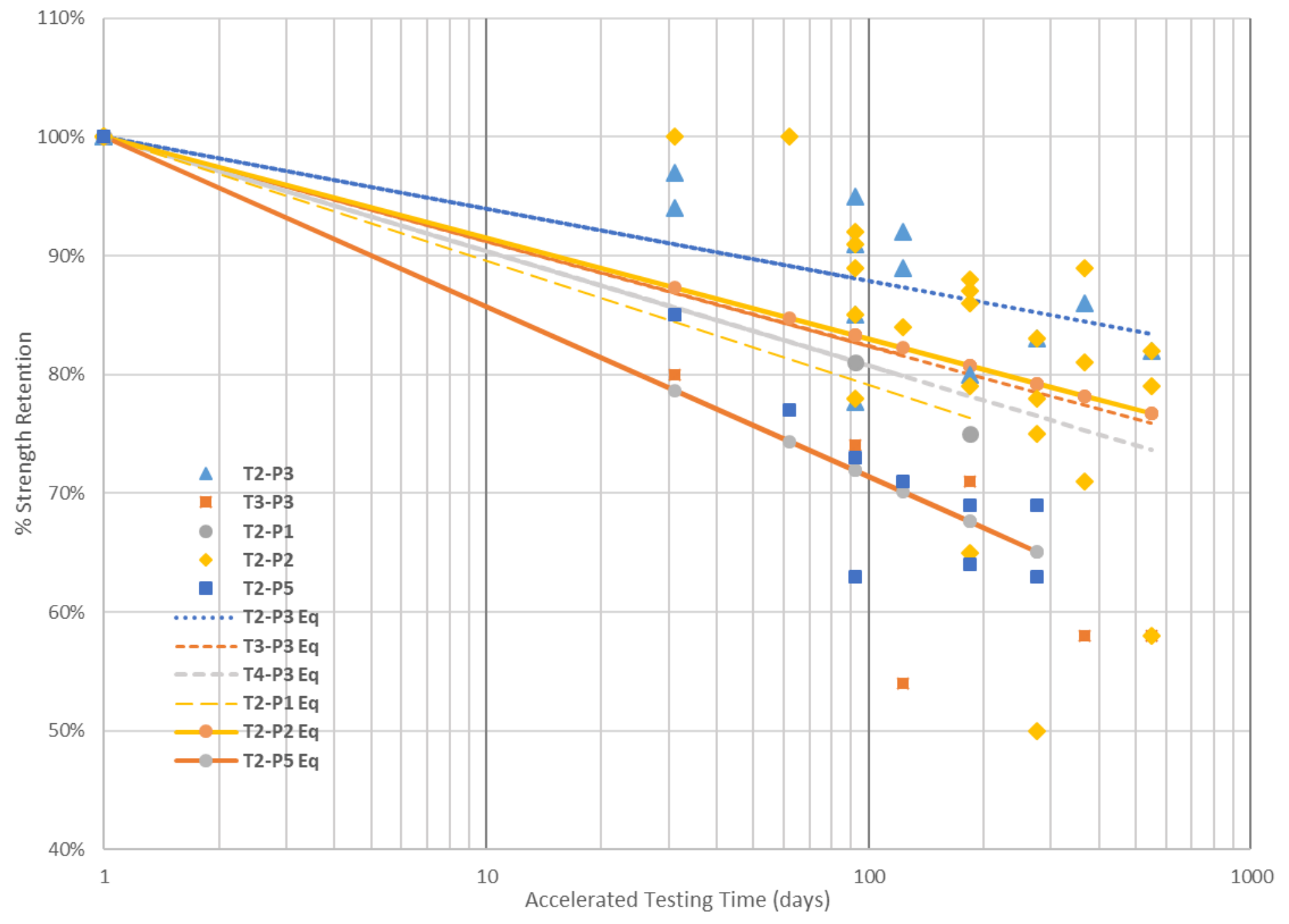


Figure B.4: Projected Normalized Flexural Strengths of Vinylester GFRP

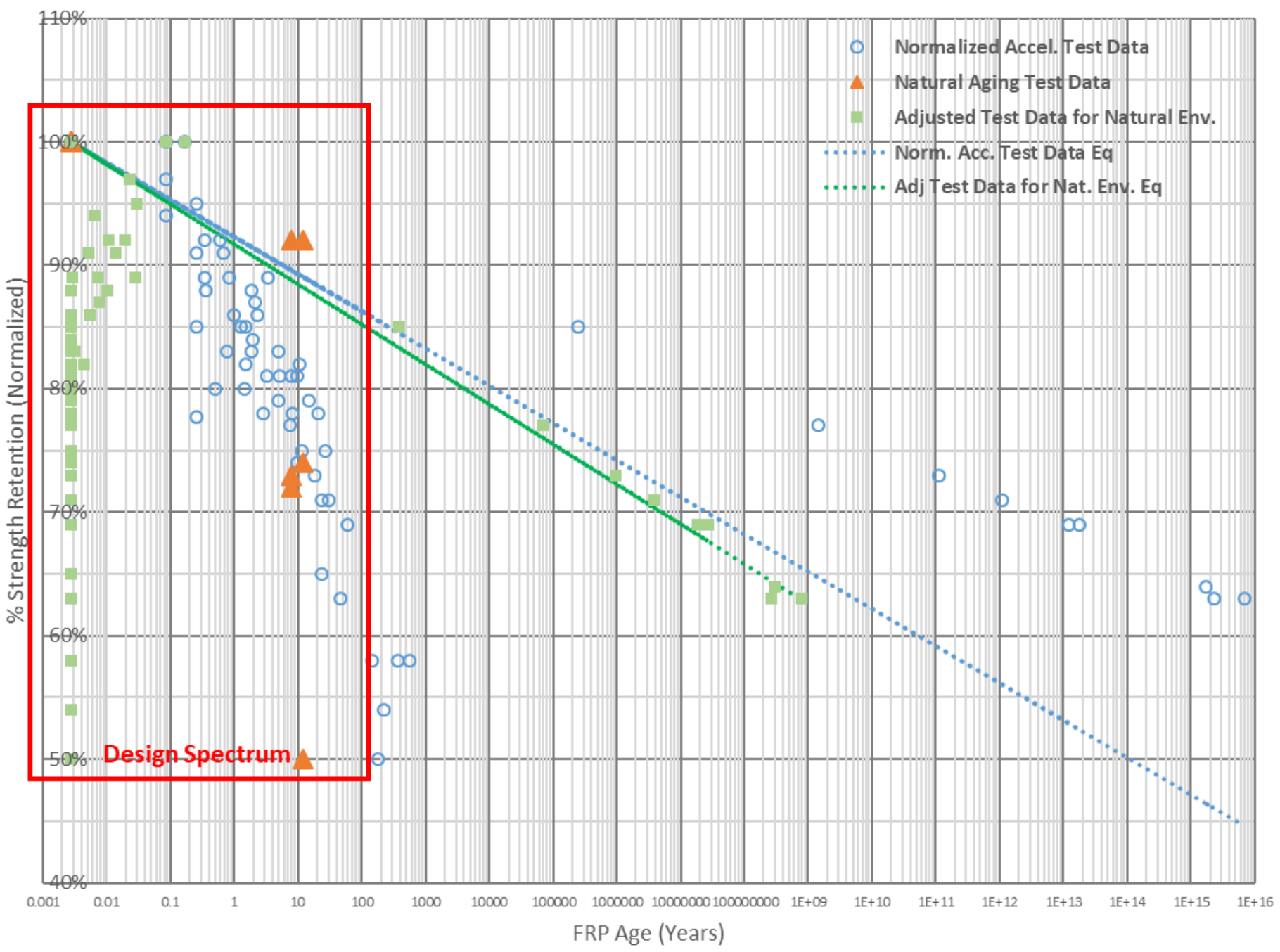

Note: Further refinement of the existing data and/or additional data will benefit this projection. 
Figure B.5: Tested ATM Shear Strengths of Vinylester GFRP

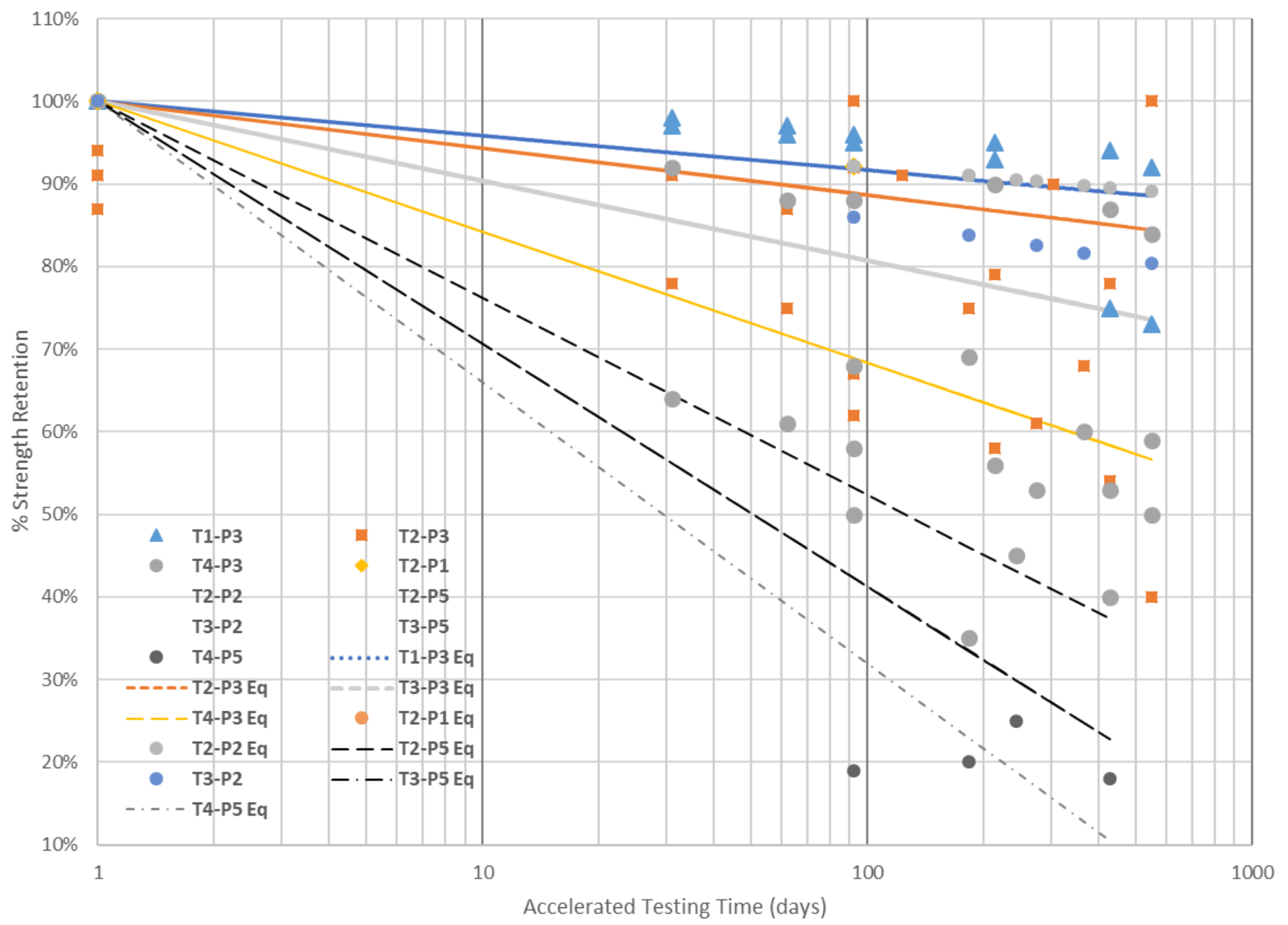


Figure B.6: Projected Normalized Shear Strengths of Vinylester GFRP

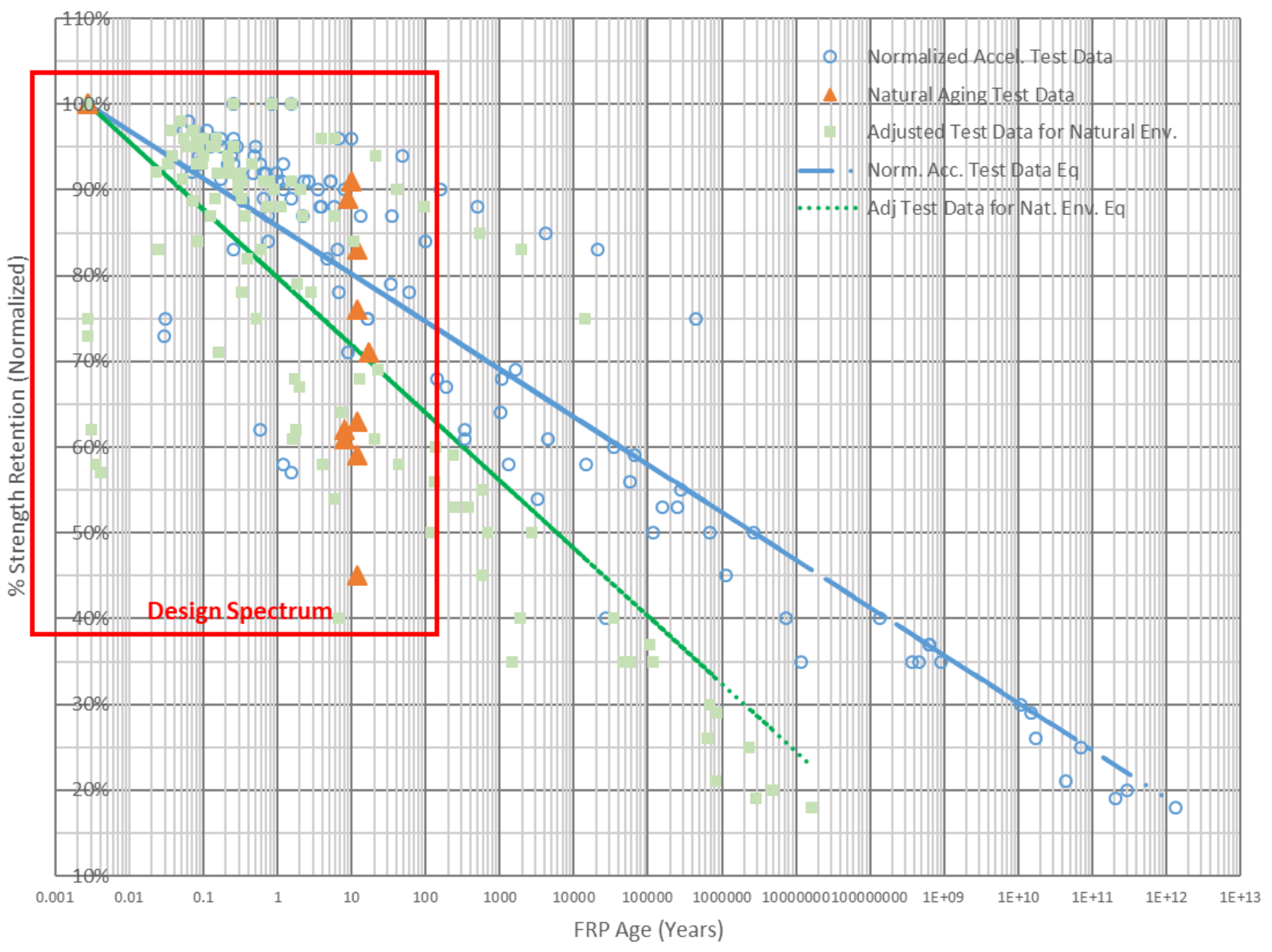

Note: Further refinement of the exiting data and/or additional data will benefit this projection. 
Figure B.7: Tested ATM Tensile Strengths of Polyester GFRP

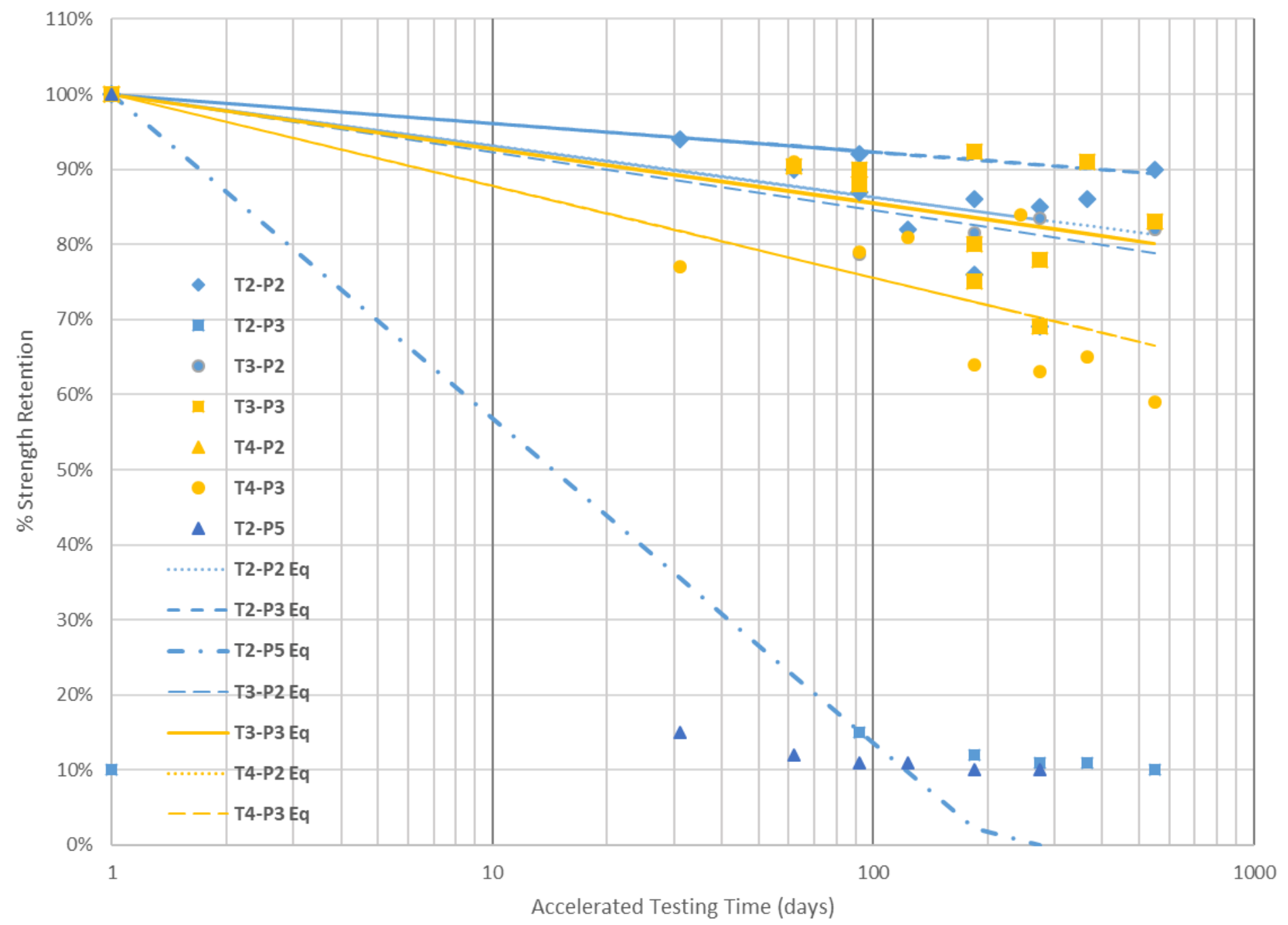


Figure B.8: Projected Normalized Tensile Strengths of Polyester GFRP

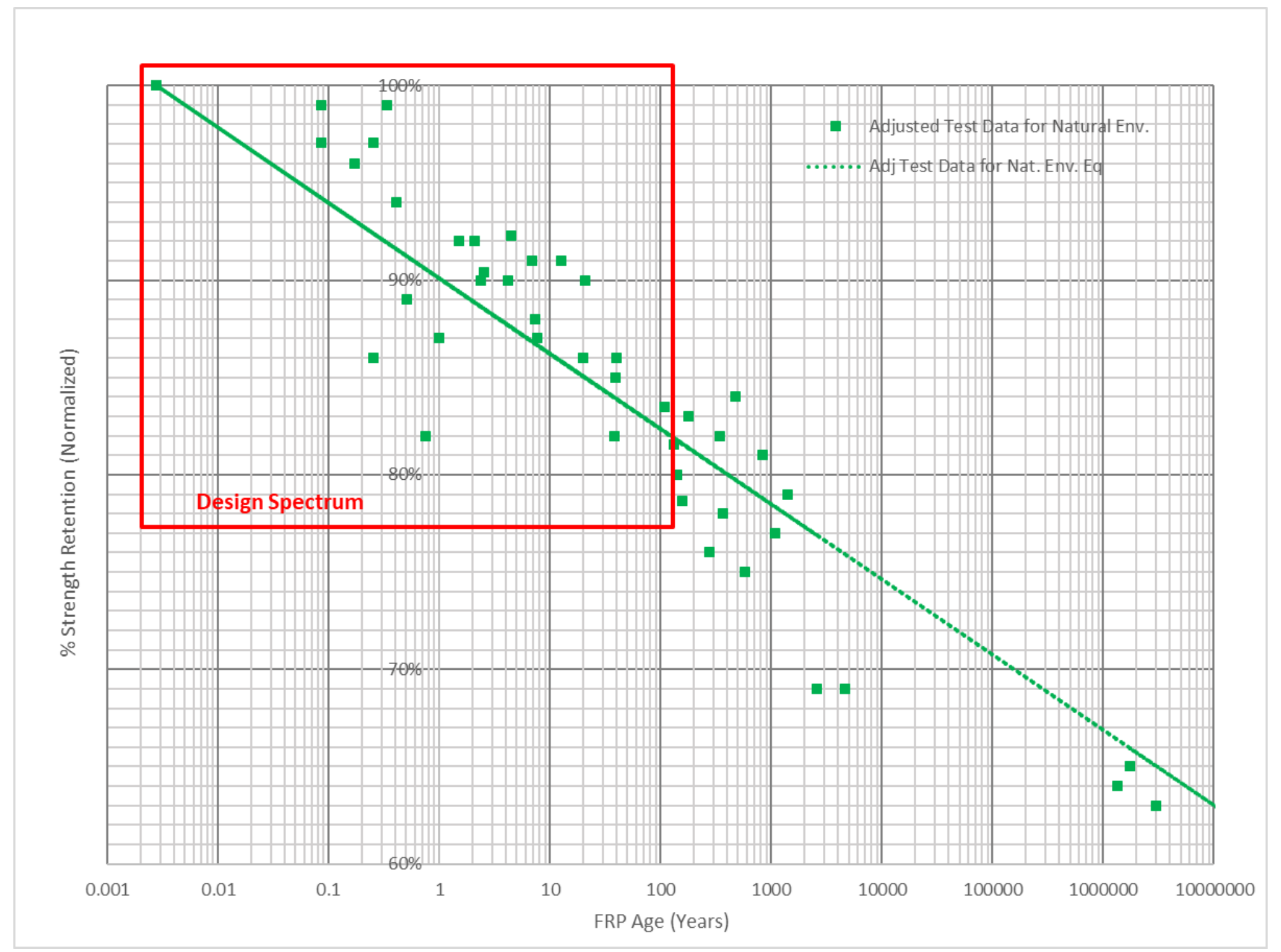

Note: Further refinement of the exiting data and/or additional data will benefit this projection. 
Figure B.9: Tested ATM Flexural Strengths of Polyester GFRP

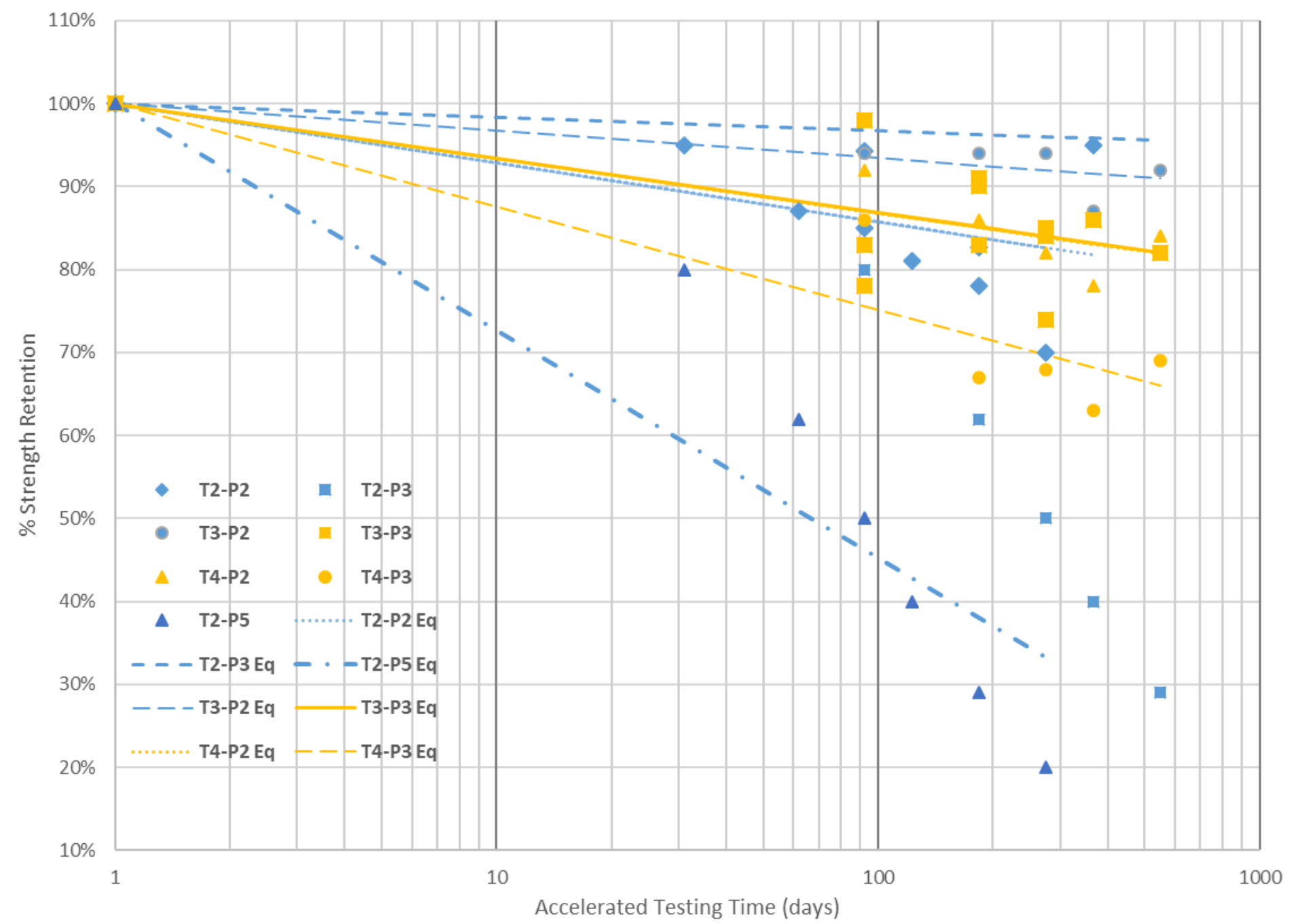


Figure B.10: Projected Normalized Flexural Strengths of Polyester GFRP

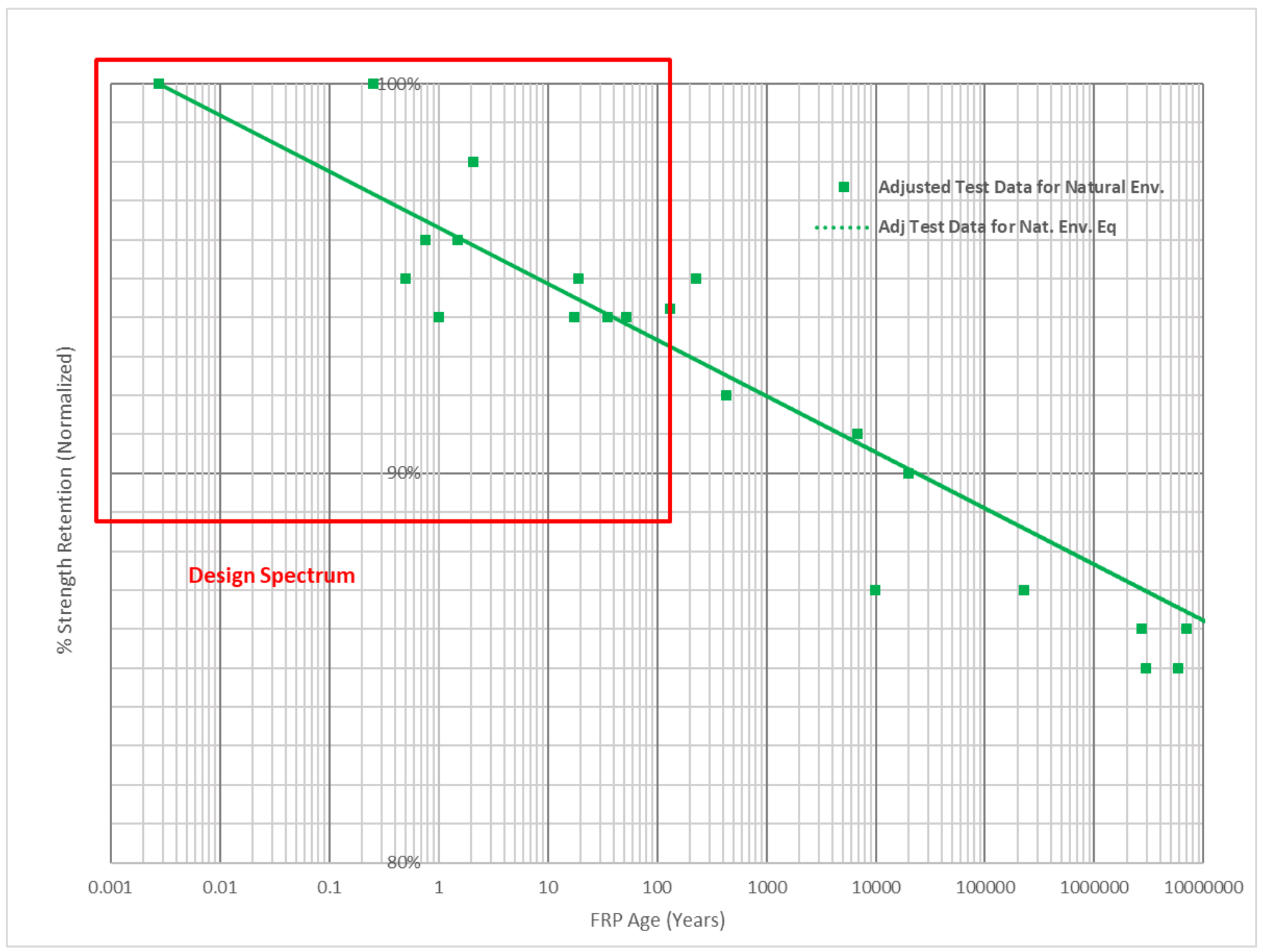

Note: Further refinement of the exiting data and/or additional data will benefit this projection. 
Figure B.11: Tested ATM Shear Strengths of Polyester GFRP

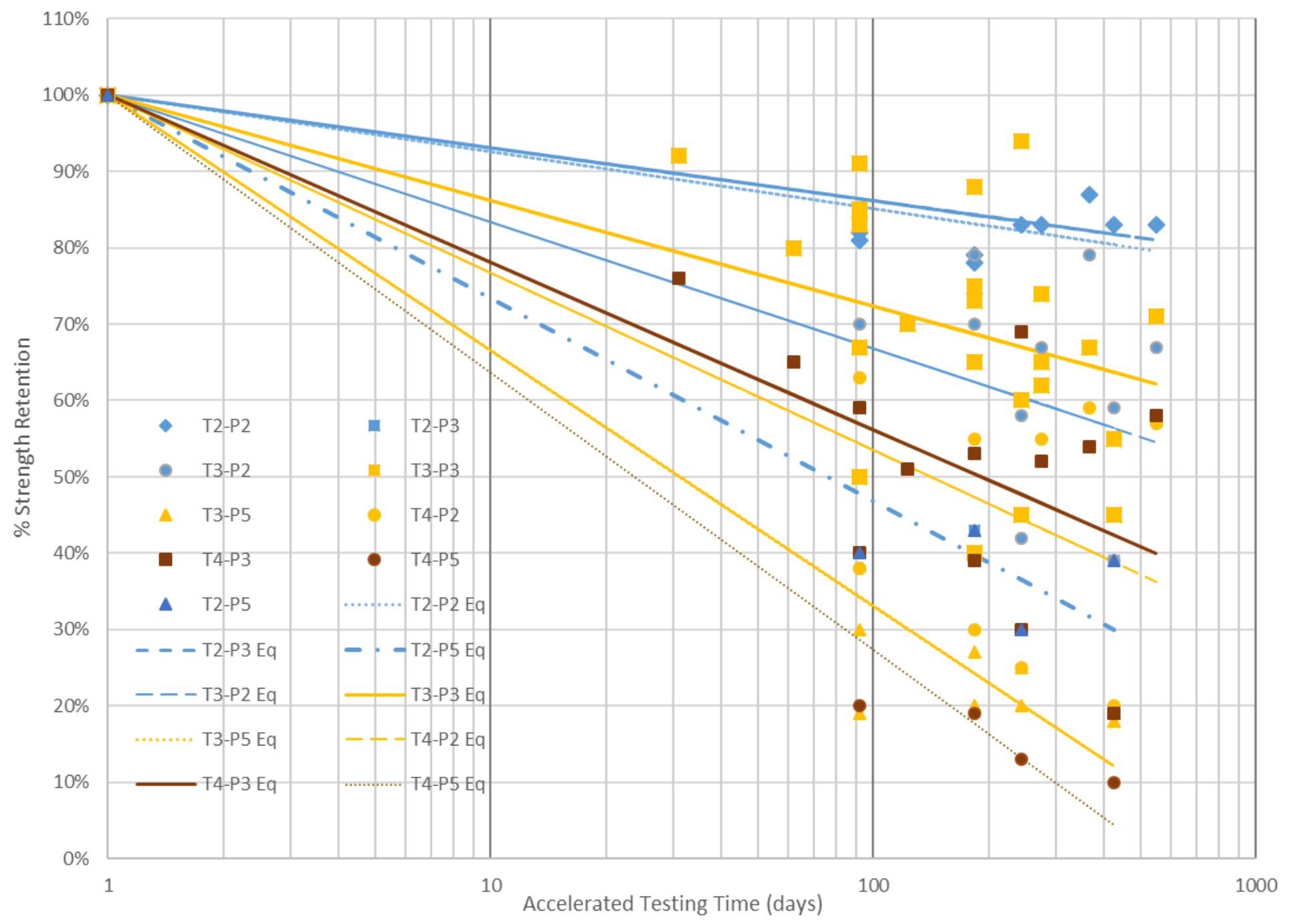


Figure B.12: Projected Normalized Shear Strengths of Polyester GFRP

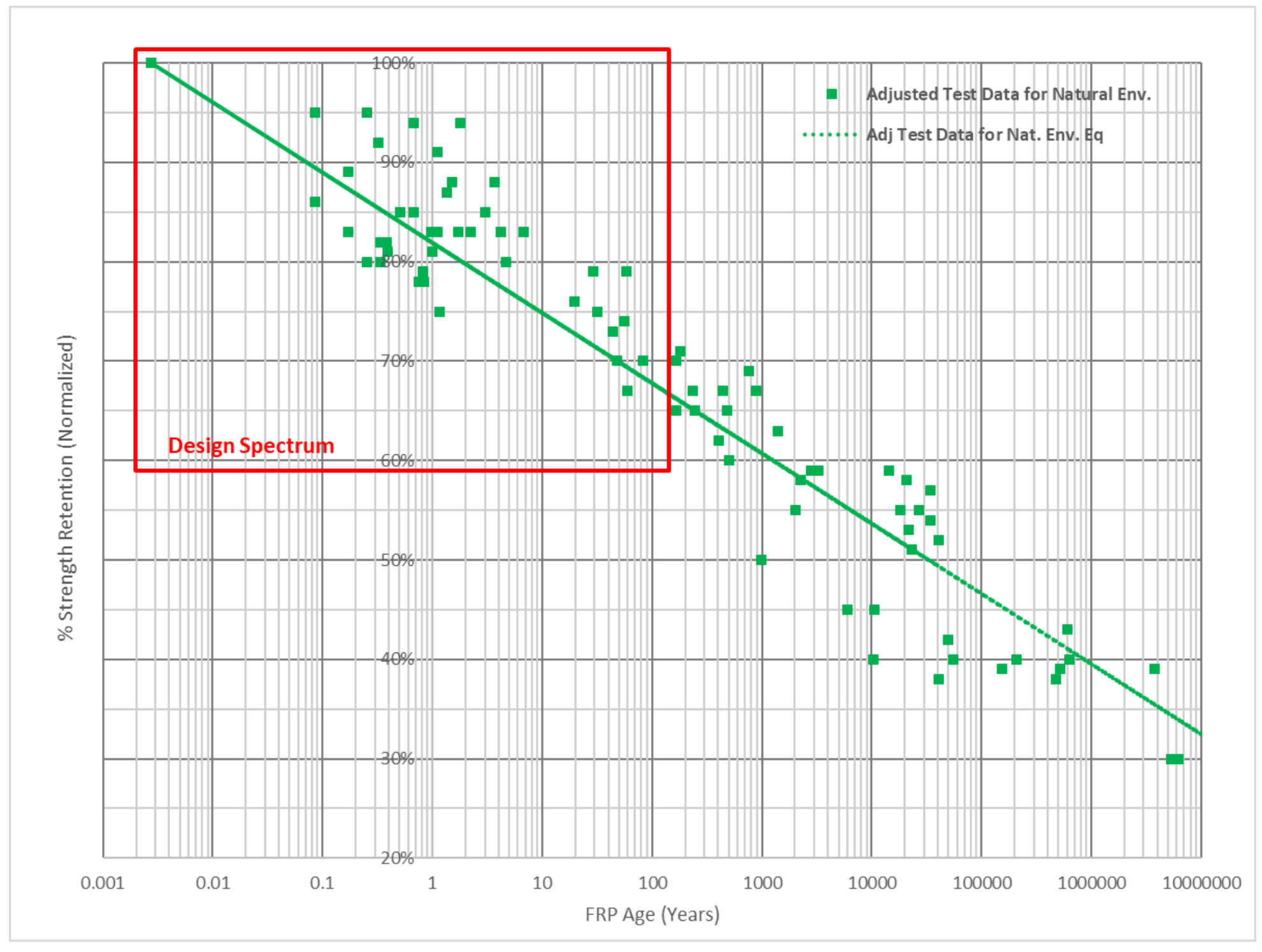

Note: Further refinement of the exiting data and/or additional data will benefit this projection. 
Figure B.13: Tested ATM Tensile Strengths of Epoxy GFRP

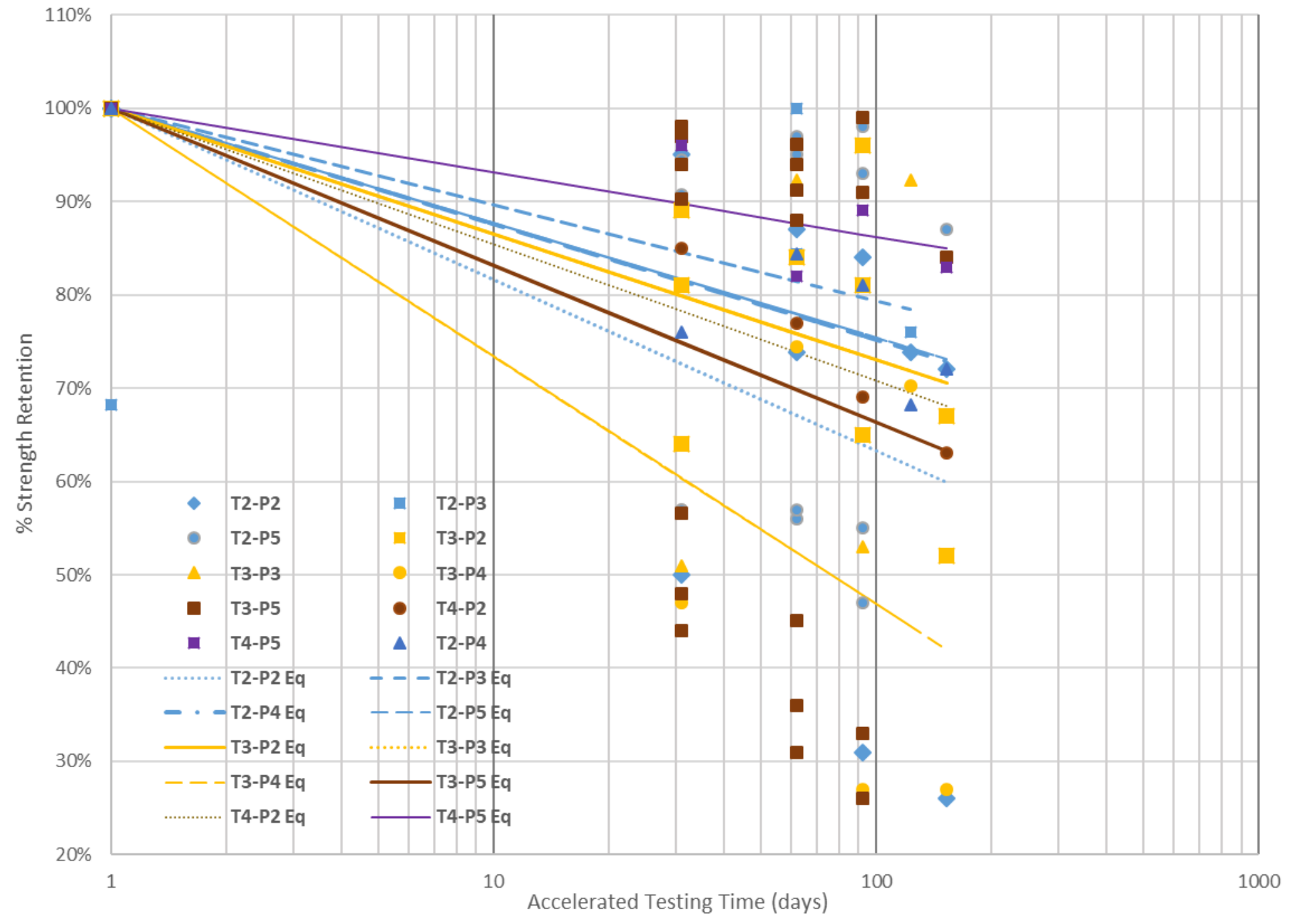


Figure B.14: Projected Normalized Tensile Strengths of Epoxy GFRP

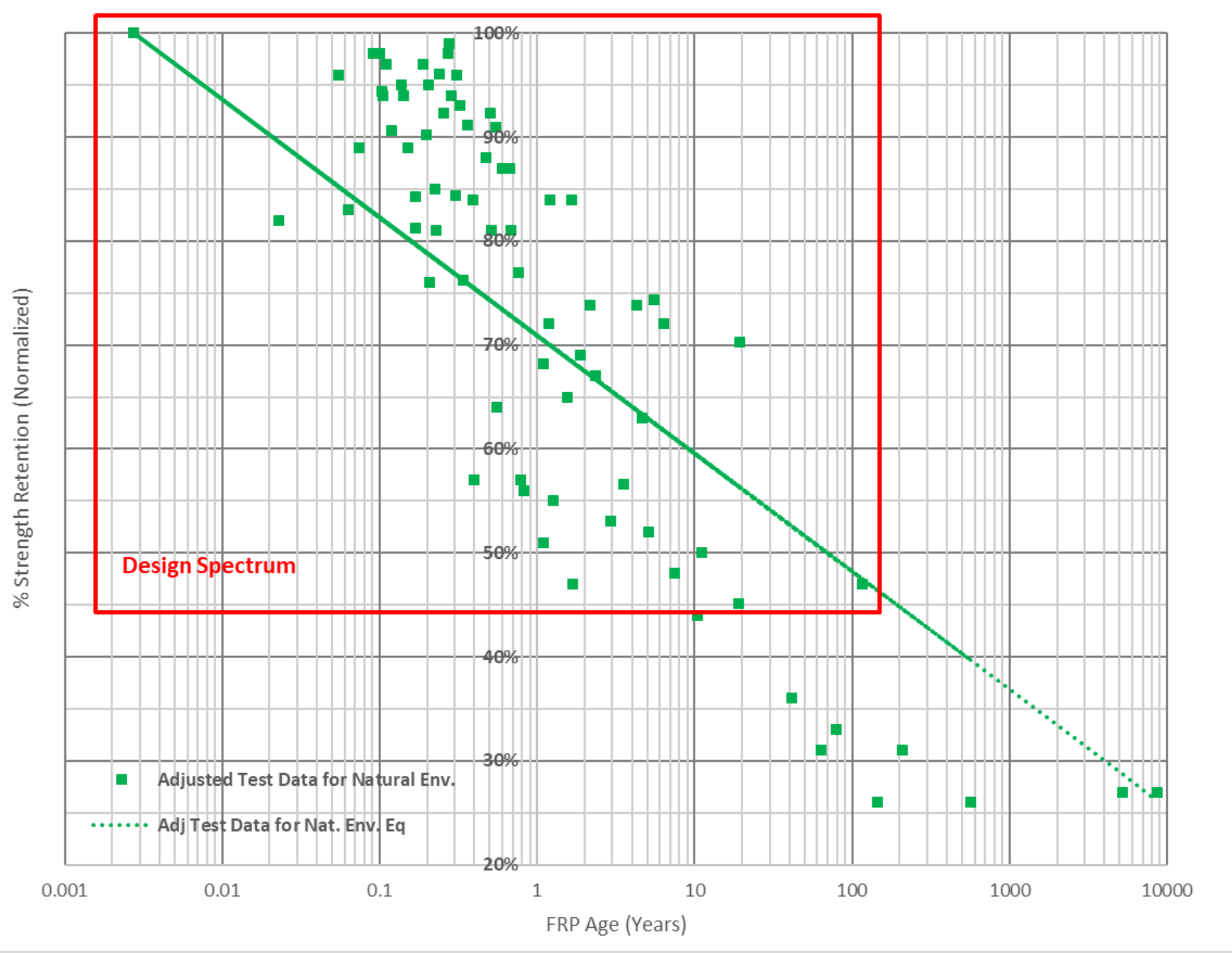

Note: Further refinement of the existing data and/or additional data will benefit this projection. 


\section{APPENDIX-C SUBSEA FRP DATABASE}

This Appendix supplement the information presented in Chapter 6.

The following pages contain strength retention plots of Accelerated Testing and projected normalized timeline. Strengths considered includes:

Glass Fiber in Vinylester Matrix:

VE-TT for Tensile Strength in Transverse Direction (Lab and Projected)

VE-TL for Tensile Strength in Longitudinal Direction (lab and Projected)

VE-FT for Flexural Strength in Transverse Direction

VE-FL for Flexural Strength in Longitudinal Direction

VE-ST for Shear Strength in Transverse Direction

VE-SL for Shear Strength in Longitudinal Direction

VE-ET for Tensile Elastic Modulus in Transverse Direction

VE-EL for Tensile Elastic Modulus in Longitudinal Direction

Glass Fiber in Polyester Matrix:

PE-TT for Tensile Strength in Transverse Direction

PE-TL for Tensile Strength in Longitudinal Direction

PE-FT for Flexural Strength in Transverse Direction

PE-FL for Flexural Strength in Longitudinal Direction

PE-ST for Shear Strength in Transverse Direction

PE-SL for Shear Strength in Longitudinal Direction

PE-ET for Tensile Elastic Modulus in Transverse Direction

PE-EL for Tensile Elastic Modulus in Longitudinal Direction 
Table C.1: Sample of Original Dataset Provided by the FRP System Supplier

Transverse \& Longitudinal Tensile test Data

\begin{tabular}{|c|c|c|c|c|c|c|c|c|c|c|c|c|c|c|c|c|c|c|c|c|c|}
\hline & \multicolumn{10}{|c|}{ Virgin Material } & \multicolumn{11}{|c|}{ Merganser Cocoon 17 Material } \\
\hline \multirow{10}{*}{$\begin{array}{c}\text { Tables } \\
6.1 \& \\
6.2\end{array}$} & \multirow{2}{*}{\multicolumn{5}{|c|}{$\begin{array}{cc}\text { Tensile (Transverse) } \\
\text { Virgin Dry VE } \\
\text { Sample ID } \\
\text { Tcknss Width Stress (MPa) E (GPa) }\end{array}$}} & \multicolumn{5}{|c|}{$\begin{array}{l}\text { Tensile (Longitudinal) } \\
\text { Virgin Dry VE }\end{array}$} & \multirow{10}{*}{$\begin{array}{l}\text { Table } \\
6.5 \& \\
6.6\end{array}$} & \multicolumn{5}{|c|}{$\begin{array}{c}\text { Tensile (Transverse) } \\
\text { Cocoon VE }\end{array}$} & \multicolumn{5}{|c|}{$\begin{array}{l}\text { Tensile (Longitudinal) } \\
\text { Cocoon VE }\end{array}$} \\
\hline & & & & & & Sample ID & Tcknss & Width & Stress (MPa) & $E(G P a)$ & & Sample ID & Tcknss & Width $\mathrm{S}$ & Stress (MPa) & $E(G P a)$ & Sample ID & Tcknss & Width $\subseteq$ & Stress (MPa) & $E(G P a)$ \\
\hline & VE-V-TT-31 & 8.99 & 13.06 & 221 & 14 & VE-V-TL-31 & 9.094 & 12.99 & 325 & 21 & & VE-C-TT-16 & 8.77 & 13.02 & 144 & 14 & VE-C-TL-16 & 10.224 & 12.97 & 225 & 18 \\
\hline & VE-V-TT-32 & 10.118 & 13.03 & 177 & 17 & VE-V-TL-32 & 10.621 & 13.02 & 290 & 19 & & VE-C-TT-17 & 8.773 & 13.08 & 147 & 13 & VE-C-TL-17 & 11.888 & 12.94 & 273 & 19 \\
\hline & VE-V-TT-33 & 9.043 & 13.02 & 205 & 16 & VE-V-TL-33 & 10.39 & 12.99 & 286 & 20 & & VE-C-TT-18 & 8.73 & 13.02 & 136 & 15 & VE-C-TL-18 & 11.894 & 12.76 & 350 & 22 \\
\hline & VE-V-TT-34 & & 13.02 & 206 & 17 & VE-V-TL-34 & 9.095 & 13.04 & 316 & 20 & & VE-C-TT-19 & 8.755 & 13.01 & 154 & 13 & VE-C-TL-19 & 8.605 & 12.93 & 249 & 17 \\
\hline & VE-V-TT-35 & 10.099 & 13.02 & 155 & 15 & VE-V-TL-35 & 10.339 & 13.01 & 295 & 19 & & VE-C-TT-20 & 8.757 & 12.99 & 138 & 17 & VE-C-TL-20 & 8.493 & 12.94 & 248 & 19 \\
\hline & Avg & & & 193 & 16 & Avg & & & 302 & 20 & & Avg & & & 144 & 14 & Avg & & & 269 & 19 \\
\hline & StDev & & & 26 & 1 & StDev & & & 17 & & & StDev & & & 7 & 2 & StDev & & & 48 & 2 \\
\hline & Cov & & & $14 \%$ & $7 \%$ & Cov & & & $6 \%$ & $4 \%$ & & Cov & & & $5 \%$ & $11 \%$ & CoV & & & $18 \%$ & $9 \%$ \\
\hline \multirow{10}{*}{$\begin{array}{l}\text { Tables } \\
6.3 \& \\
6.4\end{array}$} & \multicolumn{5}{|c|}{$\begin{array}{c}\text { Tensile (Transverse) } \\
\text { Virgin Wet VE }\end{array}$} & \multicolumn{5}{|c|}{$\begin{array}{c}\text { Tensile (Lognitudinal) } \\
\text { Virgin Wet VE }\end{array}$} & \multirow{10}{*}{$\begin{array}{c}\text { Tables } \\
6.11 \& \\
6.12\end{array}$} & \multicolumn{5}{|c|}{$\begin{array}{c}\text { Tensile (Transverse) } \\
\text { ocoon (3.7 month - } 16.8 \text { years Arrhenius ageing) V }\end{array}$} & \\
\hline & Sample ID & Tcknss & Width & Stress (MPa) & $E(G P a)$ & Sample ID & Tcknss & Width & Stress (MPa) & $E(G P a)$ & & \multicolumn{5}{|c|}{ ocoon (3.7 month - 16.8 years Arrhenius ageing) $V$} & \multicolumn{5}{|c|}{$\begin{array}{l}\text { ocoon ( } 3.7 \text { month }-16.8 \text { years Arrhenius ageing) } \\
\text { Sample ID Tcknss Width Stress (MPa) E (GPa) }\end{array}$} \\
\hline & $\overline{V E-V-T T-26}$ & 8.702 & 13.04 & 168 & 16 & VE-V-TL-26 & 10.161 & 12.95 & 231 & 17 & & VE-C-TT-11 & 8.76 & 13.09 & 122 & 18 & VE-C-TL-11 & 10.233 & 12.97 & 193 & 15 \\
\hline & VE-V-TT-27 & 8.792 & 13.06 & 162 & 16 & VE-V-TL-27 & 10.193 & 12.94 & 220 & 18 & & VE-C-TT-12 & 8.68 & 13.06 & 118 & 13 & VE-C-TL-12 & 10.149 & 12.96 & 197 & 17 \\
\hline & VE-V-TT-28 & 8.828 & 13.05 & 201 & 17 & VE-V-TL-28 & 11.583 & 12.93 & 277 & 22 & & VE-C-TT-13 & 8.845 & 13.09 & 121 & 14 & VE-C-TL-13 & 10.254 & 12.96 & 180 & 14 \\
\hline & VE-V-TT-29 & 8.856 & 13.03 & 148 & 13 & VE-V-TL-29 & 10.097 & 12.93 & 241 & 18 & & VE-C-TT-14 & 8.794 & 13.09 & 136 & 15 & VE-C-TL-14 & 10.098 & 12.96 & 206 & 16 \\
\hline & VE-V-TT-30 & 8.808 & 13.05 & 156 & 15 & VE-V-TL-30 & 9.105 & 12.93 & 247 & 20 & & VE-C-TT-15 & 8.726 & 13.07 & 134 & 15 & VE-C-TL-15 & 11.611 & 13 & 206 & 18 \\
\hline & Avg & & & 167 & 15 & Avg & & & 243 & 19 & & Avg & & & 126 & $\overline{15}$ & Avg & & & 196 & 16 \\
\hline & StDev & & & 20 & 2 & StDev & & & 21 & 2 & & StDev & & & 8 & 2 & StDev & & & 11 & \\
\hline & CoV & & & $12 \%$ & $8 \%$ & CoV & & & $9 \%$ & $11 \%$ & & CoV & & & $7 \%$ & $12 \%$ & CoV & & & $6 \%$ & $9 \%$ \\
\hline \multirow{10}{*}{$\begin{array}{l}\text { Tables } \\
6.7 \& \\
6.8\end{array}$} & Virgin $(1 \mathrm{~m}$ & $\begin{array}{l}\text { Tensile } \\
\text { nonth - } 2 \text {. }\end{array}$ & $\begin{array}{l}\text { e }(\text { Tran } \\
.5 \text { year } A\end{array}$ & $\begin{array}{l}\text { nsverse) } \\
\text { Arrhenius ageir }\end{array}$ & ng) VE & $\operatorname{Virgin}(1 \mathrm{~m}$ & $\begin{array}{l}\text { Tensile } \\
\text { nonth - } 2 \text {. }\end{array}$ & $\begin{array}{l}\text { (Longi } \\
5 \text { year } \mathrm{A}\end{array}$ & $\begin{array}{l}\text { itudinal) } \\
\text { Arrhenius agein }\end{array}$ & ing) VE & \multirow{10}{*}{$\begin{array}{c}\text { Tables } \\
6.17 \& \\
6.18\end{array}$} & \multirow{2}{*}{\multicolumn{5}{|c|}{$\begin{array}{l}\text { Tensile (Transverse) } \\
\text { ocoon (7.8 month - 27.3 years Arrhenius ageing) V } \\
\text { Sample ID Tcknss Width Stress (MPa) E (GPa) } \\
\end{array}$}} & \multirow{2}{*}{\multicolumn{5}{|c|}{$\begin{array}{l}\text { Tensile (Longitudinal) } \\
\text { ocoon (7.8 month - } 27.3 \text { years Arrhenius ageing) } \\
\text { Sample ID Tcknss Width Stress (MPa) E (GPa) }\end{array}$}} \\
\hline & Sample ID & Tcknss & Width & Stress (MPa) & E (GPa) & Sample ID & Tcknss & Width & Stress (MPa) & $\mathrm{E}(\mathrm{GPa})$ & & & & & & & & & & & \\
\hline & VE-V-TT-21 & 8.853 & 13.07 & 192 & 17 & VE-V-TL-21 & 10.224 & 12.98 & 197 & 17 & & VE-C-TT-6 & 8.666 & 13.06 & 108 & 14 & VE-C-TL-6 & 10.271 & 12.95 & 179 & 15 \\
\hline & VE-V-TT-22 & 8.796 & 13.09 & 133 & 13 & VE-V-TL-22 & 10.712 & 12.98 & 204 & 18 & & VE-C-TT-7 & 8.723 & 13.05 & 142 & 16 & VE-C-TL-7 & 8.477 & 12.95 & 197 & 19 \\
\hline & VE-V-TT-23 & 8.827 & 13.07 & 191 & 16 & VE-V-TL-23 & 9.116 & 12.97 & 221 & 20 & & VE-C-TT-8 & 8.763 & 13.04 & 113 & 16 & VE-C-TL-8 & 8.486 & 12.93 & 196 & 18 \\
\hline & VE-V-TT-24 & 8.834 & 13.08 & 148 & 14 & VE-V-TL-24 & 11.314 & 12.98 & 243 & 23 & & VE-C-TT-9 & 8.807 & 13.05 & 140 & 14 & VE-C-TL-9 & 8.51 & 12.95 & 191 & 19 \\
\hline & VE-V-TT-25 & 8.614 & 13.1 & 180 & 16 & VE-V-TL-25 & 10.413 & 12.97 & 200 & 19 & & VE-C-TT-10 & 8.777 & 13.04 & 133 & 15 & VE-C-TL-10 & 10.198 & 12.93 & 183 & 17 \\
\hline & Avg & & & 169 & $\overline{15}$ & Avg & & & 213 & 19 & & Avg & & & 127 & $\overline{15}$ & Avg & & & 189 & 18 \\
\hline & StDev & & & 27 & 2 & StDev & & & 19 & 2 & & StDev & & & 16 & 1 & StDev & & & 8 & \\
\hline & CoV & & & $16 \%$ & $9 \%$ & CoV & & & $9 \%$ & $11 \%$ & & $\mathrm{CoV}$ & & & $12 \%$ & $7 \%$ & CoV & & & $4 \%$ & $10 \%$ \\
\hline
\end{tabular}

Note: Only a sample data is shown. The database is too large for printing in Appendix 
Figure C.1: Tested Tensile-Transverse Strengths of Vinylester GFRP

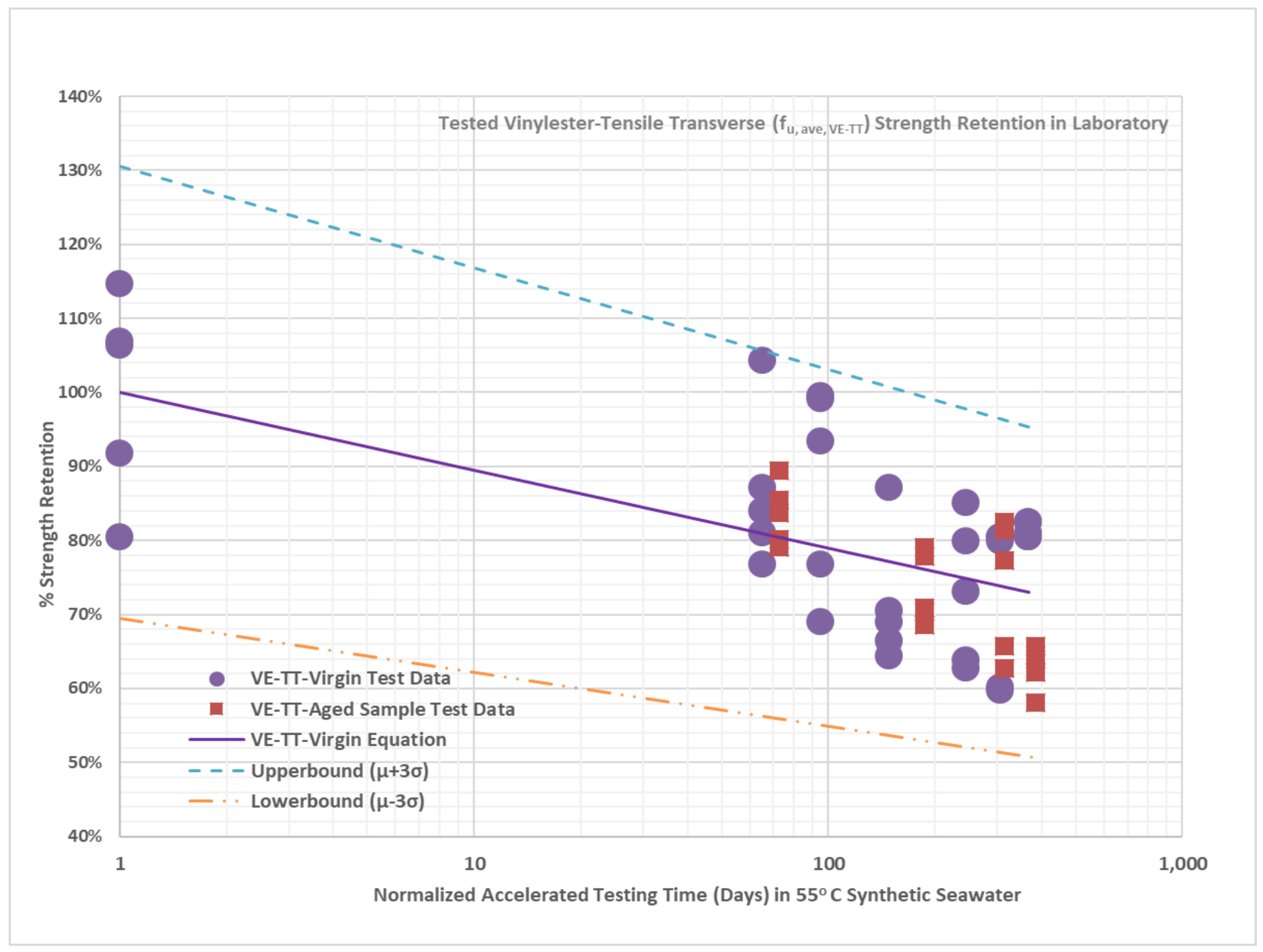


Figure C.2: Projected Tensile-Transverse Strengths of Vinylester GFRP

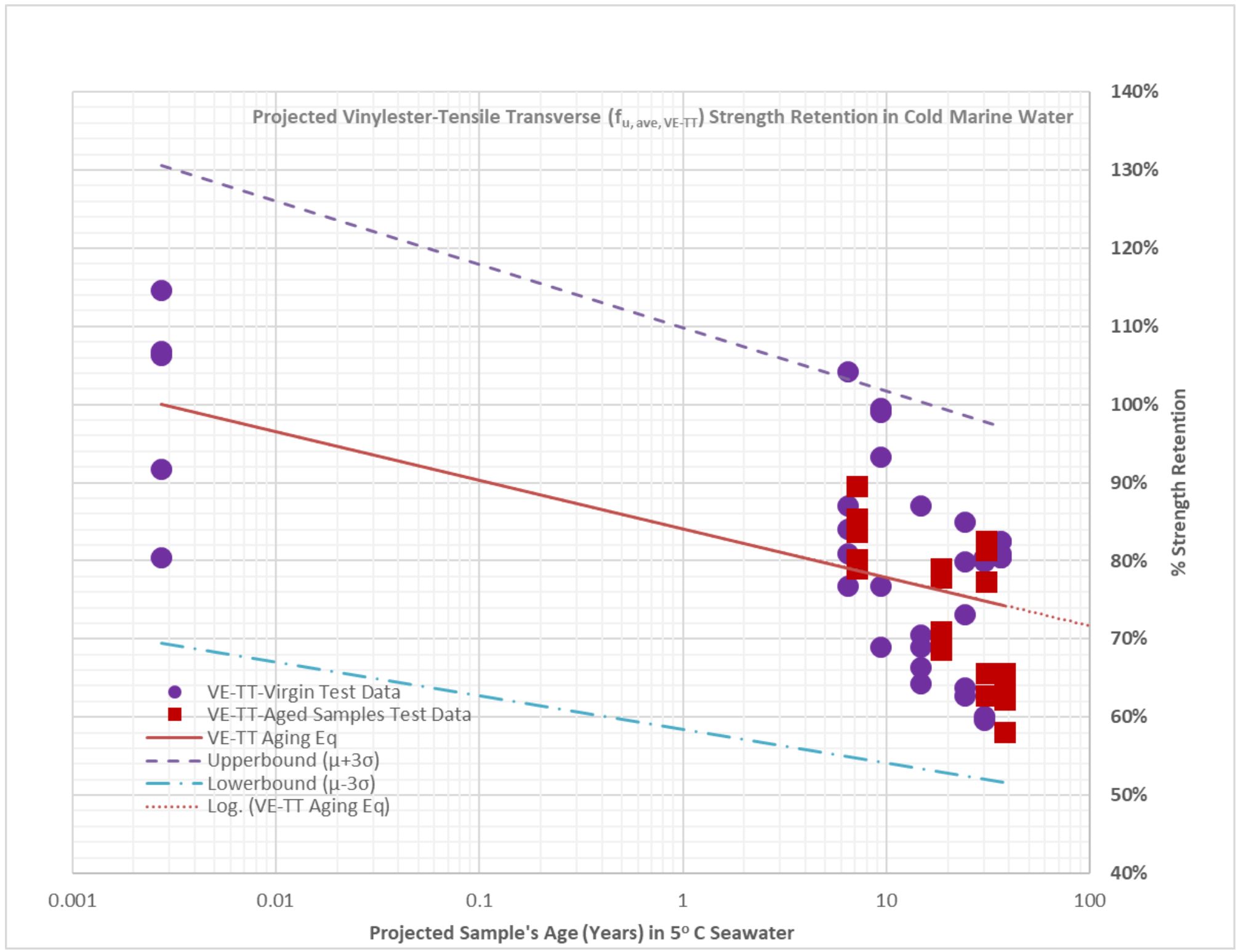




\section{Figure C.3: Tested Tensile-Longitudinal Strengths of Vinylester GFRP}

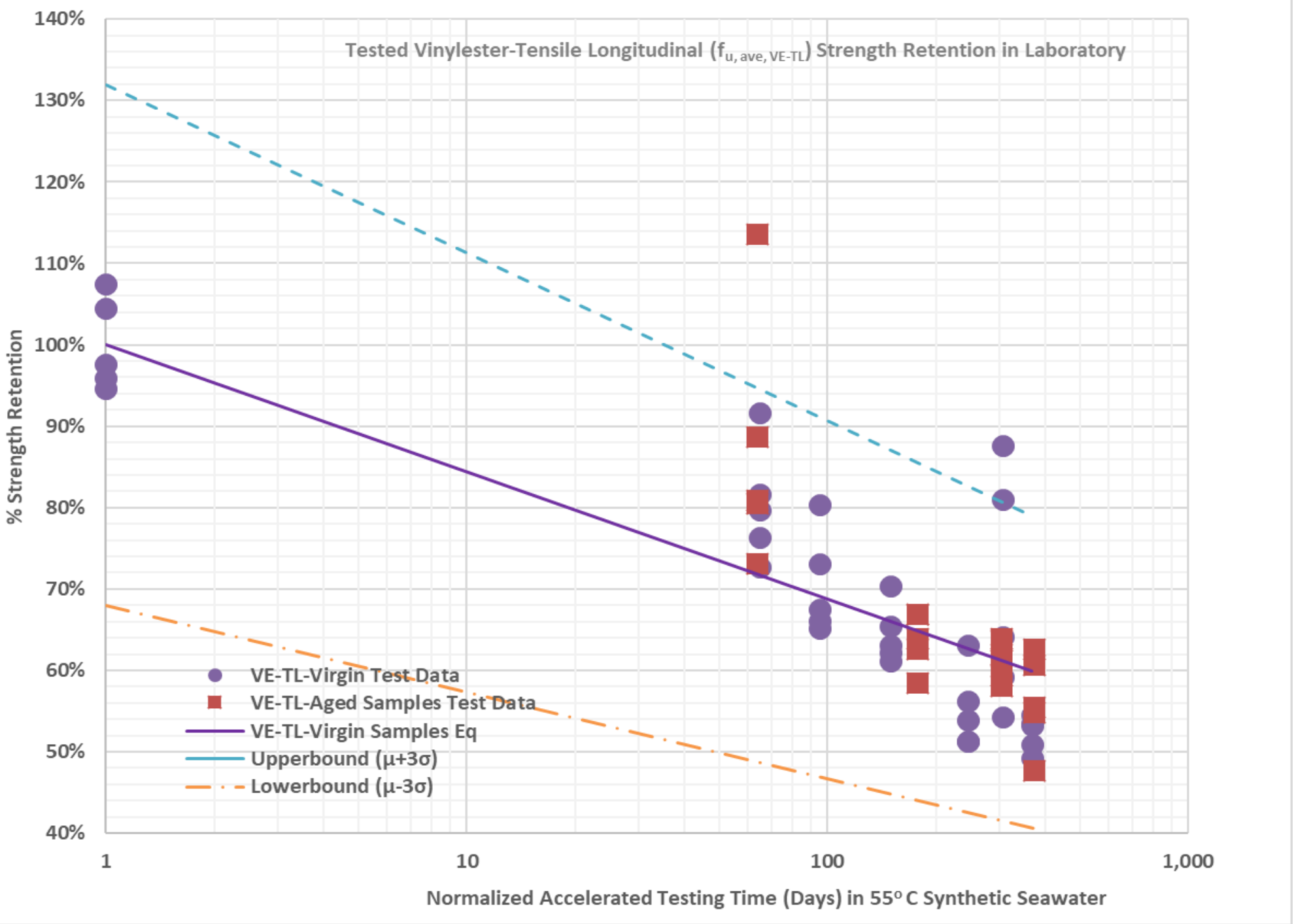


Figure C.4: Projected Tensile-Longitudinal Strengths of Vinylester GFRP

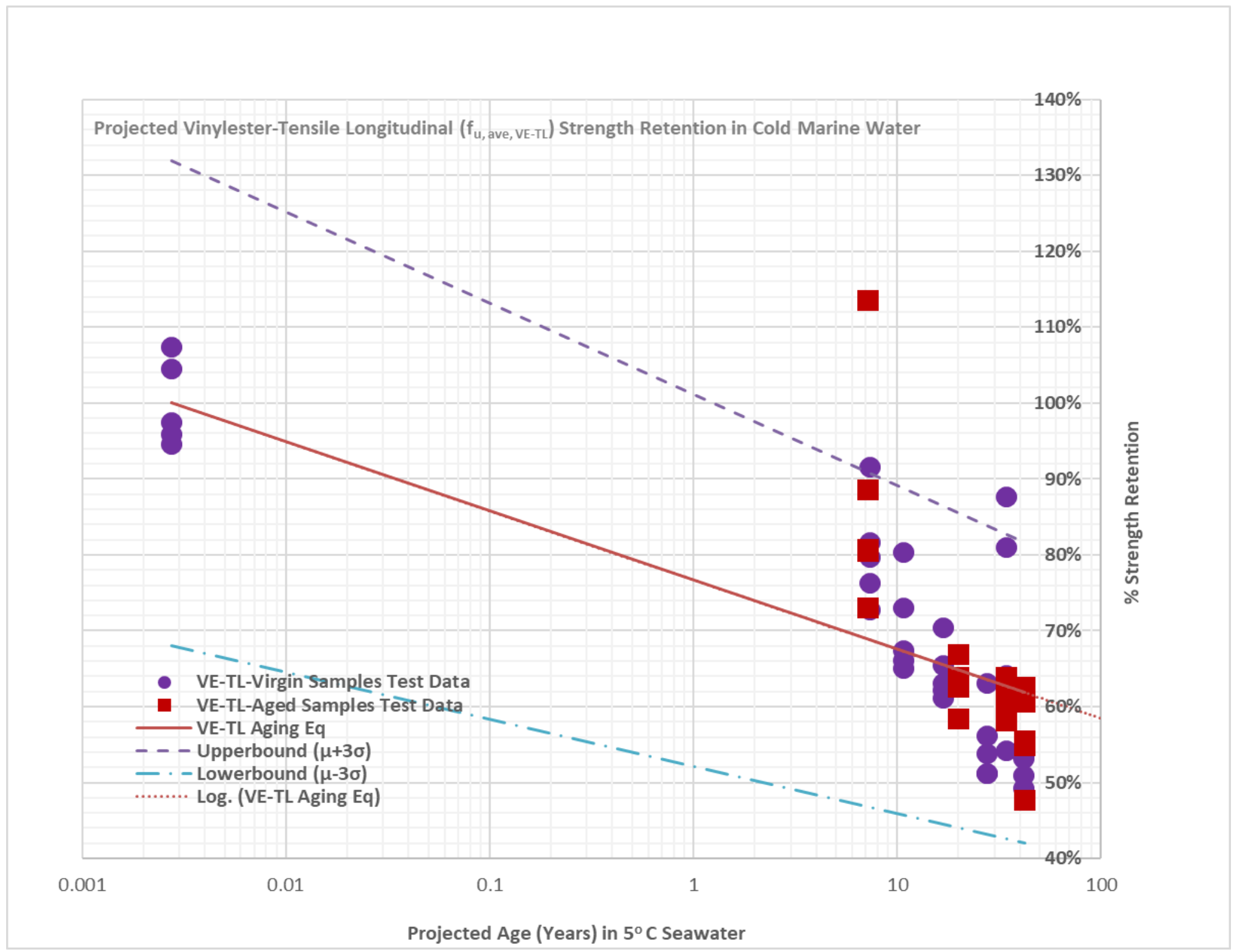


Figure C.5: Projected Flexural-Transverse Strengths of Vinylester GFRP

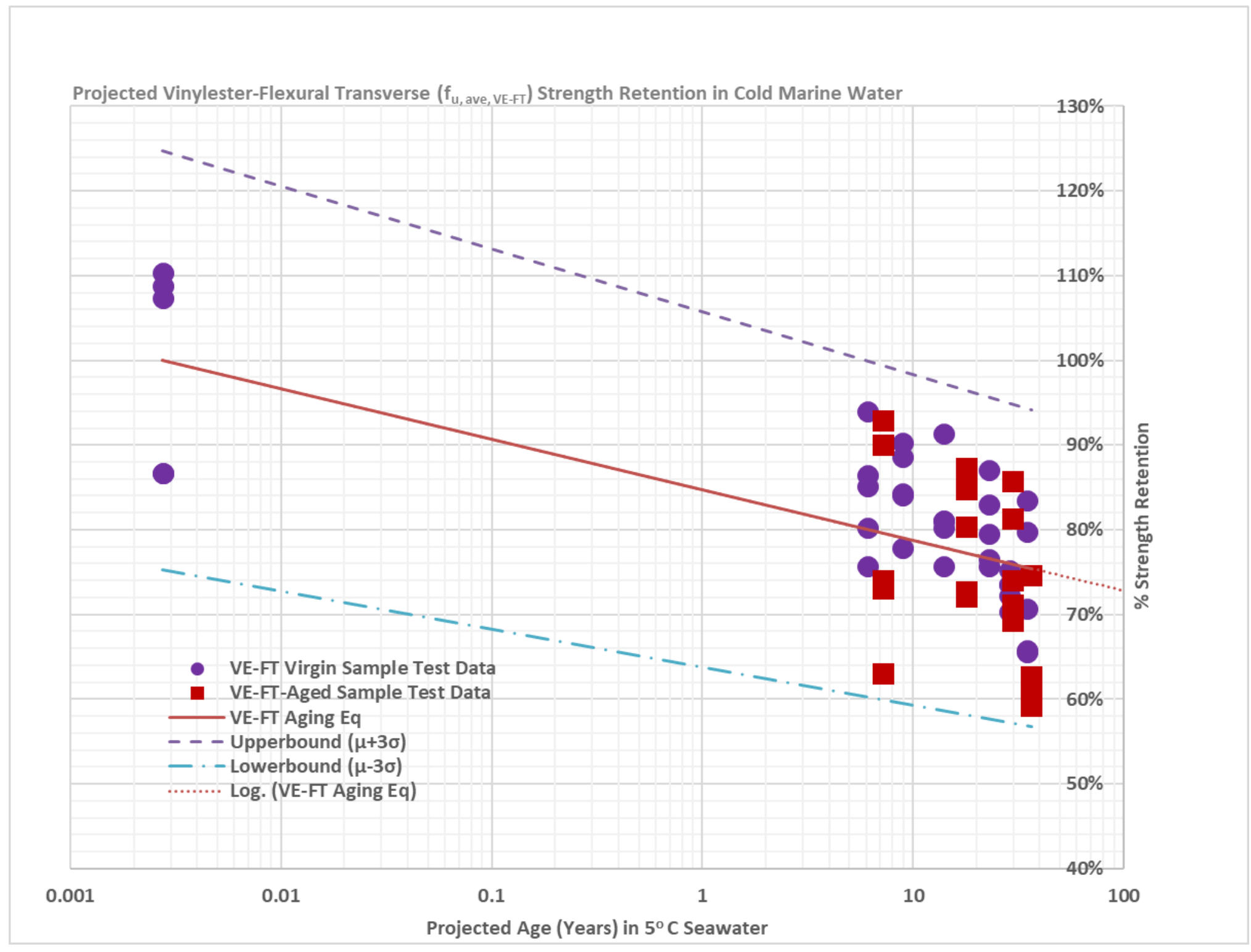


Figure C.6: Projected Flexural-Longitudinal Strengths of Vinylester GFRP

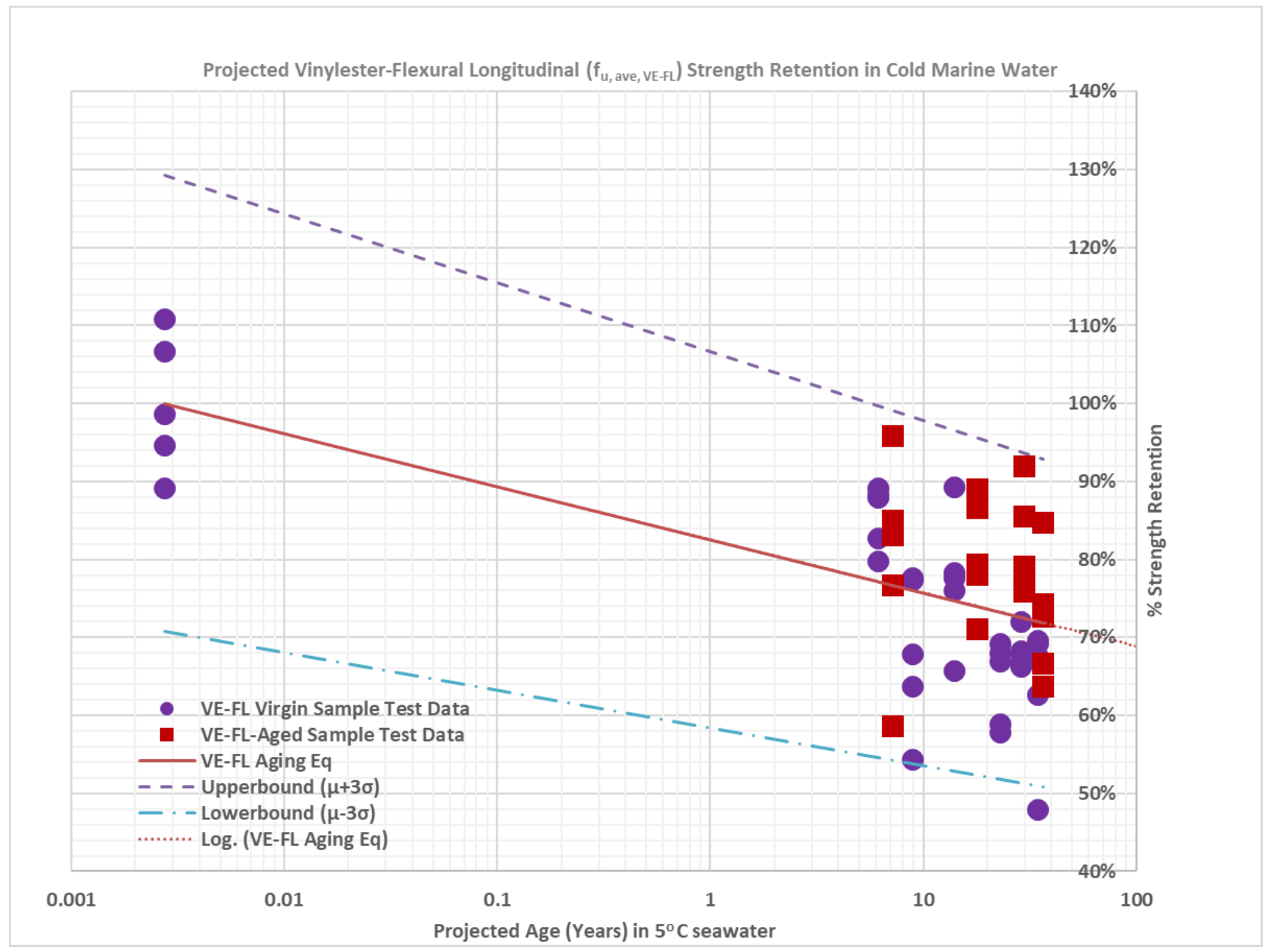


Figure C.7: Projected Shear-Transverse Strengths of Vinylester GFRP

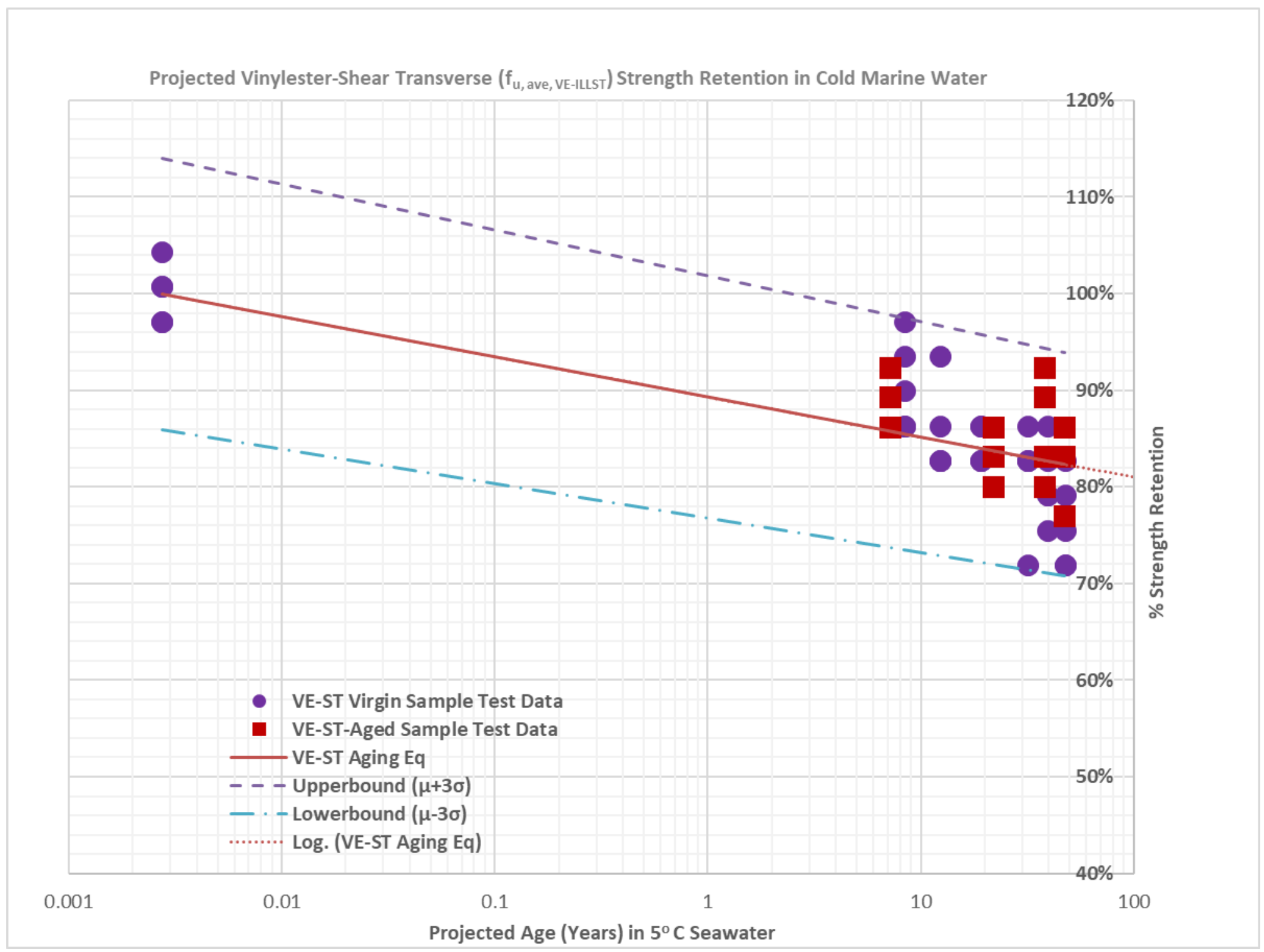


Figure C.8: Projected Shear-Longitudinal Strengths of Vinylester GFRP

Projected Vinylester-Shear Longitudinal $\left(f_{\mathrm{u}, \text { ave, VE-ILSL }}\right)$ Strength Retention in Cold Marine Water

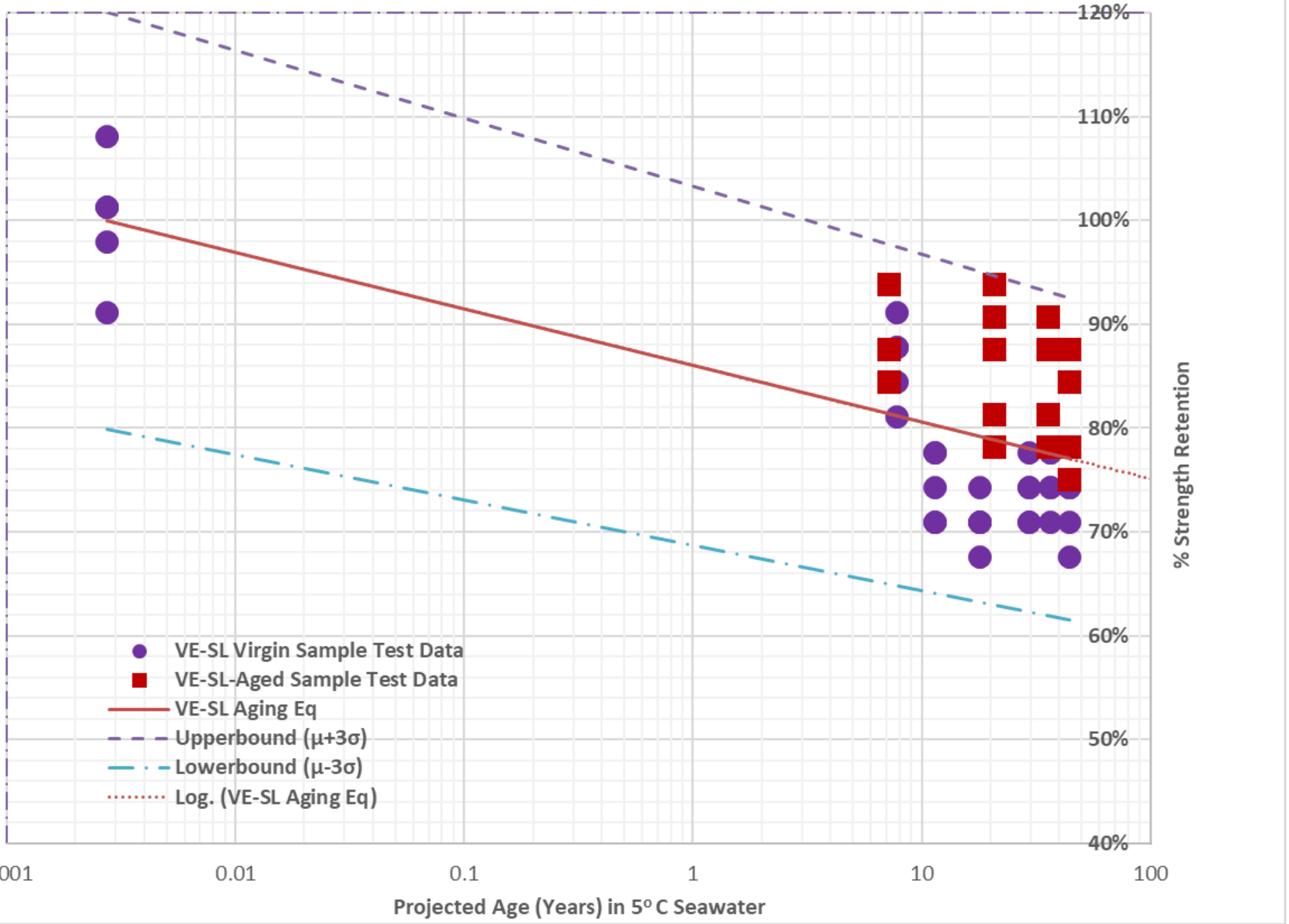


Figure C.9: Projected Tensile Modulus-Transverse Strengths of Vinylester GFRP

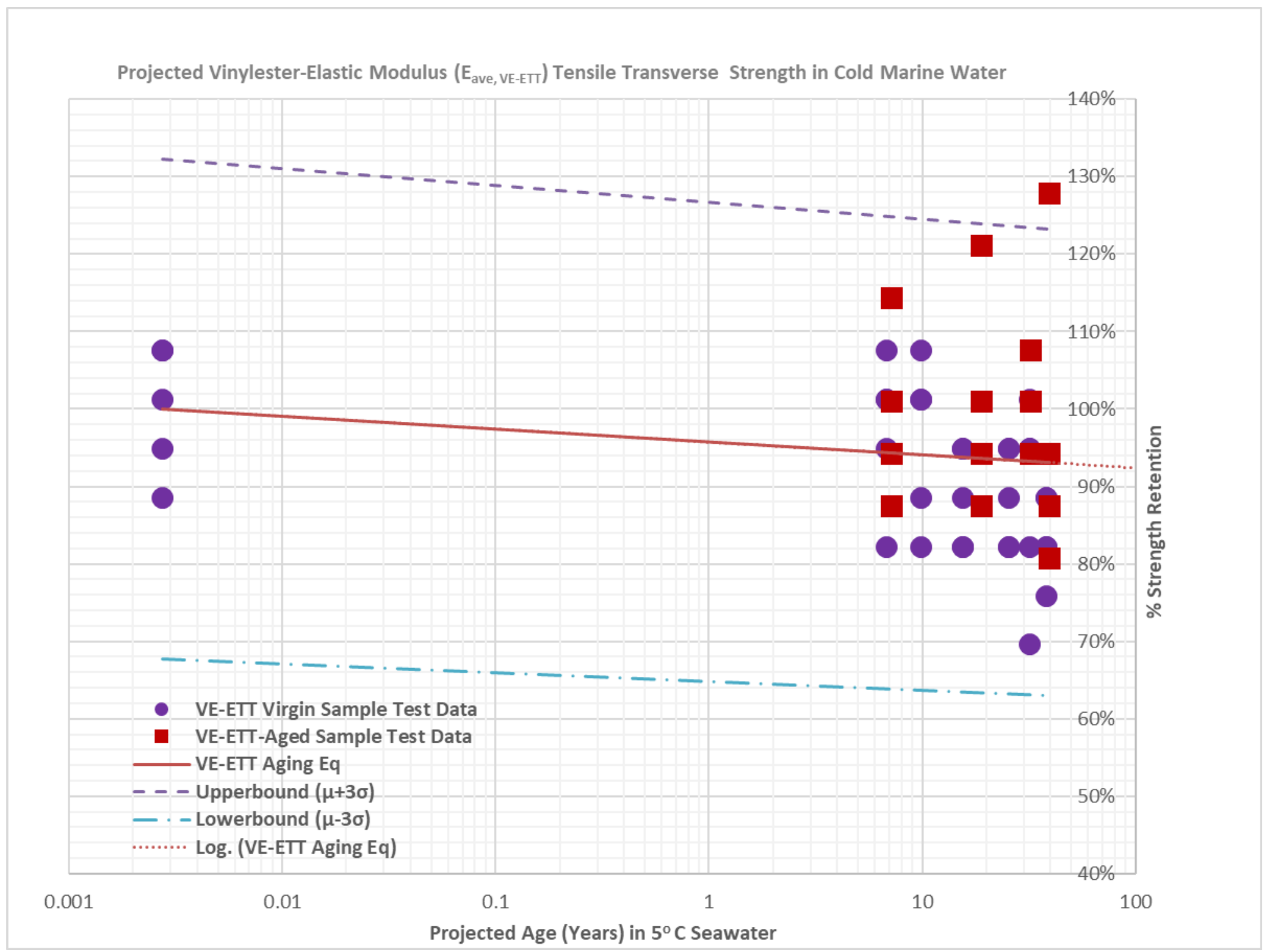


Figure C.10: Projected Tensile Modulus-Longitudinal Strengths of Vinylester GFRP

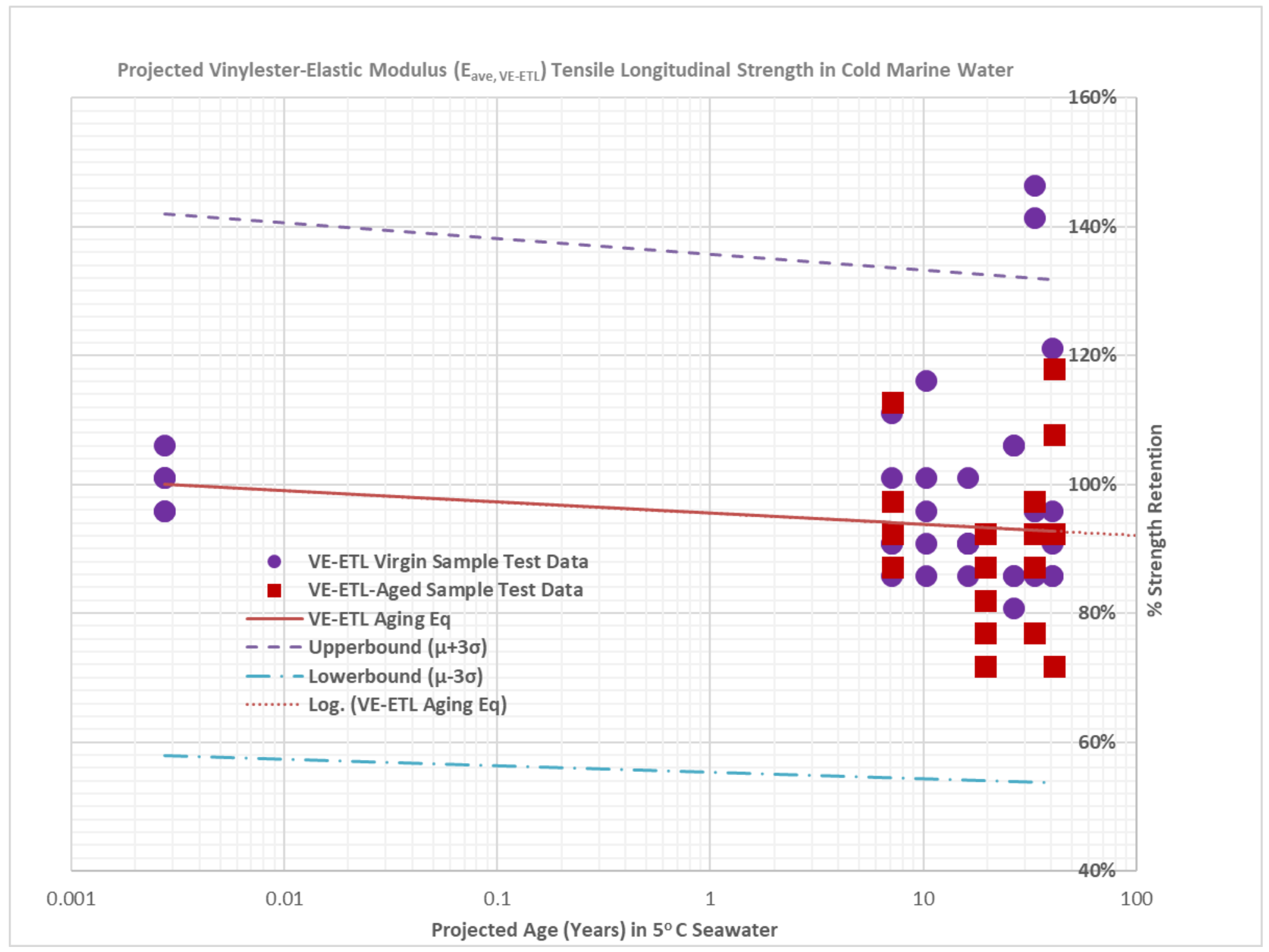


Figure C.11: Projected Tensile-Transverse Strengths of Polyester GFRP

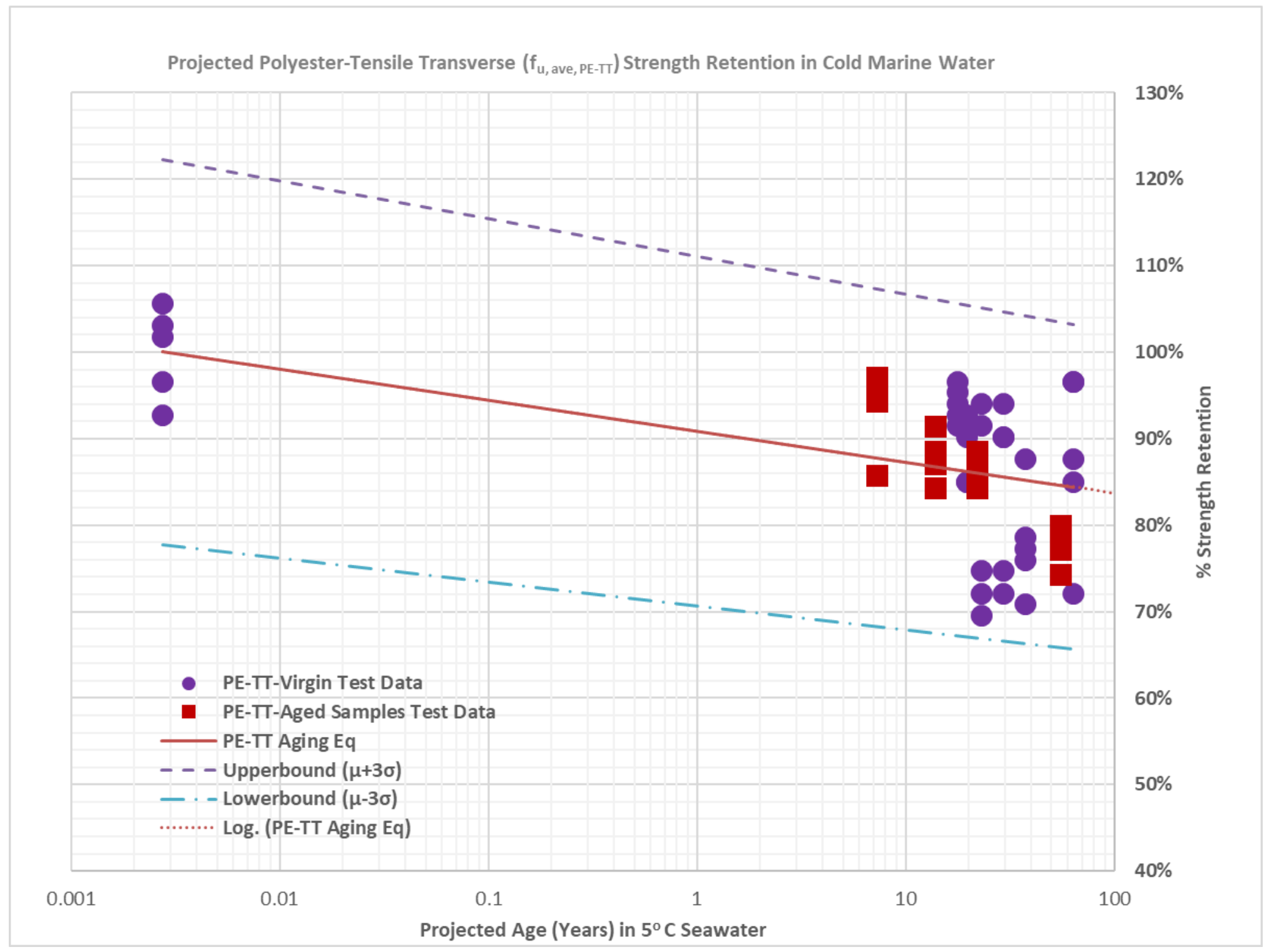


Figure C.12: Projected Tensile-Longitudinal Strengths of Polyester GFRP

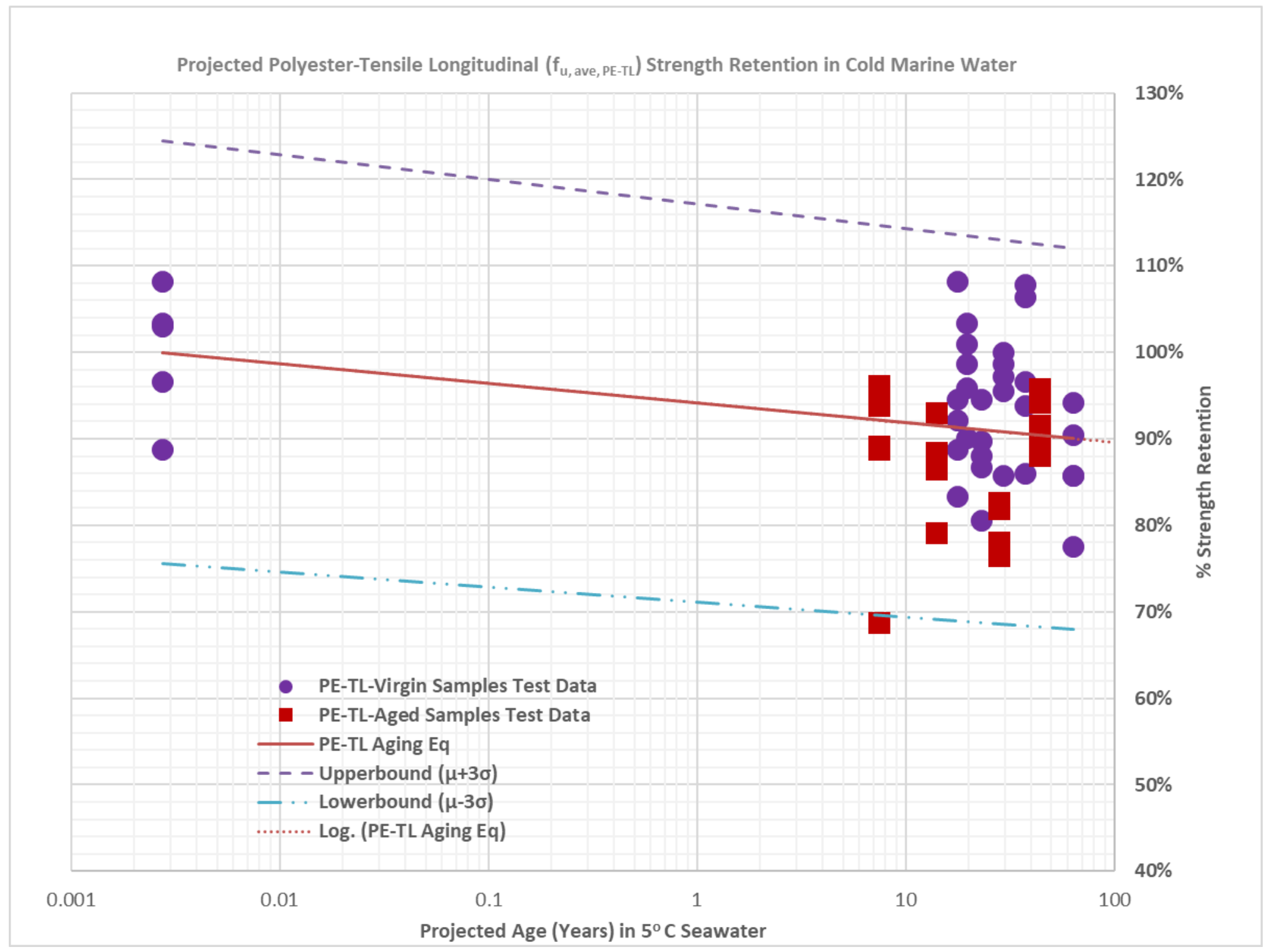


Figure C.13: Projected Flexural-Transverse Strengths of Polyester GFRP

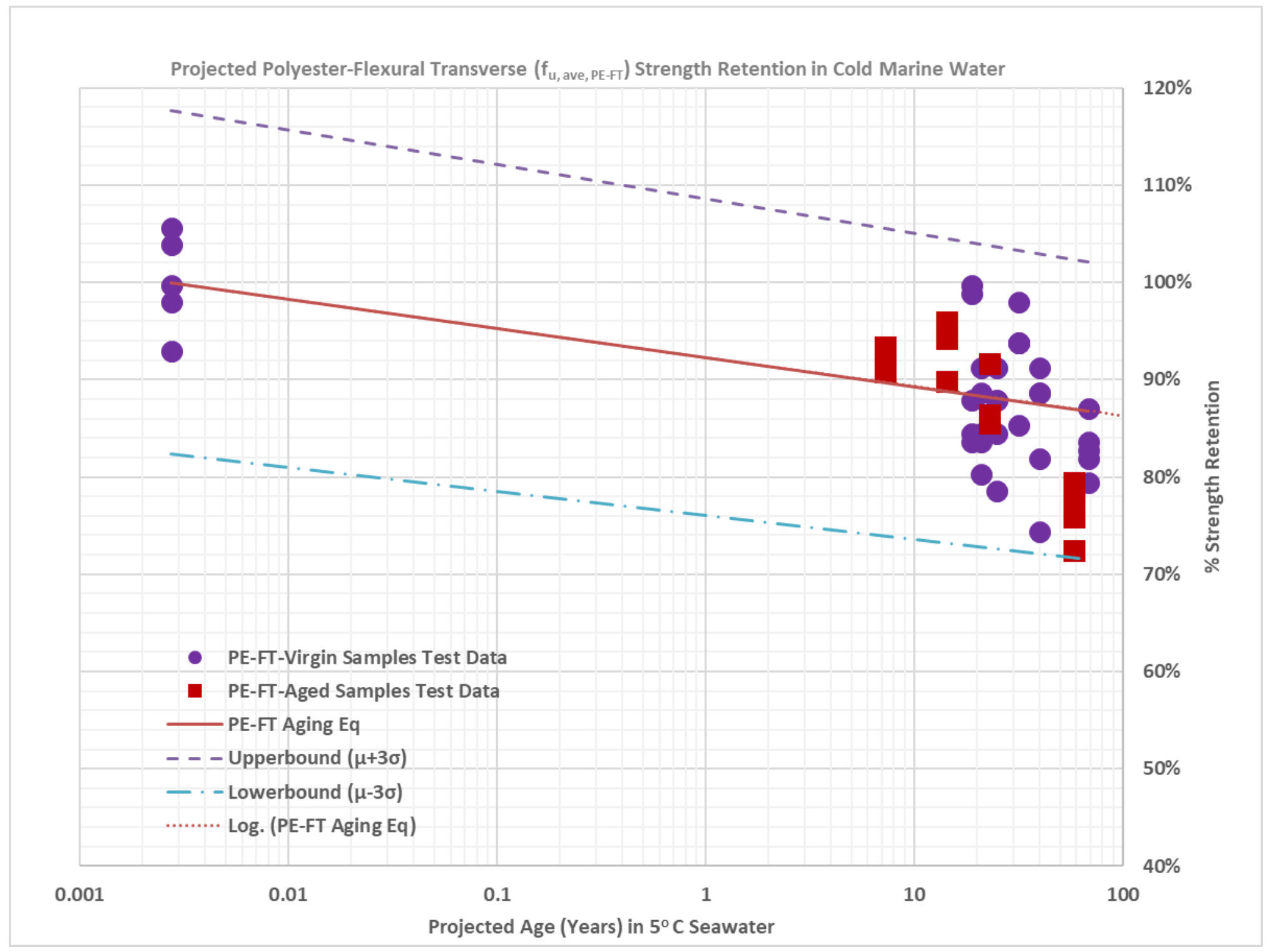


Figure C.14: Projected Flexural-Longitudinal Strengths of Polyester GFRP

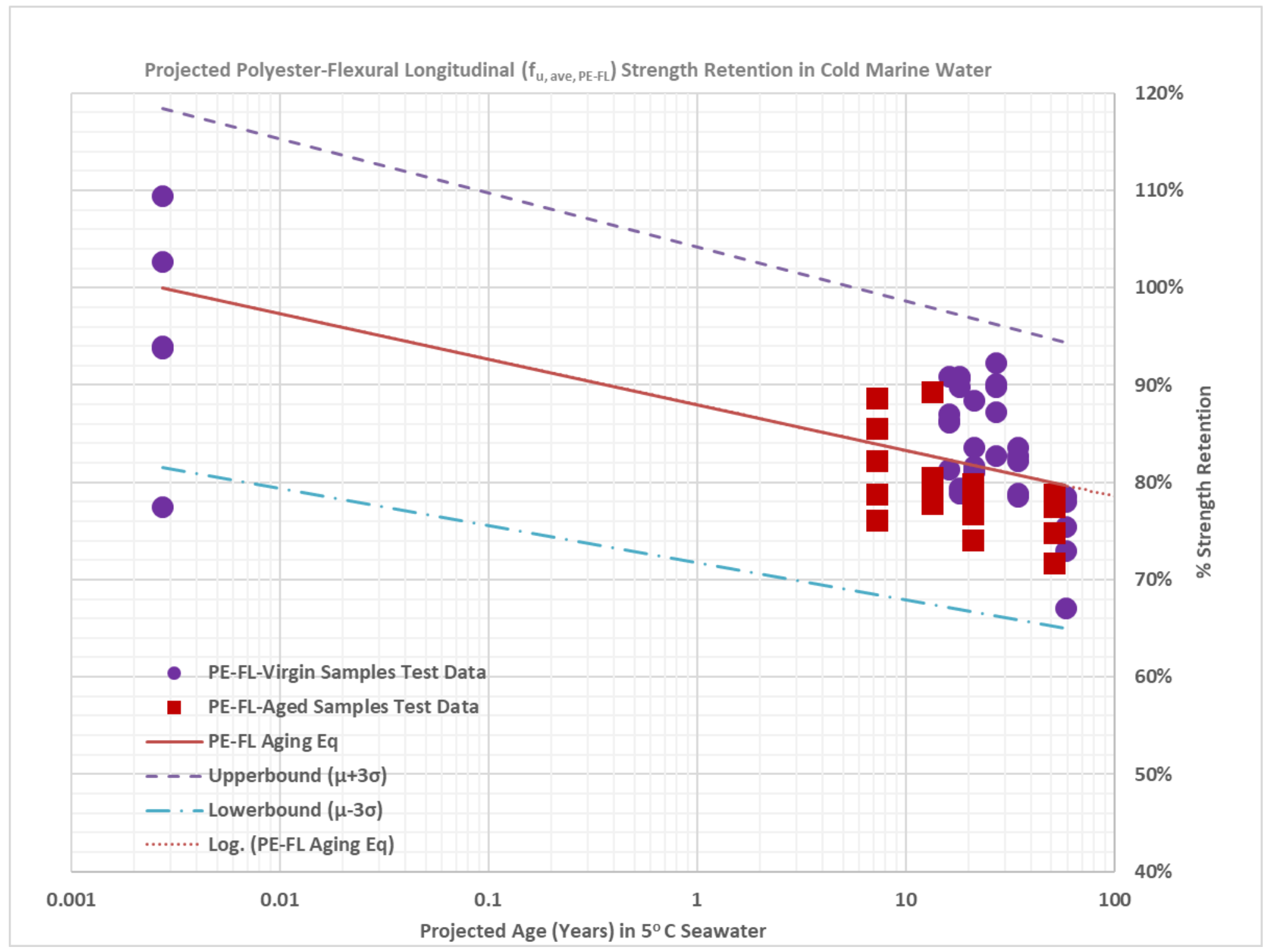


Figure C.15: Projected Shear-Transverse Strengths of Polyester GFRP

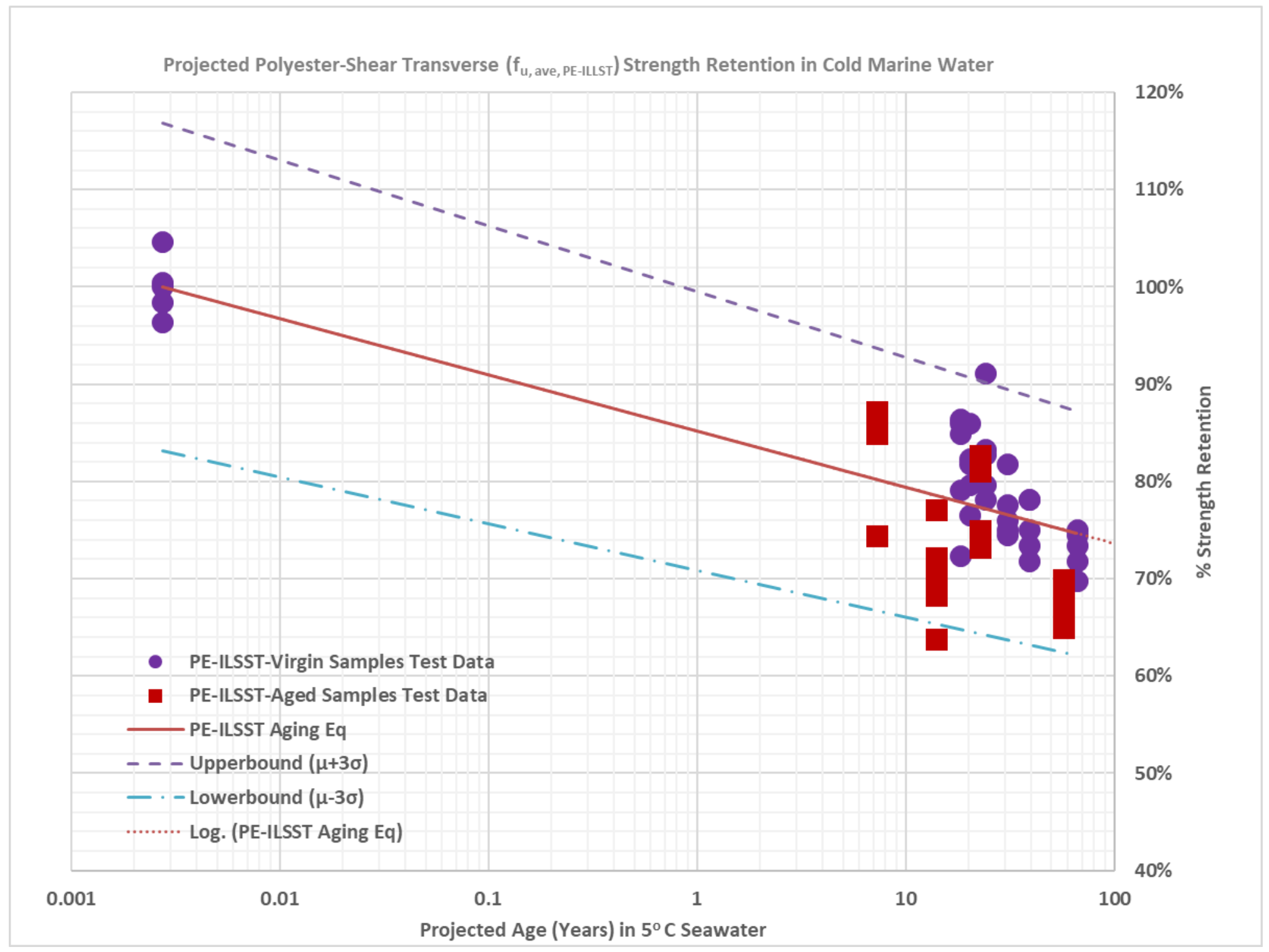


Figure C.16: Projected Shear-Longitudinal Strengths of Polyester GFRP

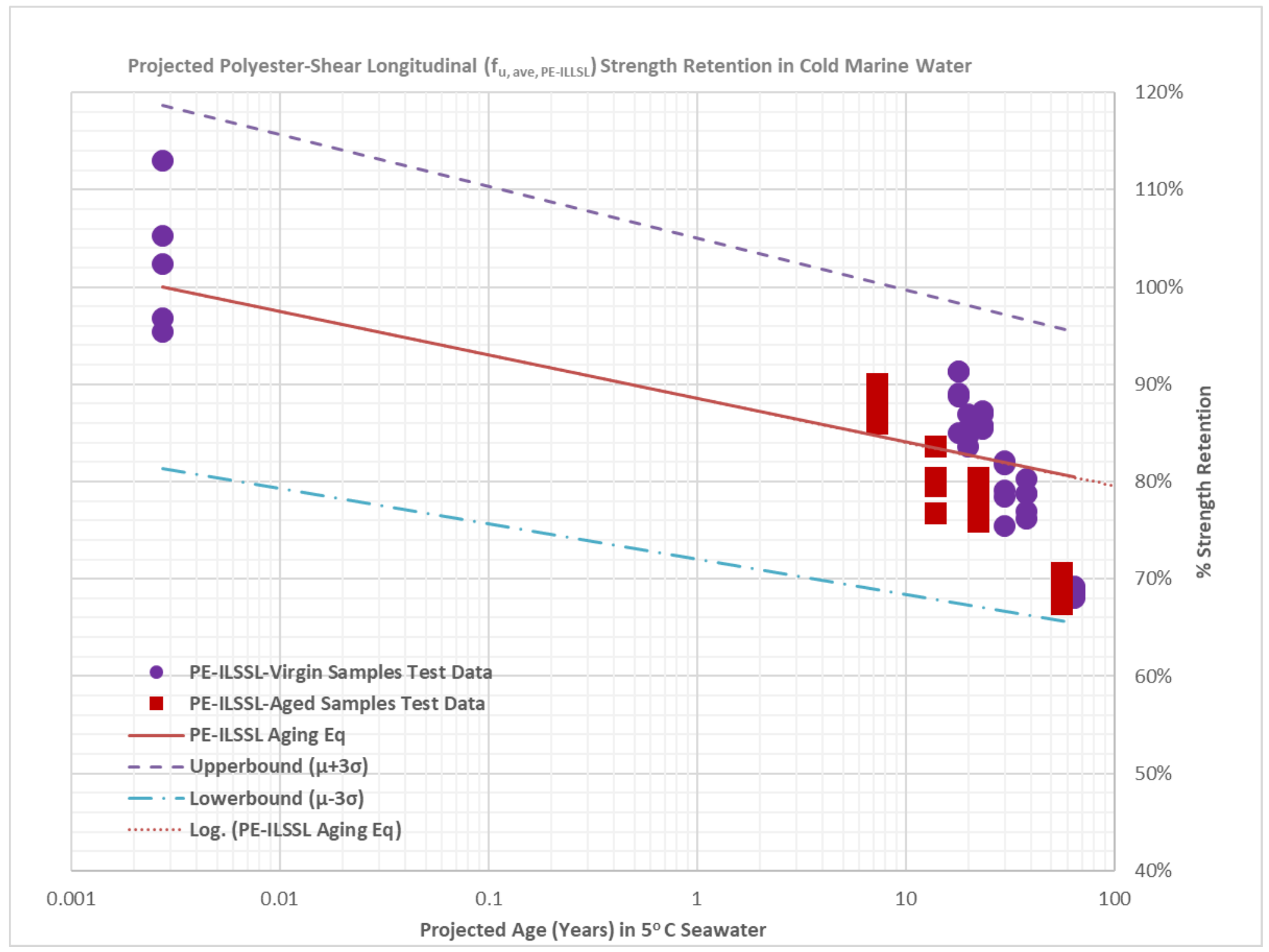


Figure C.17: Projected Tensile Modulus-Transverse Strengths of Polyester GFRP

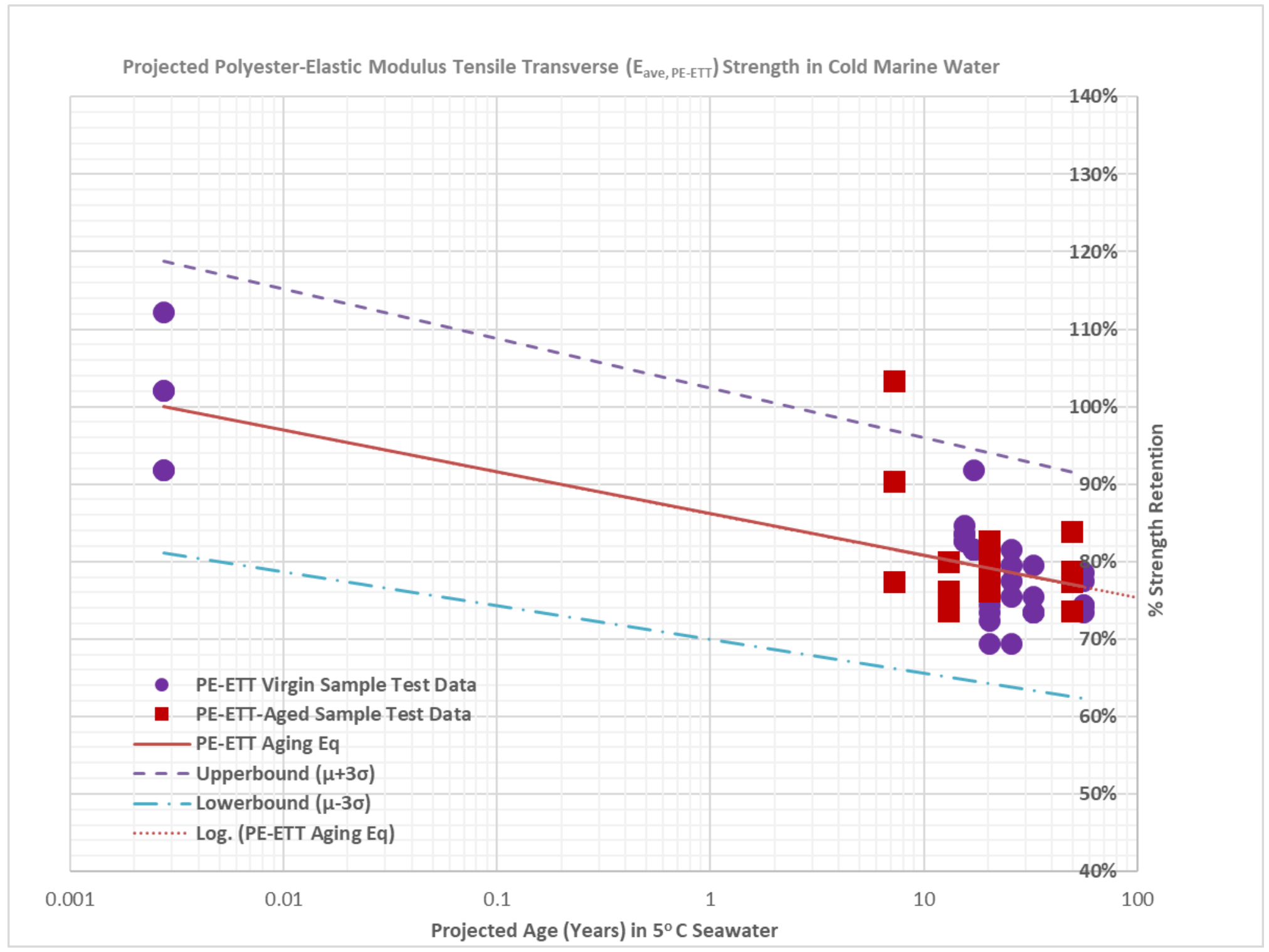


Figure C.18: Projected Tensile Modulus-Longitudinal Strengths of Polyester GFRP

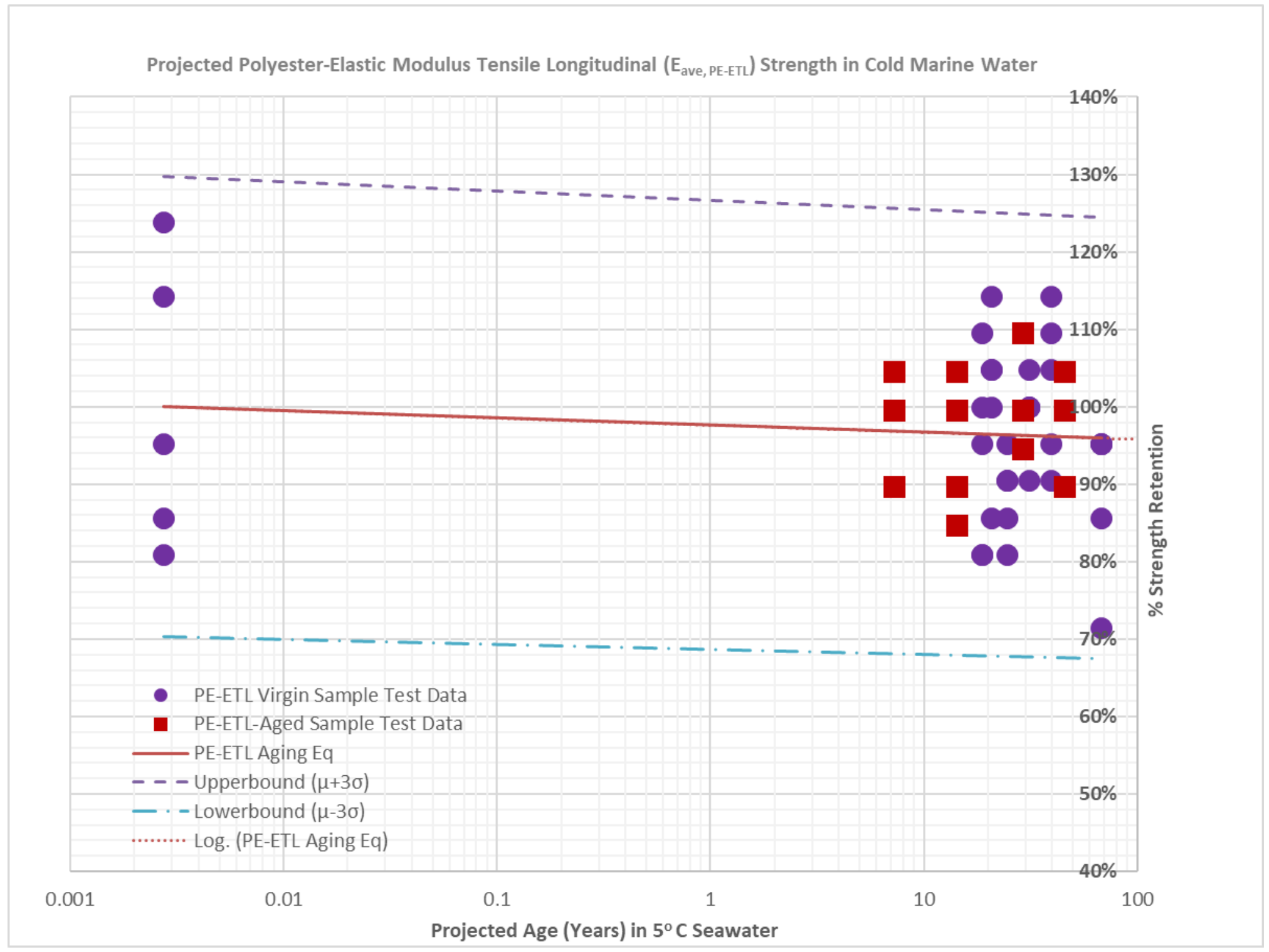




\section{APPENDIX-D SUSTAINED STRESS DATABASE}

This Appendix supplement the information presented in Chapter 5.

Data Source: University of Sherbrook (Youssef, 2010) 
Table D.1: Listing of Test Database Used in Sustained Stress Study

\begin{tabular}{|c|c|c|c|c|c|c|c|c|c|}
\hline \multicolumn{10}{|c|}{ Modified Data - Re-Tabulated \& Normalized Creep Test Data (Youssef, 2010) } \\
\hline & & & $\varepsilon_{0}^{\prime}$ & & $\varepsilon_{t}^{\prime}$ & $\varepsilon_{\text {tu }}^{\prime}$ & & $\varepsilon_{\text {to }}^{\prime}$ & \\
\hline & $(t)$ & $\left(t^{\prime}\right)$ & $\left(\varepsilon_{0} / \varepsilon_{\mathrm{fu}}\right)$ & $\left(\varepsilon_{0} / \varepsilon^{*}{ }_{f u}\right)$ & $\left(\Delta \varepsilon_{t} / \varepsilon_{0}\right)$ & $\left(\varepsilon_{t} / \varepsilon_{\mathrm{fu}}\right)$ & $\left(\varepsilon_{t} / \varepsilon^{*}{ }_{f u}\right)$ & $\left(\varepsilon_{t} / \varepsilon_{0}\right)$ & $\left(\varepsilon_{\mathrm{t}} / \varepsilon^{*}{ }_{0}\right)$ \\
\hline Sample \# & t hrs & t yrs & iSSR & Not used & cSSR & fSSR & Not used & tSSR & Not used \\
\hline $\begin{array}{c}\text { GFRP Rebar } \\
\text { Sample } \\
\text { Designation }\end{array}$ & $\begin{array}{l}\text { Test Time } \\
\text { (hrs) }\end{array}$ & $\begin{array}{l}\text { Test Time } \\
\text { (Years) }\end{array}$ & $\begin{array}{c}\text { Initial } \\
\text { Sustained } \\
\text { Strain Ratio } \\
\text { (based on } \\
\text { Ultimate } \\
\text { Strain) }\end{array}$ & $\begin{array}{l}\text { Nominal } \\
\text { Initial } \\
\text { Strain } \\
\text { Ratio } \\
(\% * f u)\end{array}$ & $\begin{array}{c}\% \text { Creep } \\
\text { Strain } \\
\text { Increase } \\
\text { Ratio }\end{array}$ & $\begin{array}{c}\text { Final } \\
\text { Sustained } \\
\text { Strain Ratio } \\
\text { (based on } \\
\text { Ultimate) }\end{array}$ & $\begin{array}{l}\text { Final Strain } \\
\text { Ratio (based } \\
\text { on Nominal) }\end{array}$ & $\begin{array}{c}\text { Total } \\
\text { Sustained } \\
\text { Strain } \\
\text { Increase } \\
\text { over } 100 \% \\
\text { initial (based } \\
\text { on Ultimate) }\end{array}$ & $\begin{array}{c}\% \text { Strain } \\
\text { over } 100 \% \\
\text { (based on } \\
\text { Nominal) }\end{array}$ \\
\hline GFRP1-15-1 & 1 & 0.000114 & $12.40 \%$ & $14.20 \%$ & $0.00 \%$ & $12.40 \%$ & $14.20 \%$ & $100.0 \%$ & $100.0 \%$ \\
\hline GFRP1-15-1 & 1000 & 0.114155 & $12.40 \%$ & $14.20 \%$ & $2.00 \%$ & $14.40 \%$ & $16.20 \%$ & $116.1 \%$ & $114.1 \%$ \\
\hline GFRP1-15-1 & 3000 & 0.342466 & $12.40 \%$ & $14.20 \%$ & $3.00 \%$ & $15.40 \%$ & $17.20 \%$ & $124.2 \%$ & $121.1 \%$ \\
\hline GFRP1-15-1 & 10000 & 1.141553 & $12.40 \%$ & $14.20 \%$ & $4.10 \%$ & $16.50 \%$ & $18.30 \%$ & $133.1 \%$ & $128.9 \%$ \\
\hline GFRP1-15-2 & 1 & 0.000114 & $12.40 \%$ & $14.20 \%$ & $0.00 \%$ & $12.40 \%$ & $14.20 \%$ & $100.0 \%$ & $100.0 \%$ \\
\hline GFRP1-15-2 & 1000 & 0.114155 & $12.40 \%$ & $14.20 \%$ & $1.20 \%$ & $13.60 \%$ & $15.40 \%$ & $109.7 \%$ & $108.5 \%$ \\
\hline GFRP1-15-2 & 3000 & 0.342466 & $12.40 \%$ & $14.20 \%$ & $4.50 \%$ & $16.90 \%$ & $18.70 \%$ & $136.3 \%$ & $131.7 \%$ \\
\hline GFRP1-15-2 & 10000 & 1.141553 & $12.40 \%$ & $14.20 \%$ & $7.80 \%$ & $20.20 \%$ & $22.00 \%$ & $162.9 \%$ & $154.9 \%$ \\
\hline GFRP1-15-3 & 1 & 0.000114 & $14.40 \%$ & $16.50 \%$ & $0.00 \%$ & $14.40 \%$ & $16.50 \%$ & $100.0 \%$ & $100.0 \%$ \\
\hline GFRP1-15-3 & 1000 & 0.114155 & $14.40 \%$ & $16.50 \%$ & $0.00 \%$ & $14.40 \%$ & $16.50 \%$ & $100.0 \%$ & $100.0 \%$ \\
\hline GFRP $1-15-3$ & 3000 & 0.342466 & $14.40 \%$ & $16.50 \%$ & $6.90 \%$ & $21.30 \%$ & $23.40 \%$ & $147.9 \%$ & $141.8 \%$ \\
\hline GFRP1-15-3 & 10000 & 1.141553 & $14.40 \%$ & $16.50 \%$ & $5.00 \%$ & $19.40 \%$ & $21.50 \%$ & $134.7 \%$ & $130.3 \%$ \\
\hline GFRP1-15-4 & 1 & 0.000114 & $12.60 \%$ & $14.50 \%$ & $0.00 \%$ & $12.60 \%$ & $14.50 \%$ & $100.0 \%$ & $100.0 \%$ \\
\hline GFRP1-15-4 & 1000 & 0.114155 & $12.60 \%$ & $14.50 \%$ & $0.30 \%$ & $12.90 \%$ & $14.80 \%$ & $102.4 \%$ & $102.1 \%$ \\
\hline GFRP1-15-4 & 3000 & 0.342466 & $12.60 \%$ & $14.50 \%$ & $3.30 \%$ & $15.90 \%$ & $17.80 \%$ & $126.2 \%$ & $122.8 \%$ \\
\hline GFRP1-15-4 & 10000 & 1.141553 & $12.60 \%$ & $14.50 \%$ & $7.20 \%$ & $19.80 \%$ & $21.70 \%$ & $157.1 \%$ & $149.7 \%$ \\
\hline GFRP1-15-5 & 1 & 0.000114 & $14.80 \%$ & $17.00 \%$ & $0.00 \%$ & $14.80 \%$ & $17.00 \%$ & $100.0 \%$ & $100.0 \%$ \\
\hline GFRP1-15-5 & 1000 & 0.114155 & $14.80 \%$ & $17.00 \%$ & $5.70 \%$ & $20.50 \%$ & $22.70 \%$ & $138.5 \%$ & $133.5 \%$ \\
\hline GFRP1-15-5 & 3000 & 0.342466 & $14.80 \%$ & $17.00 \%$ & $5.40 \%$ & $20.20 \%$ & $22.40 \%$ & $136.5 \%$ & $131.8 \%$ \\
\hline
\end{tabular}




\begin{tabular}{|c|c|c|c|c|c|c|c|c|c|}
\hline & & & $\varepsilon_{0}^{\prime}$ & & $\varepsilon_{\mathrm{t}}^{\prime}$ & $\varepsilon_{\mathrm{tu}}^{\prime}$ & & $\varepsilon_{\mathrm{t} 0}^{\prime}$ & \\
\hline & $(t)$ & $\left(t^{\prime}\right)$ & $\left(\varepsilon_{0} / \varepsilon_{f u}\right)$ & $\left(\varepsilon_{0} / \varepsilon^{*}{ }_{f u}\right)$ & $\left(\Delta \varepsilon_{t} / \varepsilon_{0}\right)$ & $\left(\varepsilon_{t} / \varepsilon_{f u}\right)$ & $\left(\varepsilon_{t} / \varepsilon^{*}{ }_{f u}\right)$ & $\left(\varepsilon_{t} / \varepsilon_{0}\right)$ & $\left(\varepsilon_{t} / \varepsilon^{*}{ }_{0}\right)$ \\
\hline Sample \# & t hrs & tyrs & iSSR & Not used & cSSR & fSSR & Not used & tSSR & Not used \\
\hline GFRP1-15-5 & 10000 & 1.141553 & $14.80 \%$ & $17.00 \%$ & $8.80 \%$ & $23.60 \%$ & $25.80 \%$ & $159.5 \%$ & $151.8 \%$ \\
\hline GFRP1-30-1 & 1 & 0.000114 & $20.90 \%$ & $23.90 \%$ & $0.00 \%$ & $20.90 \%$ & $23.90 \%$ & $100.0 \%$ & $100.0 \%$ \\
\hline GFRP1-30-1 & 1000 & 0.114155 & $20.90 \%$ & $23.90 \%$ & $-0.20 \%$ & $20.70 \%$ & $23.70 \%$ & $99.0 \%$ & $99.2 \%$ \\
\hline GFRP1-30-1 & 3000 & 0.342466 & $20.90 \%$ & $23.90 \%$ & $1.30 \%$ & $22.20 \%$ & $25.20 \%$ & $106.2 \%$ & $105.4 \%$ \\
\hline GFRP1-30-1 & 10000 & 1.141553 & $20.90 \%$ & $23.90 \%$ & $1.20 \%$ & $22.10 \%$ & $25.10 \%$ & $105.7 \%$ & $105.0 \%$ \\
\hline GFRP1-30-2 & 1 & 0.000114 & $31.10 \%$ & $35.60 \%$ & $0.00 \%$ & $31.10 \%$ & $35.60 \%$ & $100.0 \%$ & $100.0 \%$ \\
\hline GFRP1-30-2 & 1000 & 0.114155 & $31.10 \%$ & $35.60 \%$ & $-2.90 \%$ & $28.20 \%$ & $32.70 \%$ & $90.7 \%$ & $91.9 \%$ \\
\hline GFRP1-30-2 & 3000 & 0.342466 & $31.10 \%$ & $35.60 \%$ & $-2.30 \%$ & $28.80 \%$ & $33.30 \%$ & $92.6 \%$ & $93.5 \%$ \\
\hline GFRP1-30-2 & 10000 & 1.141553 & $31.10 \%$ & $35.60 \%$ & $-2.90 \%$ & $28.20 \%$ & $32.70 \%$ & $90.7 \%$ & $91.9 \%$ \\
\hline GFRP1-30-3 & 1 & 0.000114 & $28.60 \%$ & $32.70 \%$ & $0.00 \%$ & $28.60 \%$ & $32.70 \%$ & $100.0 \%$ & $100.0 \%$ \\
\hline GFRP1-30-3 & 1000 & 0.114155 & $28.60 \%$ & $32.70 \%$ & $1.40 \%$ & $30.00 \%$ & $34.10 \%$ & $104.9 \%$ & $104.3 \%$ \\
\hline GFRP1-30-3 & 3000 & 0.342466 & $28.60 \%$ & $32.70 \%$ & $0.70 \%$ & $29.30 \%$ & $33.40 \%$ & $102.4 \%$ & $102.1 \%$ \\
\hline GFRP1-30-3 & 10000 & 1.141553 & $28.60 \%$ & $32.70 \%$ & $0.60 \%$ & $29.20 \%$ & $33.30 \%$ & $102.1 \%$ & $101.8 \%$ \\
\hline GFRP1-30-4 & 1 & 0.000114 & $33.00 \%$ & $37.80 \%$ & $0.00 \%$ & $33.00 \%$ & $37.80 \%$ & $100.0 \%$ & $100.0 \%$ \\
\hline GFRP1-30-4 & 1000 & 0.114155 & $33.00 \%$ & $37.80 \%$ & $-4.50 \%$ & $28.50 \%$ & $33.30 \%$ & $86.4 \%$ & $88.1 \%$ \\
\hline GFRP1-30-4 & 3000 & 0.342466 & $33.00 \%$ & $37.80 \%$ & $-4.80 \%$ & $28.20 \%$ & $33.00 \%$ & $85.5 \%$ & $87.3 \%$ \\
\hline GFRP1-30-4 & 10000 & 1.141553 & $33.00 \%$ & $37.80 \%$ & $-4.80 \%$ & $28.20 \%$ & $33.00 \%$ & $85.5 \%$ & $87.3 \%$ \\
\hline GFRP1-30-5 & 1 & 0.000114 & $28.80 \%$ & $33.00 \%$ & $0.00 \%$ & $28.80 \%$ & $33.00 \%$ & $100.0 \%$ & $100.0 \%$ \\
\hline GFRP1-30-5 & 1000 & 0.114155 & $28.80 \%$ & $33.00 \%$ & $2.20 \%$ & $31.00 \%$ & $35.20 \%$ & $107.6 \%$ & $106.7 \%$ \\
\hline GFRP1-30-5 & 3000 & 0.342466 & $28.80 \%$ & $33.00 \%$ & $3.30 \%$ & $32.10 \%$ & $36.30 \%$ & $111.5 \%$ & $110.0 \%$ \\
\hline GFRP1-30-5 & 10000 & 1.141553 & $28.80 \%$ & $33.00 \%$ & $3.80 \%$ & $32.60 \%$ & $36.80 \%$ & $113.2 \%$ & $111.5 \%$ \\
\hline GFRP1-45a-1 & 1 & 0.000114 & $47.10 \%$ & $54.00 \%$ & $0.00 \%$ & $47.10 \%$ & $54.00 \%$ & $100.0 \%$ & $100.0 \%$ \\
\hline GFRP1-45a-1 & 1000 & 0.114155 & $47.10 \%$ & $54.00 \%$ & $1.30 \%$ & $48.40 \%$ & $55.30 \%$ & $102.8 \%$ & $102.4 \%$ \\
\hline GFRP1-45a-1 & 3000 & 0.342466 & $47.10 \%$ & $54.00 \%$ & $1.10 \%$ & $48.20 \%$ & $55.10 \%$ & $102.3 \%$ & $102.0 \%$ \\
\hline GFRP1-45a-1 & 10000 & 1.141553 & $47.10 \%$ & $54.00 \%$ & $2.70 \%$ & $49.80 \%$ & $56.70 \%$ & $105.7 \%$ & $105.0 \%$ \\
\hline GFRP1-45a-2 & 1 & 0.000114 & $44.90 \%$ & $51.40 \%$ & $0.00 \%$ & $44.90 \%$ & $51.40 \%$ & $100.0 \%$ & $100.0 \%$ \\
\hline GFRP1-45a-2 & 1000 & 0.114155 & $44.90 \%$ & $51.40 \%$ & $2.20 \%$ & $47.10 \%$ & $53.60 \%$ & $104.9 \%$ & $104.3 \%$ \\
\hline GFRP1-45a-2 & 3000 & 0.342466 & $44.90 \%$ & $51.40 \%$ & $2.80 \%$ & $47.70 \%$ & $54.20 \%$ & $106.2 \%$ & $105.4 \%$ \\
\hline
\end{tabular}




\begin{tabular}{|c|c|c|c|c|c|c|c|c|c|}
\hline & & & $\varepsilon_{0}^{\prime}$ & & $\varepsilon_{\mathrm{t}}^{\prime}$ & $\varepsilon_{\mathrm{tu}}^{\prime}$ & & $\varepsilon_{\mathrm{t} 0}^{\prime}$ & \\
\hline & $(t)$ & $\left(t^{\prime}\right)$ & $\left(\varepsilon_{0} / \varepsilon_{f u}\right)$ & $\left(\varepsilon_{0} / \varepsilon^{*}{ }_{f u}\right)$ & $\left(\Delta \varepsilon_{t} / \varepsilon_{0}\right)$ & $\left(\varepsilon_{t} / \varepsilon_{f u}\right)$ & $\left(\varepsilon_{t} / \varepsilon^{*}{ }_{f u}\right)$ & $\left(\varepsilon_{t} / \varepsilon_{0}\right)$ & $\left(\varepsilon_{t} / \varepsilon^{*}{ }_{0}\right)$ \\
\hline Sample \# & t hrs & tyrs & iSSR & Not used & cSSR & fSSR & Not used & tSSR & Not used \\
\hline GFRP1-45a-2 & 10000 & 1.141553 & $44.90 \%$ & $51.40 \%$ & $3.60 \%$ & $48.50 \%$ & $55.00 \%$ & $108.0 \%$ & $107.0 \%$ \\
\hline GFRP1-45a-3 & 1 & 0.000114 & $44.40 \%$ & $50.80 \%$ & $0.00 \%$ & $44.40 \%$ & $50.80 \%$ & $100.0 \%$ & $100.0 \%$ \\
\hline GFRP1-45a-3 & 1000 & 0.114155 & $44.40 \%$ & $50.80 \%$ & $1.60 \%$ & $46.00 \%$ & $52.40 \%$ & $103.6 \%$ & $103.1 \%$ \\
\hline GFRP1-45a-3 & 3000 & 0.342466 & $44.40 \%$ & $50.80 \%$ & $-0.30 \%$ & $44.10 \%$ & $50.50 \%$ & $99.3 \%$ & $99.4 \%$ \\
\hline GFRP1-45a-3 & 10000 & 1.141553 & $44.40 \%$ & $50.80 \%$ & $1.00 \%$ & $45.40 \%$ & $51.80 \%$ & $102.3 \%$ & $102.0 \%$ \\
\hline GFRP1-45a-4 & 1 & 0.000114 & $40.80 \%$ & $46.70 \%$ & $0.00 \%$ & $40.80 \%$ & $46.70 \%$ & $100.0 \%$ & $100.0 \%$ \\
\hline GFRP1-45a-4 & 1000 & 0.114155 & $40.80 \%$ & $46.70 \%$ & $2.60 \%$ & $43.40 \%$ & $49.30 \%$ & $106.4 \%$ & $105.6 \%$ \\
\hline GFRP1-45a-4 & 3000 & 0.342466 & $40.80 \%$ & $46.70 \%$ & $2.40 \%$ & $43.20 \%$ & $49.10 \%$ & $105.9 \%$ & $105.1 \%$ \\
\hline GFRP1-45a-4 & 10000 & 1.141553 & $40.80 \%$ & $46.70 \%$ & $4.60 \%$ & $45.40 \%$ & $51.30 \%$ & $111.3 \%$ & $109.9 \%$ \\
\hline GFRP1-45a-5 & 1 & 0.000114 & $40.80 \%$ & $55.80 \%$ & $0.00 \%$ & $40.80 \%$ & $55.80 \%$ & $100.0 \%$ & $100.0 \%$ \\
\hline GFRP1-45a-5 & 1000 & 0.114155 & $40.80 \%$ & $55.80 \%$ & $2.20 \%$ & $43.00 \%$ & $58.00 \%$ & $105.4 \%$ & $103.9 \%$ \\
\hline GFRP1-45a-5 & 3000 & 0.342466 & $40.80 \%$ & $55.80 \%$ & $1.30 \%$ & $42.10 \%$ & $57.10 \%$ & $103.2 \%$ & $102.3 \%$ \\
\hline GFRP1-45a-5 & 10000 & 1.141553 & $40.80 \%$ & $55.80 \%$ & $4.20 \%$ & $45.00 \%$ & $60.00 \%$ & $110.3 \%$ & $107.5 \%$ \\
\hline GFRP1-45b-1 & 1 & 0.000114 & $47.10 \%$ & $53.00 \%$ & $0.00 \%$ & $47.10 \%$ & $53.00 \%$ & $100.0 \%$ & $100.0 \%$ \\
\hline GFRP1-45b-1 & 1000 & 0.114155 & $47.10 \%$ & $53.00 \%$ & $2.70 \%$ & $49.80 \%$ & $55.70 \%$ & $105.7 \%$ & $105.1 \%$ \\
\hline GFRP1-45b-1 & 3000 & 0.342466 & $47.10 \%$ & $53.00 \%$ & $4.50 \%$ & $51.60 \%$ & $57.50 \%$ & $109.6 \%$ & $108.5 \%$ \\
\hline GFRP1-45b-1 & 10000 & 1.141553 & $47.10 \%$ & $53.00 \%$ & $4.80 \%$ & $51.90 \%$ & $57.80 \%$ & $110.2 \%$ & $109.1 \%$ \\
\hline GFRP1-45b-2 & 1 & 0.000114 & $44.90 \%$ & $47.10 \%$ & $0.00 \%$ & $44.90 \%$ & $47.10 \%$ & $100.0 \%$ & $100.0 \%$ \\
\hline GFRP1-45b-2 & 1000 & 0.114155 & $44.90 \%$ & $47.10 \%$ & $3.40 \%$ & $48.30 \%$ & $50.50 \%$ & $107.6 \%$ & $107.2 \%$ \\
\hline GFRP1-45b-2 & 3000 & 0.342466 & $44.90 \%$ & $47.10 \%$ & $6.50 \%$ & $51.40 \%$ & $53.60 \%$ & $114.5 \%$ & $113.8 \%$ \\
\hline GFRP1-45b-2 & 10000 & 1.141553 & $44.90 \%$ & $47.10 \%$ & $5.80 \%$ & $50.70 \%$ & $52.90 \%$ & $112.9 \%$ & $112.3 \%$ \\
\hline GFRP1-45b-3 & 1 & 0.000114 & $44.40 \%$ & $51.20 \%$ & $0.00 \%$ & $44.40 \%$ & $51.20 \%$ & $100.0 \%$ & $100.0 \%$ \\
\hline GFRP1-45b-3 & 1000 & 0.114155 & $44.40 \%$ & $51.20 \%$ & $9.20 \%$ & $53.60 \%$ & $60.40 \%$ & $120.7 \%$ & $118.0 \%$ \\
\hline GFRP1-45b-3 & 3000 & 0.342466 & $44.40 \%$ & $51.20 \%$ & $9.30 \%$ & $53.70 \%$ & $60.50 \%$ & $120.9 \%$ & $118.2 \%$ \\
\hline GFRP1-45b-3 & 10000 & 1.141553 & $44.40 \%$ & $51.20 \%$ & $7.60 \%$ & $52.00 \%$ & $58.80 \%$ & $117.1 \%$ & $114.8 \%$ \\
\hline GFRP1-45b-4 & 1 & 0.000114 & $40.80 \%$ & $49.00 \%$ & $0.00 \%$ & $40.80 \%$ & $49.00 \%$ & $100.0 \%$ & $100.0 \%$ \\
\hline GFRP1-45b-4 & 1000 & 0.114155 & $40.80 \%$ & $49.00 \%$ & $0.50 \%$ & $41.30 \%$ & $49.50 \%$ & $101.2 \%$ & $101.0 \%$ \\
\hline GFRP1-45b-4 & 3000 & 0.342466 & $40.80 \%$ & $49.00 \%$ & $0.80 \%$ & $41.60 \%$ & $49.80 \%$ & $102.0 \%$ & $101.6 \%$ \\
\hline
\end{tabular}




\begin{tabular}{|c|c|c|c|c|c|c|c|c|c|}
\hline & & & $\varepsilon_{0}^{\prime}$ & & $\varepsilon_{\mathrm{t}}^{\prime}$ & $\varepsilon_{\mathrm{tu}}^{\prime}$ & & $\varepsilon_{\mathrm{t} 0}^{\prime}$ & \\
\hline & $(t)$ & $\left(t^{\prime}\right)$ & $\left(\varepsilon_{0} / \varepsilon_{f u}\right)$ & $\left(\varepsilon_{0} / \varepsilon^{*}{ }_{f u}\right)$ & $\left(\Delta \varepsilon_{t} / \varepsilon_{0}\right)$ & $\left(\varepsilon_{t} / \varepsilon_{f u}\right)$ & $\left(\varepsilon_{t} / \varepsilon^{*}{ }_{f u}\right)$ & $\left(\varepsilon_{t} / \varepsilon_{0}\right)$ & $\left(\varepsilon_{t} / \varepsilon^{*}{ }_{0}\right)$ \\
\hline Sample \# & thrs & t yrs & iSSR & Not used & cSSR & fSSR & Not used & tSSR & Not used \\
\hline GFRP1-45b-4 & 10000 & 1.141553 & $40.80 \%$ & $49.00 \%$ & $3.00 \%$ & $43.80 \%$ & $52.00 \%$ & $107.4 \%$ & $106.1 \%$ \\
\hline GFRP1-60-1 & 1 & 0.000114 & $57.10 \%$ & $65.40 \%$ & $0.00 \%$ & $57.10 \%$ & $65.40 \%$ & $100.0 \%$ & $100.0 \%$ \\
\hline GFRP1-60-1 & 1000 & 0.114155 & $57.10 \%$ & $65.40 \%$ & $-1.00 \%$ & $56.10 \%$ & $64.40 \%$ & $98.2 \%$ & $98.5 \%$ \\
\hline GFRP1-60-1 & 3000 & 0.342466 & $57.10 \%$ & $65.40 \%$ & $1.70 \%$ & $58.80 \%$ & $67.10 \%$ & $103.0 \%$ & $102.6 \%$ \\
\hline GFRP1-60-1 & 10000 & 1.141553 & $57.10 \%$ & $65.40 \%$ & $2.80 \%$ & $59.90 \%$ & $68.20 \%$ & $104.9 \%$ & $104.3 \%$ \\
\hline GFRP1-60-2 & 1 & 0.000114 & $60.60 \%$ & $69.40 \%$ & $0.00 \%$ & $60.60 \%$ & $69.40 \%$ & $100.0 \%$ & $100.0 \%$ \\
\hline GFRP1-60-2 & 1000 & 0.114155 & $60.60 \%$ & $69.40 \%$ & $1.20 \%$ & $61.80 \%$ & $70.60 \%$ & $102.0 \%$ & $101.7 \%$ \\
\hline GFRP1-60-2 & 3000 & 0.342466 & $60.60 \%$ & $69.40 \%$ & $2.60 \%$ & $63.20 \%$ & $72.00 \%$ & $104.3 \%$ & $103.7 \%$ \\
\hline GFRP1-60-2 & 10000 & 1.141553 & $60.60 \%$ & $69.40 \%$ & $3.60 \%$ & $64.20 \%$ & $73.00 \%$ & $105.9 \%$ & $105.2 \%$ \\
\hline GFRP1-60-3 & 1 & 0.000114 & $63.40 \%$ & $72.60 \%$ & $0.00 \%$ & $63.40 \%$ & $72.60 \%$ & $100.0 \%$ & $100.0 \%$ \\
\hline GFRP1-60-3 & 1000 & 0.114155 & $63.40 \%$ & $72.60 \%$ & $3.40 \%$ & $66.80 \%$ & $76.00 \%$ & $105.4 \%$ & $104.7 \%$ \\
\hline GFRP1-60-3 & 2964 & 0.338356 & $63.40 \%$ & $72.60 \%$ & $4.30 \%$ & $67.70 \%$ & $76.90 \%$ & $106.8 \%$ & $105.9 \%$ \\
\hline GFRP2-15-1 & 1 & 0.000114 & $11.00 \%$ & $12.80 \%$ & $0.00 \%$ & $11.00 \%$ & $12.80 \%$ & $100.0 \%$ & $100.0 \%$ \\
\hline GFRP2-15-1 & 1000 & 0.114155 & $11.00 \%$ & $12.80 \%$ & $1.50 \%$ & $12.50 \%$ & $14.30 \%$ & $113.6 \%$ & $111.7 \%$ \\
\hline GFRP 2-15-1 & 3000 & 0.342466 & $11.00 \%$ & $12.80 \%$ & $-0.40 \%$ & $10.60 \%$ & $12.40 \%$ & $96.4 \%$ & $96.9 \%$ \\
\hline GFRP $2-15-1$ & 10000 & 1.141553 & $11.00 \%$ & $12.80 \%$ & $-0.60 \%$ & $10.40 \%$ & $12.20 \%$ & $94.5 \%$ & $95.3 \%$ \\
\hline GFRP2-15-2 & 1 & 0.000114 & $10.50 \%$ & $12.20 \%$ & $0.00 \%$ & $10.50 \%$ & $12.20 \%$ & $100.0 \%$ & $100.0 \%$ \\
\hline GFRP $2-15-2$ & 1000 & 0.114155 & $10.50 \%$ & $12.20 \%$ & $0.60 \%$ & $11.10 \%$ & $12.80 \%$ & $105.7 \%$ & $104.9 \%$ \\
\hline GFRP $2-15-2$ & 3000 & 0.342466 & $10.50 \%$ & $12.20 \%$ & $1.50 \%$ & $12.00 \%$ & $13.70 \%$ & $114.3 \%$ & $112.3 \%$ \\
\hline GFRP $2-15-2$ & 10000 & 1.141553 & $10.50 \%$ & $12.20 \%$ & $1.70 \%$ & $12.20 \%$ & $13.90 \%$ & $116.2 \%$ & $113.9 \%$ \\
\hline GFRP $2-15-3$ & 1 & 0.000114 & $11.40 \%$ & $13.30 \%$ & $0.00 \%$ & $11.40 \%$ & $13.30 \%$ & $100.0 \%$ & $100.0 \%$ \\
\hline GFRP2-15-3 & 1000 & 0.114155 & $11.40 \%$ & $13.30 \%$ & $1.70 \%$ & $13.10 \%$ & $15.00 \%$ & $114.9 \%$ & $112.8 \%$ \\
\hline GFRP $2-15-3$ & 3000 & 0.342466 & $11.40 \%$ & $13.30 \%$ & $1.60 \%$ & $13.00 \%$ & $14.90 \%$ & $114.0 \%$ & $112.0 \%$ \\
\hline GFRP $2-15-3$ & 10000 & 1.141553 & $11.40 \%$ & $13.30 \%$ & $2.50 \%$ & $13.90 \%$ & $15.80 \%$ & $121.9 \%$ & $118.8 \%$ \\
\hline GFRP2-30-1 & 1 & 0.000114 & $37.60 \%$ & $43.70 \%$ & $0.00 \%$ & $37.60 \%$ & $43.70 \%$ & $100.0 \%$ & $100.0 \%$ \\
\hline GFRP2-30-1 & 1000 & 0.114155 & $37.60 \%$ & $43.70 \%$ & $10.10 \%$ & $47.70 \%$ & $53.80 \%$ & $126.9 \%$ & $123.1 \%$ \\
\hline GFRP $2-30-1$ & 3000 & 0.342466 & $37.60 \%$ & $43.70 \%$ & $10.90 \%$ & $48.50 \%$ & $54.60 \%$ & $129.0 \%$ & $124.9 \%$ \\
\hline GFRP2-30-1 & 10000 & 1.141553 & $37.60 \%$ & $43.70 \%$ & $11.80 \%$ & $49.40 \%$ & $55.50 \%$ & $131.4 \%$ & $127.0 \%$ \\
\hline
\end{tabular}




\begin{tabular}{|c|c|c|c|c|c|c|c|c|c|}
\hline & & & $\varepsilon_{0}^{\prime}$ & & $\varepsilon_{\mathrm{t}}^{\prime}$ & $\varepsilon_{\mathrm{tu}}^{\prime}$ & & $\varepsilon_{\text {to }}^{\prime}$ & \\
\hline & $(t)$ & $\left(t^{\prime}\right)$ & $\left(\varepsilon_{0} / \varepsilon_{f u}\right)$ & $\left(\varepsilon_{0} / \varepsilon^{*}{ }_{f u}\right)$ & $\left(\Delta \varepsilon_{t} / \varepsilon_{0}\right)$ & $\left(\varepsilon_{t} / \varepsilon_{f u}\right)$ & $\left(\varepsilon_{t} / \varepsilon^{*}{ }_{f u}\right)$ & $\left(\varepsilon_{t} / \varepsilon_{0}\right)$ & $\left(\varepsilon_{t} / \varepsilon^{*}{ }_{0}\right)$ \\
\hline Sample \# & t hrs & tyrs & iSSR & Not used & cSSR & fSSR & Not used & tSSR & Not used \\
\hline GFRP $2-30-2$ & 1 & 0.000114 & $29.10 \%$ & $33.90 \%$ & $0.00 \%$ & $29.10 \%$ & $33.90 \%$ & $100.0 \%$ & $100.0 \%$ \\
\hline GFRP $2-30-2$ & 1000 & 0.114155 & $29.10 \%$ & $33.90 \%$ & $1.50 \%$ & $30.60 \%$ & $35.40 \%$ & $105.2 \%$ & $104.4 \%$ \\
\hline GFRP $2-30-2$ & 3000 & 0.342466 & $29.10 \%$ & $33.90 \%$ & $1.20 \%$ & $30.30 \%$ & $35.10 \%$ & $104.1 \%$ & $103.5 \%$ \\
\hline GFRP $2-30-2$ & 10000 & 1.141553 & $29.10 \%$ & $33.90 \%$ & $2.00 \%$ & $31.10 \%$ & $35.90 \%$ & $106.9 \%$ & $105.9 \%$ \\
\hline GFRP $2-30-3$ & 1 & 0.000114 & $48.70 \%$ & $56.70 \%$ & $0.00 \%$ & $48.70 \%$ & $56.70 \%$ & $100.0 \%$ & $100.0 \%$ \\
\hline GFRP $2-30-3$ & 1000 & 0.114155 & $48.70 \%$ & $56.70 \%$ & $-0.20 \%$ & $48.50 \%$ & $56.50 \%$ & $99.6 \%$ & $99.6 \%$ \\
\hline GFRP $2-30-3$ & 3000 & 0.342466 & $48.70 \%$ & $56.70 \%$ & $0.40 \%$ & $49.10 \%$ & $57.10 \%$ & $100.8 \%$ & $100.7 \%$ \\
\hline GFRP $2-30-3$ & 10000 & 1.141553 & $48.70 \%$ & $56.70 \%$ & $2.40 \%$ & $51.10 \%$ & $59.10 \%$ & $104.9 \%$ & $104.2 \%$ \\
\hline GFRP $2-30-4$ & 1 & 0.000114 & $30.30 \%$ & $35.30 \%$ & $0.00 \%$ & $30.30 \%$ & $35.30 \%$ & $100.0 \%$ & $100.0 \%$ \\
\hline GFRP $2-30-4$ & 1000 & 0.114155 & $30.30 \%$ & $35.30 \%$ & $3.70 \%$ & $34.00 \%$ & $39.00 \%$ & $112.2 \%$ & $110.5 \%$ \\
\hline GFRP $2-30-4$ & 3000 & 0.342466 & $30.30 \%$ & $35.30 \%$ & $4.10 \%$ & $34.40 \%$ & $39.40 \%$ & $113.5 \%$ & $111.6 \%$ \\
\hline GFRP $2-30-4$ & 10000 & 1.141553 & $30.30 \%$ & $35.30 \%$ & $5.50 \%$ & $35.80 \%$ & $40.80 \%$ & $118.2 \%$ & $115.6 \%$ \\
\hline GFRP $2-45-1$ & 1 & 0.000114 & $44.30 \%$ & $51.60 \%$ & $0.00 \%$ & $44.30 \%$ & $51.60 \%$ & $100.0 \%$ & $100.0 \%$ \\
\hline GFRP $2-45-1$ & 1000 & 0.114155 & $44.30 \%$ & $51.60 \%$ & $3.00 \%$ & $47.30 \%$ & $54.60 \%$ & $106.8 \%$ & $105.8 \%$ \\
\hline GFRP $2-45-1$ & 3000 & 0.342466 & $44.30 \%$ & $51.60 \%$ & $3.80 \%$ & $48.10 \%$ & $55.40 \%$ & $108.6 \%$ & $107.4 \%$ \\
\hline GFRP $2-45-1$ & 10000 & 1.141553 & $44.30 \%$ & $51.60 \%$ & $4.70 \%$ & $49.00 \%$ & $56.30 \%$ & $110.6 \%$ & $109.1 \%$ \\
\hline GFRP $2-45-2$ & 1 & 0.000114 & $48.60 \%$ & $56.50 \%$ & $0.00 \%$ & $48.60 \%$ & $56.50 \%$ & $100.0 \%$ & $100.0 \%$ \\
\hline GFRP $2-45-2$ & 1000 & 0.114155 & $48.60 \%$ & $56.50 \%$ & $7.10 \%$ & $55.70 \%$ & $63.60 \%$ & $114.6 \%$ & $112.6 \%$ \\
\hline GFRP $2-45-2$ & 3000 & 0.342466 & $48.60 \%$ & $56.50 \%$ & $8.40 \%$ & $57.00 \%$ & $64.90 \%$ & $117.3 \%$ & $114.9 \%$ \\
\hline GFRP $2-45-2$ & 10000 & 1.141553 & $48.60 \%$ & $56.50 \%$ & $9.40 \%$ & $58.00 \%$ & $65.90 \%$ & $119.3 \%$ & $116.6 \%$ \\
\hline GFRP $2-45-3$ & 1 & 0.000114 & $51.90 \%$ & $60.40 \%$ & $0.00 \%$ & $51.90 \%$ & $60.40 \%$ & $100.0 \%$ & $100.0 \%$ \\
\hline GFRP $2-45-3$ & 1000 & 0.114155 & $51.90 \%$ & $60.40 \%$ & $6.40 \%$ & $58.30 \%$ & $66.80 \%$ & $112.3 \%$ & $110.6 \%$ \\
\hline GFRP $2-45-3$ & 3000 & 0.342466 & $51.90 \%$ & $60.40 \%$ & $7.20 \%$ & $59.10 \%$ & $67.60 \%$ & $113.9 \%$ & $111.9 \%$ \\
\hline GFRP $2-45-3$ & 10000 & 1.141553 & $51.90 \%$ & $60.40 \%$ & $7.50 \%$ & $59.40 \%$ & $67.90 \%$ & $114.5 \%$ & $112.4 \%$ \\
\hline GFRP $2-45-4$ & 1 & 0.000114 & $50.00 \%$ & $58.20 \%$ & $0.00 \%$ & $50.00 \%$ & $58.20 \%$ & $100.0 \%$ & $100.0 \%$ \\
\hline GFRP $2-45-4$ & 1000 & 0.114155 & $50.00 \%$ & $58.20 \%$ & $10.20 \%$ & $60.20 \%$ & $68.40 \%$ & $120.4 \%$ & $117.5 \%$ \\
\hline GFRP $2-45-4$ & 3000 & 0.342466 & $50.00 \%$ & $58.20 \%$ & $11.50 \%$ & $61.50 \%$ & $69.70 \%$ & $123.0 \%$ & $119.8 \%$ \\
\hline GFRP $2-45-4$ & 10000 & 1.141553 & $50.00 \%$ & $58.20 \%$ & $12.00 \%$ & $62.00 \%$ & $70.20 \%$ & $124.0 \%$ & $120.6 \%$ \\
\hline
\end{tabular}




\begin{tabular}{|c|c|c|c|c|c|c|c|c|c|}
\hline & & & $\varepsilon_{0}^{\prime}$ & & $\varepsilon_{\mathrm{t}}^{\prime}$ & $\varepsilon_{\mathrm{tu}}^{\prime}$ & & $\varepsilon_{\text {to }}^{\prime}$ & \\
\hline & $(t)$ & $\left(t^{\prime}\right)$ & $\left(\varepsilon_{0} / \varepsilon_{f u}\right)$ & $\left(\varepsilon_{0} / \varepsilon^{*}{ }_{f u}\right)$ & $\left(\Delta \varepsilon_{t} / \varepsilon_{0}\right)$ & $\left(\varepsilon_{t} / \varepsilon_{f u}\right)$ & $\left(\varepsilon_{t} / \varepsilon^{*}{ }_{f u}\right)$ & $\left(\varepsilon_{t} / \varepsilon_{0}\right)$ & $\left(\varepsilon_{t} / \varepsilon^{*}{ }_{0}\right)$ \\
\hline Sample \# & t hrs & tyrs & iSSR & Not used & cSSR & fSSR & Not used & tSSR & Not used \\
\hline GFRP $2-60-1$ & 1 & 0.000114 & $80.90 \%$ & $94.20 \%$ & $0.00 \%$ & $80.90 \%$ & $94.20 \%$ & $100.0 \%$ & $100.0 \%$ \\
\hline GFRP2-60-1 & 13.8 & 0.001575 & $80.90 \%$ & $94.20 \%$ & $2.20 \%$ & $83.10 \%$ & $96.40 \%$ & $102.7 \%$ & $102.3 \%$ \\
\hline GFRP $2-60-2$ & 1 & 0.000114 & $62.30 \%$ & $72.50 \%$ & $0.00 \%$ & $62.30 \%$ & $72.50 \%$ & $100.0 \%$ & $100.0 \%$ \\
\hline GFRP $2-60-2$ & 56.8 & 0.006484 & $62.30 \%$ & $72.50 \%$ & $1.30 \%$ & $63.60 \%$ & $73.80 \%$ & $102.1 \%$ & $101.8 \%$ \\
\hline GFRP $2-60-3$ & 1 & 0.000114 & $75.70 \%$ & $88.10 \%$ & $0.00 \%$ & $75.70 \%$ & $88.10 \%$ & $100.0 \%$ & $100.0 \%$ \\
\hline GFRP $2-60-3$ & 231 & 0.02637 & $75.70 \%$ & $88.10 \%$ & $10.60 \%$ & $86.30 \%$ & $98.70 \%$ & $114.0 \%$ & $112.0 \%$ \\
\hline GFRP 2-60-4 & 1 & 0.000114 & $58.60 \%$ & $68.30 \%$ & $0.00 \%$ & $58.60 \%$ & $68.30 \%$ & $100.0 \%$ & $100.0 \%$ \\
\hline GFRP2-60-4 & 54 & 0.006164 & $58.60 \%$ & $68.30 \%$ & $6.40 \%$ & $65.00 \%$ & $74.70 \%$ & $110.9 \%$ & $109.4 \%$ \\
\hline GFRP3-15-1 & 1 & 0.000114 & $16.20 \%$ & $19.90 \%$ & $0.00 \%$ & $16.20 \%$ & $19.90 \%$ & $100.0 \%$ & $100.0 \%$ \\
\hline GFRP3-15-1 & 1000 & 0.114155 & $16.20 \%$ & $19.90 \%$ & $0.30 \%$ & $16.50 \%$ & $20.20 \%$ & $101.9 \%$ & $101.5 \%$ \\
\hline GFRP3-15-1 & 3000 & 0.342466 & $16.20 \%$ & $19.90 \%$ & $0.90 \%$ & $17.10 \%$ & $20.80 \%$ & $105.6 \%$ & $104.5 \%$ \\
\hline GFRP3-15-1 & 10000 & 1.141553 & $16.20 \%$ & $19.90 \%$ & $2.00 \%$ & $18.20 \%$ & $21.90 \%$ & $112.3 \%$ & $110.1 \%$ \\
\hline GFRP3-15-2 & 1 & 0.000114 & $16.20 \%$ & $19.80 \%$ & $0.00 \%$ & $16.20 \%$ & $19.80 \%$ & $100.0 \%$ & $100.0 \%$ \\
\hline GFRP3-15-2 & 1000 & 0.114155 & $16.20 \%$ & $19.80 \%$ & $1.00 \%$ & $17.20 \%$ & $20.80 \%$ & $106.2 \%$ & $105.1 \%$ \\
\hline GFRP3-15-2 & 3000 & 0.342466 & $16.20 \%$ & $19.80 \%$ & $1.90 \%$ & $18.10 \%$ & $21.70 \%$ & $111.7 \%$ & $109.6 \%$ \\
\hline GFRP3-15-2 & 10000 & 1.141553 & $16.20 \%$ & $19.80 \%$ & $3.60 \%$ & $19.80 \%$ & $23.40 \%$ & $122.2 \%$ & $118.2 \%$ \\
\hline GFRP3-15-3 & 1 & 0.000114 & $14.80 \%$ & $18.10 \%$ & $0.00 \%$ & $14.80 \%$ & $18.10 \%$ & $100.0 \%$ & $100.0 \%$ \\
\hline GFRP3-15-3 & 1000 & 0.114155 & $14.80 \%$ & $18.10 \%$ & $-1.50 \%$ & $13.30 \%$ & $16.60 \%$ & $89.9 \%$ & $91.7 \%$ \\
\hline GFRP3-15-3 & 3000 & 0.342466 & $14.80 \%$ & $18.10 \%$ & $-0.30 \%$ & $14.50 \%$ & $17.80 \%$ & $98.0 \%$ & $98.3 \%$ \\
\hline GFRP3-15-3 & 10000 & 1.141553 & $14.80 \%$ & $18.10 \%$ & $0.70 \%$ & $15.50 \%$ & $18.80 \%$ & $104.7 \%$ & $103.9 \%$ \\
\hline GFRP3-15-4 & 1 & 0.000114 & $16.00 \%$ & $19.60 \%$ & $0.00 \%$ & $16.00 \%$ & $19.60 \%$ & $100.0 \%$ & $100.0 \%$ \\
\hline GFRP3-15-4 & 1000 & 0.114155 & $16.00 \%$ & $19.60 \%$ & $2.00 \%$ & $18.00 \%$ & $21.60 \%$ & $112.5 \%$ & $110.2 \%$ \\
\hline GFRP3-15-4 & 3000 & 0.342466 & $16.00 \%$ & $19.60 \%$ & $3.30 \%$ & $19.30 \%$ & $22.90 \%$ & $120.6 \%$ & $116.8 \%$ \\
\hline GFRP3-15-4 & 10000 & 1.141553 & $16.00 \%$ & $19.60 \%$ & $4.10 \%$ & $20.10 \%$ & $23.70 \%$ & $125.6 \%$ & $120.9 \%$ \\
\hline GFRP4-15-1 & 1 & 0.000114 & $13.40 \%$ & $17.30 \%$ & $0.00 \%$ & $13.40 \%$ & $17.30 \%$ & $100.0 \%$ & $100.0 \%$ \\
\hline GFRP4-15-1 & 1000 & 0.114155 & $13.40 \%$ & $17.30 \%$ & $0.20 \%$ & $13.60 \%$ & $17.50 \%$ & $101.5 \%$ & $101.2 \%$ \\
\hline GFRP4-15-1 & 3000 & 0.342466 & $13.40 \%$ & $17.30 \%$ & $-0.30 \%$ & $13.10 \%$ & $17.00 \%$ & $97.8 \%$ & $98.3 \%$ \\
\hline GFRP4-15-1 & 10000 & 1.141553 & $13.40 \%$ & $17.30 \%$ & $0.70 \%$ & $14.10 \%$ & $18.00 \%$ & $105.2 \%$ & $104.0 \%$ \\
\hline
\end{tabular}




\begin{tabular}{|c|c|c|c|c|c|c|c|c|c|}
\hline & & & $\varepsilon_{0}^{\prime}$ & & $\varepsilon_{\mathrm{t}}^{\prime}$ & $\varepsilon_{\mathrm{tu}}^{\prime}$ & & $\varepsilon_{\text {to }}^{\prime}$ & \\
\hline & $(t)$ & $\left(t^{\prime}\right)$ & $\left(\varepsilon_{0} / \varepsilon_{f u}\right)$ & $\left(\varepsilon_{0} / \varepsilon^{*}{ }_{f u}\right)$ & $\left(\Delta \varepsilon_{t} / \varepsilon_{0}\right)$ & $\left(\varepsilon_{t} / \varepsilon_{f u}\right)$ & $\left(\varepsilon_{t} / \varepsilon^{*}{ }_{f u}\right)$ & $\left(\varepsilon_{t} / \varepsilon_{0}\right)$ & $\left(\varepsilon_{t} / \varepsilon^{*}{ }_{0}\right)$ \\
\hline Sample \# & t hrs & tyrs & iSSR & Not used & cSSR & fSSR & Not used & tSSR & Not used \\
\hline GFRP4-15-2 & 1 & 0.000114 & $12.90 \%$ & $16.70 \%$ & $0.00 \%$ & $12.90 \%$ & $16.70 \%$ & $100.0 \%$ & $100.0 \%$ \\
\hline GFRP4-15-2 & 1000 & 0.114155 & $12.90 \%$ & $16.70 \%$ & $0.60 \%$ & $13.50 \%$ & $17.30 \%$ & $104.7 \%$ & $103.6 \%$ \\
\hline GFRP4-15-2 & 3000 & 0.342466 & $12.90 \%$ & $16.70 \%$ & $1.60 \%$ & $14.50 \%$ & $18.30 \%$ & $112.4 \%$ & $109.6 \%$ \\
\hline GFRP4-15-2 & 10000 & 1.141553 & $12.90 \%$ & $16.70 \%$ & $2.20 \%$ & $15.10 \%$ & $18.90 \%$ & $117.1 \%$ & $113.2 \%$ \\
\hline GFRP4-15-3 & 1 & 0.000114 & $16.00 \%$ & $20.60 \%$ & $0.00 \%$ & $16.00 \%$ & $20.60 \%$ & $100.0 \%$ & $100.0 \%$ \\
\hline GFRP4-15-3 & 1000 & 0.114155 & $16.00 \%$ & $20.60 \%$ & $2.20 \%$ & $18.20 \%$ & $22.80 \%$ & $113.8 \%$ & $110.7 \%$ \\
\hline GFRP4-15-3 & 3000 & 0.342466 & $16.00 \%$ & $20.60 \%$ & $2.50 \%$ & $18.50 \%$ & $23.10 \%$ & $115.6 \%$ & $112.1 \%$ \\
\hline GFRP4-15-3 & 10000 & 1.141553 & $16.00 \%$ & $20.60 \%$ & $3.20 \%$ & $19.20 \%$ & $23.80 \%$ & $120.0 \%$ & $115.5 \%$ \\
\hline GFRP4-15-4 & 1 & 0.000114 & $13.00 \%$ & $16.80 \%$ & $0.00 \%$ & $13.00 \%$ & $16.80 \%$ & $100.0 \%$ & $100.0 \%$ \\
\hline GFRP4-15-4 & 1000 & 0.114155 & $13.00 \%$ & $16.80 \%$ & $1.50 \%$ & $14.50 \%$ & $18.30 \%$ & $111.5 \%$ & $108.9 \%$ \\
\hline GFRP4-15-4 & 3000 & 0.342466 & $13.00 \%$ & $16.80 \%$ & $2.40 \%$ & $15.40 \%$ & $19.20 \%$ & $118.5 \%$ & $114.3 \%$ \\
\hline GFRP4-15-4 & 10000 & 1.141553 & $13.00 \%$ & $16.80 \%$ & $2.60 \%$ & $15.60 \%$ & $19.40 \%$ & $120.0 \%$ & $115.5 \%$ \\
\hline GFRP5-15-1 & 1 & 0.000114 & $14.10 \%$ & $17.20 \%$ & $0.00 \%$ & $14.10 \%$ & $17.20 \%$ & $100.0 \%$ & $100.0 \%$ \\
\hline GFRP5-15-1 & 1000 & 0.114155 & $14.10 \%$ & $17.20 \%$ & $3.40 \%$ & $17.50 \%$ & $20.60 \%$ & $124.1 \%$ & $119.8 \%$ \\
\hline GFRP5-15-1 & 3000 & 0.342466 & $14.10 \%$ & $17.20 \%$ & $4.60 \%$ & $18.70 \%$ & $21.80 \%$ & $132.6 \%$ & $126.7 \%$ \\
\hline GFRP5-15-1 & 10000 & 1.141553 & $14.10 \%$ & $17.20 \%$ & $5.80 \%$ & $19.90 \%$ & $23.00 \%$ & $141.1 \%$ & $133.7 \%$ \\
\hline GFRP5-15-2 & 1 & 0.000114 & $15.70 \%$ & $19.30 \%$ & $0.00 \%$ & $15.70 \%$ & $19.30 \%$ & $100.0 \%$ & $100.0 \%$ \\
\hline GFRP5-15-2 & 1000 & 0.114155 & $15.70 \%$ & $19.30 \%$ & $1.70 \%$ & $17.40 \%$ & $21.00 \%$ & $110.8 \%$ & $108.8 \%$ \\
\hline GFRP5-15-2 & 3000 & 0.342466 & $15.70 \%$ & $19.30 \%$ & $2.80 \%$ & $18.50 \%$ & $22.10 \%$ & $117.8 \%$ & $114.5 \%$ \\
\hline GFRP5-15-2 & 10000 & 1.141553 & $15.70 \%$ & $19.30 \%$ & $4.20 \%$ & $19.90 \%$ & $23.50 \%$ & $126.8 \%$ & $121.8 \%$ \\
\hline GFRP5-15-3 & 1 & 0.000114 & $15.40 \%$ & $18.80 \%$ & $0.00 \%$ & $15.40 \%$ & $18.80 \%$ & $100.0 \%$ & $100.0 \%$ \\
\hline GFRP5-15-3 & 1000 & 0.114155 & $15.40 \%$ & $18.80 \%$ & $3.20 \%$ & $18.60 \%$ & $22.00 \%$ & $120.8 \%$ & $117.0 \%$ \\
\hline GFRP5-15-3 & 3000 & 0.342466 & $15.40 \%$ & $18.80 \%$ & $4.40 \%$ & $19.80 \%$ & $23.20 \%$ & $128.6 \%$ & $123.4 \%$ \\
\hline GFRP5-15-3 & 10000 & 1.141553 & $15.40 \%$ & $18.80 \%$ & $4.90 \%$ & $20.30 \%$ & $23.70 \%$ & $131.8 \%$ & $126.1 \%$ \\
\hline GFRP5-15-4 & 1 & 0.000114 & $14.10 \%$ & $17.30 \%$ & $0.00 \%$ & $14.10 \%$ & $17.30 \%$ & $100.0 \%$ & $100.0 \%$ \\
\hline GFRP5-15-4 & 1000 & 0.114155 & $14.10 \%$ & $17.30 \%$ & $5.30 \%$ & $19.40 \%$ & $22.60 \%$ & $137.6 \%$ & $130.6 \%$ \\
\hline GFRP5-15-4 & 3000 & 0.342466 & $14.10 \%$ & $17.30 \%$ & $6.60 \%$ & $20.70 \%$ & $23.90 \%$ & $146.8 \%$ & $138.2 \%$ \\
\hline GFRP5-15-4 & 10000 & 1.141553 & $14.10 \%$ & $17.30 \%$ & $8.30 \%$ & $22.40 \%$ & $25.60 \%$ & $158.9 \%$ & $148.0 \%$ \\
\hline
\end{tabular}




\begin{tabular}{|c|c|c|c|c|c|c|c|c|c|}
\hline & & & $\varepsilon_{0}^{\prime}$ & & $\varepsilon_{\mathrm{t}}^{\prime}$ & $\varepsilon_{\mathrm{tu}}^{\prime}$ & & $\varepsilon_{\text {to }}^{\prime}$ & \\
\hline & $(t)$ & $\left(t^{\prime}\right)$ & $\left(\varepsilon_{0} / \varepsilon_{f u}\right)$ & $\left(\varepsilon_{0} / \varepsilon^{*}{ }_{f u}\right)$ & $\left(\Delta \varepsilon_{t} / \varepsilon_{0}\right)$ & $\left(\varepsilon_{t} / \varepsilon_{f u}\right)$ & $\left(\varepsilon_{t} / \varepsilon^{*}{ }_{f u}\right)$ & $\left(\varepsilon_{t} / \varepsilon_{0}\right)$ & $\left(\varepsilon_{t} / \varepsilon^{*}{ }_{0}\right)$ \\
\hline Sample \# & t hrs & tyrs & iSSR & Not used & cSSR & fSSR & Not used & tSSR & Not used \\
\hline GFRP6-15-1 & 1 & 0.000114 & $15.40 \%$ & $16.80 \%$ & $0.00 \%$ & $15.40 \%$ & $16.80 \%$ & $100.0 \%$ & $100.0 \%$ \\
\hline GFRP6-15-1 & 1000 & 0.114155 & $15.40 \%$ & $16.80 \%$ & $11.80 \%$ & $27.20 \%$ & $28.60 \%$ & $176.6 \%$ & $170.2 \%$ \\
\hline GFRP6-15-1 & 3000 & 0.342466 & $15.40 \%$ & $16.80 \%$ & $13.30 \%$ & $28.70 \%$ & $30.10 \%$ & $186.4 \%$ & $179.2 \%$ \\
\hline GFRP6-15-1 & 10000 & 1.141553 & $15.40 \%$ & $16.80 \%$ & $15.70 \%$ & $31.10 \%$ & $32.50 \%$ & $201.9 \%$ & $193.5 \%$ \\
\hline GFRP6-15-2 & 1 & 0.000114 & $14.80 \%$ & $16.10 \%$ & $0.00 \%$ & $14.80 \%$ & $16.10 \%$ & $100.0 \%$ & $100.0 \%$ \\
\hline GFRP6-15-2 & 1000 & 0.114155 & $14.80 \%$ & $16.10 \%$ & $6.80 \%$ & $21.60 \%$ & $22.90 \%$ & $145.9 \%$ & $142.2 \%$ \\
\hline GFRP6-15-2 & 3000 & 0.342466 & $14.80 \%$ & $16.10 \%$ & $8.30 \%$ & $23.10 \%$ & $24.40 \%$ & $156.1 \%$ & $151.6 \%$ \\
\hline GFRP6-15-2 & 10000 & 1.141553 & $14.80 \%$ & $16.10 \%$ & $9.70 \%$ & $24.50 \%$ & $25.80 \%$ & $165.5 \%$ & $160.2 \%$ \\
\hline GFRP6-15-3 & 1 & 0.000114 & $19.00 \%$ & $20.70 \%$ & $0.00 \%$ & $19.00 \%$ & $20.70 \%$ & $100.0 \%$ & $100.0 \%$ \\
\hline GFRP6-15-3 & 1000 & 0.114155 & $19.00 \%$ & $20.70 \%$ & $11.60 \%$ & $30.60 \%$ & $32.30 \%$ & $161.1 \%$ & $156.0 \%$ \\
\hline GFRP6-15-3 & 3000 & 0.342466 & $19.00 \%$ & $20.70 \%$ & $13.10 \%$ & $32.10 \%$ & $33.80 \%$ & $168.9 \%$ & $163.3 \%$ \\
\hline GFRP6-15-3 & 10000 & 1.141553 & $19.00 \%$ & $20.70 \%$ & $15.70 \%$ & $34.70 \%$ & $36.40 \%$ & $182.6 \%$ & $175.8 \%$ \\
\hline GFRP6-15-4 & 1 & 0.000114 & $17.10 \%$ & $18.70 \%$ & $0.00 \%$ & $17.10 \%$ & $18.70 \%$ & $100.0 \%$ & $100.0 \%$ \\
\hline GFRP6-15-4 & 1000 & 0.114155 & $17.10 \%$ & $18.70 \%$ & $8.60 \%$ & $25.70 \%$ & $27.30 \%$ & $150.3 \%$ & $146.0 \%$ \\
\hline GFRP6-15-4 & 3000 & 0.342466 & $17.10 \%$ & $18.70 \%$ & $9.50 \%$ & $26.60 \%$ & $28.20 \%$ & $155.6 \%$ & $150.8 \%$ \\
\hline GFRP6-15-4 & 10000 & 1.141553 & $17.10 \%$ & $18.70 \%$ & $11.40 \%$ & $28.50 \%$ & $30.10 \%$ & $166.7 \%$ & $161.0 \%$ \\
\hline GFRP3a-25-1 & 1 & 0.000114 & $24.30 \%$ & $29.70 \%$ & $0.00 \%$ & $24.30 \%$ & $29.70 \%$ & $100.0 \%$ & $100.0 \%$ \\
\hline GFRP3a-25-1 & 1000 & 0.114155 & $24.30 \%$ & $29.70 \%$ & $1.50 \%$ & $25.80 \%$ & $31.20 \%$ & $106.2 \%$ & $105.1 \%$ \\
\hline GFRP3a-25-1 & 3000 & 0.342466 & $24.30 \%$ & $29.70 \%$ & $1.20 \%$ & $25.50 \%$ & $30.90 \%$ & $104.9 \%$ & $104.0 \%$ \\
\hline GFRP3a-25-1 & 10000 & 1.141553 & $24.30 \%$ & $29.70 \%$ & $5.30 \%$ & $29.60 \%$ & $35.00 \%$ & $121.8 \%$ & $117.8 \%$ \\
\hline GFRP3a-25-2 & 1 & 0.000114 & $25.10 \%$ & $30.80 \%$ & $0.00 \%$ & $25.10 \%$ & $30.80 \%$ & $100.0 \%$ & $100.0 \%$ \\
\hline GFRP3a-25-2 & 1000 & 0.114155 & $25.10 \%$ & $30.80 \%$ & $1.50 \%$ & $26.60 \%$ & $32.30 \%$ & $106.0 \%$ & $104.9 \%$ \\
\hline GFRP3a-25-2 & 3000 & 0.342466 & $25.10 \%$ & $30.80 \%$ & $8.70 \%$ & $33.80 \%$ & $39.50 \%$ & $134.7 \%$ & $128.2 \%$ \\
\hline GFRP3a-25-2 & 10000 & 1.141553 & $25.10 \%$ & $30.80 \%$ & $12.00 \%$ & $37.10 \%$ & $42.80 \%$ & $147.8 \%$ & $139.0 \%$ \\
\hline GFRP3a-25-3 & 1 & 0.000114 & $25.90 \%$ & $31.70 \%$ & $0.00 \%$ & $25.90 \%$ & $31.70 \%$ & $100.0 \%$ & $100.0 \%$ \\
\hline GFRP3a-25-3 & 1000 & 0.114155 & $25.90 \%$ & $31.70 \%$ & $2.30 \%$ & $28.20 \%$ & $34.00 \%$ & $108.9 \%$ & $107.3 \%$ \\
\hline GFRP3a-25-3 & 3000 & 0.342466 & $25.90 \%$ & $31.70 \%$ & $3.10 \%$ & $29.00 \%$ & $34.80 \%$ & $112.0 \%$ & $109.8 \%$ \\
\hline GFRP3a-25-3 & 10000 & 1.141553 & $25.90 \%$ & $31.70 \%$ & $6.30 \%$ & $32.20 \%$ & $38.00 \%$ & $124.3 \%$ & $119.9 \%$ \\
\hline
\end{tabular}




\begin{tabular}{|c|c|c|c|c|c|c|c|c|c|}
\hline & & & $\varepsilon_{0}^{\prime}$ & & $\varepsilon_{\mathrm{t}}^{\prime}$ & $\varepsilon_{\text {tu }}^{\prime}$ & & $\varepsilon_{\text {to }}^{\prime}$ & \\
\hline & $(t)$ & $\left(t^{\prime}\right)$ & $\left(\varepsilon_{0} / \varepsilon_{f u}\right)$ & $\left(\varepsilon_{0} / \varepsilon^{*}{ }_{f u}\right)$ & $\left(\Delta \varepsilon_{t} / \varepsilon_{0}\right)$ & $\left(\varepsilon_{\mathrm{t}} / \varepsilon_{\mathrm{fu}}\right)$ & $\left(\varepsilon_{t} / \varepsilon^{*}{ }_{f u}\right)$ & $\left(\varepsilon_{t} / \varepsilon_{0}\right)$ & $\left(\varepsilon_{\mathrm{t}} / \varepsilon^{*}{ }_{0}\right)$ \\
\hline Sample \# & t hrs & tyrs & iSSR & Not used & cSSR & fSSR & Not used & tSSR & Not used \\
\hline GFRP3a-30-4 & 1 & 0.000114 & $29.10 \%$ & $35.60 \%$ & $0.00 \%$ & $29.10 \%$ & $35.60 \%$ & $100.0 \%$ & $100.0 \%$ \\
\hline GFRP3a-30-4 & 1000 & 0.114155 & $29.10 \%$ & $35.60 \%$ & $6.00 \%$ & $35.10 \%$ & $41.60 \%$ & $120.6 \%$ & $116.9 \%$ \\
\hline GFRP3a-30-4 & 3000 & 0.342466 & $29.10 \%$ & $35.60 \%$ & $5.80 \%$ & $34.90 \%$ & $41.40 \%$ & $119.9 \%$ & $116.3 \%$ \\
\hline GFRP3a-30-4 & 10000 & 1.141553 & $29.10 \%$ & $35.60 \%$ & $5.00 \%$ & $34.10 \%$ & $40.60 \%$ & $117.2 \%$ & $114.0 \%$ \\
\hline GFRP3b-25-1 & 1 & 0.000114 & $26.50 \%$ & $32.50 \%$ & $0.00 \%$ & $26.50 \%$ & $32.50 \%$ & $100.0 \%$ & $100.0 \%$ \\
\hline GFRP3b-25-1 & 1000 & 0.114155 & $26.50 \%$ & $32.50 \%$ & $0.10 \%$ & $26.60 \%$ & $32.60 \%$ & $100.4 \%$ & $100.3 \%$ \\
\hline GFRP3b-25-1 & 3000 & 0.342466 & $26.50 \%$ & $32.50 \%$ & $-2.90 \%$ & $23.60 \%$ & $29.60 \%$ & $89.1 \%$ & $91.1 \%$ \\
\hline GFRP3b-25-1 & 10000 & 1.141553 & $26.50 \%$ & $32.50 \%$ & $-2.00 \%$ & $24.50 \%$ & $30.50 \%$ & $92.5 \%$ & $93.8 \%$ \\
\hline GFRP3b-25-2 & 1 & 0.000114 & $24.60 \%$ & $30.10 \%$ & $0.00 \%$ & $24.60 \%$ & $30.10 \%$ & $100.0 \%$ & $100.0 \%$ \\
\hline GFRP3b-25-2 & 1000 & 0.114155 & $24.60 \%$ & $30.10 \%$ & $2.70 \%$ & $27.30 \%$ & $32.80 \%$ & $111.0 \%$ & $109.0 \%$ \\
\hline GFRP3b-25-2 & 3000 & 0.342466 & $24.60 \%$ & $30.10 \%$ & $1.50 \%$ & $26.10 \%$ & $31.60 \%$ & $106.1 \%$ & $105.0 \%$ \\
\hline GFRP3b-25-2 & 10000 & 1.141553 & $24.60 \%$ & $30.10 \%$ & $1.60 \%$ & $26.20 \%$ & $31.70 \%$ & $106.5 \%$ & $105.3 \%$ \\
\hline GFRP3b-25-3 & 1 & 0.000114 & $25.60 \%$ & $31.40 \%$ & $0.00 \%$ & $25.60 \%$ & $31.40 \%$ & $100.0 \%$ & $100.0 \%$ \\
\hline GFRP3b-25-3 & 1000 & 0.114155 & $25.60 \%$ & $31.40 \%$ & $1.10 \%$ & $26.70 \%$ & $32.50 \%$ & $104.3 \%$ & $103.5 \%$ \\
\hline GFRP3b-25-3 & 3000 & 0.342466 & $25.60 \%$ & $31.40 \%$ & $1.50 \%$ & $27.10 \%$ & $32.90 \%$ & $105.9 \%$ & $104.8 \%$ \\
\hline GFRP4-20-1 & 1 & 0.000114 & $20.30 \%$ & $26.10 \%$ & $0.00 \%$ & $20.30 \%$ & $26.10 \%$ & $100.0 \%$ & $100.0 \%$ \\
\hline GFRP4-20-1 & 1000 & 0.114155 & $20.30 \%$ & $26.10 \%$ & $-0.20 \%$ & $20.10 \%$ & $25.90 \%$ & $99.0 \%$ & $99.2 \%$ \\
\hline GFRP4-20-1 & 3000 & 0.342466 & $20.30 \%$ & $26.10 \%$ & $4.20 \%$ & $24.50 \%$ & $30.30 \%$ & $120.7 \%$ & $116.1 \%$ \\
\hline GFRP4-20-1 & 10000 & 1.141553 & $20.30 \%$ & $26.10 \%$ & $3.80 \%$ & $24.10 \%$ & $29.90 \%$ & $118.7 \%$ & $114.6 \%$ \\
\hline GFRP4-20-2 & 1 & 0.000114 & $20.00 \%$ & $25.80 \%$ & $0.00 \%$ & $20.00 \%$ & $25.80 \%$ & $100.0 \%$ & $100.0 \%$ \\
\hline GFRP4-20-2 & 1000 & 0.114155 & $20.00 \%$ & $25.80 \%$ & $2.60 \%$ & $22.60 \%$ & $28.40 \%$ & $113.0 \%$ & $110.1 \%$ \\
\hline GFRP4-20-2 & 3000 & 0.342466 & $20.00 \%$ & $25.80 \%$ & $3.70 \%$ & $23.70 \%$ & $29.50 \%$ & $118.5 \%$ & $114.3 \%$ \\
\hline GFRP4-20-2 & 10000 & 1.141553 & $20.00 \%$ & $25.80 \%$ & $4.70 \%$ & $24.70 \%$ & $30.50 \%$ & $123.5 \%$ & $118.2 \%$ \\
\hline GFRP4-20-3 & 1 & 0.000114 & $17.20 \%$ & $22.20 \%$ & $0.00 \%$ & $17.20 \%$ & $22.20 \%$ & $100.0 \%$ & $100.0 \%$ \\
\hline GFRP4-20-3 & 1000 & 0.114155 & $17.20 \%$ & $22.20 \%$ & $-0.50 \%$ & $16.70 \%$ & $21.70 \%$ & $97.1 \%$ & $97.7 \%$ \\
\hline GFRP4-20-3 & 3000 & 0.342466 & $17.20 \%$ & $22.20 \%$ & $1.50 \%$ & $18.70 \%$ & $23.70 \%$ & $108.7 \%$ & $106.8 \%$ \\
\hline GFRP4-20-3 & 10000 & 1.141553 & $17.20 \%$ & $22.20 \%$ & $1.70 \%$ & $18.90 \%$ & $23.90 \%$ & $109.9 \%$ & $107.7 \%$ \\
\hline GFRP4-20-4 & 1 & 0.000114 & $18.80 \%$ & $24.30 \%$ & $0.00 \%$ & $18.80 \%$ & $24.30 \%$ & $100.0 \%$ & $100.0 \%$ \\
\hline
\end{tabular}




\begin{tabular}{|c|c|c|c|c|c|c|c|c|c|}
\hline & & & $\varepsilon_{0}^{\prime}$ & & $\varepsilon_{t}^{\prime}$ & $\varepsilon_{\text {tu }}^{\prime}$ & & $\varepsilon_{\text {to }}^{\prime}$ & \\
\hline & (t) & $\left(t^{\prime}\right)$ & $\left(\varepsilon_{0} / \varepsilon_{f u}\right)$ & $\left(\varepsilon_{0} / \varepsilon^{*}{ }_{f u}\right)$ & $\left(\Delta \varepsilon_{t} / \varepsilon_{0}\right)$ & $\left(\varepsilon_{t} / \varepsilon_{f u}\right)$ & $\left(\varepsilon_{t} / \varepsilon^{*}{ }_{f u}\right)$ & $\left(\varepsilon_{t} / \varepsilon_{0}\right)$ & $\left(\varepsilon_{t} / \varepsilon^{*}{ }_{0}\right)$ \\
\hline Sample \# & thrs & t yrs & iSSR & Not used & cSSR & fSSR & Not used & tSSR & Not used \\
\hline GFRP4-20-4 & 1000 & 0.114155 & $18.80 \%$ & $24.30 \%$ & $1.50 \%$ & $20.30 \%$ & $25.80 \%$ & $108.0 \%$ & $106.2 \%$ \\
\hline GFRP4-20-4 & 3000 & 0.342466 & $18.80 \%$ & $24.30 \%$ & $2.80 \%$ & $21.60 \%$ & $27.10 \%$ & $114.9 \%$ & $111.5 \%$ \\
\hline GFRP4-20-4 & 10000 & 1.141553 & $18.80 \%$ & $24.30 \%$ & $1.90 \%$ & $20.70 \%$ & $26.20 \%$ & $110.1 \%$ & $107.8 \%$ \\
\hline GFRP4-20-5 & 1 & 0.000114 & $19.10 \%$ & $24.70 \%$ & $0.00 \%$ & $19.10 \%$ & $24.70 \%$ & $100.0 \%$ & $100.0 \%$ \\
\hline GFRP4-20-5 & 1000 & 0.114155 & $19.10 \%$ & $24.70 \%$ & $4.60 \%$ & $23.70 \%$ & $29.30 \%$ & $124.1 \%$ & $118.6 \%$ \\
\hline GFRP4-20-5 & 3000 & 0.342466 & $19.10 \%$ & $24.70 \%$ & $4.90 \%$ & $24.00 \%$ & $29.60 \%$ & $125.7 \%$ & $119.8 \%$ \\
\hline GFRP4-20-5 & 10000 & 1.141553 & $19.10 \%$ & $24.70 \%$ & $5.60 \%$ & $24.70 \%$ & $30.30 \%$ & $129.3 \%$ & $122.7 \%$ \\
\hline GFRP5-30-1 & 1 & 0.000114 & $37.30 \%$ & $45.70 \%$ & $0.00 \%$ & $37.30 \%$ & $45.70 \%$ & $100.0 \%$ & $100.0 \%$ \\
\hline GFRP5-30-1 & 1000 & 0.114155 & $37.30 \%$ & $45.70 \%$ & $0.70 \%$ & $38.00 \%$ & $46.40 \%$ & $101.9 \%$ & $101.5 \%$ \\
\hline GFRP5-30-1 & 3000 & 0.342466 & $37.30 \%$ & $45.70 \%$ & $1.50 \%$ & $38.80 \%$ & $47.20 \%$ & $104.0 \%$ & $103.3 \%$ \\
\hline GFRP5-30-1 & 10000 & 1.141553 & $37.30 \%$ & $45.70 \%$ & $3.20 \%$ & $40.50 \%$ & $48.90 \%$ & $108.6 \%$ & $107.0 \%$ \\
\hline GFRP5-30-2 & 1 & 0.000114 & $30.90 \%$ & $37.80 \%$ & $0.00 \%$ & $30.90 \%$ & $37.80 \%$ & $100.0 \%$ & $100.0 \%$ \\
\hline GFRP5-30-2 & 1000 & 0.114155 & $30.90 \%$ & $37.80 \%$ & $4.70 \%$ & $35.60 \%$ & $42.50 \%$ & $115.2 \%$ & $112.4 \%$ \\
\hline GFRP5-30-2 & 3000 & 0.342466 & $30.90 \%$ & $37.80 \%$ & $4.50 \%$ & $35.40 \%$ & $42.30 \%$ & $114.6 \%$ & $111.9 \%$ \\
\hline GFRP5-30-2 & 10000 & 1.141553 & $30.90 \%$ & $37.80 \%$ & $8.60 \%$ & $39.50 \%$ & $46.40 \%$ & $127.8 \%$ & $122.8 \%$ \\
\hline GFRP5-30-3 & 1 & 0.000114 & $29.10 \%$ & $35.70 \%$ & $0.00 \%$ & $29.10 \%$ & $35.70 \%$ & $100.0 \%$ & $100.0 \%$ \\
\hline GFRP5-30-3 & 1000 & 0.114155 & $29.10 \%$ & $35.70 \%$ & $5.70 \%$ & $34.80 \%$ & $41.40 \%$ & $119.6 \%$ & $116.0 \%$ \\
\hline GFRP5-30-3 & 3000 & 0.342466 & $29.10 \%$ & $35.70 \%$ & $6.20 \%$ & $35.30 \%$ & $41.90 \%$ & $121.3 \%$ & $117.4 \%$ \\
\hline GFRP5-30-3 & 10000 & 1.141553 & $29.10 \%$ & $35.70 \%$ & $6.60 \%$ & $35.70 \%$ & $42.30 \%$ & $122.7 \%$ & $118.5 \%$ \\
\hline GFRP5-30-4 & 1 & 0.000114 & $29.40 \%$ & $36.00 \%$ & $0.00 \%$ & $29.40 \%$ & $36.00 \%$ & $100.0 \%$ & $100.0 \%$ \\
\hline GFRP5-30-4 & 1000 & 0.114155 & $29.40 \%$ & $36.00 \%$ & $3.00 \%$ & $32.40 \%$ & $39.00 \%$ & $110.2 \%$ & $108.3 \%$ \\
\hline GFRP5-30-4 & 3000 & 0.342466 & $29.40 \%$ & $36.00 \%$ & $4.90 \%$ & $34.30 \%$ & $40.90 \%$ & $116.7 \%$ & $113.6 \%$ \\
\hline GFRP5-30-4 & 10000 & 1.141553 & $29.40 \%$ & $36.00 \%$ & $6.20 \%$ & $35.60 \%$ & $42.20 \%$ & $121.1 \%$ & $117.2 \%$ \\
\hline GFRP6-30-1 & 1 & 0.000114 & $37.60 \%$ & $43.70 \%$ & $0.00 \%$ & $37.60 \%$ & $43.70 \%$ & $100.0 \%$ & $100.0 \%$ \\
\hline GFRP6-30-1 & 1000 & 0.114155 & $37.60 \%$ & $43.70 \%$ & $10.10 \%$ & $47.70 \%$ & $53.80 \%$ & $126.9 \%$ & $123.1 \%$ \\
\hline GFRP6-30-1 & 3000 & 0.342466 & $37.60 \%$ & $43.70 \%$ & $10.90 \%$ & $48.50 \%$ & $54.60 \%$ & $129.0 \%$ & $124.9 \%$ \\
\hline GFRP6-30-1 & 10000 & 1.141553 & $37.60 \%$ & $43.70 \%$ & $11.80 \%$ & $49.40 \%$ & $55.50 \%$ & $131.4 \%$ & $127.0 \%$ \\
\hline GFRP $6-30-2$ & 1 & 0.000114 & $29.10 \%$ & $33.90 \%$ & $0.00 \%$ & $29.10 \%$ & $33.90 \%$ & $100.0 \%$ & $100.0 \%$ \\
\hline
\end{tabular}




\begin{tabular}{|c|c|c|c|c|c|c|c|c|c|}
\hline & & & $\varepsilon_{0}^{\prime}$ & & $\varepsilon_{\mathrm{t}}^{\prime}$ & $\varepsilon_{\text {tu }}^{\prime}$ & & $\varepsilon_{\text {to }}^{\prime}$ & \\
\hline & $(t)$ & $\left(t^{\prime}\right)$ & $\left(\varepsilon_{0} / \varepsilon_{\mathrm{fu}}\right)$ & $\left(\varepsilon_{0} / \varepsilon^{*}{ }_{f u}\right)$ & $\left(\Delta \varepsilon_{t} / \varepsilon_{0}\right)$ & $\left(\varepsilon_{t} / \varepsilon_{f u}\right)$ & $\left(\varepsilon_{\mathrm{t}} / \varepsilon_{\mathrm{fu}}^{*}\right)$ & $\left(\varepsilon_{t} / \varepsilon_{0}\right)$ & $\left(\varepsilon_{t} / \varepsilon^{*}{ }_{0}\right)$ \\
\hline Sample \# & thrs & tyrs & iSSR & Not used & cSSR & fSSR & Not used & tSSR & Not used \\
\hline GFRP6-30-2 & 1000 & 0.114155 & $29.10 \%$ & $33.90 \%$ & $1.50 \%$ & $30.60 \%$ & $35.40 \%$ & $105.2 \%$ & $104.4 \%$ \\
\hline GFRP6-30-2 & 3000 & 0.342466 & $29.10 \%$ & $33.90 \%$ & $1.20 \%$ & $30.30 \%$ & $35.10 \%$ & $104.1 \%$ & $103.5 \%$ \\
\hline GFRP6-30-2 & 10000 & 1.141553 & $29.10 \%$ & $33.90 \%$ & $2.00 \%$ & $31.10 \%$ & $35.90 \%$ & $106.9 \%$ & $105.9 \%$ \\
\hline GFRP6-30-3 & 1 & 0.000114 & $48.70 \%$ & $56.70 \%$ & $0.00 \%$ & $48.70 \%$ & $56.70 \%$ & $100.0 \%$ & $100.0 \%$ \\
\hline GFRP6-30-3 & 1000 & 0.114155 & $48.70 \%$ & $56.70 \%$ & $-0.20 \%$ & $48.50 \%$ & $56.50 \%$ & $99.6 \%$ & $99.6 \%$ \\
\hline GFRP6-30-3 & 3000 & 0.342466 & $48.70 \%$ & $56.70 \%$ & $0.40 \%$ & $49.10 \%$ & $57.10 \%$ & $100.8 \%$ & $100.7 \%$ \\
\hline GFRP6-30-3 & 10000 & 1.141553 & $48.70 \%$ & $56.70 \%$ & $2.40 \%$ & $51.10 \%$ & $59.10 \%$ & $104.9 \%$ & $104.2 \%$ \\
\hline GFRP6-30-4 & 1 & 0.000114 & $30.30 \%$ & $35.30 \%$ & $0.00 \%$ & $30.30 \%$ & $35.30 \%$ & $100.0 \%$ & $100.0 \%$ \\
\hline GFRP6-30-4 & 1000 & 0.114155 & $30.30 \%$ & $35.30 \%$ & $3.70 \%$ & $34.00 \%$ & $39.00 \%$ & $112.2 \%$ & $110.5 \%$ \\
\hline GFRP6-30-4 & 3000 & 0.342466 & $30.30 \%$ & $35.30 \%$ & $4.10 \%$ & $34.40 \%$ & $39.40 \%$ & $113.5 \%$ & $111.6 \%$ \\
\hline GFRP6-30-4 & 10000 & 1.141553 & $30.30 \%$ & $35.30 \%$ & $5.50 \%$ & $35.80 \%$ & $40.80 \%$ & $118.2 \%$ & $115.6 \%$ \\
\hline
\end{tabular}




\section{APPENDIX-E DURABILITY TEST DATABASE}

This Appendix supplement the information presented in Chapter 4.

Data Source: Various (listed with each test data)

Acknowledgement: The original database assembled by Lorenzo (2018)

Acknowledgment: The original database assembled by Lorenzo (2018) 
Table E.1: Listing of Test Database Used in Durability Study

Listing of Accelerated and Natural Aging Test Data Used in the Study (Chapter 4)

\section{LEGEND:}

$\mathrm{VE}=$ Vinylester; $\mathrm{PE}=$ Polyester; $\mathrm{EN}=$ Epoxy;

$\mathrm{GL}=$ Glass FRP; $\mathrm{CR}=$ Carbon FRP

TS = Tensile Strength; ILSS = Interlaminar Shear Strength; FS = Flexural Strength

T0, T2, T2, T3, T4, and T5 = Temperature Groups: Natural, 1, 2, 3, 4, 5

P0, P1, P2, P3, P4, and P5 = pH Groups: Neutral, 1, 2, 3, 4, and 5

\begin{tabular}{|c|c|c|c|c|c|c|c|c|}
\hline Sample Designation & Matrix & $\begin{array}{l}\text { Reinforce } \\
\text { ment }\end{array}$ & $\begin{array}{l}\text { Property } \\
\text { Tested }\end{array}$ & $\begin{array}{l}\text { Temp. } \\
\text { Group }\end{array}$ & $\begin{array}{l}\text { pH } \\
\text { Groups }\end{array}$ & $\begin{array}{l}\text { Time in } \\
\text { Aging } \\
\text { Condition } \\
\text { (months) }\end{array}$ & $\begin{array}{l}\text { SR - \% } \\
\text { Strength } \\
\text { Reduction }\end{array}$ & Data Source Ref. \\
\hline VE-TS-T2-P2 & VE & $\mathrm{GL}$ & TS & $\mathrm{T} 2$ & P2 & 0 & $100 \%$ & (Vijay, 1999) \\
\hline VE-TS-T2-P2 & VE & GL & TS & $\mathrm{T} 2$ & P2 & 3 & $98 \%$ & (Vijay, 1999) \\
\hline VE-TS-T2-P2 & VE & GL & TS & $\mathrm{T} 2$ & P2 & 8 & $94 \%$ & (Vijay, 1999) \\
\hline VE-TS-T2-P2 & VE & $\mathrm{GL}$ & TS & $\mathrm{T} 2$ & P2 & 15 & $92 \%$ & (Vijay, 1999) \\
\hline VE-TS-T2-P5 & VE & GL & TS & $\mathrm{T} 2$ & P5 & 0 & $100 \%$ & (Vijay, 1999) \\
\hline VE-TS-T2-P5 & VE & GL & TS & $\mathrm{T} 2$ & P5 & 3 & $93 \%$ & (Vijay, 1999) \\
\hline VE-TS-T2-P5 & VE & GL & TS & $\mathrm{T} 2$ & P5 & 8 & $92 \%$ & (Vijay, 1999) \\
\hline VE-TS-T2-P5 & VE & $\mathrm{GL}$ & TS & $\mathrm{T} 2$ & P5 & 15 & $67 \%$ & (Vijay, 1999) \\
\hline VE-TS-T2-P2 & VE & GL & TS & $\mathrm{T} 2$ & P2 & 0 & $100 \%$ & (Vijay, 1999) \\
\hline VE-TS-T2-P2 & VE & $\mathrm{GL}$ & TS & $\mathrm{T} 2$ & P2 & 3 & $99 \%$ & (Vijay, 1999) \\
\hline VE-TS-T2-P2 & VE & GL & TS & $\mathrm{T} 2$ & P2 & 8 & $98 \%$ & (Vijay, 1999) \\
\hline VE-TS-T2-P2 & VE & GL & TS & $\mathrm{T} 2$ & P2 & 12 & $95 \%$ & (Vijay, 1999) \\
\hline VE-TS-T2-P5 & VE & GL & TS & $\mathrm{T} 2$ & P5 & 0 & $100 \%$ & (Vijay, 1999) \\
\hline VE-TS-T2-P5 & VE & $\mathrm{GL}$ & TS & $\mathrm{T} 2$ & P5 & 3 & $95 \%$ & (Vijay, 1999) \\
\hline VE-TS-T2-P5 & VE & GL & TS & $\mathrm{T} 2$ & P5 & 8 & $92 \%$ & (Vijay, 1999) \\
\hline VE-TS-T2-P5 & VE & $\mathrm{GL}$ & TS & $\mathrm{T} 2$ & P5 & 12 & $87 \%$ & (Vijay, 1999) \\
\hline VE-TS-T2-P2 & VE & $\mathrm{GL}$ & TS & $\mathrm{T} 2$ & P2 & 0 & $100 \%$ & (Sonawala \& Spontak, 1996) \\
\hline VE-TS-T2-P2 & VE & $\mathrm{GL}$ & TS & $\mathrm{T} 2$ & P2 & 1 & $95 \%$ & (Sonawala \& Spontak, 1996) \\
\hline
\end{tabular}




\begin{tabular}{|c|c|c|c|c|c|c|c|c|}
\hline Sample Designation & Matrix & $\begin{array}{l}\text { Reinforce } \\
\text { ment }\end{array}$ & $\begin{array}{l}\text { Property } \\
\text { Tested }\end{array}$ & $\begin{array}{l}\text { Temp. } \\
\text { Group }\end{array}$ & \begin{tabular}{|l} 
pH \\
Groups
\end{tabular} & $\begin{array}{l}\text { Time in } \\
\text { Aging } \\
\text { Condition } \\
\text { (months) }\end{array}$ & \begin{tabular}{|l} 
SR - \% \\
Strength \\
Reduction
\end{tabular} & Data Source Ref. \\
\hline VE-TS-T2-P2 & VE & $\mathrm{GL}$ & TS & $\mathrm{T} 2$ & P2 & 2 & $90 \%$ & (Sonawala \& Spontak, 1996) \\
\hline VE-TS-T2-P2 & VE & GL & TS & $\mathrm{T} 2$ & P2 & 3 & $88 \%$ & (Sonawala \& Spontak, 1996) \\
\hline VE-TS-T2-P2 & VE & GL & TS & $\mathrm{T} 2$ & P2 & 4 & $86 \%$ & (Sonawala \& Spontak, 1996) \\
\hline VE-TS-T2-P2 & VE & GL & TS & $\mathrm{T} 2$ & P2 & 6 & $85 \%$ & (Sonawala \& Spontak, 1996) \\
\hline VE-TS-T2-P2 & VE & $\mathrm{GL}$ & TS & $\mathrm{T} 2$ & P2 & 9 & $84 \%$ & (Sonawala \& Spontak, 1996) \\
\hline VE-TS-T2-P5 & VE & $\mathrm{GL}$ & TS & $\mathrm{T} 2$ & P5 & 0 & $100 \%$ & (Sonawala \& Spontak, 1996) \\
\hline VE-TS-T2-P5 & VE & GL & TS & $\mathrm{T} 2$ & P5 & 1 & $92 \%$ & (Sonawala \& Spontak, 1996) \\
\hline VE-TS-T2-P5 & VE & $\mathrm{GL}$ & TS & $\mathrm{T} 2$ & P5 & 2 & $90 \%$ & (Sonawala \& Spontak, 1996) \\
\hline VE-TS-T2-P5 & VE & $\mathrm{GL}$ & TS & $\mathrm{T} 2$ & P5 & 3 & $89 \%$ & (Sonawala \& Spontak, 1996) \\
\hline VE-TS-T2-P5 & VE & $\mathrm{GL}$ & TS & $\mathrm{T} 2$ & P5 & 4 & $88 \%$ & (Sonawala \& Spontak, 1996) \\
\hline VE-TS-T2-P5 & VE & $\mathrm{GL}$ & TS & $\mathrm{T} 2$ & P5 & 6 & $88 \%$ & (Sonawala \& Spontak, 1996) \\
\hline VE-TS-T2-P5 & VE & $\mathrm{GL}$ & TS & $\mathrm{T} 2$ & P5 & 9 & $89 \%$ & (Sonawala \& Spontak, 1996) \\
\hline VE-TS-T2-P5 & VE & GL & TS & $\mathrm{T} 2$ & P5 & 0 & $100 \%$ & (Chen et. al., 2006) \\
\hline VE-TS-T2-P5 & VE & $\mathrm{GL}$ & TS & $\mathrm{T} 2$ & P5 & 2 & $82 \%$ & (Chen et. al., 2006) \\
\hline VE-TS-T2-P5 & VE & GL & TS & $\mathrm{T} 2$ & P5 & 3 & $64 \%$ & (Chen et. al., 2006) \\
\hline VE-TS-T2-P5 & VE & $\mathrm{GL}$ & TS & $\mathrm{T} 2$ & P5 & 4 & $55 \%$ & (Chen et. al., 2006) \\
\hline VE-TS-T2-P5 & VE & $\mathrm{GL}$ & TS & $\mathrm{T} 2$ & P5 & 8 & $43 \%$ & (Chen et. al., 2006) \\
\hline VE-TS-T3-P5 & VE & GL & TS & T3 & P5 & 0 & $100 \%$ & (Chen et. al., 2006) \\
\hline VE-TS-T3-P5 & VE & $\mathrm{GL}$ & TS & T3 & P5 & 2 & $70 \%$ & (Chen et. al., 2006) \\
\hline VE-TS-T3-P5 & VE & $\mathrm{GL}$ & TS & T3 & P5 & 3 & $60 \%$ & (Chen et. al., 2006) \\
\hline VE-TS-T3-P5 & VE & $\mathrm{GL}$ & TS & T3 & P5 & 4 & $51 \%$ & (Chen et. al., 2006) \\
\hline VE-TS-T3-P5 & VE & $\mathrm{GL}$ & TS & T3 & P5 & 8 & $31 \%$ & (Chen et. al., 2006) \\
\hline VE-TS-T4-P5 & VE & $\mathrm{GL}$ & TS & T4 & P5 & 0 & $100 \%$ & (Chen et. al., 2006) \\
\hline VE-TS-T4-P5 & VE & $\mathrm{GL}$ & TS & T4 & P5 & 2 & $50 \%$ & (Chen et. al., 2006) \\
\hline VE-TS-T4-P5 & VE & $\mathrm{GL}$ & TS & T4 & P5 & 3 & $47 \%$ & (Chen et. al., 2006) \\
\hline VE-TS-T4-P5 & VE & $\mathrm{GL}$ & TS & T4 & P5 & 4 & $38 \%$ & (Chen et. al., 2006) \\
\hline VE-TS-T4-P5 & VE & $\mathrm{GL}$ & TS & T4 & P5 & 8 & $25 \%$ & (Chen et. al., 2006) \\
\hline VE-TS-T2-P5 & VE & $\overline{\mathrm{GL}}$ & $\overline{T S}$ & $\mathrm{~T} 2$ & $\overline{P 5}$ & 0 & $100 \%$ & (Won et. al., 2007) \\
\hline VE-TS-T2-P5 & VE & $\mathrm{GL}$ & TS & $\mathrm{T} 2$ & P5 & 1 & $98 \%$ & (Won et. al., 2007) \\
\hline VE-TS-T2-P5 & VE & $\mathrm{GL}$ & TS & $\mathrm{T} 2$ & P5 & 2 & $95 \%$ & (Won et. al., 2007) \\
\hline
\end{tabular}




\begin{tabular}{|c|c|c|c|c|c|c|c|c|}
\hline Sample Designation & Matrix & $\begin{array}{l}\text { Reinforce } \\
\text { ment }\end{array}$ & $\begin{array}{l}\text { Property } \\
\text { Tested }\end{array}$ & $\begin{array}{l}\text { Temp. } \\
\text { Group }\end{array}$ & $\begin{array}{l}\text { pH } \\
\text { Groups }\end{array}$ & $\begin{array}{l}\text { Time in } \\
\text { Aging } \\
\text { Condition } \\
\text { (months) }\end{array}$ & $\begin{array}{l}\text { SR - \% } \\
\text { Strength } \\
\text { Reduction }\end{array}$ & Data Source Ref. \\
\hline VE-TS-T2-P5 & VE & $\mathrm{GL}$ & TS & $\mathrm{T} 2$ & P5 & 3 & $93 \%$ & (Won et. al., 2007) \\
\hline VE-TS-T2-P5 & VE & $\mathrm{GL}$ & TS & $\mathrm{T} 2$ & P5 & 6 & $86 \%$ & (Won et. al., 2007) \\
\hline VE-TS-T2-P5 & VE & $\mathrm{GL}$ & TS & $\mathrm{T} 2$ & P5 & 8 & $86 \%$ & (Won et. al., 2007) \\
\hline VE-TS-T2-P5 & VE & $\mathrm{GL}$ & TS & $\mathrm{T} 2$ & P5 & 10 & $82 \%$ & (Won et. al., 2007) \\
\hline VE-TS-T3-P5 & VE & $\mathrm{GL}$ & TS & T3 & P5 & 0 & $100 \%$ & (Won et. al., 2007) \\
\hline VE-TS-T3-P5 & VE & $\mathrm{GL}$ & TS & T3 & P5 & 1 & $97 \%$ & (Won et. al., 2007) \\
\hline VE-TS-T3-P5 & VE & $\mathrm{GL}$ & TS & T3 & P5 & 2 & $93 \%$ & (Won et. al., 2007) \\
\hline VE-TS-T3-P5 & VE & $\mathrm{GL}$ & TS & T3 & P5 & 3 & $93 \%$ & (Won et. al., 2007) \\
\hline VE-TS-T3-P5 & VE & $\mathrm{GL}$ & TS & T3 & P5 & 6 & $85 \%$ & (Won et. al., 2007) \\
\hline VE-TS-T3-P5 & VE & $\mathrm{GL}$ & TS & T3 & P5 & 8 & $79 \%$ & (Won et. al., 2007) \\
\hline VE-TS-T3-P5 & VE & $\mathrm{GL}$ & TS & T3 & P5 & 10 & $70 \%$ & (Won et. al., 2007) \\
\hline VE-TS-T3-P5 & VE & $\mathrm{GL}$ & TS & T3 & P5 & 0 & $100 \%$ & (Won et. al., 2007) \\
\hline VE-TS-T3-P5 & VE & $\mathrm{GL}$ & TS & T3 & P5 & 1 & $90 \%$ & (Won et. al., 2007) \\
\hline VE-TS-T3-P5 & VE & $\mathrm{GL}$ & TS & T3 & P5 & 2 & $86 \%$ & (Won et. al., 2007) \\
\hline VE-TS-T3-P5 & VE & GL & TS & T3 & P5 & 3 & $75 \%$ & (Won et. al., 2007) \\
\hline VE-TS-T3-P5 & VE & $\mathrm{GL}$ & TS & T3 & P5 & 6 & $74 \%$ & (Won et. al., 2007) \\
\hline VE-TS-T3-P5 & VE & GL & TS & T3 & P5 & 8 & $73 \%$ & (Won et. al., 2007) \\
\hline VE-TS-T3-P5 & VE & $\mathrm{GL}$ & TS & T3 & P5 & 10 & $70 \%$ & (Won et. al., 2007) \\
\hline VE-TS-T4-P5 & VE & $\overline{G L}$ & TS & T4 & P5 & 0 & $100 \%$ & (Won et. al., 2007) \\
\hline VE-TS-T4-P5 & VE & $\mathrm{GL}$ & TS & T4 & P5 & 1 & $83 \%$ & (Won et. al., 2007) \\
\hline VE-TS-T4-P5 & VE & $\mathrm{GL}$ & TS & T4 & P5 & 2 & $80 \%$ & (Won et. al., 2007) \\
\hline VE-TS-T4-P5 & VE & GL & TS & T4 & P5 & 3 & $75 \%$ & (Won et. al., 2007) \\
\hline VE-TS-T4-P5 & VE & $\mathrm{GL}$ & TS & T4 & P5 & 6 & $70 \%$ & (Won et. al., 2007) \\
\hline VE-TS-T4-P5 & VE & $\mathrm{GL}$ & TS & T4 & P5 & 8 & $69 \%$ & (Won et. al., 2007) \\
\hline VE-TS-T4-P5 & VE & $\mathrm{GL}$ & TS & T4 & P5 & 10 & $64 \%$ & (Won et. al., 2007) \\
\hline VE-TS-T2-P3 & VE & $\overline{G L}$ & TS & $\mathrm{T2}$ & P3 & 0 & $100 \%$ & (Kim, 2007) \\
\hline VE-TS-T2-P3 & VE & $\mathrm{GL}$ & TS & $\mathrm{T} 2$ & P3 & 1 & $92 \%$ & (Kim, 2007) \\
\hline VE-TS-T2-P3 & VE & $\mathrm{GL}$ & TS & $\mathrm{T} 2$ & P3 & 2 & $85 \%$ & (Kim, 2007) \\
\hline VE-TS-T2-P3 & VE & $\mathrm{GL}$ & TS & $\mathrm{T} 2$ & P3 & 3 & $80 \%$ & (Kim, 2007) \\
\hline VE-TS-T2-P3 & VE & $\mathrm{GL}$ & TS & $\mathrm{T} 2$ & P3 & 5 & $89 \%$ & (Kim, 2007) \\
\hline
\end{tabular}




\begin{tabular}{|c|c|c|c|c|c|c|c|c|}
\hline Sample Designation & Matrix & $\begin{array}{l}\text { Reinforce } \\
\text { ment }\end{array}$ & $\begin{array}{l}\text { Property } \\
\text { Tested }\end{array}$ & $\begin{array}{l}\text { Temp. } \\
\text { Group }\end{array}$ & \begin{tabular}{|l} 
pH \\
Groups
\end{tabular} & $\begin{array}{l}\text { Time in } \\
\text { Aging } \\
\text { Condition } \\
\text { (months) }\end{array}$ & $\begin{array}{l}\text { SR - \% } \\
\text { Strength } \\
\text { Reduction }\end{array}$ & Data Source Ref. \\
\hline VE-TS-T3-P3 & VE & $\mathrm{GL}$ & TS & T3 & P3 & 0 & $100 \%$ & (Kim, 2007) \\
\hline VE-TS-T3-P3 & VE & GL & TS & T3 & P3 & 1 & $89 \%$ & (Kim, 2007) \\
\hline VE-TS-T3-P3 & VE & $\mathrm{GL}$ & TS & T3 & P3 & 2 & $84 \%$ & (Kim, 2007) \\
\hline VE-TS-T3-P3 & VE & GL & TS & T3 & P3 & 3 & $82 \%$ & (Kim, 2007) \\
\hline VE-TS-T3-P3 & VE & $\mathrm{GL}$ & TS & T3 & P3 & 5 & $83 \%$ & (Kim, 2007) \\
\hline VE-TS-T4-P3 & VE & $\mathrm{GL}$ & TS & T4 & P3 & 0 & $100 \%$ & (Kim, 2007) \\
\hline VE-TS-T4-P3 & VE & GL & TS & T4 & P3 & 1 & $85 \%$ & (Kim, 2007) \\
\hline VE-TS-T4-P3 & VE & $\mathrm{GL}$ & TS & T4 & P3 & 2 & $76 \%$ & (Kim, 2007) \\
\hline VE-TS-T4-P3 & VE & $\mathrm{GL}$ & TS & T4 & P3 & 3 & $79 \%$ & (Kim, 2007) \\
\hline VE-TS-T4-P3 & VE & GL & TS & $\mathrm{T} 4$ & P3 & 5 & $78 \%$ & (Kim, 2007) \\
\hline VE-TS-T2-P2 & VE & $\mathrm{GL}$ & TS & $\mathrm{T} 2$ & P2 & 0 & $100 \%$ & (Kim, 2007) \\
\hline VE-TS-T2-P2 & VE & $\mathrm{GL}$ & TS & $\mathrm{T} 2$ & P2 & 1 & $93 \%$ & (Kim, 2007) \\
\hline VE-TS-T2-P2 & VE & $\mathrm{GL}$ & TS & $\mathrm{T} 2$ & P2 & 2 & $82 \%$ & (Kim, 2007) \\
\hline VE-TS-T2-P2 & VE & $\mathrm{GL}$ & TS & $\mathrm{T} 2$ & P2 & 3 & $83 \%$ & (Kim, 2007) \\
\hline VE-TS-T2-P2 & VE & $\mathrm{GL}$ & TS & $\mathrm{T} 2$ & P2 & 5 & $86 \%$ & (Kim, 2007) \\
\hline VE-TS-T3-P2 & VE & $\mathrm{GL}$ & TS & T3 & P2 & 0 & $100 \%$ & (Kim, 2007) \\
\hline VE-TS-T3-P2 & VE & $\mathrm{GL}$ & TS & T3 & P2 & 1 & $91 \%$ & (Kim, 2007) \\
\hline VE-TS-T3-P2 & VE & $\mathrm{GL}$ & TS & T3 & P2 & 2 & $85 \%$ & (Kim, 2007) \\
\hline VE-TS-T3-P2 & VE & $\mathrm{GL}$ & TS & T3 & P2 & 3 & $85 \%$ & (Kim, 2007) \\
\hline VE-TS-T3-P2 & VE & $\mathrm{GL}$ & TS & T3 & P2 & 5 & $88 \%$ & (Kim, 2007) \\
\hline VE-TS-T4-P2 & VE & $\mathrm{GL}$ & TS & T4 & P2 & 0 & $100 \%$ & (Kim, 2007) \\
\hline VE-TS-T4-P2 & VE & $\mathrm{GL}$ & TS & T4 & P2 & 1 & $90 \%$ & (Kim, 2007) \\
\hline VE-TS-T4-P2 & VE & $\mathrm{GL}$ & TS & T4 & P2 & 2 & $81 \%$ & (Kim, 2007) \\
\hline VE-TS-T4-P2 & VE & $\mathrm{GL}$ & TS & T4 & P2 & 3 & $80 \%$ & (Kim, 2007) \\
\hline VE-TS-T4-P2 & VE & $\mathrm{GL}$ & TS & T4 & P2 & 5 & $81 \%$ & (Kim, 2007) \\
\hline VE-TS-T2-P5 & VE & $\mathrm{GL}$ & TS & $\mathrm{T} 2$ & P5 & 0 & $100 \%$ & (Kim, 2007) \\
\hline VE-TS-T2-P5 & VE & $\mathrm{GL}$ & TS & $\mathrm{T} 2$ & P5 & 1 & $76 \%$ & (Kim, 2007) \\
\hline VE-TS-T2-P5 & VE & $\mathrm{GL}$ & TS & $\mathrm{T} 2$ & P5 & 2 & $67 \%$ & (Kim, 2007) \\
\hline VE-TS-T2-P5 & VE & $\mathrm{GL}$ & TS & $\mathrm{T} 2$ & P5 & 3 & $66 \%$ & (Kim, 2007) \\
\hline VE-TS-T3-P5 & VE & $\mathrm{GL}$ & TS & T3 & P5 & 0 & $100 \%$ & (Kim, 2007) \\
\hline
\end{tabular}




\begin{tabular}{|c|c|c|c|c|c|c|c|c|}
\hline Sample Designation & Matrix & $\begin{array}{l}\text { Reinforce } \\
\text { ment }\end{array}$ & $\begin{array}{l}\text { Property } \\
\text { Tested }\end{array}$ & $\begin{array}{l}\text { Temp. } \\
\text { Group }\end{array}$ & $\begin{array}{l}\text { pH } \\
\text { Groups }\end{array}$ & $\begin{array}{l}\text { Time in } \\
\text { Aging } \\
\text { Condition } \\
\text { (months) }\end{array}$ & $\begin{array}{l}\text { SR - \% } \\
\text { Strength } \\
\text { Reduction }\end{array}$ & Data Source Ref. \\
\hline VE-TS-T3-P5 & VE & $\mathrm{GL}$ & TS & T3 & P5 & 1 & $78 \%$ & $($ Kim, 2007) \\
\hline VE-TS-T3-P5 & VE & $\mathrm{GL}$ & TS & T3 & P5 & 2 & $71 \%$ & (Kim, 2007) \\
\hline VE-TS-T3-P5 & VE & $\mathrm{GL}$ & TS & T3 & P5 & 3 & $68 \%$ & (Kim, 2007) \\
\hline VE-TS-T4-P5 & VE & $\mathrm{GL}$ & TS & $\mathrm{T} 4$ & P5 & 0 & $100 \%$ & (Kim, 2007) \\
\hline VE-TS-T4-P5 & VE & GL & TS & $\mathrm{T} 4$ & P5 & 1 & $64 \%$ & (Kim, 2007) \\
\hline VE-TS-T4-P5 & VE & GL & TS & $\mathrm{T} 4$ & P5 & 2 & $60 \%$ & (Kim, 2007) \\
\hline VE-TS-T2-P3 & VE & GL & TS & $\mathrm{T} 2$ & P3 & 0 & $100 \%$ & (Kim, 2007) \\
\hline VE-TS-T2-P3 & VE & $\mathrm{GL}$ & TS & $\mathrm{T} 2$ & P3 & 1 & $95 \%$ & (Kim, 2007) \\
\hline VE-TS-T2-P3 & VE & $\mathrm{GL}$ & TS & $\mathrm{T} 2$ & P3 & 2 & $75 \%$ & (Kim, 2007) \\
\hline VE-TS-T2-P3 & VE & $\mathrm{GL}$ & TS & $\mathrm{T} 2$ & P3 & 3 & $82 \%$ & (Kim, 2007) \\
\hline VE-TS-T2-P3 & VE & $\mathrm{GL}$ & TS & $\mathrm{T} 2$ & P3 & 5 & $87 \%$ & (Kim, 2007) \\
\hline VE-TS-T3-P3 & VE & $\mathrm{GL}$ & TS & T3 & P3 & 0 & $100 \%$ & (Kim, 2007) \\
\hline VE-TS-T3-P3 & VE & $\mathrm{GL}$ & TS & T3 & P3 & 1 & $92 \%$ & (Kim, 2007) \\
\hline VE-TS-T3-P3 & VE & $\mathrm{GL}$ & TS & T3 & P3 & 2 & $86 \%$ & (Kim, 2007) \\
\hline VE-TS-T3-P3 & VE & $\mathrm{GL}$ & TS & T3 & P3 & 3 & $78 \%$ & (Kim, 2007) \\
\hline VE-TS-T3-P3 & VE & $\mathrm{GL}$ & TS & T3 & P3 & 5 & $81 \%$ & (Kim, 2007) \\
\hline VE-TS-T4-P3 & VE & $\mathrm{GL}$ & TS & T4 & P3 & 0 & $100 \%$ & (Kim, 2007) \\
\hline VE-TS-T4-P3 & VE & $\mathrm{GL}$ & TS & $\mathrm{T} 4$ & P3 & 1 & $66 \%$ & (Kim, 2007) \\
\hline VE-TS-T4-P3 & VE & $\mathrm{GL}$ & TS & T4 & P3 & 2 & $55 \%$ & (Kim, 2007) \\
\hline VE-TS-T4-P3 & VE & $\mathrm{GL}$ & TS & $\mathrm{T} 4$ & P3 & 3 & $49 \%$ & (Kim, 2007) \\
\hline VE-TS-T4-P3 & VE & $\mathrm{GL}$ & TS & $\mathrm{T} 4$ & P3 & 5 & $44 \%$ & (Kim, 2007) \\
\hline VE-TS-T2-P2 & VE & $\mathrm{GL}$ & TS & $\mathrm{T} 2$ & P2 & 0 & $100 \%$ & (Kim, 2007) \\
\hline VE-TS-T2-P2 & VE & $\mathrm{GL}$ & TS & $\mathrm{T} 2$ & P2 & 1 & $95 \%$ & (Kim, 2007) \\
\hline VE-TS-T2-P2 & VE & $\mathrm{GL}$ & TS & $\mathrm{T} 2$ & P2 & 2 & $84 \%$ & (Kim, 2007) \\
\hline VE-TS-T2-P2 & VE & $\mathrm{GL}$ & TS & $\mathrm{T} 2$ & P2 & 3 & $83 \%$ & (Kim, 2007) \\
\hline VE-TS-T2-P2 & VE & $\mathrm{GL}$ & TS & $\mathrm{T} 2$ & P2 & 5 & $81 \%$ & (Kim, 2007) \\
\hline VE-TS-T3-P2 & VE & $\mathrm{GL}$ & TS & T3 & P2 & 0 & $100 \%$ & (Kim, 2007) \\
\hline VE-TS-T3-P2 & VE & $\mathrm{GL}$ & TS & T3 & P2 & 1 & $90 \%$ & (Kim, 2007) \\
\hline VE-TS-T3-P2 & VE & GL & TS & $\mathrm{T} 3$ & P2 & 2 & $77 \%$ & (Kim, 2007) \\
\hline VE-TS-T3-P2 & VE & $\mathrm{GL}$ & TS & T3 & P2 & 3 & $82 \%$ & (Kim, 2007) \\
\hline
\end{tabular}




\begin{tabular}{|c|c|c|c|c|c|c|c|c|}
\hline Sample Designation & Matrix & $\begin{array}{l}\text { Reinforce } \\
\text { ment }\end{array}$ & $\begin{array}{l}\text { Property } \\
\text { Tested }\end{array}$ & $\begin{array}{l}\text { Temp. } \\
\text { Group }\end{array}$ & $\begin{array}{l}\text { pH } \\
\text { Groups }\end{array}$ & $\begin{array}{l}\text { Time in } \\
\text { Aging } \\
\text { Condition } \\
\text { (months) }\end{array}$ & $\begin{array}{l}\text { SR - \% } \\
\text { Strength } \\
\text { Reduction }\end{array}$ & Data Source Ref. \\
\hline VE-TS-T4-P2 & VE & $\mathrm{GL}$ & TS & T4 & P2 & 0 & $100 \%$ & (Kim, 2007) \\
\hline VE-TS-T4-P2 & VE & $\mathrm{GL}$ & TS & T4 & P2 & 1 & $65 \%$ & (Kim, 2007) \\
\hline VE-TS-T4-P2 & VE & $\mathrm{GL}$ & TS & T4 & P2 & 2 & $54 \%$ & (Kim, 2007) \\
\hline VE-TS-T4-P2 & VE & $\mathrm{GL}$ & TS & T4 & P2 & 5 & $42 \%$ & (Kim, 2007) \\
\hline VE-TS-T2-P5 & VE & GL & TS & $\mathrm{T} 2$ & P5 & 0 & $100 \%$ & (Kim, 2007) \\
\hline VE-TS-T2-P5 & VE & $\mathrm{GL}$ & TS & $\mathrm{T} 2$ & P5 & 1 & $93 \%$ & (Kim, 2007) \\
\hline VE-TS-T2-P5 & VE & $\mathrm{GL}$ & TS & $\mathrm{T} 2$ & P5 & 2 & $82 \%$ & (Kim, 2007) \\
\hline VE-TS-T3-P5 & VE & $\mathrm{GL}$ & TS & T3 & P5 & 0 & $100 \%$ & (Kim, 2007) \\
\hline VE-TS-T3-P5 & VE & $\mathrm{GL}$ & TS & T3 & P5 & 1 & $86 \%$ & (Kim, 2007) \\
\hline VE-TS-T4-P5 & VE & $\mathrm{GL}$ & TS & T4 & P5 & 1 & $62 \%$ & (Kim, 2007) \\
\hline VE-TS-T4-P5 & VE & $\mathrm{GL}$ & TS & T4 & P5 & 2 & $50 \%$ & (Kim, 2007) \\
\hline VE-TS-T2-P3 & VE & $\mathrm{GL}$ & TS & $\mathrm{T} 2$ & P3 & 0 & $100 \%$ & (Cabral-Fonseca, 2012) \\
\hline VE-TS-T2-P3 & VE & $\mathrm{GL}$ & TS & $\mathrm{T} 2$ & P3 & 3 & $96 \%$ & (Cabral-Fonseca, 2012) \\
\hline VE-TS-T2-P3 & VE & $\mathrm{GL}$ & TS & $\mathrm{T} 2$ & P3 & 6 & $97 \%$ & (Cabral-Fonseca, 2012) \\
\hline VE-TS-T2-P3 & VE & $\mathrm{GL}$ & TS & $\mathrm{T} 2$ & P3 & 9 & $87 \%$ & (Cabral-Fonseca, 2012) \\
\hline VE-TS-T3-P3 & VE & $\mathrm{GL}$ & TS & T3 & P3 & 0 & $100 \%$ & (Cabral-Fonseca, 2012) \\
\hline VE-TS-T3-P3 & VE & $\mathrm{GL}$ & TS & $\mathrm{T} 3$ & P3 & 3 & $89 \%$ & (Cabral-Fonseca, 2012) \\
\hline VE-TS-T3-P3 & VE & $\mathrm{GL}$ & TS & T3 & P3 & 6 & $83 \%$ & (Cabral-Fonseca, 2012) \\
\hline VE-TS-T3-P3 & VE & $\mathrm{GL}$ & TS & T3 & P3 & 9 & $77 \%$ & (Cabral-Fonseca, 2012) \\
\hline VE-TS-T3-P3 & VE & $\mathrm{GL}$ & TS & T3 & P3 & 15 & $84 \%$ & (Cabral-Fonseca, 2012) \\
\hline
\end{tabular}




\begin{tabular}{|c|c|c|c|c|c|c|c|c|}
\hline Sample Designation & Matrix & $\begin{array}{l}\text { Reinforce } \\
\text { ment }\end{array}$ & $\begin{array}{l}\text { Property } \\
\text { Tested }\end{array}$ & $\begin{array}{l}\text { Temp. } \\
\text { Group }\end{array}$ & $\begin{array}{l}\text { pH } \\
\text { Groups }\end{array}$ & $\begin{array}{l}\text { Time in } \\
\text { Aging } \\
\text { Condition } \\
\text { (months) }\end{array}$ & $\begin{array}{l}\text { SR - \% } \\
\text { Strength } \\
\text { Reduction }\end{array}$ & Data Source Ref. \\
\hline VE-TS-T2-P2 & VE & $\mathrm{GL}$ & TS & $\mathrm{T} 2$ & P2 & 0 & $100 \%$ & (Cabral-Fonseca, 2012) \\
\hline VE-TS-T2-P2 & VE & $\mathrm{GL}$ & TS & $\mathrm{T} 2$ & P2 & 3 & $90 \%$ & (Cabral-Fonseca, 2012) \\
\hline VE-TS-T2-P2 & VE & GL & TS & $\mathrm{T} 2$ & P2 & 6 & $77 \%$ & (Cabral-Fonseca, 2012) \\
\hline VE-TS-T2-P2 & VE & $\mathrm{GL}$ & TS & $\mathrm{T} 2$ & P2 & 9 & $90 \%$ & (Cabral-Fonseca, 2012) \\
\hline VE-TS-T2-P2 & VE & GL & TS & $\mathrm{T} 2$ & P2 & 15 & $96 \%$ & (Cabral-Fonseca, 2012) \\
\hline VE-TS-T3-P2 & VE & GL & TS & T3 & P2 & 0 & $100 \%$ & (Cabral-Fonseca, 2012) \\
\hline VE-TS-T3-P2 & VE & GL & TS & T3 & P2 & 3 & $99 \%$ & (Cabral-Fonseca, 2012) \\
\hline VE-TS-T3-P2 & VE & $\mathrm{GL}$ & TS & T3 & P2 & 6 & $87 \%$ & (Cabral-Fonseca, 2012) \\
\hline VE-TS-T3-P2 & VE & $\mathrm{GL}$ & TS & T3 & P2 & 9 & $88 \%$ & (Cabral-Fonseca, 2012) \\
\hline VE-TS-T3-P2 & VE & $\mathrm{GL}$ & TS & T3 & P2 & 12 & $89 \%$ & (Cabral-Fonseca, 2012) \\
\hline VE-TS-T3-P2 & VE & $\mathrm{GL}$ & TS & T3 & P2 & 18 & $90 \%$ & (Cabral-Fonseca, 2012) \\
\hline VE-TS-T4-P2 & VE & $\mathrm{GL}$ & TS & T4 & P2 & 0 & $100 \%$ & (Cabral-Fonseca, 2012) \\
\hline VE-TS-T4-P2 & VE & $\mathrm{GL}$ & TS & T4 & P2 & 3 & $96 \%$ & (Cabral-Fonseca, 2012) \\
\hline VE-TS-T4-P2 & VE & GL & TS & $\mathrm{T} 4$ & P2 & 6 & $82 \%$ & (Cabral-Fonseca, 2012) \\
\hline VE-TS-T4-P2 & VE & GL & TS & T4 & P2 & 9 & $71 \%$ & (Cabral-Fonseca, 2012) \\
\hline VE-TS-T4-P2 & VE & $\mathrm{GL}$ & TS & T4 & P2 & 12 & $84 \%$ & (Cabral-Fonseca, 2012) \\
\hline VE-TS-T4-P2 & VE & $\mathrm{GL}$ & TS & $\mathrm{T} 4$ & $\mathrm{P} 2$ & 18 & $86 \%$ & (Cabral-Fonseca, 2012) \\
\hline VE-TS-T3-P3 & VE & $\mathrm{GL}$ & TS & $\mathrm{T} 3$ & P3 & 3 & $100 \%$ & (Cabral-Fonseca, 2012) \\
\hline VE-TS-T3-P3 & VE & GL & TS & T3 & P3 & 6 & $91 \%$ & (Cabral-Fonseca, 2012) \\
\hline VE-TS-T3-P3 & VE & $\mathrm{GL}$ & TS & T3 & P3 & 9 & $89 \%$ & (Cabral-Fonseca, 2012) \\
\hline VE-TS-T4-P5 & VE & $\mathrm{GL}$ & TS & $\mathrm{T} 4$ & P5 & 0 & $100 \%$ & (Dejke, 2001) \\
\hline VE-TS-T4-P5 & VE & GL & TS & T4 & P5 & 1 & $70 \%$ & (Dejke, 2001) \\
\hline VE-TS-T4-P5 & VE & $\mathrm{GL}$ & TS & $\mathrm{T} 4$ & P5 & 2 & $67 \%$ & (Dejke, 2001) \\
\hline VE-TS-T4-P5 & VE & $\mathrm{GL}$ & TS & $\mathrm{T} 4$ & P5 & 6 & $56 \%$ & (Dejke, 2001) \\
\hline VE-TS-T4-P5 & VE & $\mathrm{GL}$ & TS & T4 & P5 & 12 & $52 \%$ & (Dejke, 2001) \\
\hline VE-TS-T4-P3 & VE & $\mathrm{GL}$ & TS & T4 & P3 & 0 & $100 \%$ & (Dejke, 2001) \\
\hline VE-TS-T4-P3 & VE & $\mathrm{GL}$ & TS & $\mathrm{T} 4$ & P3 & 1 & $79 \%$ & (Dejke, 2001) \\
\hline VE-TS-T4-P3 & VE & $\mathrm{GL}$ & TS & T4 & P3 & 6 & $64 \%$ & (Dejke, 2001) \\
\hline VE-TS-T4-P4 & VE & $\mathrm{GL}$ & TS & $\mathrm{T} 4$ & P4 & 0 & $100 \%$ & (Dejke, 2001) \\
\hline VE-TS-T4-P4 & VE & GL & TS & T4 & P4 & 1 & $75 \%$ & (Dejke, 2001) \\
\hline
\end{tabular}




\begin{tabular}{|c|c|c|c|c|c|c|c|c|}
\hline Sample Designation & Matrix & $\begin{array}{l}\text { Reinforce } \\
\text { ment }\end{array}$ & $\begin{array}{l}\text { Property } \\
\text { Tested }\end{array}$ & $\begin{array}{l}\text { Temp. } \\
\text { Group }\end{array}$ & $\begin{array}{l}\text { pH } \\
\text { Groups }\end{array}$ & $\begin{array}{l}\text { Time in } \\
\text { Aging } \\
\text { Condition } \\
\text { (months) }\end{array}$ & $\begin{array}{l}\text { SR - \% } \\
\text { Strength } \\
\text { Reduction }\end{array}$ & Data Source Ref. \\
\hline VE-TS-T4-P4 & VE & $\mathrm{GL}$ & TS & T4 & P4 & 2 & $67 \%$ & (Dejke, 2001) \\
\hline VE-TS-T4-P4 & VE & $\mathrm{GL}$ & TS & T4 & P4 & 6 & $70 \%$ & (Dejke, 2001) \\
\hline VE-TS-T4-P4 & VE & $\mathrm{GL}$ & TS & $\mathrm{T} 4$ & P4 & 12 & $60 \%$ & (Dejke, 2001) \\
\hline VE-TS-T2-P5 & VE & GL & TS & $\mathrm{T} 2$ & P5 & 0 & $100 \%$ & (Dejke, 2001) \\
\hline VE-TS-T2-P5 & VE & GL & TS & $\mathrm{T} 2$ & P5 & 8 & $80 \%$ & (Dejke, 2001) \\
\hline VE-TS-T2-P5 & VE & $\mathrm{GL}$ & TS & $\mathrm{T} 2$ & P5 & 12 & $70 \%$ & (Dejke, 2001) \\
\hline VE-TS-T2-P3 & VE & GL & TS & $\mathrm{T} 2$ & P3 & 0 & $100 \%$ & (Dejke, 2001) \\
\hline VE-TS-T2-P3 & VE & $\mathrm{GL}$ & TS & $\mathrm{T} 2$ & P3 & 8 & $100 \%$ & (Dejke, 2001) \\
\hline VE-TS-T2-P3 & VE & $\mathrm{GL}$ & TS & $\mathrm{T} 2$ & P3 & 12 & $82 \%$ & (Dejke, 2001) \\
\hline VE-TS-T2-P4 & VE & $\mathrm{GL}$ & TS & $\mathrm{T} 2$ & P4 & 0 & $100 \%$ & (Dejke, 2001) \\
\hline VE-TS-T2-P4 & VE & $\mathrm{GL}$ & TS & $\mathrm{T} 2$ & P4 & 2 & $93 \%$ & (Dejke, 2001) \\
\hline VE-TS-T2-P4 & VE & GL & TS & $\mathrm{T} 2$ & P4 & 8 & $80 \%$ & (Dejke, 2001) \\
\hline VE-TS-T2-P4 & VE & $\mathrm{GL}$ & TS & $\mathrm{T} 2$ & P4 & 12 & $80 \%$ & (Dejke, 2001) \\
\hline VE-TS-T2-P4 & VE & $\mathrm{GL}$ & TS & $\mathrm{T} 2$ & P4 & 14 & $80 \%$ & (Dejke, 2001) \\
\hline VE-TS-TO-PO & VE & $\overline{G L}$ & TS & TO & $\mathrm{PO}$ & 0 & $100 \%$ & (Dittenber/WVU-CFC, 2013) \\
\hline VE-TS-TO-PO & VE & GL & TS & TO & PO & 96 & $75 \%$ & (Dittenber/WVU-CFC, 2013) \\
\hline VE-TS-TO-PO & VE & GL & TS & TO & PO & 0 & $100 \%$ & (Dittenber/WVU-CFC, 2013) \\
\hline VE-TS-TO-PO & VE & $\mathrm{GL}$ & TS & TO & PO & 96 & $89 \%$ & (Dittenber/WVU-CFC, 2013) \\
\hline VE-TS-TO-PO & VE & $\mathrm{GL}$ & TS & TO & $\mathrm{PO}$ & 0 & $100 \%$ & (Dittenber/WVU-CFC, 2013) \\
\hline VE-TS-TO-PO & VE & $\mathrm{GL}$ & TS & TO & $\mathrm{PO}$ & 144 & $89 \%$ & (Dittenber/WVU-CFC, 2013) \\
\hline VE-TS-TO-PO & VE & $\mathrm{GL}$ & TS & TO & PO & 0 & $100 \%$ & (Dittenber/WVU-CFC, 2013) \\
\hline VE-TS-TO-PO & VE & $\mathrm{GL}$ & TS & TO & PO & 144 & $83 \%$ & (Dittenber/WVU-CFC, 2013) \\
\hline VE-TS-TO-PO & VE & $\mathrm{GL}$ & TS & TO & PO & 0 & $100 \%$ & (Dittenber/WVU-CFC, 2013) \\
\hline VE-TS-TO-PO & VE & GL & TS & TO & PO & 144 & $81 \%$ & (Dittenber/WVU-CFC, 2013) \\
\hline VE-TS-TO-PO & VE & $\mathrm{GL}$ & TS & TO & PO & 0 & $100 \%$ & (Dittenber/WVU-CFC, 2013) \\
\hline VE-TS-TO-PO & VE & $\mathrm{GL}$ & TS & TO & PO & 96 & $80 \%$ & (Dittenber/WVU-CFC, 2013) \\
\hline VE-TS-TO-PO & VE & $\mathrm{GL}$ & TS & TO & PO & 0 & $100 \%$ & (Dittenber/WVU-CFC, 2013) \\
\hline VE-TS-TO-PO & VE & $\mathrm{GL}$ & TS & TO & PO & 96 & $75 \%$ & (Dittenber/WVU-CFC, 2013) \\
\hline
\end{tabular}




\begin{tabular}{|c|c|c|c|c|c|c|c|c|}
\hline Sample Designation & Matrix & $\begin{array}{l}\text { Reinforce } \\
\text { ment }\end{array}$ & $\begin{array}{l}\text { Property } \\
\text { Tested }\end{array}$ & $\begin{array}{l}\text { Temp. } \\
\text { Group } \\
\end{array}$ & $\begin{array}{l}\text { pH } \\
\text { Groups } \\
\end{array}$ & $\begin{array}{l}\text { Time in } \\
\text { Aging } \\
\text { Condition } \\
\text { (months) } \\
\end{array}$ & \begin{tabular}{|l} 
SR - \% \\
Strength \\
Reduction
\end{tabular} & Data Source Ref. \\
\hline \multicolumn{9}{|c|}{ VE-GL-ILSS: Vinylester - Glass FRP - Interlaminar Shear Strength Testing Database } \\
\hline VE-ILSS-T2-P3 & VE & GL & ILSS & $\mathrm{T} 2$ & P3 & 0 & $100 \%$ & (Chin et. al., 2001) \\
\hline VE-ILSS-T2-P3 & VE & GL & ILSS & $\mathrm{T} 2$ & P3 & 2 & $91 \%$ & (Chin et. al., 2001) \\
\hline VE-ILSS-T2-P3 & VE & $\mathrm{GL}$ & ILSS & $\mathrm{T} 2$ & P3 & 4 & $89 \%$ & (Chin et. al., 2001) \\
\hline VE-ILSS-T2-P3 & VE & $\mathrm{GL}$ & ILSS & $\mathrm{T} 2$ & P3 & 10 & $100 \%$ & (Chin et. al., 2001) \\
\hline VE-ILSS-T3-P3 & VE & $\mathrm{GL}$ & ILSS & T3 & P3 & 0 & $100 \%$ & (Chin et. al., 2001) \\
\hline VE-ILSS-T3-P3 & VE & $\mathrm{GL}$ & ILSS & T3 & P3 & 3 & $87 \%$ & (Chin et. al., 2001) \\
\hline VE-ILSS-T3-P3 & VE & $\mathrm{GL}$ & ILSS & T3 & P3 & 6 & $91 \%$ & (Chin et. al., 2001) \\
\hline VE-ILSS-T3-P3 & VE & GL & ILSS & T3 & P3 & 8 & $90 \%$ & (Chin et. al., 2001) \\
\hline VE-ILSS-T3-P3 & VE & GL & ILSS & T3 & P3 & 14 & $91 \%$ & (Chin et. al., 2001) \\
\hline VE-ILSS-T3-P3 & VE & $\mathrm{GL}$ & ILSS & T3 & P3 & 0 & $100 \%$ & (Chin et. al., 2001) \\
\hline VE-ILSS-T3-P3 & VE & $\mathrm{GL}$ & ILSS & T3 & P3 & 3 & $75 \%$ & (Chin et. al., 2001) \\
\hline VE-ILSS-T3-P3 & VE & $\mathrm{GL}$ & ILSS & T3 & P3 & 6 & $61 \%$ & (Chin et. al., 2001) \\
\hline VE-ILSS-T3-P3 & VE & $\mathrm{GL}$ & ILSS & T3 & P3 & 8 & $68 \%$ & (Chin et. al., 2001) \\
\hline VE-ILSS-T3-P3 & VE & $\mathrm{GL}$ & ILSS & T3 & P3 & 14 & $40 \%$ & (Chin et. al., 2001) \\
\hline VE-ILSS-T4-P3 & VE & GL & ILSS & $\mathrm{T} 4$ & P3 & 0 & $100 \%$ & (Chin et. al., 2001) \\
\hline VE-ILSS-T4-P3 & VE & $\mathrm{GL}$ & ILSS & T4 & P3 & 3 & $50 \%$ & (Chin et. al., 2001) \\
\hline VE-ILSS-T4-P3 & VE & $\mathrm{GL}$ & ILSS & T4 & P3 & 6 & $35 \%$ & (Chin et. al., 2001) \\
\hline VE-ILSS-T4-P3 & VE & $\mathrm{GL}$ & ILSS & T4 & P3 & 8 & $45 \%$ & (Chin et. al., 2001) \\
\hline VE-ILSS-T4-P3 & VE & $\mathrm{GL}$ & ILSS & T4 & P3 & 14 & $40 \%$ & (Chin et. al., 2001) \\
\hline VE-ILSS-T2-P2 & VE & $\mathrm{GL}$ & ILSS & $\mathrm{T} 2$ & P2 & 0 & $100 \%$ & (Chin et. al., 2001) \\
\hline VE-ILSS-T2-P2 & VE & $\mathrm{GL}$ & ILSS & $\mathrm{T} 2$ & P2 & 3 & $75 \%$ & (Chin et. al., 2001) \\
\hline VE-ILSS-T2-P2 & VE & $\mathrm{GL}$ & ILSS & $\mathrm{T} 2$ & P2 & 6 & $90 \%$ & (Chin et. al., 2001) \\
\hline VE-ILSS-T2-P2 & VE & $\mathrm{GL}$ & ILSS & $\mathrm{T} 2$ & P2 & 8 & $96 \%$ & (Chin et. al., 2001) \\
\hline VE-ILSS-T2-P2 & VE & $\mathrm{GL}$ & ILSS & $\mathrm{T} 2$ & P2 & 14 & $83 \%$ & (Chin et. al., 2001) \\
\hline VE-ILSS-T3-P2 & VE & $\mathrm{GL}$ & ILSS & T3 & P2 & 0 & $100 \%$ & (Chin et. al., 2001) \\
\hline VE-ILSS-T3-P2 & VE & $\mathrm{GL}$ & ILSS & T3 & P2 & 3 & $80 \%$ & (Chin et. al., 2001) \\
\hline VE-ILSS-T3-P2 & VE & $\mathrm{GL}$ & ILSS & T3 & P2 & 6 & $79 \%$ & (Chin et. al., 2001) \\
\hline VE-ILSS-T3-P2 & VE & $\mathrm{GL}$ & ILSS & T3 & P2 & 8 & $72 \%$ & (Chin et. al., 2001) \\
\hline VE-ILSS-T3-P2 & VE & $\mathrm{GL}$ & ILSS & T3 & P2 & 14 & $81 \%$ & (Chin et. al., 2001) \\
\hline
\end{tabular}




\begin{tabular}{|c|c|c|c|c|c|c|c|c|}
\hline Sample Designation & Matrix & $\begin{array}{l}\text { Reinforce } \\
\text { ment }\end{array}$ & $\begin{array}{l}\text { Property } \\
\text { Tested }\end{array}$ & $\begin{array}{l}\text { Temp. } \\
\text { Group }\end{array}$ & \begin{tabular}{|l} 
pH \\
Groups
\end{tabular} & $\begin{array}{l}\text { Time in } \\
\text { Aging } \\
\text { Condition } \\
\text { (months) }\end{array}$ & $\begin{array}{l}\text { SR - \% } \\
\text { Strength } \\
\text { Reduction }\end{array}$ & Data Source Ref. \\
\hline VE-ILSS-T3-P2 & VE & $\mathrm{GL}$ & ILSS & T3 & P2 & 0 & $100 \%$ & (Chin et. al., 2001) \\
\hline VE-ILSS-T3-P2 & VE & GL & ILSS & T3 & P2 & 3 & $62 \%$ & (Chin et. al., 2001) \\
\hline VE-ILSS-T3-P2 & VE & $\mathrm{GL}$ & ILSS & T3 & P2 & 6 & $70 \%$ & (Chin et. al., 2001) \\
\hline VE-ILSS-T3-P2 & VE & $\mathrm{GL}$ & ILSS & T3 & P2 & 8 & $63 \%$ & (Chin et. al., 2001) \\
\hline VE-ILSS-T3-P2 & VE & $\mathrm{GL}$ & ILSS & T3 & P2 & 14 & $65 \%$ & (Chin et. al., 2001) \\
\hline VE-ILSS-T4-P2 & VE & $\mathrm{GL}$ & ILSS & T4 & P2 & 0 & $100 \%$ & (Chin et. al., 2001) \\
\hline VE-ILSS-T4-P2 & VE & $\mathrm{GL}$ & ILSS & T4 & P2 & 3 & $70 \%$ & (Chin et. al., 2001) \\
\hline VE-ILSS-T4-P2 & VE & $\mathrm{GL}$ & ILSS & T4 & P2 & 6 & $60 \%$ & (Chin et. al., 2001) \\
\hline VE-ILSS-T4-P2 & VE & $\mathrm{GL}$ & ILSS & T4 & P2 & 8 & $55 \%$ & (Chin et. al., 2001) \\
\hline VE-ILSS-T4-P2 & VE & GL & ILSS & $\mathrm{T} 4$ & P2 & 14 & $47 \%$ & (Chin et. al., 2001) \\
\hline VE-ILSS-T2-P5 & VE & $\mathrm{GL}$ & ILSS & $\mathrm{T} 2$ & P5 & 0 & $100 \%$ & (Chin et. al., 2001) \\
\hline VE-ILSS-T2-P5 & VE & $\mathrm{GL}$ & ILSS & $\mathrm{T} 2$ & P5 & 3 & $55 \%$ & (Chin et. al., 2001) \\
\hline VE-ILSS-T2-P5 & VE & $\mathrm{GL}$ & ILSS & $\mathrm{T} 2$ & P5 & 6 & $50 \%$ & (Chin et. al., 2001) \\
\hline VE-ILSS-T2-P5 & VE & $\mathrm{GL}$ & ILSS & $\mathrm{T} 2$ & P5 & 8 & $35 \%$ & (Chin et. al., 2001) \\
\hline VE-ILSS-T2-P5 & VE & $\mathrm{GL}$ & ILSS & $\mathrm{T} 2$ & P5 & 14 & $40 \%$ & (Chin et. al., 2001) \\
\hline VE-ILSS-T3-P5 & VE & $\mathrm{GL}$ & ILSS & T3 & P5 & 0 & $100 \%$ & (Chin et. al., 2001) \\
\hline VE-ILSS-T3-P5 & VE & $\mathrm{GL}$ & ILSS & T3 & P5 & 3 & $35 \%$ & (Chin et. al., 2001) \\
\hline VE-ILSS-T3-P5 & VE & GL & ILSS & T3 & P5 & 6 & $35 \%$ & (Chin et. al., 2001) \\
\hline VE-ILSS-T3-P5 & VE & $\mathrm{GL}$ & ILSS & T3 & P5 & 8 & $37 \%$ & (Chin et. al., 2001) \\
\hline VE-ILSS-T3-P5 & VE & $\mathrm{GL}$ & ILSS & T3 & P5 & 14 & $30 \%$ & (Chin et. al., 2001) \\
\hline VE-ILSS-T3-P5 & VE & $\mathrm{GL}$ & ILSS & T3 & P5 & 0 & $100 \%$ & (Chin et. al., 2001) \\
\hline VE-ILSS-T3-P5 & VE & $\mathrm{GL}$ & ILSS & T3 & P5 & 3 & $21 \%$ & (Chin et. al., 2001) \\
\hline VE-ILSS-T3-P5 & VE & $\mathrm{GL}$ & ILSS & T3 & P5 & 6 & $26 \%$ & (Chin et. al., 2001) \\
\hline VE-ILSS-T3-P5 & VE & $\mathrm{GL}$ & ILSS & T3 & P5 & 8 & $37 \%$ & (Chin et. al., 2001) \\
\hline VE-ILSS-T3-P5 & VE & $\mathrm{GL}$ & ILSS & T3 & P5 & 14 & $29 \%$ & (Chin et. al., 2001) \\
\hline VE-ILSS-T4-P5 & VE & $\mathrm{GL}$ & ILSS & T4 & P5 & 0 & $100 \%$ & (Chin et. al., 2001) \\
\hline VE-ILSS-T4-P5 & VE & $\mathrm{GL}$ & ILSS & T4 & P5 & 3 & $19 \%$ & (Chin et. al., 2001) \\
\hline VE-ILSS-T4-P5 & VE & $\mathrm{GL}$ & ILSS & T4 & P5 & 6 & $20 \%$ & (Chin et. al., 2001) \\
\hline VE-ILSS-T4-P5 & VE & GL & ILSS & T4 & P5 & 8 & $25 \%$ & (Chin et. al., 2001) \\
\hline VE-ILSS-T4-P5 & VE & $\mathrm{GL}$ & ILSS & T4 & P5 & 14 & $18 \%$ & (Chin et. al., 2001) \\
\hline
\end{tabular}




\begin{tabular}{|c|c|c|c|c|c|c|c|c|}
\hline Sample Designation & Matrix & $\begin{array}{l}\text { Reinforce } \\
\text { ment }\end{array}$ & $\begin{array}{l}\text { Property } \\
\text { Tested }\end{array}$ & $\begin{array}{l}\text { Temp. } \\
\text { Group }\end{array}$ & \begin{tabular}{|l} 
pH \\
Groups
\end{tabular} & $\begin{array}{l}\text { Time in } \\
\text { Aging } \\
\text { Condition } \\
\text { (months) }\end{array}$ & \begin{tabular}{|l} 
SR - \% \\
Strength \\
Reduction
\end{tabular} & Data Source Ref. \\
\hline VE-ILSS-T2-P3 & VE & $\mathrm{GL}$ & ILSS & $\mathrm{T} 2$ & P3 & 0 & $100 \%$ & (Cabral-Fonseca, 2012) \\
\hline VE-ILSS-T2-P3 & VE & GL & ILSS & $\mathrm{T} 2$ & P3 & 3 & $96 \%$ & (Cabral-Fonseca, 2012) \\
\hline VE-ILSS-T2-P3 & VE & $\mathrm{GL}$ & ILSS & $\mathrm{T} 2$ & P3 & 6 & $95 \%$ & (Cabral-Fonseca, 2012) \\
\hline VE-ILSS-T2-P3 & VE & $\mathrm{GL}$ & ILSS & $\mathrm{T} 2$ & P3 & 9 & $84 \%$ & (Cabral-Fonseca, 2012) \\
\hline VE-ILSS-T2-P3 & VE & $\mathrm{GL}$ & ILSS & $\mathrm{T} 2$ & P3 & 12 & $91 \%$ & (Cabral-Fonseca, 2012) \\
\hline VE-ILSS-T2-P3 & VE & $\mathrm{GL}$ & ILSS & $\mathrm{T} 2$ & P3 & 18 & $100 \%$ & (Cabral-Fonseca, 2012) \\
\hline VE-ILSS-T3-P3 & VE & $\mathrm{GL}$ & ILSS & T3 & P3 & 0 & $100 \%$ & (Cabral-Fonseca, 2012) \\
\hline VE-ILSS-T3-P3 & VE & $\mathrm{GL}$ & ILSS & T3 & P3 & 3 & $91 \%$ & (Cabral-Fonseca, 2012) \\
\hline VE-ILSS-T3-P3 & VE & $\mathrm{GL}$ & ILSS & T3 & P3 & 6 & $88 \%$ & (Cabral-Fonseca, 2012) \\
\hline VE-ILSS-T3-P3 & VE & GL & ILSS & T3 & P3 & 9 & $67 \%$ & (Cabral-Fonseca, 2012) \\
\hline VE-ILSS-T3-P3 & VE & $\mathrm{GL}$ & ILSS & T3 & P3 & 12 & $79 \%$ & (Cabral-Fonseca, 2012) \\
\hline VE-ILSS-T3-P3 & VE & $\mathrm{GL}$ & ILSS & T3 & P3 & 18 & $78 \%$ & (Cabral-Fonseca, 2012) \\
\hline VE-ILSS-T4-P3 & VE & $\mathrm{GL}$ & ILSS & T4 & P3 & 0 & $100 \%$ & (Cabral-Fonseca, 2012) \\
\hline VE-ILSS-T4-P3 & VE & $\mathrm{GL}$ & ILSS & T4 & P3 & 3 & $68 \%$ & (Cabral-Fonseca, 2012) \\
\hline VE-ILSS-T4-P3 & VE & GL & ILSS & T4 & P3 & 6 & $69 \%$ & (Cabral-Fonseca, 2012) \\
\hline VE-ILSS-T4-P3 & VE & $\mathrm{GL}$ & ILSS & T4 & P3 & 9 & $53 \%$ & (Cabral-Fonseca, 2012) \\
\hline VE-ILSS-T4-P3 & VE & $\mathrm{GL}$ & ILSS & T4 & P3 & 12 & $60 \%$ & (Cabral-Fonseca, 2012) \\
\hline VE-ILSS-T4-P3 & VE & $\mathrm{GL}$ & ILSS & T4 & P3 & 18 & $59 \%$ & (Cabral-Fonseca, 2012) \\
\hline VE-ILSS-T2-P2 & VE & $\overline{G L}$ & ILSS & $\mathrm{T} 2$ & $\mathrm{P} 2$ & 0 & $100 \%$ & (Cabral-Fonseca, 2012) \\
\hline VE-ILSS-T2-P2 & VE & $\mathrm{GL}$ & ILSS & $\mathrm{T} 2$ & P2 & 3 & $100 \%$ & (Cabral-Fonseca, 2012) \\
\hline VE-ILSS-T2-P2 & VE & $\mathrm{GL}$ & ILSS & $\mathrm{T} 2$ & P2 & 6 & $88 \%$ & (Cabral-Fonseca, 2012) \\
\hline VE-ILSS-T2-P2 & VE & $\mathrm{GL}$ & ILSS & $\mathrm{T} 2$ & P2 & 9 & $85 \%$ & (Cabral-Fonseca, 2012) \\
\hline VE-ILSS-T2-P2 & VE & $\mathrm{GL}$ & ILSS & $\mathrm{T} 2$ & P2 & 12 & $96 \%$ & (Cabral-Fonseca, 2012) \\
\hline VE-ILSS-T2-P2 & VE & $\mathrm{GL}$ & ILSS & $\mathrm{T} 2$ & P2 & 18 & $94 \%$ & (Cabral-Fonseca, 2012) \\
\hline VE-ILSS-T3-P2 & VE & $\mathrm{GL}$ & ILSS & T3 & P2 & 0 & $100 \%$ & (Cabral-Fonseca, 2012) \\
\hline VE-ILSS-T3-P2 & VE & $\mathrm{GL}$ & ILSS & T3 & P2 & 3 & $89 \%$ & (Cabral-Fonseca, 2012) \\
\hline VE-ILSS-T3-P2 & VE & $\mathrm{GL}$ & ILSS & T3 & P2 & 6 & $90 \%$ & (Cabral-Fonseca, 2012) \\
\hline VE-ILSS-T3-P2 & VE & $\mathrm{GL}$ & ILSS & T3 & P2 & 9 & $71 \%$ & (Cabral-Fonseca, 2012) \\
\hline VE-ILSS-T3-P2 & VE & GL & ILSS & T3 & P2 & 12 & $82 \%$ & (Cabral-Fonseca, 2012) \\
\hline VE-ILSS-T3-P2 & VE & $\mathrm{GL}$ & ILSS & T3 & P2 & 18 & $83 \%$ & (Cabral-Fonseca, 2012) \\
\hline
\end{tabular}




\begin{tabular}{|c|c|c|c|c|c|c|c|c|}
\hline Sample Designation & Matrix & $\begin{array}{l}\text { Reinforce } \\
\text { ment }\end{array}$ & $\begin{array}{l}\text { Property } \\
\text { Tested }\end{array}$ & $\begin{array}{l}\text { Temp. } \\
\text { Group }\end{array}$ & $\begin{array}{l}\text { pH } \\
\text { Groups }\end{array}$ & $\begin{array}{l}\text { Time in } \\
\text { Aging } \\
\text { Condition } \\
\text { (months) }\end{array}$ & $\begin{array}{l}\text { SR - \% } \\
\text { Strength } \\
\text { Reduction }\end{array}$ & Data Source Ref. \\
\hline VE-ILSS-T4-P2 & VE & $\mathrm{GL}$ & ILSS & T4 & P2 & 3 & $81 \%$ & (Cabral-Fonseca, 2012) \\
\hline VE-ILSS-T4-P2 & VE & $\mathrm{GL}$ & ILSS & T4 & P2 & 6 & $69 \%$ & (Cabral-Fonseca, 2012) \\
\hline VE-ILSS-T4-P2 & VE & $\mathrm{GL}$ & ILSS & T4 & P2 & 9 & $60 \%$ & (Cabral-Fonseca, 2012) \\
\hline VE-ILSS-T4-P2 & VE & $\mathrm{GL}$ & ILSS & T4 & P2 & 18 & $61 \%$ & (Cabral-Fonseca, 2012) \\
\hline VE-ILSS-TO-PO & $\mathrm{VE}$ & $\mathrm{GL}$ & ILSS & TO & $\mathrm{PO}$ & 0 & $100 \%$ & (Karbhari, 2004) \\
\hline VE-ILSS-TO-PO & VE & $\mathrm{GL}$ & ILSS & TO & PO & 7 & $100 \%$ & (Karbhari, 2004) \\
\hline VE-ILSS-TO-PO & VE & $\mathrm{GL}$ & ILSS & TO & PO & 11 & $100 \%$ & (Karbhari, 2004) \\
\hline VE-ILSS-T2-P3 & VE & $\mathrm{GL}$ & ILSS & $\mathrm{T} 2$ & P3 & 0 & $100 \%$ & (Karbhari, 2004) \\
\hline VE-ILSS-T2-P3 & VE & $\mathrm{GL}$ & ILSS & $\mathrm{T} 2$ & P3 & 1 & $93 \%$ & (Karbhari, 2004) \\
\hline VE-ILSS-T2-P3 & VE & $\mathrm{GL}$ & ILSS & $\mathrm{T} 2$ & P3 & 14 & $58 \%$ & (Karbhari, 2004) \\
\hline VE-ILSS-T2-P3 & VE & $\mathrm{GL}$ & ILSS & T2 & P3 & 18 & $57 \%$ & (Karbhari, 2004) \\
\hline VE-ILSS-T3-P3 & VE & $\mathrm{GL}$ & ILSS & T3 & P3 & 0 & $100 \%$ & (Karbhari, 2004) \\
\hline VE-ILSS-T3-P3 & VE & $\mathrm{GL}$ & ILSS & T3 & P3 & 1 & $87 \%$ & (Karbhari, 2004) \\
\hline VE-ILSS-T3-P3 & VE & $\mathrm{GL}$ & ILSS & T3 & P3 & 2 & $78 \%$ & (Karbhari, 2004) \\
\hline VE-ILSS-T3-P3 & VE & $\mathrm{GL}$ & ILSS & T3 & P3 & 3 & $75 \%$ & (Karbhari, 2004) \\
\hline VE-ILSS-T3-P3 & VE & $\mathrm{GL}$ & ILSS & T3 & P3 & 7 & $62 \%$ & (Karbhari, 2004) \\
\hline VE-ILSS-T3-P3 & VE & $\mathrm{GL}$ & ILSS & T3 & P3 & 14 & $58 \%$ & (Karbhari, 2004) \\
\hline VE-ILSS-T3-P3 & VE & $\mathrm{GL}$ & ILSS & T3 & P3 & 18 & $54 \%$ & (Karbhari, 2004) \\
\hline VE-ILSS-T4-P3 & VE & $\mathrm{GL}$ & ILSS & T4 & P3 & 0 & $100 \%$ & (Karbhari, 2004) \\
\hline VE-ILSS-T4-P3 & VE & $\mathrm{GL}$ & ILSS & T4 & P3 & 1 & $64 \%$ & (Karbhari, 2004) \\
\hline
\end{tabular}




\begin{tabular}{|c|c|c|c|c|c|c|c|c|}
\hline Sample Designation & Matrix & $\begin{array}{l}\text { Reinforce } \\
\text { ment }\end{array}$ & $\begin{array}{l}\text { Property } \\
\text { Tested }\end{array}$ & $\begin{array}{l}\text { Temp. } \\
\text { Group }\end{array}$ & $\begin{array}{l}\text { pH } \\
\text { Groups }\end{array}$ & $\begin{array}{l}\text { Time in } \\
\text { Aging } \\
\text { Condition } \\
\text { (months) }\end{array}$ & $\begin{array}{l}\text { SR - \% } \\
\text { Strength } \\
\text { Reduction }\end{array}$ & Data Source Ref. \\
\hline VE-ILSS-TO-PO & VE & $\mathrm{GL}$ & ILSS & TO & PO & 0 & $100 \%$ & (Karbhari, 2004) \\
\hline VE-ILSS-TO-PO & VE & GL & ILSS & TO & PO & 7 & $100 \%$ & (Karbhari, 2004) \\
\hline VE-ILSS-TO-PO & VE & $\mathrm{GL}$ & ILSS & TO & PO & 11 & $100 \%$ & (Karbhari, 2004) \\
\hline VE-ILSS-T2-P3 & VE & $\mathrm{GL}$ & ILSS & $\mathrm{T} 2$ & P3 & 0 & $100 \%$ & (Karbhari, 2004) \\
\hline VE-ILSS-T2-P3 & VE & $\mathrm{GL}$ & ILSS & $\mathrm{T} 2$ & P3 & 1 & $94 \%$ & (Karbhari, 2004) \\
\hline VE-ILSS-T2-P3 & VE & $\mathrm{GL}$ & ILSS & $\mathrm{T} 2$ & P3 & 2 & $95 \%$ & (Karbhari, 2004) \\
\hline VE-ILSS-T2-P3 & VE & $\mathrm{GL}$ & ILSS & $\mathrm{T} 2$ & P3 & 3 & $95 \%$ & (Karbhari, 2004) \\
\hline VE-ILSS-T2-P3 & VE & $\mathrm{GL}$ & ILSS & $\mathrm{T} 2$ & P3 & 7 & $93 \%$ & (Karbhari, 2004) \\
\hline VE-ILSS-T2-P3 & VE & $\mathrm{GL}$ & ILSS & $\mathrm{T} 2$ & P3 & 14 & $93 \%$ & (Karbhari, 2004) \\
\hline VE-ILSS-T2-P3 & VE & $\mathrm{GL}$ & ILSS & $\mathrm{T} 2$ & P3 & 18 & $89 \%$ & (Karbhari, 2004) \\
\hline VE-ILSS-T3-P3 & VE & $\mathrm{GL}$ & ILSS & T3 & P3 & 0 & $100 \%$ & (Karbhari, 2004) \\
\hline VE-ILSS-T3-P3 & VE & GL & ILSS & T3 & P3 & 1 & $94 \%$ & (Karbhari, 2004) \\
\hline VE-ILSS-T3-P3 & VE & $\mathrm{GL}$ & ILSS & T3 & P3 & 2 & $92 \%$ & (Karbhari, 2004) \\
\hline VE-ILSS-T3-P3 & VE & $\mathrm{GL}$ & ILSS & T3 & P3 & 3 & $92 \%$ & (Karbhari, 2004) \\
\hline VE-ILSS-T3-P3 & VE & $\mathrm{GL}$ & ILSS & T3 & P3 & 7 & $91 \%$ & (Karbhari, 2004) \\
\hline VE-ILSS-T3-P3 & VE & $\mathrm{GL}$ & ILSS & T3 & P3 & 14 & $91 \%$ & (Karbhari, 2004) \\
\hline VE-ILSS-T3-P3 & VE & GL & ILSS & T3 & P3 & 18 & $87 \%$ & (Karbhari, 2004) \\
\hline VE-ILSS-T4-P3 & VE & $\mathrm{GL}$ & ILSS & T4 & P3 & 0 & $100 \%$ & (Karbhari, 2004) \\
\hline VE-ILSS-T4-P3 & VE & $\mathrm{GL}$ & ILSS & T4 & P3 & 1 & $92 \%$ & (Karbhari, 2004) \\
\hline VE-ILSS-T4-P3 & VE & $\mathrm{GL}$ & ILSS & T4 & P3 & 2 & $88 \%$ & (Karbhari, 2004) \\
\hline VE-ILSS-T4-P3 & VE & $\mathrm{GL}$ & ILSS & T4 & P3 & 3 & $88 \%$ & (Karbhari, 2004) \\
\hline VE-ILSS-T4-P3 & VE & $\mathrm{GL}$ & ILSS & T4 & P3 & 7 & $90 \%$ & (Karbhari, 2004) \\
\hline VE-ILSS-T4-P3 & VE & $\mathrm{GL}$ & ILSS & T4 & P3 & 14 & $87 \%$ & (Karbhari, 2004) \\
\hline VE-ILSS-T4-P3 & VE & $\mathrm{GL}$ & ILSS & T4 & P3 & 18 & $84 \%$ & (Karbhari, 2004) \\
\hline VE-ILSS-T2-P3 & $\overline{V E}$ & $\overline{\mathrm{GL}}$ & ILSS & $\mathrm{T2}$ & $\mathrm{P3}$ & 0 & $100 \%$ & (Hammami, 2004) \\
\hline VE-ILSS-T2-P3 & VE & $\mathrm{GL}$ & ILSS & $\mathrm{T} 2$ & P3 & 3 & $93 \%$ & (Hammami, 2004) \\
\hline VE-ILSS-T2-P2 & VE & $\mathrm{GL}$ & ILSS & $\mathrm{T} 2$ & P2 & 0 & $100 \%$ & (Hammami, 2004) \\
\hline VE-ILSS-T2-P2 & VE & $\mathrm{GL}$ & ILSS & $\mathrm{T} 2$ & P2 & 3 & $100 \%$ & (Hammami, 2004) \\
\hline VE-ILSS-T2-P1 & VE & $\mathrm{GL}$ & ILSS & $\mathrm{T} 2$ & P1 & 0 & $100 \%$ & (Hammami, 2004) \\
\hline VE-ILSS-T2-P1 & VE & $\mathrm{GL}$ & ILSS & $\mathrm{T} 2$ & P1 & 3 & $92 \%$ & (Hammami, 2004) \\
\hline
\end{tabular}




\begin{tabular}{|c|c|c|c|c|c|c|c|c|}
\hline Sample Designation & Matrix & $\begin{array}{l}\text { Reinforce } \\
\text { ment }\end{array}$ & $\begin{array}{l}\text { Property } \\
\text { Tested }\end{array}$ & $\begin{array}{l}\text { Temp. } \\
\text { Group }\end{array}$ & $\begin{array}{l}\text { pH } \\
\text { Groups }\end{array}$ & $\begin{array}{l}\text { Time in } \\
\text { Aging } \\
\text { Condition } \\
\text { (months) }\end{array}$ & $\begin{array}{l}\text { SR - \% } \\
\text { Strength } \\
\text { Reduction }\end{array}$ & Data Source Ref. \\
\hline VE-ILSS-TO-PO & VE & $\mathrm{GL}$ & ILSS & TO & PO & 0 & $100 \%$ & (Dittenber/WVU-CFC, 2013) \\
\hline VE-ILSS-TO-PO & VE & GL & ILSS & TO & PO & 96 & $62 \%$ & (Dittenber/WVU-CFC, 2013) \\
\hline VE-ILSS-TO-PO & VE & $\mathrm{GL}$ & ILSS & TO & PO & 0 & $100 \%$ & (Dittenber/WVU-CFC, 2013) \\
\hline VE-ILSS-TO-PO & VE & GL & ILSS & TO & PO & 96 & $61 \%$ & (Dittenber/WVU-CFC, 2013) \\
\hline VE-ILSS-TO-PO & VE & $\mathrm{GL}$ & ILSS & TO & $\mathrm{PO}$ & 0 & $100 \%$ & (Dittenber/WVU-CFC, 2013) \\
\hline VE-ILSS-TO-PO & VE & $\mathrm{GL}$ & ILSS & TO & PO & 144 & $83 \%$ & (Dittenber/WVU-CFC, 2013) \\
\hline VE-ILSS-TO-PO & VE & $\mathrm{GL}$ & ILSS & TO & $\mathrm{PO}$ & 144 & $71 \%$ & (Baskar, 2018) \\
\hline VE-ILSS-TO-PO & VE & $\mathrm{GL}$ & ILSS & TO & $\mathrm{PO}$ & 0 & $100 \%$ & (Baskar, 2018) \\
\hline VE-ILSS-TO-PO & VE & $\mathrm{GL}$ & ILSS & TO & $\mathrm{PO}$ & 72 & $77 \%$ & (Baskar, 2018) \\
\hline VE-ILSS-TO-PO & VE & $\mathrm{GL}$ & ILSS & TO & PO & 0 & $100 \%$ & (Dittenber/WVU-CFC, 2013) \\
\hline VE-ILSS-TO-PO & VE & $\mathrm{GL}$ & ILSS & TO & PO & 144 & $45 \%$ & (Dittenber/WVU-CFC, 2013) \\
\hline VE-ILSS-TO-PO & VE & $\mathrm{GL}$ & ILSS & TO & PO & 0 & $100 \%$ & (Dittenber/WVU-CFC, 2013) \\
\hline VE-ILSS-TO-PO & VE & $\mathrm{GL}$ & ILSS & TO & PO & 144 & $63 \%$ & (Dittenber/WVU-CFC, 2013) \\
\hline VE-ILSS-TO-PO & VE & $\mathrm{GL}$ & ILSS & TO & PO & 0 & $100 \%$ & (Dittenber/WVU-CFC, 2013) \\
\hline VE-ILSS-TO-PO & VE & $\mathrm{GL}$ & ILSS & TO & PO & 144 & $76 \%$ & (Dittenber/WVU-CFC, 2013) \\
\hline VE-ILSS-TO-PO & VE & $\mathrm{GL}$ & ILSS & TO & PO & 0 & $100 \%$ & (Dittenber/WVU-CFC, 2013) \\
\hline VE-ILSS-TO-PO & VE & GL & ILSS & TO & PO & 144 & $59 \%$ & (Dittenber/WVU-CFC, 2013) \\
\hline VE-ILSS-TO-PO & VE & $\mathrm{GL}$ & ILSS & TO & $\mathrm{PO}$ & 0 & $100 \%$ & (Dittenber/WVU-CFC, 2013) \\
\hline VE-ILSS-TO-PO & VE & $\mathrm{GL}$ & ILSS & TO & PO & 108 & $89 \%$ & (Dittenber/WVU-CFC, 2013) \\
\hline VE-ILSS-TO-PO & VE & $\mathrm{GL}$ & ILSS & TO & PO & 0 & $100 \%$ & (Dittenber/WVU-CFC, 2013) \\
\hline VE-ILSS-TO-PO & VE & $\mathrm{GL}$ & ILSS & TO & PO & 120 & $91 \%$ & (Dittenber/WVU-CFC, 2013) \\
\hline
\end{tabular}

\begin{tabular}{|lllllllll|}
\hline VE-GL-FS: Vinylester - Glass FRP - Flexural Strength Testing Database \\
\hline VE-FS-T2-P2 & VE & GL & FS & T2 & P2 & 0 & $100 \%$ & (Sonawala \& Spontak, 1996) \\
\hline VE-FS-T2-P2 & VE & GL & FS & T2 & P2 & 1 & $100 \%$ & (Sonawala \& Spontak, 1996) \\
\hline VE-FS-T2-P2 & VE & GL & FS & T2 & P2 & 2 & $100 \%$ & (Sonawala \& Spontak, 1996) \\
\hline VE-FS-T2-P2 & VE & GL & FS & T2 & P2 & 3 & $92 \%$ & (Sonawala \& Spontak, 1996) \\
\hline VE-FS-T2-P2 & VE & GL & FS & T2 & P2 & 4 & $84 \%$ & (Sonawala \& Spontak, 1996) \\
\hline VE-FS-T2-P2 & VE & GL & FS & T2 & P2 & 6 & $65 \%$ & (Sonawala \& Spontak, 1996) \\
\hline VE-FS-T2-P2 & VE & GL & FS & T2 & P2 & 9 & $50 \%$ & (Sonawala \& Spontak, 1996) \\
\hline
\end{tabular}




\begin{tabular}{|c|c|c|c|c|c|c|c|c|}
\hline Sample Designation & Matrix & $\begin{array}{l}\text { Reinforce } \\
\text { ment }\end{array}$ & $\begin{array}{l}\text { Property } \\
\text { Tested }\end{array}$ & $\begin{array}{l}\text { Temp. } \\
\text { Group }\end{array}$ & \begin{tabular}{|l} 
pH \\
Groups
\end{tabular} & $\begin{array}{l}\text { Time in } \\
\text { Aging } \\
\text { Condition } \\
\text { (months) }\end{array}$ & \begin{tabular}{|l} 
SR - \% \\
Strength \\
Reduction
\end{tabular} & Data Source Ref. \\
\hline VE-FS-T2-P5 & VE & $\mathrm{GL}$ & FS & $\mathrm{T} 2$ & P5 & 0 & $100 \%$ & (Sonawala \& Spontak, 1996) \\
\hline VE-FS-T2-P5 & VE & GL & FS & $\mathrm{T} 2$ & P5 & 1 & $85 \%$ & (Sonawala \& Spontak, 1996) \\
\hline VE-FS-T2-P5 & VE & $\mathrm{GL}$ & FS & $\mathrm{T} 2$ & P5 & 2 & $77 \%$ & (Sonawala \& Spontak, 1996) \\
\hline VE-FS-T2-P5 & VE & $\mathrm{GL}$ & FS & $\mathrm{T} 2$ & P5 & 3 & $73 \%$ & (Sonawala \& Spontak, 1996) \\
\hline VE-FS-T2-P5 & VE & GL & FS & $\mathrm{T} 2$ & P5 & 4 & $71 \%$ & (Sonawala \& Spontak, 1996) \\
\hline VE-FS-T2-P5 & VE & $\mathrm{GL}$ & FS & $\mathrm{T} 2$ & P5 & 6 & $69 \%$ & (Sonawala \& Spontak, 1996) \\
\hline VE-FS-T2-P5 & VE & $\mathrm{GL}$ & FS & $\mathrm{T} 2$ & P5 & 9 & $69 \%$ & (Sonawala \& Spontak, 1996) \\
\hline VE-FS-T2-P3 & VE & GL & FS & $\mathrm{T} 2$ & P3 & 0 & $100 \%$ & (Robert, 2010) \\
\hline VE-FS-T2-P3 & VE & $\mathrm{GL}$ & FS & $\mathrm{T} 2$ & P3 & 1 & $97 \%$ & (Robert, 2010) \\
\hline VE-FS-T2-P3 & VE & $\mathrm{GL}$ & FS & $\mathrm{T} 2$ & P3 & 3 & $95 \%$ & (Robert, 2010) \\
\hline VE-FS-T2-P3 & VE & $\mathrm{GL}$ & FS & $\mathrm{T} 2$ & P3 & 4 & $92 \%$ & (Robert, 2010) \\
\hline VE-FS-T2-P3 & VE & $\mathrm{GL}$ & FS & $\mathrm{T} 2$ & P3 & 0 & $100 \%$ & (Robert, 2010) \\
\hline VE-FS-T2-P3 & VE & $\mathrm{GL}$ & FS & $\mathrm{T} 2$ & P3 & 1 & $94 \%$ & (Robert, 2010) \\
\hline VE-FS-T2-P3 & VE & $\mathrm{GL}$ & FS & $\mathrm{T} 2$ & P3 & 3 & $91 \%$ & (Robert, 2010) \\
\hline VE-FS-T2-P3 & VE & $\mathrm{GL}$ & FS & $\mathrm{T} 2$ & P3 & 4 & $89 \%$ & (Robert, 2010) \\
\hline VE-FS-T3-P3 & VE & $\mathrm{GL}$ & FS & T3 & P3 & 0 & $100 \%$ & (Robert, 2010) \\
\hline VE-FS-T3-P3 & VE & $\mathrm{GL}$ & FS & T3 & P3 & 1 & $88 \%$ & (Robert, 2010) \\
\hline VE-FS-T3-P3 & VE & GL & FS & T3 & P3 & 3 & $85 \%$ & (Robert, 2010) \\
\hline VE-FS-T3-P3 & VE & $\mathrm{GL}$ & FS & T3 & P3 & 4 & $81 \%$ & (Robert, 2010) \\
\hline VE-FS-T4-P3 & VE & $\mathrm{GL}$ & FS & T4 & P3 & 0 & $100 \%$ & (Robert, 2010) \\
\hline VE-FS-T4-P3 & VE & $\mathrm{GL}$ & FS & T4 & P3 & 1 & $80 \%$ & (Robert, 2010) \\
\hline VE-FS-T4-P3 & VE & $\mathrm{GL}$ & FS & T4 & P3 & 3 & $63 \%$ & (Robert, 2010) \\
\hline VE-FS-T4-P3 & VE & $\mathrm{GL}$ & FS & $\mathrm{T} 4$ & P3 & 4 & $54 \%$ & (Robert, 2010) \\
\hline VE-FS-T2-P3 & $\overline{V E}$ & $\overline{\mathrm{GL}}$ & FS & $\mathrm{T2}$ & P3 & $\overline{0}$ & $100 \%$ & (Cabral-Fonseca, 2012) \\
\hline VE-FS-T2-P3 & VE & $\mathrm{GL}$ & FS & $\mathrm{T} 2$ & P3 & 3 & $85 \%$ & (Cabral-Fonseca, 2012) \\
\hline VE-FS-T2-P3 & VE & $\mathrm{GL}$ & FS & $\mathrm{T} 2$ & P3 & 6 & $80 \%$ & (Cabral-Fonseca, 2012) \\
\hline VE-FS-T2-P3 & VE & GL & FS & $\mathrm{T} 2$ & P3 & 9 & $83 \%$ & (Cabral-Fonseca, 2012) \\
\hline VE-FS-T2-P3 & VE & $\mathrm{GL}$ & FS & $\mathrm{T} 2$ & P3 & 12 & $86 \%$ & (Cabral-Fonseca, 2012) \\
\hline VE-FS-T2-P3 & VE & $\mathrm{GL}$ & FS & $\mathrm{T} 2$ & P3 & 18 & $82 \%$ & (Cabral-Fonseca, 2012) \\
\hline VE-FS-T3-P3 & VE & $\mathrm{GL}$ & FS & T3 & P3 & 0 & $100 \%$ & (Cabral-Fonseca, 2012) \\
\hline
\end{tabular}




\begin{tabular}{|c|c|c|c|c|c|c|c|c|}
\hline Sample Designation & Matrix & $\begin{array}{l}\text { Reinforce } \\
\text { ment }\end{array}$ & $\begin{array}{l}\text { Property } \\
\text { Tested }\end{array}$ & $\begin{array}{l}\text { Temp. } \\
\text { Group }\end{array}$ & \begin{tabular}{|l} 
pH \\
Groups
\end{tabular} & $\begin{array}{l}\text { Time in } \\
\text { Aging } \\
\text { Condition } \\
\text { (months) }\end{array}$ & \begin{tabular}{|l} 
SR - \% \\
Strength \\
Reduction
\end{tabular} & Data Source Ref. \\
\hline VE-FS-T3-P3 & VE & $\mathrm{GL}$ & FS & T3 & P3 & 3 & $83 \%$ & (Cabral-Fonseca, 2012) \\
\hline VE-FS-T3-P3 & VE & GL & FS & T3 & P3 & 6 & $77 \%$ & (Cabral-Fonseca, 2012) \\
\hline VE-FS-T3-P3 & VE & $\mathrm{GL}$ & FS & T3 & P3 & 9 & $73 \%$ & (Cabral-Fonseca, 2012) \\
\hline VE-FS-T3-P3 & VE & $\mathrm{GL}$ & FS & T3 & P3 & 12 & $81 \%$ & (Cabral-Fonseca, 2012) \\
\hline VE-FS-T3-P3 & VE & $\mathrm{GL}$ & FS & T3 & P3 & 18 & $78 \%$ & (Cabral-Fonseca, 2012) \\
\hline VE-FS-T4-P3 & VE & $\mathrm{GL}$ & FS & T4 & P3 & 0 & $100 \%$ & (Cabral-Fonseca, 2012) \\
\hline VE-FS-T4-P3 & VE & $\mathrm{GL}$ & FS & T4 & P3 & 3 & $74 \%$ & (Cabral-Fonseca, 2012) \\
\hline VE-FS-T4-P3 & VE & $\mathrm{GL}$ & FS & T4 & P3 & 6 & $71 \%$ & (Cabral-Fonseca, 2012) \\
\hline VE-FS-T4-P3 & VE & $\mathrm{GL}$ & FS & T4 & P3 & 9 & $69 \%$ & (Cabral-Fonseca, 2012) \\
\hline VE-FS-T4-P3 & VE & GL & FS & $\mathrm{T} 4$ & P3 & 12 & $58 \%$ & (Cabral-Fonseca, 2012) \\
\hline VE-FS-T4-P3 & VE & $\mathrm{GL}$ & FS & T4 & P3 & 18 & $58 \%$ & (Cabral-Fonseca, 2012) \\
\hline VE-FS-T2-P2 & VE & $\overline{\mathrm{GL}}$ & FS & $\mathrm{T2}$ & $\overline{P 2}$ & 0 & $100 \%$ & (Cabral-Fonseca, 2012) \\
\hline VE-FS-T2-P2 & VE & $\mathrm{GL}$ & FS & $\mathrm{T} 2$ & P2 & 3 & $91 \%$ & (Cabral-Fonseca, 2012) \\
\hline VE-FS-T2-P2 & VE & $\mathrm{GL}$ & FS & $\mathrm{T} 2$ & P2 & 6 & $88 \%$ & (Cabral-Fonseca, 2012) \\
\hline VE-FS-T2-P2 & VE & GL & FS & $\mathrm{T} 2$ & P2 & 9 & $83 \%$ & (Cabral-Fonseca, 2012) \\
\hline VE-FS-T2-P2 & VE & $\mathrm{GL}$ & FS & $\mathrm{T} 2$ & P2 & 12 & $89 \%$ & (Cabral-Fonseca, 2012) \\
\hline VE-FS-T2-P2 & VE & $\mathrm{GL}$ & FS & $\mathrm{T} 2$ & P2 & 18 & $82 \%$ & (Cabral-Fonseca, 2012) \\
\hline VE-FS-T3-P2 & VE & GL & FS & T3 & P2 & 0 & $100 \%$ & (Cabral-Fonseca, 2012) \\
\hline VE-FS-T3-P2 & VE & $\mathrm{GL}$ & FS & T3 & P2 & 3 & $78 \%$ & (Cabral-Fonseca, 2012) \\
\hline VE-FS-T3-P2 & VE & $\mathrm{GL}$ & FS & T3 & P2 & 6 & $87 \%$ & (Cabral-Fonseca, 2012) \\
\hline VE-FS-T3-P2 & VE & $\mathrm{GL}$ & FS & T3 & P2 & 9 & $78 \%$ & (Cabral-Fonseca, 2012) \\
\hline VE-FS-T3-P2 & VE & $\mathrm{GL}$ & FS & T3 & P2 & 12 & $81 \%$ & (Cabral-Fonseca, 2012) \\
\hline VE-FS-T3-P2 & VE & $\mathrm{GL}$ & FS & T3 & P2 & 18 & $79 \%$ & (Cabral-Fonseca, 2012) \\
\hline VE-FS-T4-P2 & VE & $\mathrm{GL}$ & FS & T4 & P2 & 0 & $100 \%$ & (Cabral-Fonseca, 2012) \\
\hline VE-FS-T4-P2 & VE & $\mathrm{GL}$ & FS & T4 & P2 & 3 & $85 \%$ & (Cabral-Fonseca, 2012) \\
\hline VE-FS-T4-P2 & VE & $\mathrm{GL}$ & FS & T4 & P2 & 6 & $79 \%$ & (Cabral-Fonseca, 2012) \\
\hline VE-FS-T4-P2 & VE & $\mathrm{GL}$ & FS & T4 & P2 & 9 & $75 \%$ & (Cabral-Fonseca, 2012) \\
\hline VE-FS-T4-P2 & VE & $\mathrm{GL}$ & FS & T4 & P2 & 12 & $71 \%$ & (Cabral-Fonseca, 2012) \\
\hline VE-FS-T4-P2 & VE & GL & FS & T4 & P2 & 18 & $58 \%$ & (Cabral-Fonseca, 2012) \\
\hline VE-FS-T2-P5 & VE & $\overline{\mathrm{GL}}$ & FS & $\mathrm{T} 2$ & P5 & 0 & $100 \%$ & $(\operatorname{Sen}, 2002)$ \\
\hline
\end{tabular}




\begin{tabular}{|c|c|c|c|c|c|c|c|c|}
\hline Sample Designation & Matrix & $\begin{array}{l}\text { Reinforce } \\
\text { ment }\end{array}$ & $\begin{array}{l}\text { Property } \\
\text { Tested }\end{array}$ & $\begin{array}{l}\text { Temp. } \\
\text { Group }\end{array}$ & $\begin{array}{l}\text { pH } \\
\text { Groups }\end{array}$ & $\begin{array}{l}\text { Time in } \\
\text { Aging } \\
\text { Condition } \\
\text { (months) }\end{array}$ & $\begin{array}{l}\text { SR - \% } \\
\text { Strength } \\
\text { Reduction }\end{array}$ & Data Source Ref. \\
\hline VE-FS-T2-P5 & VE & GL & FS & $\mathrm{T} 2$ & P5 & 3 & $63 \%$ & (Sen, 2002) \\
\hline VE-FS-T2-P5 & VE & GL & FS & $\mathrm{T} 2$ & P5 & 6 & $64 \%$ & (Sen, 2002) \\
\hline VE-FS-T2-P5 & VE & $\mathrm{GL}$ & FS & $\mathrm{T} 2$ & P5 & 9 & $63 \%$ & $($ Sen, 2002) \\
\hline VE-FS-T2-P3 & VE & $\mathrm{GL}$ & FS & $\mathrm{T} 2$ & P3 & 0 & $100 \%$ & (Hammami, 2004) \\
\hline VE-FS-T2-P3 & VE & GL & FS & $\mathrm{T} 2$ & P3 & 3 & $78 \%$ & (Hammami, 2004) \\
\hline VE-FS-T2-P2 & VE & GL & FS & $\mathrm{T} 2$ & P2 & 0 & $100 \%$ & (Hammami, 2004) \\
\hline VE-FS-T2-P2 & VE & GL & FS & $\mathrm{T} 2$ & P2 & 3 & $89 \%$ & (Hammami, 2004) \\
\hline VE-FS-T2-P2 & VE & GL & FS & $\mathrm{T} 2$ & P2 & 6 & $86 \%$ & (Hammami, 2004) \\
\hline VE-FS-T2-P1 & VE & GL & FS & $\mathrm{T} 2$ & P1 & 0 & $100 \%$ & (Hammami, 2004) \\
\hline VE-FS-T2-P1 & VE & $\mathrm{GL}$ & FS & $\mathrm{T} 2$ & P1 & 3 & $81 \%$ & (Hammami, 2004) \\
\hline VE-FS-T2-P1 & VE & $\mathrm{GL}$ & FS & $\mathrm{T} 2$ & P1 & 6 & $75 \%$ & (Hammami, 2004) \\
\hline VE-FS-TO-PO & VE & $\mathrm{GL}$ & FS & TO & PO & 0 & $100 \%$ & (Dittenber/WVU-CFC, 2013) \\
\hline VE-FS-TO-PO & VE & $\mathrm{GL}$ & FS & TO & PO & 96 & $92 \%$ & (Dittenber/WVU-CFC, 2013) \\
\hline VE-FS-TO-PO & VE & GL & FS & TO & PO & 0 & $100 \%$ & (Dittenber/WVU-CFC, 2013) \\
\hline VE-FS-TO-PO & VE & GL & FS & TO & PO & 144 & $92 \%$ & (Dittenber/WVU-CFC, 2013) \\
\hline VE-FS-TO-PO & VE & GL & FS & TO & PO & 0 & $100 \%$ & (Dittenber/WVU-CFC, 2013) \\
\hline VE-FS-TO-PO & VE & GL & FS & TO & PO & 144 & $50 \%$ & (Dittenber/WVU-CFC, 2013) \\
\hline VE-FS-TO-PO & VE & GL & FS & TO & PO & 0 & $100 \%$ & (Dittenber/WVU-CFC, 2013) \\
\hline VE-FS-TO-PO & VE & GL & FS & TO & $\mathrm{PO}$ & 144 & $74 \%$ & (Dittenber/WVU-CFC, 2013) \\
\hline VE-FS-TO-PO & VE & GL & FS & TO & $\mathrm{PO}$ & 0 & $100 \%$ & (Dittenber/WVU-CFC, 2013) \\
\hline VE-FS-TO-PO & VE & GL & FS & TO & PO & 96 & $73 \%$ & (Dittenber/WVU-CFC, 2013) \\
\hline VE-FS-TO-PO & VE & GL & FS & TO & PO & 0 & $100 \%$ & (Dittenber/WVU-CFC, 2013) \\
\hline VE-FS-TO-PO & VE & GL & FS & TO & PO & 96 & $72 \%$ & (Dittenber/WVU-CFC, 2013) \\
\hline
\end{tabular}

\begin{tabular}{|lllllllll|}
\hline PE-GL-TS: Polyester - Glass FRP - Tension Strength Testing Database & & & \\
\hline PE-TS-T2-P2 & PE & GL & TS & T2 & P2 & 0 & $100 \%$ & (Sonawala \& Spontak, 1996) \\
PE-TS-T2-P2 & PE & GL & TS & T2 & P2 & 1 & $94 \%$ & (Sonawala \& Spontak, 1996) \\
PE-TS-T2-P2 & PE & GL & TS & T2 & P2 & 2 & $90 \%$ & (Sonawala \& Spontak, 1996) \\
PE-TS-T2-P2 & PE & GL & TS & T2 & P2 & 3 & $87 \%$ & (Sonawala \& Spontak, 1996) \\
\hline PE-TS-T2-P2 & PE & GL & TS & T2 & P2 & 4 & $82 \%$ & (Sonawala \& Spontak, 1996) \\
\hline
\end{tabular}




\begin{tabular}{|c|c|c|c|c|c|c|c|c|}
\hline Sample Designation & Matrix & $\begin{array}{l}\text { Reinforce } \\
\text { ment }\end{array}$ & $\begin{array}{l}\text { Property } \\
\text { Tested }\end{array}$ & $\begin{array}{l}\text { Temp. } \\
\text { Group }\end{array}$ & \begin{tabular}{|l} 
pH \\
Groups
\end{tabular} & $\begin{array}{l}\text { Time in } \\
\text { Aging } \\
\text { Condition } \\
\text { (months) }\end{array}$ & $\begin{array}{l}\text { SR - \% } \\
\text { Strength } \\
\text { Reduction }\end{array}$ & Data Source Ref. \\
\hline PE-TS-T2-P2 & $\mathrm{PE}$ & $\mathrm{GL}$ & TS & $\mathrm{T} 2$ & P2 & 6 & $76 \%$ & (Sonawala \& Spontak, 1996) \\
\hline PE-TS-T2-P2 & PE & GL & TS & $\mathrm{T} 2$ & P2 & 9 & $69 \%$ & (Sonawala \& Spontak, 1996) \\
\hline PE-TS-T2-P5 & $\mathrm{PE}$ & $\mathrm{GL}$ & TS & $\mathrm{T} 2$ & P5 & 0 & $100 \%$ & (Sonawala \& Spontak, 1996) \\
\hline PE-TS-T2-P5 & PE & $\mathrm{GL}$ & TS & $\mathrm{T} 2$ & P5 & 1 & $15 \%$ & (Sonawala \& Spontak, 1996) \\
\hline PE-TS-T2-P5 & $\mathrm{PE}$ & GL & TS & $\mathrm{T} 2$ & P5 & 2 & $12 \%$ & (Sonawala \& Spontak, 1996) \\
\hline PE-TS-T2-P5 & PE & $\mathrm{GL}$ & TS & $\mathrm{T} 2$ & P5 & 3 & $11 \%$ & (Sonawala \& Spontak, 1996) \\
\hline PE-TS-T2-P5 & PE & $\mathrm{GL}$ & TS & $\mathrm{T} 2$ & P5 & 4 & $11 \%$ & (Sonawala \& Spontak, 1996) \\
\hline PE-TS-T2-P5 & $P E$ & $\mathrm{GL}$ & TS & $\mathrm{T} 2$ & P5 & 6 & $10 \%$ & (Sonawala \& Spontak, 1996) \\
\hline PE-TS-T2-P5 & PE & $\mathrm{GL}$ & TS & $\mathrm{T} 2$ & P5 & 9 & $10 \%$ & (Sonawala \& Spontak, 1996) \\
\hline PE-TS-T2-P3 & $P E$ & GL & TS & $\mathrm{T} 2$ & P3 & 0 & $100 \%$ & (Cabral-Fonseca, 2012) \\
\hline PE-TS-T2-P3 & $\mathrm{PE}$ & $\mathrm{GL}$ & TS & $\mathrm{T} 2$ & P3 & 3 & $86 \%$ & (Cabral-Fonseca, 2012) \\
\hline PE-TS-T2-P3 & PE & $\mathrm{GL}$ & TS & $\mathrm{T} 2$ & P3 & 6 & $89 \%$ & (Cabral-Fonseca, 2012) \\
\hline PE-TS-T2-P3 & PE & $\mathrm{GL}$ & TS & $\mathrm{T} 2$ & P3 & 9 & $82 \%$ & (Cabral-Fonseca, 2012) \\
\hline PE-TS-T2-P3 & PE & GL & TS & $\mathrm{T} 2$ & P3 & 12 & $87 \%$ & (Cabral-Fonseca, 2012) \\
\hline PE-TS-T2-P3 & PE & GL & TS & $\mathrm{T} 2$ & P3 & 18 & $92 \%$ & (Cabral-Fonseca, 2012) \\
\hline PE-TS-T3-P3 & PE & $\mathrm{GL}$ & TS & T3 & P3 & 0 & $100 \%$ & (Cabral-Fonseca, 2012) \\
\hline PE-TS-T3-P3 & $\mathrm{PE}$ & GL & TS & T3 & P3 & 3 & $90 \%$ & (Cabral-Fonseca, 2012) \\
\hline PE-TS-T3-P3 & $\mathrm{PE}$ & GL & TS & T3 & P3 & 6 & $80 \%$ & (Cabral-Fonseca, 2012) \\
\hline PE-TS-T3-P3 & $\mathrm{PE}$ & $\mathrm{GL}$ & TS & T3 & P3 & 9 & $78 \%$ & (Cabral-Fonseca, 2012) \\
\hline PE-TS-T3-P3 & PE & $\mathrm{GL}$ & TS & T3 & P3 & 12 & $91 \%$ & (Cabral-Fonseca, 2012) \\
\hline PE-TS-T3-P3 & $\mathrm{PE}$ & $\mathrm{GL}$ & TS & T3 & P3 & 18 & $83 \%$ & (Cabral-Fonseca, 2012) \\
\hline PE-TS-T4-P3 & PE & GL & TS & T4 & P3 & 0 & $100 \%$ & (Cabral-Fonseca, 2012) \\
\hline PE-TS-T4-P3 & PE & $\mathrm{GL}$ & TS & $\mathrm{T} 4$ & P3 & 3 & $79 \%$ & (Cabral-Fonseca, 2012) \\
\hline PE-TS-T4-P3 & PE & $\mathrm{GL}$ & TS & T4 & P3 & 6 & $64 \%$ & (Cabral-Fonseca, 2012) \\
\hline PE-TS-T4-P3 & $\mathrm{PE}$ & $\mathrm{GL}$ & TS & T4 & P3 & 9 & $63 \%$ & (Cabral-Fonseca, 2012) \\
\hline PE-TS-T4-P3 & $\mathrm{PE}$ & $\mathrm{GL}$ & TS & T4 & P3 & 12 & $65 \%$ & (Cabral-Fonseca, 2012) \\
\hline PE-TS-T4-P3 & $\mathrm{PE}$ & $\mathrm{GL}$ & TS & T4 & P3 & 18 & $59 \%$ & (Cabral-Fonseca, 2012) \\
\hline PE-TS-T2-P2 & $\mathrm{PE}$ & $\mathrm{GL}$ & TS & $\mathrm{T} 2$ & P2 & 0 & $100 \%$ & (Cabral-Fonseca, 2012) \\
\hline PE-TS-T2-P2 & $\mathrm{PE}$ & GL & TS & $\mathrm{T} 2$ & P2 & 3 & $92 \%$ & (Cabral-Fonseca, 2012) \\
\hline PE-TS-T2-P2 & $\mathrm{PE}$ & $\mathrm{GL}$ & TS & $\mathrm{T} 2$ & P2 & 6 & $86 \%$ & (Cabral-Fonseca, 2012) \\
\hline
\end{tabular}




\begin{tabular}{|c|c|c|c|c|c|c|c|c|}
\hline Sample Designation & Matrix & $\begin{array}{l}\text { Reinforce } \\
\text { ment }\end{array}$ & $\begin{array}{l}\text { Property } \\
\text { Tested }\end{array}$ & $\begin{array}{l}\text { Temp. } \\
\text { Group }\end{array}$ & $\begin{array}{l}\mathrm{pH} \\
\text { Groups }\end{array}$ & $\begin{array}{l}\text { Time in } \\
\text { Aging } \\
\text { Condition } \\
\text { (months) } \\
\end{array}$ & \begin{tabular}{|l} 
SR - \% \\
Strength \\
Reduction
\end{tabular} & Data Source Ref. \\
\hline PE-TS-T2-P2 & $\mathrm{PE}$ & $\mathrm{GL}$ & TS & $\mathrm{T} 2$ & P2 & 12 & $86 \%$ & (Cabral-Fonseca, 2012) \\
\hline PE-TS-T2-P2 & $\mathrm{PE}$ & $\mathrm{GL}$ & TS & $\mathrm{T} 2$ & $\mathrm{P} 2$ & 18 & $90 \%$ & (Cabral-Fonseca, 2012) \\
\hline PE-TS-T3-P2 & $\mathrm{PE}$ & $\mathrm{GL}$ & TS & T3 & P2 & 0 & $100 \%$ & (Cabral-Fonseca, 2012) \\
\hline PE-TS-T3-P2 & $\mathrm{PE}$ & GL & TS & T3 & P2 & 6 & $82 \%$ & (Cabral-Fonseca, 2012) \\
\hline PE-TS-T3-P2 & $\mathrm{PE}$ & GL & TS & T3 & $\mathrm{P} 2$ & 9 & $83 \%$ & (Cabral-Fonseca, 2012) \\
\hline PE-TS-T3-P2 & PE & $\mathrm{GL}$ & TS & T3 & P2 & 18 & $82 \%$ & (Cabral-Fonseca, 2012) \\
\hline PE-TS-T4-P2 & $\mathrm{PE}$ & GL & TS & T4 & P2 & 0 & $100 \%$ & (Cabral-Fonseca, 2012) \\
\hline PE-TS-T4-P2 & PE & GL & TS & T4 & P2 & 3 & $91 \%$ & (Cabral-Fonseca, 2012) \\
\hline PE-TS-T4-P2 & $\mathrm{PE}$ & $\mathrm{GL}$ & TS & T4 & P2 & 6 & $80 \%$ & (Cabral-Fonseca, 2012) \\
\hline PE-TS-T3-P3 & $\overline{P E}$ & $\overline{G L}$ & TS & T3 & P3 & 0 & $100 \%$ & (Cabral-Fonseca, 2012) \\
\hline PE-TS-T3-P3 & $\mathrm{PE}$ & $\mathrm{GL}$ & TS & T3 & P3 & 3 & $88 \%$ & (Cabral-Fonseca, 2012) \\
\hline PE-TS-T3-P3 & $\mathrm{PE}$ & GL & TS & T3 & P3 & 6 & $75 \%$ & (Cabral-Fonseca, 2012) \\
\hline PE-TS-T3-P3 & $\mathrm{PE}$ & $\mathrm{GL}$ & TS & T3 & P3 & 9 & $69 \%$ & (Cabral-Fonseca, 2012) \\
\hline PE-TS-T2-P3 & $\mathrm{PE}$ & $\mathrm{GL}$ & TS & $\mathrm{T} 2$ & P3 & 0 & $100 \%$ & (Grammatikos, 2016) \\
\hline PE-TS-T2-P3 & PE & GL & TS & $\mathrm{T} 2$ & P3 & 1 & $99 \%$ & (Grammatikos, 2016) \\
\hline PE-TS-T2-P3 & $\mathrm{PE}$ & GL & TS & $\mathrm{T} 2$ & P3 & 2 & $96 \%$ & (Grammatikos, 2016) \\
\hline PE-TS-T2-P3 & PE & $\mathrm{GL}$ & TS & $\mathrm{T} 2$ & P3 & 4 & $99 \%$ & (Grammatikos, 2016) \\
\hline PE-TS-T2-P3 & $\mathrm{PE}$ & $\mathrm{GL}$ & TS & T2 & P3 & 1 & $100 \%$ & (Grammatikos, 2016) \\
\hline PE-TS-T2-P3 & $\mathrm{PE}$ & $\mathrm{GL}$ & TS & $\mathrm{T} 2$ & P3 & 2 & $97 \%$ & (Grammatikos, 2016) \\
\hline PE-TS-T2-P3 & $\mathrm{PE}$ & $\mathrm{GL}$ & TS & T2 & P3 & 4 & $97 \%$ & (Grammatikos, 2016) \\
\hline
\end{tabular}




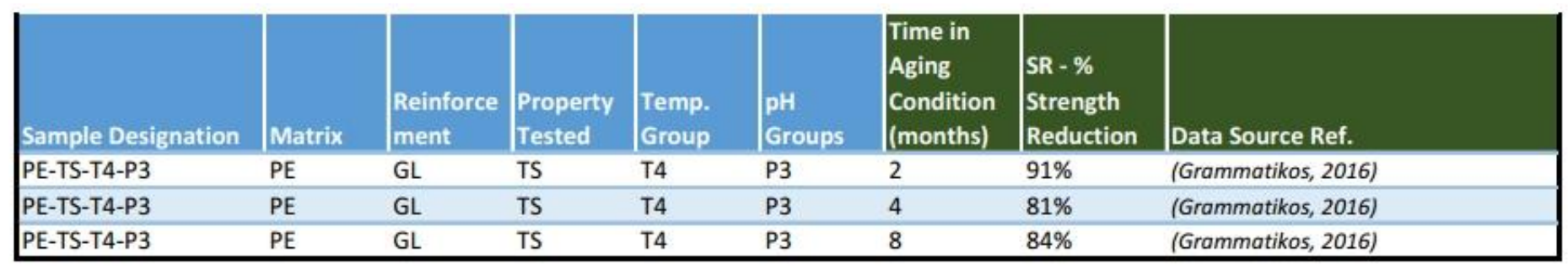

\begin{tabular}{|c|c|c|c|c|c|c|c|c|}
\hline \multicolumn{9}{|c|}{ PE-GL-ILSS: Polyester - Glass FRP - Interlaminar Shear Strength Testing Database } \\
\hline PE-ILSS-T2-P3 & $\mathrm{PE}$ & GL & ILSS & $\mathrm{T} 2$ & P3 & 0 & $100 \%$ & (Chin et. al., 2001) \\
\hline PE-ILSS-T2-P3 & PE & GL & ILSS & $\mathrm{T} 2$ & P3 & 3 & $95 \%$ & (Chin et. al., 2001) \\
\hline PE-ILSS-T2-P3 & PE & $\mathrm{GL}$ & ILSS & $\mathrm{T} 2$ & P3 & 6 & $85 \%$ & (Chin et. al., 2001) \\
\hline PE-ILSS-T2-P3 & $\mathrm{PE}$ & GL & ILSS & $\mathrm{T} 2$ & P3 & 8 & $85 \%$ & (Chin et. al., 2001) \\
\hline PE-ILSS-T2-P3 & $\mathrm{PE}$ & $\mathrm{GL}$ & ILSS & $\mathrm{T} 2$ & P3 & 14 & $75 \%$ & (Chin et. al., 2001) \\
\hline PE-ILSS-T3-P3 & $\mathrm{PE}$ & GL & ILSS & T3 & P3 & 0 & $100 \%$ & (Chin et. al., 2001) \\
\hline PE-ILSS-T3-P3 & PE & GL & ILSS & T3 & P3 & 3 & $83 \%$ & (Chin et. al., 2001) \\
\hline PE-ILSS-T3-P3 & $\mathrm{PE}$ & $\mathrm{GL}$ & ILSS & T3 & P3 & 6 & $65 \%$ & (Chin et. al., 2001) \\
\hline PE-ILSS-T3-P3 & PE & GL & ILSS & T3 & P3 & 8 & $60 \%$ & (Chin et. al., 2001) \\
\hline PE-ILSS-T3-P3 & $\mathrm{PE}$ & $\mathrm{GL}$ & ILSS & T3 & P3 & 14 & $55 \%$ & (Chin et. al., 2001) \\
\hline PE-ILSS-T3-P3 & $\mathrm{PE}$ & $\mathrm{GL}$ & ILSS & T3 & P3 & 0 & $100 \%$ & (Chin et. al., 2001) \\
\hline PE-ILSS-T3-P3 & $\mathrm{PE}$ & GL & ILSS & T3 & P3 & 3 & $50 \%$ & (Chin et. al., 2001) \\
\hline PE-ILSS-T3-P3 & $\mathrm{PE}$ & GL & ILSS & T3 & P3 & 6 & $40 \%$ & (Chin et. al., 2001) \\
\hline PE-ILSS-T3-P3 & $\mathrm{PE}$ & $\mathrm{GL}$ & ILSS & T3 & P3 & 8 & $45 \%$ & (Chin et. al., 2001) \\
\hline PE-ILSS-T3-P3 & PE & $\mathrm{GL}$ & ILSS & T3 & P3 & 14 & $45 \%$ & (Chin et. al., 2001) \\
\hline PE-ILSS-T4-P3 & $\mathrm{PE}$ & GL & ILSS & T4 & P3 & 0 & $100 \%$ & (Chin et. al., 2001) \\
\hline PE-ILSS-T4-P3 & PE & GL & ILSS & T4 & P3 & 3 & $40 \%$ & (Chin et. al., 2001) \\
\hline PE-ILSS-T4-P3 & $\mathrm{PE}$ & $\mathrm{GL}$ & ILSS & T4 & P3 & 6 & $39 \%$ & (Chin et. al., 2001) \\
\hline PE-ILSS-T4-P3 & $\mathrm{PE}$ & $\mathrm{GL}$ & ILSS & T4 & P3 & 8 & $30 \%$ & (Chin et. al., 2001) \\
\hline PE-ILSS-T4-P3 & $\mathrm{PE}$ & $\mathrm{GL}$ & ILSS & $\mathrm{T} 4$ & P3 & 14 & $19 \%$ & (Chin et. al., 2001) \\
\hline PE-ILSS-T2-P2 & $\mathrm{PE}$ & $\mathrm{GL}$ & ILSS & $\mathrm{T} 2$ & P2 & 0 & $100 \%$ & (Chin et. al., 2001) \\
\hline PE-ILSS-T2-P2 & $\mathrm{PE}$ & GL & ILSS & $\mathrm{T} 2$ & P2 & 3 & $82 \%$ & (Chin et. al., 2001) \\
\hline PE-ILSS-T2-P2 & $\mathrm{PE}$ & $\mathrm{GL}$ & ILSS & $\mathrm{T} 2$ & P2 & 6 & $79 \%$ & (Chin et. al., 2001) \\
\hline PE-ILSS-T2-P2 & $\mathrm{PE}$ & $\mathrm{GL}$ & ILSS & $\mathrm{T} 2$ & P2 & 8 & $83 \%$ & (Chin et. al., 2001) \\
\hline PE-ILSS-T2-P2 & $\mathrm{PE}$ & $\mathrm{GL}$ & ILSS & $\mathrm{T} 2$ & P2 & 14 & $83 \%$ & (Chin et. al., 2001) \\
\hline
\end{tabular}




\begin{tabular}{|c|c|c|c|c|c|c|c|c|}
\hline Sample Designation & Matrix & $\begin{array}{l}\text { Reinforce } \\
\text { ment }\end{array}$ & $\begin{array}{l}\text { Property } \\
\text { Tested }\end{array}$ & $\begin{array}{l}\text { Temp. } \\
\text { Group }\end{array}$ & \begin{tabular}{|l} 
pH \\
Groups
\end{tabular} & $\begin{array}{l}\text { Time in } \\
\text { Aging } \\
\text { Condition } \\
\text { (months) }\end{array}$ & $\begin{array}{l}\text { SR - \% } \\
\text { Strength } \\
\text { Reduction }\end{array}$ & Data Source Ref. \\
\hline PE-ILSS-T3-P2 & $\mathrm{PE}$ & $\mathrm{GL}$ & ILSS & T3 & P2 & 0 & $100 \%$ & (Chin et. al., 2001) \\
\hline PE-ILSS-T3-P2 & PE & GL & ILSS & T3 & P2 & 3 & $70 \%$ & (Chin et. al., 2001) \\
\hline PE-ILSS-T3-P2 & PE & $\mathrm{GL}$ & ILSS & T3 & P2 & 6 & $70 \%$ & (Chin et. al., 2001) \\
\hline PE-ILSS-T3-P2 & PE & $\mathrm{GL}$ & ILSS & T3 & P2 & 8 & $58 \%$ & (Chin et. al., 2001) \\
\hline PE-ILSS-T3-P2 & $\mathrm{PE}$ & $\mathrm{GL}$ & ILSS & T3 & P2 & 14 & $59 \%$ & (Chin et. al., 2001) \\
\hline PE-ILSS-T3-P2 & $\mathrm{PE}$ & $\mathrm{GL}$ & ILSS & T3 & P2 & 0 & $100 \%$ & (Chin et. al., 2001) \\
\hline PE-ILSS-T3-P2 & PE & $\mathrm{GL}$ & ILSS & T3 & P2 & 3 & $38 \%$ & (Chin et. al., 2001) \\
\hline PE-ILSS-T3-P2 & $P E$ & $\mathrm{GL}$ & ILSS & T3 & P2 & 6 & $40 \%$ & (Chin et. al., 2001) \\
\hline PE-ILSS-T3-P2 & PE & $\mathrm{GL}$ & ILSS & T3 & P2 & 8 & $42 \%$ & (Chin et. al., 2001) \\
\hline PE-ILSS-T3-P2 & $P E$ & GL & ILSS & T3 & P2 & 14 & $39 \%$ & (Chin et. al., 2001) \\
\hline PE-ILSS-T4-P2 & PE & $\mathrm{GL}$ & ILSS & T4 & P2 & 0 & $100 \%$ & (Chin et. al., 2001) \\
\hline PE-ILSS-T4-P2 & PE & $\mathrm{GL}$ & ILSS & T4 & P2 & 3 & $38 \%$ & (Chin et. al., 2001) \\
\hline PE-ILSS-T4-P2 & PE & $\mathrm{GL}$ & ILSS & T4 & P2 & 6 & $30 \%$ & (Chin et. al., 2001) \\
\hline PE-ILSS-T4-P2 & PE & GL & ILSS & T4 & P2 & 8 & $25 \%$ & (Chin et. al., 2001) \\
\hline PE-ILSS-T4-P2 & PE & GL & ILSS & T4 & P2 & 14 & $20 \%$ & (Chin et. al., 2001) \\
\hline PE-ILSS-T2-P5 & PE & $\mathrm{GL}$ & ILSS & $\mathrm{T} 2$ & P5 & 0 & $100 \%$ & (Chin et. al., 2001) \\
\hline PE-ILSS-T2-P5 & $\mathrm{PE}$ & $\mathrm{GL}$ & ILSS & $\mathrm{T} 2$ & P5 & 3 & $40 \%$ & (Chin et. al., 2001) \\
\hline PE-ILSS-T2-P5 & $\mathrm{PE}$ & GL & ILSS & $\mathrm{T} 2$ & P5 & 6 & $43 \%$ & (Chin et. al., 2001) \\
\hline PE-ILSS-T2-P5 & $\mathrm{PE}$ & $\mathrm{GL}$ & ILSS & $\mathrm{T} 2$ & P5 & 8 & $30 \%$ & (Chin et. al., 2001) \\
\hline PE-ILSS-T2-P5 & PE & $\mathrm{GL}$ & ILSS & $\mathrm{T} 2$ & P5 & 14 & $39 \%$ & (Chin et. al., 2001) \\
\hline PE-ILSS-T3-P5 & $\mathrm{PE}$ & $\mathrm{GL}$ & ILSS & T3 & P5 & 0 & $100 \%$ & (Chin et. al., 2001) \\
\hline PE-ILSS-T3-P5 & PE & $\mathrm{GL}$ & ILSS & T3 & P5 & 3 & $19 \%$ & (Chin et. al., 2001) \\
\hline PE-ILSS-T3-P5 & PE & $\mathrm{GL}$ & ILSS & T3 & P5 & 6 & $20 \%$ & (Chin et. al., 2001) \\
\hline PE-ILSS-T3-P5 & PE & $\mathrm{GL}$ & ILSS & T3 & P5 & 8 & $25 \%$ & (Chin et. al., 2001) \\
\hline PE-ILSS-T3-P5 & $\mathrm{PE}$ & $\mathrm{GL}$ & ILSS & T3 & P5 & 14 & $18 \%$ & (Chin et. al., 2001) \\
\hline PE-ILSS-T3-P5 & $\mathrm{PE}$ & $\mathrm{GL}$ & ILSS & T3 & P5 & 0 & $100 \%$ & (Chin et. al., 2001) \\
\hline PE-ILSS-T3-P5 & $\mathrm{PE}$ & $\mathrm{GL}$ & ILSS & T3 & P5 & 3 & $30 \%$ & (Chin et. al., 2001) \\
\hline PE-ILSS-T3-P5 & $\mathrm{PE}$ & $\mathrm{GL}$ & ILSS & T3 & P5 & 6 & $27 \%$ & (Chin et. al., 2001) \\
\hline PE-ILSS-T3-P5 & $\mathrm{PE}$ & GL & ILSS & T3 & P5 & 8 & $20 \%$ & (Chin et. al., 2001) \\
\hline PE-ILSS-T3-P5 & $\mathrm{PE}$ & $\mathrm{GL}$ & ILSS & T3 & P5 & 14 & $18 \%$ & (Chin et. al., 2001) \\
\hline
\end{tabular}




\begin{tabular}{|c|c|c|c|c|c|c|c|c|}
\hline Sample Designation & Matrix & $\begin{array}{l}\text { Reinforce } \\
\text { ment }\end{array}$ & $\begin{array}{l}\text { Property } \\
\text { Tested }\end{array}$ & $\begin{array}{l}\text { Temp. } \\
\text { Group }\end{array}$ & $\begin{array}{l}\text { pH } \\
\text { Groups }\end{array}$ & $\begin{array}{l}\text { Time in } \\
\text { Aging } \\
\text { Condition } \\
\text { (months) }\end{array}$ & $\begin{array}{l}\text { SR - \% } \\
\text { Strength } \\
\text { Reduction }\end{array}$ & Data Source Ref. \\
\hline PE-ILSS-T4-P5 & $\mathrm{PE}$ & $\mathrm{GL}$ & ILSS & T4 & P5 & 3 & $20 \%$ & (Chin et. al., 2001) \\
\hline PE-ILSS-T4-P5 & PE & $\mathrm{GL}$ & ILSS & T4 & P5 & 6 & $19 \%$ & (Chin et. al., 2001) \\
\hline PE-ILSS-T4-P5 & PE & $\mathrm{GL}$ & ILSS & T4 & P5 & 8 & $13 \%$ & (Chin et. al., 2001) \\
\hline PE-ILSS-T2-P3 & $\overline{P E}$ & $\mathrm{GL}$ & ILSS & $\mathrm{T} 2$ & P3 & 0 & $100 \%$ & (Cabral-Fonseca, 2012) \\
\hline PE-ILSS-T2-P3 & $\mathrm{PE}$ & $\mathrm{GL}$ & ILSS & $\mathrm{T} 2$ & P3 & 3 & $80 \%$ & (Cabral-Fonseca, 2012) \\
\hline PE-ILSS-T2-P3 & $P E$ & $\mathrm{GL}$ & ILSS & $\mathrm{T} 2$ & P3 & 6 & $85 \%$ & (Cabral-Fonseca, 2012) \\
\hline PE-ILSS-T2-P3 & $\mathrm{PE}$ & $\mathrm{GL}$ & ILSS & $\mathrm{T} 2$ & P3 & 9 & $78 \%$ & (Cabral-Fonseca, 2012) \\
\hline PE-ILSS-T2-P3 & $P E$ & $\mathrm{GL}$ & ILSS & $\mathrm{T} 2$ & P3 & 12 & $81 \%$ & (Cabral-Fonseca, 2012) \\
\hline PE-ILSS-T2-P3 & $\mathrm{PE}$ & $\mathrm{GL}$ & ILSS & $\mathrm{T} 2$ & P3 & 18 & $88 \%$ & (Cabral-Fonseca, 2012) \\
\hline PE-ILSS-T3-P3 & $\mathrm{PE}$ & $\mathrm{GL}$ & ILSS & T3 & P3 & 9 & $62 \%$ & (Cabral-Fonseca, 2012) \\
\hline PE-ILSS-T3-P3 & PE & GL & ILSS & T3 & P3 & 12 & $67 \%$ & (Cabral-Fonseca, 2012) \\
\hline PE-ILSS-T3-P3 & PE & $\mathrm{GL}$ & ILSS & T3 & P3 & 18 & $71 \%$ & (Cabral-Fonseca, 2012) \\
\hline PE-ILSS-T4-P3 & $P E$ & $\mathrm{GL}$ & ILSS & T4 & P3 & 0 & $100 \%$ & (Cabral-Fonseca, 2012) \\
\hline PE-ILSS-T4-P3 & $\mathrm{PE}$ & $\mathrm{GL}$ & ILSS & T4 & P3 & 3 & $59 \%$ & (Cabral-Fonseca, 2012) \\
\hline PE-ILSS-T4-P3 & PE & $\mathrm{GL}$ & ILSS & T4 & P3 & 6 & $53 \%$ & (Cabral-Fonseca, 2012) \\
\hline PE-ILSS-T4-P3 & $\mathrm{PE}$ & $\mathrm{GL}$ & ILSS & T4 & P3 & 9 & $52 \%$ & (Cabral-Fonseca, 2012) \\
\hline PE-ILSS-T4-P3 & PE & $\mathrm{GL}$ & ILSS & T4 & P3 & 12 & $54 \%$ & (Cabral-Fonseca, 2012) \\
\hline PE-ILSS-T4-P3 & $\mathrm{PE}$ & $\mathrm{GL}$ & ILSS & $\mathrm{T} 4$ & P3 & 18 & $58 \%$ & (Cabral-Fonseca, 2012) \\
\hline PE-ILSS-T2-P2 & $\mathrm{PE}$ & $\overline{G L}$ & ILSS & $\mathrm{T} 2$ & P2 & 0 & $100 \%$ & (Cabral-Fonseca, 2012) \\
\hline PE-ILSS-T2-P2 & PE & $\mathrm{GL}$ & ILSS & $\mathrm{T} 2$ & P2 & 3 & $81 \%$ & (Cabral-Fonseca, 2012) \\
\hline
\end{tabular}




\begin{tabular}{|c|c|c|c|c|c|c|c|c|}
\hline Sample Designation & Matrix & $\begin{array}{l}\text { Reinforce } \\
\text { ment }\end{array}$ & $\begin{array}{l}\text { Property } \\
\text { Tested }\end{array}$ & $\begin{array}{l}\text { Temp. } \\
\text { Group }\end{array}$ & $\begin{array}{l}\text { pH } \\
\text { Groups }\end{array}$ & \begin{tabular}{|l} 
Time in \\
Aging \\
Condition \\
(months)
\end{tabular} & $\begin{array}{l}\text { SR - \% } \\
\text { Strength } \\
\text { Reduction }\end{array}$ & Data Source Ref. \\
\hline PE-ILSS-T3-P2 & PE & $\mathrm{GL}$ & ILSS & T3 & P2 & 6 & $79 \%$ & (Cabral-Fonseca, 2012) \\
\hline PE-ILSS-T3-P2 & PE & $\mathrm{GL}$ & ILSS & T3 & P2 & 9 & $67 \%$ & (Cabral-Fonseca, 2012) \\
\hline PE-ILSS-T3-P2 & PE & $\mathrm{GL}$ & ILSS & T3 & P2 & 12 & $79 \%$ & (Cabral-Fonseca, 2012) \\
\hline PE-ILSS-T4-P2 & $\mathrm{PE}$ & $\mathrm{GL}$ & ILSS & T4 & P2 & 0 & $100 \%$ & (Cabral-Fonseca, 2012) \\
\hline PE-ILSS-T4-P2 & $\mathrm{PE}$ & $\mathrm{GL}$ & ILSS & T4 & P2 & 3 & $63 \%$ & (Cabral-Fonseca, 2012) \\
\hline PE-ILSS-T4-P2 & PE & $\mathrm{GL}$ & ILSS & T4 & P2 & 6 & $55 \%$ & (Cabral-Fonseca, 2012) \\
\hline PE-ILSS-T4-P2 & PE & $\mathrm{GL}$ & ILSS & T4 & P2 & 9 & $55 \%$ & (Cabral-Fonseca, 2012) \\
\hline PE-ILSS-T4-P2 & PE & GL & ILSS & T4 & P2 & 12 & $59 \%$ & (Cabral-Fonseca, 2012) \\
\hline PE-ILSS-T4-P2 & $\mathrm{PE}$ & $\mathrm{GL}$ & ILSS & $\mathrm{T} 4$ & $\mathrm{P} 2$ & 18 & $57 \%$ & (Cabral-Fonseca, 2012) \\
\hline PE-ILSS-T3-P3 & PE & $\mathrm{GL}$ & ILSS & T3 & P3 & 9 & $65 \%$ & (Cabral-Fonseca, 2012) \\
\hline PE-ILSS-T3-P3 & PE & $\mathrm{GL}$ & ILSS & T3 & P3 & 0 & $100 \%$ & (Cabral-Fonseca, 2012) \\
\hline PE-ILSS-T3-P3 & PE & GL & ILSS & T3 & P3 & 3 & $91 \%$ & (Cabral-Fonseca, 2012) \\
\hline PE-ILSS-T3-P3 & PE & $\mathrm{GL}$ & ILSS & T3 & P3 & 6 & $88 \%$ & (Cabral-Fonseca, 2012) \\
\hline PE-ILSS-T3-P3 & $\mathrm{PE}$ & $\mathrm{GL}$ & ILSS & T3 & P3 & 9 & $74 \%$ & (Cabral-Fonseca, 2012) \\
\hline PE-ILSS-T2-P3 & $\mathrm{PE}$ & $\mathrm{GL}$ & ILSS & T2 & P3 & 0 & $100 \%$ & (Grammatikos, 2016) \\
\hline PE-ILSS-T2-P3 & $\mathrm{PE}$ & GL & ILSS & T2 & P3 & 1 & $86 \%$ & (Grammatikos, 2016) \\
\hline PE-ILSS-T2-P3 & PE & $\mathrm{GL}$ & ILSS & $\mathrm{T} 2$ & P3 & 2 & $83 \%$ & (Grammatikos, 2016) \\
\hline PE-ILSS-T2-P3 & $\mathrm{PE}$ & $\mathrm{GL}$ & ILSS & T2 & P3 & 4 & $80 \%$ & (Grammatikos, 2016) \\
\hline PE-ILSS-T2-P3 & $\mathrm{PE}$ & $\mathrm{GL}$ & ILSS & $\mathrm{T} 2$ & P3 & 0 & $100 \%$ & (Grammatikos, 2016) \\
\hline PE-ILSS-T2-P3 & PE & $\mathrm{GL}$ & ILSS & $\mathrm{T} 2$ & P3 & 1 & $95 \%$ & (Grammatikos, 2016) \\
\hline
\end{tabular}




\begin{tabular}{|c|c|c|c|c|c|c|c|c|}
\hline Sample Designation & Matrix & $\begin{array}{l}\text { Reinforce } \\
\text { ment }\end{array}$ & $\begin{array}{l}\text { Property } \\
\text { Tested }\end{array}$ & $\begin{array}{l}\text { Temp. } \\
\text { Group }\end{array}$ & \begin{tabular}{|l} 
pH \\
Groups
\end{tabular} & $\begin{array}{l}\text { Time in } \\
\text { Aging } \\
\text { Condition } \\
\text { (months) }\end{array}$ & $\begin{array}{l}\text { SR - \% } \\
\text { Strength } \\
\text { Reduction }\end{array}$ & Data Source Ref. \\
\hline PE-ILSS-T3-P3 & $\mathrm{PE}$ & $\mathrm{GL}$ & ILSS & T3 & P3 & 2 & $80 \%$ & (Grammatikos, 2016) \\
\hline PE-ILSS-T3-P3 & $\mathrm{PE}$ & GL & ILSS & T3 & P3 & 4 & $70 \%$ & (Grammatikos, 2016) \\
\hline PE-ILSS-T3-P3 & $\mathrm{PE}$ & GL & ILSS & T3 & P3 & 8 & $94 \%$ & (Grammatikos, 2016) \\
\hline PE-ILSS-T4-P3 & $\mathrm{PE}$ & $\mathrm{GL}$ & ILSS & $\mathrm{T} 4$ & P3 & 0 & $100 \%$ & (Grammatikos, 2016) \\
\hline PE-ILSS-T4-P3 & $\mathrm{PE}$ & GL & ILSS & $\mathrm{T} 4$ & P3 & 1 & $76 \%$ & (Grammatikos, 2016) \\
\hline PE-ILSS-T4-P3 & $\mathrm{PE}$ & GL & ILSS & T4 & P3 & 2 & $65 \%$ & (Grammatikos, 2016) \\
\hline PE-ILSS-T4-P3 & $\mathrm{PE}$ & GL & ILSS & $\mathrm{T} 4$ & P3 & 4 & $51 \%$ & (Grammatikos, 2016) \\
\hline PE-ILSS-T4-P3 & PE & $\mathrm{GL}$ & ILSS & T4 & P3 & 8 & $69 \%$ & (Grammatikos, 2016) \\
\hline
\end{tabular}

\begin{tabular}{|c|c|c|c|c|c|c|c|c|}
\hline \multicolumn{9}{|c|}{ PE-GL-FS:: Polyester - Glass FRP - Flexural Strength Testing Database } \\
\hline PE-FS-T2-P2 & PE & GL & FS & $\mathrm{T} 2$ & P2 & 0 & $100 \%$ & (Sonawala \& Spontak, 1996) \\
\hline PE-FS-T2-P2 & $\mathrm{PE}$ & GL & FS & $\mathrm{T} 2$ & P2 & 1 & $95 \%$ & (Sonawala \& Spontak, 1996) \\
\hline PE-FS-T2-P2 & $\mathrm{PE}$ & GL & FS & $\mathrm{T} 2$ & P2 & 2 & $87 \%$ & (Sonawala \& Spontak, 1996) \\
\hline PE-FS-T2-P2 & PE & GL & FS & $\mathrm{T} 2$ & P2 & 3 & $85 \%$ & (Sonawala \& Spontak, 1996) \\
\hline PE-FS-T2-P2 & $\mathrm{PE}$ & $\mathrm{GL}$ & FS & $\mathrm{T} 2$ & P2 & 4 & $81 \%$ & (Sonawala \& Spontak, 1996) \\
\hline PE-FS-T2-P2 & $\mathrm{PE}$ & $\mathrm{GL}$ & FS & $\mathrm{T} 2$ & P2 & 6 & $78 \%$ & (Sonawala \& Spontak, 1996) \\
\hline PE-FS-T2-P2 & $\mathrm{PE}$ & GL & FS & $\mathrm{T} 2$ & P2 & 9 & $70 \%$ & (Sonawala \& Spontak, 1996) \\
\hline PE-FS-T2-P5 & $\mathrm{PE}$ & GL & FS & $\mathrm{T} 2$ & P5 & 0 & $100 \%$ & (Sonawala \& Spontak, 1996) \\
\hline PE-FS-T2-P5 & PE & $\mathrm{GL}$ & FS & $\mathrm{T} 2$ & P5 & 1 & $80 \%$ & (Sonawala \& Spontak, 1996) \\
\hline PE-FS-T2-P5 & $\mathrm{PE}$ & $\mathrm{GL}$ & FS & $\mathrm{T} 2$ & P5 & 2 & $62 \%$ & (Sonawala \& Spontak, 1996) \\
\hline PE-FS-T2-P5 & $\mathrm{PE}$ & $\mathrm{GL}$ & FS & $\mathrm{T} 2$ & P5 & 3 & $50 \%$ & (Sonawala \& Spontak, 1996) \\
\hline PE-FS-T2-P5 & PE & $\mathrm{GL}$ & FS & $\mathrm{T} 2$ & P5 & 4 & $40 \%$ & (Sonawala \& Spontak, 1996) \\
\hline PE-FS-T2-P5 & PE & GL & FS & $\mathrm{T} 2$ & P5 & 6 & $29 \%$ & (Sonawala \& Spontak, 1996) \\
\hline PE-FS-T2-P5 & $\mathrm{PE}$ & $\mathrm{GL}$ & FS & $\mathrm{T} 2$ & P5 & 9 & $20 \%$ & (Sonawala \& Spontak, 1996) \\
\hline PE-FS-T2-P3 & $\mathrm{PE}$ & $\mathrm{GL}$ & FS & $\mathrm{T} 2$ & P3 & 0 & $100 \%$ & (Cabral-Fonseca, 2012) \\
\hline PE-FS-T2-P3 & PE & GL & FS & $\mathrm{T} 2$ & P3 & 3 & $100 \%$ & (Cabral-Fonseca, 2012) \\
\hline PE-FS-T2-P3 & $\mathrm{PE}$ & $\mathrm{GL}$ & FS & $\mathrm{T} 2$ & P3 & 6 & $95 \%$ & (Cabral-Fonseca, 2012) \\
\hline PE-FS-T2-P3 & PE & $\mathrm{GL}$ & FS & $\mathrm{T} 2$ & P3 & 9 & $96 \%$ & (Cabral-Fonseca, 2012) \\
\hline PE-FS-T2-P3 & PE & $\mathrm{GL}$ & FS & $\mathrm{T} 2$ & P3 & 12 & $94 \%$ & (Cabral-Fonseca, 2012) \\
\hline PE-FS-T2-P3 & PE & $\mathrm{GL}$ & FS & $\mathrm{T} 2$ & P3 & 18 & $96 \%$ & (Cabral-Fonseca, 2012) \\
\hline
\end{tabular}




\begin{tabular}{|c|c|c|c|c|c|c|c|c|}
\hline Sample Designation & Matrix & $\begin{array}{l}\text { Reinforce } \\
\text { ment }\end{array}$ & $\begin{array}{l}\text { Property } \\
\text { Tested }\end{array}$ & $\begin{array}{l}\text { Temp. } \\
\text { Group }\end{array}$ & \begin{tabular}{|l} 
pH \\
Groups
\end{tabular} & $\begin{array}{l}\text { Time in } \\
\text { Aging } \\
\text { Condition } \\
\text { (months) }\end{array}$ & $\begin{array}{l}\text { SR - \% } \\
\text { Strength } \\
\text { Reduction }\end{array}$ & Data Source Ref. \\
\hline PE-FS-T3-P3 & $\mathrm{PE}$ & $\mathrm{GL}$ & FS & T3 & P3 & 3 & $98 \%$ & (Cabral-Fonseca, 2012) \\
\hline PE-FS-T3-P3 & $\mathrm{PE}$ & $\mathrm{GL}$ & FS & T3 & P3 & 6 & $91 \%$ & (Cabral-Fonseca, 2012) \\
\hline PE-FS-T3-P3 & PE & $\mathrm{GL}$ & FS & T3 & P3 & 9 & $84 \%$ & (Cabral-Fonseca, 2012) \\
\hline PE-FS-T3-P3 & $\mathrm{PE}$ & $\mathrm{GL}$ & FS & T3 & P3 & 18 & $82 \%$ & (Cabral-Fonseca, 2012) \\
\hline PE-FS-T4-P3 & $\mathrm{PE}$ & $\mathrm{GL}$ & FS & T4 & P3 & 0 & $100 \%$ & (Cabral-Fonseca, 2012) \\
\hline PE-FS-T4-P3 & $\mathrm{PE}$ & $\mathrm{GL}$ & FS & T4 & P3 & 3 & $86 \%$ & (Cabral-Fonseca, 2012) \\
\hline PE-FS-T4-P3 & $\mathrm{PE}$ & $\mathrm{GL}$ & FS & T4 & P3 & 6 & $67 \%$ & (Cabral-Fonseca, 2012) \\
\hline PE-FS-T4-P3 & $P E$ & $\mathrm{GL}$ & FS & T4 & P3 & 9 & $68 \%$ & (Cabral-Fonseca, 2012) \\
\hline PE-FS-T4-P3 & $\mathrm{PE}$ & $\mathrm{GL}$ & FS & T4 & P3 & 12 & $63 \%$ & (Cabral-Fonseca, 2012) \\
\hline PE-FS-T2-P2 & $P E$ & $\mathrm{GL}$ & FS & $\mathrm{T} 2$ & P2 & 6 & $83 \%$ & (Cabral-Fonseca, 2012) \\
\hline PE-FS-T2-P2 & $\mathrm{PE}$ & $\mathrm{GL}$ & FS & $\mathrm{T} 2$ & P2 & 12 & $95 \%$ & (Cabral-Fonseca, 2012) \\
\hline PE-FS-T3-P2 & $\mathrm{PE}$ & $\mathrm{GL}$ & FS & T3 & P2 & 0 & $100 \%$ & (Cabral-Fonseca, 2012) \\
\hline PE-FS-T3-P2 & $\mathrm{PE}$ & $\mathrm{GL}$ & FS & T3 & P2 & 3 & $94 \%$ & (Cabral-Fonseca, 2012) \\
\hline PE-FS-T3-P2 & PE & $\mathrm{GL}$ & FS & T3 & P2 & 6 & $94 \%$ & (Cabral-Fonseca, 2012) \\
\hline PE-FS-T3-P2 & PE & $\mathrm{GL}$ & FS & T3 & P2 & 9 & $100 \%$ & (Cabral-Fonseca, 2012) \\
\hline PE-FS-T3-P2 & PE & $\mathrm{GL}$ & FS & T3 & P2 & 12 & $87 \%$ & (Cabral-Fonseca, 2012) \\
\hline PE-FS-T3-P2 & PE & $\mathrm{GL}$ & FS & T3 & P2 & 18 & $92 \%$ & (Cabral-Fonseca, 2012) \\
\hline PE-FS-T4-P2 & $\mathrm{PE}$ & $\mathrm{GL}$ & FS & T4 & P2 & 0 & $100 \%$ & (Cabral-Fonseca, 2012) \\
\hline PE-FS-T4-P2 & PE & $\mathrm{GL}$ & FS & T4 & P2 & 3 & $92 \%$ & (Cabral-Fonseca, 2012) \\
\hline PE-FS-T4-P2 & $\mathrm{PE}$ & $\mathrm{GL}$ & FS & T4 & P2 & 6 & $86 \%$ & (Cabral-Fonseca, 2012) \\
\hline
\end{tabular}




\begin{tabular}{|c|c|c|c|c|c|c|c|c|}
\hline Sample Designation & Matrix & $\begin{array}{l}\text { Reinforce } \\
\text { ment }\end{array}$ & $\begin{array}{l}\text { Property } \\
\text { Tested }\end{array}$ & $\begin{array}{l}\text { Temp. } \\
\text { Group }\end{array}$ & $\begin{array}{l}\text { pH } \\
\text { Groups }\end{array}$ & $\begin{array}{l}\text { Time in } \\
\text { Aging } \\
\text { Condition } \\
\text { (months) }\end{array}$ & $\begin{array}{l}\text { SR - \% } \\
\text { Strength } \\
\text { Reduction }\end{array}$ & Data Source Ref. \\
\hline PE-FS-T3-P3 & $\mathrm{PE}$ & $\mathrm{GL}$ & FS & T3 & P3 & 6 & $90 \%$ & (Cabral-Fonseca, 2012) \\
\hline PE-FS-T3-P3 & PE & $\mathrm{GL}$ & FS & T3 & P3 & 9 & $85 \%$ & (Cabral-Fonseca, 2012) \\
\hline PE-FS-T3-P3 & PE & $\mathrm{GL}$ & FS & T3 & P3 & 0 & $100 \%$ & (Cabral-Fonseca, 2012) \\
\hline PE-FS-T3-P3 & PE & GL & FS & T3 & P3 & 3 & $83 \%$ & (Cabral-fonseca, 2012) \\
\hline PE-FS-T3-P3 & PE & GL & FS & T3 & P3 & 6 & $83 \%$ & (Cabral-Fonseca, 2012) \\
\hline PE-FS-T3-P3 & $\mathrm{PE}$ & $\mathrm{GL}$ & FS & T3 & P3 & 9 & $74 \%$ & (Cabral-Fonseca, 2012) \\
\hline
\end{tabular}

\begin{tabular}{|c|c|c|c|c|c|c|c|c|}
\hline \multicolumn{9}{|c|}{ EN-GL-TS: Epoxy - Glass FRP - Tension Strength Testing Database } \\
\hline EN-TS-T2-P4 & EN & GL & TS & T2 & P4 & 0 & $100 \%$ & (Kajorncheappunngam, 1999) \\
\hline EN-TS-T2-P4 & EN & GL & TS & $\mathrm{T} 2$ & P4 & 2 & $84 \%$ & (Kajorncheappunngam, 1999) \\
\hline EN-TS-T2-P4 & EN & GL & TS & $\mathrm{T} 2$ & P4 & 4 & $68 \%$ & (Kajorncheappunngam, 1999) \\
\hline EN-TS-T2-P3 & EN & GL & TS & $\mathrm{T} 2$ & P3 & 0 & $100 \%$ & (Kajorncheappunngam, 1999) \\
\hline EN-TS-T2-P3 & EN & $\mathrm{GL}$ & TS & $\mathrm{T} 2$ & P3 & 2 & $84 \%$ & (Kajorncheappunngam, 1999) \\
\hline EN-TS-T2-P2 & EN & GL & TS & $\mathrm{T} 2$ & P2 & 0 & $100 \%$ & (Kajorncheappunngam, 1999) \\
\hline EN-TS-T2-P2 & EN & GL & TS & $\mathrm{T} 2$ & P2 & 2 & $74 \%$ & (Kajorncheappunngam, 1999) \\
\hline EN-TS-T2-P2 & EN & GL & TS & $\mathrm{T} 2$ & P2 & 4 & $74 \%$ & (Kajorncheappunngam, 1999) \\
\hline EN-TS-T3-P4 & EN & GL & TS & T3 & P4 & 0 & $100 \%$ & (Kajorncheappunngam, 1999) \\
\hline EN-TS-T3-P4 & EN & GL & TS & T3 & P4 & 2 & $74 \%$ & (Kajorncheappunngam, 1999) \\
\hline EN-TS-T3-P4 & EN & GL & TS & T3 & P4 & 4 & $70 \%$ & (Kajorncheappunngam, 1999) \\
\hline EN-TS-T3-P3 & EN & GL & TS & T3 & P3 & 0 & $100 \%$ & (Kajorncheappunngam, 1999) \\
\hline EN-TS-T3-P3 & EN & GL & TS & T3 & P3 & 2 & $92 \%$ & (Kajorncheappunngam, 1999) \\
\hline EN-TS-T3-P3 & EN & GL & TS & T3 & P3 & 4 & $92 \%$ & (Kajorncheappunngam, 1999) \\
\hline EN-TS-T3-P2 & EN & GL & TS & T3 & P2 & 0 & $100 \%$ & (Kajorncheappunngam, 1999) \\
\hline EN-TS-T3-P2 & EN & $\mathrm{GL}$ & TS & T3 & P2 & 1 & $81 \%$ & (Kajorncheappunngam, 1999) \\
\hline EN-TS-T3-P2 & EN & GL & TS & T3 & P2 & 3 & $96 \%$ & (Kajorncheappunngam, 1999) \\
\hline EN-TS-T2-P4 & EN & GL & TS & $\mathrm{T} 2$ & P4 & 0 & $100 \%$ & (Kajorncheappunngam, 1999) \\
\hline EN-TS-T2-P4 & EN & GL & TS & $\mathrm{T} 2$ & P4 & 1 & $76 \%$ & (Kajorncheappunngam, 1999) \\
\hline EN-TS-T2-P4 & EN & GL & TS & $\mathrm{T} 2$ & P4 & 3 & $81 \%$ & (Kajorncheappunngam, 1999) \\
\hline EN-TS-T2-P4 & EN & GL & TS & $\mathrm{T} 2$ & P4 & 5 & $72 \%$ & (Kajorncheappunngam, 1999) \\
\hline EN-TS-T2-P3 & EN & GL & TS & $\mathrm{T} 2$ & P3 & 0 & $100 \%$ & (Kajorncheappunngam, 1999) \\
\hline
\end{tabular}




\begin{tabular}{|c|c|c|c|c|c|c|c|c|}
\hline Sample Designation & Matrix & $\begin{array}{l}\text { Reinforce } \\
\text { ment }\end{array}$ & $\begin{array}{l}\text { Property } \\
\text { Tested }\end{array}$ & $\begin{array}{l}\text { Temp. } \\
\text { Group }\end{array}$ & \begin{tabular}{|l} 
pH \\
Groups
\end{tabular} & $\begin{array}{l}\text { Time in } \\
\text { Aging } \\
\text { Condition } \\
\text { (months) }\end{array}$ & \begin{tabular}{|l} 
SR - \% \\
Strength \\
Reduction
\end{tabular} & Data Source Ref. \\
\hline EN-TS-T2-P3 & EN & $\mathrm{GL}$ & TS & $\mathrm{T} 2$ & P3 & 2 & $81 \%$ & (Kajorncheappunngam, 1999) \\
\hline EN-TS-T2-P3 & EN & GL & TS & $\mathrm{T} 2$ & P3 & 4 & $76 \%$ & (Kajorncheappunngam, 1999) \\
\hline EN-TS-T2-P2 & EN & GL & TS & $\mathrm{T} 2$ & P2 & 0 & $100 \%$ & (Kajorncheappunngam, 1999) \\
\hline EN-TS-T2-P2 & EN & GL & TS & $\mathrm{T} 2$ & P2 & 1 & $50 \%$ & (Kajorncheappunngam, 1999) \\
\hline EN-TS-T2-P2 & EN & $\mathrm{GL}$ & TS & $\mathrm{T} 2$ & P2 & 3 & $31 \%$ & (Kajorncheappunngam, 1999) \\
\hline EN-TS-T2-P2 & EN & $\mathrm{GL}$ & TS & $\mathrm{T} 2$ & P2 & 5 & $26 \%$ & (Kajorncheappunngam, 1999) \\
\hline EN-TS-T3-P4 & EN & GL & TS & T3 & P4 & 0 & $100 \%$ & (Kajorncheappunngam, 1999) \\
\hline EN-TS-T3-P4 & EN & GL & TS & T3 & P4 & 1 & $47 \%$ & (Kajorncheappunngam, 1999) \\
\hline EN-TS-T3-P4 & EN & $\mathrm{GL}$ & TS & T3 & P4 & 3 & $27 \%$ & (Kajorncheappunngam, 1999) \\
\hline EN-TS-T3-P4 & EN & $\mathrm{GL}$ & TS & T3 & P4 & 5 & $27 \%$ & (Kajorncheappunngam, 1999) \\
\hline EN-TS-T3-P3 & EN & GL & TS & T3 & P3 & 0 & $100 \%$ & (Kajorncheappunngam, 1999) \\
\hline EN-TS-T3-P3 & EN & $\mathrm{GL}$ & TS & T3 & P3 & 1 & $51 \%$ & (Kajorncheappunngam, 1999) \\
\hline EN-TS-T3-P3 & EN & GL & TS & T3 & P3 & 3 & $53 \%$ & (Kajorncheappunngam, 1999) \\
\hline EN-TS-T3-P3 & EN & GL & TS & T3 & P3 & 5 & $52 \%$ & (Kajorncheappunngam, 1999) \\
\hline EN-TS-T3-P2 & EN & GL & TS & T3 & P2 & 0 & $100 \%$ & (Kajorncheappunngam, 1999) \\
\hline EN-TS-T3-P2 & EN & $\mathrm{GL}$ & TS & T3 & P2 & 1 & $64 \%$ & (Kajorncheappunngam, 1999) \\
\hline EN-TS-T3-P2 & EN & GL & TS & T3 & P2 & 3 & $65 \%$ & (Kajorncheappunngam, 1999) \\
\hline EN-TS-T3-P2 & EN & GL & TS & T3 & P2 & 5 & $52 \%$ & (Kajorncheappunngam, 1999) \\
\hline EN-TS-T2-P5 & $\overline{E N}$ & $\overline{\mathrm{GL}}$ & TS & $\mathrm{T2}$ & $\overline{P 5}$ & $\overline{0}$ & $100 \%$ & (Shi et. al., 2011) \\
\hline EN-TS-T2-P5 & EN & $\mathrm{GL}$ & TS & $\mathrm{T} 2$ & P5 & 1 & $57 \%$ & (Shi et. al., 2011) \\
\hline EN-TS-T2-P5 & EN & GL & TS & $\mathrm{T} 2$ & P5 & 2 & $56 \%$ & (Shi et. al., 2011) \\
\hline EN-TS-T2-P5 & EN & $\mathrm{GL}$ & TS & $\mathrm{T} 2$ & P5 & 3 & $55 \%$ & (Shi et. al., 2011) \\
\hline EN-TS-T3-P5 & EN & GL & TS & T3 & P5 & 0 & $100 \%$ & (Shi et. al., 2011) \\
\hline EN-TS-T3-P5 & EN & GL & TS & T3 & P5 & 1 & $44 \%$ & (Shi et. al., 2011) \\
\hline EN-TS-T3-P5 & EN & $\mathrm{GL}$ & TS & T3 & P5 & 2 & $31 \%$ & (Shi et. al., 2011) \\
\hline EN-TS-T3-P5 & EN & $\mathrm{GL}$ & TS & T3 & P5 & 3 & $26 \%$ & (Shiet. al., 2011) \\
\hline
\end{tabular}

